UCRL--94820

DE86 012819

\title{
Atlas of Photoneutron Cross Sections \\ obtained with Monoenergecic Photons
}

Final Edition, 1986

\author{
S. S. Dietrich \\ Lawrence Livermore National Laboratory \\ University of California \\ Livermore, California 94550 \\ B. L. Berman \\ Department of Physics \\ The George Washington University \\ Washington, D. C. 20052
}

June 1986

\section{Abstract}

Photoneutron cross-section and integrated cross-section data obtained with monoenergetic photons are presented in a uniform format. This compilation is current of as Jume 1986. 


\section{DISCLAIMER}

This report was prepared as an account of work sponsored by an agenty of the United Staics Government. Neither the United States Government nor any agency thereof, nor any of their employees, makes any warranty, expross or implied, or assumes any legal liability or responsibility for the accuracy, completeness, or usefulows of any aformation, apparatus, product, of provess disclosed, or represents that ius use would not infringe privately owned rights Refesence herein to any specilic commescial product, process, or servix by trade name, trademark, manufacturer, or otherwise dow not necesearily constitute or imply its endotsement, recommendation, or favoring by the United States Goverament or any ageney theicof. The views and opinions of authors expressed herein do -tot nesessarily state or reflact those of the United States Government or any agency thereof.

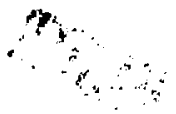




\section{Introduction}

Over the years, a considerable boay of data on photoneutron cross sections obtained with monoenergetic photons has been acquired at the Livermore, Saclay, and several other laboratories. In view of the need for a comprehensive compilation of photoneutron cross-section data, these monoenergetic-photon data are gathered together here and presented in a uniform format. This compilation updates and supersedes the earlier editions of this Atlas.' A more complete compilation (which includes data obtained with continuous bremsstrahlung radiation sources as well), is being assembled by the photonuclear group at the National Bureau of Standards.2 A critique of monoenergetic-photon tecnniques and the data obtained therefrom is included in Ref. 3. The data, here made available in graphical form, also are available upon request in digital form for purposes of theoretical analysis and for those asscciated with applications to other scientific disciplines and technologies.

\section{The Annihilation-Photon Technique}

The experimental methods for producing beams of monoenergetic photons which have been used for photonuclear-reaction stuoies include the annihilation in tlight of fast positrons, tagged bremsstrahlung, and nuclear $\gamma$-rays from the capture of protons or neutrons. Since most of the work surveyed here has been oone with annihilation photons, a brief description of this method is given.

The annihilation-photon method for obtaining a monoenergetic photon beam was first suggested by $\mathrm{Tzara}^{4}$ and has been developed or studied at several laboratories since, principally at Saclay and at Livermore. The method consists of producing positrons by pair production, transporting a beam of fast positrons having the desired energy. $E_{e+}$ to the experimental area, and allowing it to strike a thin, low- $Z$ target, thus producing, in the forward direction, a monoenergetic beam of annihilation photons (where the photon energy $E_{Y}=E_{e+}+0.76 \mathrm{MeV}$, the latter term being $3 / 4$ of the rest mass of the annihilating pair). 
Figure $A$ is a schematic experimental diagran fllustrating the tecinnique. Positrons are produced from the bremsstrahlung created when the intense beam of high-energy electrons from the first section of a high-current 1 inac strikes a thick, high- $Z$ converter. The energent positrons are captured into the second section, with the radio frequency $180^{\circ}$ out of phase with that normally used for electrons, and are accelerated to the desired energy. The positron beam is energy-analyzed to the desired momentum resolution with a bending magnet and slit, is transported to the experimental area, and is focused onto the thin, low- $Z$ annihilation target. Some of the positrons annihilate in flight, producing the monoenergetic photon beam; some produce brensstrahlung, whose effects must be subtracted out by repeating the measurement with electrons instead of positrons; and the rest are swept away from the photon bean $l$ ine and into a dunip hole by a magnet. The collimated photon beam passes through a transmission ion chamber beam monitor and strikes the nuclear sar.ple under study.

This photoneutron sample is located in the center of a highly efficient $4 \pi$ neutron detector, whose electronic scaling circuitry is triggered after each accelerator beam burst. The rumber of neutrons detected for each beam burst is recorded, and this "multiplicity-counting" system, together with a knowledge of the neutron detector efficiency, allows one to obtain the single, double, triple, etc., photoneutron cross sections simultaneously and independently. This is essential if one is to obtain directly the total photoneutron cross section, which is nearly the same as the total photonuclear absorption cross section for medium and heavy nuclei, where proton (and other charged-particle) emission at giant-resonance energies is inhibited by the Coulomb barrier.

The ion chamber photon beam monitor is calibrated against a NaI ( $T 1$ ) photon spectrometer whose response functions and absolute efficiency are measured separately. The energy resolution of the photan beam is determineo primarily by the monentum spread of the positron beam, together with the $d E / d x$ losses suffered by the positrons before annihilation, which result from the finite thickness of the annihilation target. The energy scale is set with reference to known nuclear energy levels ano thresholds. Figure $A$ also shows sample HaI photon spectra for incident positrons, incident electrons, and their difference. 


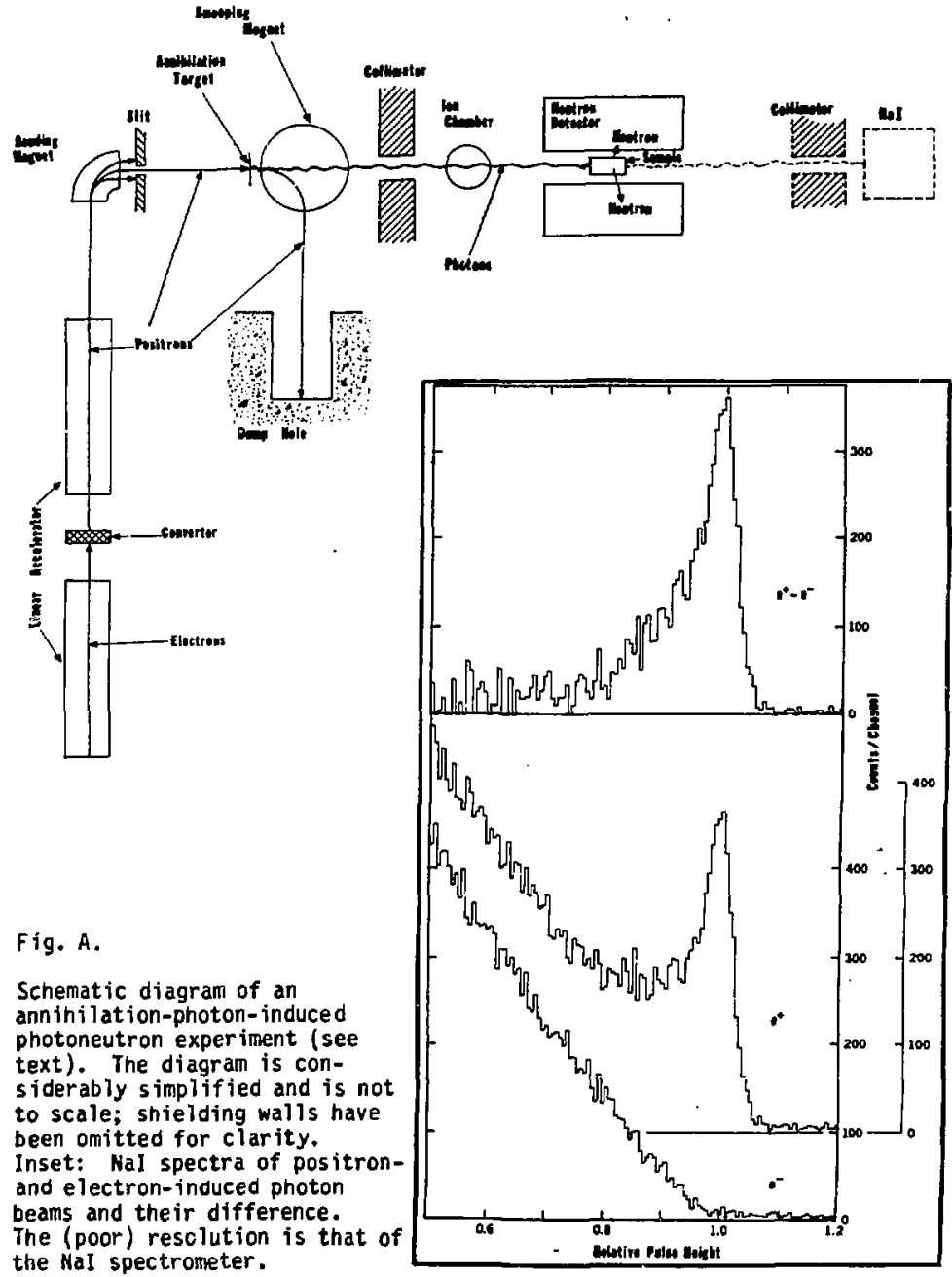




\section{Data}

The data are presented graphically, generally in arder of increasing mass number. Figure $B$ (p. 27), in the form of a chart of the stable (or long-lived) nuclei, constitutes an index of the isotopes studied to date with monoenergetic photons. When two or more laboratories have measured the same nucleus, the data are presented in chronological order. The top data plot for each nucleus is the photoneutron yield cross section $o[(\gamma, n]+(\gamma, p n)+2(\gamma, 2 n)+2(\gamma, p 2 n)+3(\gamma, 3 n)+\ldots]$; below it are the total photoneutron cross section $\sigma[(\gamma, n)+(\gamma, p n)+(\gamma, 2 n)+(\gamma, p 2 n)+(\gamma, 3 n)+\ldots]$. the single photoneutron cross section $\sigma[(\gamma, n)+(\gamma, p n)]$, the double photoneutron cross section $o[(\gamma, 2 n)+(\gamma, p 2 n)]$, and the triple photoneutron cross section $c(\gamma, 3 \pi)$. [Sometimes another cross section is substituted for $o(\gamma, 3 n)$-- see Explanation of the Grapis.] Most of the total photoneutron data plots for medium and heavy nuclei have Lorentz-curve $f i t s$ superposed.

A table of properties of the giant dipole resonance derived from measurements made with monoenergetic photon beams also is included. Integrated cross sections and their monents, derived directly from the data, constitute about half the table, and the parameters of Lorentz curves fitted to the giant-resonance data the other half. Numerous quantities of physical interest can be derived from these integrated cross sections and Lorentz parameters; a detailed exposition of these is given in Ref. 3 . The . "Reference" and "Laboratory" columns constitute an index to the monoenergetic-photon-data references. The references to the tables (through 1982) follow the notation of Ref, 2.

In the semiclassical theory of the interaction of photons with nuclei, the shape of a fundamental resonance in the absorption cross section is that 0 : the Lorentz curve $:^{5,6}$

$$
O(E)=\frac{\sigma_{m}}{1+\left[\left(E^{2}-E_{m}^{2}\right)^{2} / E^{2} r^{2}\right]}
$$


where the Lorentz parameters $E_{m}, \sigma_{m}$, and $r$ are the resonance energy, peak cross section, and full width at ha!f-maximum, respectively. In the hydrodymamic theory of photonuclear reactions, the giant dipole resonance consists of one such Lorentz line for spherical nuclei, ${ }^{5,7}$ corresponding to the absorption of photons which induce oscillations of the neutron ano proton fluids in the nucleus against each other, and the superposition of two such 1 ines for statically deforwed spheroidal nuclei, ${ }^{6,8}$ corresporiding to oscillations along each of the nondegenerate axes of the spheroid. (The lower-energy line corresponds to oscillations along the longer axis and the higher-energy line along the shorter, since the absorption frequency decreases with increasing nuclear dimensions.)

$$
O(E)=\sum_{i=?}^{2} \frac{\sigma_{m i}}{1+\left[\left(E^{2}-E_{m i}^{2}\right)^{2} / E^{2} I_{i}^{2} j\right.}
$$

where $i=1,2$ corresponds to the lower- and higher-energy lines. Note that the two Lorentz lines are noninterfering and that the damping parameters $I_{i}$ are not energy-dependent.

The Thomas-Reiche-Kuhn (TRK) sum rule ${ }^{9}$ is an expression giving the total integrated cross section for electric-dipole photon absorption, in the absence of exchange forces, and is given by:

$$
\int_{0}^{\infty} c(E) d E=\frac{2 \pi^{2} e^{2} h}{M C} \frac{N Z}{A}=60\left(\frac{N Z}{A}\right) M e V-m b
$$

where $M$ is the nucleon mass and the integration is over all energies for which dipole absorption can occur. It should be noted that the area under the Lorentz curve (from Eq. 1 or 2) is given by:

$$
\int_{0}^{\infty} O(E) d E=\frac{\pi}{2} c_{m} \Gamma\left(O r \frac{\pi}{2} \sum_{i=7}^{2} o_{m i} \Gamma_{i}\right) .
$$


The first moment of the integrated cross section, also known as the "bremsstrahlung-weighted" cross section (since the energy dependence of the bremsstrahlung spectrum of ten is spproxtmated by $1 / E$ ), is given by:

$$
a-1=\int_{0}^{\infty}[O(E) / E] d E=\frac{4 \pi^{2}}{3} \frac{e^{2}}{h c} \frac{H Z}{A-1}\left\langle r^{2}\right\rangle
$$

for light nuclei, ${ }^{9}$ where $\left\langle r^{2}\right\rangle$ is the mean-square radius of the nuclear charge distribution.

The second moment of the integrated cross section $a_{-2}$ is proportional to the nuclear polarizability $p=\left(e^{2} R^{2} A / 40 K\right)^{-2}$ and hence increases with the diffuseness of the nucleus. Here $R$ is the radius of the equivalent spherical nucleus and $K$ is the nuclear symmetry energy. Then the Migal sum rule 9,10 states that:

$$
0_{-2}=\int_{0}^{\alpha}\left[c(E) / E^{2}\right] d E=\frac{2 \pi^{2}}{h c} p=\frac{n^{2}}{20} \frac{e^{2}}{h c} \frac{R^{2} A}{k} .
$$

\section{Higher-Energy Data}

A few photoneutron cross-section measurements with monoenergetic photons have been performed at energies substantially above the giant dipole resonance, notably at Saclay. These are not presented here; one should refer to the original data references. ${ }^{17-13}$ Some additional implications of these higher-energy data are discussed in Refs. 14 and 15 as well. 


\section{Policy}

No attempt is made here to evaluate the data; thai is, to choose tetween two sets of data for the same nucleus measured at different laboratories or to compromise between them by presenting a set of reconmended intermediate values. It should be noted, however, that the overall agreement and consistency between measurements made with monoenergetic photons are very good indeed; when they are not, one usually (but not always) is best advised to weigh more recent data more heavily than earlier data.

\section{Notes on References}

When data from this Atlas are cited, reference should be made to the original publication as well.

\section{Acknowledgments}

Thanks are due to D. R. Bergère, Dr. R. E. Sund, Dr. U. Kneissel, ainci the late Professor $P$. Axel for making available the data from their laboratories.

This work was performed under the auspices of the U. S. Department of Eneryy at the Lawrence Livermore National Laboratory under contract number W-7405-Eng-48.

$0802 A-6 / 9 / 86$ 
1. B. L. Berman, At las of Photoneutron Cross Sections Obtained with Monoenergetic Photons: Atomi Data and Nuclear Tables T5, 319 (1975); Bicentennial Edition, University of California, Lawrence Livermore Natjonal Laboratory, Report No. UCRL-78482 (1976); Supplement (19/9).

2. E. G. Fuller and H. H. Gerstenberg. National Bureau of Standards, Report No. NBSIR 83-2742 (1983-86).

3. B. L. Berman and S. C. Fuitz, Rev, Mod. Phys. 47, 713 (1975).

4. C. Tzara, Compt, Rend. 56, 245 (1957).

5. H. Steinwedel and J. H, O, J'nsen, 2. Haturforsch, $\underline{5 a}, 413$ (1950).

6. M. Danos, Nuc1. Phys. 5,23 (1958).

7. H. Goldhaber and E. Teller, Phys, Rev. 74. 1046 (1948).

8. K. Okamoto, Progr. Theoret. Phys. (Kyoto) 15, 75L (1956).

9. J. 5. Levinger, Nuclear Photo-Disintegration, Oxford University Press, London (1960).

10. A. B. Migdal, A. '. Lushnikov, and D. F. Zaretsky, Nucl. Phys. 66, 193 $(1965)$.

11. A. Leprètre, H. Beil, R. Bergère, P. Carlos, J. Fagot, A. óeMiniac, ano A. Veyssière, Nuc1. Phys. A367, 237 (1987).

12. P. Carlos, H. Beil, R. Bergère, B. L, Berman, A. Lepretre and A. Veyssière, Nuc?. Phys. A379, 317 (1982).

13. A. Yeyssière, H. Beî, R. Bergère, P. Carlos, J. Fagot, A. Leprêtre, and $A$. deminiac, $Z$. Phys. A 3C5, 139 (7982).

14. B. L. Berman, R. Bergère, and P. Carlos, Phys, Rev. C 26, 304 (198c).

15. M. Blann, B. L. Berman, and T. T. Konoto, Phys. Rev. C 28, 2286 (1983). 


\section{Extizanation of the Table}

$E_{\gamma \max } \quad$ The upper-energy limit of the data

$\sigma_{\text {int }}(1, \mathrm{Sn}) \quad$ The integrated phutoneutron yield cross section:

$\sigma_{i n t}(\gamma, j n)=\int_{E_{t h r}^{\prime}(\gamma, n)}^{E_{\gamma \max }} \sigma(\gamma, S n) d:_{\gamma}$

where $E_{t h r}(Y, n)$ is the photoneutron threshold energy,

$\sigma(\gamma, S n)=\sigma(\gamma$, tot $)+o(\gamma, 2 n)+2 c(\gamma, 3 n j+(\nu-1) o(\gamma, f)$,

$o(\gamma, t, c t)=\sigma(\gamma, i n)+\sigma(\gamma, 2 n)+\sigma(\gamma, 3 n)+c(\gamma, f)$,

$\sigma(\gamma, l n) \cdot(\gamma, n)+\sigma(\gamma, p n)$,

$c(\gamma, 2 \pi)=c(\gamma, 2 n)+c(\gamma, p 2 n)$,

$\sigma(\gamma, f)=c(\gamma, F)+\sigma(\gamma, n F)$, and

$\checkmark$ is the average prompt photofission neutron multiplicity.

$\sigma_{\text {int }}(\gamma$, tot $)$ The integrated total photoneutron cross section:

$\sigma_{\text {int }}(\gamma, t \sigma c)=\int_{E_{t h r}(\gamma, n)}^{E_{\gamma \max }} \sigma(\gamma, t o t) d E_{\gamma}$

$\sigma_{\text {int }}(\gamma$, in $) \quad$ The integrated single photoneutron cross section:

$\sigma_{i n c}(\gamma, l n)=\int_{E_{t h r}(\gamma, n)}^{E_{\gamma \max }} \sigma(\gamma, \ln ) d E_{\gamma}$ 
$0_{\text {int }}(\gamma, 2 n)$ The integrated double photoneutron cross section:

$$
o_{i n t}(\gamma, 2 n)=\int_{E_{t h r}(\gamma, 2 n)}^{E_{\gamma \max }} \sigma(\gamma, 2 n) d E_{\gamma}
$$

where $E_{\text {the }}(\gamma, 2 n)$ is the threshold energy for the photoejection of two neutrons

${ }^{0}{ }_{\text {int }}(r, 3 n) \quad$ The integrated triple photoneutron cross section:

$$
o_{i n t}(\gamma, 3 n)+\int_{E_{t h r}(\gamma, 3 n)}^{E} o_{\max } o(\gamma, 3 n) d E_{\gamma}
$$

where $E_{\text {the }}\left(\gamma_{2} 3 n\right)$ is the threshold energy for the photoejection of three neutrons. For ${ }^{26} \hat{G},{ }^{32} \mathrm{P},{ }^{32} \mathrm{~S}$. and ${ }^{39} \mathrm{~K}, \mathrm{o}_{\text {int }}(\gamma, \mathrm{pn})$ appears in this column; for ${ }^{18} 0, c_{\text {int }}(\gamma, p)$ appears here; and for the actinide nuclei:

$$
\sigma_{i n t}(\gamma, f)=\int_{B_{F}}^{E_{\gamma} \max } o(\gamma, f) d E_{\gamma}
$$

where $c(\gamma, f)$ is the sum of the first- and secono-chance photofission cross sections o $(\gamma, F)$ and $c(\gamma, n F)$, respective lg, and $B_{F}$ is the energy of the photofission barrier

$$
\text { ci }
$$

The first moment of the integrated total photoneutron cross section:

$$
\sigma_{-1}=\int_{E_{t h r}(\gamma, n)}^{E_{\gamma \max }}\left[\sigma(\gamma, t o t) / E_{\gamma}\right] d E_{\gamma}
$$

$-10-$ 
The second moment of the integrated total photoneutron cross section:

$\sigma_{-2}=\int_{E_{t h r}(\gamma, n)}^{E_{\gamma} \max }\left[(\gamma, t o t) / E_{\gamma}^{2}\right] d E_{\gamma}$

Fitting The photon energy interval over which the Lorentz-curve Interval fits to the data were inade

$E_{m i}, O_{m i}, I_{i} \quad$ The parameters of the Lorentz curves fitted to the data within the fitting interval given in the preceding calumn. The Lorentz curves are of the form:

$o\left(E_{\gamma}\right)=\sum_{i} o_{m i} /\left\{1+\left[\left(E_{\gamma}^{2}-E_{m i}^{2}\right)^{2} / E_{\gamma}^{2} \Gamma_{i}^{2}\right]\right\} \quad ;$

for spherical nuclei, $i=1$; for deformed nuclei, $i=1,2$. Here "spherical" merely indicates that a one-component Lorentz curve gives a better fit to the data than a two-component one, and "deformed" indicates the opposite. The fitting procedure used is the standard bestileast-squares method, whereir the squares of the residuals are weighted with the inverse squares of the statistical uncertainties.

Feference The data references follow the table.

Laboratory The laboratory at which the data were obtained:

L - Livermore

S - Saclay

G - GiEssen

GA - General Atomic

I - Illinois

P - Pennsylvania 


\begin{tabular}{|c|c|c|c|c|c|c|c|c|c|c|c|c|c|c|c|c|c|c|}
\hline \multirow[b]{2}{*}{ Mucleus } & \multirow{2}{*}{\begin{tabular}{|l|}
$E_{Y \max }$ \\
$(\mathrm{MeV})$
\end{tabular}} & \multirow{2}{*}{ 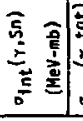 } & \multirow{2}{*}{ 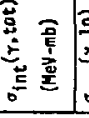 } & \multirow{2}{*}{ 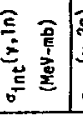 } & \multirow{2}{*}{ 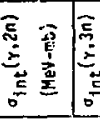 } & \multirow{2}{*}{ 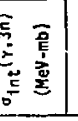 } & \multirow{2}{*}{ 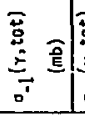 } & \multirow{2}{*}{ 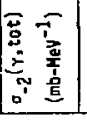 } & \multirow[b]{2}{*}{$\begin{array}{c}\text { Fitting } \\
\text { Intervof } \\
(\mathrm{HeV})\end{array}$} & \multicolumn{6}{|c|}{ Lorentz Porameters } & \multirow{2}{*}{$\stackrel{\mathrm{z}}{\mathrm{z}}$} & \multirow{2}{*}{ 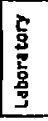 } & \multirow{2}{*}{ 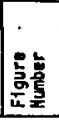 } \\
\hline & & & & & & & & & & $\begin{array}{c}E_{m 1} \\
(\mathrm{MeV})\end{array}$ & $\begin{array}{c}a_{m x} \\
\text { (mb) }\end{array}$ & $\begin{array}{c}\mathbf{r}_{1} \\
(\mathrm{HeV})\end{array}$ & $\begin{array}{c}E_{m_{2}} \\
(\mathrm{MeV})\end{array}$ & $\begin{array}{c}\sigma_{m_{2}} \\
(m b)\end{array}$ & $\begin{array}{c}r_{2} \\
(M E V)\end{array}$ & & & \\
\hline${ }^{3} \mathrm{H}$ & 23.4 & 32.3 & 22.0 & 11.7 & $10.2^{h}$ & & 1.48 & 0.11 & & & & & & & & 8ifal & $\mathbf{L}$ & 1 \\
\hline${ }^{3} \mathrm{He}$ & 30.2 & 13.0 & 13.0 & 13.0 & & & 0.77 & 0.05 & & & & & . & & & 74BeB & $\mathbf{L}$ & i \\
\hline 'He & 25.8 & 14.1 & 14.7 & 14.1 & & & 0.83 & 0.05 & & & & & & & & BlFal & $\mathbf{L}$ & 3 \\
\hline "He & 31.4 & 1.9 & 1.9 & 7.9 & 0 & & 0.30 & 0.01 & & & & & & & & illes & l & $n$ \\
\hline "He & 47.3 & 23.5 & 23.5 & 23.5 & & & 0.73 & 0.02 & & & & & & & & AOBEI & $\mathbf{L}$ & 5 \\
\hline 'L & 32.0 & 28.2 & 27.8 & 27.4 & 0.4 & & 1.91 & 0.16 & & & & & & & & o5gel & $\mathbf{L}$ & $i$ \\
\hline 'Li & 30.5 & 30.2 & 20.1 & 10.0 & 10.1 & & 1.15 & 0.07 & & & & & & & & $738 \mathrm{r} 11$ & $\mathbf{L}$ & 7 \\
\hline "Be & 37.0 & 83.7 & 58.1 & 32.5 & 25.6 & & 2.52 & 0.13 & & & & & & & & $75 \mathrm{Kn} 5$ & G & $b$ \\
\hline 198 & 35.1 & 84.3 & 82.3 & 80.4 & 1.9 & & 3.69 & 0.18 & & & & & · & & : & $76 \mathrm{Kn} 3$ & $\mathbf{G}$ & 9 \\
\hline $11_{B}$ & 35.1 & 84.1 & 69.2 & 54.3 & 14.9 & & 2.93 & 0.13 & & & & & & & & $76 \mathrm{Kn} 3$ & $G$ & in \\
\hline${ }^{12} \mathrm{C}$ & 26.7 & 34.5 & 34.5 & 34.5 & & & 1.47 & 0.063 & & & & & & & & $\left\{\begin{array}{l}06101 \\
6\end{array}\right.$ & $P$ & 11 \\
\hline${ }^{12} \mathrm{C}$ & 25.5 & 29.4 & 29.4 & 29.4 & & & & & & & & & & & & $66 \mathrm{Mi} 2$ & $s$ & \\
\hline${ }^{12} \mathrm{C}$ & 37.4 & 46.8 & 46.8 & 46.8 & 0 & & 1.83 & 0.073 & & & & & & & & $66 \mathrm{Ful}$ & $\iota$ & 12 \\
\hline${ }^{12} \mathrm{C}$ & 32.1 & 51.6 & $51.6^{\mathrm{B}}$ & & & & $2.08^{\circ}$ & $0.085^{a}$ & & & & & & & & $75 \mathrm{kn} 8$ & $G$ & ذا \\
\hline${ }^{13} \mathrm{C}$ & 41.8 & 131 & 126 & 121 & 4.7 & & 5.72 & 0.306 & & & & & & & & 79uus & $L$ & 14 \\
\hline 1 "C & 36.2 & 163 & 126 & 89.7 & 36.6 & & 6.09 & 0.322 & & & & & & & $\cdot$ & 65Pyl & $\mathrm{L}$ & is \\
\hline $1 " \mathrm{H}$ & 29.5 & 98 & 98 & 98 & & & 4.36 & 0.20 & & & & . & & & & $70 \mathrm{Be} 8$ & $L$ & 16 \\
\hline $1 s_{\mathrm{N}}$ & 38.0 & 114 & 106 & 98.8 & 7.6 & & 4.65 & 0.218 & & & & & & & & b2Jü & $\mathbf{l}$ & 17 \\
\hline $1 \% 0$ & 26.5 & 41.5 & 41.5 & 41.5 & & & & & & & & & & & & $\begin{array}{r}66 M 12 \\
\text { fo48r }\end{array}$ & 5 & \\
\hline 10 & 28.0 & 41.5 & 41.5 & 41.5 & & & 1.76 & 0.075 & & & & & & & & Gofcai & $\mathbf{L}$ & 16 \\
\hline 10 & 37.1 & 80.1 & 79.6 & 78.9 & 0.6 & $11^{p}$ & 3.04 & 0.119 & & & & & & & & 7avel & 5 & 19 \\
\hline 10 & 37.0 & 62.9 & 62.9 & 62.9 & 0 & $\because \cdot$ & 2.40 & 0.094 & & & & & & & & $75 \mathrm{KnB}$ & $G$ & $<0$ \\
\hline $1 \times 0$ & 33.1 & 61.5 & 61.5 & 61.5 & & & 2.51 & 0.105 & & & & & & & & 83Bel & $\mathbf{L}$ & $i 1$ \\
\hline${ }^{120}$ & 39.7 & 128 & 118 & 109 & 9.3 & & 5.22 & 0.266 & & & & & & & & 80งu4 & $\mathbf{L}$ & $2 L$ \\
\hline 10 & 41.8 & 275 & 198 & 121 & 77 & $44^{9}$ & 9.08 & 0.483 & & & & & & & & 79Hol & $\mathbf{L}$ & is \\
\hline 10 & 33.0 & 191 & 142 & 93 & 49 & & 6.90 & 0.38 & & & & & & & & $76 \mathrm{Kn} 4$ & G & $24-$ \\
\hline $1 P_{F}$ & 27.8 & 109 & 104 & 99 & 5.2 & & 4.95 & 0.25 & & & & & & & & 74Ve 1 & $s$ & 23 \\
\hline
\end{tabular}




\begin{tabular}{|c|c|c|c|c|c|c|c|c|c|c|c|c|c|c|c|c|c|c|}
\hline \multirow[b]{2}{*}{ Hocleus } & \multirow[b]{2}{*}{$\left|\begin{array}{l}E_{y} \max \\
(\operatorname{Ma} 2)\end{array}\right|$} & \multirow{2}{*}{ 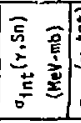 } & \multirow{2}{*}{ 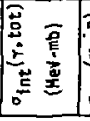 } & \multirow{2}{*}{ 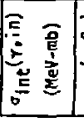 } & \multirow{2}{*}{ 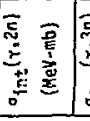 } & \multirow{2}{*}{ 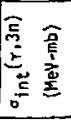 } & \multirow{2}{*}{ 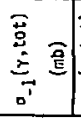 } & \multirow{2}{*}{ 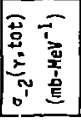 } & \multirow[b]{2}{*}{$\begin{array}{c}\text { Getting } \\
\text { 1nterval } \\
(\text { Mer) } \\
\end{array}$} & \multicolumn{6}{|c|}{ Lorentz Parameters } & \multirow{2}{*}{ 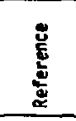 } & \multirow{2}{*}{ 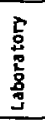 } & \multirow[b]{2}{*}{ 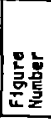 } \\
\hline & & & & & & & & & & $\begin{array}{c}\varepsilon_{\mathrm{m}_{1}} \\
(\mathrm{MeV})\end{array}$ & $\begin{array}{c}v_{\mathrm{ma}} \\
(\mathrm{mb})\end{array}$ & $\begin{array}{c}r_{1} \\
(\mathrm{MeV}) \\
\end{array}$ & $\begin{array}{c}E_{m_{2}} \\
(\mathrm{MeV})\end{array}$ & $\begin{array}{c}a_{\mathrm{m}_{2}} \\
(\mathrm{mb})\end{array}$ & $\begin{array}{c}r_{2} \\
(\mathrm{MEV})\end{array}$ & & & \\
\hline Nat $_{\text {He }}$ & 25.9 & 46 & 43 & 40 & 3.1 & & 1.98 & 0.09 & & & & & & & & Iavel & 5 & $2 b$ \\
\hline $2 \mathrm{Mz}$ & 27.1 & 120 & 119 & 118 & 0.6 & & 5.74 & 0.29 & & & & & & & & 71911 & l & 27 \\
\hline${ }^{3} \mathrm{JMa}$ & 30.1 & 139 & 136 & 133 & 2.7 & & 6.18 & 0.29 & & & & & . & & & 7avel & $s$ & 28 \\
\hline Nat $_{\text {Hg }}$ & 26.0 & 58 & $58^{\circ}$ & & $\ldots$ & & & & & & & & & & & ธ64і2 & $s$ & \\
\hline mat $_{M g}$ & 28.0 & 75.6 & 75.6 & 75.6 & & & & & & & & & & & & $21 F u 2$ & l & \\
\hline $24 \mathrm{Mg}$ & 28.3 & 51.9 & 51.9 & 51.9 & & & $2.3 ?$ & 0.11 & & & & & & & & $71 F u 2$ & $\mathbf{L}$ & 29 \\
\hline${ }^{25} \mathrm{Mg}$ & 28.9 & 248 & 247 & 245 & 1.5 & & 11.5 & 0.56 & & & & & & & & .1911 & $\mathrm{l}$ & jo \\
\hline $2 \varepsilon_{\mathrm{Mg}}$ & 28.6 & 308 & 236 & 164 & 72 & & 11.5 & 0.59 & & & & & & & & $21 \mathrm{Fu}$ & $\mathrm{l}$ & jו \\
\hline${ }^{27} \mathrm{AI}$ & 36.7 & 175 & 167 & 159 & 7.6 & & 7.17 & 0.32 & & & & & & & & 6oFul & l & $\dot{x}$ \\
\hline $27_{A 1}$ & 30.3 & 152 & 151 & 150 & 0.6 & & 6.77 & 0.31 & & & & & & & & 74rel & 5 & 33 \\
\hline $\mathrm{Nat}_{\mathrm{SH}}$ & 30.0 & 95 & 95 & 95 & & & 4.25 & 0.19 & & & & & & & & iavel & $s$ & 34 \\
\hline$z=5 i$ & 321 & 105 & 105 & 105 & 0 & & 4.46 & 0.20 & & & & & & & & 83Py1 & $\mathbf{L}$ & 35 \\
\hline$=31$ & 33.1 & 199 & 199 & 199 & 0 & & 9.66 & 0.51 & & & & & & & & 83Py1 & L & $x$ \\
\hline .051 & 33.1 & 316 & 249 & 181 & 67 & & 12.1 & 0.64 & & & & & & & & 83Pyl & L & 37 \\
\hline $\mathrm{s}_{\mathrm{p}}$ & 28.6 & 180 & 179 & 178 & 1.1 & $44^{p}$ & 8.34 & 0.40 & & & & & & & & iavel & 5 & $s$ \\
\hline $2 z_{s}$ & 32.2 & 98 & 98 & 98 & 0.3 & ${ }_{14^{r}}$ & 4.24 & 0.19 & & & & & & & & 7avel & $s$ & 39 \\
\hline${ }^{\mathrm{Nat}} \mathrm{C} 1$ & 27.6 & 292 & 273 & 254 & 19 & & 13.3 & 0.67 & & & & & & & & Tavel & $s$ & 40 \\
\hline$H=E_{x}$ & 31.6 & 218 & 217 & 216 & 1.1 & $39^{5}$ & 9.7 & 0.45 & & & & $\cdot$ & & & & Bavel & $\mathbf{s}$ & ai \\
\hline${ }^{-\mathrm{Ar}}$ & 26.8 & 538 & 390 & 242 & 148 & & 21.0 & 1.18 & & & & & & & & zavel & 5 & 42 \\
\hline${ }^{\circ} \mathrm{Ca}$ & 26.0 & 73 & 73 & 73 & & & & & & & & & & & & LSMI2 & 5 & \\
\hline${ }^{\circ} \mathrm{Ca}$ & 29.5 & 100 & 100 & 100 & & & 4.55 & 0.21 & & & & & & & & 7avel & $s$ & 43 \\
\hline "sc & 28.1 & 399 & 382 & 365 & 17 & & 19.6 & 1.05 & & & & & & & & iavel & 5 & 44 \\
\hline$=9$ & 27.8 & 654 & 552 & 450 & 102 & & 28.9 & 1.56 & $14-23$ & 17.86 & 58.8 & 4.42 & 21.22 & 28.8 & 5.10 & 62Ful & $\mathbf{l}$ & 45 \\
\hline $21 y$ & 27.8 & 689 & 610 & 531 & 79 & & 31.2 & 1.65 & $14-23$ & 17.93 & 53.3 & 3.62 & 20.95 & 40.7 & 7.15 & jayel & 3 & 40 \\
\hline${ }^{3} \mathrm{Mn}_{\mathrm{Hn}}$ & 36.5 & 902 & 733 & 567 & 163 & 3 & 36.4 & 1.93 & $14-21$ & 16.82 & 51.4 & 4.33 & 20.09 & 45.2 & 4.09 & $79 a_{12}$ & $\mathbf{L}$ & 47 \\
\hline$=9 \mathrm{Mi}$ & 33.5 & $2 \llbracket 4$ & 286 & 278 & 7.7 & & 13.8 & 0.70 & & & & & & & & $74 F \cup 3$ & L & 46 \\
\hline
\end{tabular}




\begin{tabular}{|c|c|c|c|c|c|c|c|c|c|c|c|c|c|c|c|c|c|c|}
\hline \multirow[b]{2}{*}{ Hutieus } & \multirow[b]{2}{*}{$\begin{array}{c}E_{\text {max }} \\
\left(\mathrm{HeV}_{\mathrm{m}}\right)\end{array}$} & \multirow{2}{*}{ 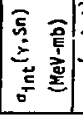 } & \multirow{2}{*}{ 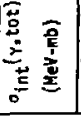 } & \multirow{2}{*}{ 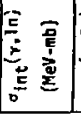 } & \multirow{2}{*}{ 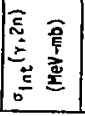 } & \multirow{2}{*}{ 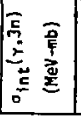 } & \multirow{2}{*}{$\begin{array}{ll}\overrightarrow{\mathbf{\Xi}} & \\
\overrightarrow{\mathbf{E}} & \overline{\mathbf{E}} \\
\overrightarrow{\mathrm{b}} & \end{array}$} & \multirow{2}{*}{ 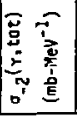 } & \multirow[b]{2}{*}{$\begin{array}{c}\text { Fitting } \\
\text { Interval } \\
\text { (MeV) }\end{array}$} & \multicolumn{6}{|c|}{ Lorentz Parameters } & \multirow{2}{*}{ 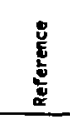 } & \multirow{2}{*}{ 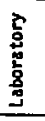 } & \multirow[b]{2}{*}{ 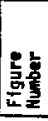 } \\
\hline & & & & & & & & & & $\begin{array}{c}E_{m_{1}} \\
(\mathrm{MeV})\end{array}$ & $\begin{array}{r}\sigma_{\mathrm{mL}} \\
(\mathrm{mb}) \\
\end{array}$ & $\begin{array}{c}\mathbf{r}_{\mathbf{1}} \\
(\mathrm{MeV})\end{array}$ & $\begin{array}{c}\mathbf{E}_{\mathbf{m}_{2}} \\
(\mathrm{MeV})\end{array}$ & $\begin{array}{c}0_{\mathbf{m}_{2}} \\
(\mathrm{mb})\end{array}$ & $\begin{array}{c}r_{2} \\
\text { (MeV) }\end{array}$ & & & \\
\hline "Co & 36.5 & 965 & 807 & 653 & 150 & 4 & 40.1 & 2.14 & $14-21$ & 16.43 & 28.3 & 2.73 & 18.66 & 58.4 & 7.38 & $79 A 12$ & $\mathbf{L}$ & 49 \\
\hline "MI & 33.2 & 772 & 700 & 628 & 72 & 0 & 35.4 & 1.89 & $14-21$ & 16.30 & 34.1 & 2.44 & 18.51 & 55.2 & 6.37 & iAFus & $\mathbf{L}$ & 50 \\
\hline Hat $_{\mathrm{Cu}}$ & 19.6 & 450 & $450^{\circ}$ & & & & & & & & & . & & & & 62HI3 & $\mathbf{s}$ & \\
\hline${ }_{\mathrm{Mat}}^{\mathrm{Cu}}$ & 27.8 & 710 & 604 & 498 & 106 & & 33.8 & 1.97 & & & & & & & & GAFul & $\mathbf{L}$ & 5 \\
\hline${ }^{2} \mathrm{Cu}$ & 27.8 & 680 & 604 & 528 & 76 & & 33.4 & 1.92 & $14-21$ & 16.24 & 60.8 & 4.65 & 19.65 & 26.8 & 4.59 & GAFul & $\mathbf{L}$ & 52 \\
\hline${ }^{3} \mathrm{Cu}$ & 25.1 & & & 498 & 43 & & & & $14-21$ & 16.72 & 66.1 & 4.19 & 19.10 & 30.1 & 3.56 & 68Sul & GA & $5 j$ \\
\hline$" 2 n$ & 29.5 & 791 & 747 & 703 & 44 & & 38.1 & 2.03 & $|4-2|$ & 16.23 & 41.4 & 3.27 & 14.19 & $56.1=$ & 5.98 & $76 \mathrm{Cal}$ & $s$ & 54 \\
\hline${ }^{\circ} \mathrm{Cu}$ & 27.8 & 817 & 619 & 421 & 198 & & 36.0 & 2.18 & $14-20$ & 16.70 & 75.2 & 6.89 & & & & 64Ful & $\mathbf{L}$ & 55 \\
\hline $\mathrm{Hat}_{\mathrm{Ga}}$ & 26.5 & 1108 & 912 & 716 & S.0 & & 52.0 & 3.09 & $|3-2|$ & 16.69 & 107 & 6.91 & & & & $76 \mathrm{Cal}$ & $s$ & 50 \\
\hline${ }^{70} \mathrm{Ge}$ & 26.5 & 855 & 771 & 687 & 84 & & 43.2 & 2.51 & $13-21$ & 16.79 & 89.4 & 7.66 & & & & $76 \mathrm{Cal}$ & 5 & 57 \\
\hline${ }^{72} \mathrm{Ge}$ & 26.5 & 1133 & 935 & 737 & 198 & & 53.5 & 3.19 & $13-21$ & 16.67 & 107 & 7.68 & & & & $76 \mathrm{Cal}$ & s & So \\
\hline "Ge & 26.5 & 1322 & 1013 & 704 & 309 & & 58.8 & 3.56 & $|3-2|$ & 14.52 & 23.9 & 1.92 & 17.02 & 102.3 & 8.18 & $76 \mathrm{Cal}$ & $S$ & 59 \\
\hline $2{ }_{A S}$ & 29.5 & 1130 & 909 & 688 & 221 & 0 & 51.4 & 3.05 & $13-21$ & 11.98 & 41.1 & 3.64 & 17.61 & 76.9 & 7.26 & 698el & L & 60 \\
\hline$"{ }^{\mathrm{As}}$ & 26.2 & 1306 & 1089 & 872 & 217 & & 62.7 & 3.76 & $13-21$ & 15.23 & 69.2 & 4.52 & 18.24 & 74.6 & 7.75 & $76 \mathrm{Cal}$ & $s$ & ol \\
\hline "Ge & 26.5 & 1487 & 1110 & 733 & 377 & 0 & 63.8 & 3.86 & $13-21$ & 15.51 & 59.4 & 4.36 & 18.83 & 73.0 & 11.44 & $76 \mathrm{Cal}$ & $s$ & $\dot{b c}$ \\
\hline 245 & 26.5 & 1177 & 996 & 815 & 181 & & 57.0 & 3.39 & $|3-2|$ & 14.95 & 68.2 & 3.84 & 18.40 & 81.8 & 6.47 & $76 \mathrm{La} 1$ & $S$ & 63 \\
\hline "Se & 26.5 & 1322 & 1050 & 778 & 272 & & 61.4 & 3.74 & $13-21$ & 15.23 & 35.8 & 2.58 & 17.18 & 161.8 & 8.55 & $76 C_{a l}$ & $\mathbf{s}$ & on \\
\hline "se & 28.1 & 1527 & 1138 & 749 & 389 & 0 & 65.9 & 3.99 & $13-21$ & 16.53 & 136 & 6.90 & & & & 760.1 & s & b5 \\
\hline $12 \mathrm{sc}$ & 26.5 & 1521 & 1124 & 727 & 397 & 0 & 66.4 & 4.11 & $13-21$ & 16.65 & 148 & 5.91 & & & & $76 \mathrm{Cal}$ & $s$ & ib \\
\hline $\mathrm{Mat}_{\mathrm{Rb}}$ & 24.3 & 1242 & 1147 & 1052 & 95 & & 67.1 & 4.04 & $14-19$ & 16.80 & 190 & 4.47 & & & & IILel & 5 & 67 \\
\hline Hat $_{\text {Sr }}$ & 27.0 & 1553 & 1432 & 1311 & 121 & & 80.3 & 4.68 & $14-19$ & 16.84 & 206 & 4.50 & & & & 7 ILeI & $s$ & 66 \\
\hline "Y & 28.0 & 1158 & 1059 & 960 & 99 & & 59.8 & 3.48 & $14-19$ & 16.79 & 185 & 3.95 & & & & $678 e 2$ & $\mathbf{L}$ & by \\
\hline$"{ }_{Y}$ & 27.0 & 1427 & 1353 & 1279 & 74 & & 76.5 & 4.46 & $14-19$ & 16.74 & 226 & 4.25 & & & & IILeI & $s$ & 70 \\
\hline "MY & $18 . i$ & 641 & 641 & & & & 40.0 & 2.52 & $14-19^{1 j}$ & 16.83 & 205 & 3.69 & & & & $72 Y c$ & 1 & 71 \\
\hline $\mathrm{Nat}_{2 r}$ & 19.7 & 1079 & 991 & 903 & 88 & & 68 & 4.9 & $14-19$ & 16.52 & 174 & 4.41 & & & & BbBel & $\mathbf{L}$ & \\
\hline$" \mathrm{zr}$ & 27.6 & 1158 & 1060 & 962 & 98 & & 59.1 & $3.3 \mathrm{~B}$ & $14-19$ & 16.85 & 185 & 4.02 & & & & 67 ber & $\mathbf{L}$ & 72 \\
\hline
\end{tabular}




\begin{tabular}{|c|c|c|c|c|c|c|c|c|c|c|c|c|c|c|c|c|c|c|}
\hline \multirow[b]{2}{*}{ Rucleus } & \multirow[b]{2}{*}{$\begin{array}{l}E_{\gamma \max } \\
(M e V)\end{array}$} & \multirow{2}{*}{ 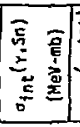 } & \multirow{2}{*}{ 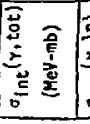 } & \multirow{2}{*}{ 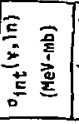 } & \multirow{2}{*}{ 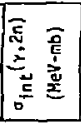 } & \multirow{2}{*}{ 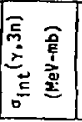 } & \multirow{2}{*}{ 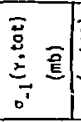 } & \multirow{2}{*}{ 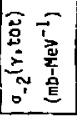 } & \multirow[b]{2}{*}{$\begin{array}{c}\text { Fittina } \\
\text { interubi } \\
\text { (MeV) }\end{array}$} & \multicolumn{6}{|c|}{ Lorentz Parameters } & \multirow{2}{*}{ 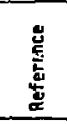 } & \multirow{2}{*}{ 窎 } & \multirow[b]{2}{*}{$\frac{85}{5}$} \\
\hline & & & & & & & & & & {$\left[\begin{array}{c}E_{m 1} \\
(M c V)\end{array}\right.$} & $\begin{array}{r}o_{m 2} \\
(m b) \\
\end{array}$ & $\begin{array}{c}\mathrm{r}_{3} \\
(\mathrm{MeV})\end{array}$ & $\begin{array}{c}E_{\mathrm{m}_{2}} \\
(\mathrm{MeV})\end{array}$ & $\begin{array}{c}\sigma_{m_{2}} \\
(m b)\end{array}$ & $\begin{array}{c}I_{2} \\
(M e V)\end{array}$ & & & \\
\hline$" z r$ & 25.9 & 1309 & 1260 & 1211 & 49 & & 70.8 & 4.08 & $14-19$ & 16.74 & 211 & 4.16 & & & & 7lleel & $s$ & 73 \\
\hline$' t z r$ & 30.0 & 1303 & 1103 & 903 & 200 & 0 & 65.4 & 4.07 & $14-19$ & 16.58 & 184 & 4,20 & & & & 67Be2 & $\iota$ & 74 \\
\hline$' 2 z r$ & 27.8 & $154 ?$ & 1091 & 639 & 452 & 0 & $6 a .2$ & 3.92 & $14-79$ & 16.26 & 266 & 4.68 & · & & & 678ez & $L$ & 75 \\
\hline${ }^{2} \mathrm{mo}_{\mathrm{O}}$ & 29.5 & 1109 & 1079 & 1049 & 30 & & 57.4 & 3.16 & $14-19$ & 16.82 & $16 ?$ & 4.14 & & & & $748 \mathrm{e} 3$ & s & 76 \\
\hline $9 \mathrm{HB}$ & 24.3 & 1610 & 1331 & 1052 & 279 & & 76.5 & 4.80 & $14-19$ & 16.59 & 200 & 5.05 & & & & 21Le1 & 5 & 77 \\
\hline 94 & 31.1 & 1767 & 1121 & 508 & 580 & 33 & 68.5 & 4.40 & $84-19$ & 16.22 & 161 & 5.29 & & & & $67 \mathrm{Be} 2$ & $\mathbf{L}$ & 78 \\
\hline " Mo & 28.4 & 1665 & 1352 & 1039 & 313 & & 78.3 & 4.73 & $14-19$ & 16.36 & 185 & 5.50 & & . & & $7^{\wedge} \mathrm{Be} 3$ & 5 & $7 y$ \\
\hline "'Mo & 27.8 & 1921 & 1483 & 1045 & 438 & $t$ & 87.6 & 5.44 & $13-19$ & 16.20 & 185 & 6.01 & & & & $74 \mathrm{Be} 3$ & 5 & 80 \\
\hline "Mo & 26.8 & 2100 & 1518 & 940 & 574 & $4^{\prime \prime}$ & 92.1 & 5.89 & $13-19$ & 15.80 & 189 & 5.94 & & & & $748 \mathrm{e} 3$ & 5 & 81 \\
\hline $10 \mathrm{CMO}^{\mathrm{Mo}}$ & 27.0 & 2270 & 1528 & 811 & 692 & $25^{8}$ & 93.6 & 6.06 & $12-20$ & 15.74 & 171 & 7.81 & & & & 74be's & 5 & 82 \\
\hline${ }^{10} \mathrm{kh}$ & 25.8 & 1948 & $1568^{b}$ & 1180 & 380 & & 94.7 & 5.97 & $13-19$ & 16.16 & 191 & 7.26 & & & & 7ALel & $s$ & 85 \\
\hline Nat $_{\text {Pd }}$ & 21.3 & 1651 & 1381 & $m$ & 270 & & 88.5 & 5.88 & $13-19$ & 15.92 & 199 & 7.18 & & & & 7ALEI & 5 & 84 \\
\hline $10{ }^{\prime} \mathrm{Ag}$ & 29.5 & 1619 & 1956 & 1093 & 263 & 0 & 78.7 & 4.82 & $13-19$ & 15.90 & 150 & 6.71 & & & & 69Bel & $\mathrm{L}$ & 85 \\
\hline Met $_{A g}$ & 24.9 & 1922 & $1643^{c}$ & 1364 & 279 & & 98.8 & 36.20 & $13-19$ & 16.07 & 199 & 7.38 & & & & 74LEe I & 5 & 80 \\
\hline $\mathrm{Hat}_{\mathrm{Cd}}$ & 24.6 & 2016 & $1685^{d}$ & 1324 & 361 & 0 & 105 & 6.96 & 13-19 & 15.81 & 227 & 6.22 & & & . & .74Lel & 5 & bi \\
\hline${ }^{11} s_{\text {in }}$ & 31.1 & 2409 & 1875 & 1354 & 508 & 13 & 113 & 7.13 & $13-18$ & 15.63 & 266 & 5.24 & & & & $69 \mathrm{Ful}$ & $L$ & 88 \\
\hline${ }^{113}$ In & 24.1 & 2026 & $1748^{c}$ & 1470 & 278 & & 108 & 5.91 & 13-18 & 15.72 & 247 & 5.60 & & & & 74LEI & 5 & $8 y$ \\
\hline $116 \mathrm{sn}$ & 29.6 & 2083 & ใ669 & 1255 & 414 & 0 & 99 & 6.13 & $13-18$ & 15.68 & 265 & 4.19 & & & & 69ful & 1 & $90^{\circ}$ \\
\hline masn & 22.1 & 1823 & $1630^{c}$ & 1437 & 193 & & 104 & 6.78 & $13-18$ & 15.56 & 271 & 5.08 & & & & 7alel & $s$ & 91 \\
\hline $115 n$ & 31.1 & 2446 & 1894 & 1380 & 475 & 38 & 114 & 7.30 & $13-18$ & 15.66 & 254 & 5.02 & & & & 69ful & $l$ & 92 \\
\hline $1175 n$ & 21.1 & 1774 & 1554 & 1334 & 220 & & 102 & 7.05 & $13-18$ & 15.64 & 259 & 5.04 & & & & 7alel & $s$ & 93 \\
\hline $11 \mathrm{~s} 5 \mathrm{n}$ & 30.8 & 2424 & 1853 & 1302 & 531 & 20. & 110 & 6.83 & $13-18$ & 15.59 & 250 & 4,77 & & & & 69Ful & $\mathbf{L}$ & 94 \\
\hline $1 s_{5 n}$ & 21.6 & 1893 & 1535 & 1377 & 258 & & 106 & 7.07 & $13-18$ & 15.44 & 275 & 4.86 & & & & 74LE? & $s$ & 95 \\
\hline " 'sn & 31.1 & 2728 & 1993 & 1326 & 597 & 65 & 118 & 7.55 & $13-18$ & 15.53 & 253 & 4.81 & & & & 69Ful & l & 46 \\
\hline $1 \times 9 \mathrm{sn}$ & 29.9 & 2771 & 2074 & 1389 & 673 & 12 & 124 & 7.79 & $13-18$ & 15.40 & 280 & 4.89 & & & & 69FuI & $\mathbf{L}$ & 97 \\
\hline${ }^{120}{ }^{5 n}$ & 22.4 & $21 \in 9$ & $1770^{c}$ & 1371 & 399 & & 113 & 7.49 & $13-18$ & 15.37 & 285 & 5.10 & & & & 74LeI & $s$ & 98 \\
\hline
\end{tabular}




\begin{tabular}{|c|c|c|c|c|c|c|c|c|c|c|c|c|c|c|c|c|c|c|}
\hline \multirow[b]{2}{*}{ Mucleus } & \multirow[b]{2}{*}{$\begin{array}{c}E_{y \text { max }} \\
(\operatorname{MeV})\end{array}$} & \multirow{2}{*}{ 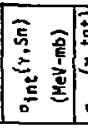 } & \multirow{2}{*}{ 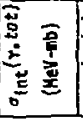 } & \multirow{2}{*}{ 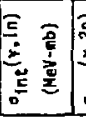 } & \multirow{2}{*}{ 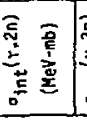 } & \multirow{2}{*}{ 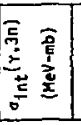 } & \multirow{2}{*}{ 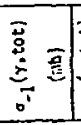 } & \multirow{2}{*}{ 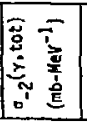 } & \multirow[b]{2}{*}{$\begin{array}{c}\text { Fitting } \\
\text { Intervo } \\
(\mathrm{MeV})\end{array}$} & \multicolumn{6}{|c|}{ Lorentz Parimeters } & \multirow{2}{*}{ 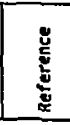 } & \multirow{2}{*}{ 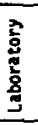 } & \multirow[b]{2}{*}{ 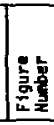 } \\
\hline & & & & & & & & & & $\begin{array}{c}E_{\mathrm{ml}} \\
(\mathrm{MeV})\end{array}$ & $\begin{array}{c}\sigma_{\mathrm{mm}} \\
(\mathrm{mb})\end{array}$ & $\begin{array}{c}\Gamma_{1} \\
(\mathrm{MeV})\end{array}$ & $\begin{array}{c}E_{\mathrm{m}_{2}} \\
\text { (MeV) }\end{array}$ & $\sigma_{(m b)}$ & $\begin{array}{c}r_{2} \\
(m+v)\end{array}$ & & & \\
\hline $1245 n$ & 31.1 & 2790 & 2010 & 1285 & 670 & 55 & 123 & 8.02 & $13-18$ & 15.19 & 283 & 4.81 & & & & 69Ful & L & yy \\
\hline $1245 n$ & 21.6 & 2060 & $1558^{\circ}$ & 1056 & 502 & & 101 & 6.74 & $13-18$ & 15.28 & 276 & 4.80 & & & & 74Le 1 & 5 & 100 \\
\hline $\mathrm{Hat}_{S b}$ & 25.7 & 2315 & $1927^{C}$ & 1539 & 388 & 0 & 119 & 7.69 & $13-1 B$ & 15.48 & 277 & 5.05 & & & & 74Lel & 5 & וסו \\
\hline $120 \mathrm{Te}$ & 26.5 & 2498 & 2022 & 1546 & 476 & 0 & 127 & 8.29 & $12-19$ & 15.24 & 281 & 5.56 & & & & 76Le2 & $\mathbf{s}$ & 102 \\
\hline 124 Te & 24.8 & 2533 & 2023 & 1513 & 510 & & 129 & 8.56 & $12-19$ & 15.17 & 296 & 5.44 & & & & 76Lé & 5 & 103 \\
\hline$x+1$ & 29.5 & $2 m$ & 1728 & 1285 & 443 & $<20$ & 105 & 6.70 & $12-20$ & 14.29 & 118 & 3.24 & 16.27 & 149 & 5.27 & $66 \mathrm{Br})$ & $\mathbf{L}$ & 104 \\
\hline${ }^{127} 1$ & 24.9 & 2380 & 1989 & 1601 & $390^{1}$ & $*$ & 128 & 8.53 & $12-20$ & 14.57 & 239 & 4.08 & 16.69 & 118 & 4.92 & $698 \mathrm{e} 6$ & 5 & lo5 \\
\hline 127 & 26.9 & 1043 & 1036 & 7030 & 6 & & 74 & 5,4 & $12 \cdot 20^{j j}$ & 14.74 & 111 & 3.34 & 15.88 & 156 & 6.44 & 86Bel & L & \\
\hline 121 Te & 26.2 & 2732 & 2093 & 1454 & 639 & 0 & 134 & 8.92 & $12-19$ & 15.13 & 305 & 5.36 & & & & 76Le2 & s & 106 \\
\hline isste & 25.9 & 2893 & 2180 & 1467 & 713 & 0 & 139 & 9.27 & $12-19$ & 15.12 & 320 & 5.03 & & & & $761 \mathrm{e} 2$ & $s$ & 107 \\
\hline Hat $_{T e}$ & 25.7 & 2636 & 2112 & 1588 & 524 & 0 & 134 & 9.05 & $12-19$ & 15.46 & 297 & 5.32 & & & & 7^Lel & 5 & 108 \\
\hline "'cs & 29.5 & 2505 & 1986 & 1475 & 503 & 8 & 124 & 8.09 & $12-19$ & 15.25 & 287 & 5.01 & & & & $698 \mathrm{e}$ & $L$ & loy \\
\hline${ }^{139} \mathrm{Cs}$ & 24.2 & 2484 & 2156 & 1828 & 328 & & 137 & 9.09 & $12-19$ & 15.34 & 317 & 5.31 & & & & 74Lel & 5 & 110 \\
\hline Nat $_{80}$ & 24.3 & 2619 & 2248 & 187 & 371 & 0 & 146 & 9.94 & $12-19$ & 15.29 & 356 & 4.89 & & & & 71 Ge4 & $s$ & $M$ \\
\hline $190 \mathrm{Ba}$ & 27.1 & 2536 & 2090 & 1547 & 490 & $3^{x}$ & 130 & 8.71 & $12-19$ & 15.26 & 327 & 4.61 & & & & 70Be8 & $\mathbf{L}$ & Mz \\
\hline "spo & 21.2 & 1910 & $1910^{\circ}$ & & & & & & & & & & & & & $\begin{array}{r}\text { b2W13 } \\
\text { j68Be5 }\end{array}$ & S & \\
\hline 13'LA & 24.3 & 2269 & 1978 & 1687 & $291^{j}$ & $y$ & 128 & B.54 & $12-19$ & 15.24 & 336 & 4.47 & & & & filbet & 5 & 112 \\
\hline${ }^{\text {hatce }}$ & 21.2 & 1890 & $1880^{\circ}$ & & & & & & & & & & & & & $62 \mathrm{Hi3}$ & $\mathbf{s}$ & \\
\hline $10{ }^{10} \mathrm{ce}$ & 26.5 & 2855 & 2398 & 1941 & 457 & 0 & 153 & 10.3 & $12 \cdot 19$ & 15.04 & 383 & 4.41 & & & & $76 \mathrm{le} 2$ & $s$ & 114 \\
\hline$w p r$ & 29.8 & 2412 & 2062 & 1717 & 340 & $5^{2}$ & 128 & 8.37 & $12-19$ & 15.15 & 324 & 4.42 & & & & $668 \mathrm{~T} 1$ & $\mathbf{L}$ & 115 \\
\hline "Wpr & 23.7 & & & $1713^{9}$ & & & & & $12-19$ & 15.23 & 341 & 4.00 & & & & $205 u 1$ & GA & 116 \\
\hline${ }^{1+1} \mathrm{pr}$ & 16.9 & 1422 & 1422 & $142 \hat{z}$ & & & 101 & 7.31 & $12 \cdot 19^{j j}$ & 15.04 & 347 & 4.49 & & & & FiBe4 & $s$ & 11 \\
\hline $1+1 \mathrm{Pr}$ & 18.1 & 1395 & $1395^{\circ}$ & & & & $94^{d}$ & $6.41^{\mathrm{a}}$ & $12-19^{j \mathrm{j}}$ & 15.36 & 332 & 4.07 & & & & $\begin{array}{l}72 y o \\
\text { frocal }\end{array}$ & I & 118 \\
\hline $1+1 \mathrm{pr}$ & 16.2 & 1252 & 1252 & $125 i$ & & & 93 & 7.0 & $12-19^{j j}$ & 15.19 & 344 & 4.24 & & & & $\left\{\begin{array}{l}\text { \{ } \\
\text { B6Be2 }\end{array}\right.$ & $\mathbf{l}$ & \\
\hline $1 * 1 r r$ & 16.9 & 1254 & 1264 & 1264 & & & 89 & 6.4 & $12-19^{\mathfrak{j} \mathbf{j}}$ & 15.19 & 340 & 4.15 & & & & $868 \mathrm{e} 1$ & 2 & \\
\hline
\end{tabular}




\begin{tabular}{|c|c|c|c|c|c|c|c|c|c|c|c|c|c|c|c|c|c|c|}
\hline \multirow[b]{2}{*}{ icleus } & \multirow[b]{2}{*}{$\mid \begin{array}{l}E_{Y \max } \\
{[(\mathrm{feV})}\end{array}$} & \multirow{2}{*}{ 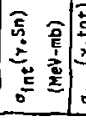 } & \multirow{2}{*}{ 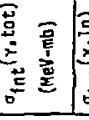 } & \multirow{2}{*}{ 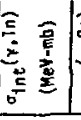 } & \multirow{2}{*}{ 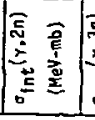 } & \multirow{2}{*}{ 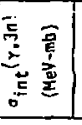 } & \multirow{2}{*}{ 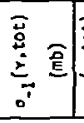 } & \multirow{2}{*}{ 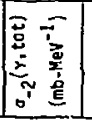 } & \multirow[b]{2}{*}{$\begin{array}{c}\begin{array}{c}\text { Fitting } \\
\text { Intervai } \\
\text { (MeV) }\end{array} \\
\end{array}$} & \multicolumn{6}{|c|}{ Lorentz Paremeters } & \multirow{2}{*}{ 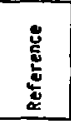 } & \multirow{2}{*}{ 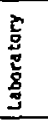 } & \multirow[b]{2}{*}{ 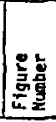 } \\
\hline & & & & & & & & & & $\begin{array}{r}5 \\
m 1 \\
(\mathrm{MeV}) \\
\end{array}$ & $\begin{array}{c}\sigma_{m 1} \\
\text { (mo) }\end{array}$ & $\left.\mid \begin{array}{c}r_{2} \\
(M \in V)\end{array}\right]$ & $\begin{array}{c}E_{m_{2}} \\
(\mathrm{MeV})\end{array}$ & $\begin{array}{l}{ }^{0} \mathrm{mz}_{2} \\
(\mathrm{mb})\end{array}$ & $\begin{array}{c}T_{2} \\
(M \in V)\end{array}$ & & & \\
\hline${ }^{\prime \prime} \mathrm{ce}$ & 23.5 & 3394 & 2206 & 1022 & 1186 & 0 & 150 & 10.7 & $12-19$ & $14.8 E$ & 332 & 5.10 & & & & 761.2 & 5 & ny \\
\hline Mat $_{\text {Nd }}$ & 18.0 & 1882 & 1559 & 1236 & 323 & & 112 & 8.46 & $12-i 3^{i j}$ & 14.92 & 315 & 4.70 & & & & $718 \mathrm{Be}$ & $s$ & 120 \\
\hline $102 \mathrm{No}$ & 20.2 & 1918 & 1873 & 1828 & 45 & & 126 & 8.66 & $12-19$ & 14.94 & 359 & 4.44 & & & & Mlal & 5 & 121 \\
\hline 1 "an $\mathrm{Md}$ & 19.8 & 2054 & 1875 & 1696 & 179 & & 130 & 9.39 & $12-19$ & 15.01 & 349 & 4.75 & & & . & IICal & $s$ & laz \\
\hline m.no & 20.2 & 2445 & 1882 & 1319 & 563 & & 128 & 9.01 & $12-19$ & 35.05 & $3 ! 7$ & 5.28 & & & & $7 \mathrm{Cas}_{\mathrm{S}}$ & 5 & 123 \\
\hline $10 \mathrm{sm}$ & 20.8 & 1970 & 1935 & 1900 & 35 & & 126 & 8.41 & $12-19$ & 15.32 & 383 & 4.45 & & & & 74 a a5 & $\mathbf{s}$ & 124 \\
\hline $105_{\text {Wd }}$ & 20.2 & 2694 & 2037 & 1380 & 657 & 0 & 147 & 11.3 & $12-19$ & 34.95 & 296 & 6.31 & & & & $716 \pm 1$ & $s$ & 125 \\
\hline $10 \times$ Hd & 20.2 & 2587 & 1920 & 1253 & 667 & & 133 & 9.60 & $12-19$ & 14.74 & 310 & 5.78 & & & & 7X[a] & 5 & 126 \\
\hline $1 " 1 \mathrm{Md}$ & 18.8 & 2537 & 1702 & 867 & 835 & & 122 & 9.02 & $10.8-18.8$ & 12.76 & ng? & 3.97 & 15.48 & 220 & 5.30 & 7lcal & 5 & 127 \\
\hline${ }^{\prime \prime} \mathrm{sm}_{\mathrm{m}}$ & 20.0 & 2498 & 1942 & 1386 & 556 & & 134 & 9.51. & - $12-! 3$ & 14.82 & 339 & 5.09 & & & & $745 a 5$ & 5 & Ikg \\
\hline $15 \mathrm{kd}$ & 20.2 & 3185 & 2011 & 1174 & 837 & 0 & $\because$ & 10.4 & $10.8-18.8$ & 12.30 & 175 & 3.38 & 16.04 & 223 & 5.17 & ncal & $s$ & $12 y$ \\
\hline isosm & 19.8 & 2687 & 1991 & 1295 & 696 & & 141 & 10.3 & $12-19$ & 14.61 & 312 & 5.97 & & & & $74 c 25$ & $s$ & 130 \\
\hline $132 \mathrm{sm}$ & 20.0 & 2707 & 2026 & 1345 & 681 & & 143 & 10.5 & $10.8-18.8$ & $12.3 \mathrm{~B}$ & 176 & 2.97 & 15.74 & 234 & 5.22 & 74 Las & $\mathbf{s}$ & 131 \\
\hline " "'EU & 28.9 & 3017 & 2273 & 1566 & 670 & 37 & 148 & 10.2 & $10.8-18.8$ & 12.33 & 185 & 2.75 & 15.79 & 222 & 5.83 & 696ев & $L$ & $13 \bar{z}$ \\
\hline${ }^{134} \mathrm{Sm}$ & 21.1 & $2 e^{x} 1$ & 2059 & 1277 & 782 & & 144 & 10.6 & $10.8-18.8$ & 12.27 & 181 & 2.95 & 15.94 & 215 & 5.70 & $.74 C 25$ & $s$ & 133 \\
\hline $\mathrm{Notsm}_{\mathrm{m}}$ & 25.2 & 3247 & 2425 & 1628 & 772 & $25^{2 a}$ & 164 & 11.7 & & & & & & & & 698e6 & 5 & 134 \\
\hline $139 \mathrm{~T}$ & 28.0 & 3187 & 2300 & 1413 & $897^{k}$ & & 151 & 20.5 & $10.8-18.8$ & 12.22 & 181 & 2.64 & 15.67 & 220 & 4.97 & 648ri & 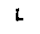 & 135 \\
\hline 159 & 27.4 & 3194 & 2557 & 1936 & 605 & $16^{66}$ & 170 & 12.0 & $10.8-18.8$ & 12.07 & 196 & 2.98 & 15.88 & 248 & 5.10 & 688e5 & 5 & 136 \\
\hline $160_{\text {Gd }}$ & 29.5 & 3748 & 2533 & 1398 & 1055 & 80 & 169 & 12.1 & $10.8-18.8$ & 12.23 & 215 & 2.77 & 15.96 & 233 & 5.28 & 698e8 & $L$ & 137 \\
\hline 10 "Ho & 19.6 & $5 \quad 2540$ & $2540^{\circ}$ & & & & & & & 12.02 & 238 & 2.35 & 15.59 & 308 & 4.85 & 66AK1 & 5 & \\
\hline 163HO & 28.9 & 3355 & 2523 & 1735 & 744 & 44 & 166 & 11.6 & $10.3-18.8$ & 12.28 & 274 & 2.57 & 15.78 & 246 & 5.00 & 698e8 & b & 136 \\
\hline 10 " Но & 26.8 & $8 \quad 3657$ & 2871 & 2090 & 766 & $15^{\mathrm{cc}}$ & 194 & 13.9 & $10.8-18.8$ & $3 \quad 12.01$ & 239 & 2.52 & 15.59 & 291 & 5.12 & 68Be5 & 5 & 139 \\
\hline${ }^{\text {Mat }} \mathrm{Er}$ & 21.1 & $\quad 2973$ & 2387 & 1801 & $58 \varepsilon^{1}$ & do & 172 & 12.9 & $10.8-18.8$ & 12.12 & 242 & 2.76 & 15.58 & 259 & 4.74 & $698 \mathrm{Be}$ & $s$ & 140 \\
\hline "'LU & 23.0 & 3142 & 2507 & 1872 & $635^{m}$ & ee & 173 & 12.5 & $10.8-18.8$ & 12.32 & 217 & 2.57 & 15.47 & 287 & 4.70 & s98ed & 5 & 141 \\
\hline 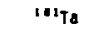 & 22.0 & 2970 & $2970^{\circ}$ & & & & & & & & & & & & & $62 h+3$ & s & \\
\hline גדי & 24.6 & 53062 & 2181 & 1300 & $881^{k}$ & & 349 & 10.7 & $10.8-18.8$ & 12.54 & 154 & 1.67 & 34.95 & 273 & 5.23 & $63 \mathrm{Br} 1$ & I & $14 i$ \\
\hline
\end{tabular}




\begin{tabular}{|c|c|c|c|c|c|c|c|c|c|c|c|c|c|c|c|c|c|c|}
\hline \multirow[b]{2}{*}{ Mucleus } & \multirow[b]{2}{*}{$\begin{array}{l}E_{y} \max \\
(\mathrm{MeV})\end{array}$} & \multirow{2}{*}{ 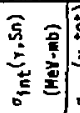 } & \multirow{2}{*}{ 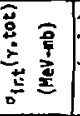 } & \multirow{2}{*}{ 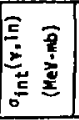 } & \multirow{2}{*}{ 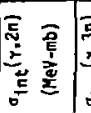 } & \multirow{2}{*}{ 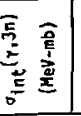 } & \multirow{2}{*}{ 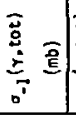 } & \multirow{2}{*}{ 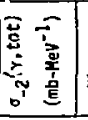 } & \multirow[b]{2}{*}{$\begin{array}{c}\text { Fitting } \\
\text { intervai } \\
\text { (MeV) }\end{array}$} & \multicolumn{6}{|c|}{ Loruntz Parameters } & \multirow{2}{*}{ 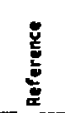 } & \multirow{2}{*}{ 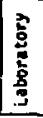 } & \multirow[b]{2}{*}{ 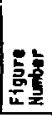 } \\
\hline & & & & & & & & & & $\begin{array}{c}E_{\max } \\
(\mathrm{HeV})\end{array}$ & $\begin{array}{c}\sigma_{\mathrm{ma}} \\
(\mathrm{mb})\end{array}$ & $\begin{array}{c}s_{2} \\
(K \in V)\end{array}$ & $\begin{array}{c}\mathbf{E}_{\mathbf{m}_{2}} \\
(\mathrm{HeV})\end{array}$ & $\begin{array}{c}a_{m_{2}} \\
(m b)\end{array}$ & $\begin{array}{c}r_{\mathbf{z}} \\
\text { (MeV) }\end{array}$ & & & \\
\hline${ }^{101} \mathrm{Ta}$ & 25.2 & 3799 & 2983 & 2180 & 790 & $13^{f f}$ & 205 & 19.8 & $10.8-18.8$ & 12.30 & 259 & 2.43 & 15.23 & 341 & 4.48 & OBBe5 & $s$ & 143 \\
\hline Mat $_{H}$ & 21.3 & 3848 & 2854 & 1860 & 994 & 0 & 203 & 15.0 & $10.8-18.8$ & 12.56 & 268 & 2.66 & 15.17 & 328 & 4.69 & isyes & $s$ & 144 \\
\hline $106 \mathrm{~W}$ & 28.6 & 4502 & 3004 & 1655 & 1200 & 149 & 203 & 14.5 & $10.8-i 8,8$ & 12.59 & 211 & 2.29 & 14.68 & 334 & 5.18 & $69 \mathrm{~B} e 8$ & $\mathbf{L}$ & 145 \\
\hline Wat & 21.6 & 3991 & 3226 & 2461 & 765 & 0 & 227 & 16.6 & $10.8-18.8$ & 12.63 & 279 & 2.77 & 15.24 & 375 & 4.69 & $75 Y e 5$ & $s$ & 146 \\
\hline $18 \mathrm{es}_{5}$ & 19.7 & 2964 & 2501 & 2038 & 463 & & 179 & 13.2 & $10.8-10.8$ & 13.03 & 308 & 3.13 & 15.26 & 302 & 3.38 & 798ed & $\mathbf{L}$ & 147 \\
\hline${ }^{1 s 4} 0 \mathrm{~s}$ & 30,4 & 4731 & 3616 & 2616 & 885 & 115 & 239 & 16.7 & $10.8-18.8$ & 12.81 & 260 & 2.76 & 14.88 & 390 & 4.19 & i98et & $\mathbf{L}$ & 148 \\
\hline 1090 & 29.9 & 4722 & 3326 & 2130 & 996 & 200 & 228 & 16.7 & $10.8-18.8$ & 12.68 & 268 & 2.71 & 14.68 & $3 \div 5$ & 3.62 & 798ed & $\mathbf{L}$ & 149 \\
\hline${ }^{19005}$ & $? 3.4$ & 4602 & 3236 & 2013 & 1080 & 142 & 220 & 15.8 & $10.8-18.8$ & 12.68 & 206 & 2.60 & 14.40 & 401 & 4.16 & 798ed & $L$ & 150 \\
\hline${ }^{192} 0 \mathrm{~s}$ & 29.9 & 4900 & 3315 & 1923 & 1200 & 192 & 224 & 16.0 & $10.8-18.8$ & 12.68 & 206 & 2.49 & 14.35 & 389 & 4.41 & 79 bet & $\mathbf{L}$ & 151 \\
\hline Wot ${ }_{1 r}$ & 21.9 & 3596 & 2965 & 2334 & E31 & & 211 & 15.5 & $11-17$ & 13.80 & 487 & 5.08 & & & & 75Yeb & $s$ & 152 \\
\hline Hat $_{p t}$ & 20.3 & 3575 & 3056 & 2537 & 519 & & 228 & 17.8 & $11-17$ & 13.72 & .512 & 4.99 & & & & 75Ves & s & 153 \\
\hline${ }^{197}{ }_{A \nu}$ & 22.0 & 3000 & $3000^{\circ}$ & & & & & & & & & & & & & $62 M+3$ & $s$ & \\
\hline$"$ "'Au & 24.7 & 3744 & 2967 & 2190 & $777^{k}$ & & 205 & 14.7 & $11-17$ & 13.82 & 560 & 3.84 & & & & 62Fúc & $\mathbf{L}$ & 154 \\
\hline $197_{\mathrm{AU}}$ & 21.7 & 3546 & 3067 & 2588 & $479^{n}$ & 99 & 217 & 15.9 & $11-17$ & 13.72 & 541 & 4.61 & & & & 7ovel & $\mathbf{s}$ & 155 \\
\hline $197 \mathrm{Au}$ & 16.9 & 2605 & 2491 & 2376 & 116 & & 194 & 15.6 & $11-17^{\mathrm{Jj}}$ & 13.73 & 502 & 4.76 & & & & $868 e 1$ & $L$ & \\
\hline Nat $_{\mathrm{Hg}}$ & 21.1 & 3678 & 3133 & 2588 & 545 & 0 & 227 & 17.2 & $17-17$ & 13.72 & 582 & 4.39 & & & . & 75Ves & 5 & 156 \\
\hline $204 \mathrm{pb}$ & 26.4 & 3441 & 2909 & 2377 & $532^{k}$ & & 203 & 15.0 & $10-17$ & 13.59 & 514 & 3.85 & & & & GAHAZ & $\mathbf{L}$ & $\mid b 7$ \\
\hline${ }^{201} \mathrm{~Pb}$ & 26.4 & 3267 & 2718 & 2169 & $549^{k}$ & & 191 & 14.2 & $10-17$ & 13.56 & 481 & 3.96 & & & & $64 \mathrm{Ha2}$ & $\mathbf{L}$ & 158 \\
\hline $210 p t$ & 26.4 & 3496 & 2636 & 1776 & $860^{k}$ & & 189 & 14.2 & $10-17$ & 13.46 & 491 & 3.90 & & & & $64 \mathrm{HaZ}$ & $\mathbf{L}$ & 159 \\
\hline $200 \mathrm{pb}$ & 18.9 & 3387 & 3059 & 2731 & $328^{\circ}$ & hh & 229 & 17.6 & $10-17$ & 13.43 & 639 & 4.07 & & & & 70Vel & 5 & 160 \\
\hline $210 P b$ & 14.9 & 2090 & $2090^{\circ}$ & & & & $165^{a}$ & $13.3^{\mathrm{d}}$ & $10.17^{j J}$ & 13.63 & 645 & 3.94 & & & & $72 Y 0$ & 1 & 161 \\
\hline Nat $t_{b}$ & 22.0 & 4100 & $4100^{\circ}$ & & & . . & & & & & & & & & & 62m13 & 5 & \\
\hline Hat $_{\mathrm{Pb}}$ & 16.9 & S192 & 3047 & 2902 & 145 & & 249 & 21.1 & $10-17^{\mathrm{Jj}}$ & 13.48 & 602 & 4.20 & & & & $86 \mathrm{Bel}$ & $\mathbf{L}$ & \\
\hline 20134 & 22.0 & 3730 & $3730^{\circ}$ & & & & & & & & & & & & & $62 \mathrm{H13}$ & 5 & \\
\hline 20181 & 26.4 & 3772 & 3058 & 2344 & $714^{k}$ & & 214 & 15.8 & $10-17$ & 13.45 & 523 & 3.97 & & & & 64Haz & $L$ & $i 62$ \\
\hline $211_{B 1}$ & 14.8 & 2129 & $2129^{\circ}$ & & & & $170^{a}$ & $13.8^{\circ}$ & $10-17^{\mathbf{J j}}$ & 13.56 & 648 & 3.72 & & & & $22 \% 0$ & I & 163 \\
\hline
\end{tabular}




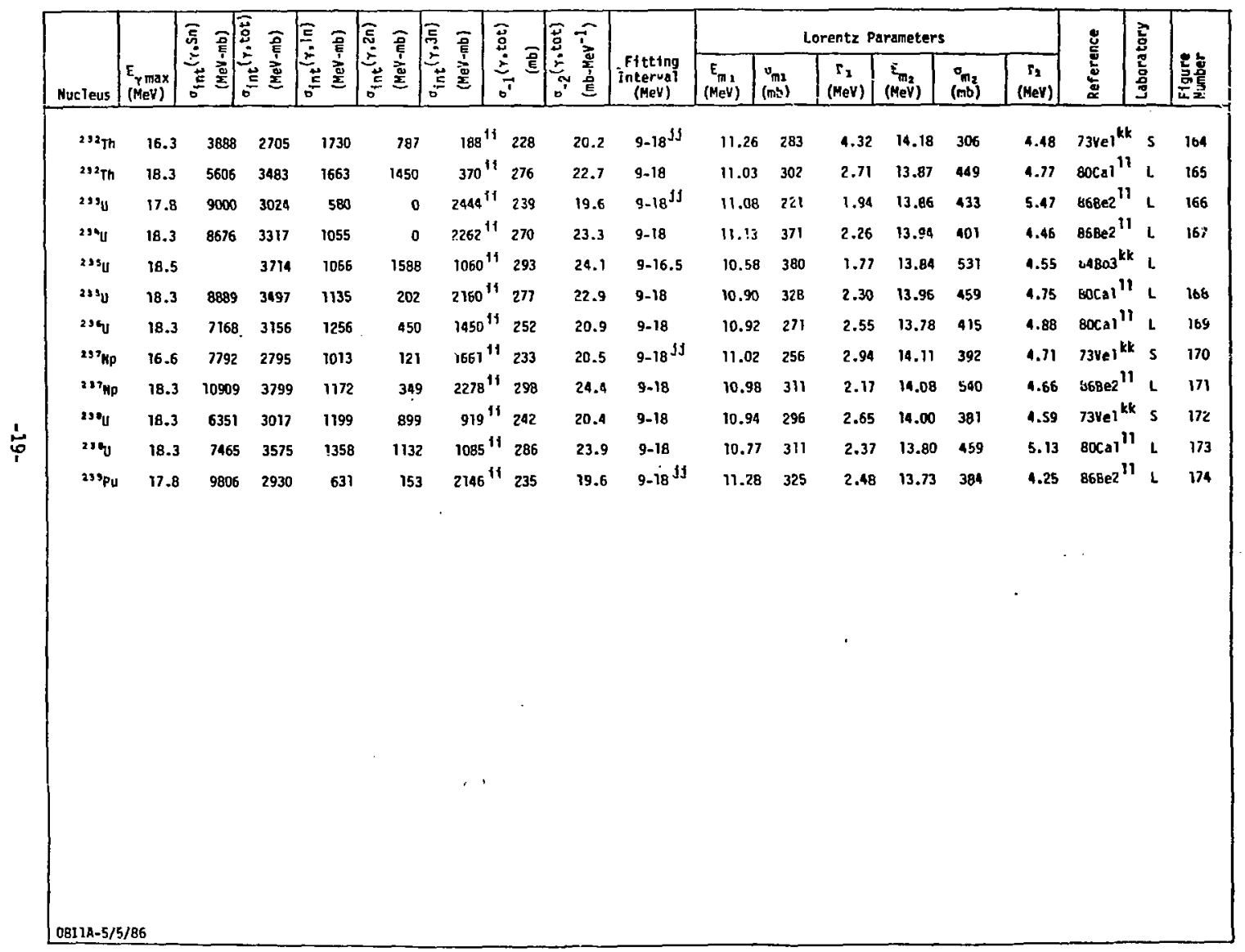




\section{Footnotes to the Table}

Pot corrected for $(\gamma, 2 n)$ events; the figures given are for the photoneutron yiela cross section $\sigma[(\gamma, n)+(\gamma, p n)+2(\gamma, 2 n)]$.

bSone $(\gamma$, tot) data up to $30.1 \mathrm{MeV}$.

CSome $(\gamma$, tot) data up to $29.5 \mathrm{MeV}$.

OSome $(\gamma$, tot) data up to $26.2 \mathrm{MeV}$.

eSome ( $r$, tot) data up to $22.7 \mathrm{MeV}$.

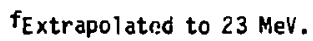

gDoes not include $(\gamma, p n)$ events.

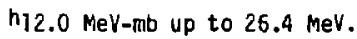

i508 MeV-mb up to $31.2 \mathrm{MeV}$.

jSome $(r, 2 n)$ data up to $29 \mathrm{MeV}$.

kIncludes $(3 / 2) \sigma_{\text {int }}(\gamma, 3 n)$.

$1768 \mathrm{MeV}-m b$ up to $27.4 \mathrm{MeV}$.

m742 MeV-mb up to $28.5 \mathrm{MeV}$.

$n_{671} \mathrm{MeV}-m b$ up to $27.1 \mathrm{meV}$.

D6 $14 \mathrm{MeV}-\mathrm{mb}$ up to $26.3 \mathrm{MeV}$.

$P_{\text {int }}(r, p n)$.

$q_{c_{\text {int }}}(r, p)$.

$r_{G_{j n t}}(Y, p n)$ up to $30.0 \mathrm{MeV}$.

$s_{C_{\text {int }}}(\gamma, p n)$ up to $27.8 \mathrm{MeV}$.

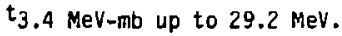

u22 MeV-mb up to $28.9 \mathrm{MeV}$.

$v_{49} \mathrm{MeV}-\mathrm{mb}$ up to $28.4 \mathrm{MeV}$.

W31 MeV-mb up to $31.2 \mathrm{MeV}$.

x $13 \mathrm{MeV}-\mathrm{mb}$ up to $28.6 \mathrm{MeV}$.

ysome $(r, 3 n$ ) data up to $30 \mathrm{meV}$.

$236 \mathrm{NeV}-m b$ up to $33.0 \mathrm{MeV}$. 
aa59 MeV-mb up to $27.4 \mathrm{MeV}$.

bb46 MeV-mb up to $29.3 \mathrm{MeV}$.

c $45 \mathrm{MeV}-\mathrm{mb}$ up to $28.5 \mathrm{HieV}$.

dd52 MeV-mb up to $27.7 \mathrm{MeV}$.

ee65 MeV-mb up to $28.5 \mathrm{MeV}$.

$f_{1} 137 \mathrm{MeV}-\mathrm{mb}$ up to $36.4 \mathrm{MeV}$; some $(\gamma, 4 n)$ data up to $36.4 \mathrm{MeV}$.

$9924 \mathrm{MeV}-\mathrm{mb}$ up to $27.1 \mathrm{MeV}$.

hhr $97 \mathrm{MeV}-\mathrm{mb}$ up to $37.8 \mathrm{MeV}$; some $(r, 411)$ data up to $37.8 \mathrm{MeV}$.

$i i_{\sigma_{\text {int }}}(\gamma, f)$.

jjoata oo not exteno to upper limit of fitting interval.

$k k_{\bar{v}}\left\langle E_{\gamma}\right\rangle$ estimated.

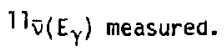




\section{References to the Table}

620el W. E. Del Bianco, W. E. Stephens; Phys. Rev. 126, 709 (1962).

62ful S. C. Fultz, R. L. Bramblett, J. T. Caldwe11, N. E. Hansen, C. P. Jupiter; Phys. Rev. 12B, 2345 (1962).

$62 F u 2$ 5. C. Fuliz, R. L. Bramblatt, J. T. Caldwell, N. A. Kerr; Phys. Rev. 127. 1273 (1962).

62 Mi3 J. Hiller, C. Schuhl, C. Tzara; Nucl. Phys. 32, 236 (196c).

63Erl R. L. Bramblett, J. T. Caldwe11, G. F. Auchampaugh, S. C. Fultz; Phys. Rev. 129, $2723(1963)$.

63Cal J. T. Caldwell, R. R. Hervey, R. L. Bramblett, S. C. Fultz; Phys. Lett. $6,213(1963)$.

64803 C. D. Bowman, G. F. Auchampaugh, S. C. Fuitz; Phys. Rev. 133, 8676 (i964).

64Brl R. L. Bramblett, J. T. Caldwe17, R. R. Harvey, S. C. Fultz; Phys. Rev. 133, B869 (1964).

64ful S. C. Fultz, R. L. Bramblett, J. T. Caldwell, R. R. Harvey; Phys. Rev. 133, 81149 (1964).

$64 \mathrm{Ha} 2$ R. R. Marvey, J. T. Caldwrll, R. L. Branblett, S. C. Fultz; Phys. Rev. 136, B126 (1964).

65Bel B. L. Berman, R. L. Bramblett, J. T. Caldwell, R. R. Harvey, S. C. Fultz; Phys. Rev. Lett. 15, 727 (1965).

65 Cal J. T. Caldwell, R. L. Bramblett, B. L. Berman, R. R. Harvey, S. C. Fultz; Phys. Rev. Lett. 15, 976 (1965).

66Axl F. Axel, J. Miller, C. Schuh7, G. Tamas, C. Tzarä; J. Physique 27, $262(7966)$.

$66 \mathrm{Br} 7$ R. L. Bramblett, J. T. Caldwell, B. L. Berman, R. R. Harvey, S. C. Fultz; Phys. Rev. 1488, 1198 (1966).

66Ful S. C. Fultz, J. T. Caldweil, B. L. Berman, R. L. Bramblett, R. R. Harvey; Phys. Rev. 143, 790 (1966).

66Lol W. A. Lochstet, W. E. Stephens; Phys. Rev. 141, 1002 (1966).

66Mi2 J. Milier, C. Schuh1, G. Tamas, C. Tzara; J. Phjsique 27, 8 (1966).

67 Be2 B. L. Berman, J. T. Caldwell, R. R. Karvey, M. A. Kelly,

R. L. Bramblett, S. C. Fultz; Phys. Rev. 162, 1098 (1967).

68Be5 R. Bergère, H. Beil, A. Veyssière; Nucl. Phys. Al2l, 463 (1968). 
68Sul R. E. Fand, M. P. Baker, L. A. Kull, R. B. Halton; Phys. Rev. 176, 1366 (1968).

69Bel B. L. Berman, R. L. Bramblett, J. T. Caldwell, H. S. Dai is, M. A. Kelly, S. C. Fultz; Phys. Rev. 177, 1745 (1969).

698e6 R. Bergère, H. Beil, P. Carlos, A. Veyssière, Nucl. Phys. Al33, 417 (1969).

$698 \mathrm{~B} 8$ B. L. Berman, M. A. Kelly, R. L. Bramblett, J. T. Caldwell,

H. S. Davis, S. C. Fultz; Phys. Rev. 185, 1576 (1969).

69Ful S. C. Fultz, B. L. Berman, J. T. Caldwell, R. L. Bramblett,

M. A. Kelly; Phys. Rev. 186, 1255 (1969;.

70 Be8 B. L. Berman, S. C. Fultz, J. T. Caldwell, M. A. Kelly,

S. S. Dietrich; Phys. Rev. C 2, 2318 (1970).

70Sul R. E. Sund, V. V. Verbinski, H. Weber, L. A. Kull; Phys. Rev. C 2, $1129(1970)$.

7ovel A. Veyssière, H, Beil, R. Bergère, P. Carlos, A. Leprêtre; èucl. Phys. Al59, 561 (1970).

$71 A 17$ R. A. Alvarez, B. L. Parman, D. R. Lasher, T. W. Phillips,

S. C. Fultz; Phys. Re'. C 4, 1673 (1971).

$71 \mathrm{Be} 3$ B. L, Berman, S. C. Fultz, M. A. Kelly; Phys. Rev. C 4, 723 (1971).

7lBe4 H. Beil, R. Bergère, P. Sarlos, A. Lepretre, A. Veyssière,

A. Parlag; Nuc 1. Phys. A172, 426 (1971).

TlCal P. Carlos, H, Beil, R. Bergère, A. Leprêtre, A. Veyssière; Nucl. Phys. Al72, 437 (1971).

7lFuz S. C. Fultz, R. A. Alvarez, B. L. Berman, H. A. Kelly, D. R. Lasher, T. W. Phillips, J. McElhinney; Phys. Rev. C 4, 149 (1971).

7ll.el A. Leprêtre, H. Beil, R. Bergère, P. Carlos, A. Veyssière, M. Sugawara, Nuc1. Phys. A775, 609 (1971).

72Yo L. M. Young. Ph.D. Thesis, U-iversity of Illinois (1972), unpubl ished.

$73 \mathrm{Br} l l$ R. L. Bramblett, B. L. Berman, H. A. Kelly, J. T. Caldwell, S. C. Fultz; Proc. Int. Conf. Photonuclear Reactions and Applications (1973), p. 175.

73vel A. Veyssière, H. Beil, R. Beirsère, P. Carlos, A. Leprêtre, K. Kernbach; Nuc 1. Phys. A]99, 45 (1973).

74Be3 H. Beil, R. Bergère, P. Carlos, A. Leprêtre, A. de Miniac, A. Veyssière; Nucl. Phys. A227, $427\left(19^{7} 4\right)$. 
74Be8 B. L. Berman, S. C. Fultz, P. F. Yergin; Phys. Rev. C 10, ¿221 (1974).

74 Ca5 P. Carlos, H. Beil, R. Bergere, A. Lepretre, A. de Miniac,

A. Veyssière; Nucl. Phys. A?.25, 171 (1974).

74fu3 S. C. Fultz, R. A. Aivarez, B. L. Berman, P. Meyer; Phys. Rev, C 10, 608 (1974).

74Lel A. Lepretre, H. Beil, R. Bergère, P. Carlos, A. de Miniac,

A. Veyssière, K. Kernbach; Nucl. Phys. A279, 39 (1974).

74Vel A. Veyssière, H. Beil, R. Bergère, P. Carlos, A. Leprêtre,

A. de Miniac; Nucl. Phys. A227, 513 (1974).

75Kn5 U. Kneiss1, G. Kuhl, K. H. Leister, A. Heller; Nucl. Phys. A247, 91 (1.79).

75kn8 U. Kneiss1, E. A. Koop, G. Kuhl, K. H. Leister, A. Weller; Nuct. Instrum. Methods 127, 1 (1975).

75ves A. Veyssière, H. Bei1, R. Bergère, P. Car1os, A. Leprêtre,

A. de Miniac; J. Physique 36, L-267 (1975).

76Cal P. Carlos, H. Beil, R. Bergère, J. Fagot, A. Leprêtre,

A. Veyssière, G. V. Solodukhov; Nuc1. Phys. A258, 365 (1976).

$76 \mathrm{Kn3}$ U. Kneissl, K. H. Leister, H. O. Neidel, A. Weller; Nucl. Phys. A264, 30 (1976).

$76 \mathrm{Kn4}$ U. Kneiss1, K. H. Leister, H. O. Neidel, A. Weller; Nucl. Phys. A272, 125 (1976).

76Le2 A. Leprêtre, H. Beil, R. Bergère, P. Carlos, J. Fagot, A. de Miniac, A. Veyssiere, H. Miyase; Nuc 1. Phys. A258, 350 (1976).

79 Al2 R. A. Alvarez, B. L. Berman, D. D. Faui, F. H. Lewis, Jr.,

P. Meyer; Phys. Rev. C 20, 128 (1979).

79 Be 4 B. L. Berman, D. D. Faul, R. A. Alvarez, P. Meyer, D. L. 0lson;

Phys. Rev. C 19, 1205 (1979).

79Ju3 J. H. Jury, B. L. Berman, D. D. Faul, P. Meyer, K. G. MoNeill,

J. G. Woodworth; Phys. Rev. C 19, 1684 (1979).

79Wo? J. E. Woodworth, K. G. MoNeill, J. W. Jury, R. A. Alvarez,

B. L. Berman, D. D. Faul, P. Meyer, Phys. Rev. C 19, 1667 (1979).

80Be1 B. L. Berman, D. D. Faul: P. Meyer, D. L. 01son; Phys. Rev. C 2̌́, 2273 (1980).

80Ca 7 J. T. Caldwell, E. J. Dowdy, B. L. Berman, R. A. Alvarez,

P. Meyer; Phys. Rev. C 21, 503 (1980). 
$80 J u 4$ J. W. Jury, B. L. Berman, D. D. Faul, P. Meyer, J. G. Woodworth; Phys. Rev. C 21, 503 (1980).

BlFal D. D. Faul, B. L. Berman, P. Meyer, D. L. 01son; Phys. Rev. C 24 , 849 (1981).

Q2Ju2 J. W. Jury, B. L. Berman, J. G. Woodworth, M. N. Thompson, R. E. Pywe 1], K. G. Mcleill; Phys. Rev. C 26, 777 (1982).

83Be] B. L. Berman, J. W. Jury, J. G. Woodworth, R. E. Pywel1, K. G. McNeil1, M. N. Thompson; Phys. Rev. C 27, 1 (1983).

83Pyl R. E. Pywell, B. L. Berman, J. H. Jury, J. G. Woodworth, K. G. McNeill, M. K. Thompson; Phys. Rev. C 27, 960 (1983).

85Pyl R. E. Pywell, B. L. Berman, J. G. Woodworth, J. W. Jury, K. G. Heil1, M. N. Thompson; Phys. Rev. C 32, 384 (1985).

86Bel B. L. Berman, R. E. Pywell, M. N. Thompson, K. G. McNeil1, J. W. Jury, J. G. Woodworth; Bull. Am. Phys. Soc. 31, 855 (1986).

86Be2 B. L. Berman, J. T. Caldwel], 5. J. Dowdy, S. S. Dietrich, P. Meyer, R. A. Alvarez, Bull. Am. Phys. Soc. 31, 855 (1986). 
The graphs presented are 1 isted in the table with relevant figure numbers. Figure $B$, on the following page, shows, in diagr ammatic style, the stable nuclei for which giant-resonance cross-section sęasurements have been made with monoenergetic photons.

The following designations are usea on the graphs:

(a) Photoneutron yield cross section

$$
o(r, S n)=o[(r, i n)+2(\gamma, 2 n)+3(\gamma, 3 n)+\bar{v}(r, f)]
$$

(b) Total photoneutron cross section $o(r$, tot $)=c[(\gamma, \ln )+(\gamma, 2 n)+(\gamma, 3 n)+(\gamma, f)]$.

(c) Single photoneutron cross section $c(\gamma, l n)=c[(\gamma, n)+(\gamma, p n)]$.

(d) Double photoneutron cross section $o(r, 2 n)=o[(r, 2 n)+(r, p 2 n)]$.

(e) Triple photoneutron cross section $c(\gamma, 3 n)$. For the ruclei ${ }^{16} 0,{ }^{31} \mathrm{P},{ }^{32} \mathrm{~S}$, and ${ }^{39} \mathrm{~K}$, part $(\mathrm{e})$ is $\sigma(\mathrm{r}, \mathrm{pn})$; ior ${ }^{10} 0$, it is $o(i, D)$; ano for the actinide nuclei, it is $o(y, f)$.

Threshold Indicated on the graphs by arrows; values are taken from Energies A. H. Wapstra and K. Bos, Atomic Data and Nuclear Data Tables 19. 215 (1977).

References for the data and the laboratories where the measurements wert made are listed in the table.

The solid curves, drawr on the graphs of the total photoneutron cross sections for most medium and heavy nuclei, are lorentz curves fitted to the giant-resonance data, whose parameters are given in the table. 


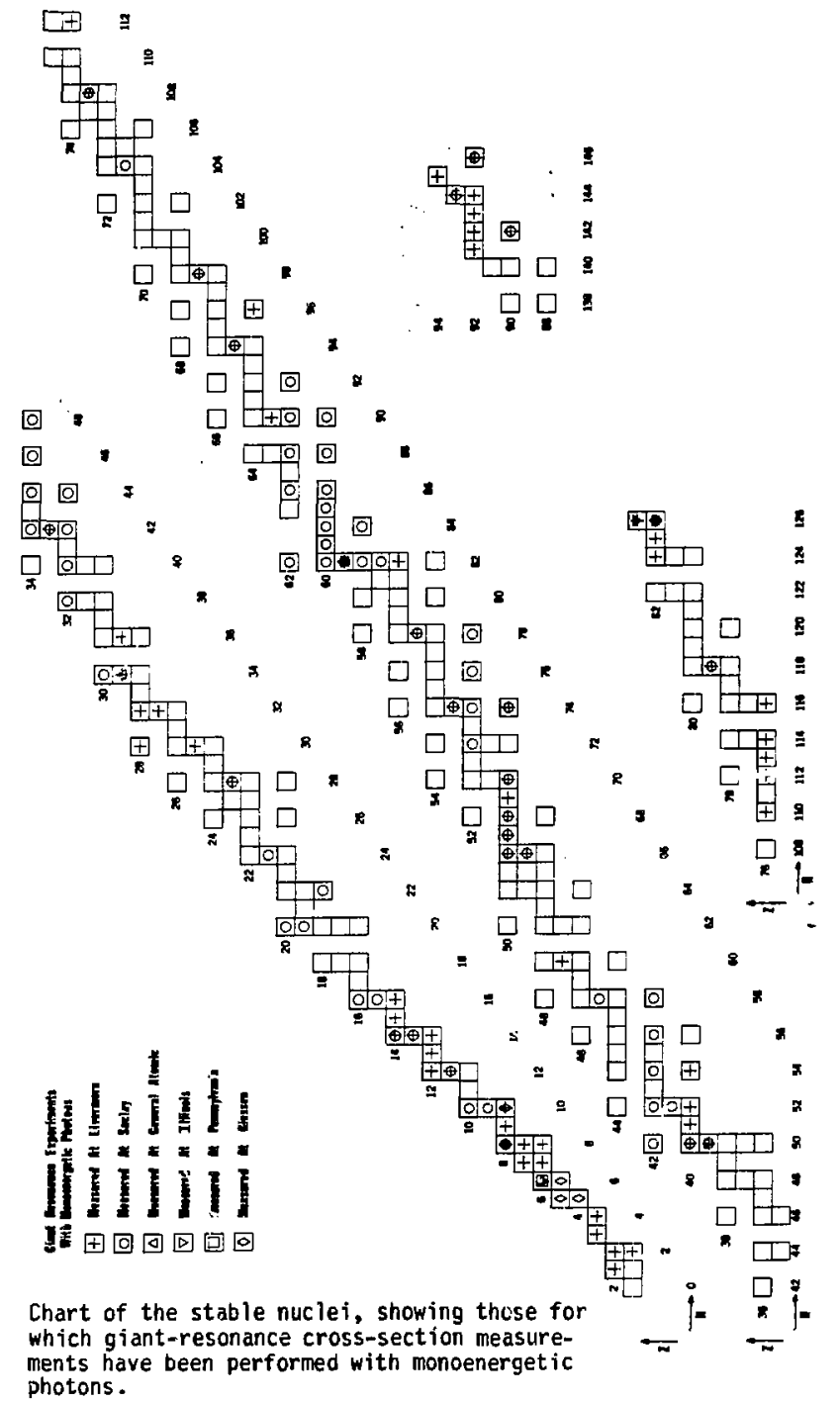

Fig. B. Chart of the stable nuclei, showing those for ments have been performed with monoenerget ic 

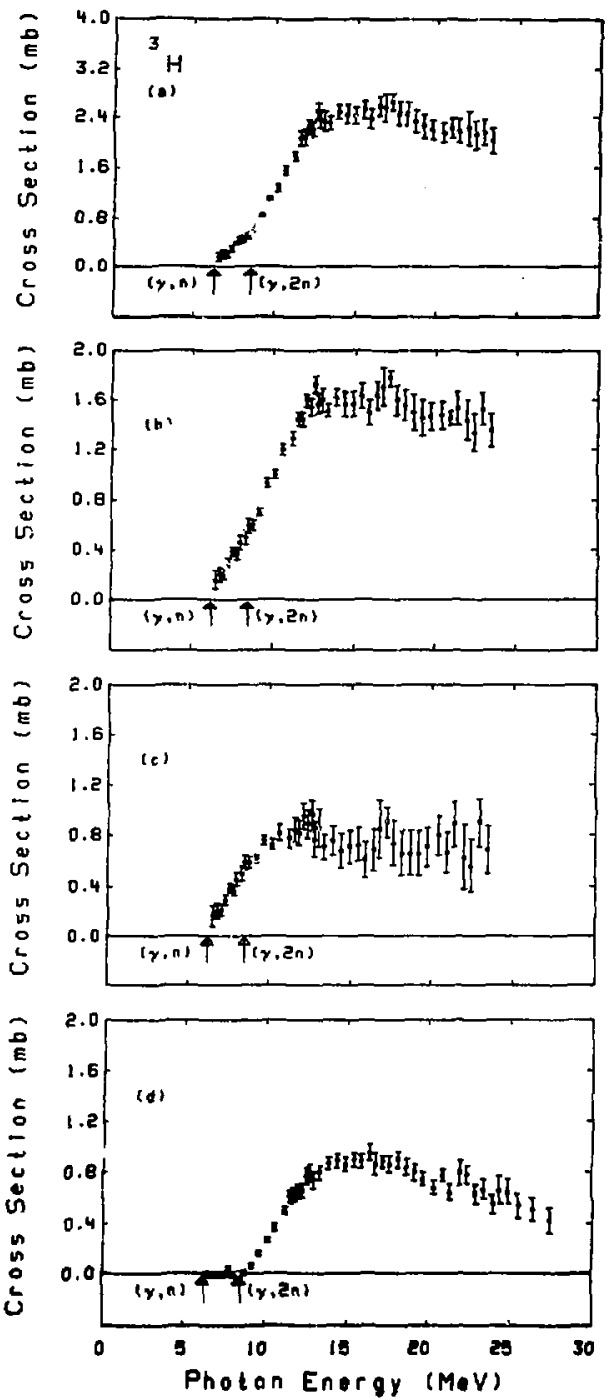

Fig. $1 A$ 


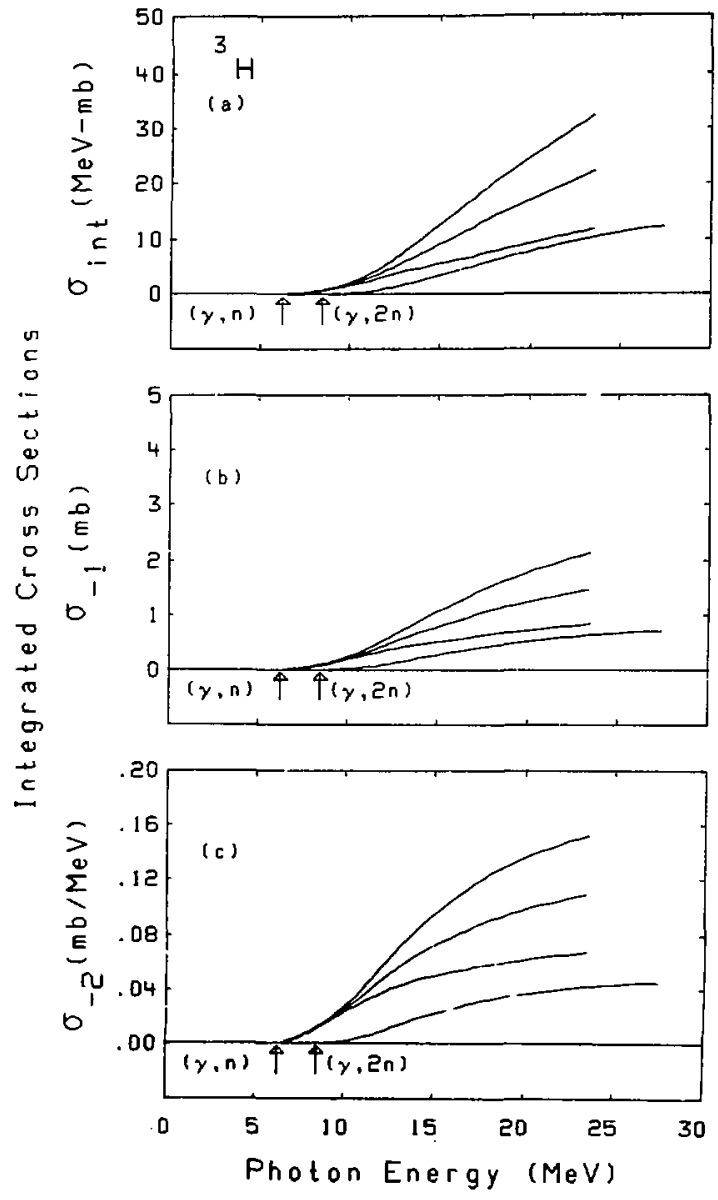

Fig. 1B 


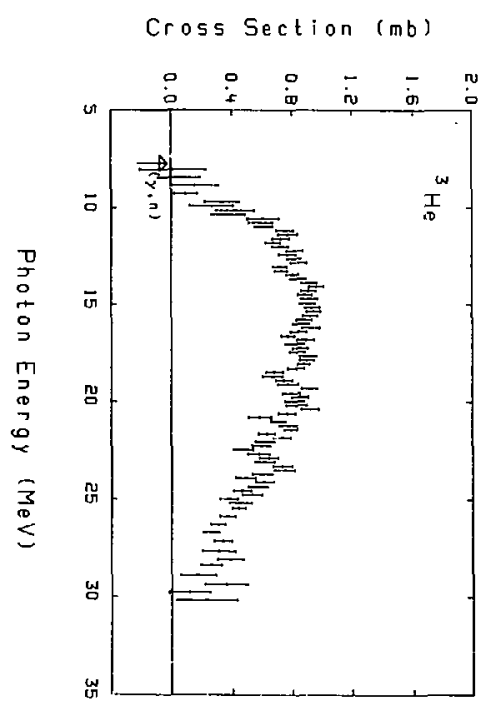




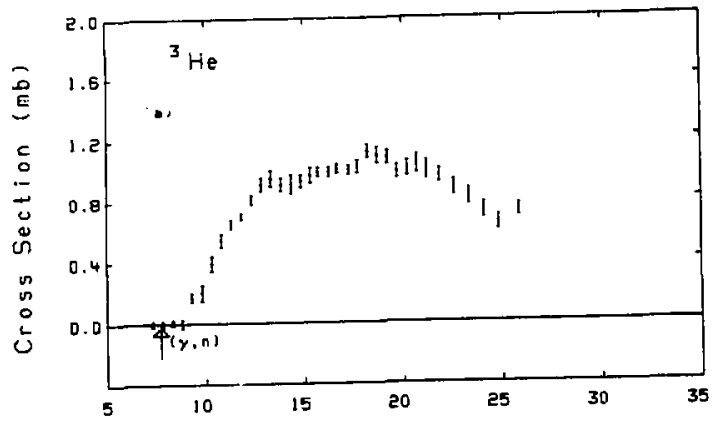

Phaton Energy (MeV)

Fig. 3A 


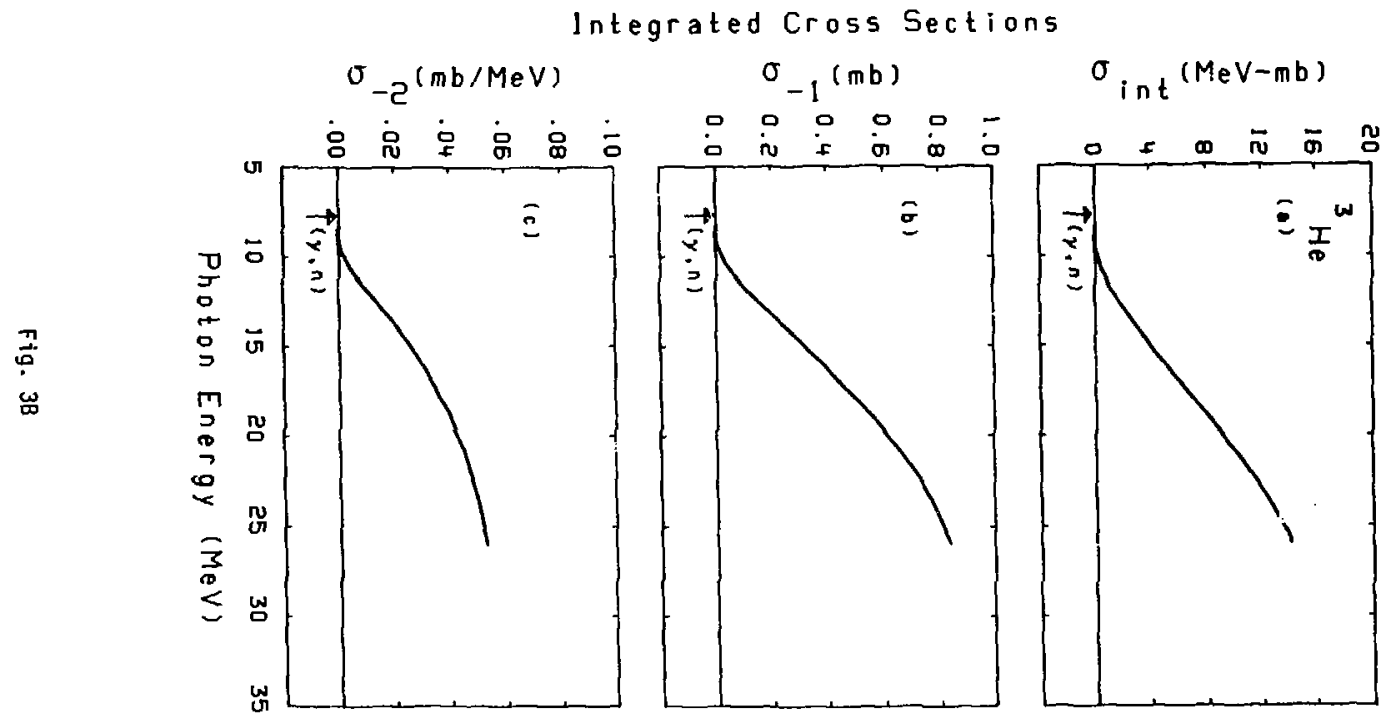




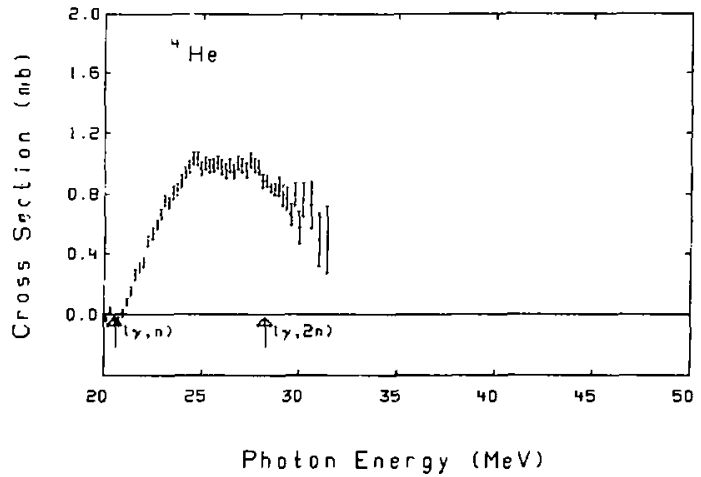

Fig. $4 A$ 


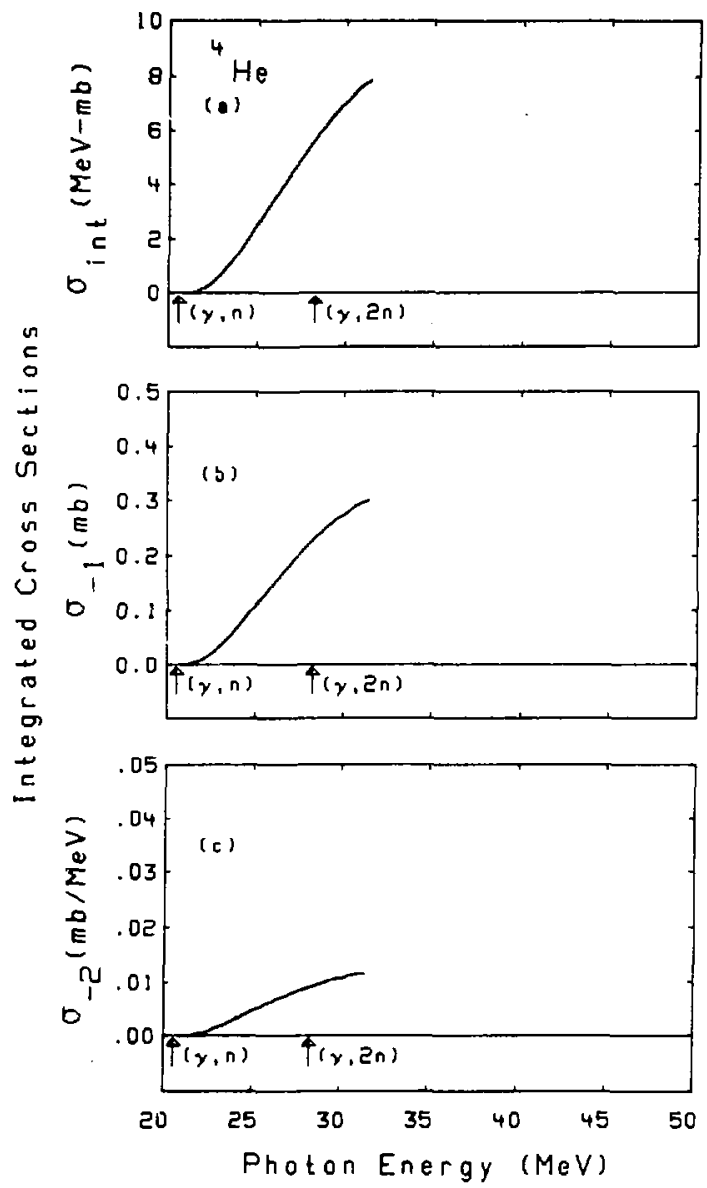

Fig. 48 


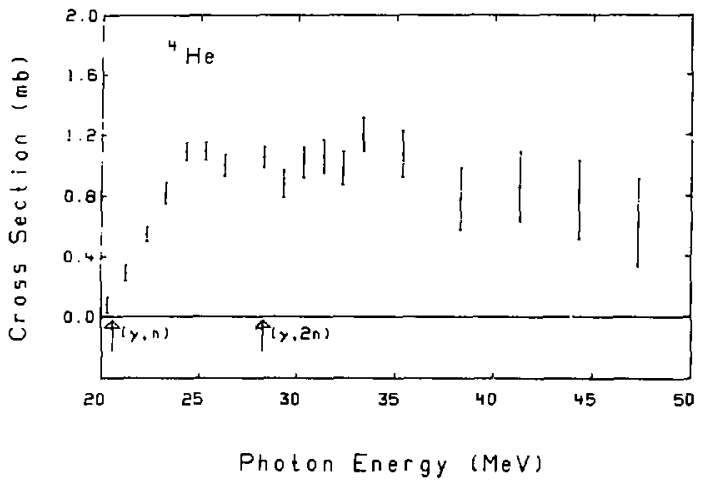

Fig. $5 A$ 


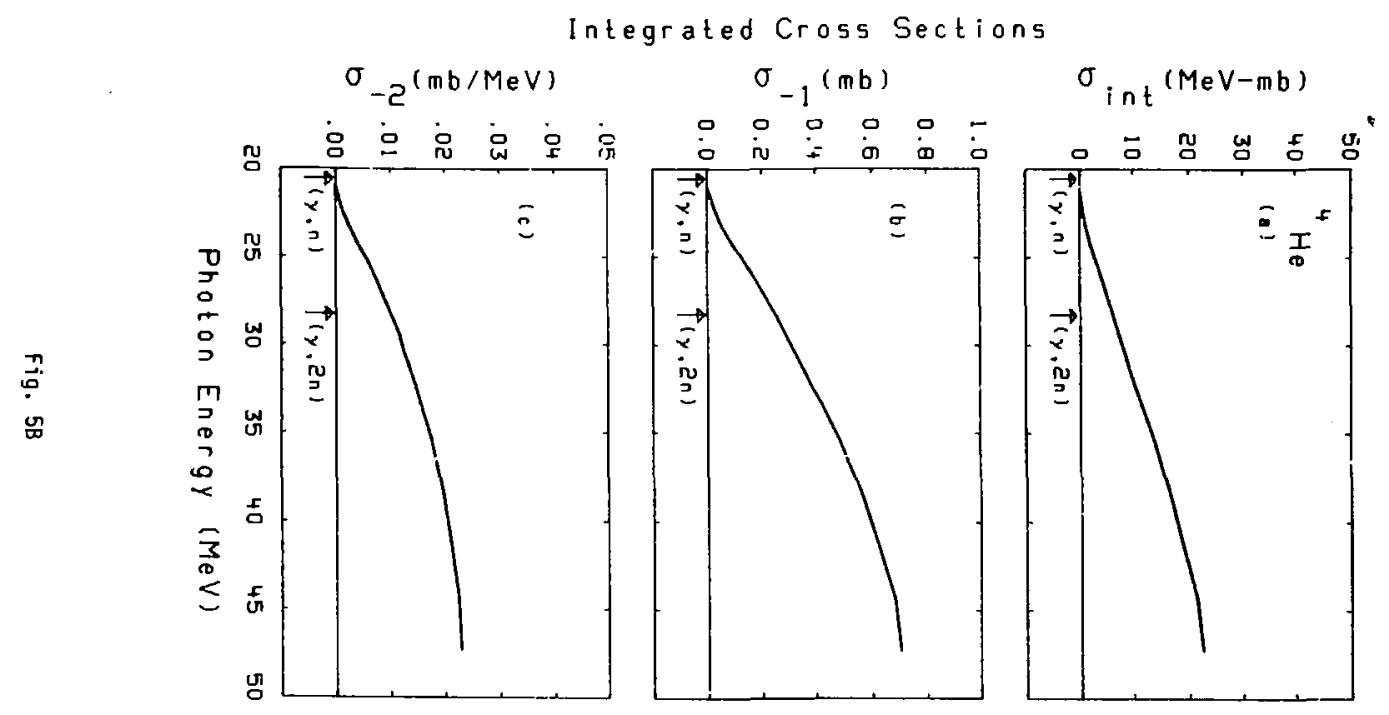



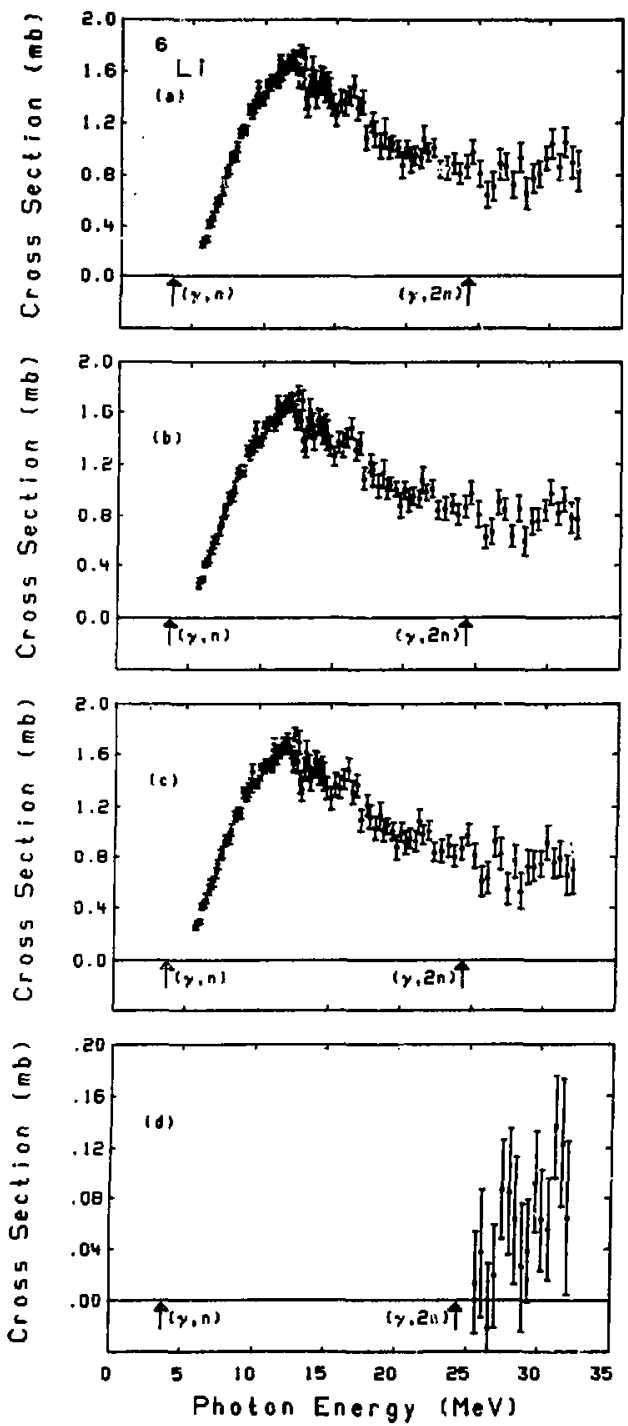

Fig. $6 \mathrm{~A}$ 


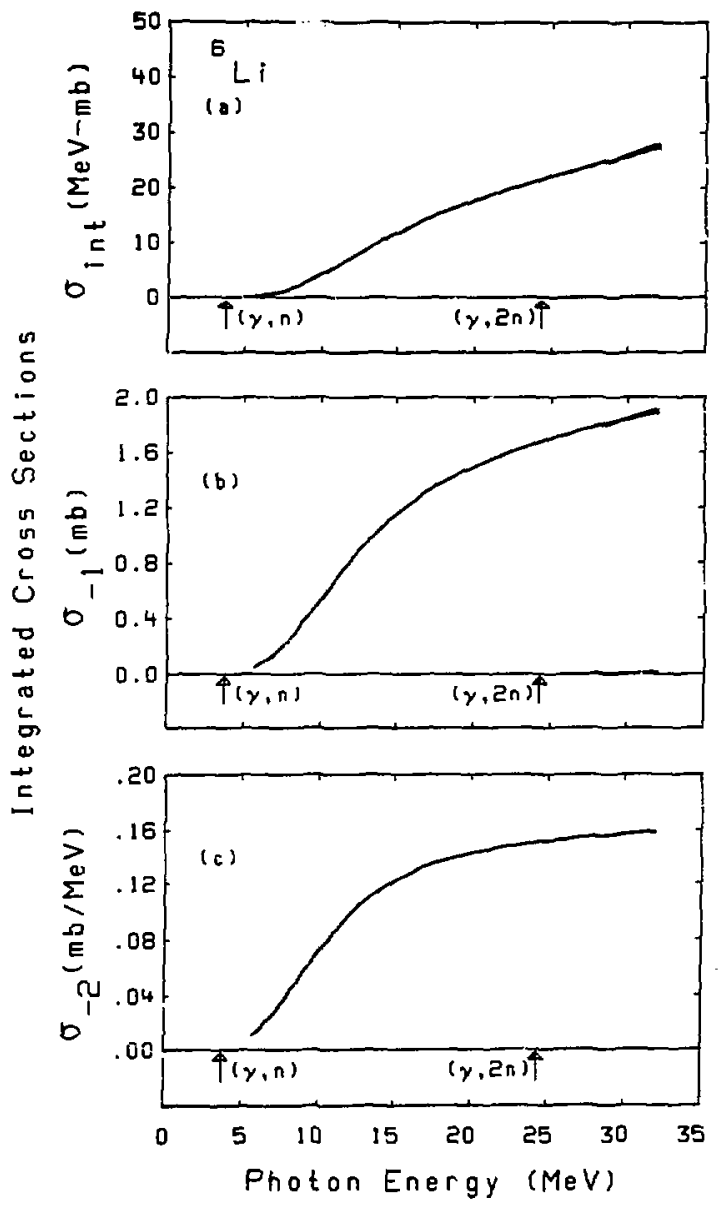

Fig. $6 \mathrm{~B}$ 

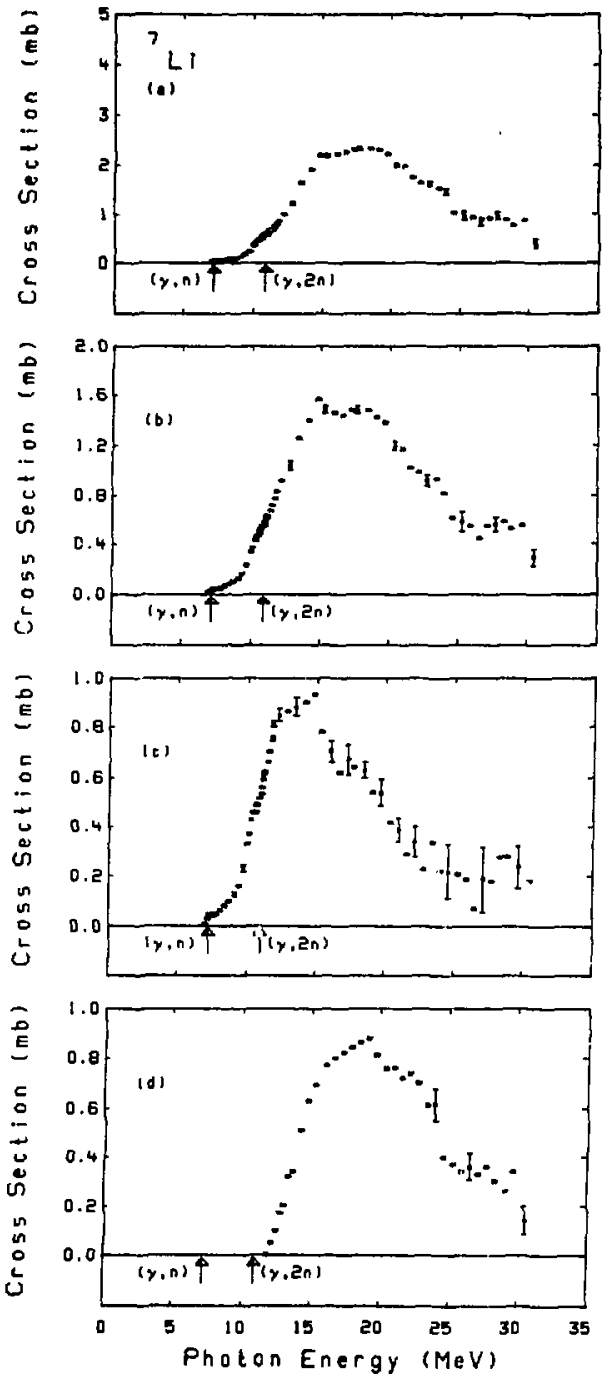

Fig. 7A 


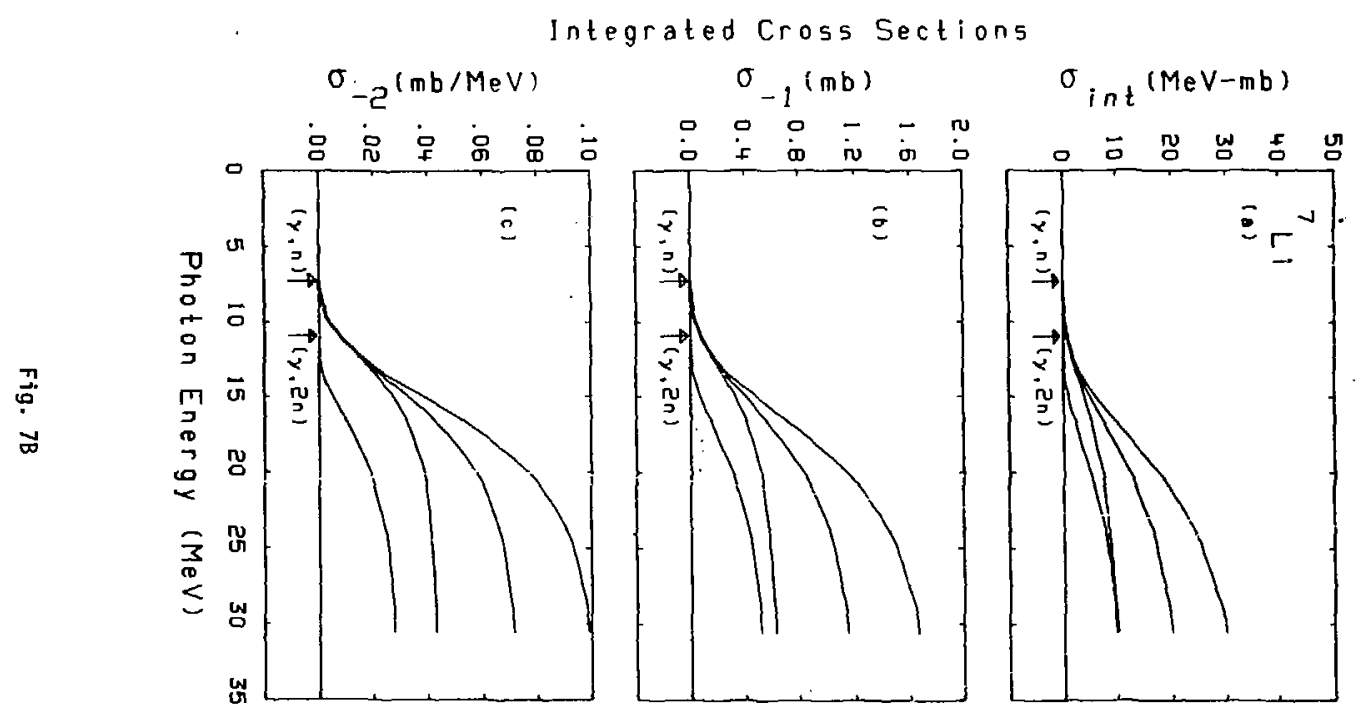



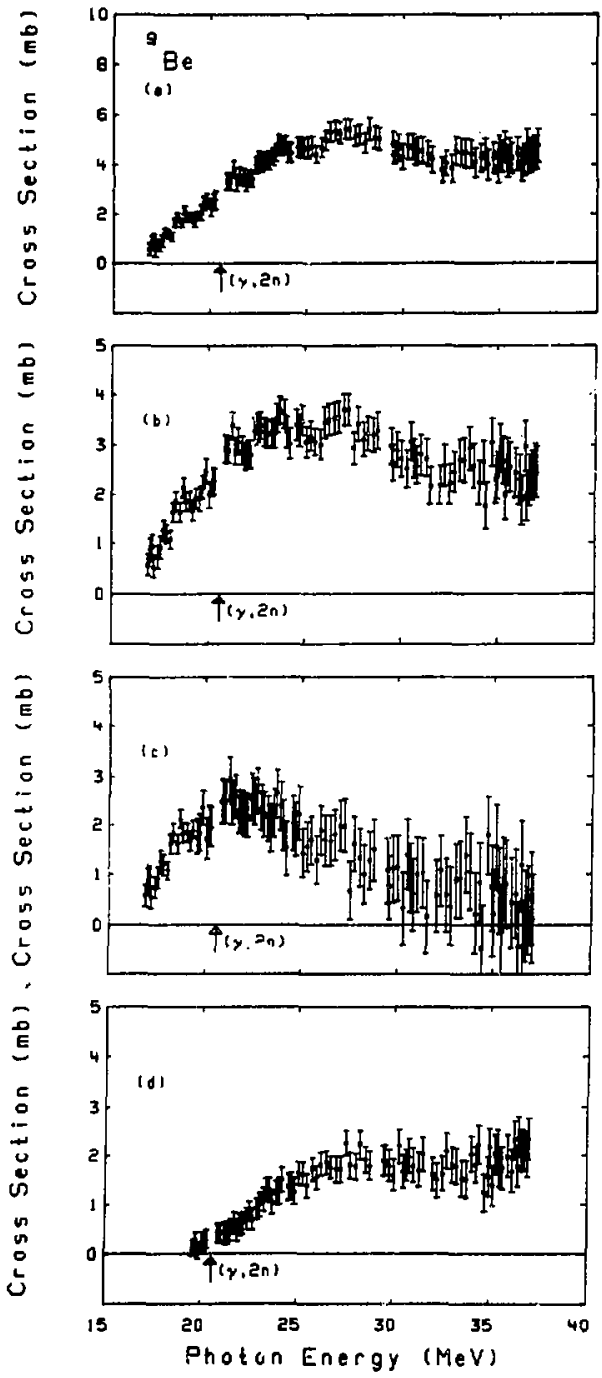

fig. $8 A$ 


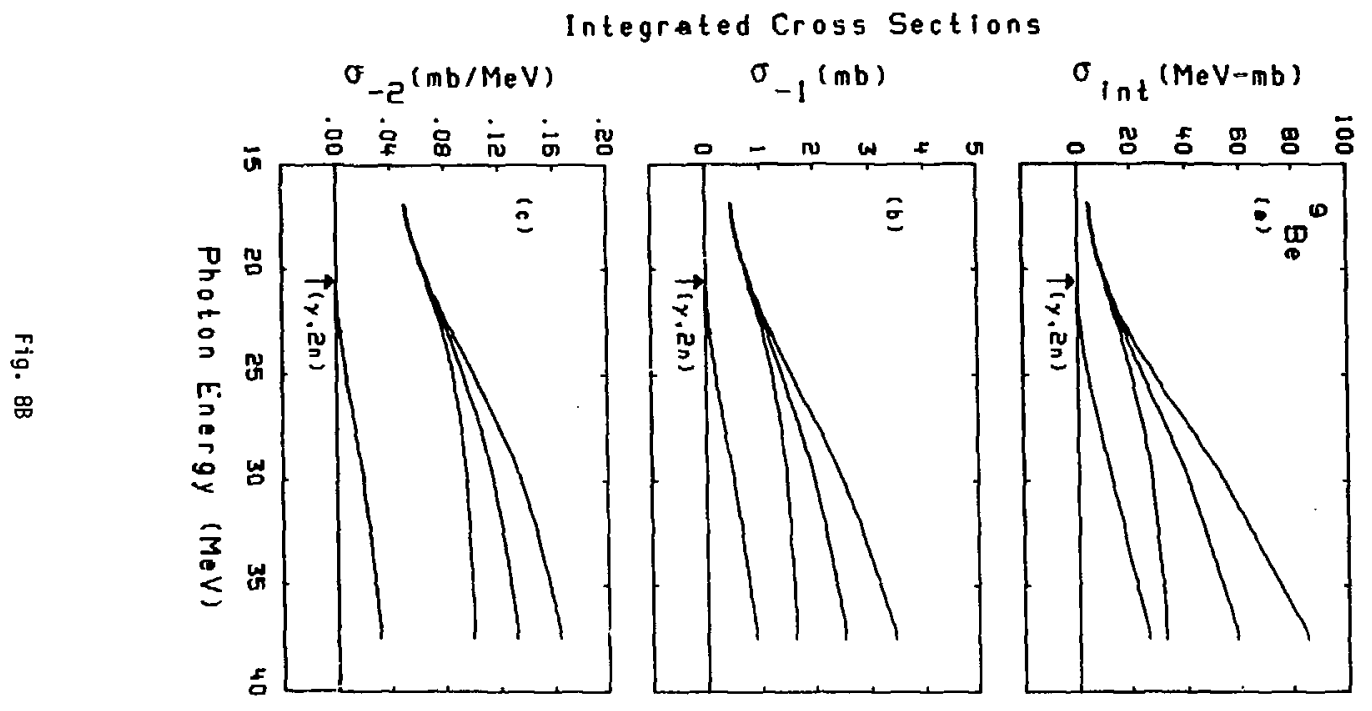



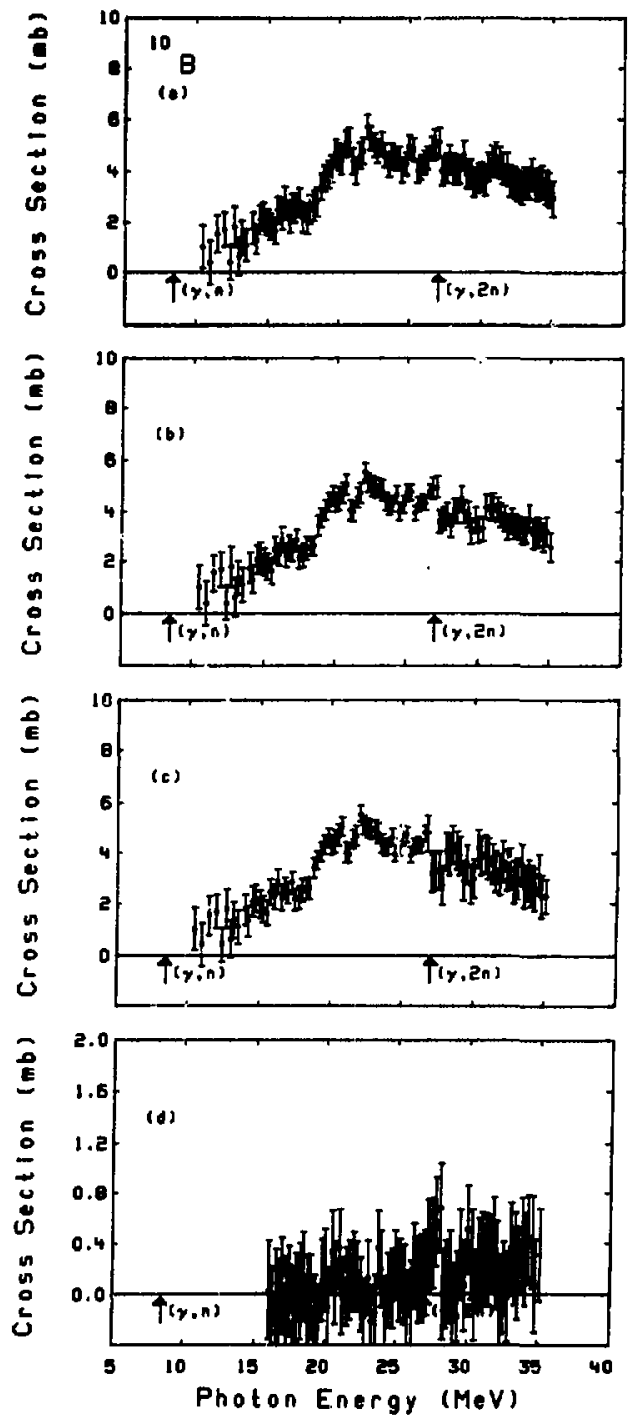

Fig. 9A 

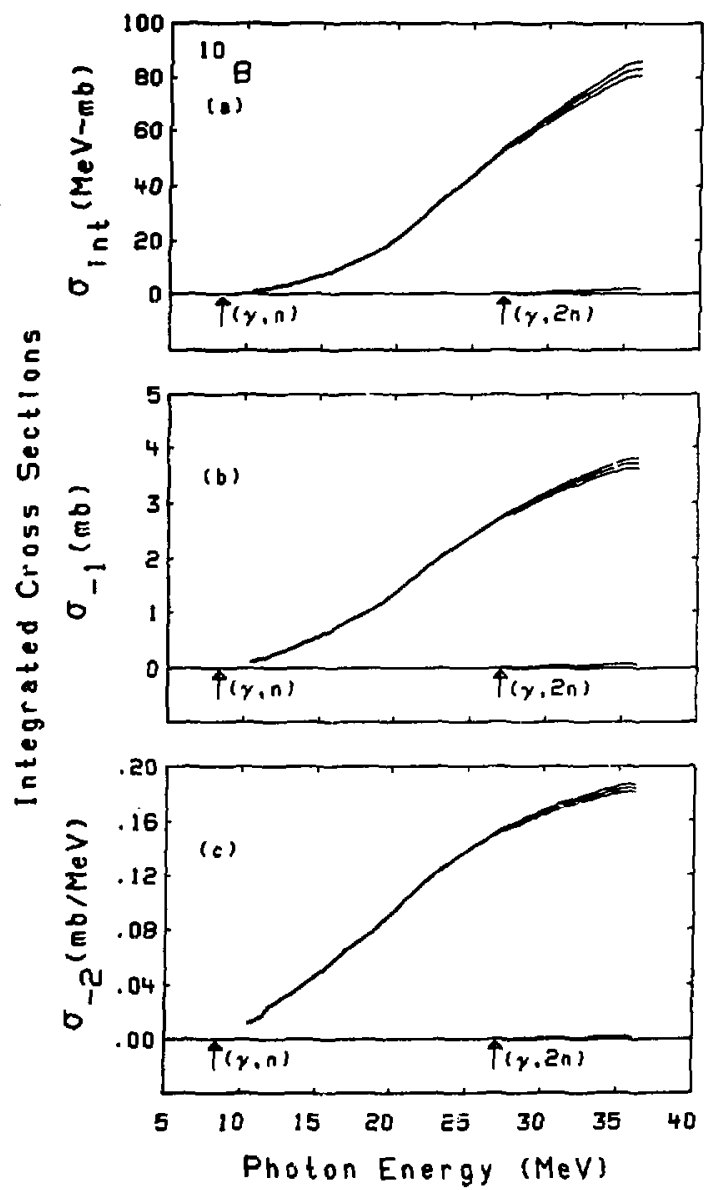

Fig. $9 B$ 

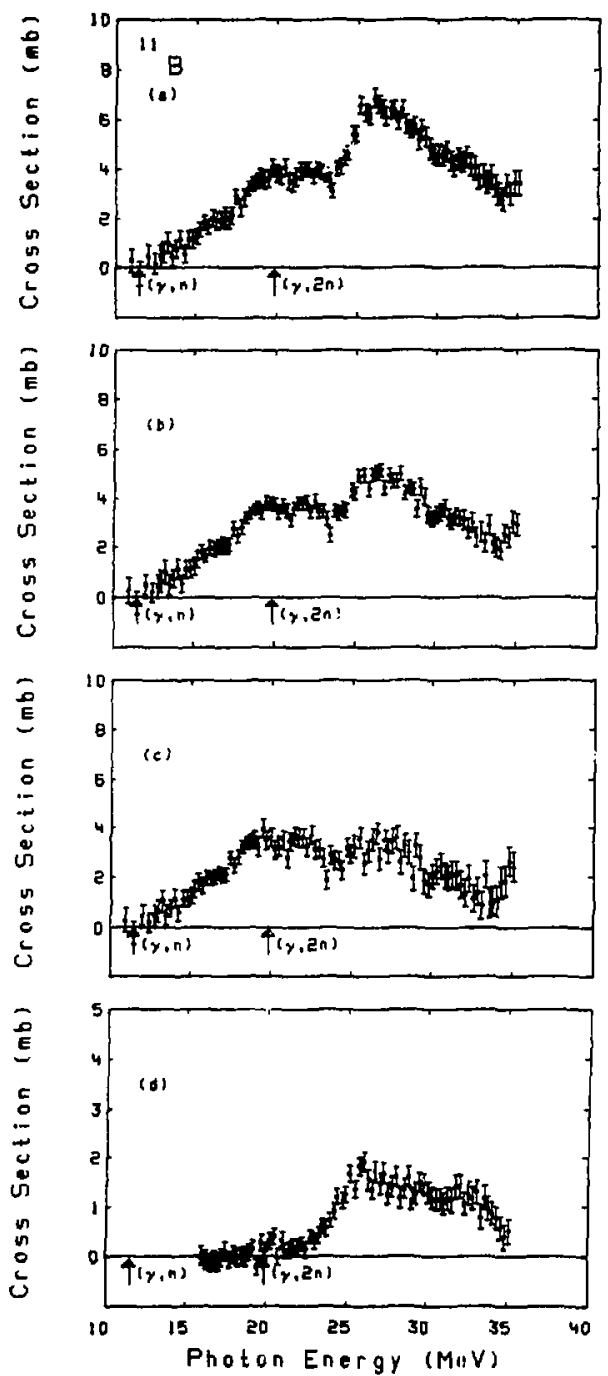

Fig. 10A 


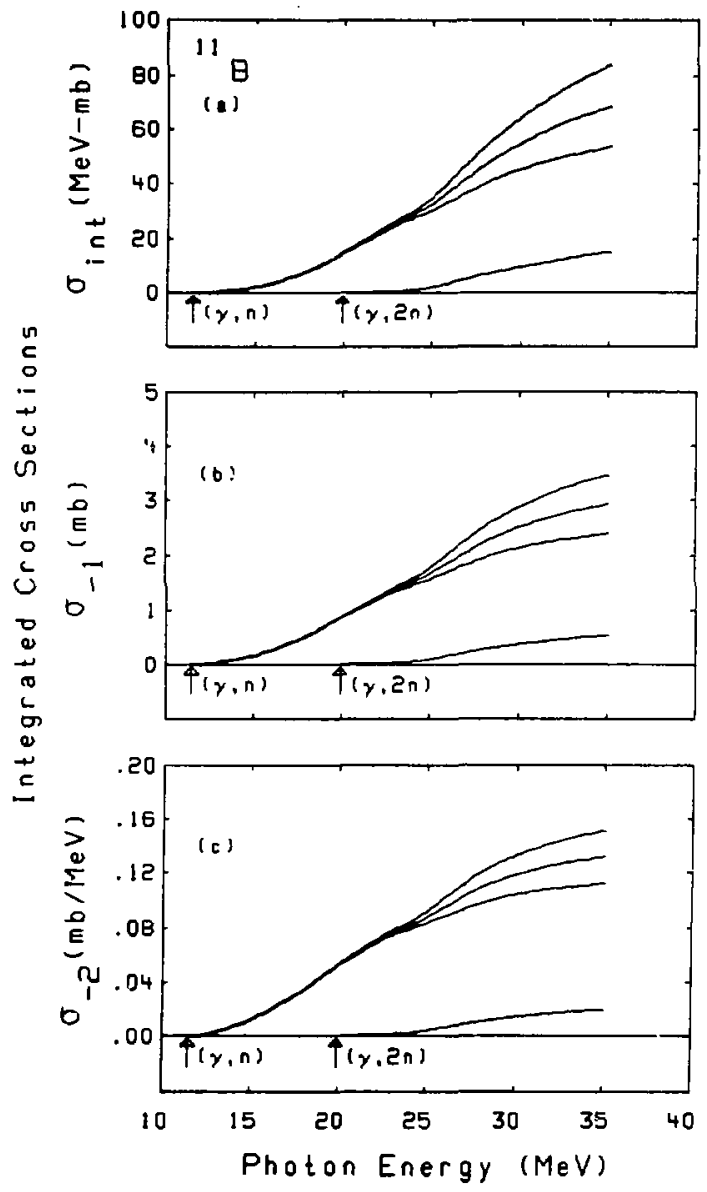

Fig. 108 


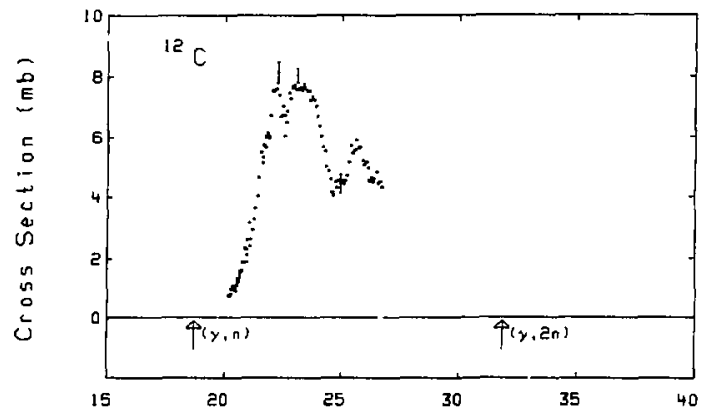

Photon Energy (MeV)

Fig. IIA 


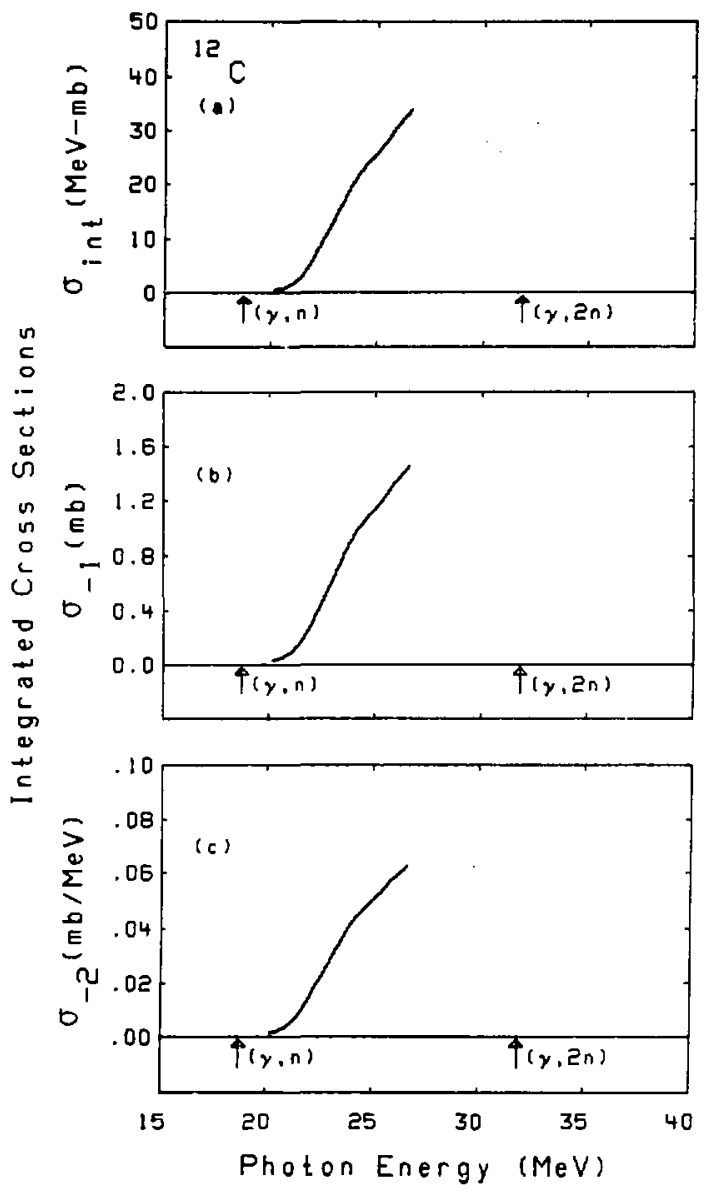

Fig. 11B 


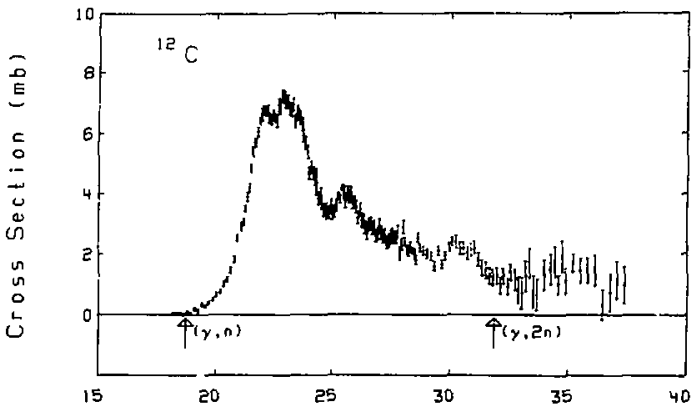

Photon Energy (MeV)

Fig. $12 \mathrm{~A}$ 


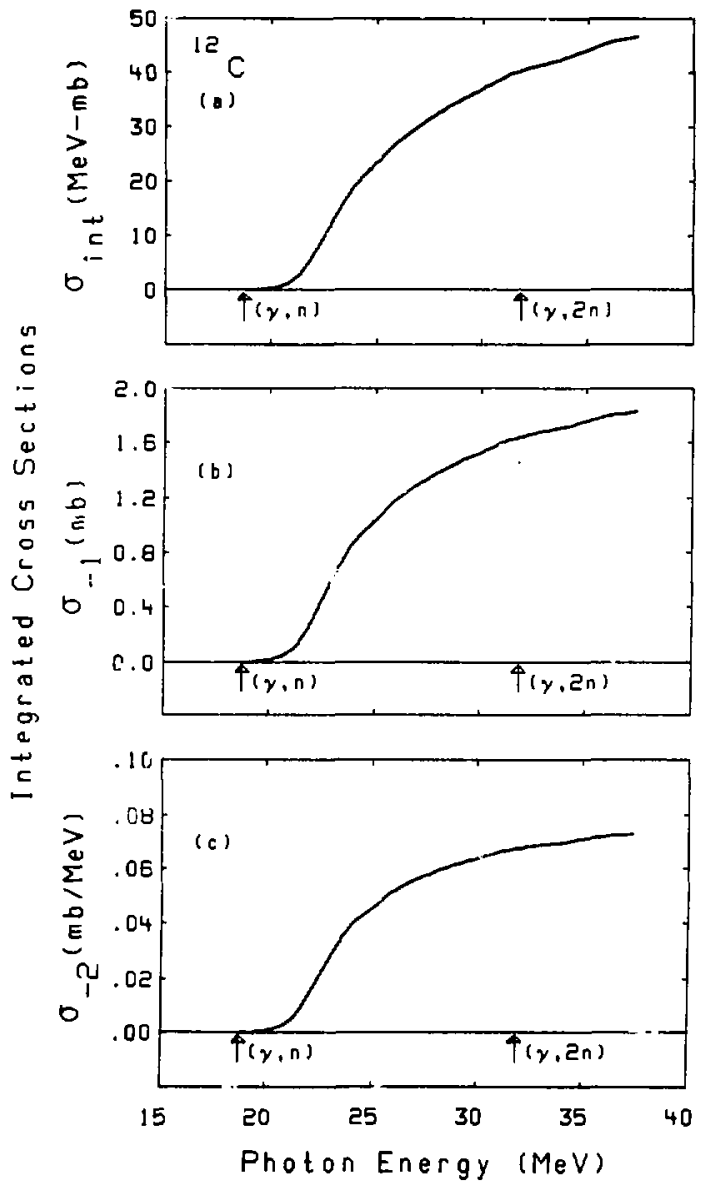

Fig. 12B 


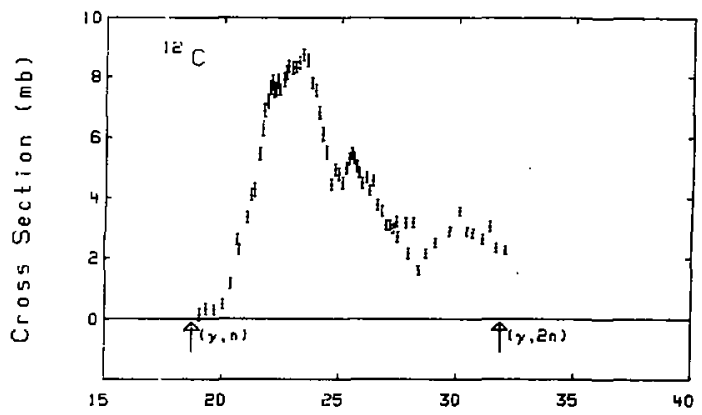

Photon Energy (MeV)

Fig. 13A 


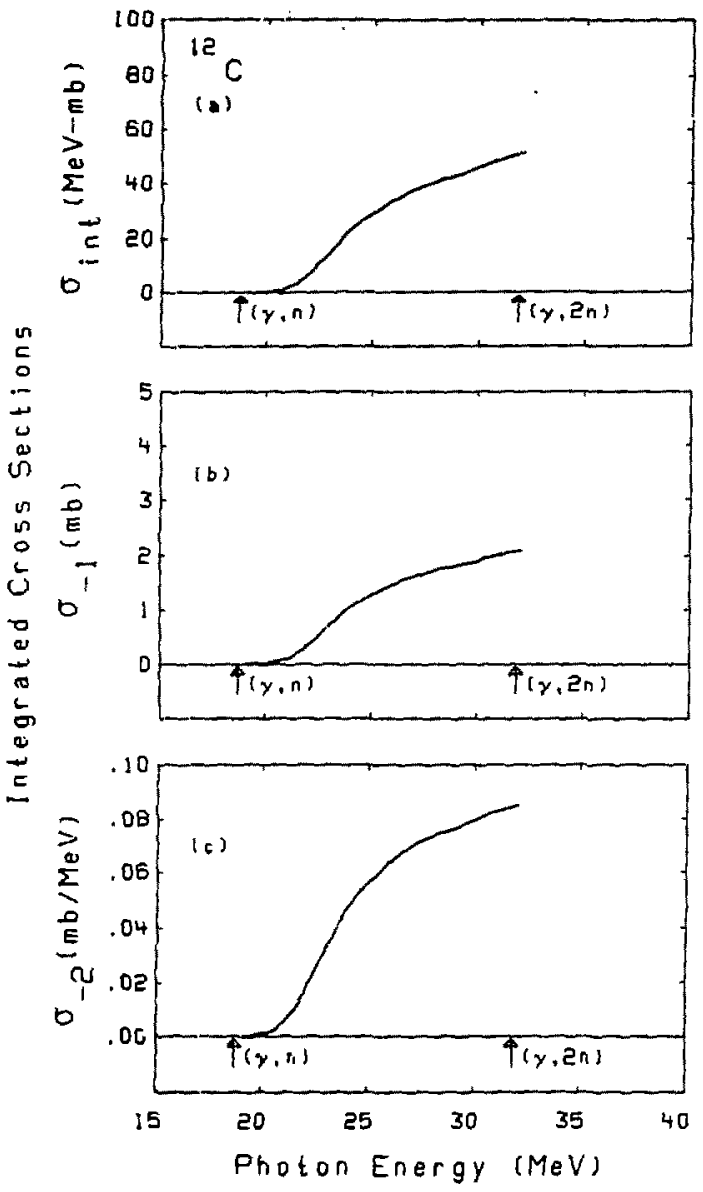

Fig. 138 

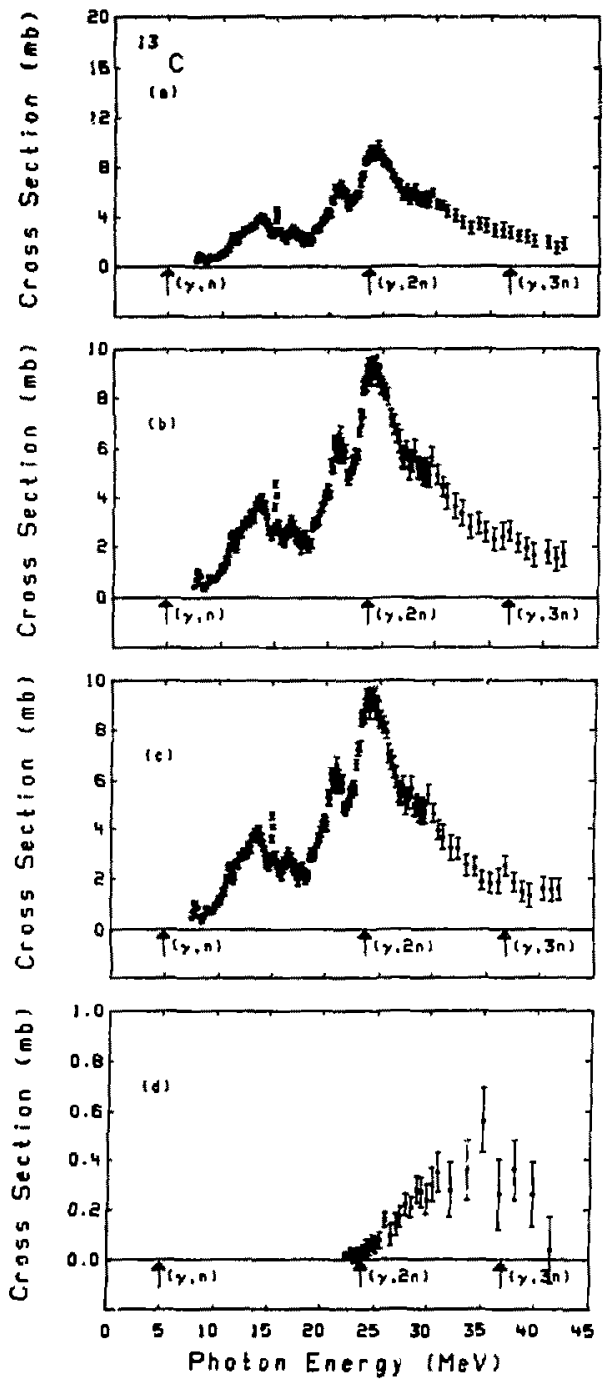


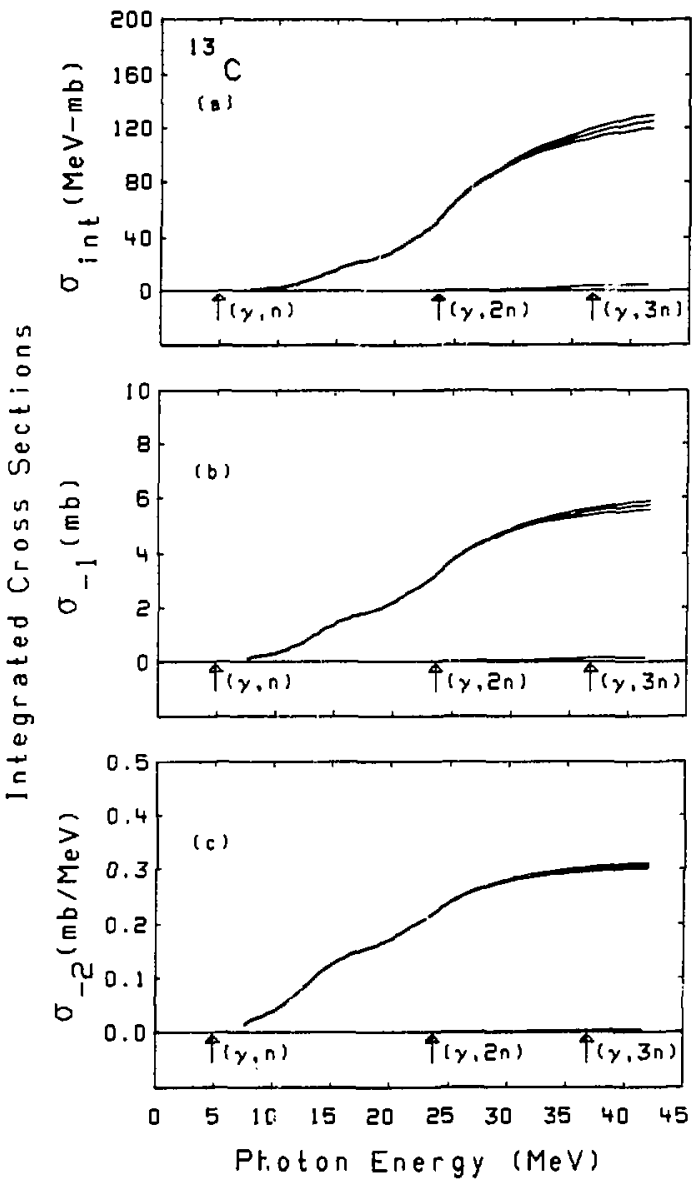

Fig. 14B 

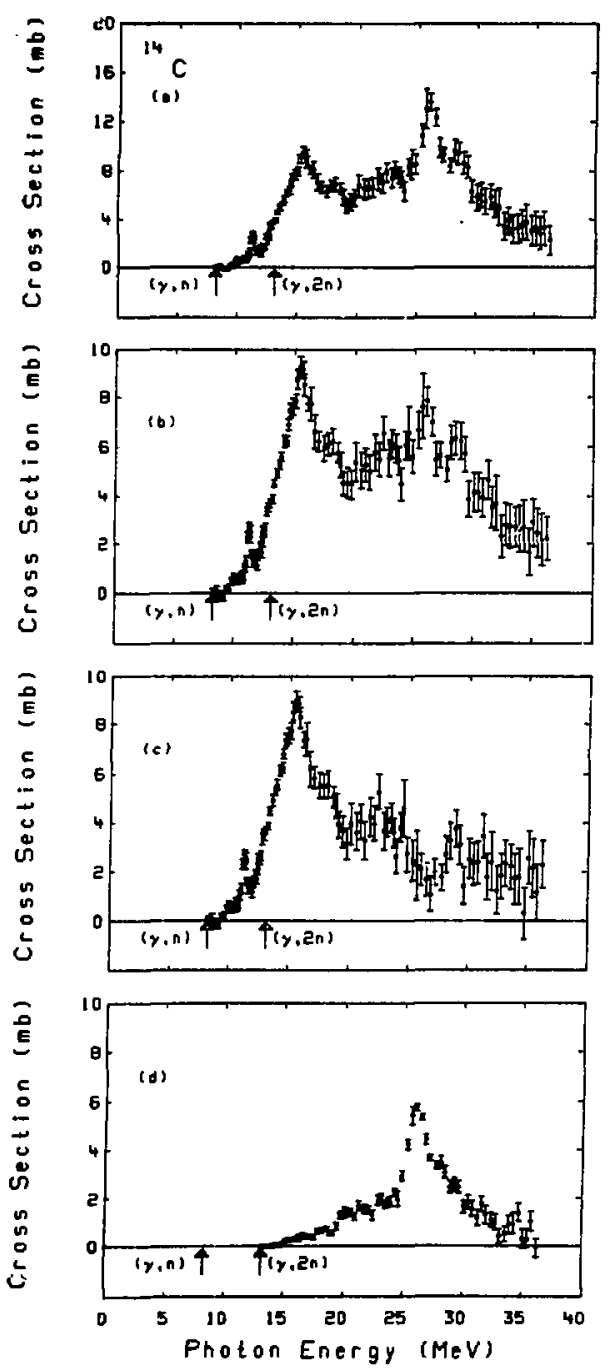

Fig. $15 \mathrm{~A}$ 


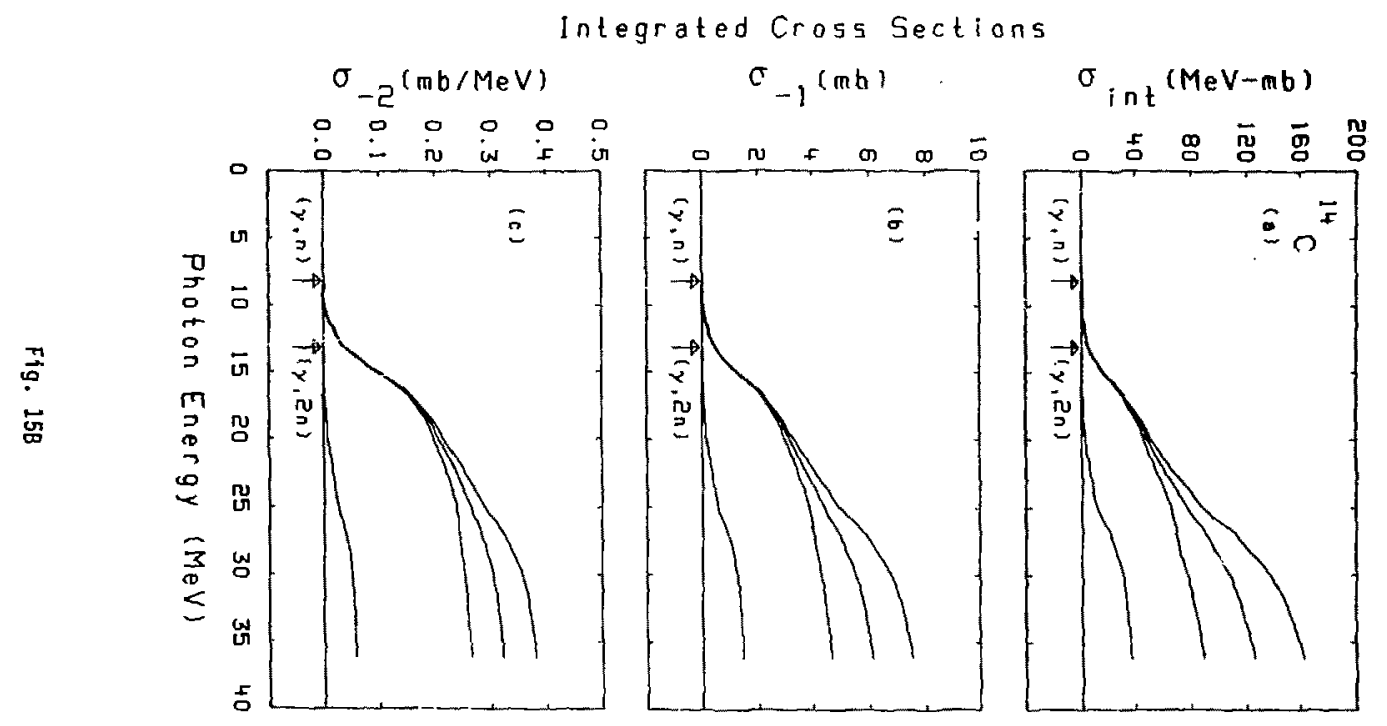




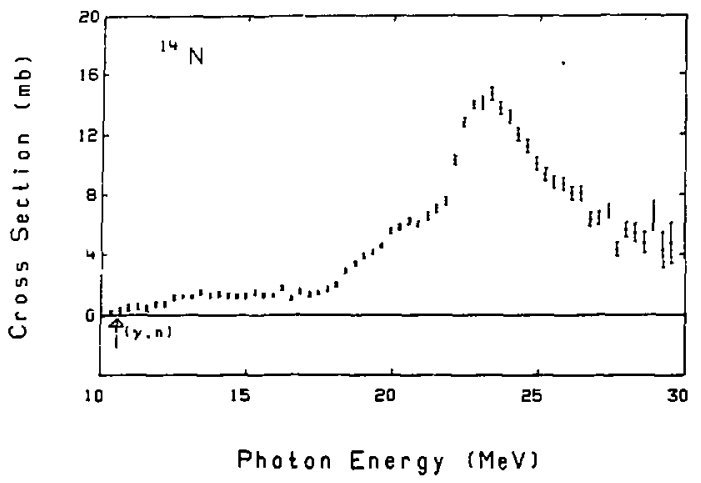

Fig. $16 \mathrm{~A}$ 


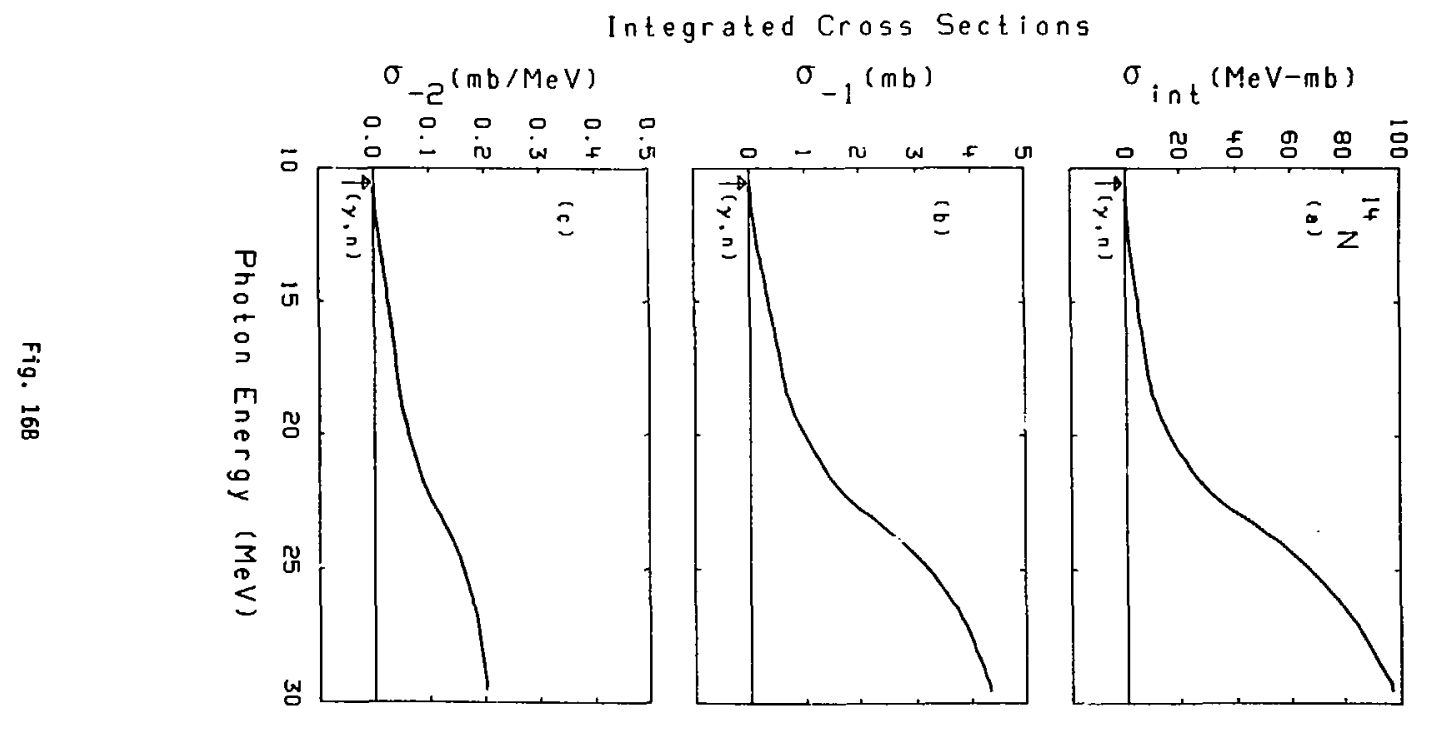



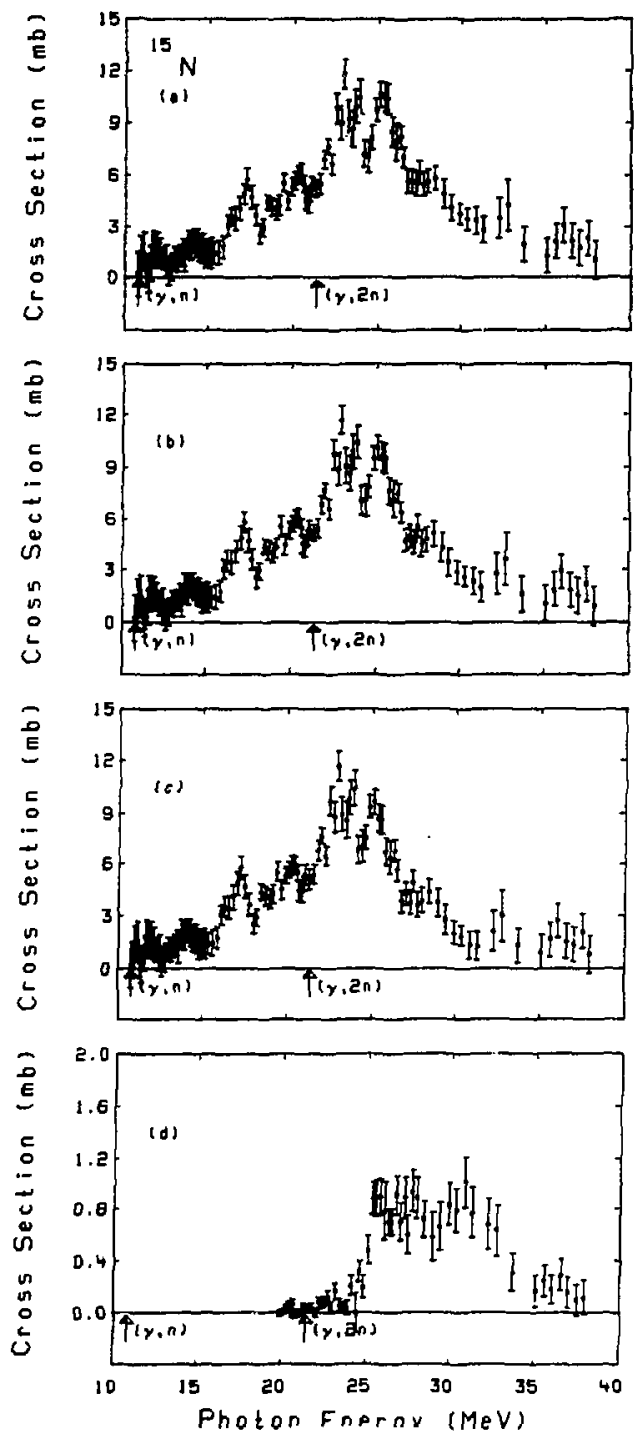

Fig. $17 A$ 


$$
\text { D }
$$




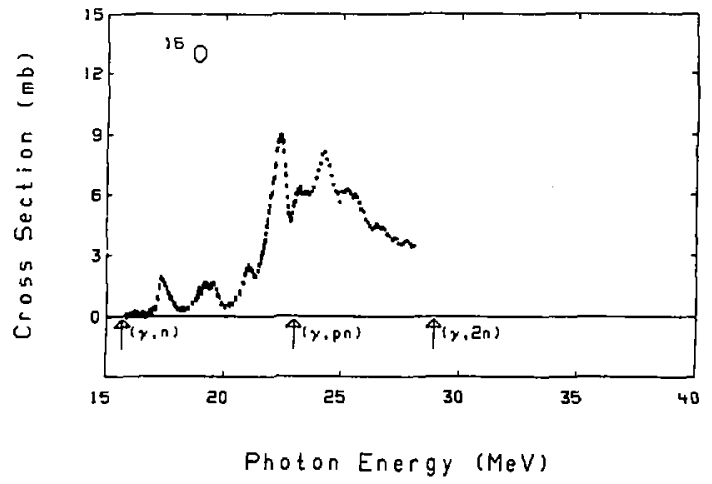

Fig. 18A 


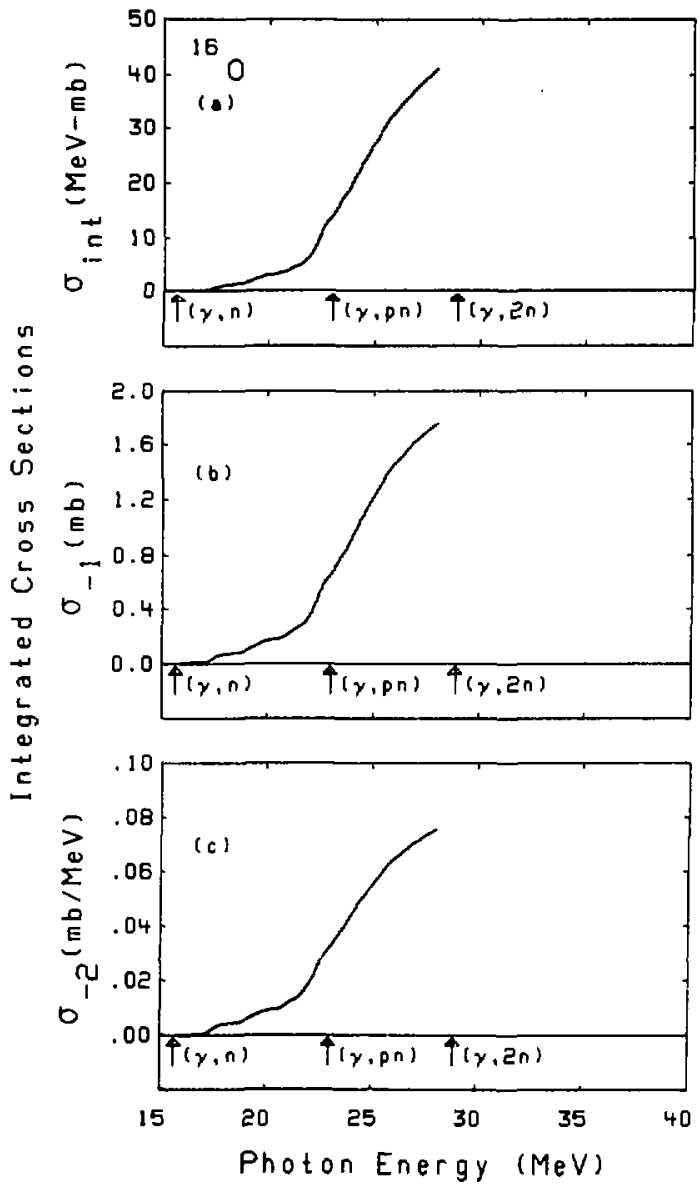

Fig. 18B 

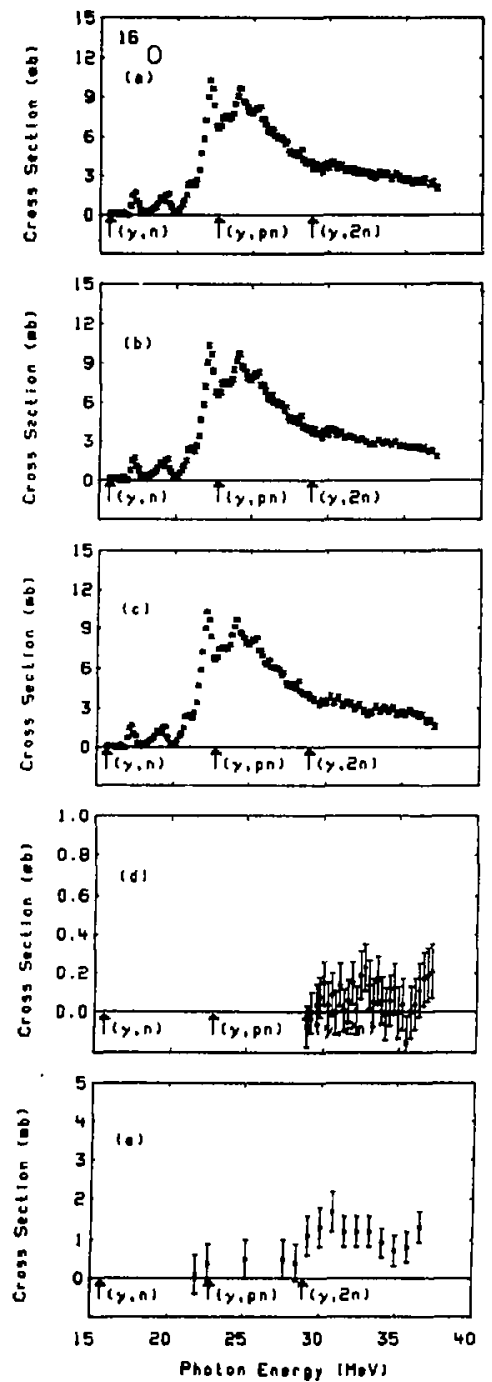

Fig. $19 A$ 


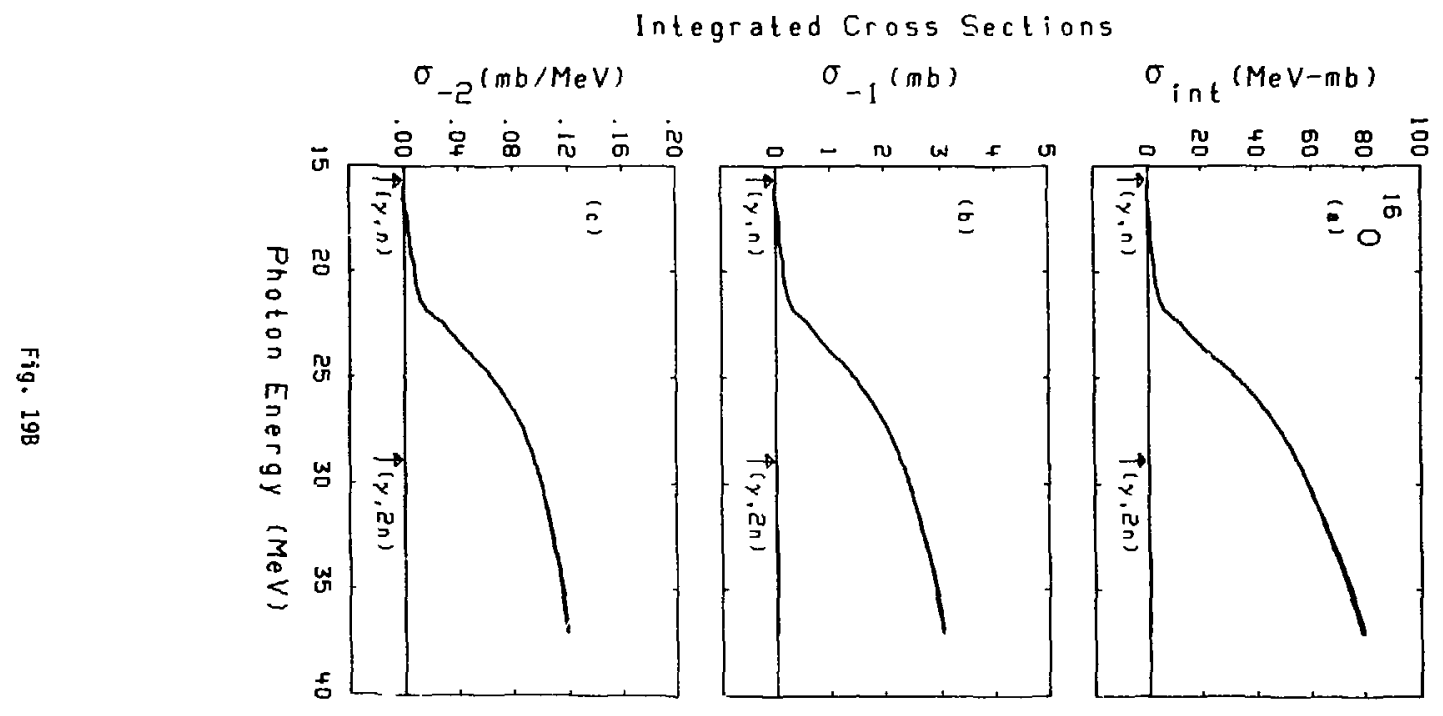




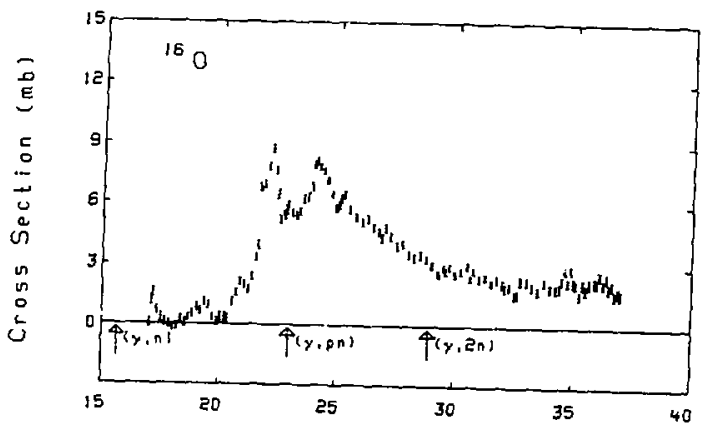

Photon Energy (MeV) 


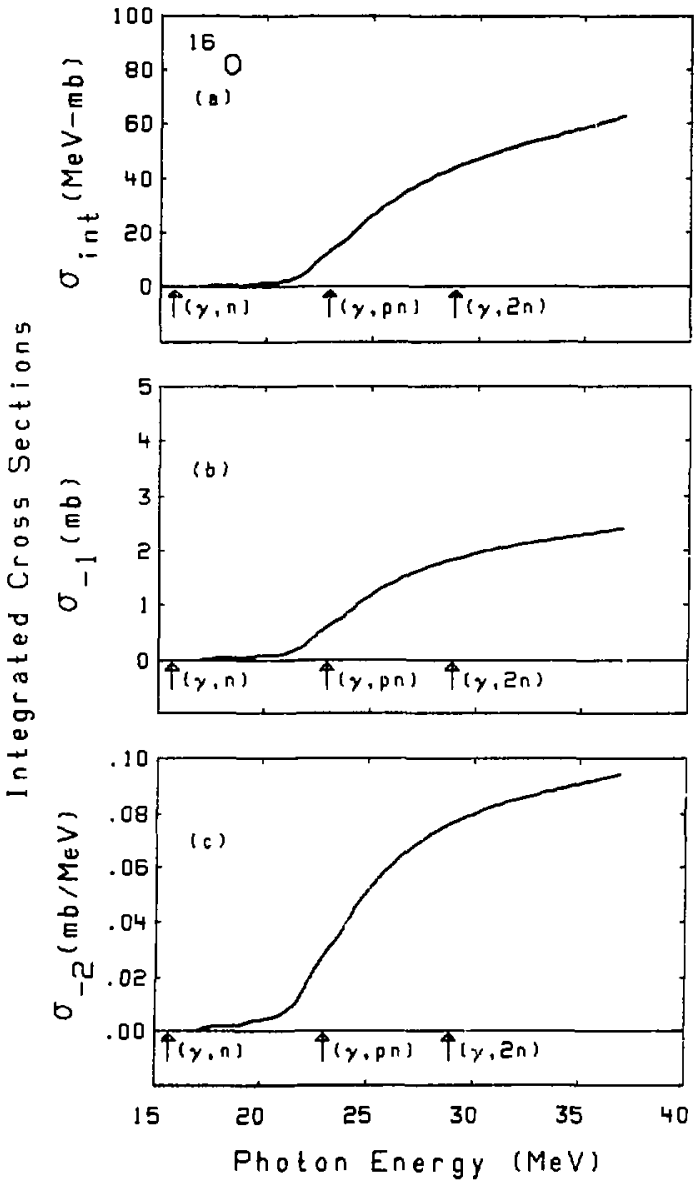

Fig. $20 B$ 


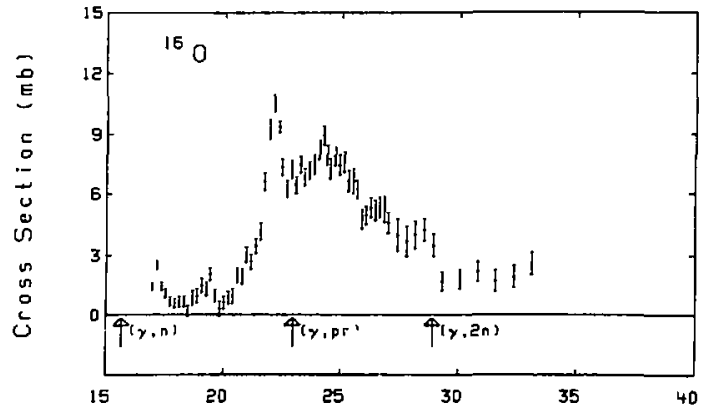

Photon Energy (MeV)

Fig. 21A 


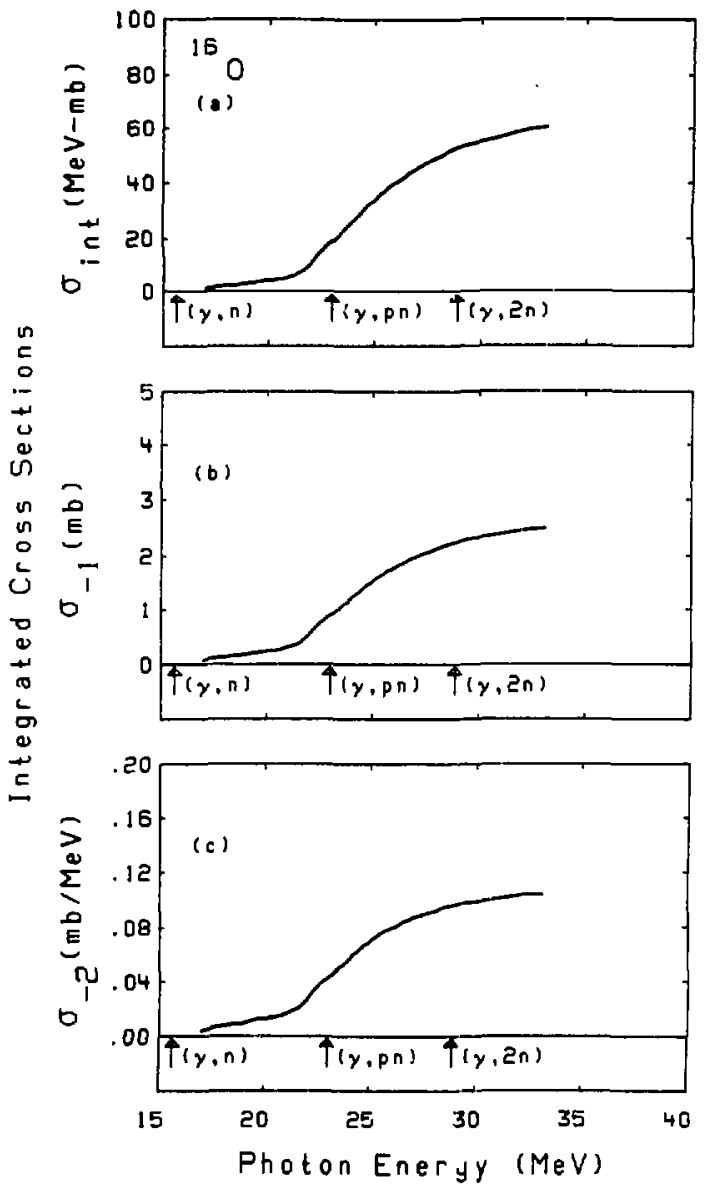

Fig. 21B 

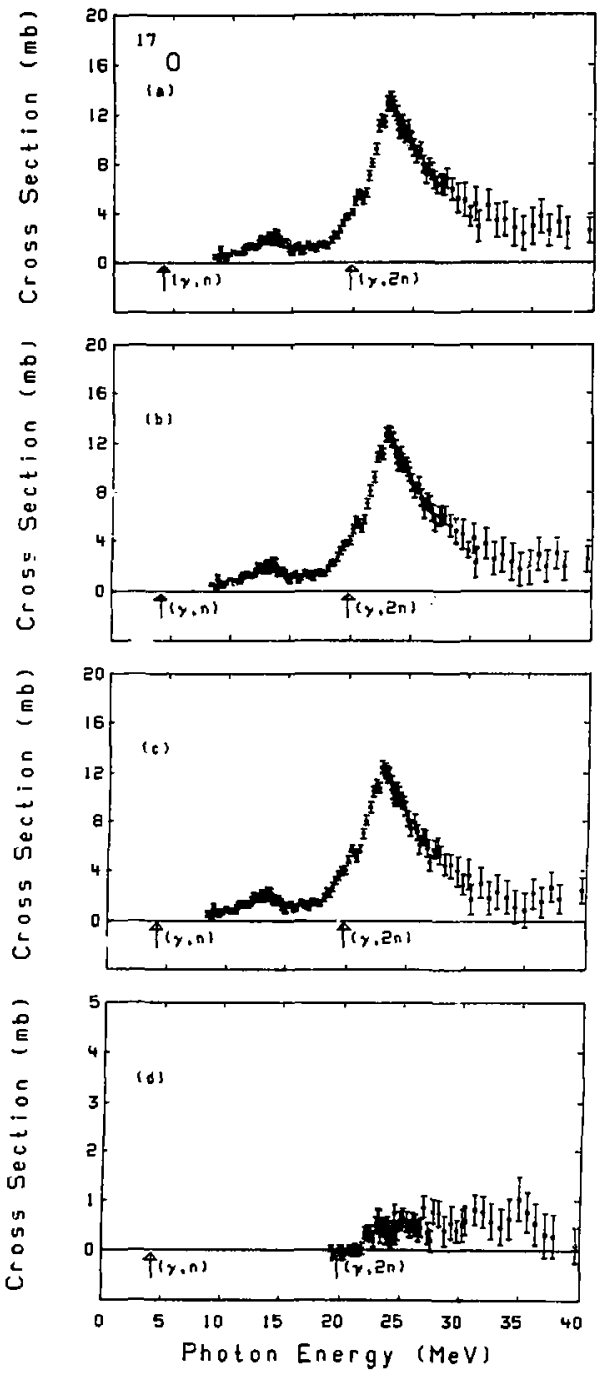

F1g. 22A 


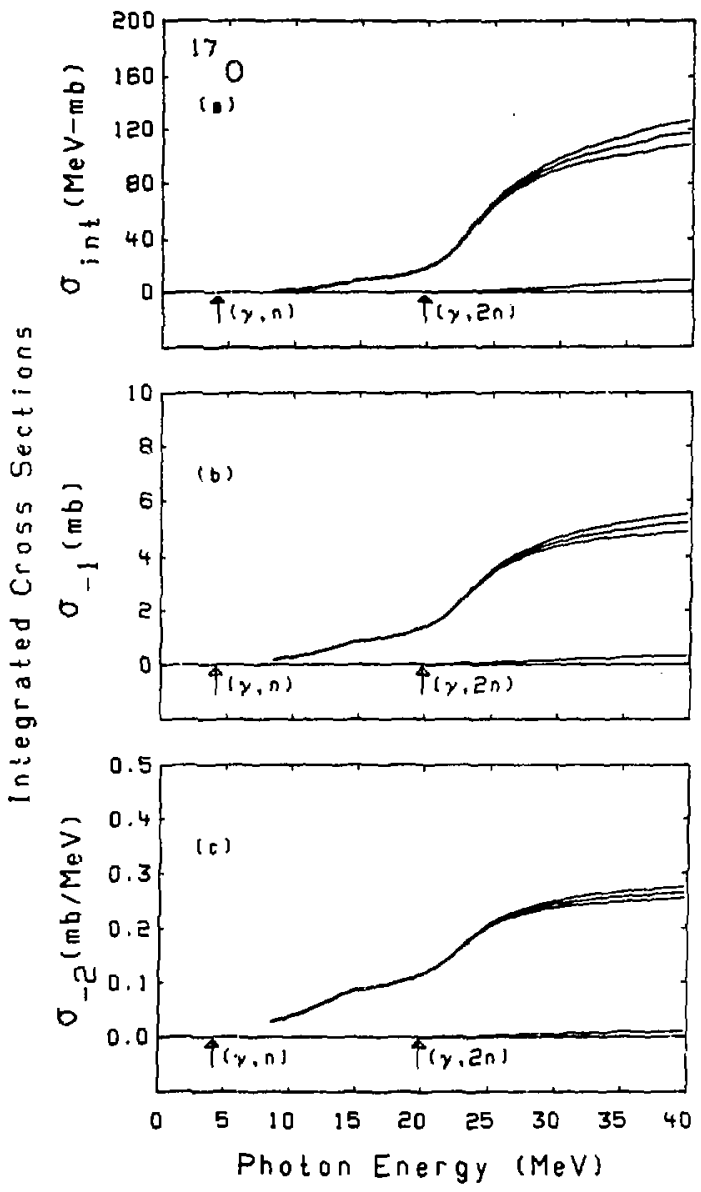

Fig. 228 

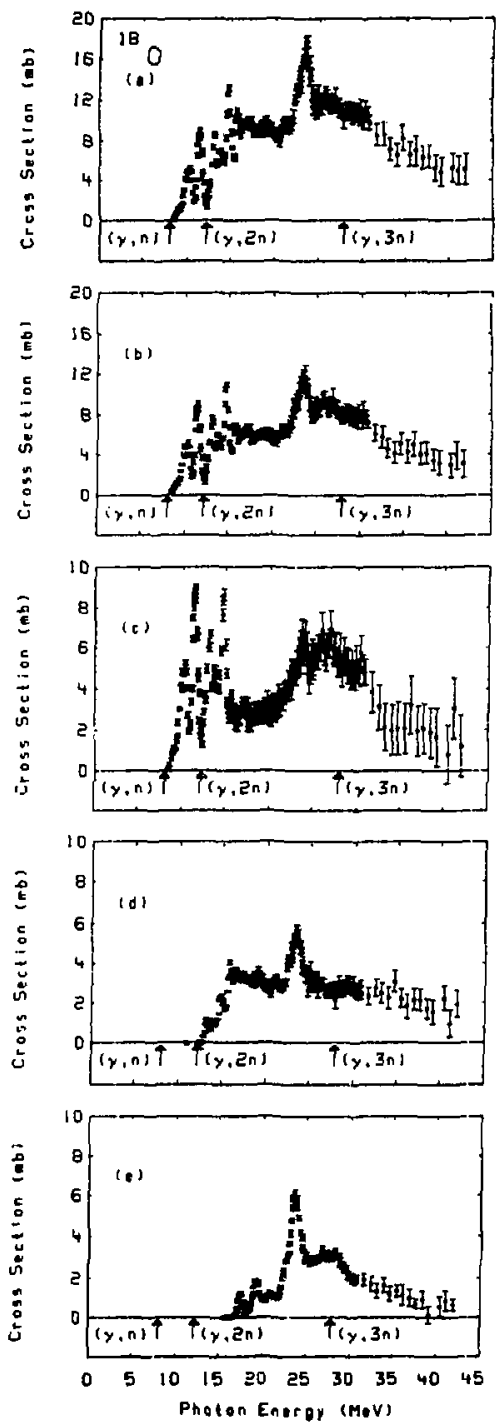

Fig. $23 \mathrm{~A}$ 


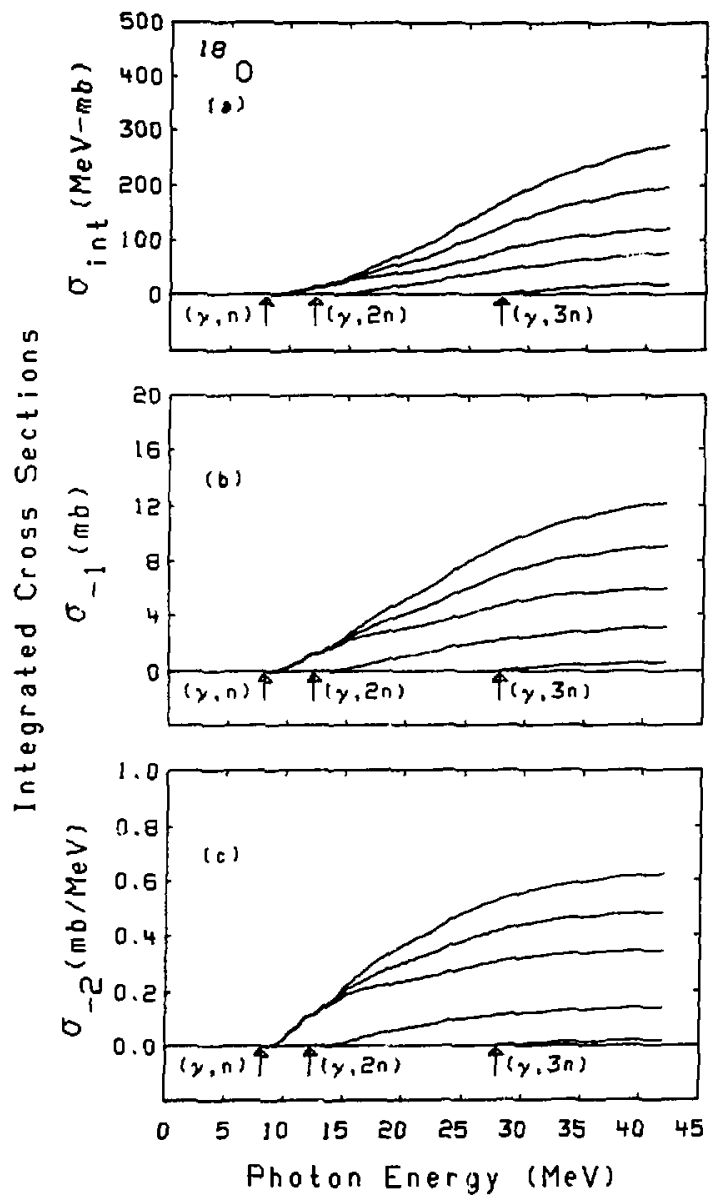

Fig. $23 B$ 

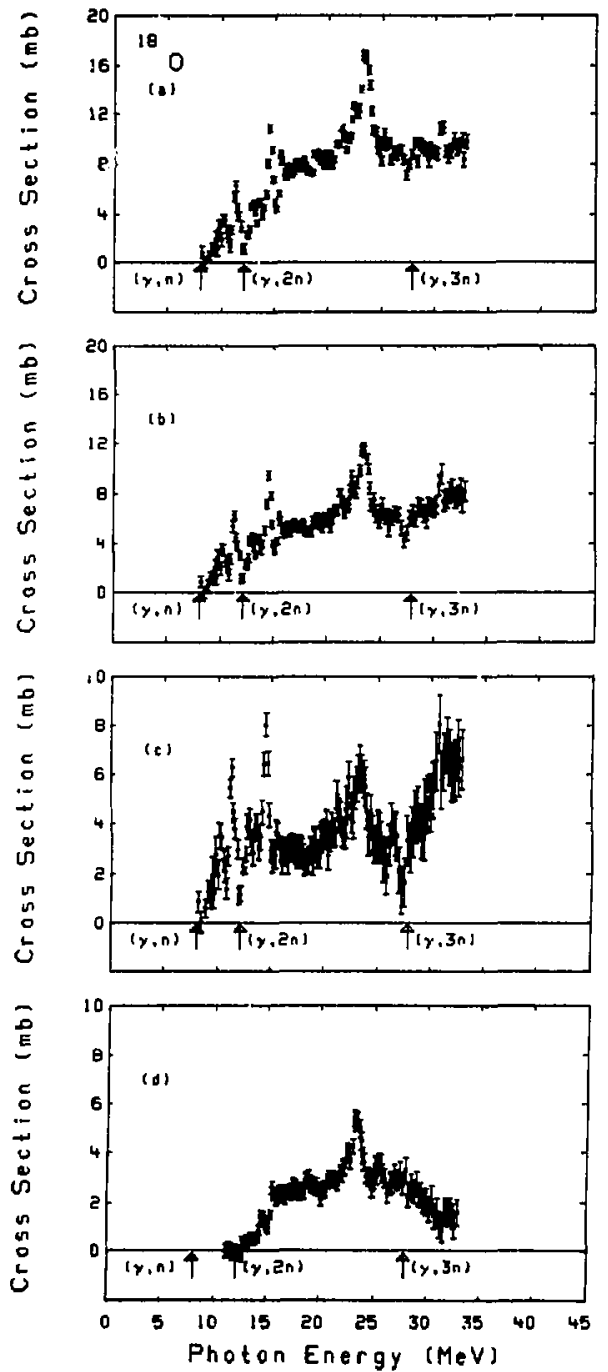

Fig. 24A 

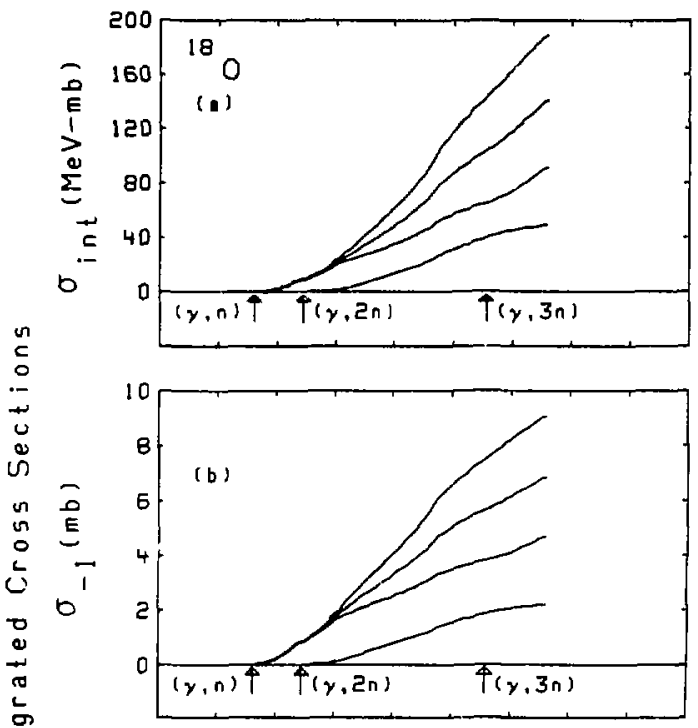

$\stackrel{5}{5}$

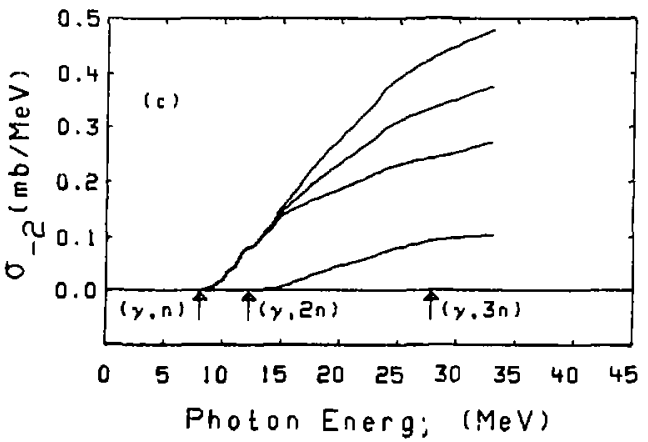

Fig. 24B 

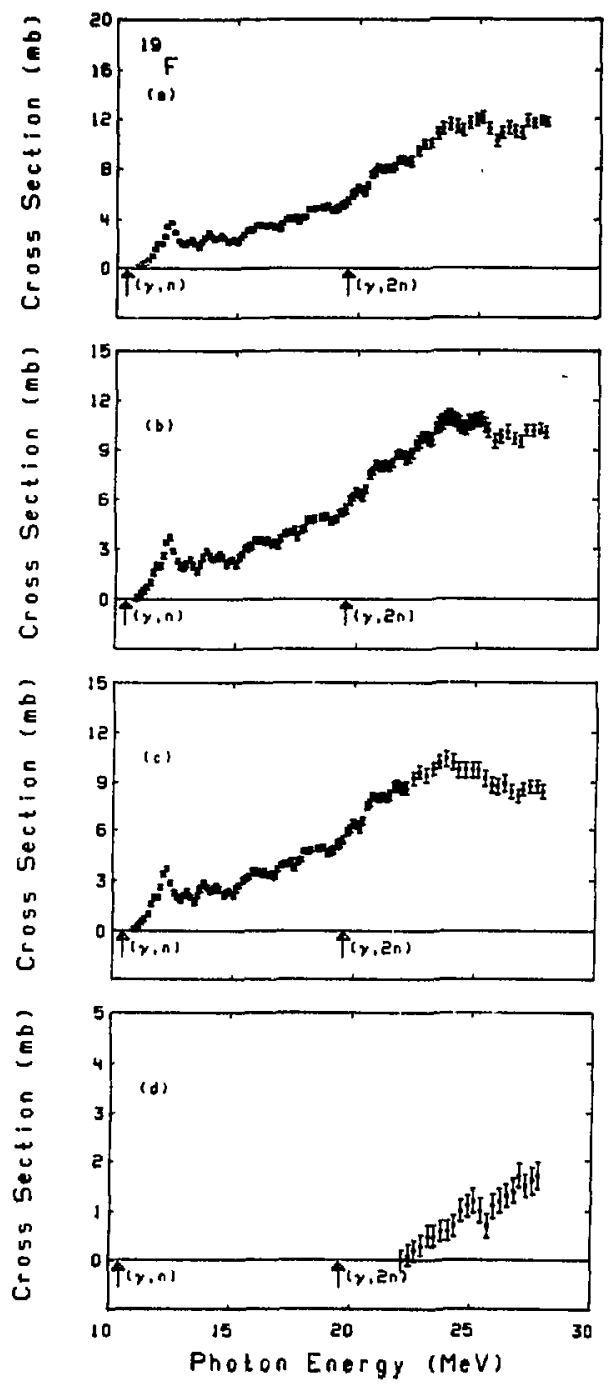

Fig. 25A 


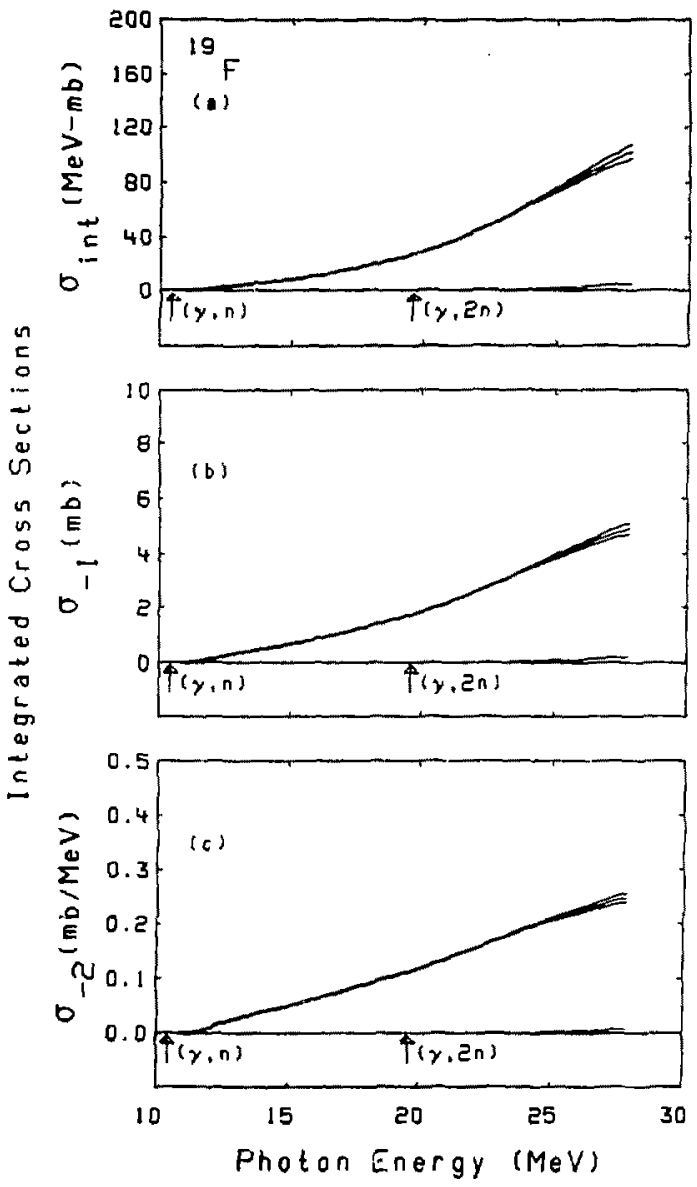

Fig. 25B 

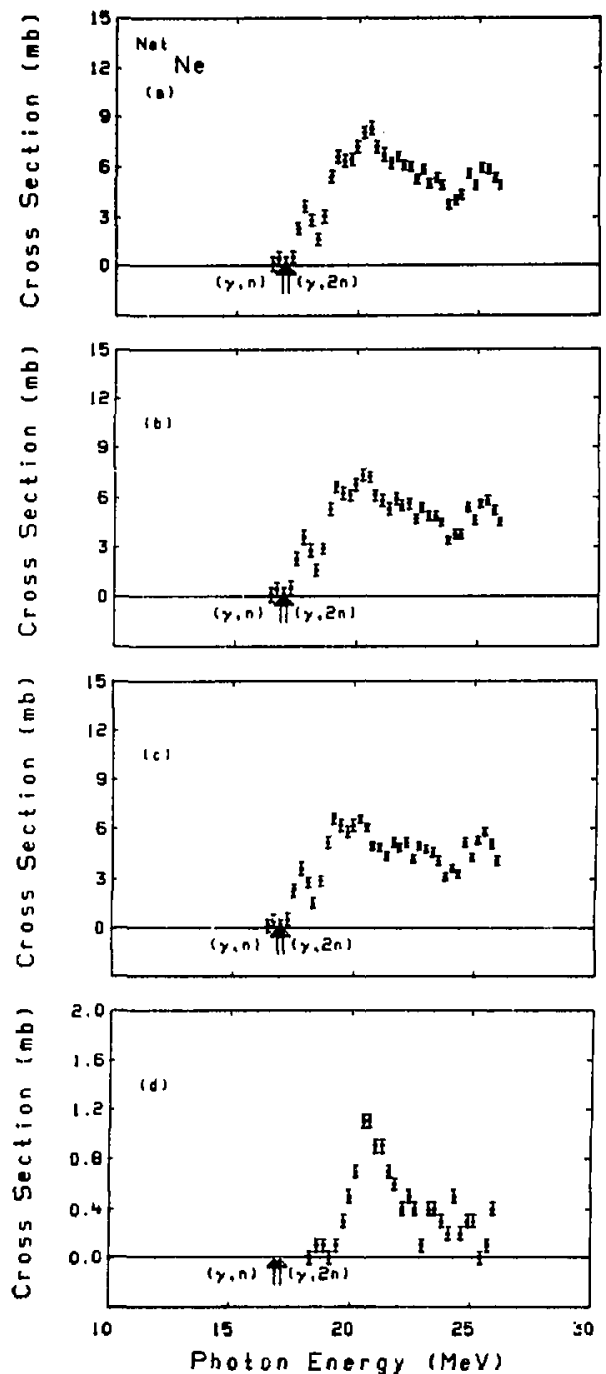

Fig. $20 A$ 


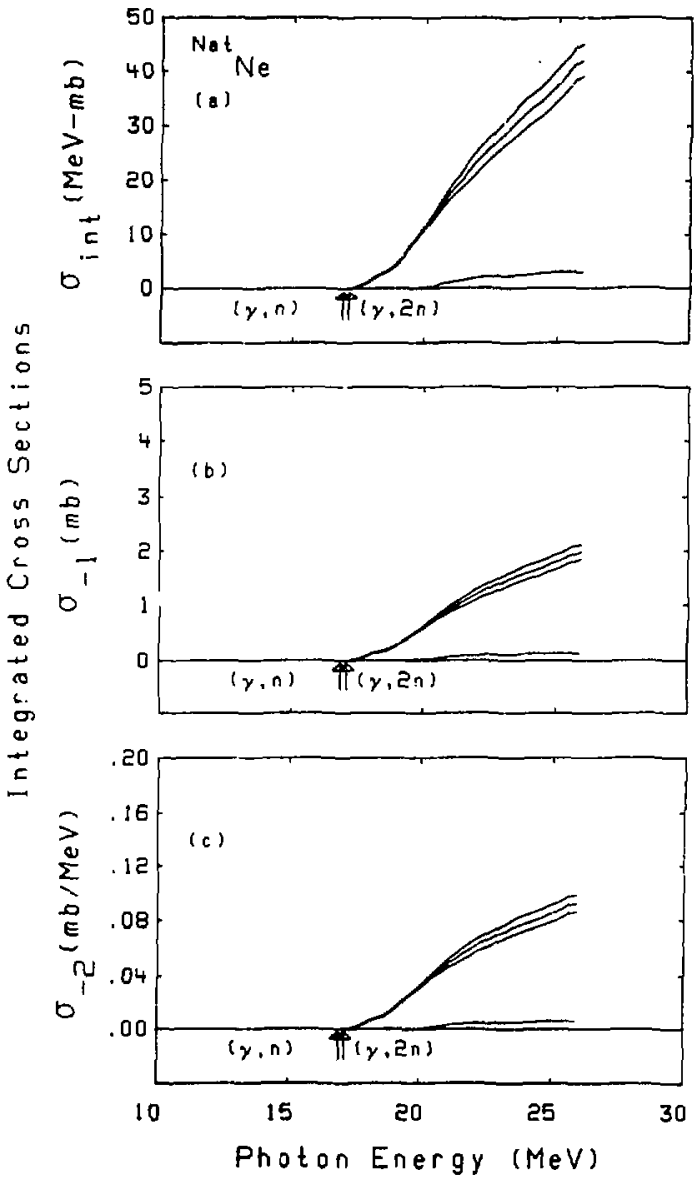

Fig. 268 

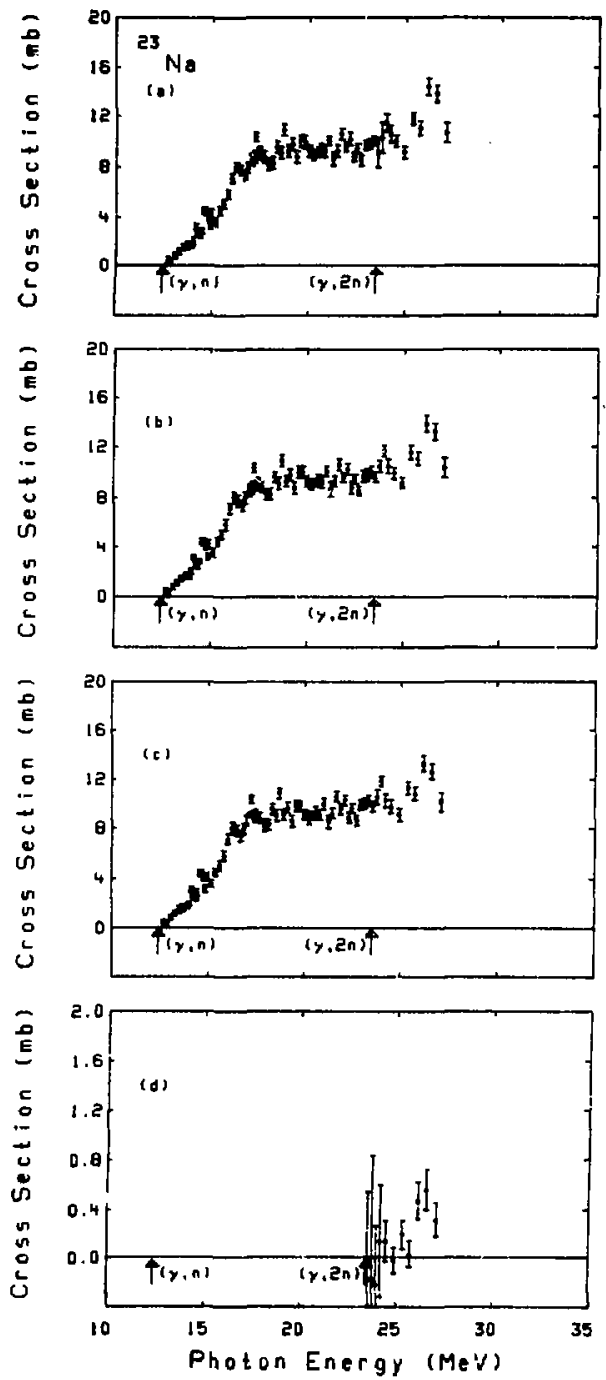

Fig. $27 \mathrm{~A}$ 


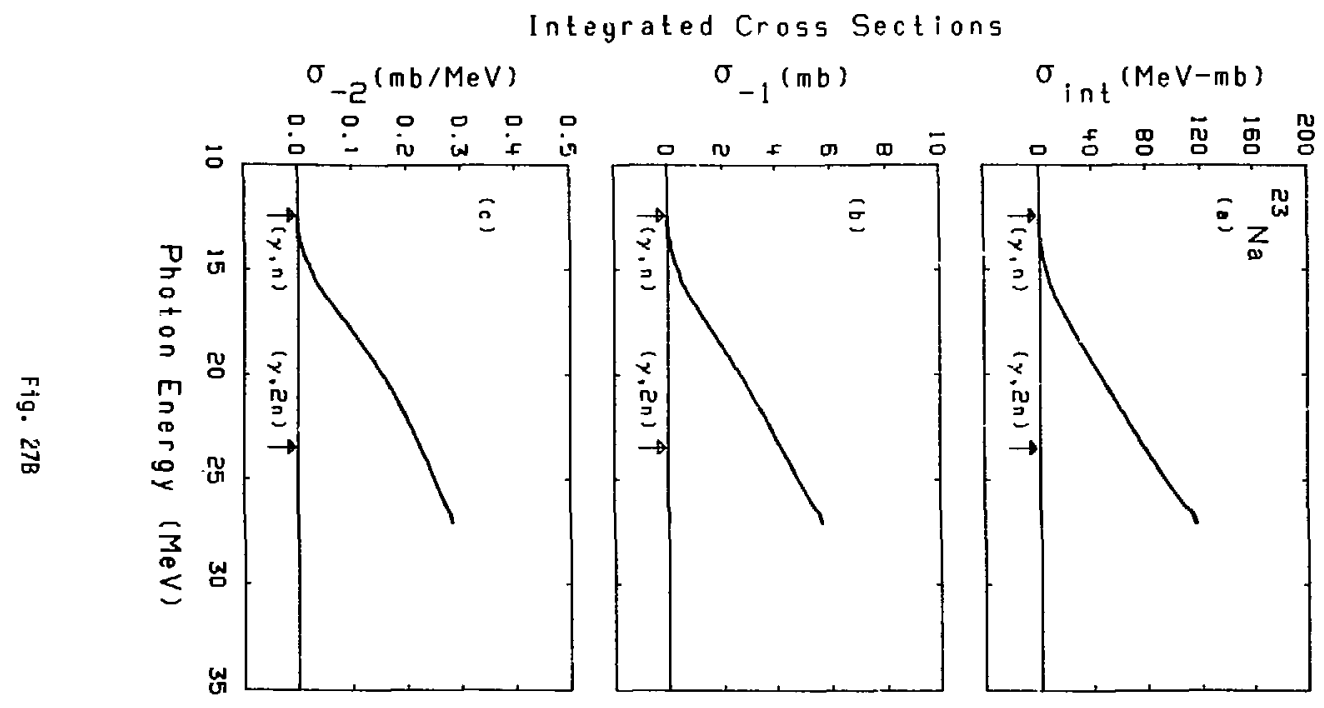




$$
(x) \sqrt{3}
$$




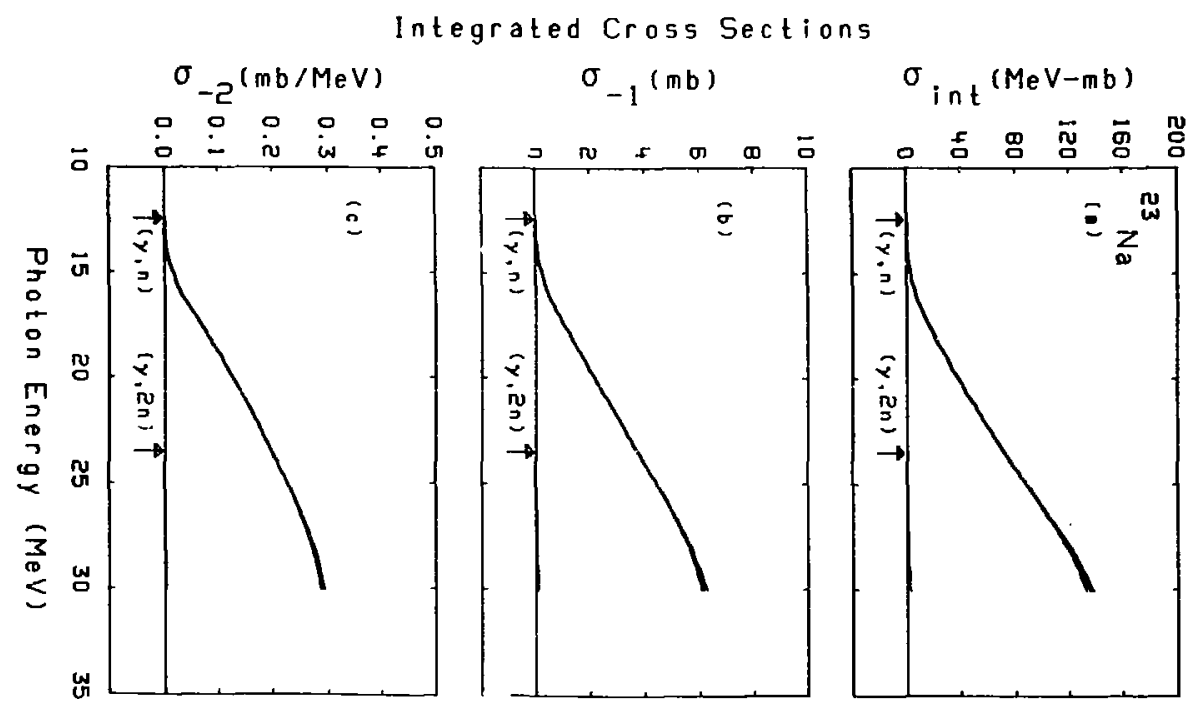




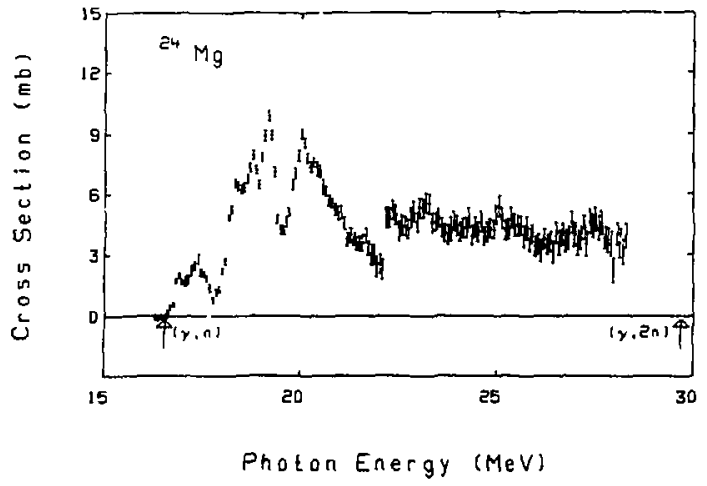

Fig. $29 A$ 


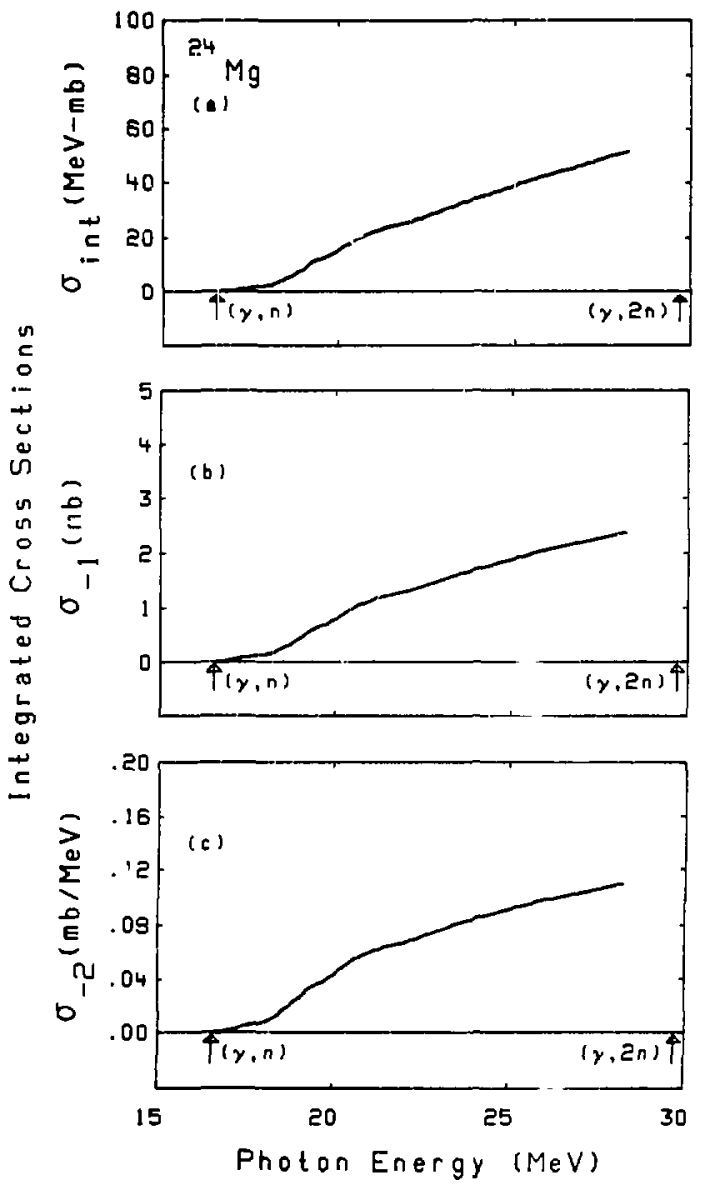

Fig. $29 B$ 

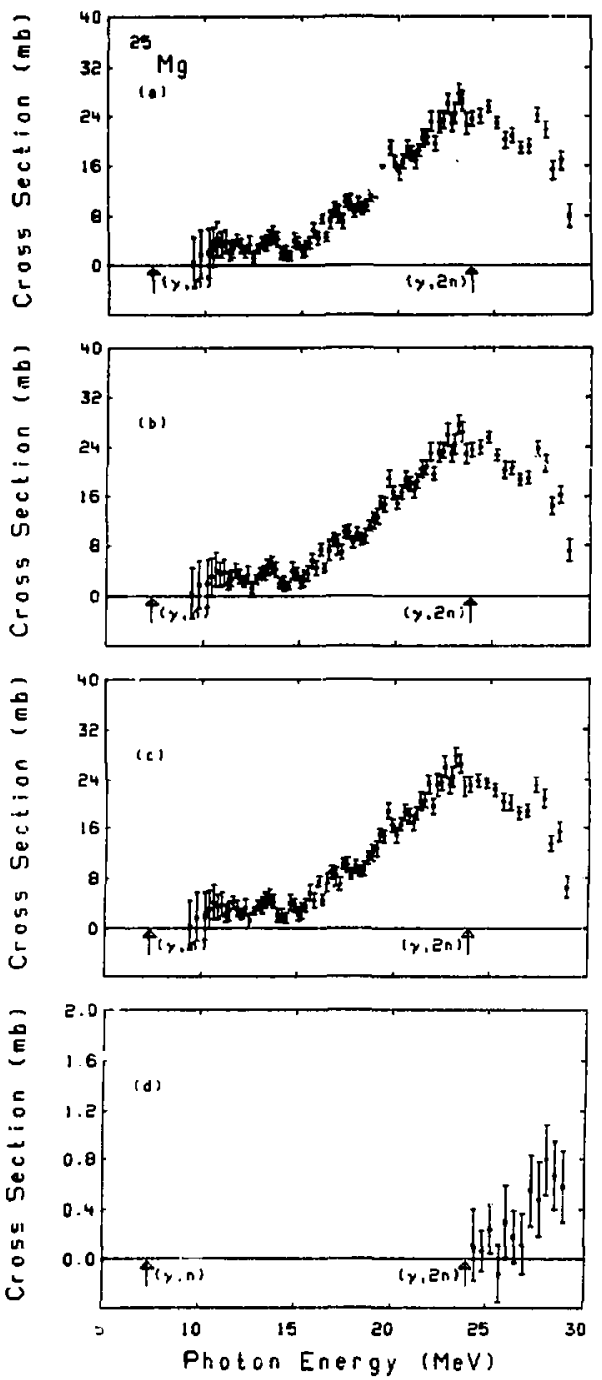

ig. $30 \mathrm{~A}$ 


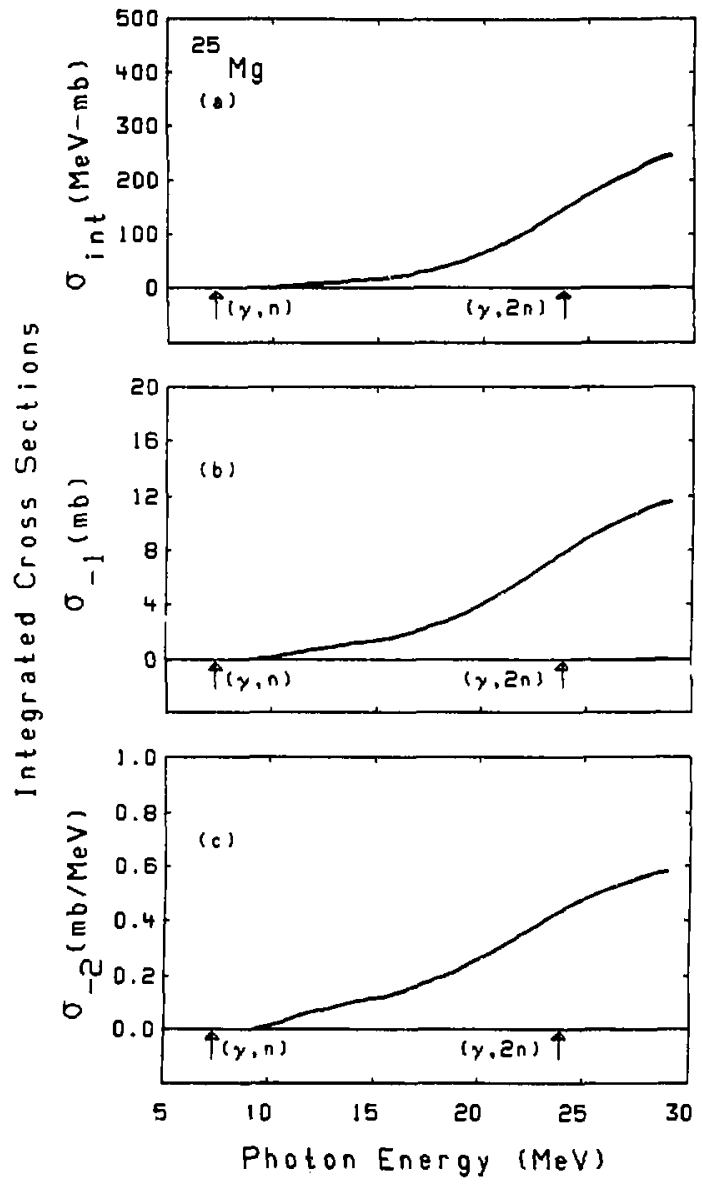

Fig. 308 

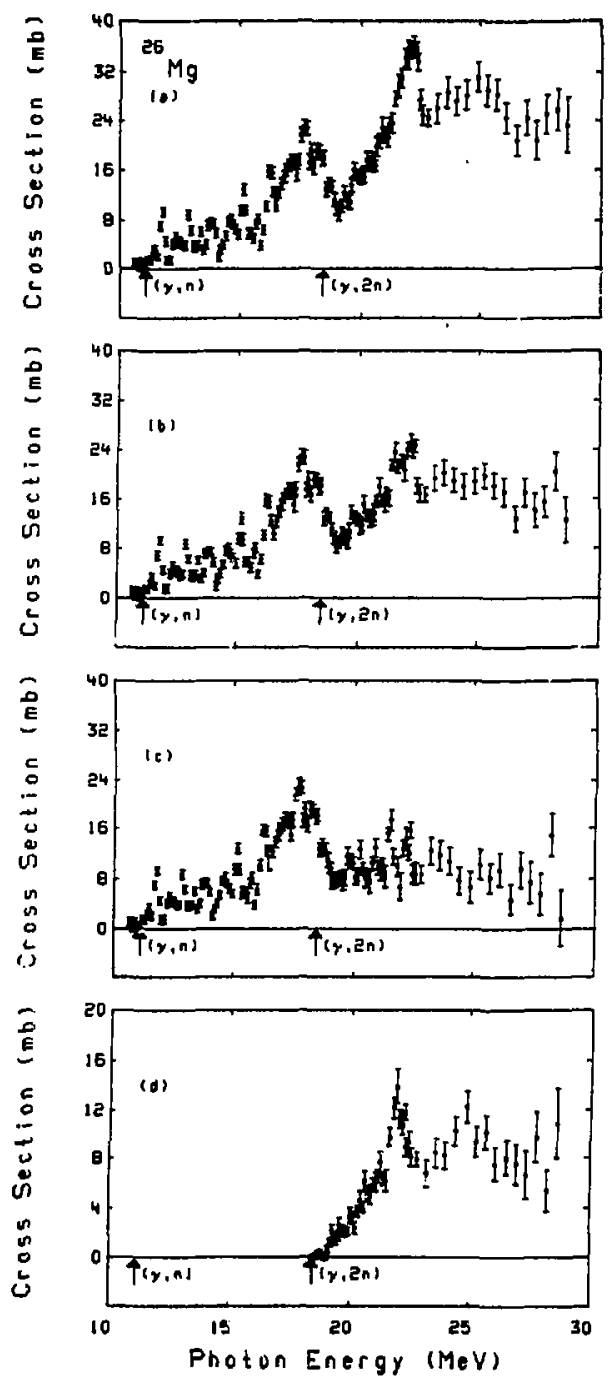

Fig. 31A 


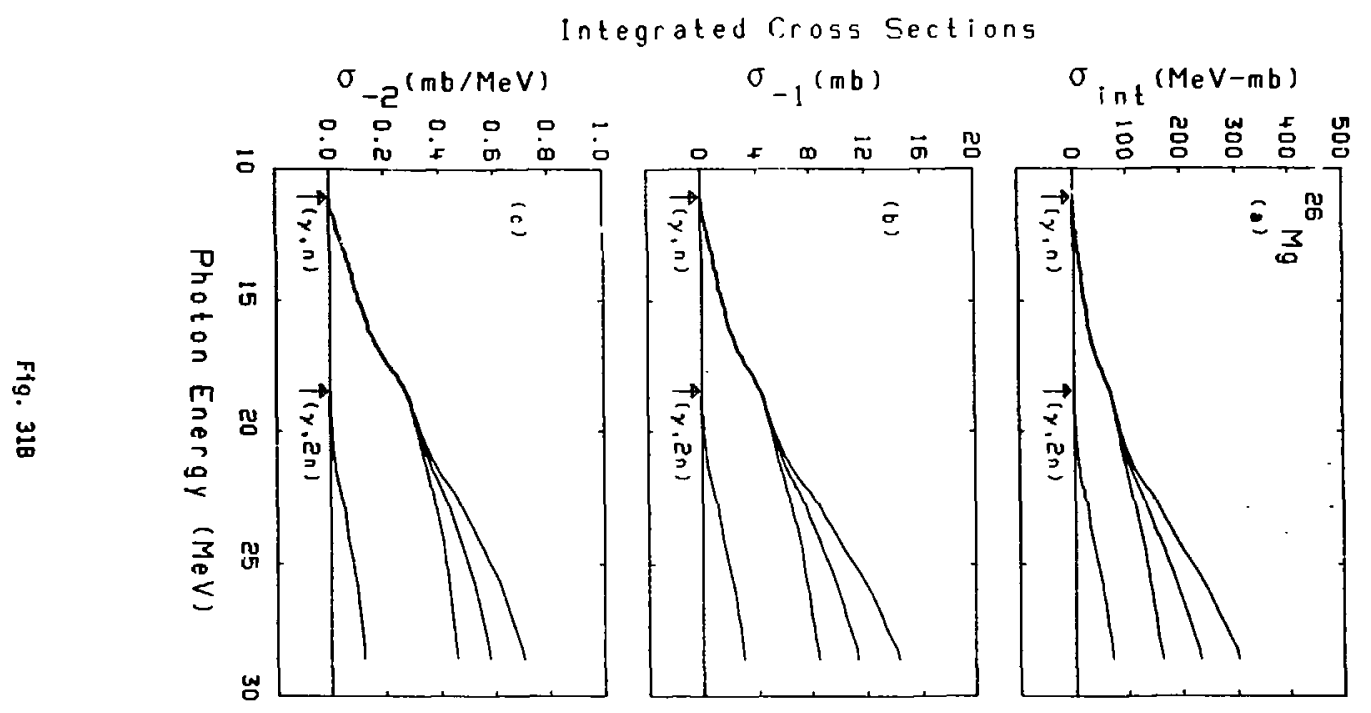



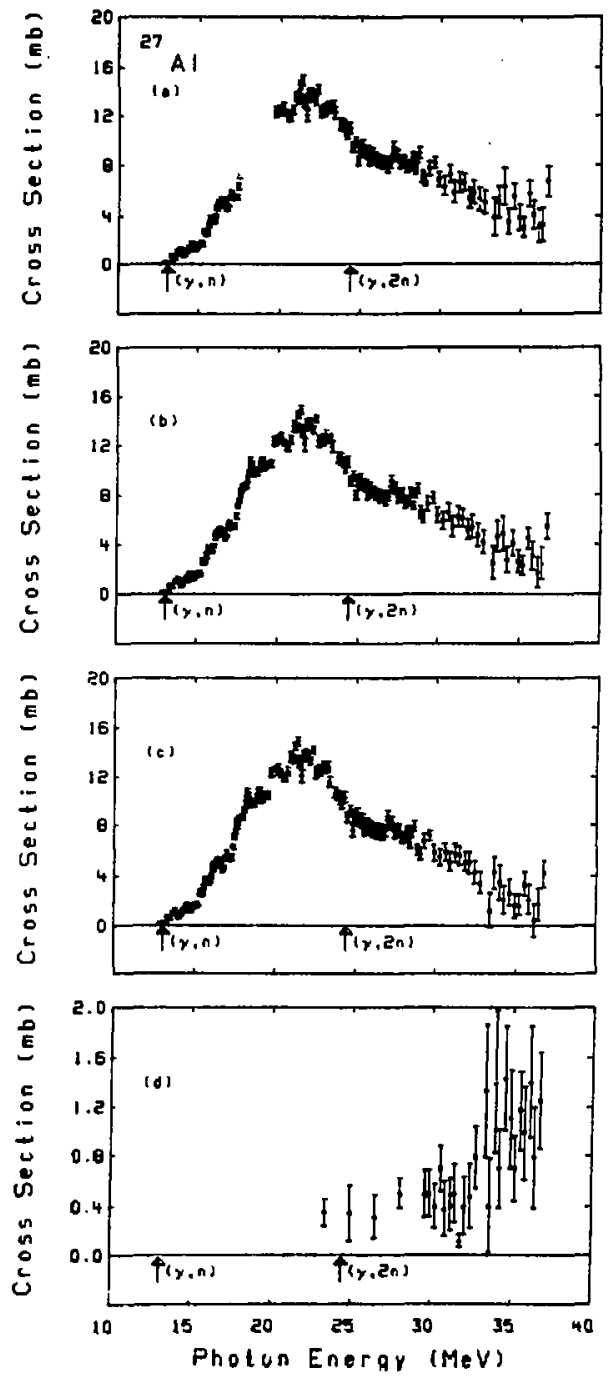

Fig. $32 A$ 


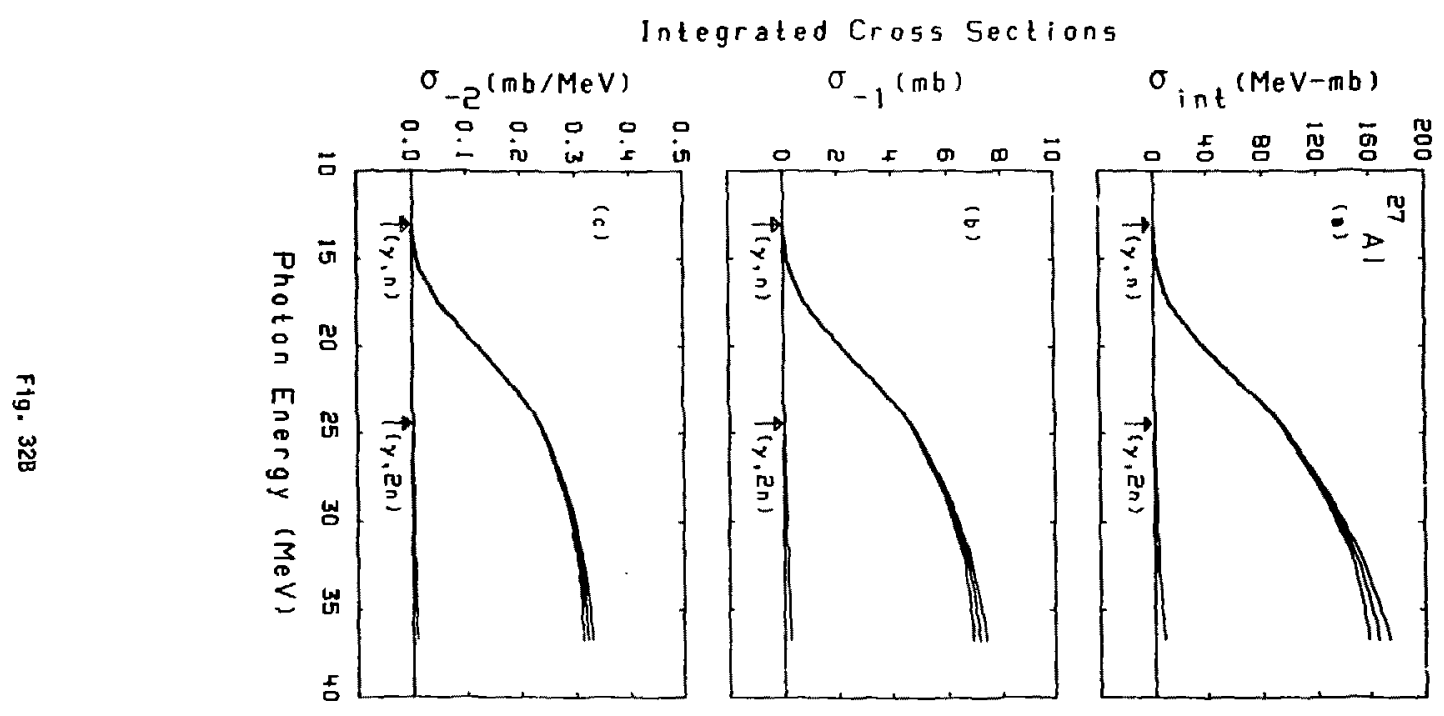



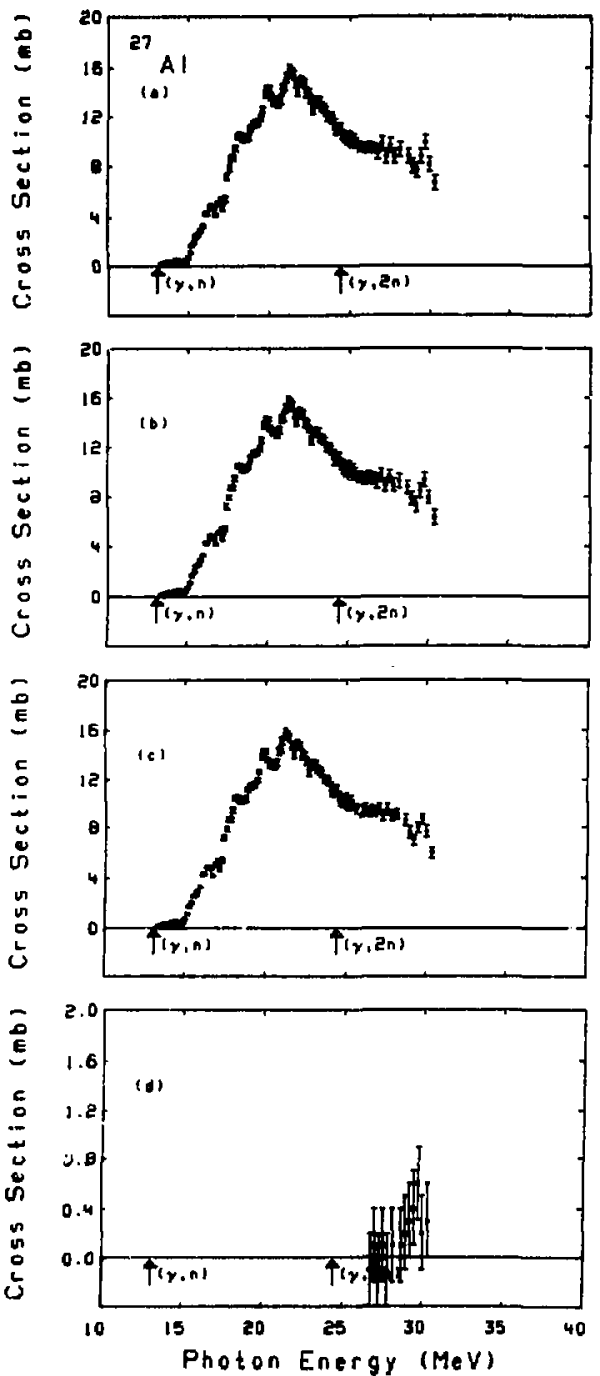

Fig. $33 \mathrm{~A}$ 


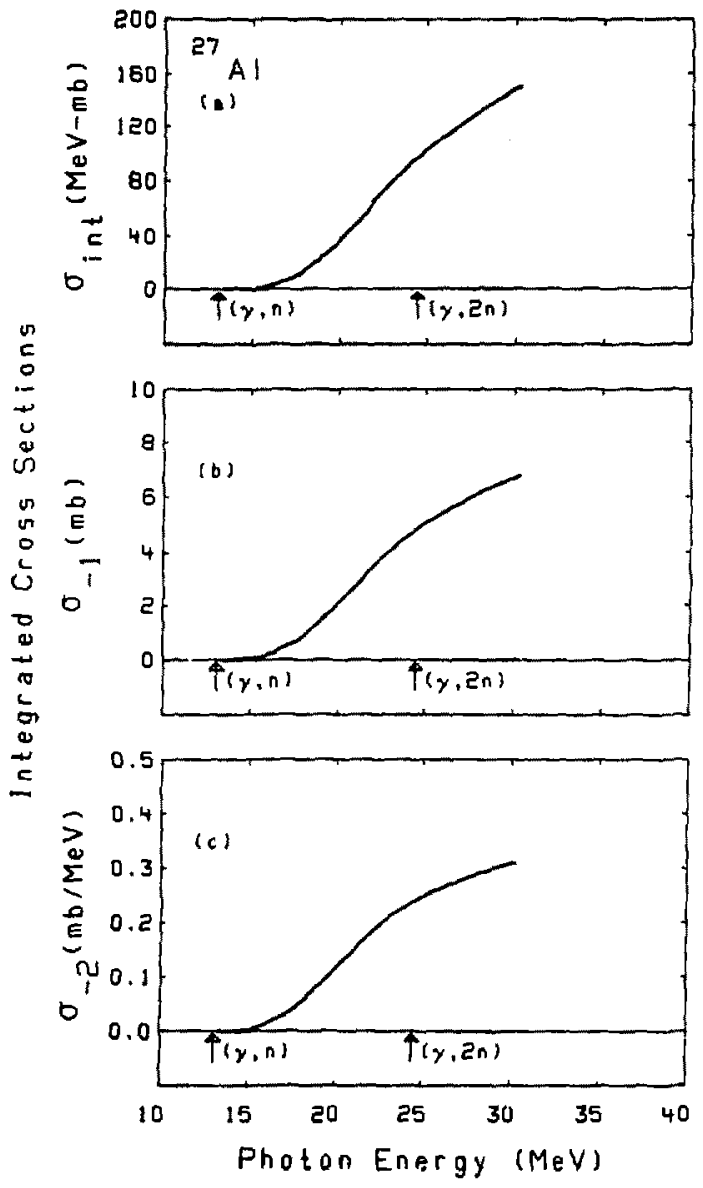

Fig. 338 


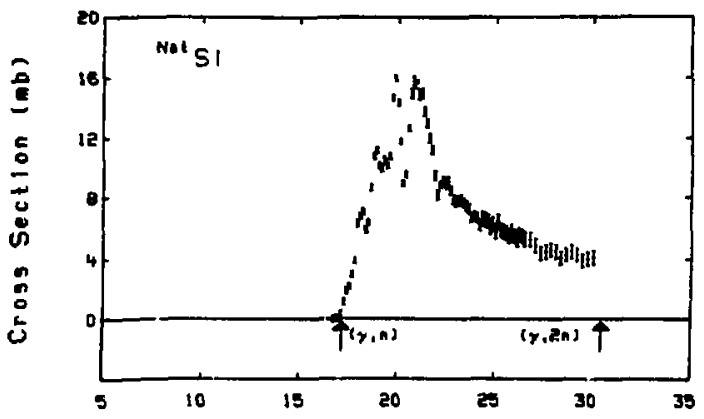

Photon Enorgy (MoV)

Fig. $34 \mathrm{~A}$ 


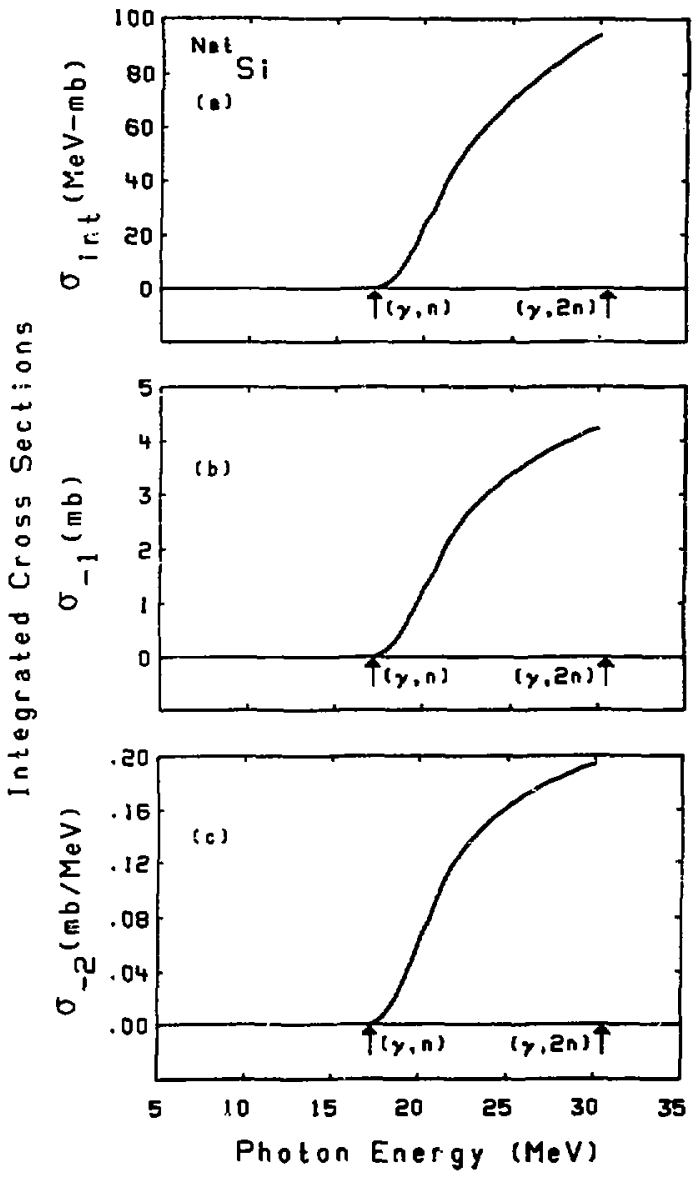

Fig. 34B 


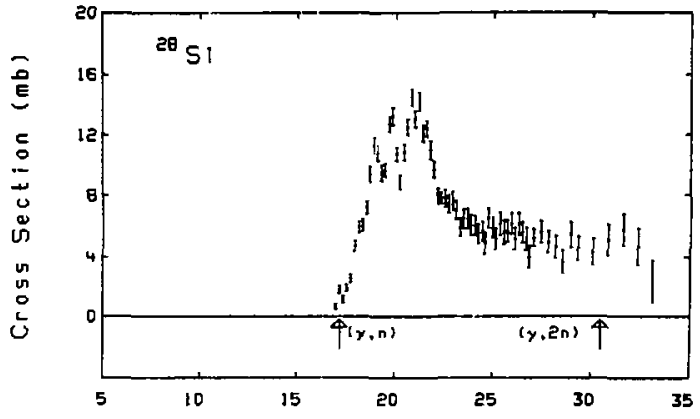

Photon Energy (MeV)

Fig. $35 \mathrm{~A}$ 


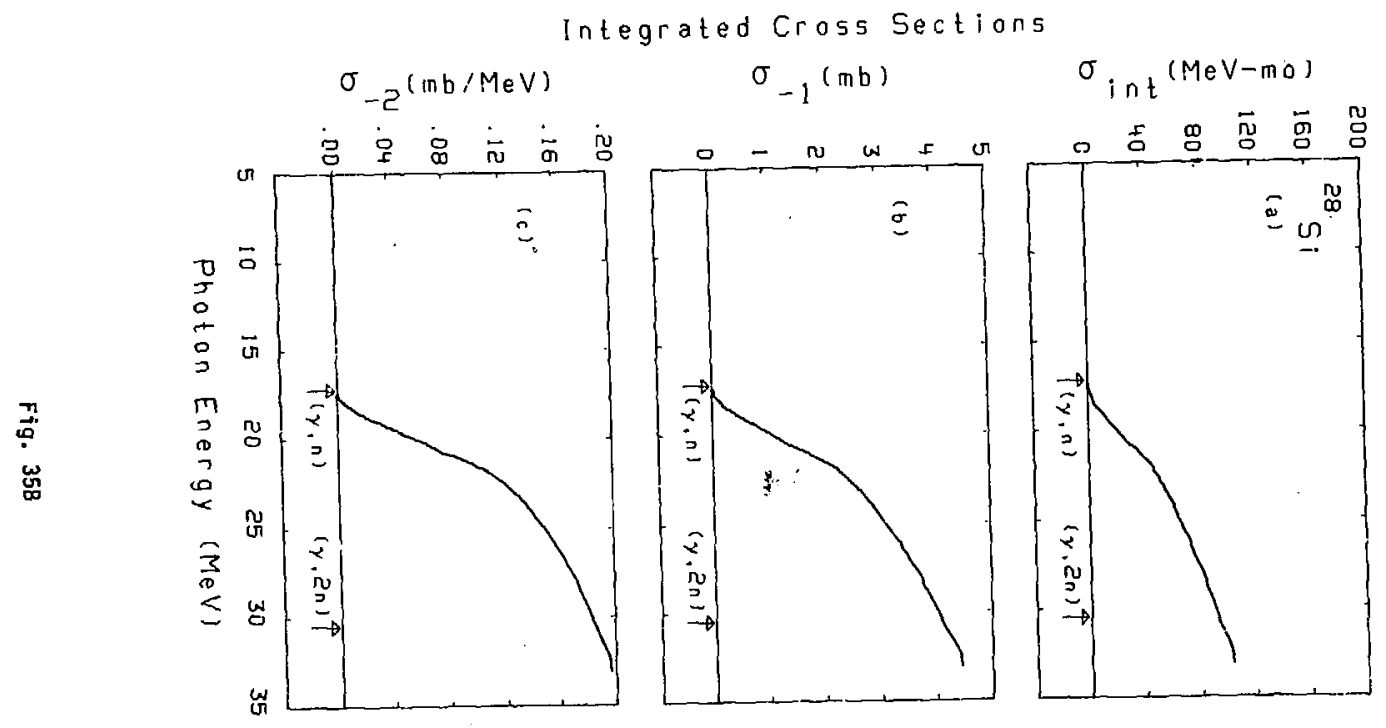




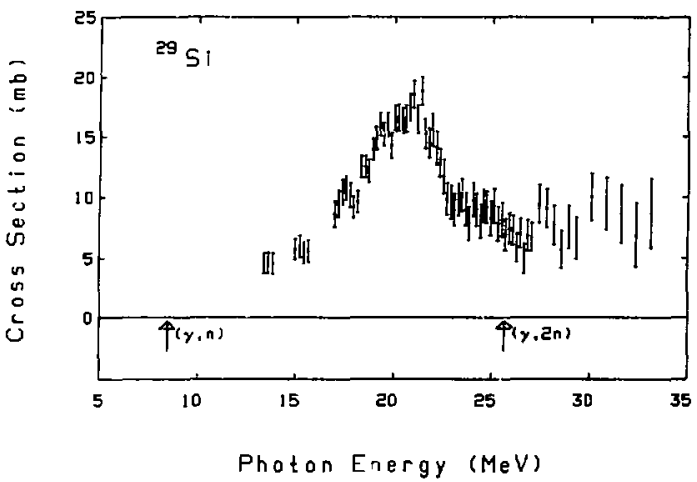

Fig. $36 \mathrm{~A}$ 


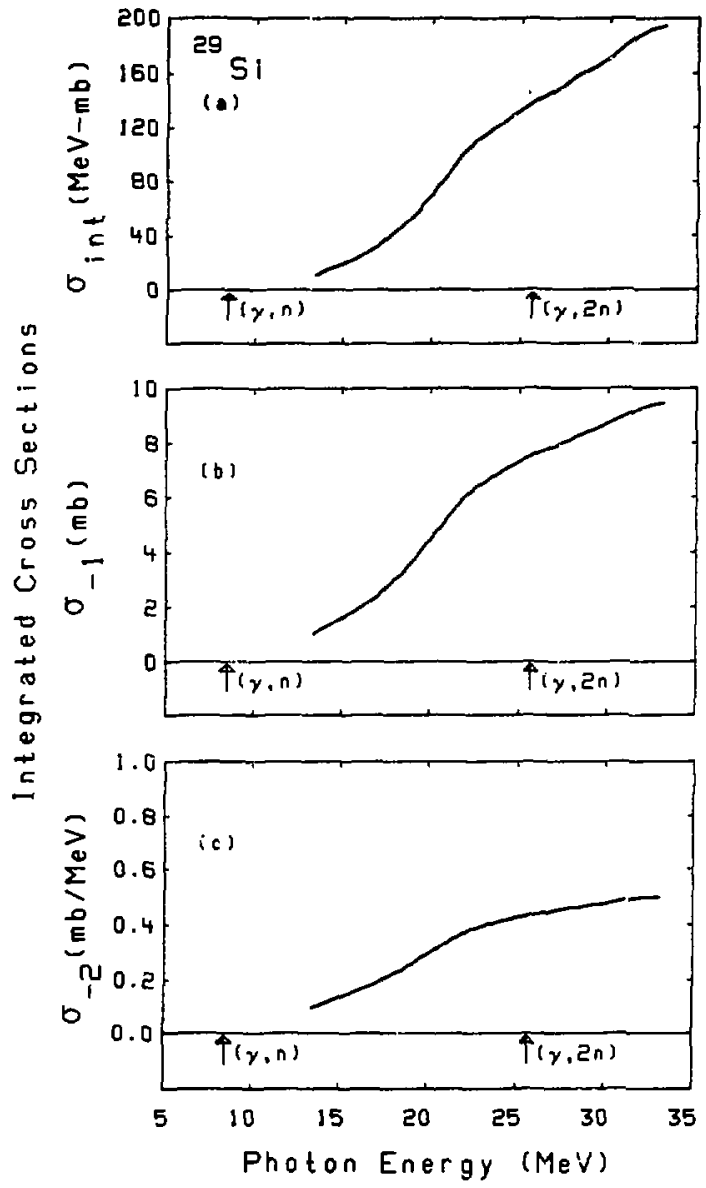

fig. 368 

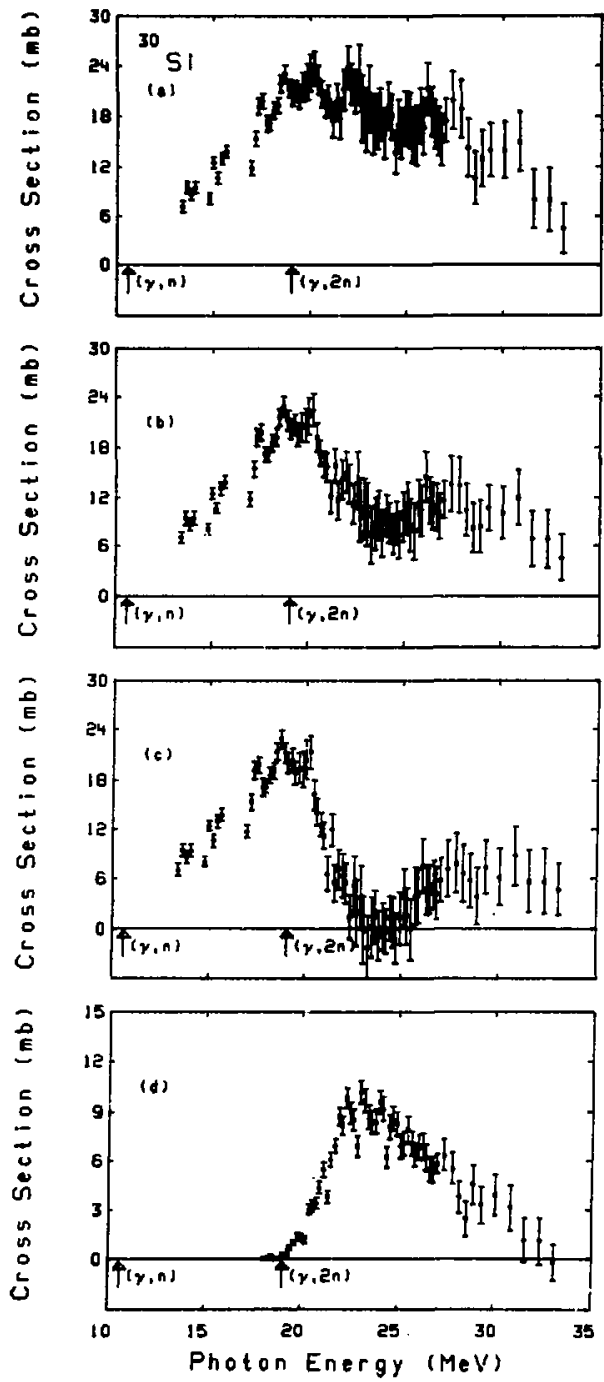

Fig. $37 \mathrm{~A}$ 


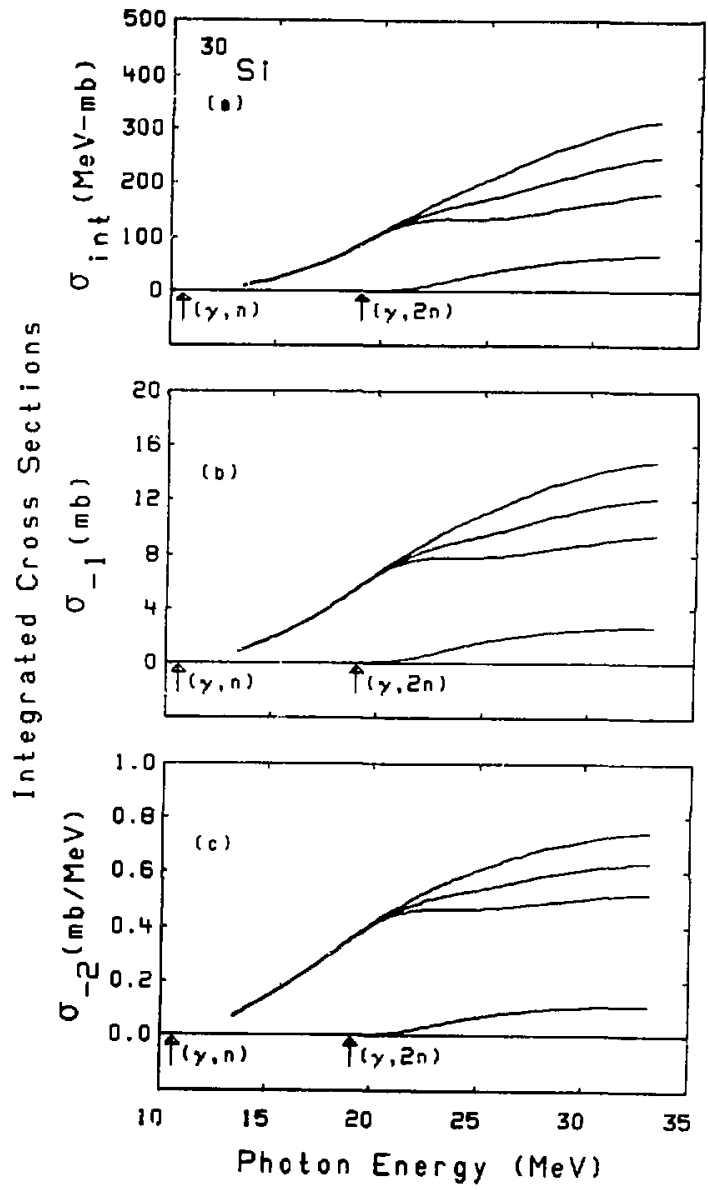

fig. 37B 

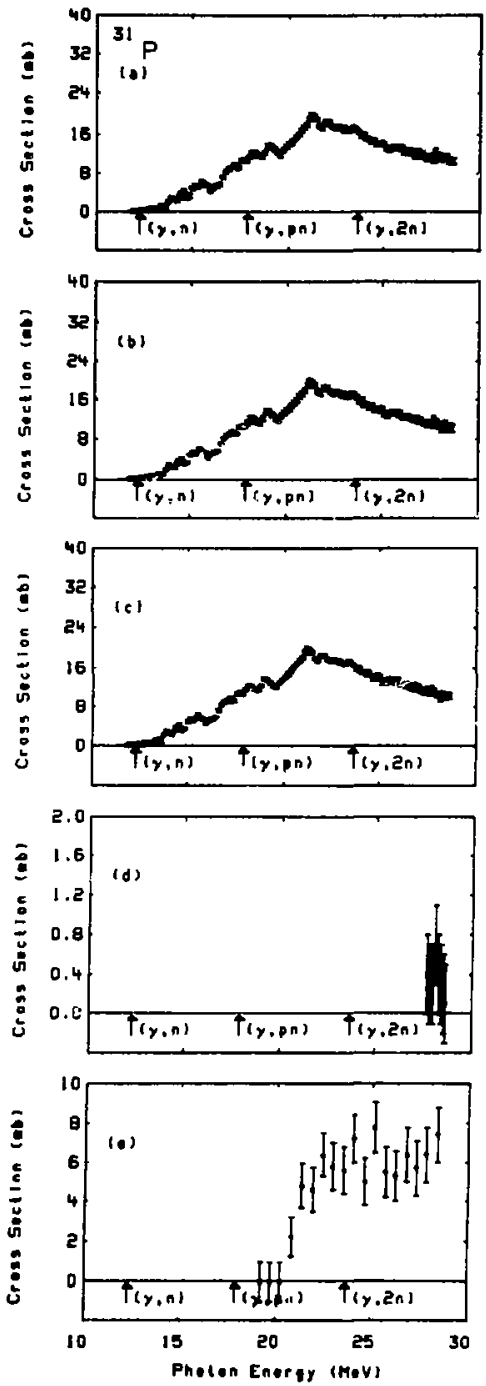

Fig. $38 A$ 


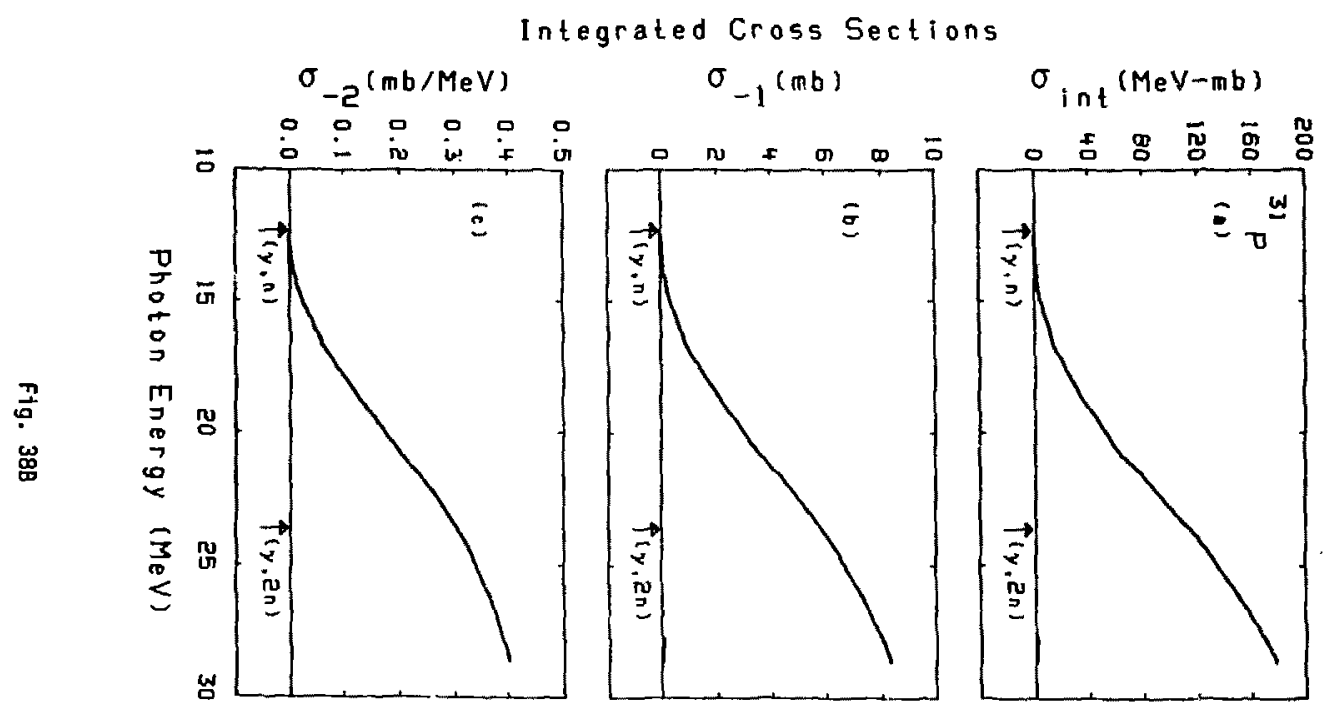



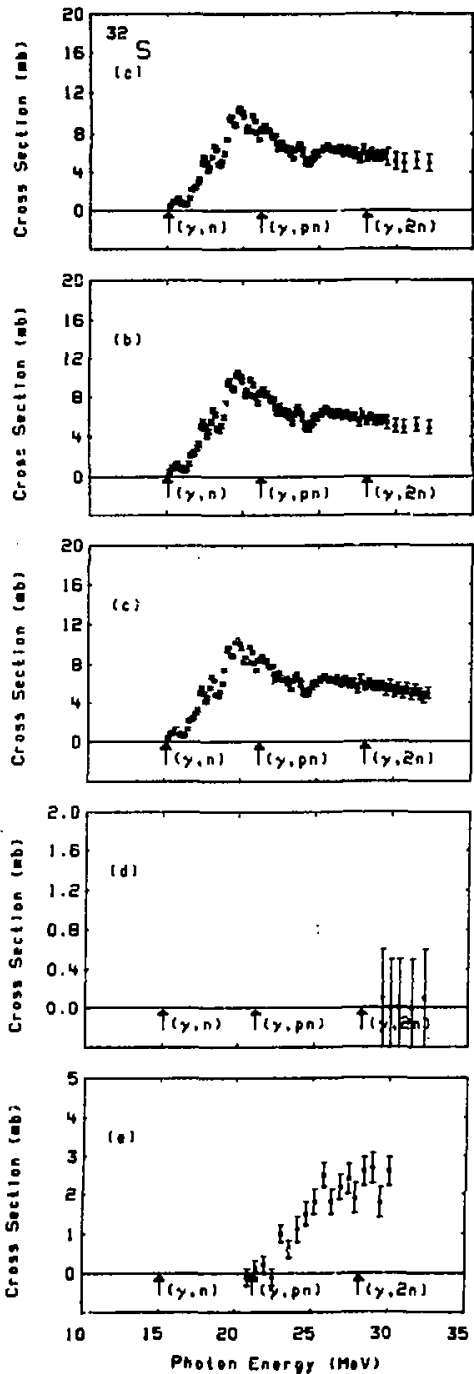

Fig. 39A 


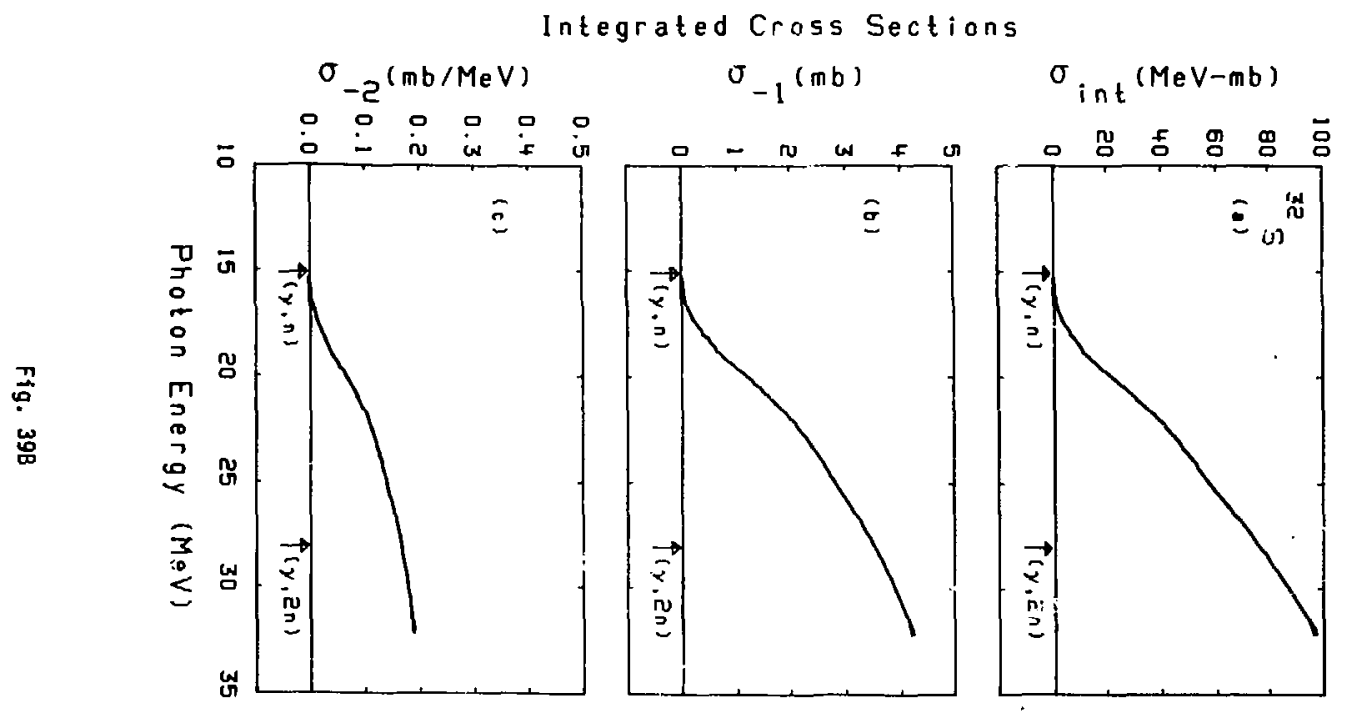


Cross Section (mb) Cross Section (mb) Cross Section (mb) Cross Section (mb)

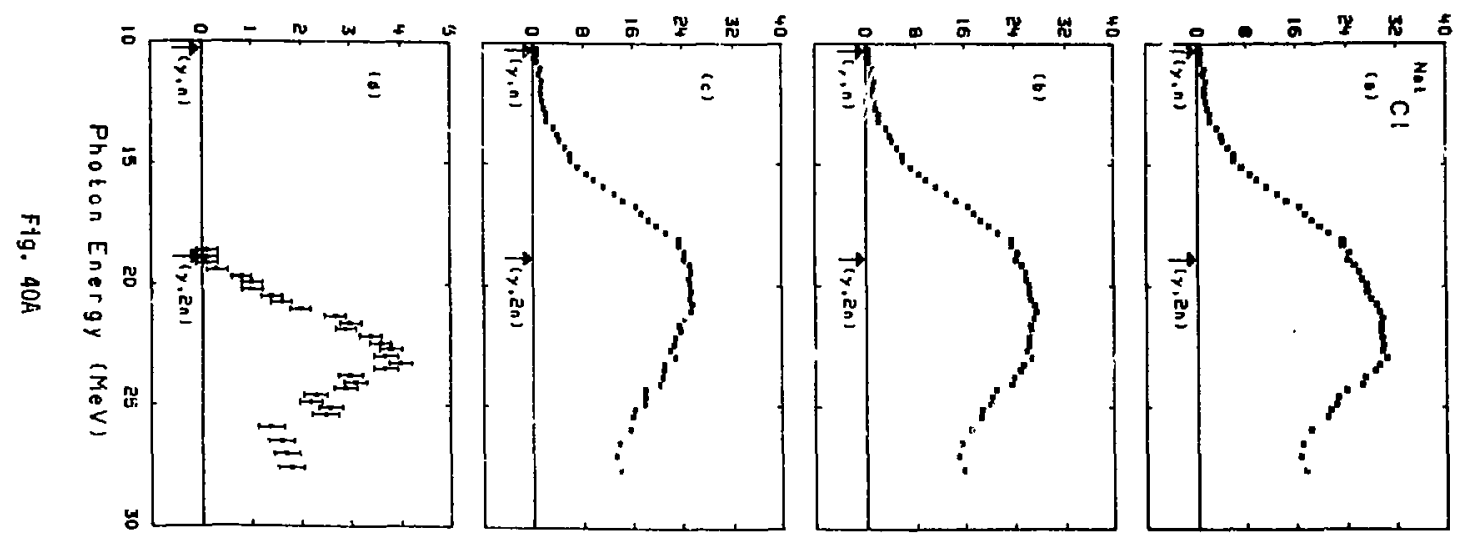




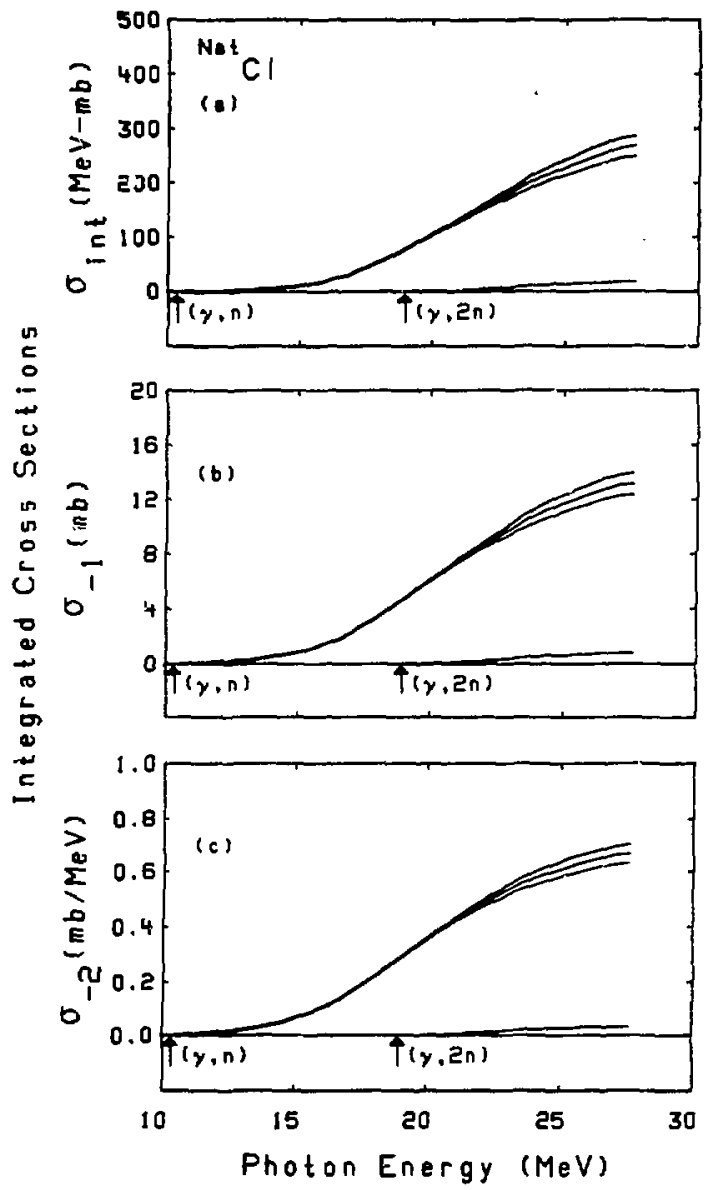

Fig. $40 B$ 


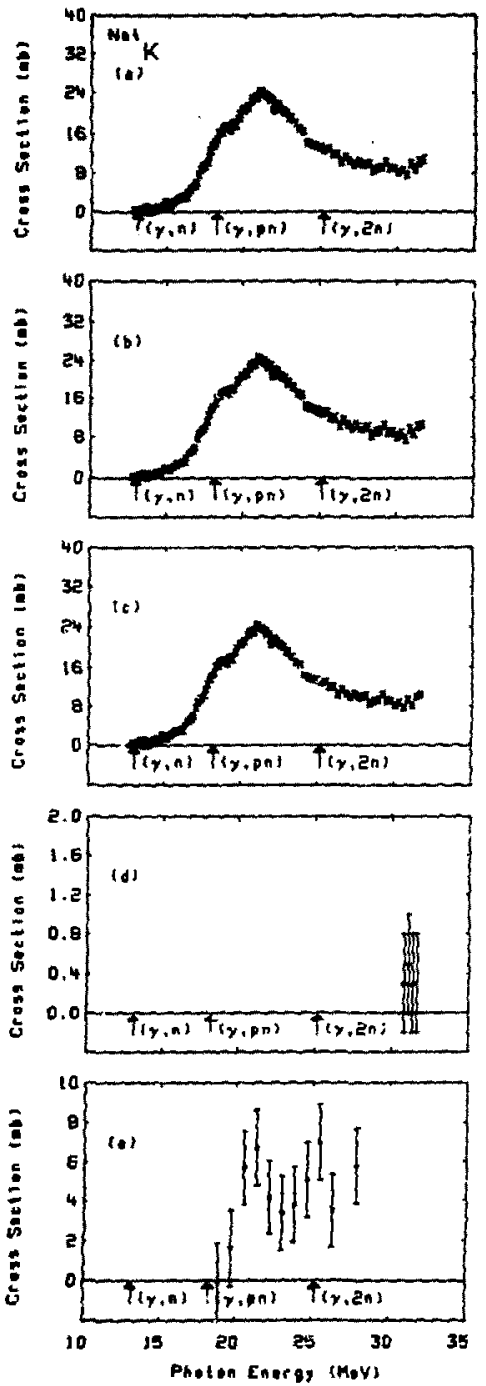

Fig. 4IA 


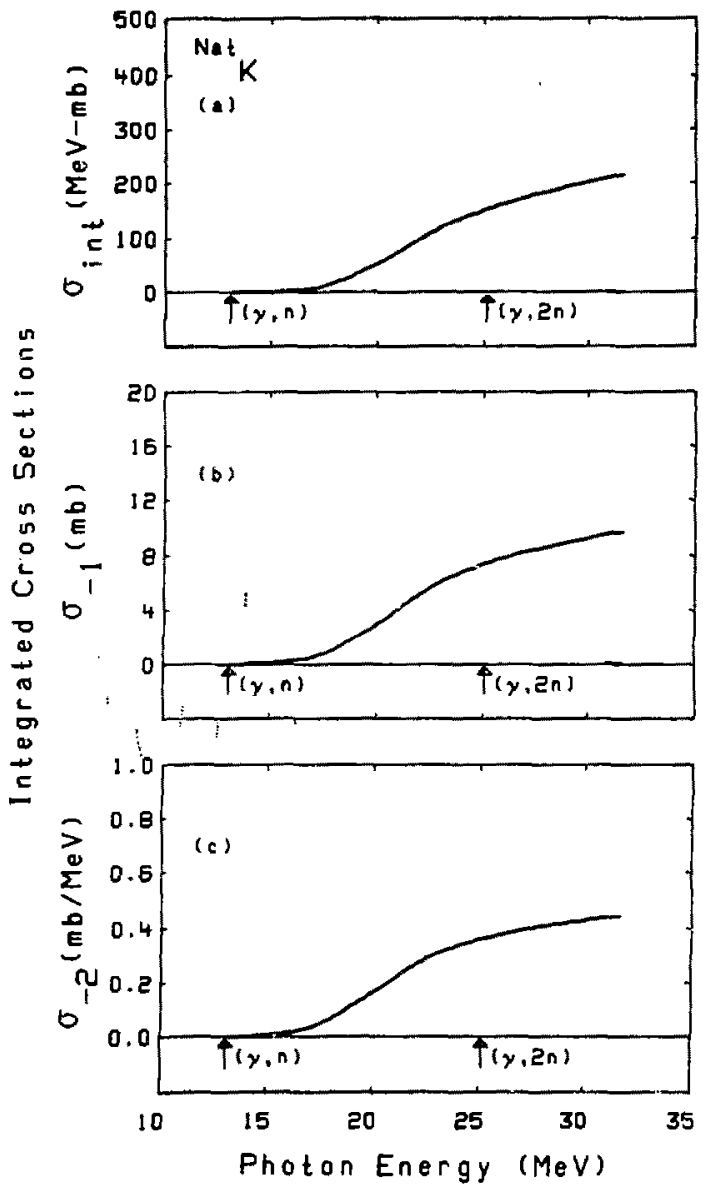

Fig. 41B 

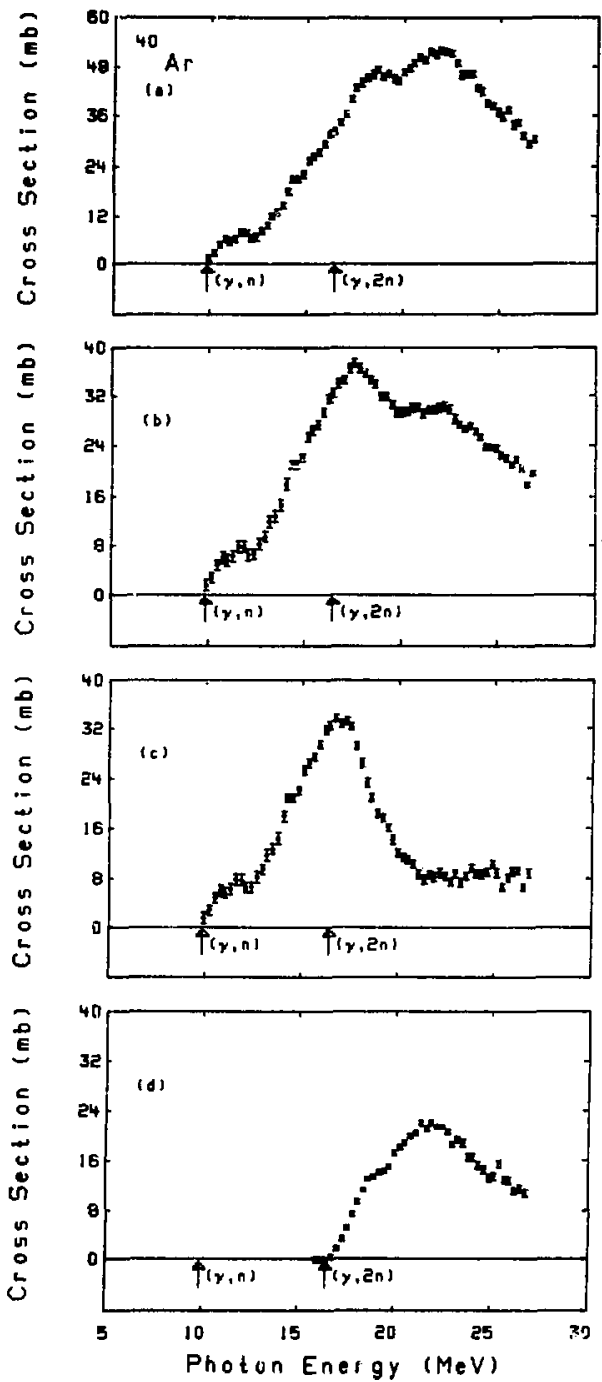

Fig. $42 \mathrm{~A}$ 


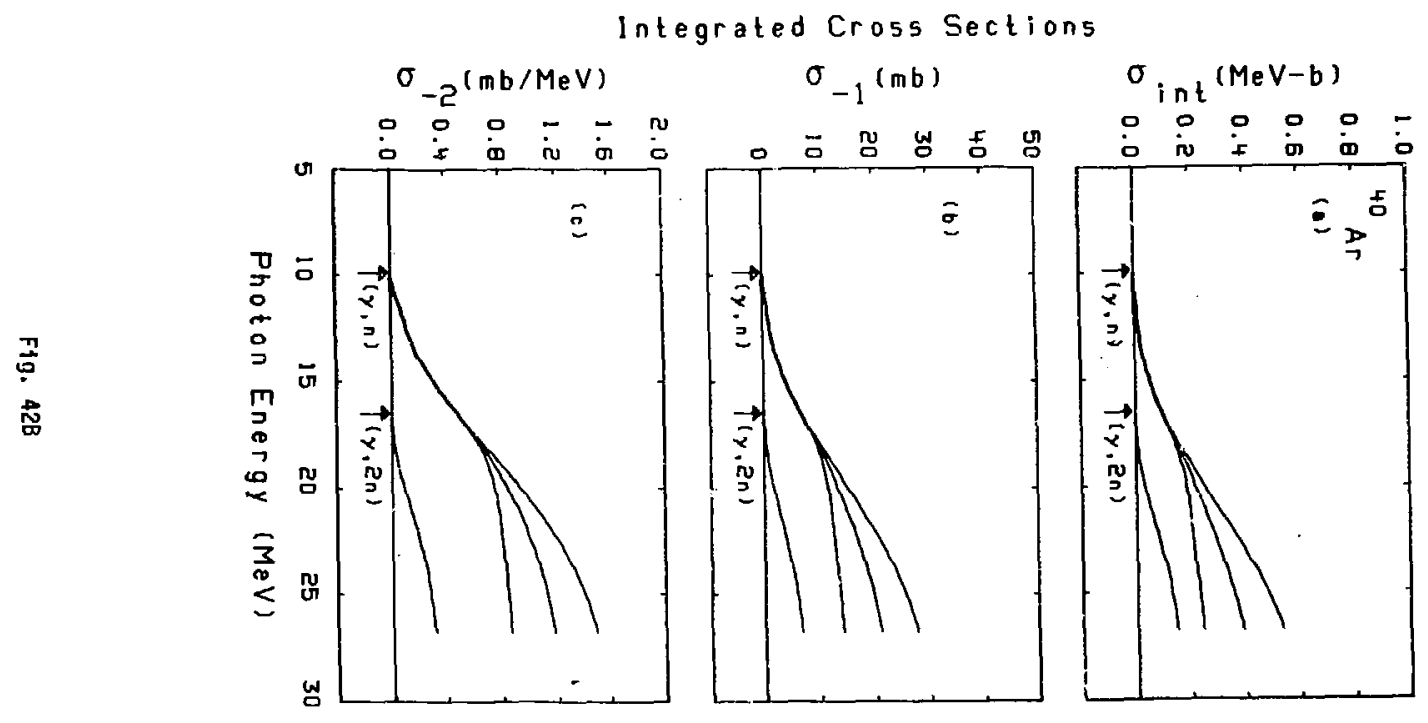




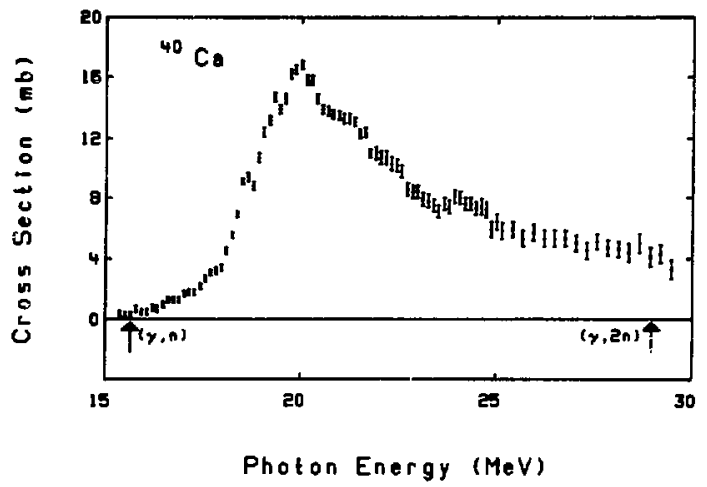

Fig. 43A 


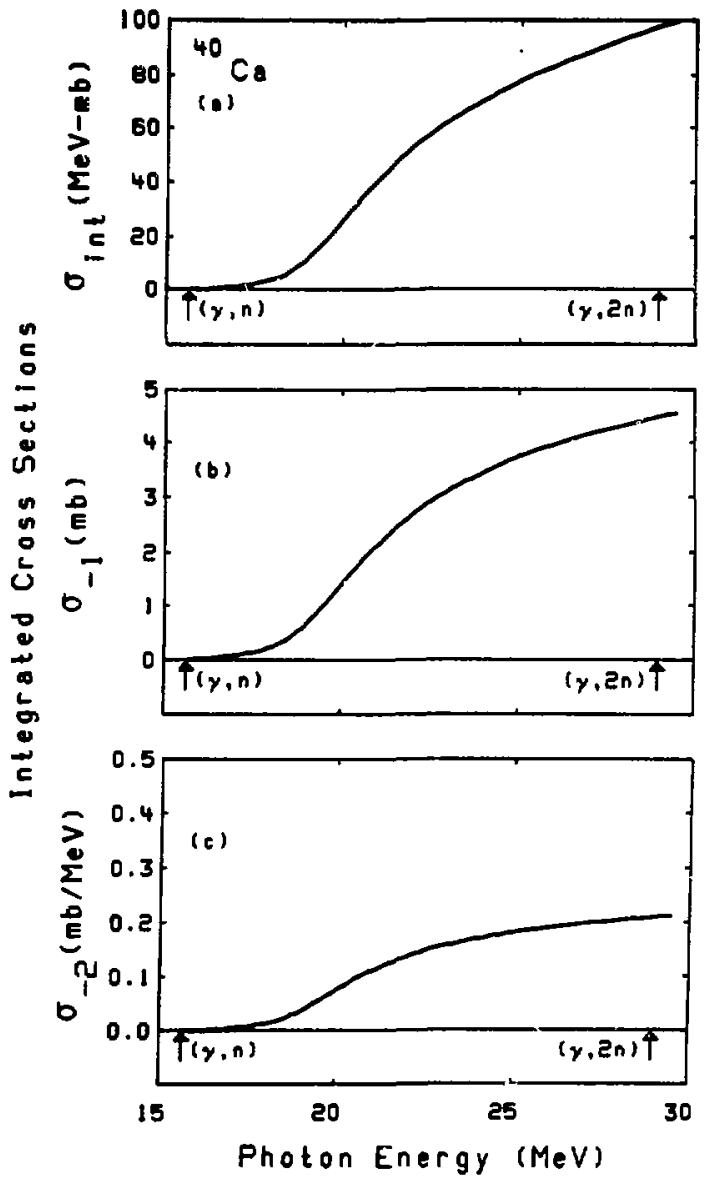

F1g. 438 

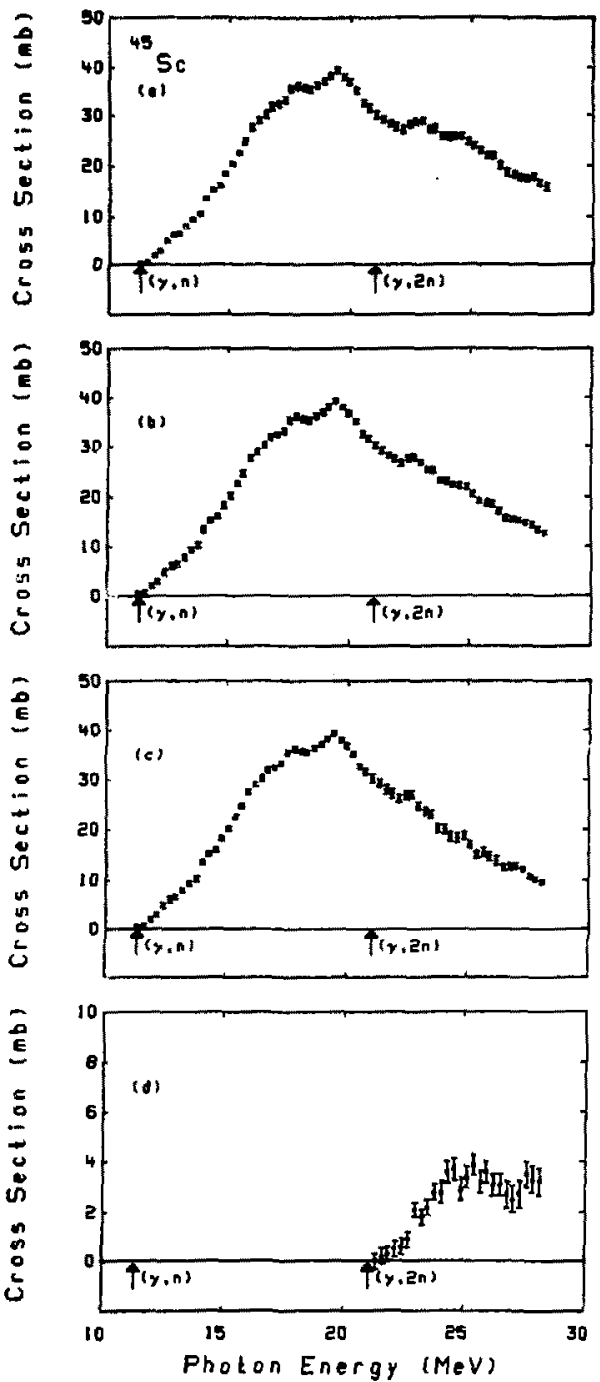

Fig. $44 A$ 

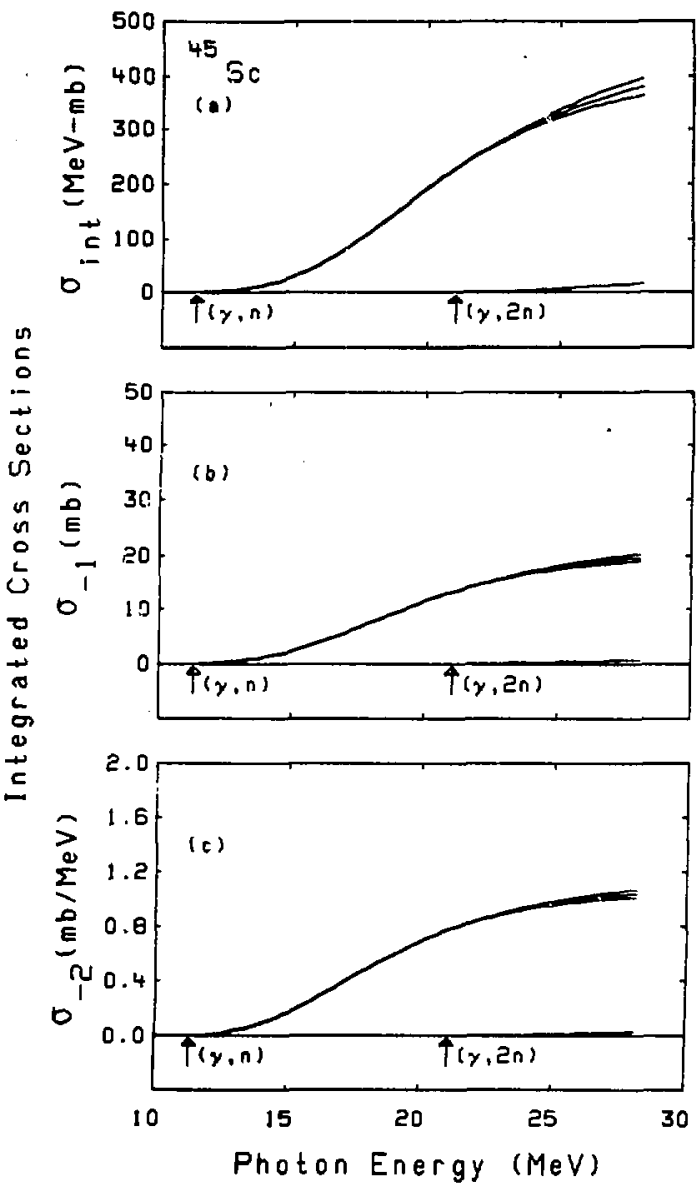

Fig. 44B 

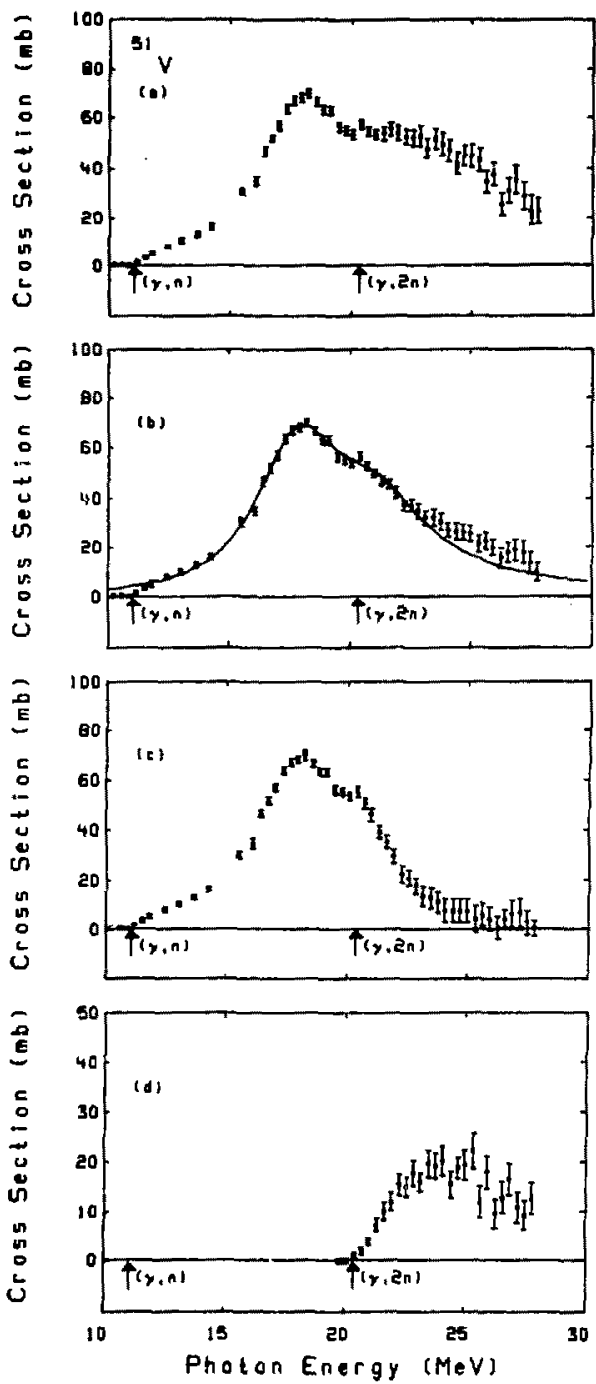

Fig. $45 \mathrm{~A}$ 

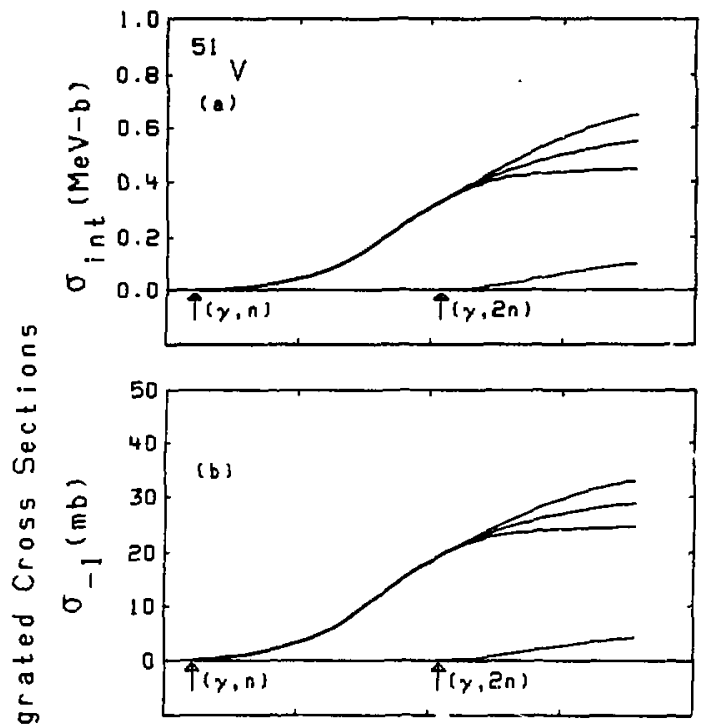

$\stackrel{ \pm}{5}$

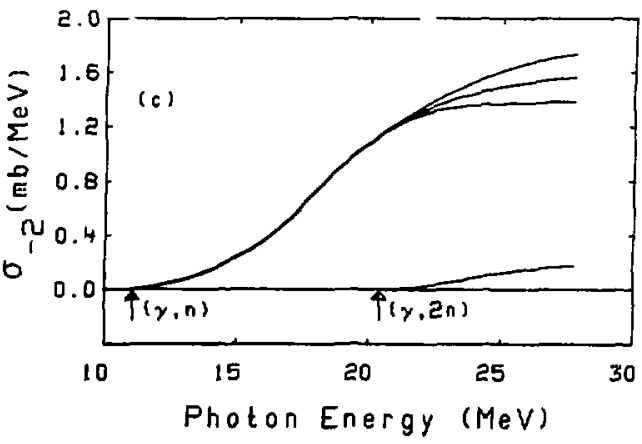

Fig. $45 B$ 

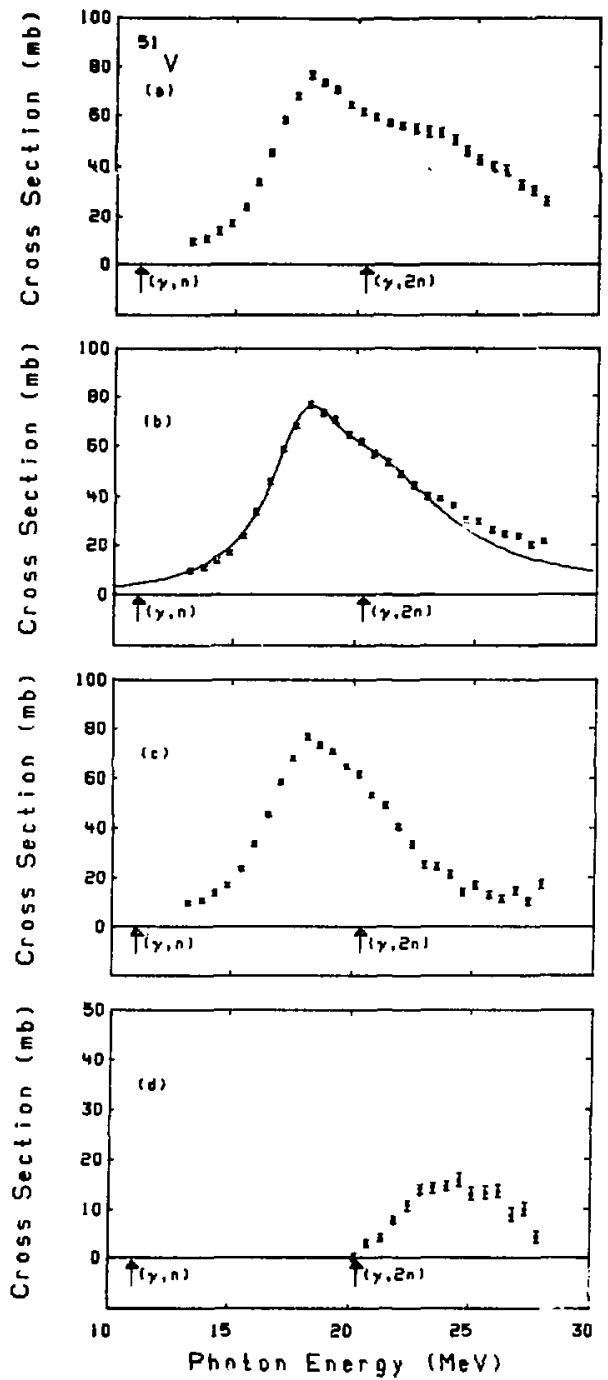

Fig. $46 \mathrm{~A}$ 

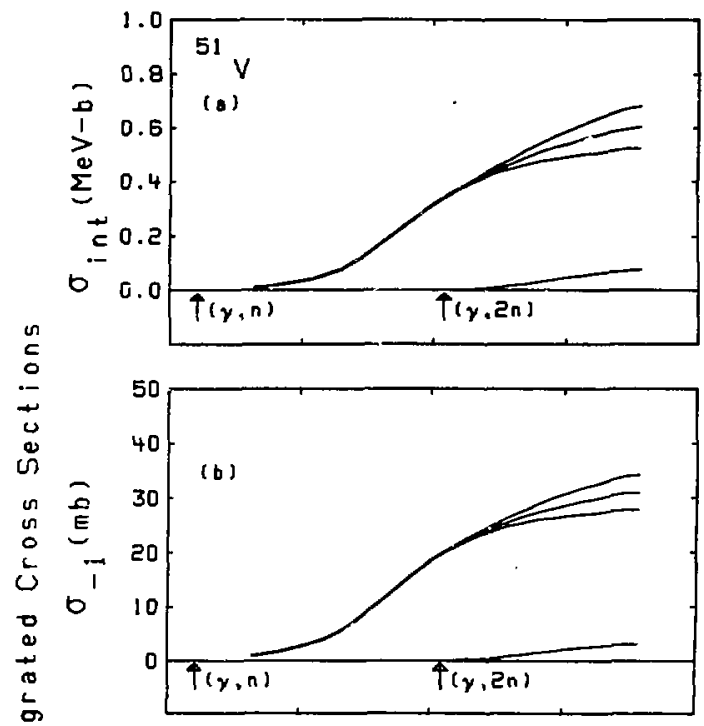

$\stackrel{5}{5}$

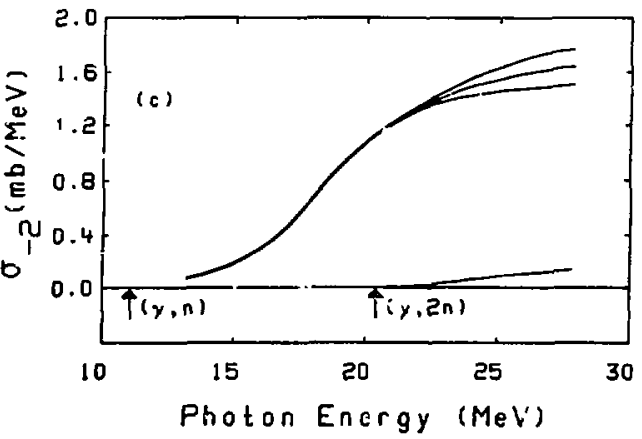

Fig. 46B 

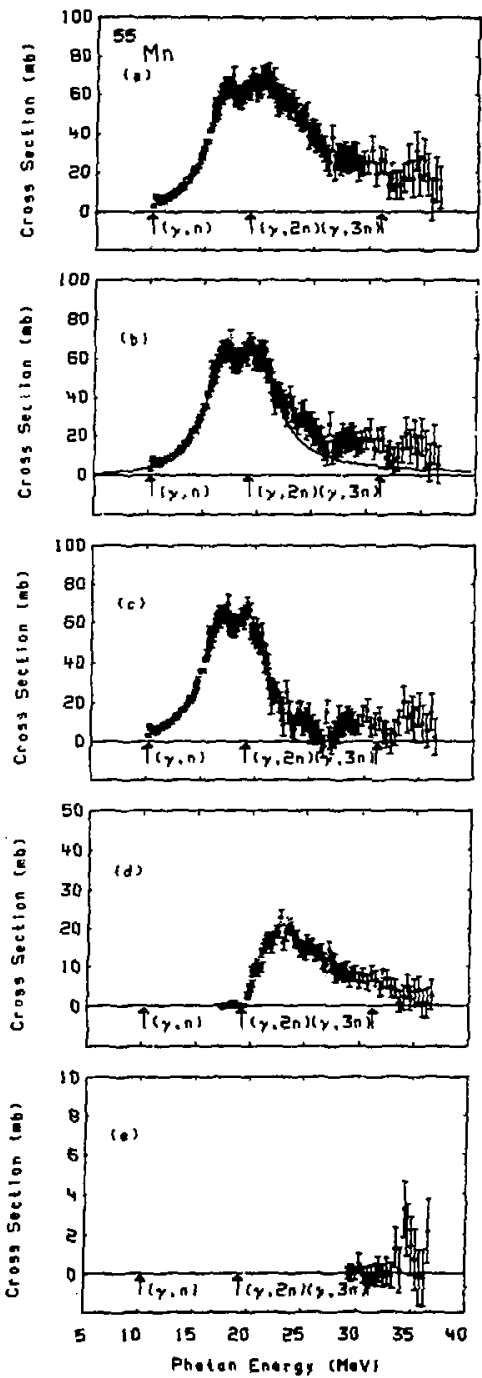

Fig. $47 A$ 

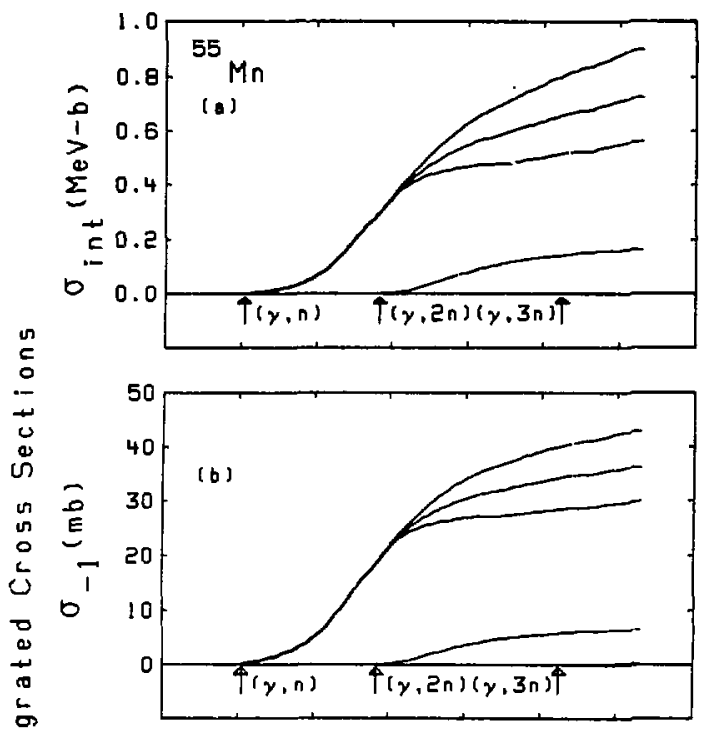

$\stackrel{0}{5}$

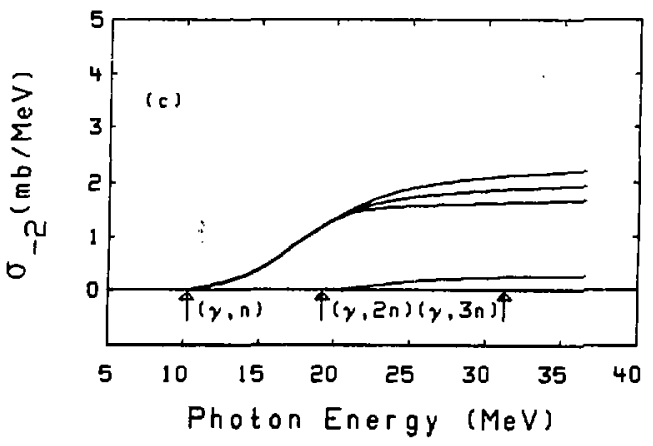

Fig. 47B 
Cross Section (mb) Cross Section (mb) Cross Section (mb) Cross Section (mb)

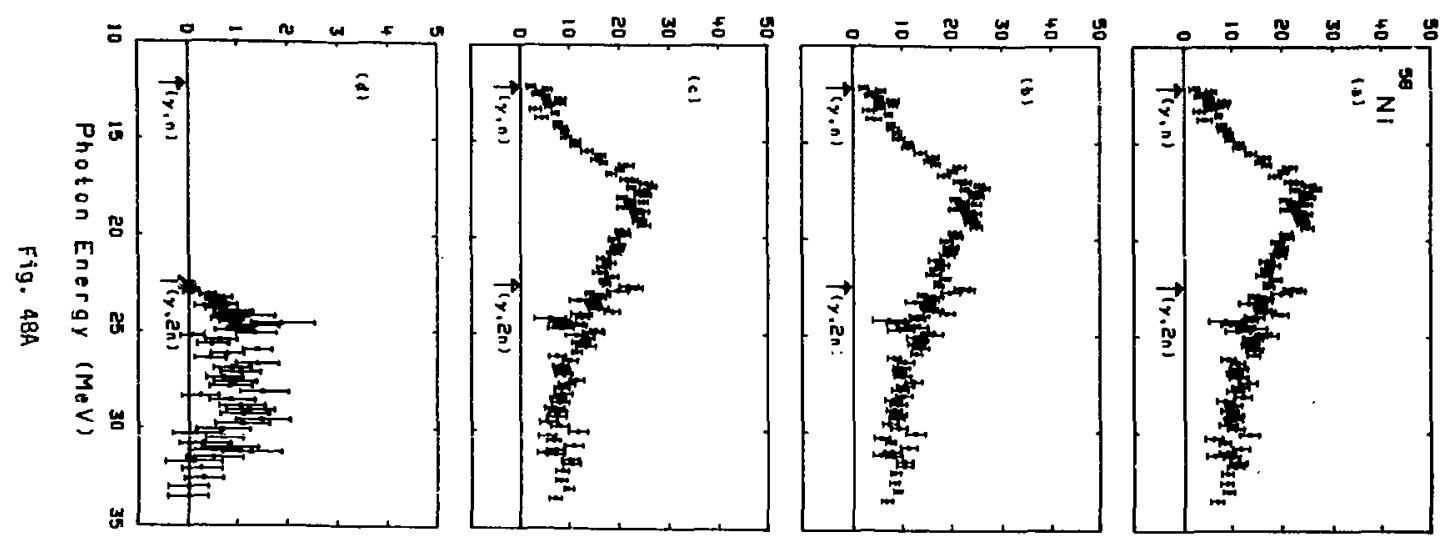




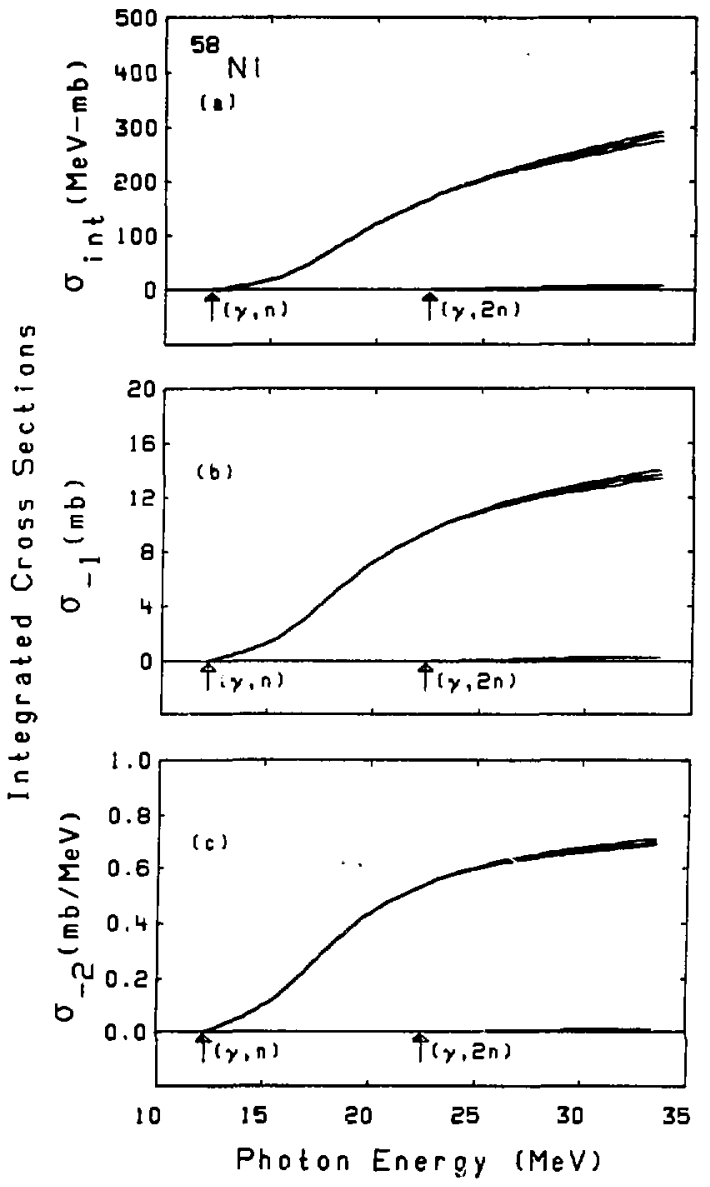

Fig. $48 B$ 

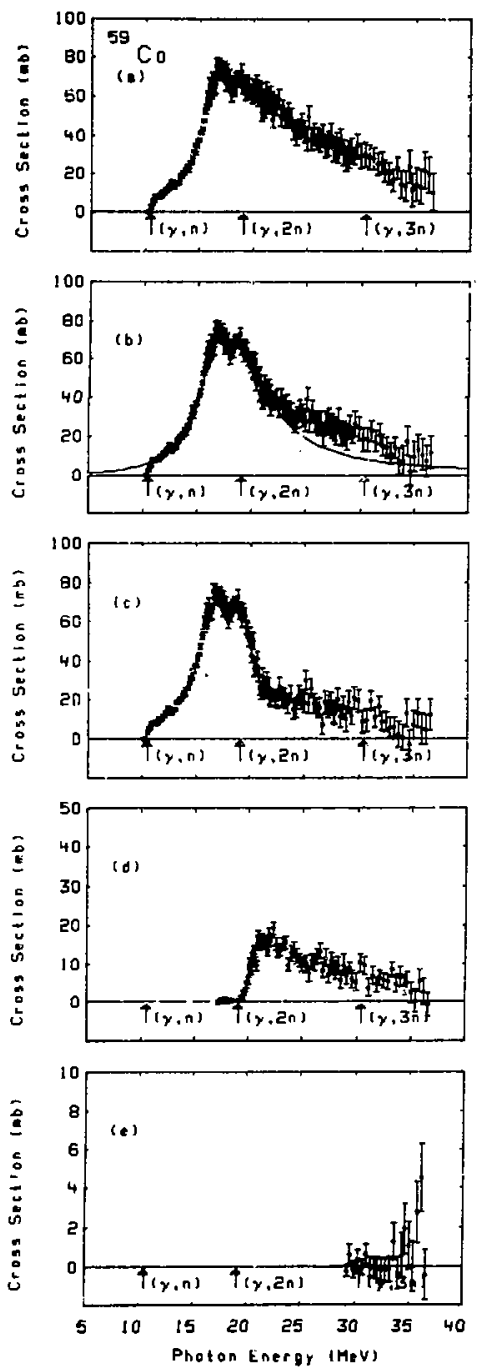

Fig. $19 \mathrm{~A}$ 

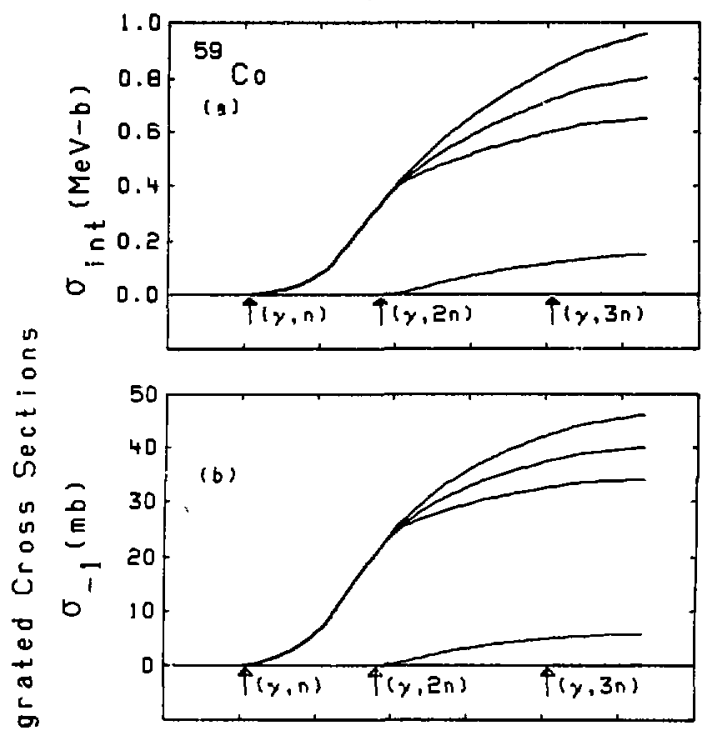

$\pm$

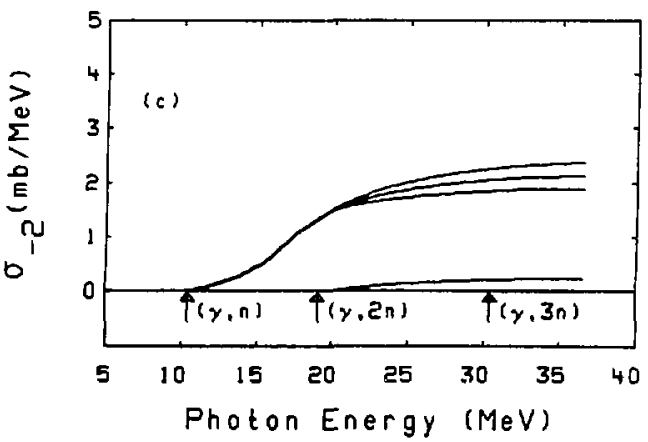

Fig. $49 B$ 


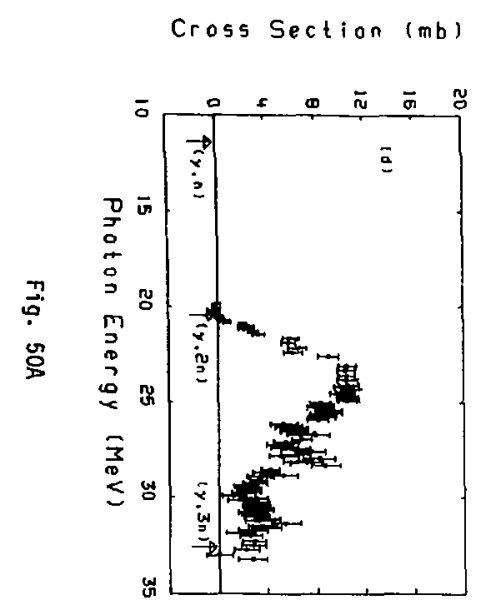

Cross Section (mb)

Cross Section (mb) Cross Section (mb)
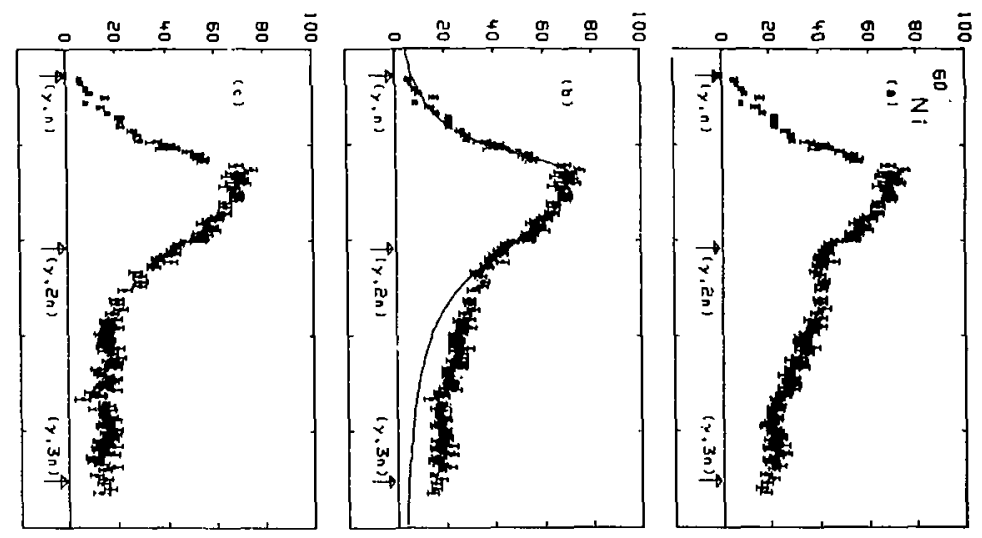


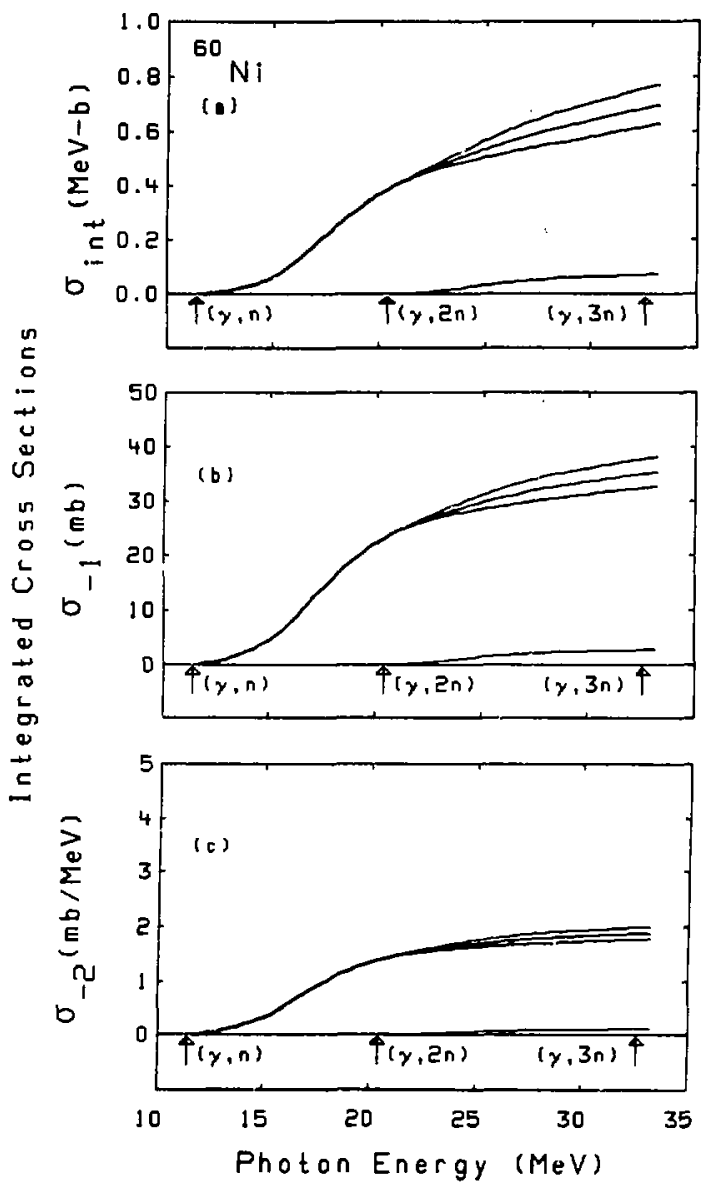

Fig. 50B 

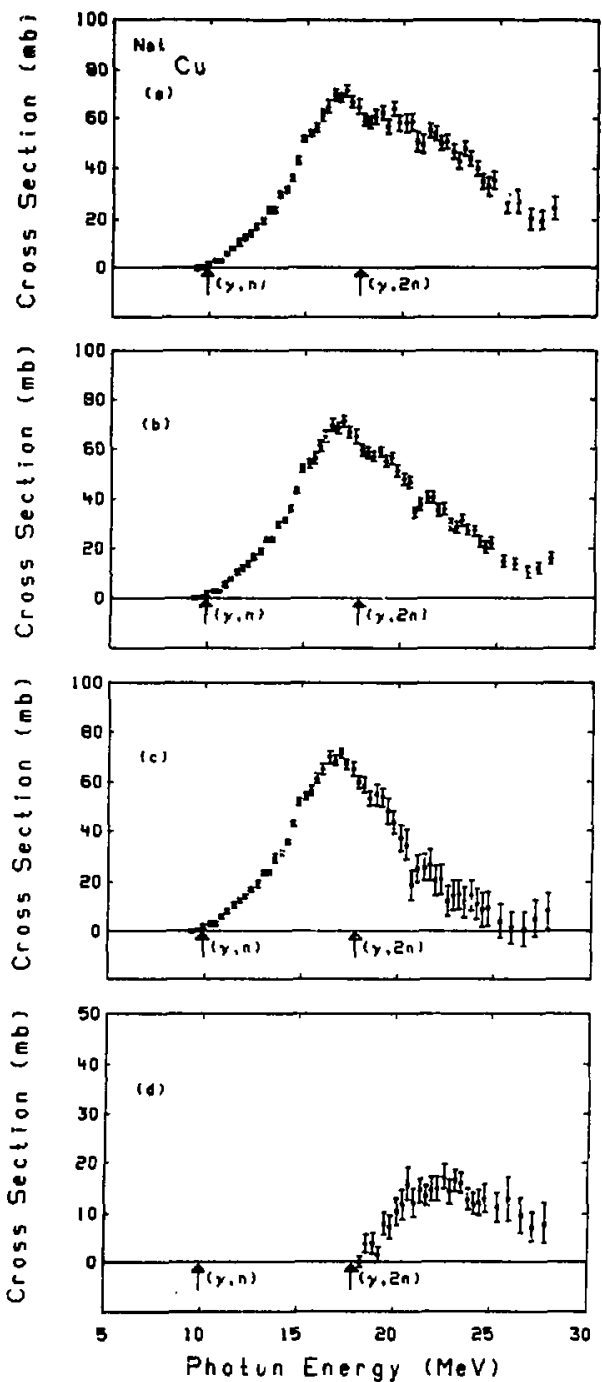

F1g. 51A 


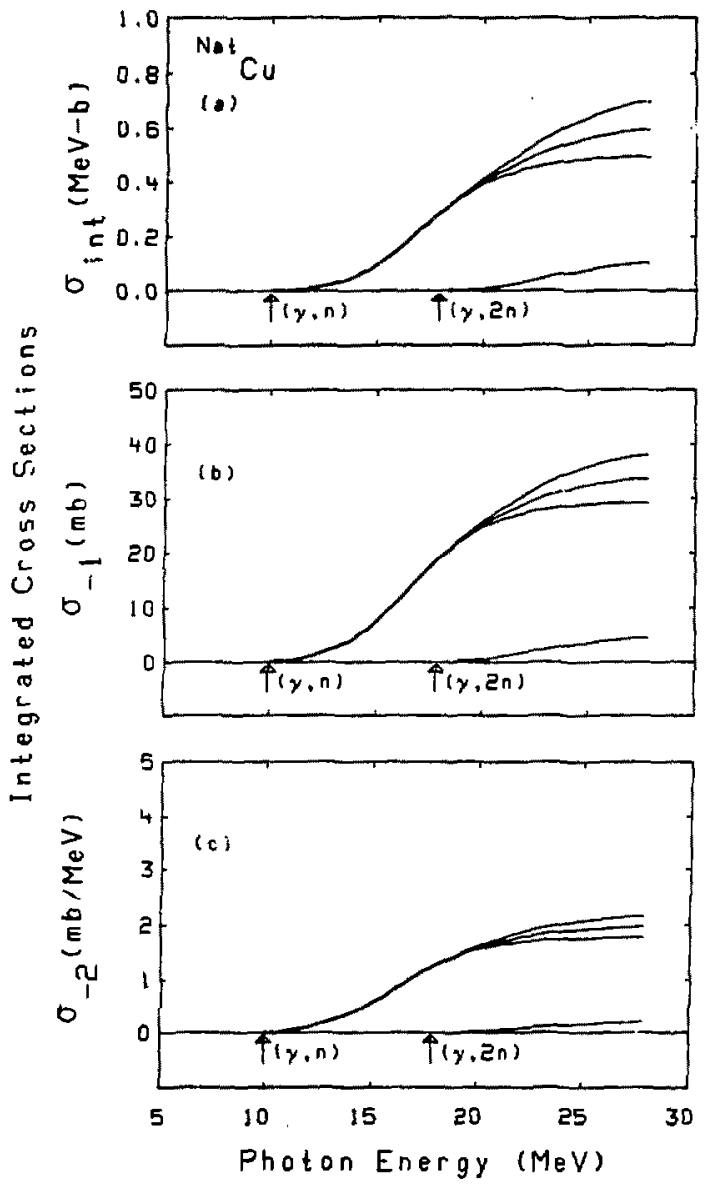

Fig. $51 B$ 

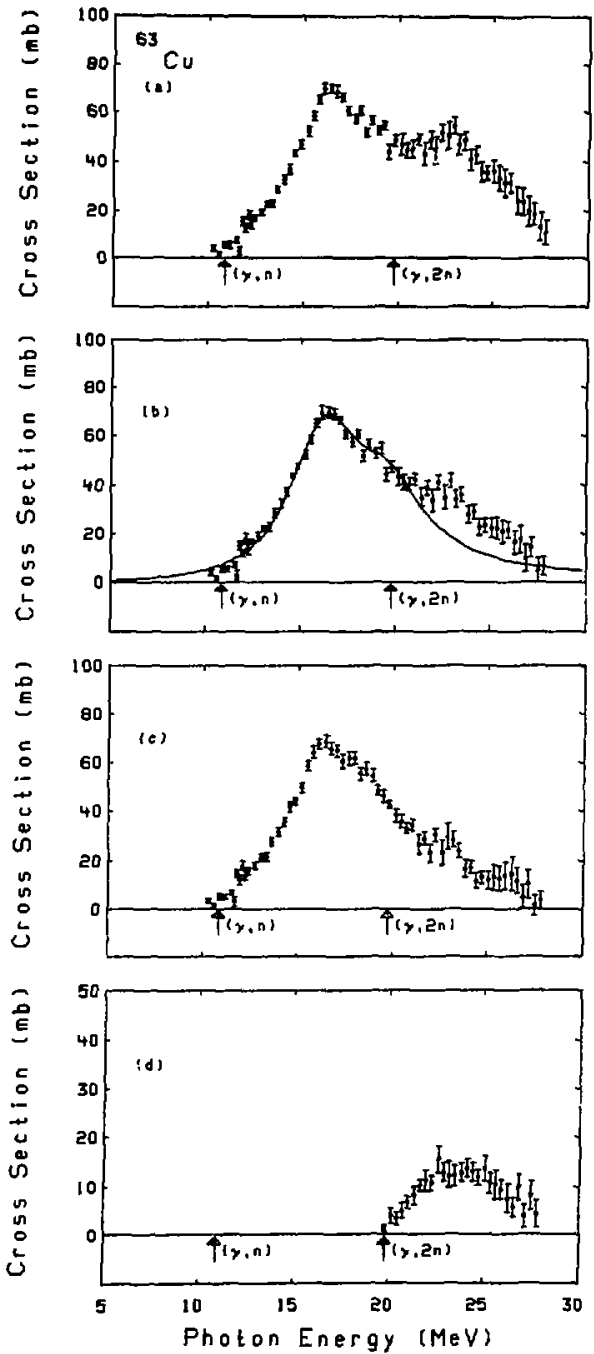

Fig. $52 \mathrm{~A}$ 


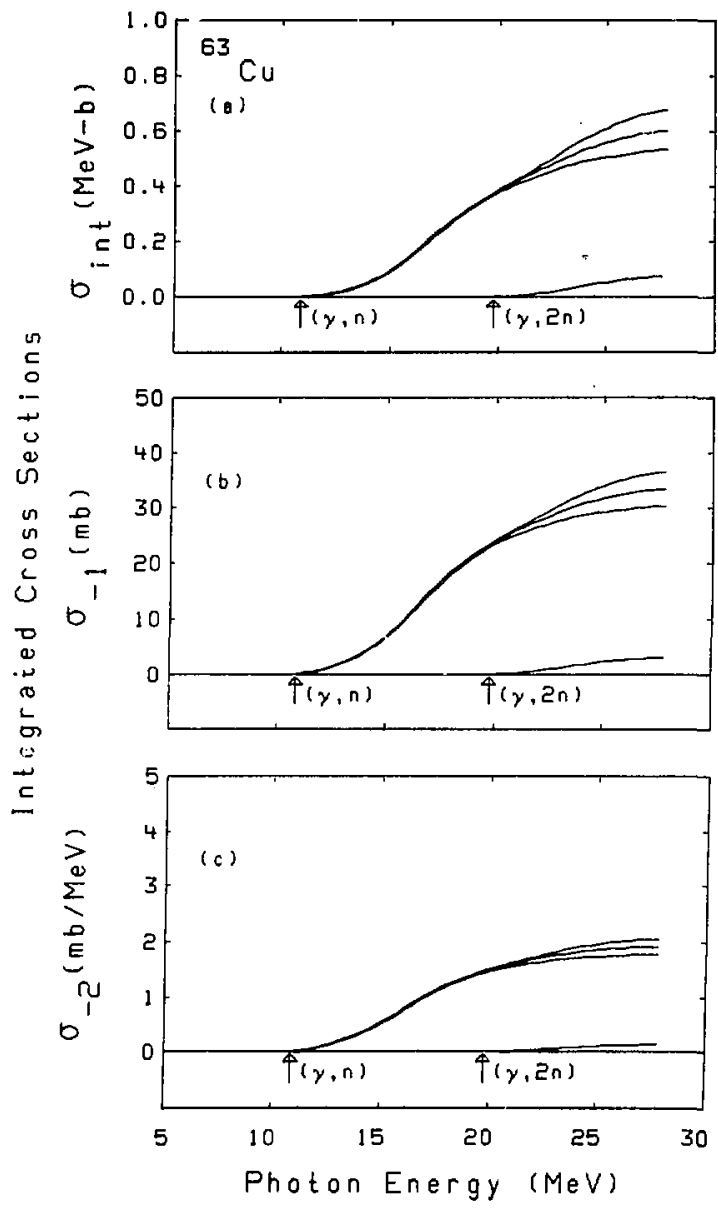

Fig. $52 B$ 

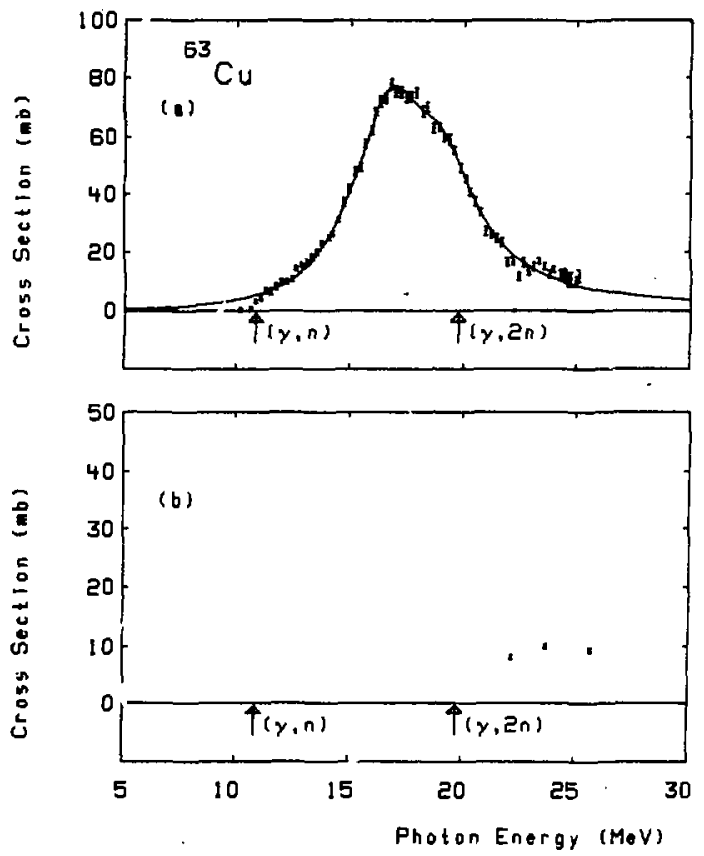

Fig. $53 \mathrm{~A}$ 


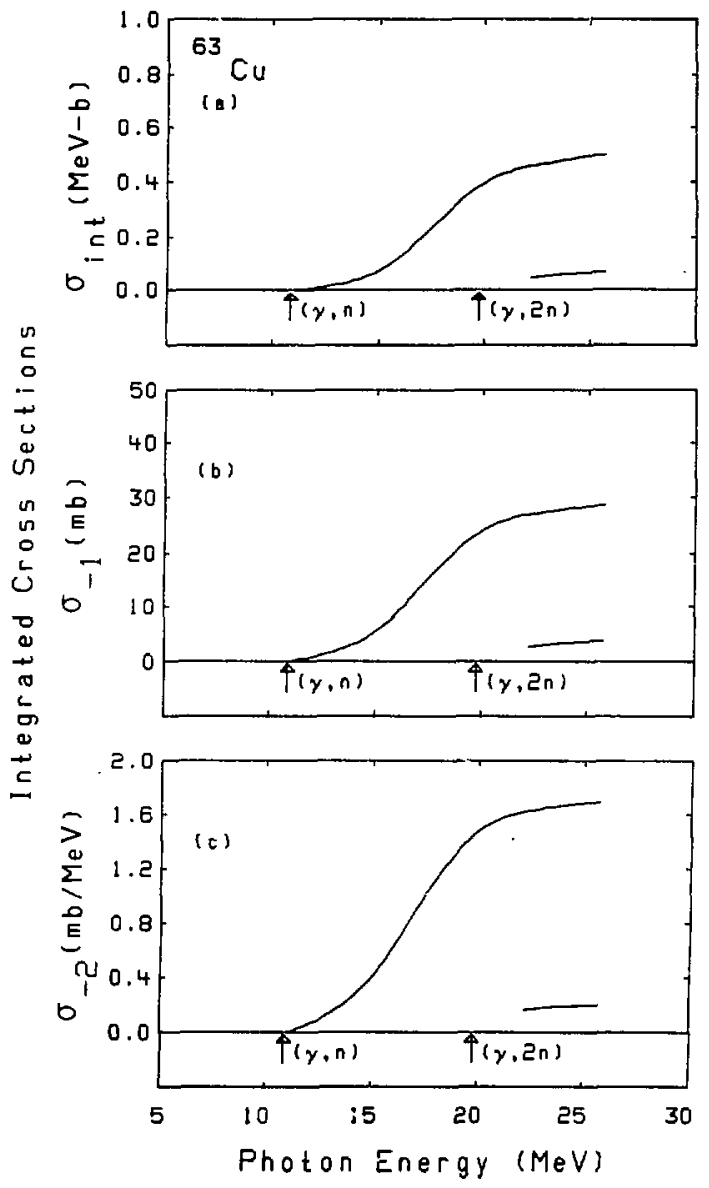

Fig. 538 

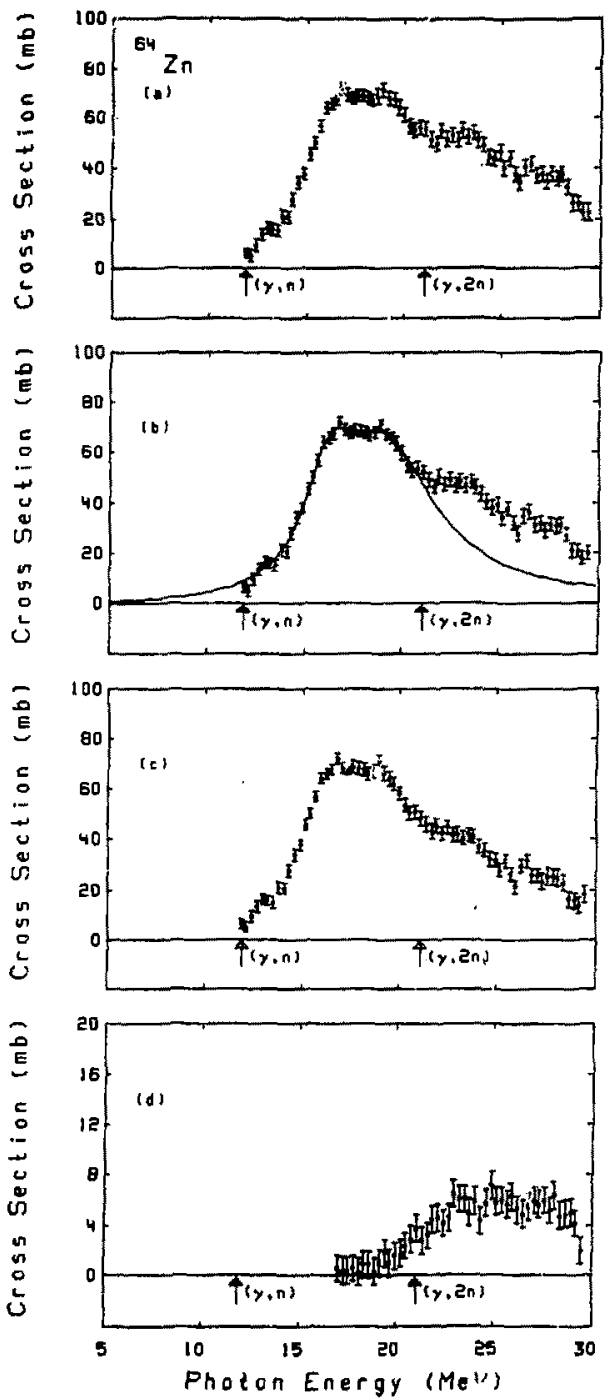

Fig. $54 A$ 


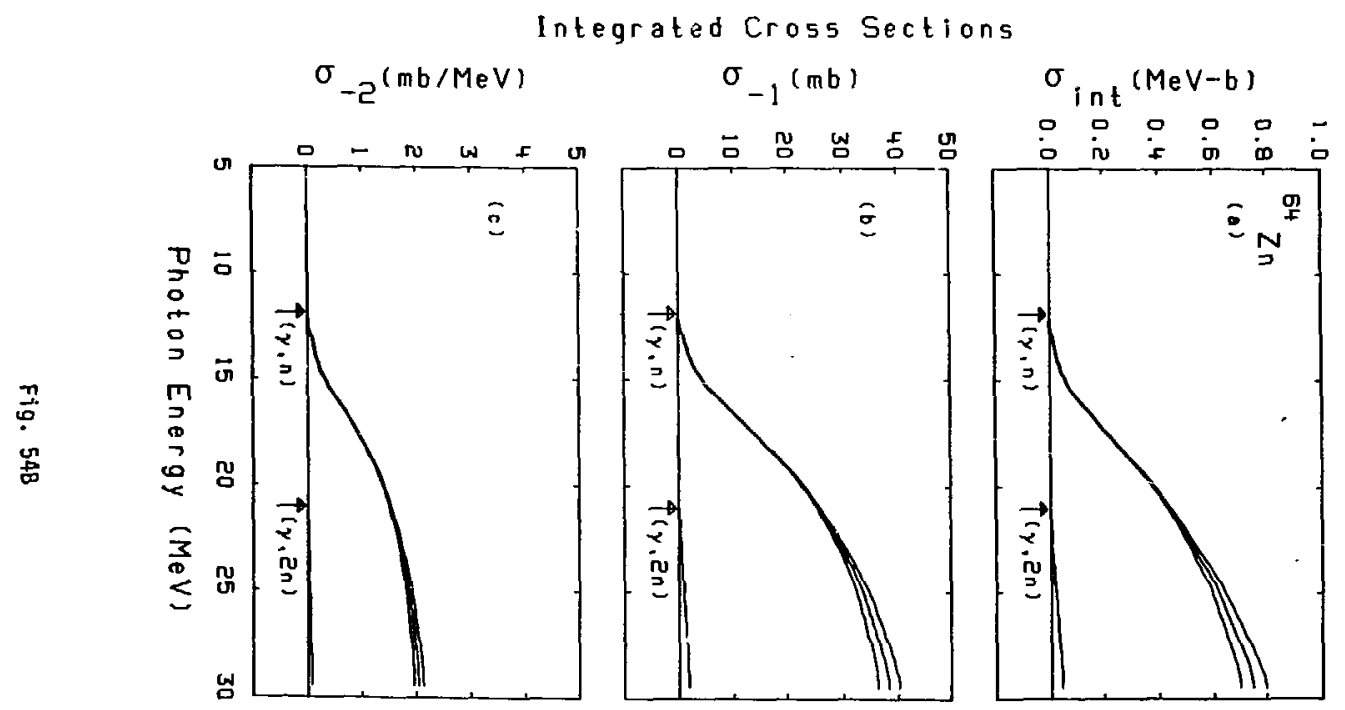



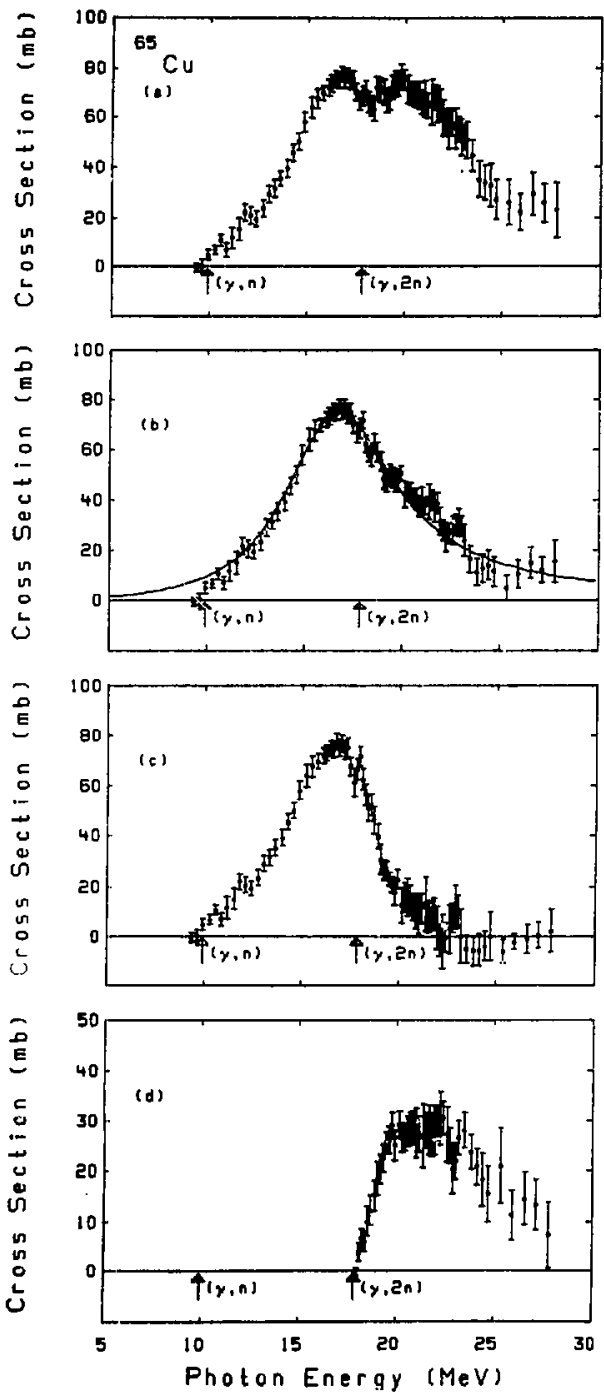

Fig. $55 \mathrm{~A}$ 

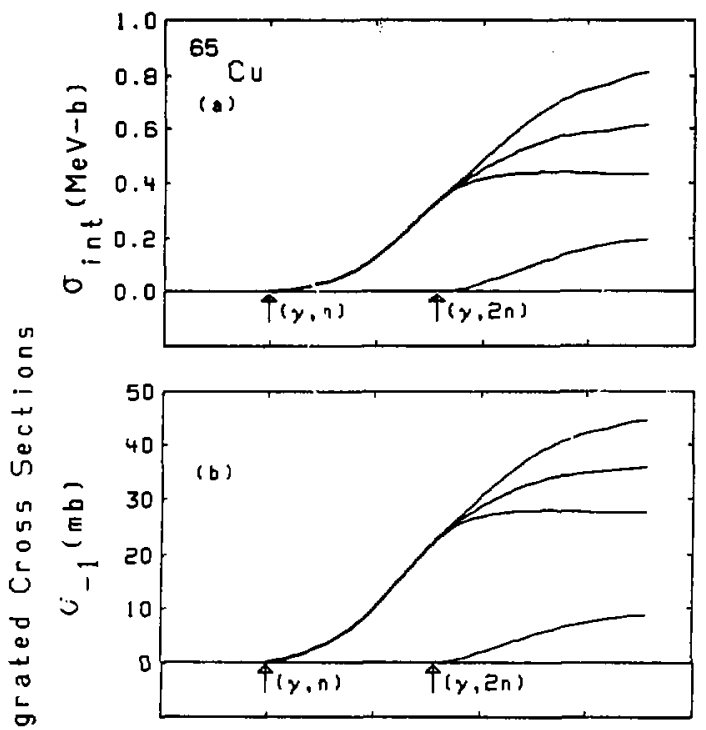

$\stackrel{\circ}{5}$

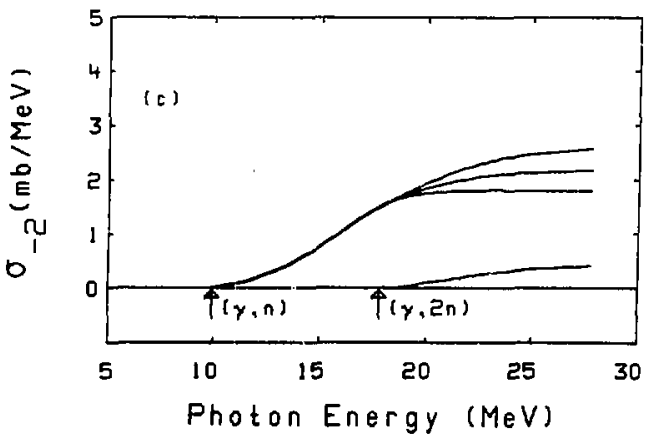

Fic. 358 

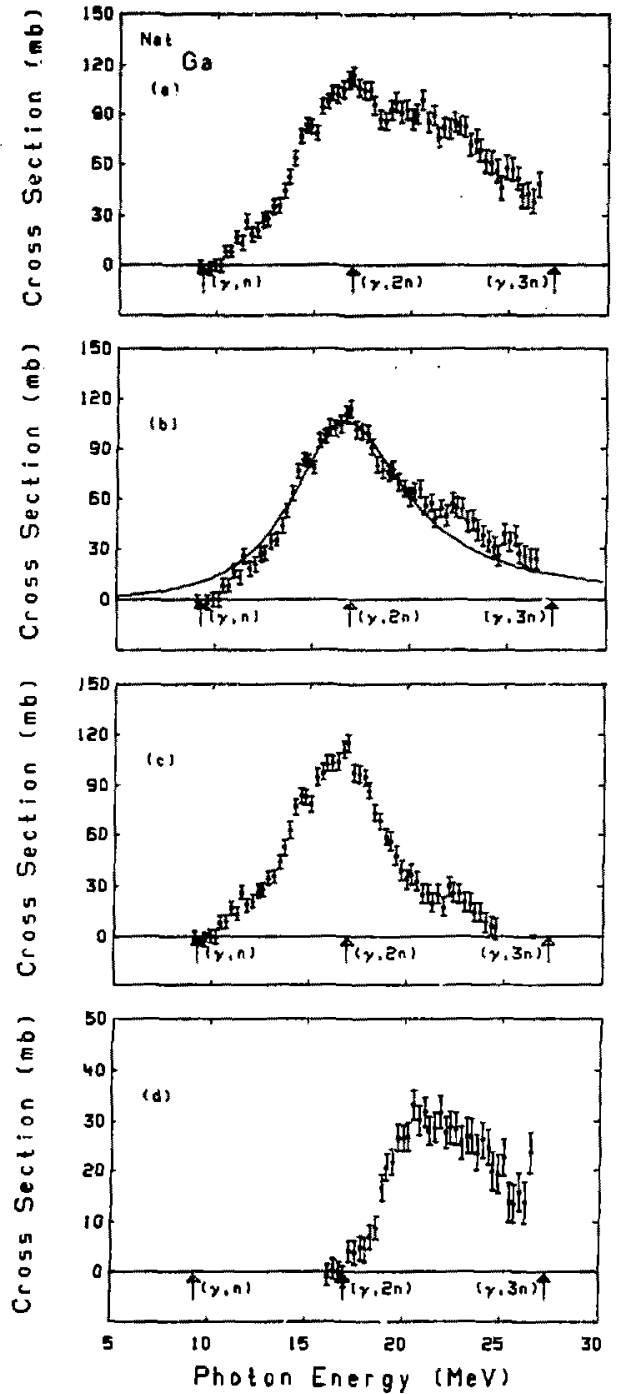

Fig. 56A 


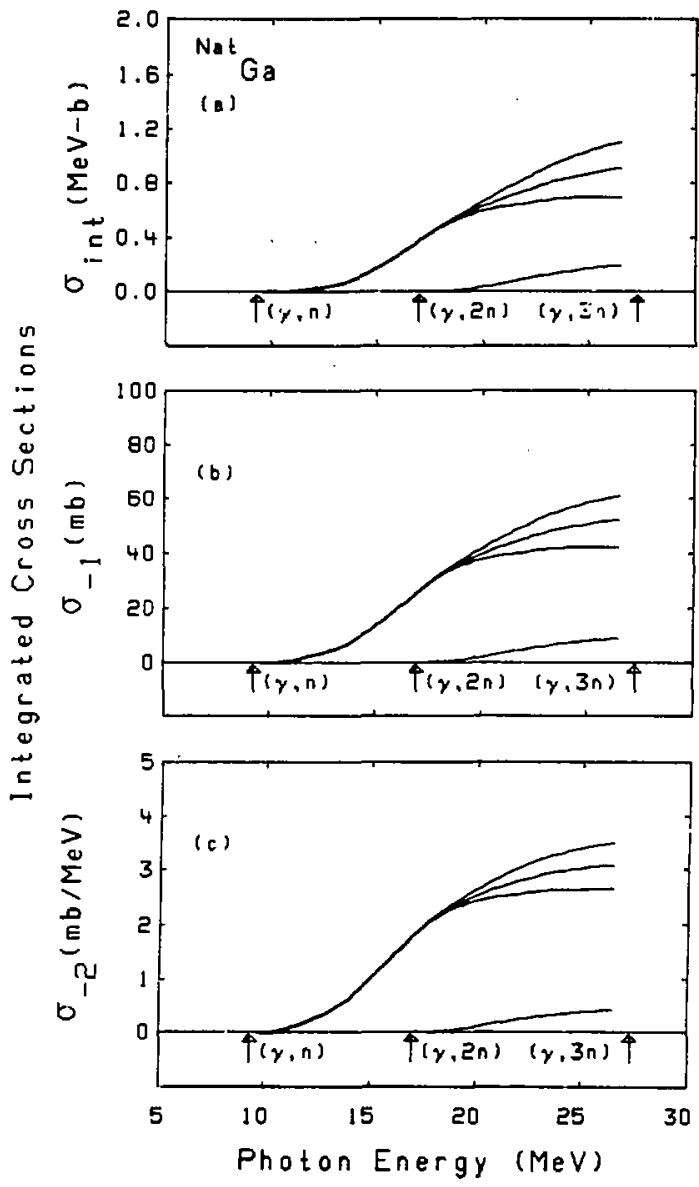

Fig. 568 

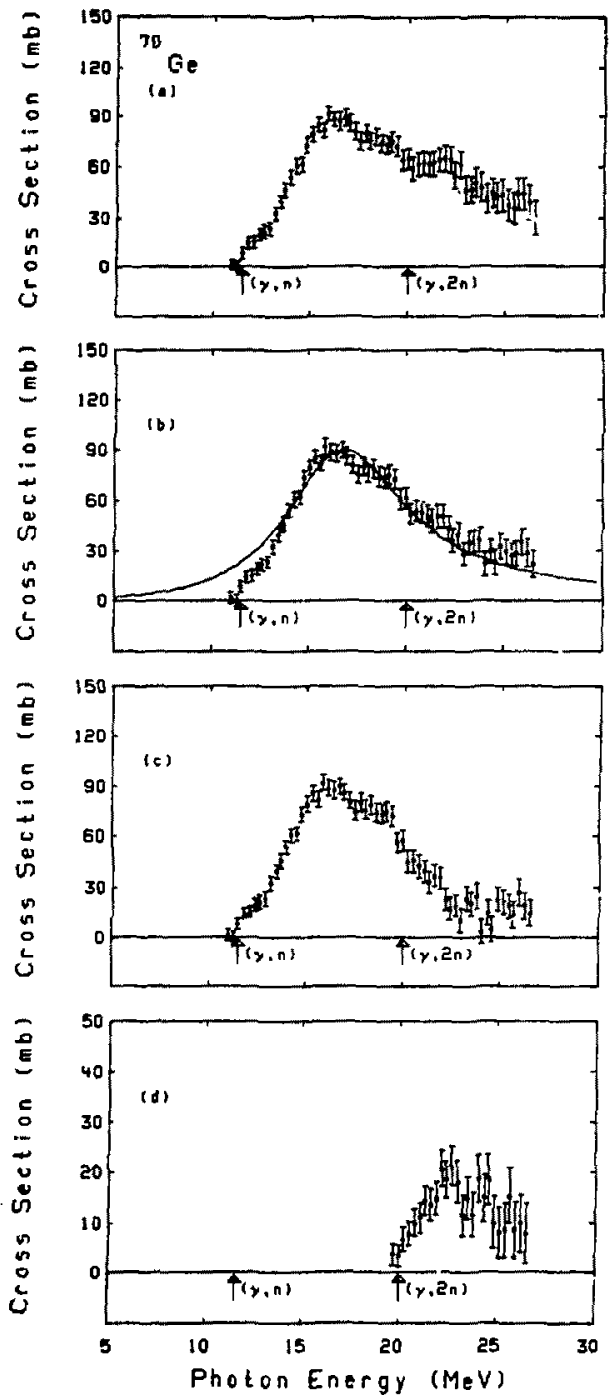

Fig. 57A 

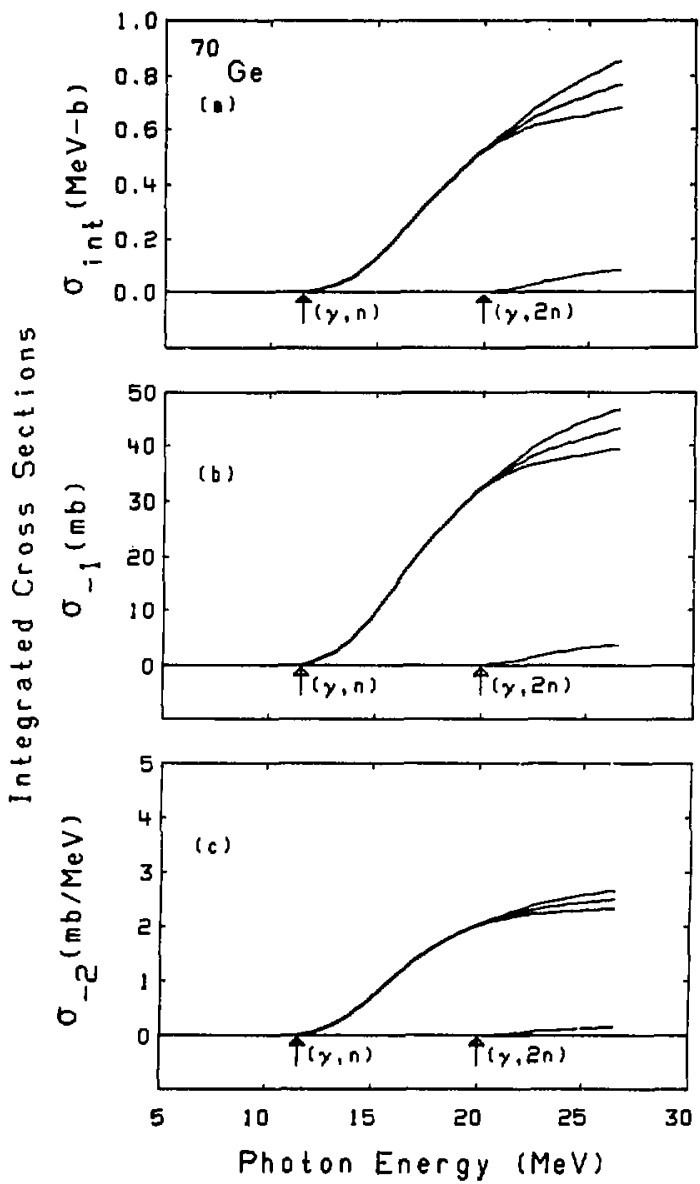

Fig. 57B 


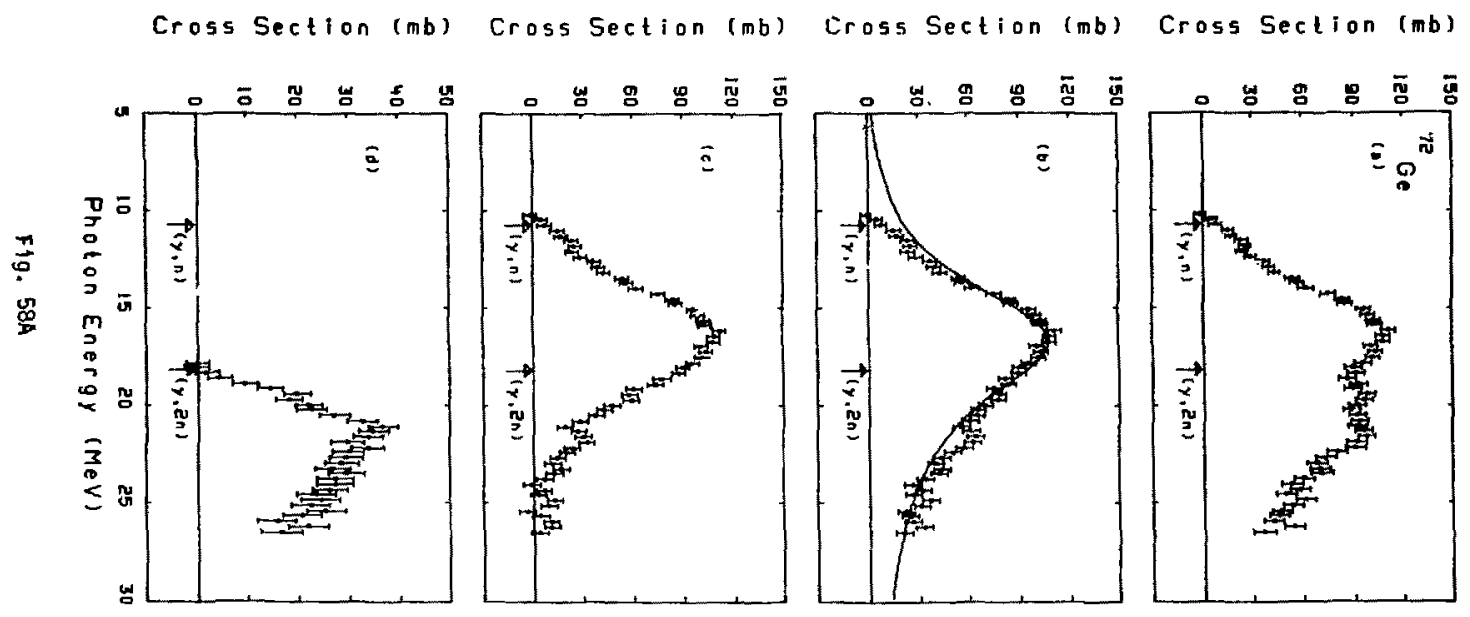




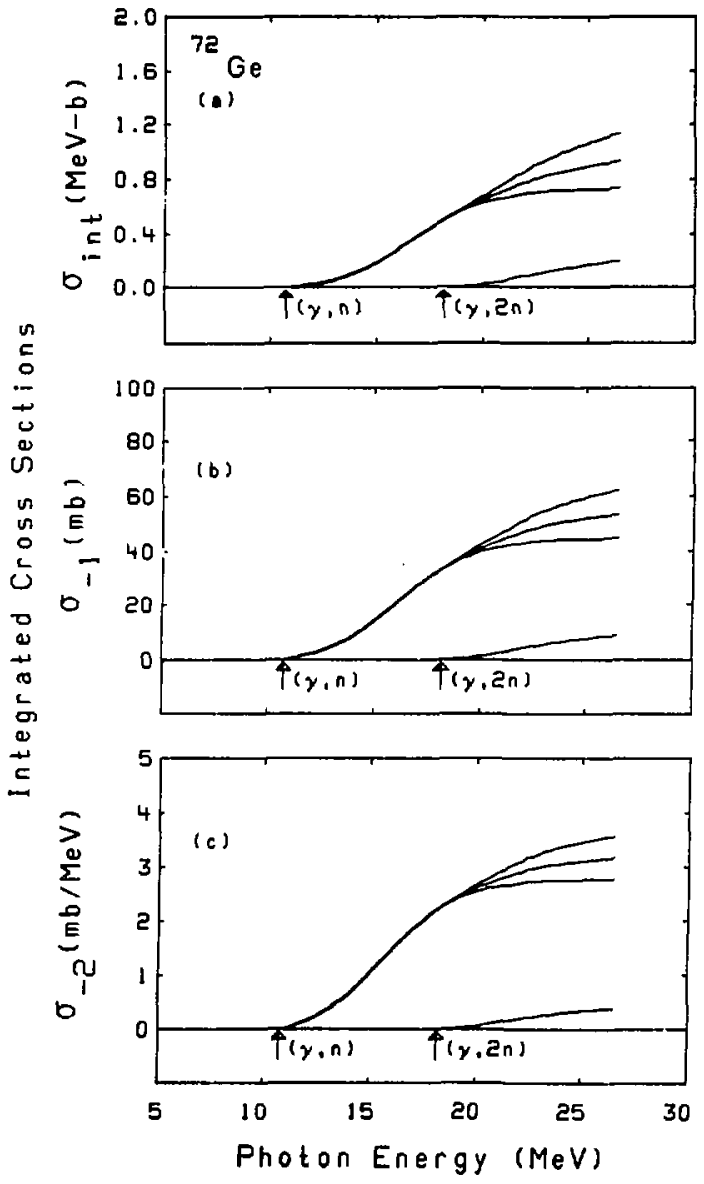

Fig. $58 B$ 

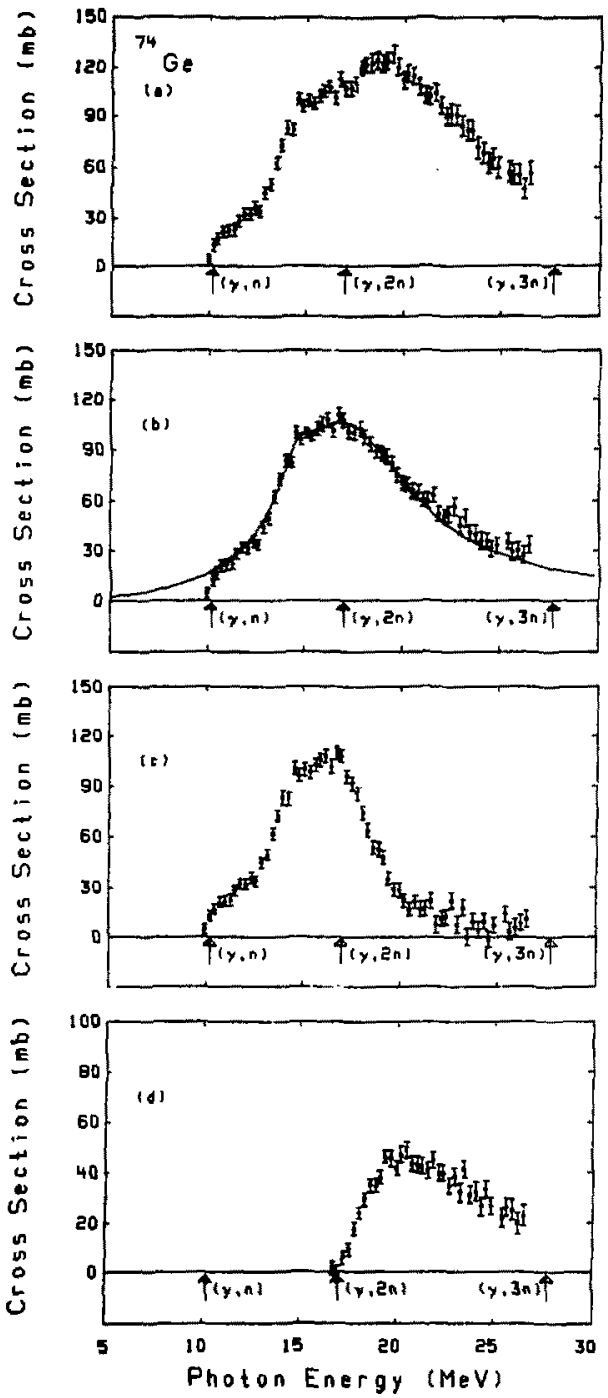

F1g. 59A 


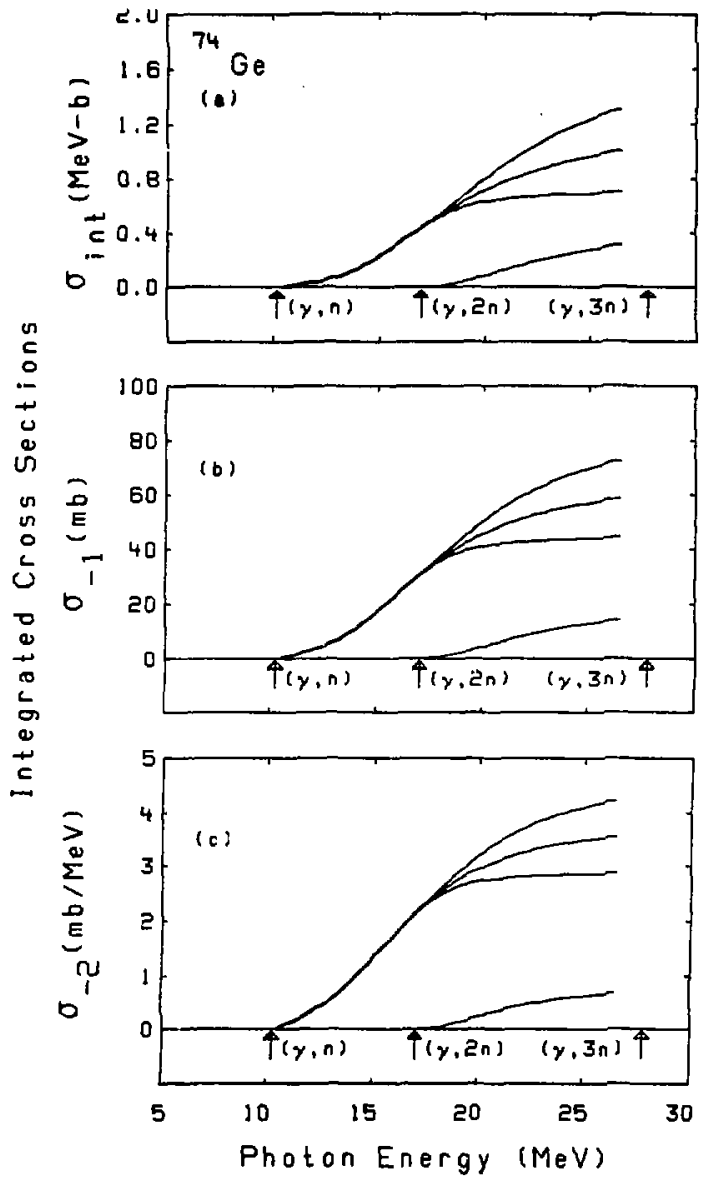

Fig. 59B 

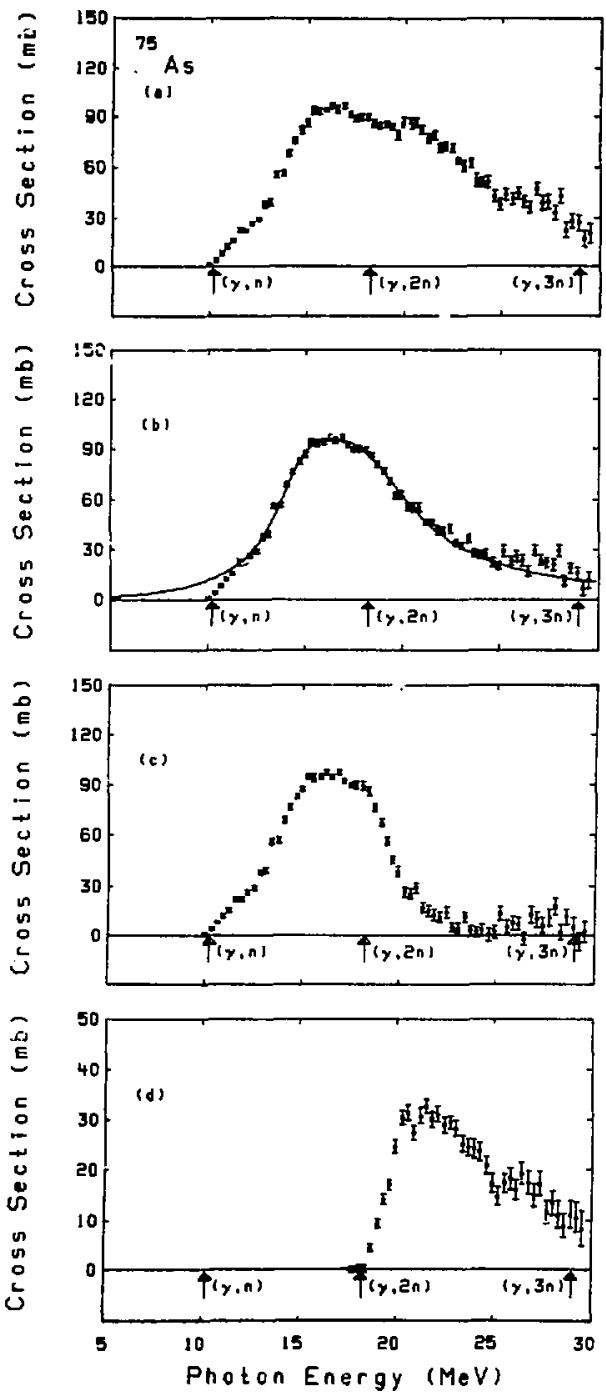

Fig. $60 \mathrm{~A}$ 


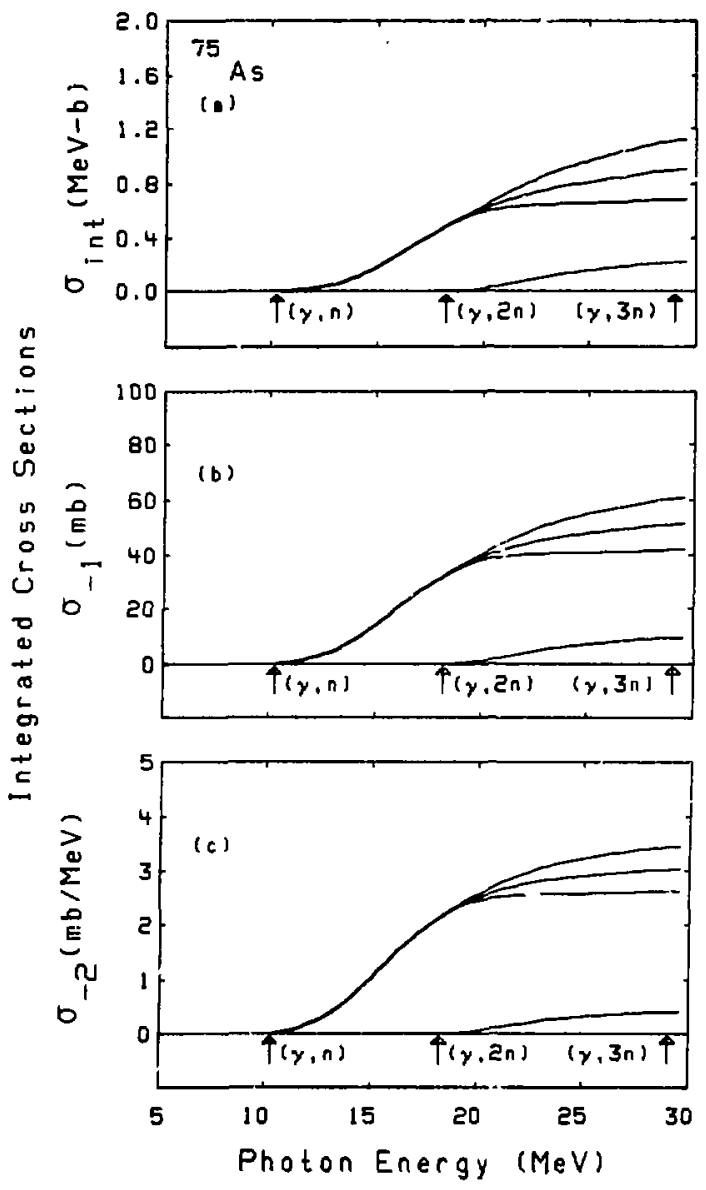

Fig. $60 \mathrm{~B}$ 

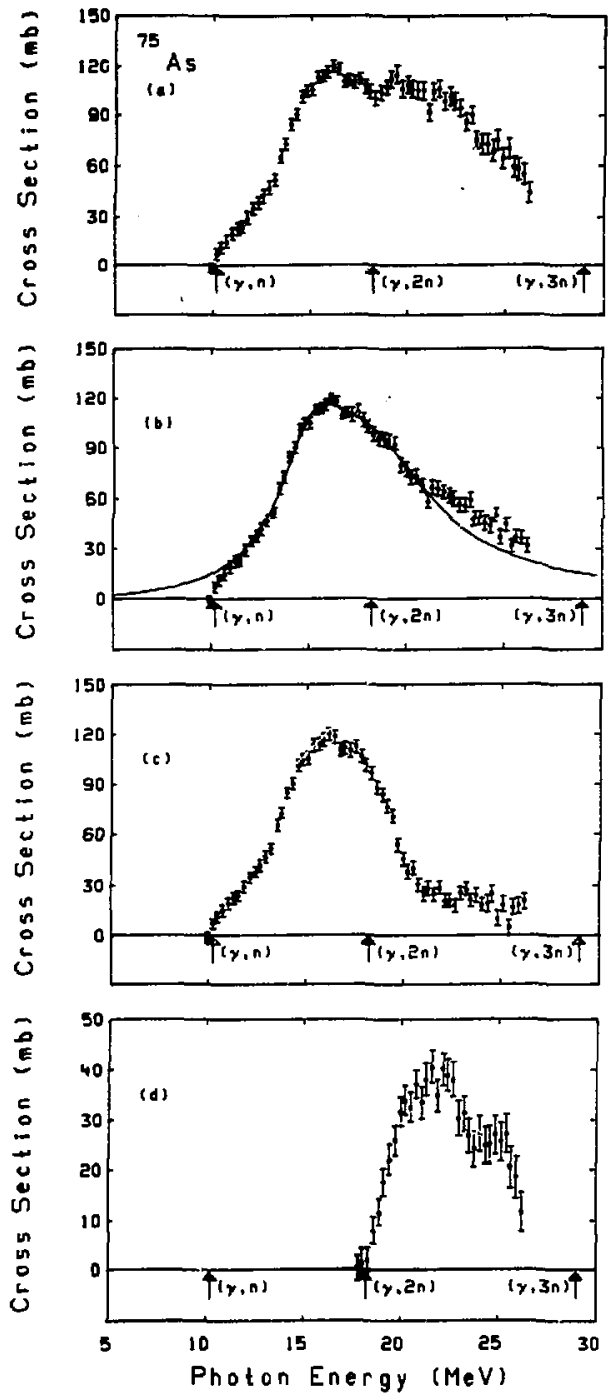

Fig. $61 \mathrm{~A}$ 


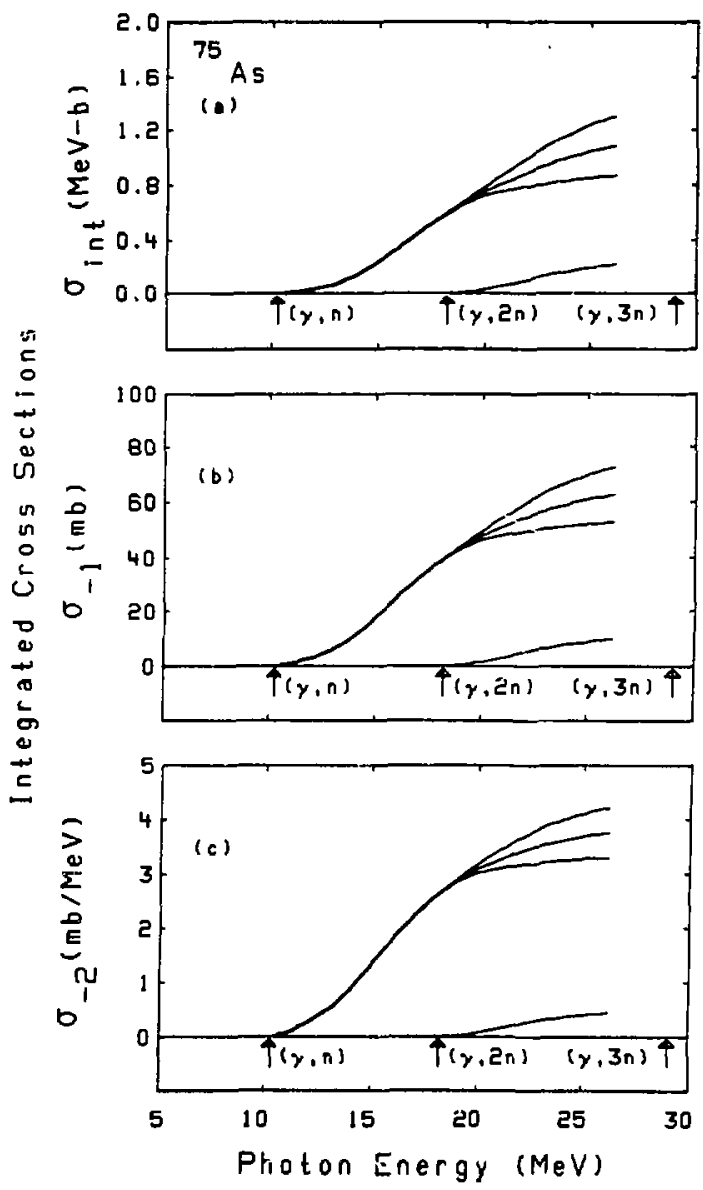

Fig. 61B 

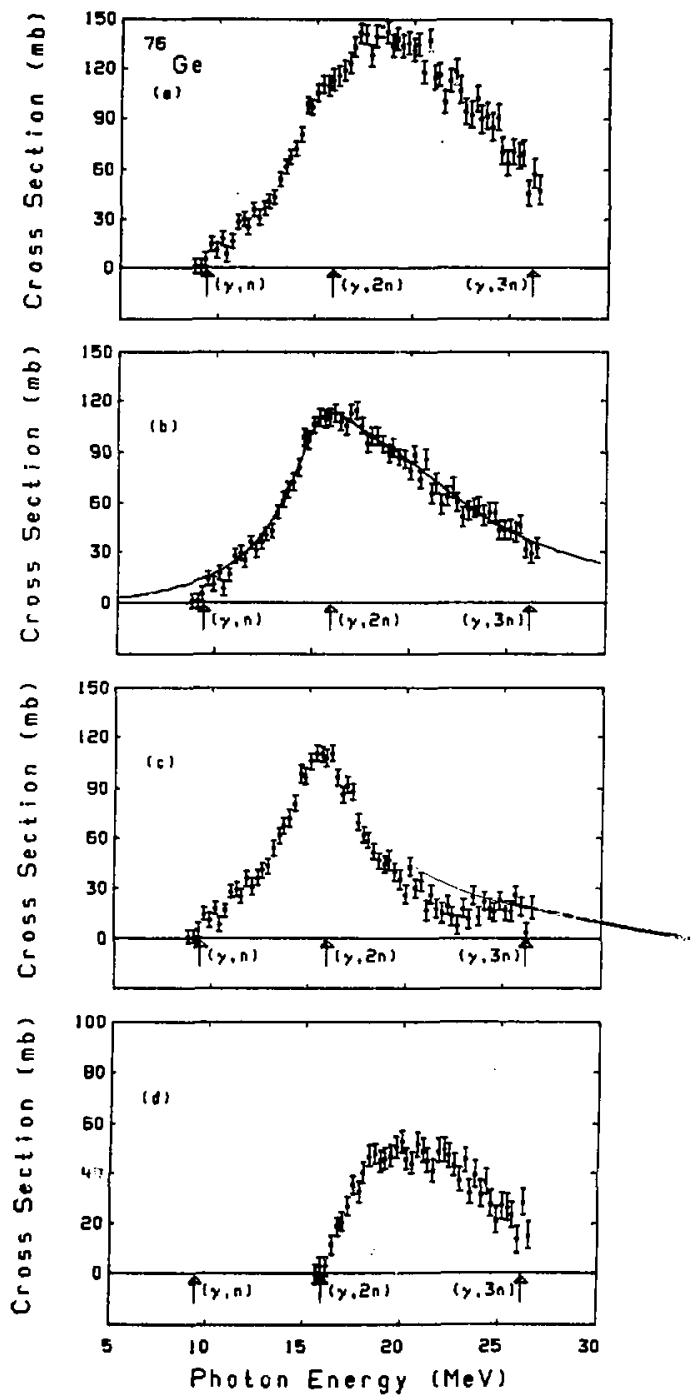

Fig. $62 \mathrm{~A}$ 


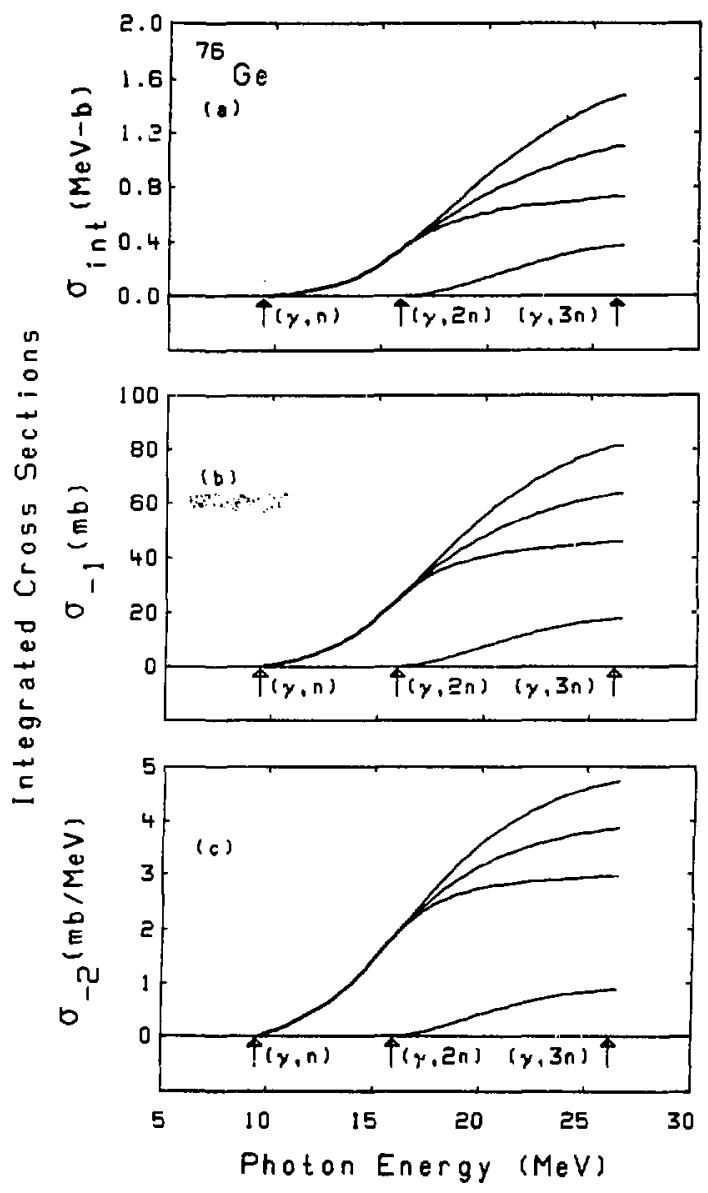

Fig. 62B 


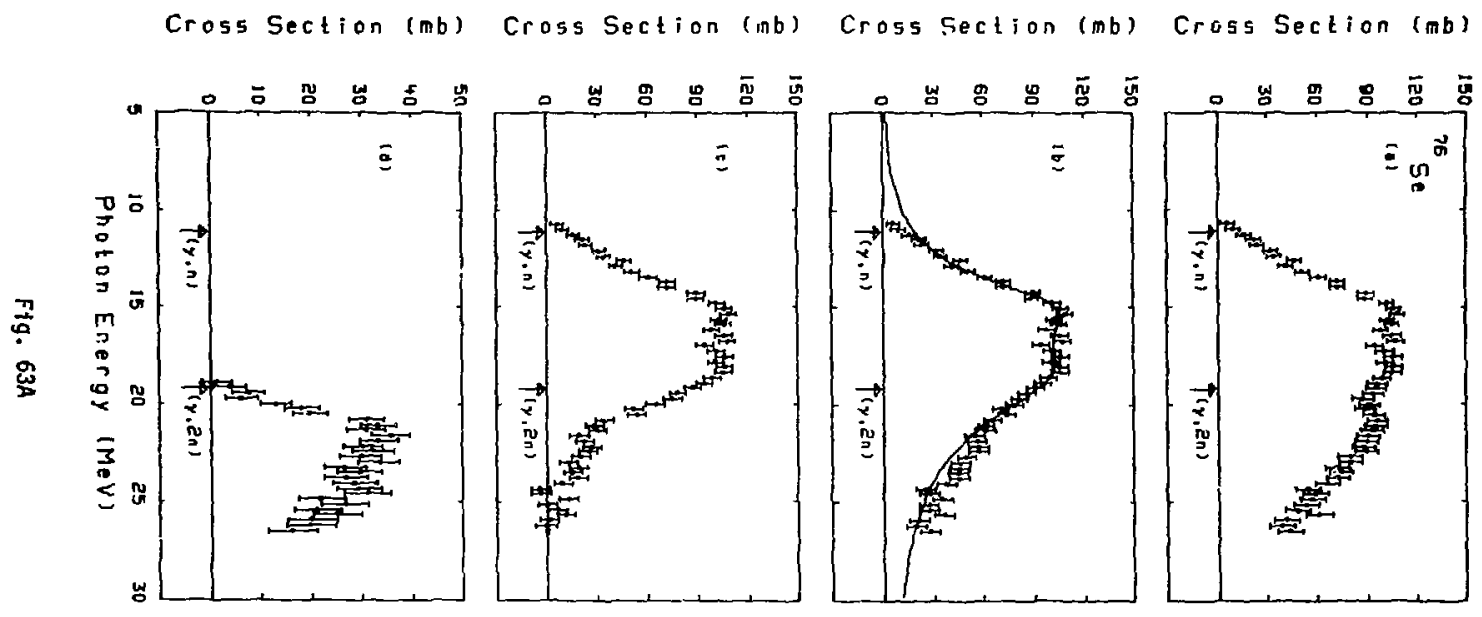




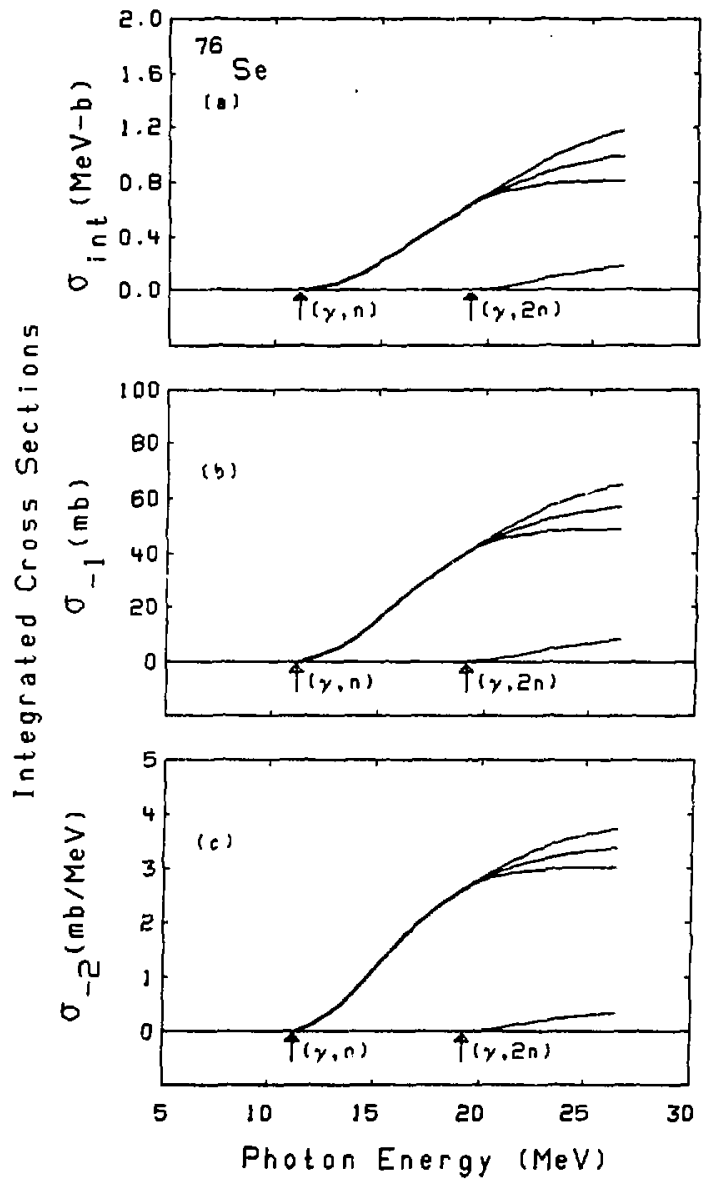

Fig. $63 B$ 

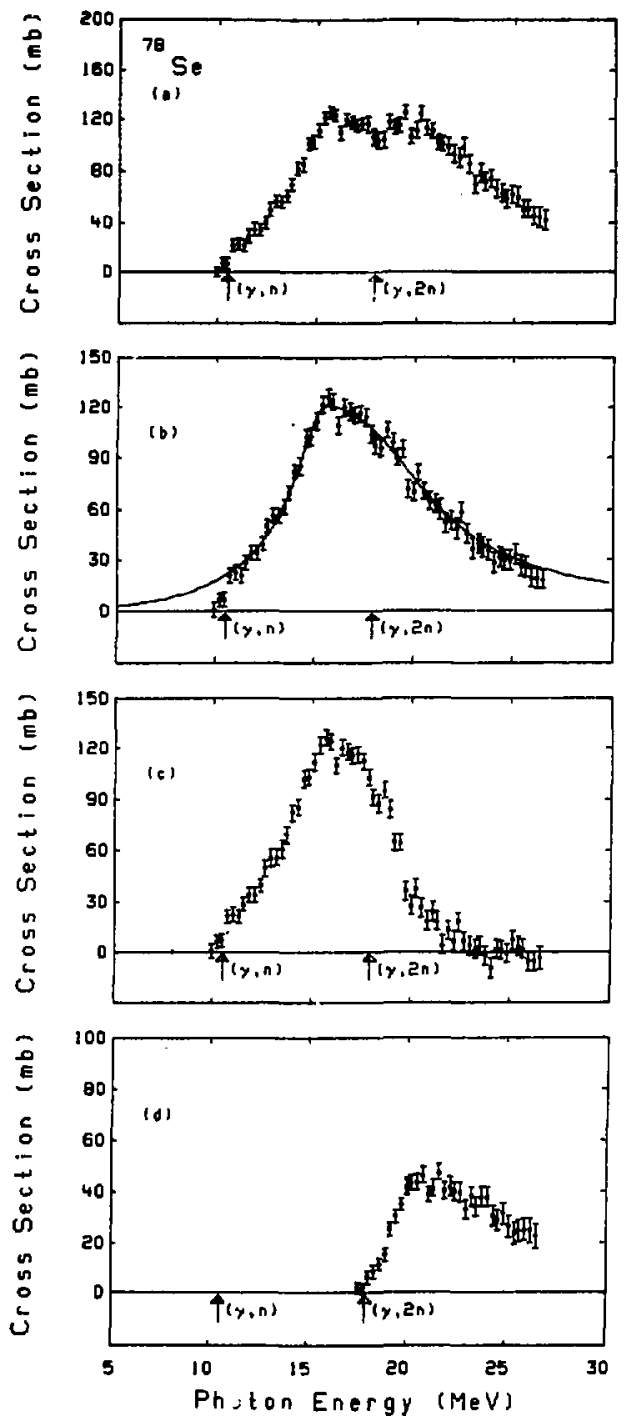

Fig. $64 A$ 


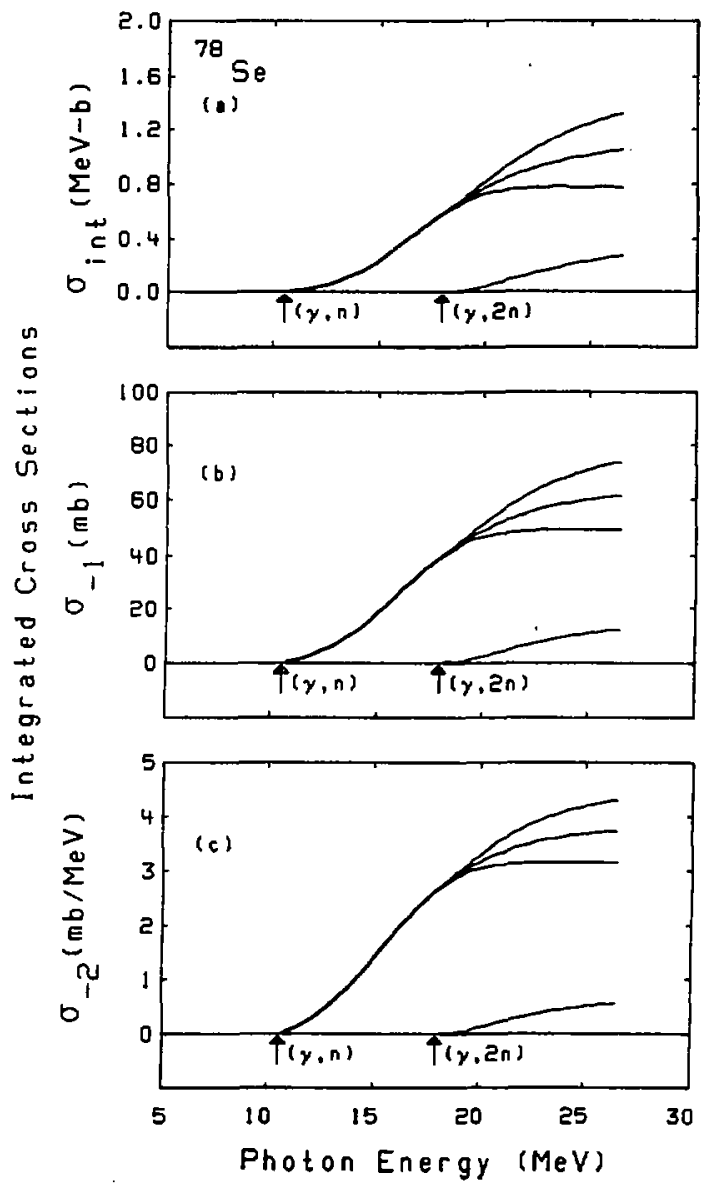

F1g. 64B 

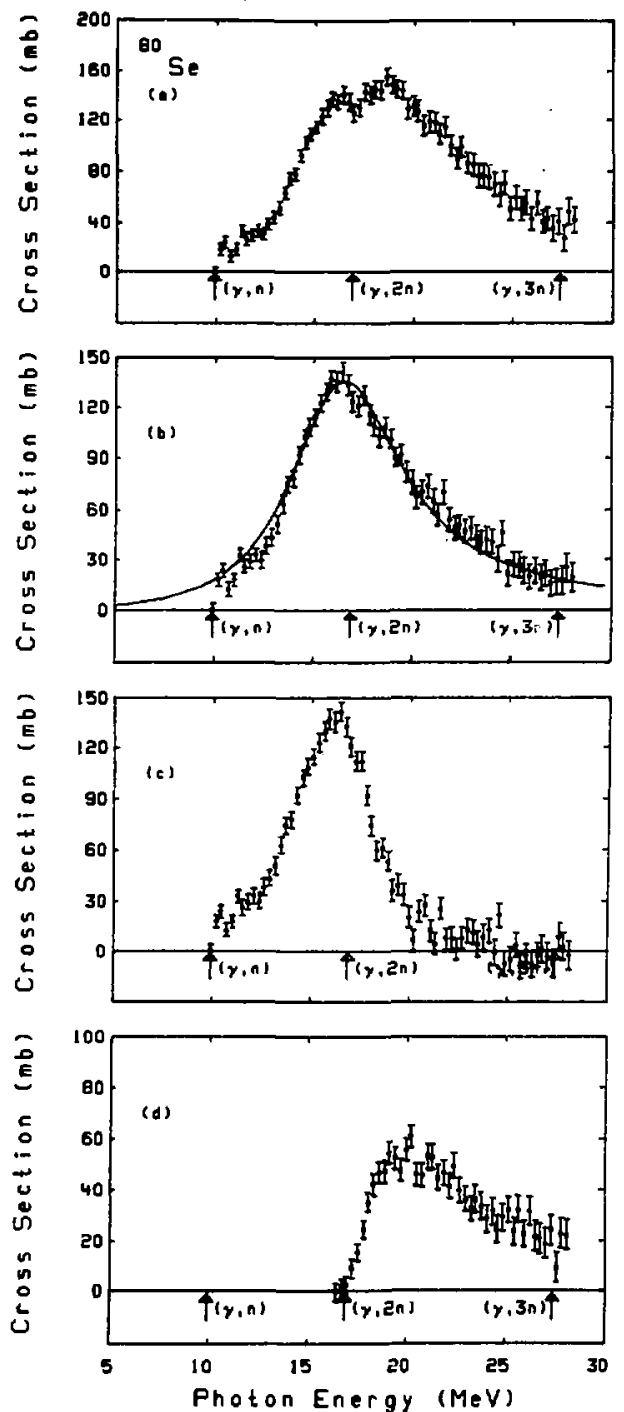

F1g. $65 \mathrm{~A}$ 


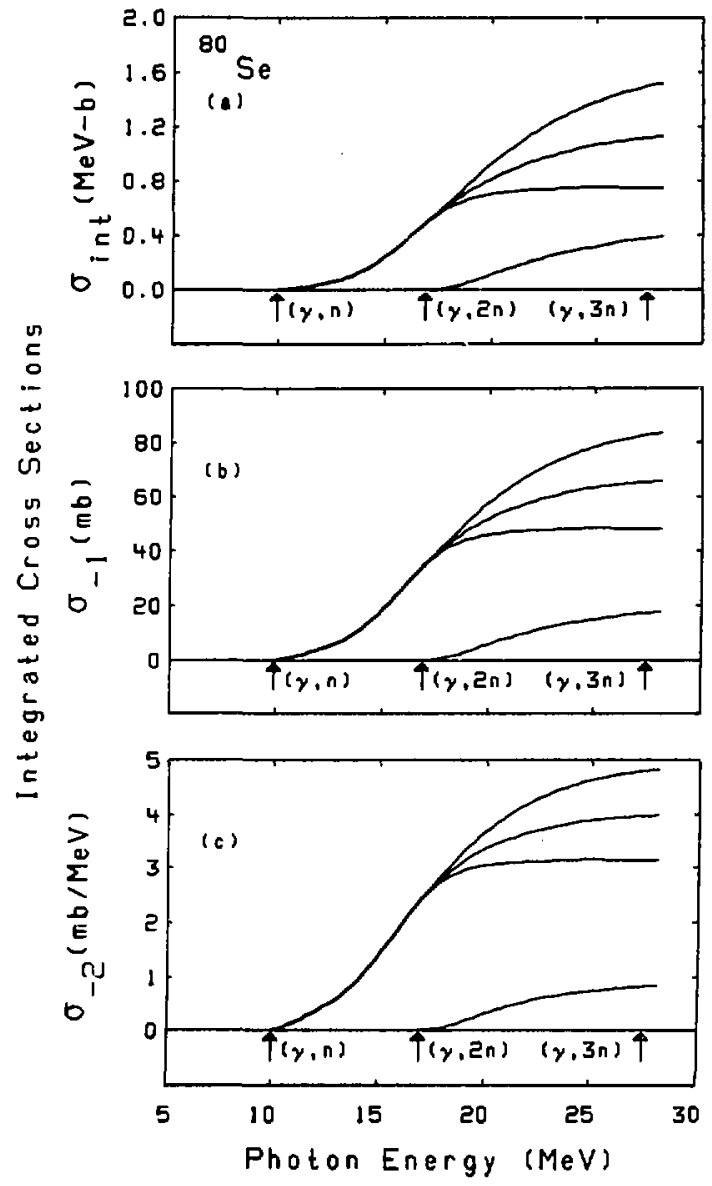

Fig. 658 

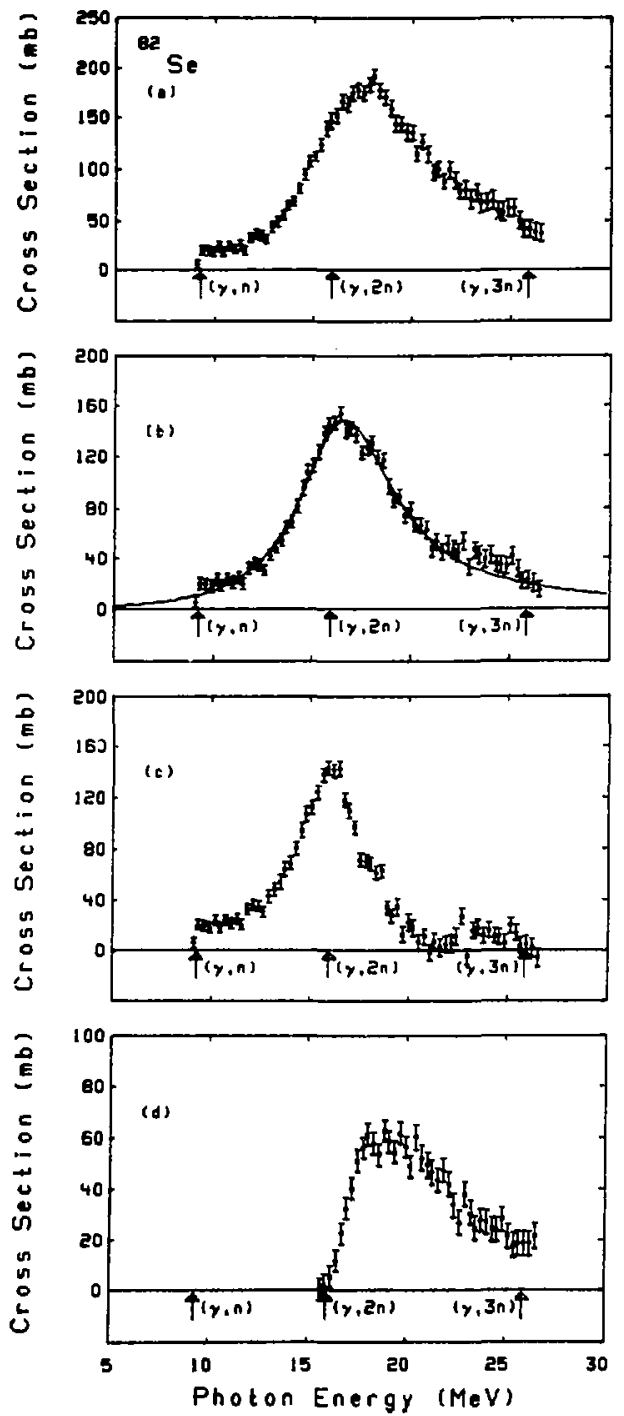

Fig. $66 \mathrm{~A}$ 

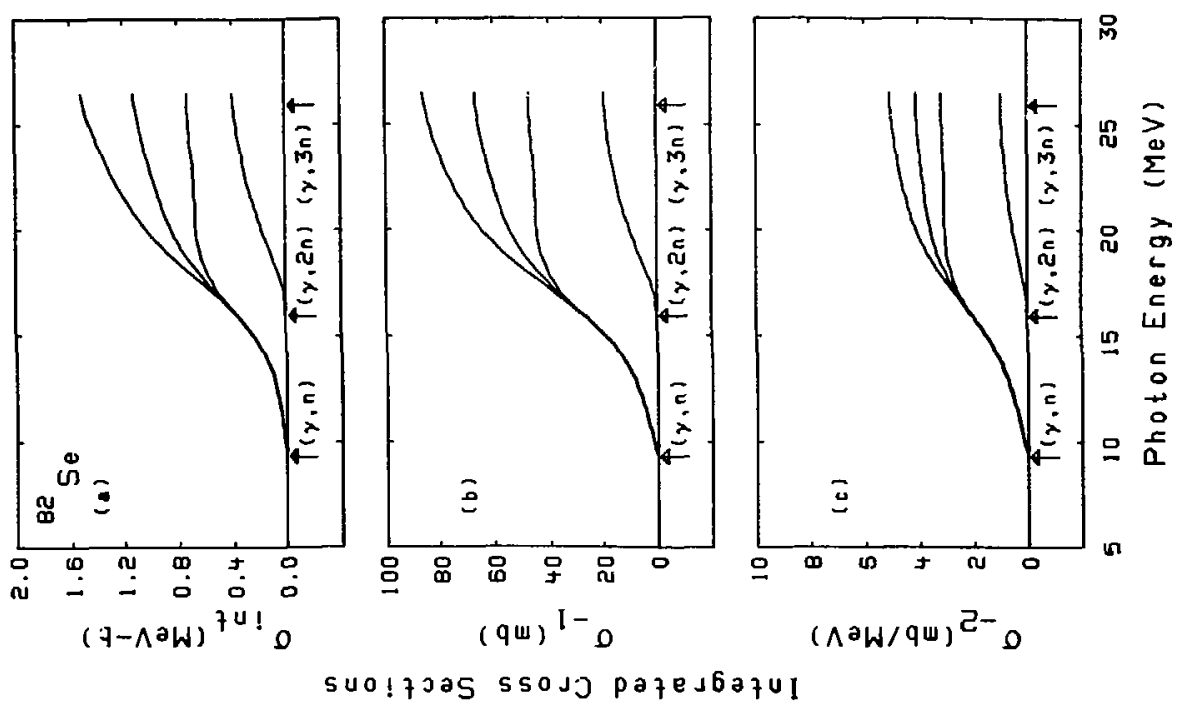

兽

suo!zoas ssojo pazejazul 

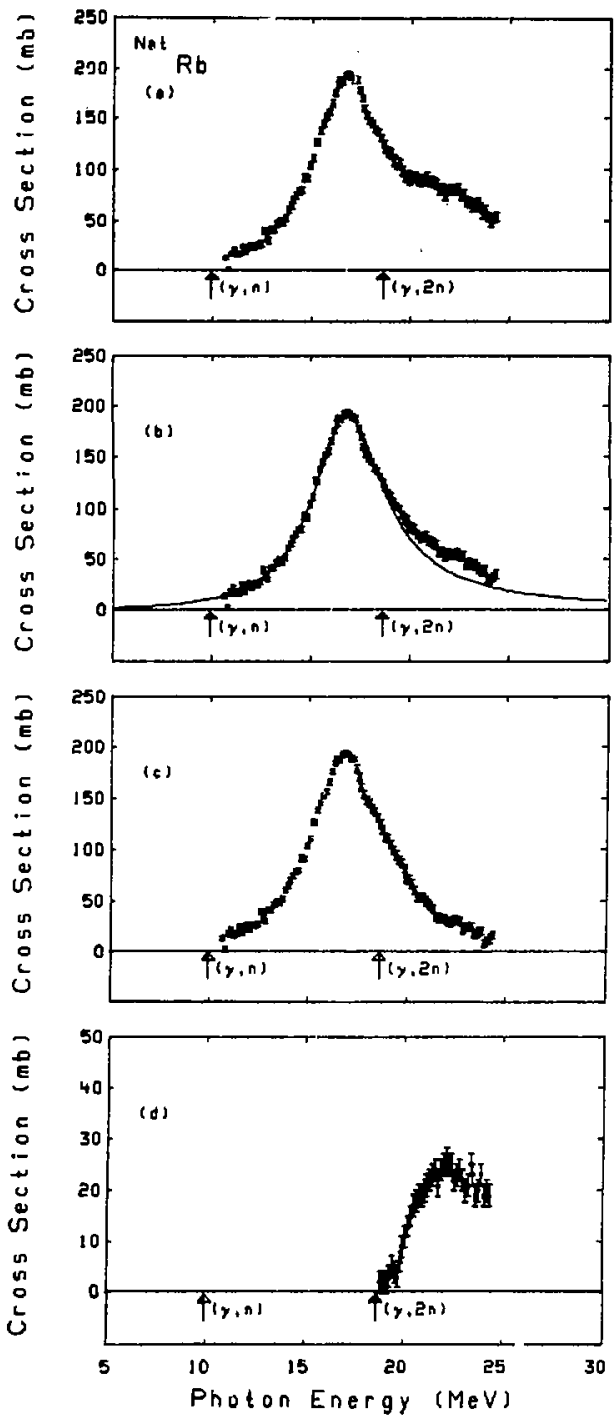

Fig. 67A 

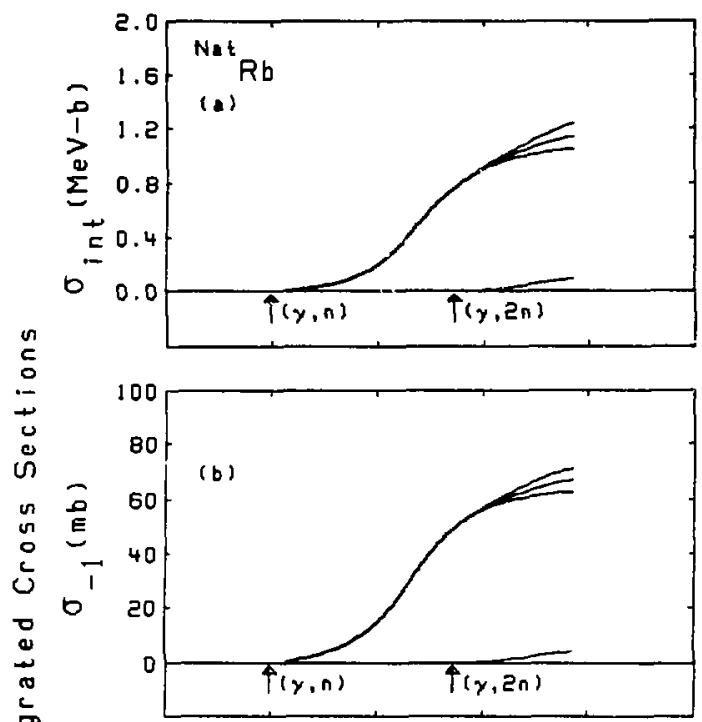

$\stackrel{+}{5}$

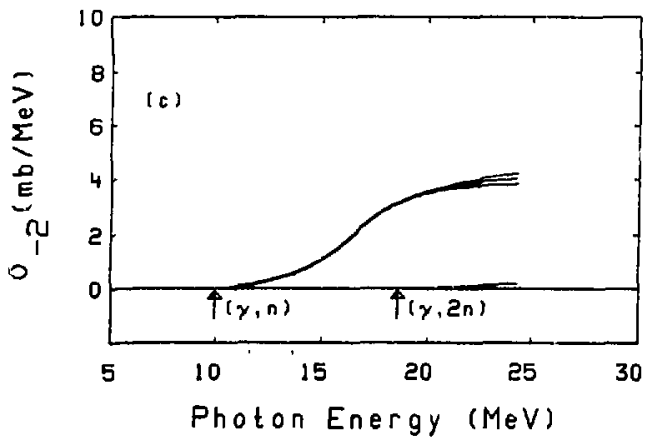

Fig. $67 \mathrm{~B}$ 

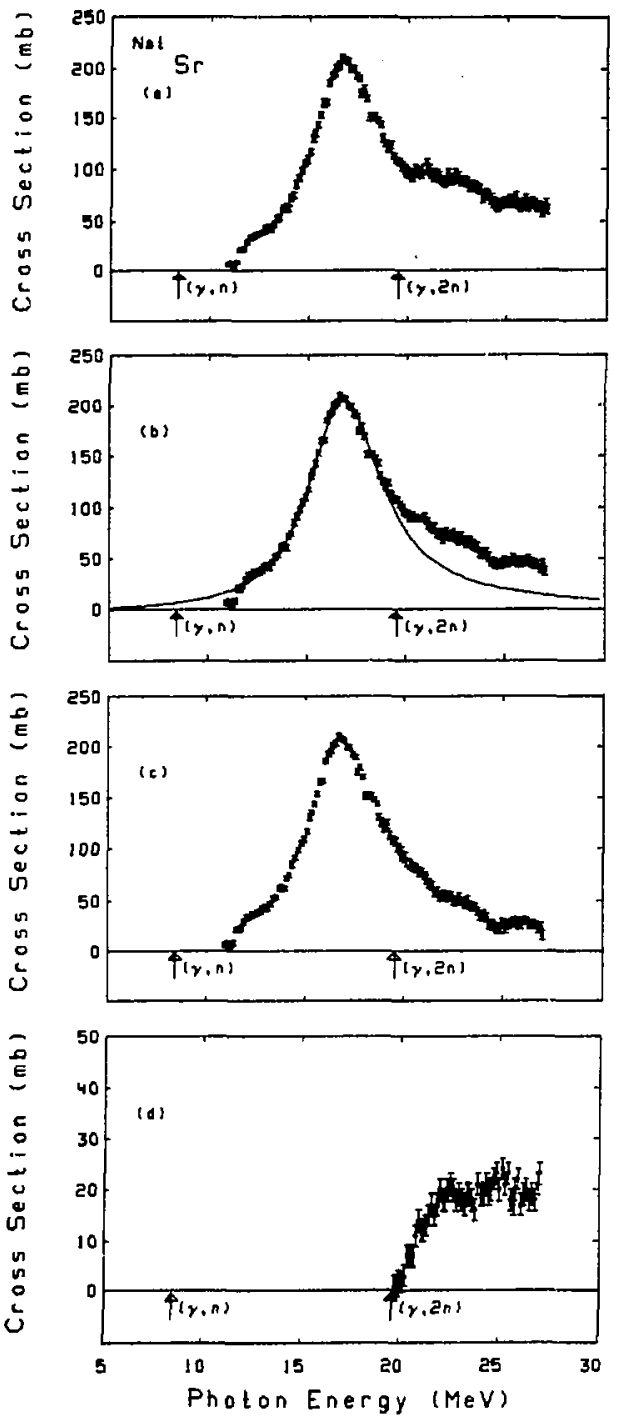

Fig. $68 \mathrm{~A}$ 


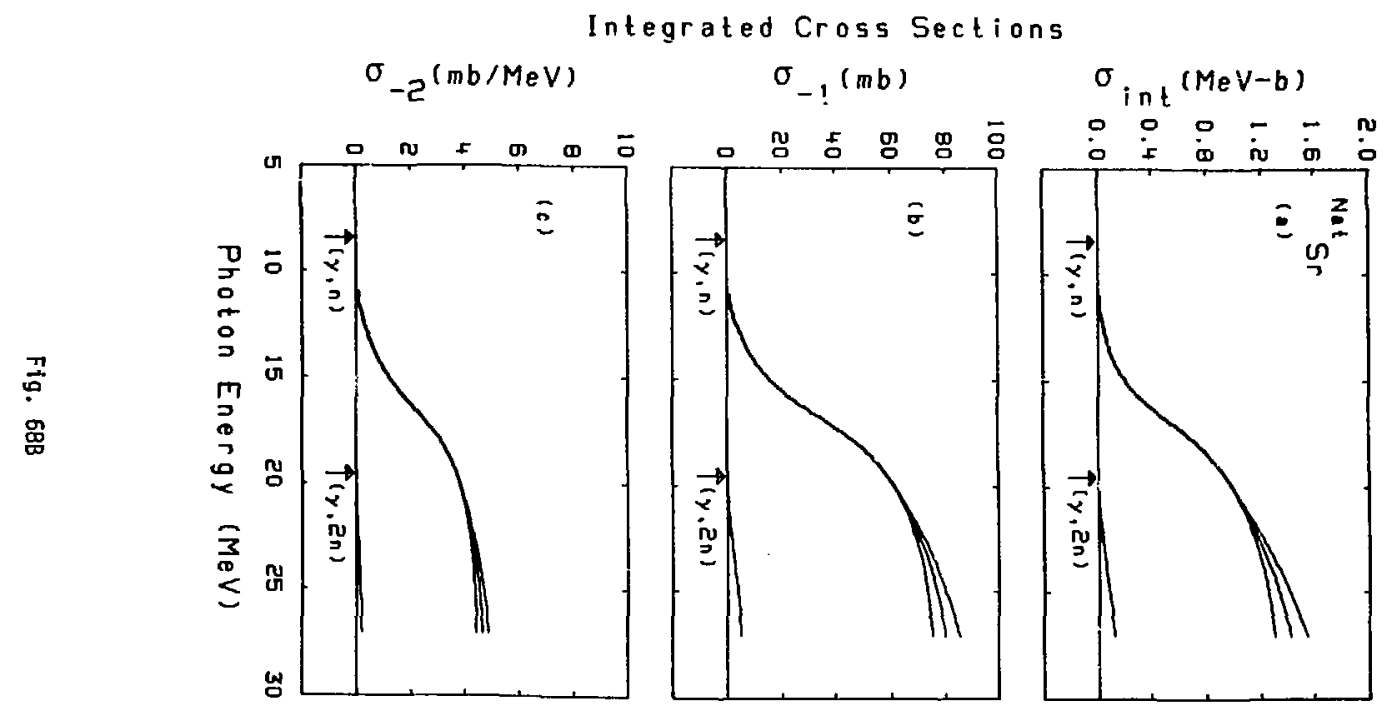



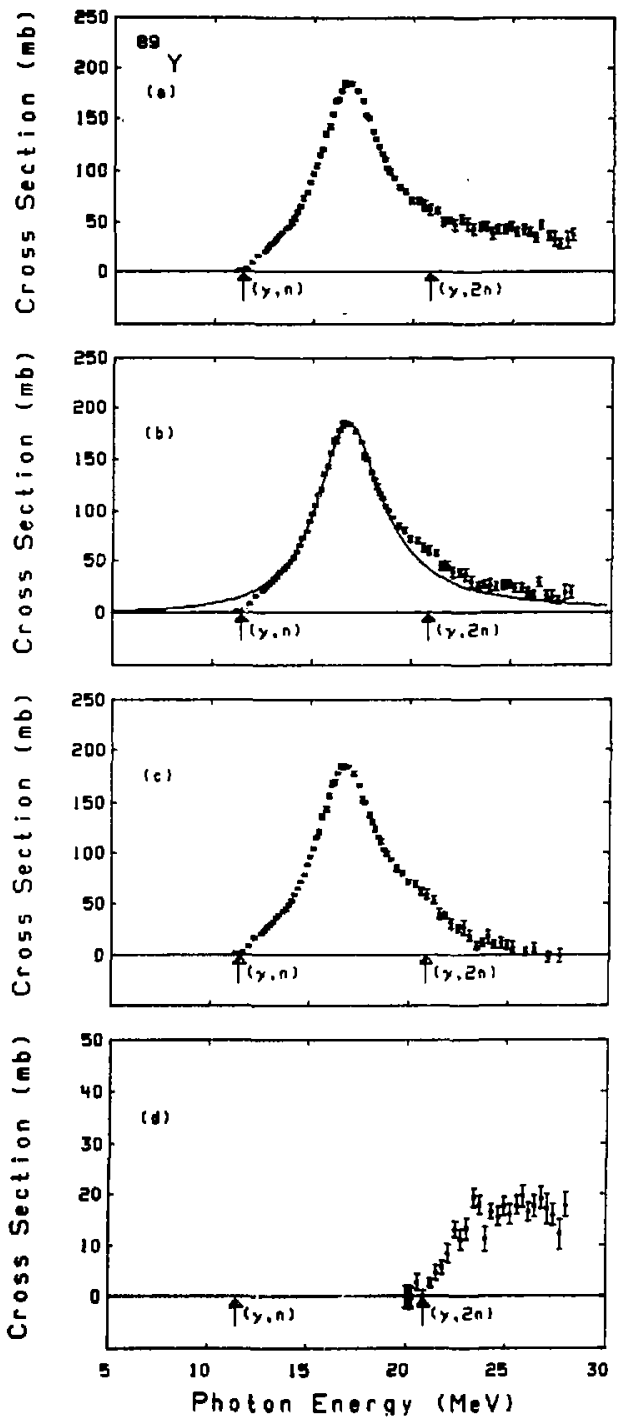

Fig. 69A 


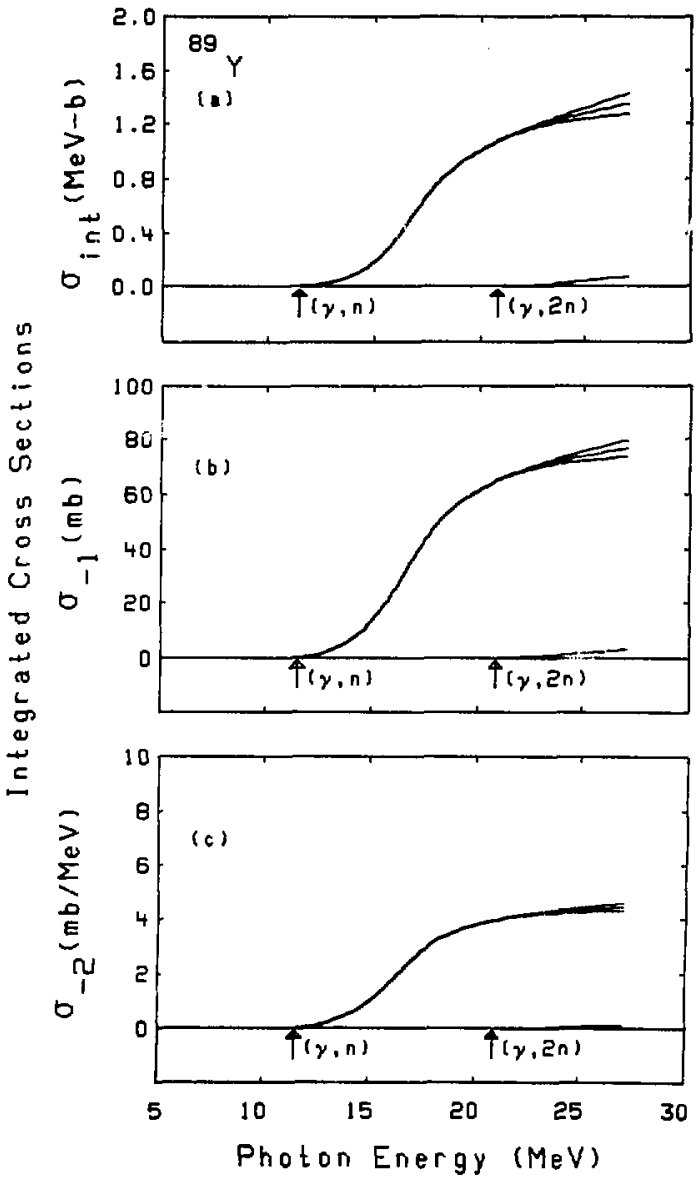

F1g. $69 \mathrm{~B}$ 

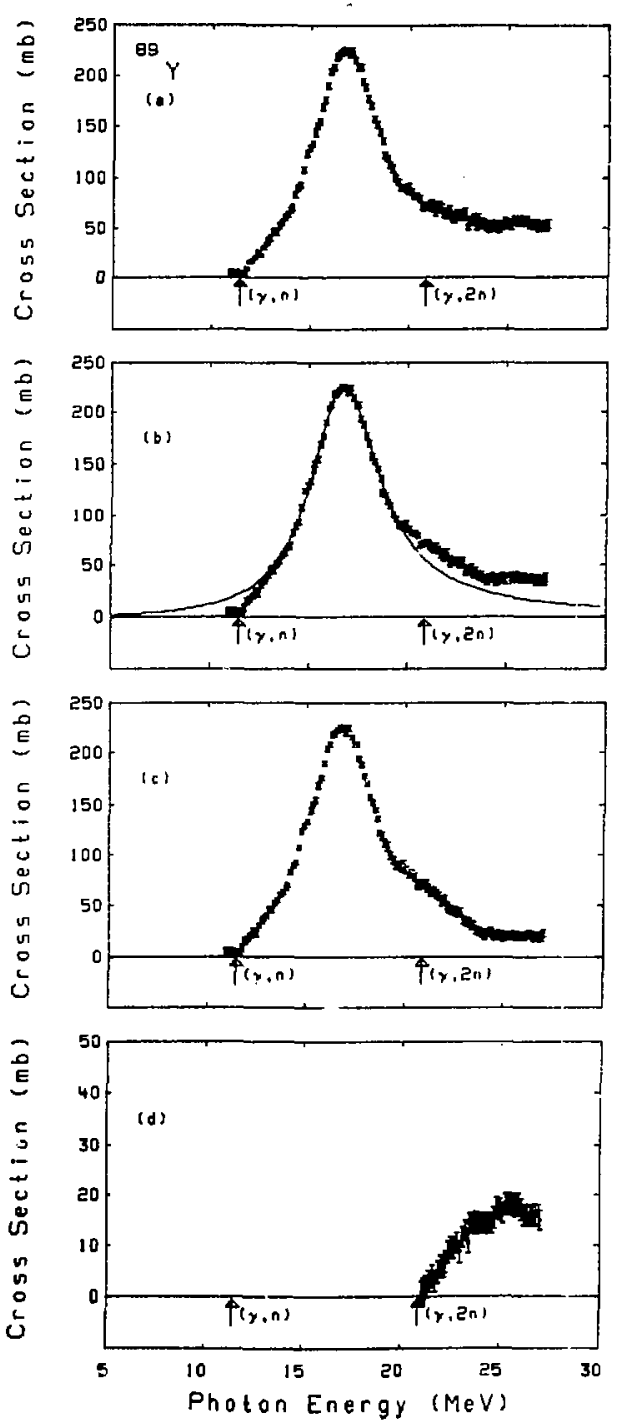

Fig. $70 \mathrm{~A}$ 


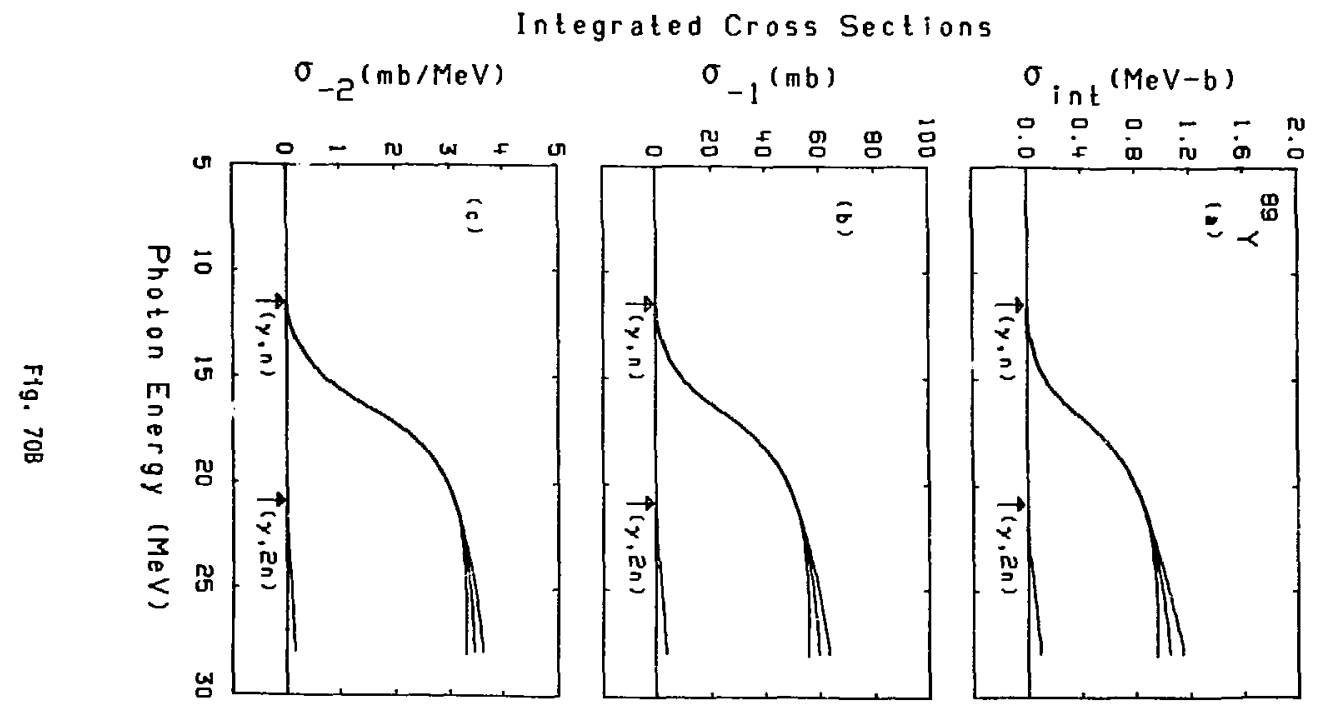




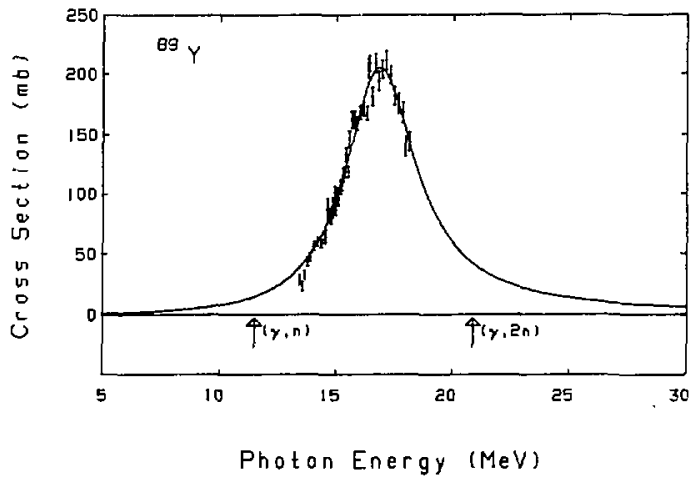

Fig. 71A 


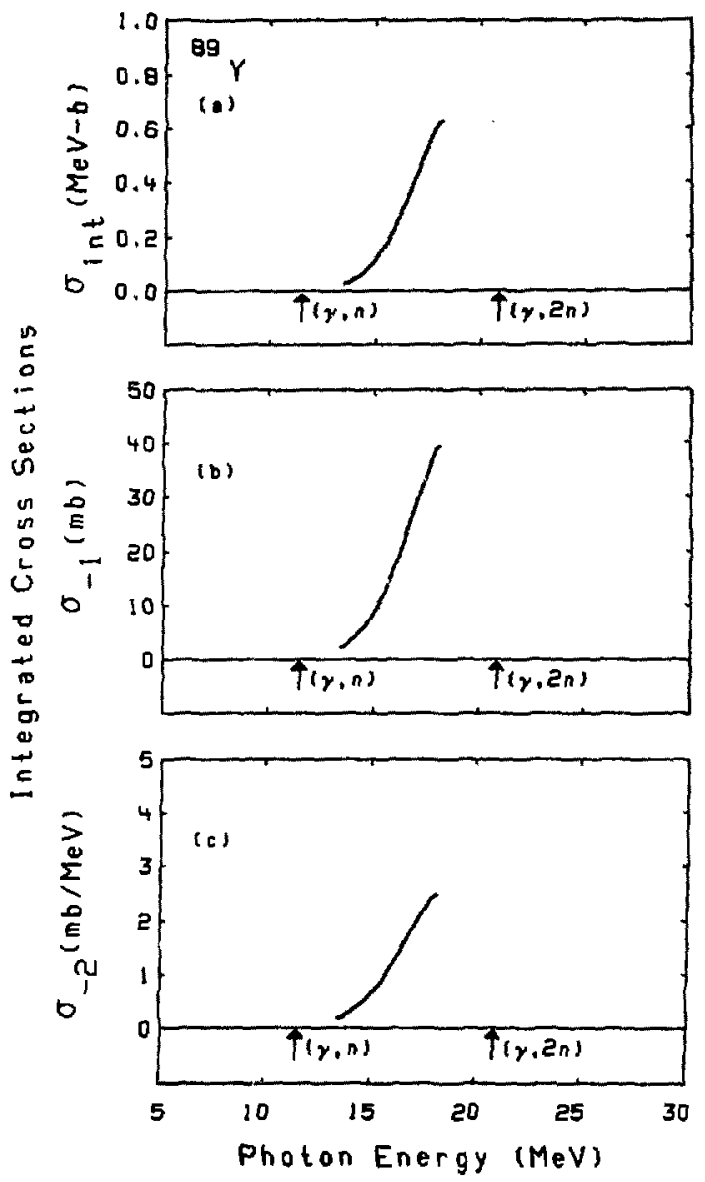

Fig. $71 B$ 

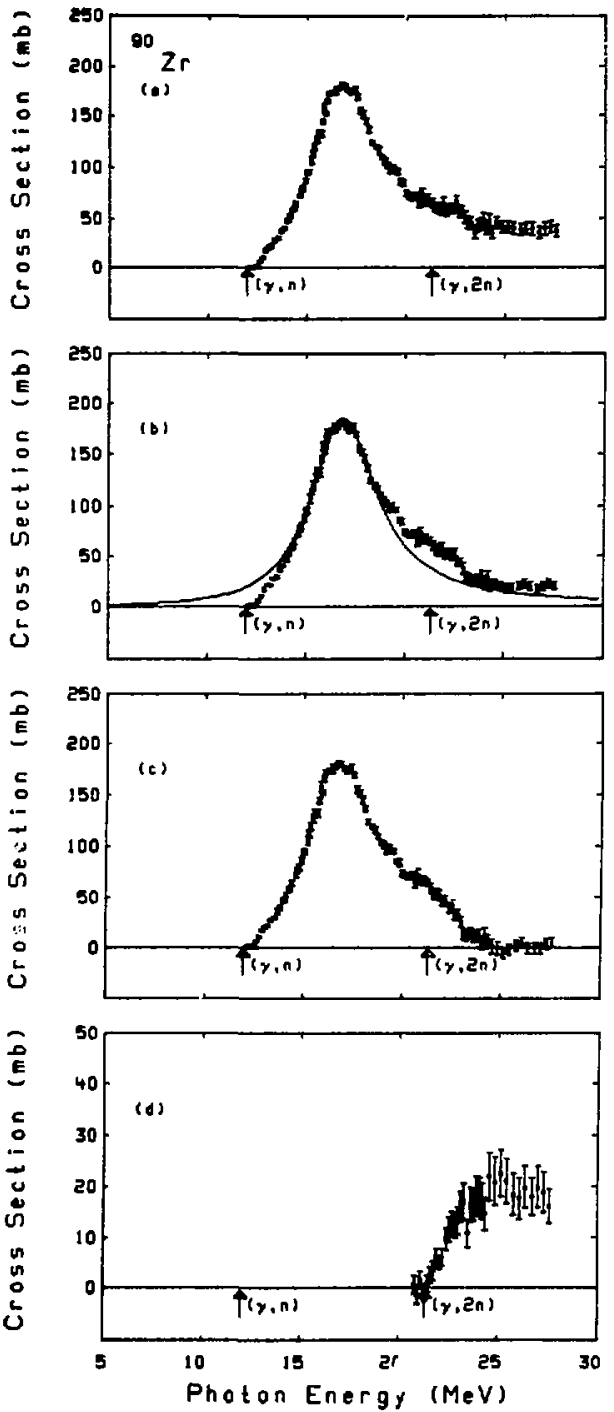

Fig. 72A 


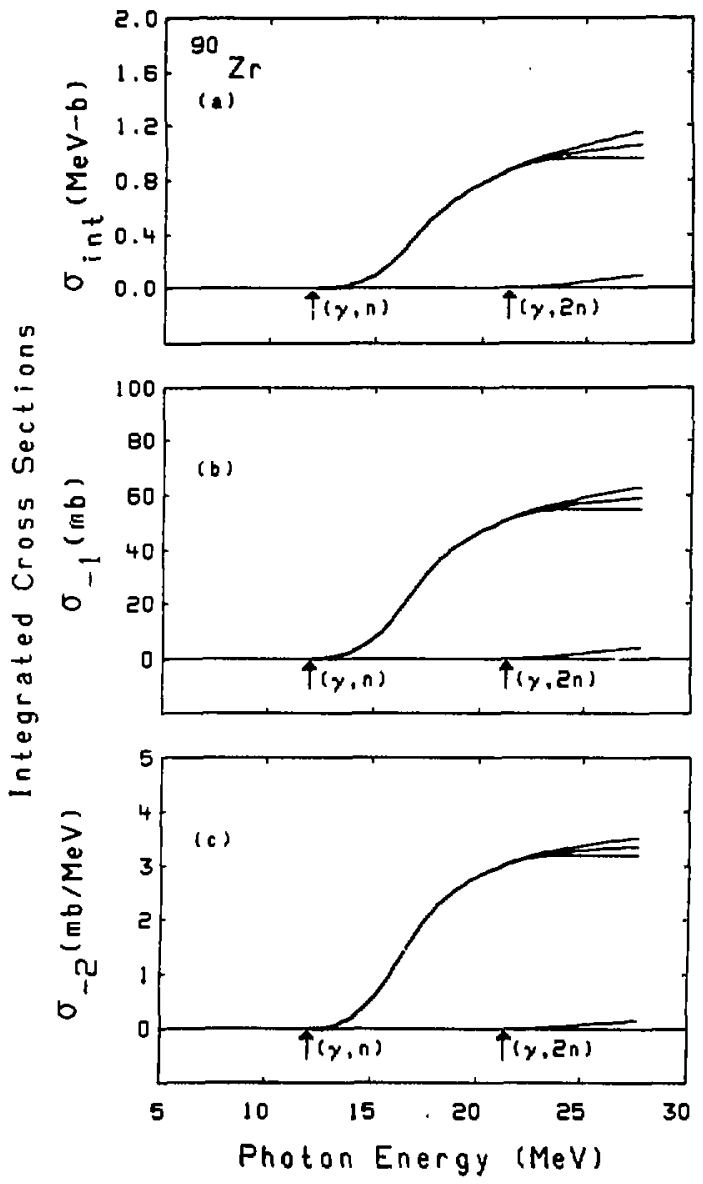

fig. 72B 

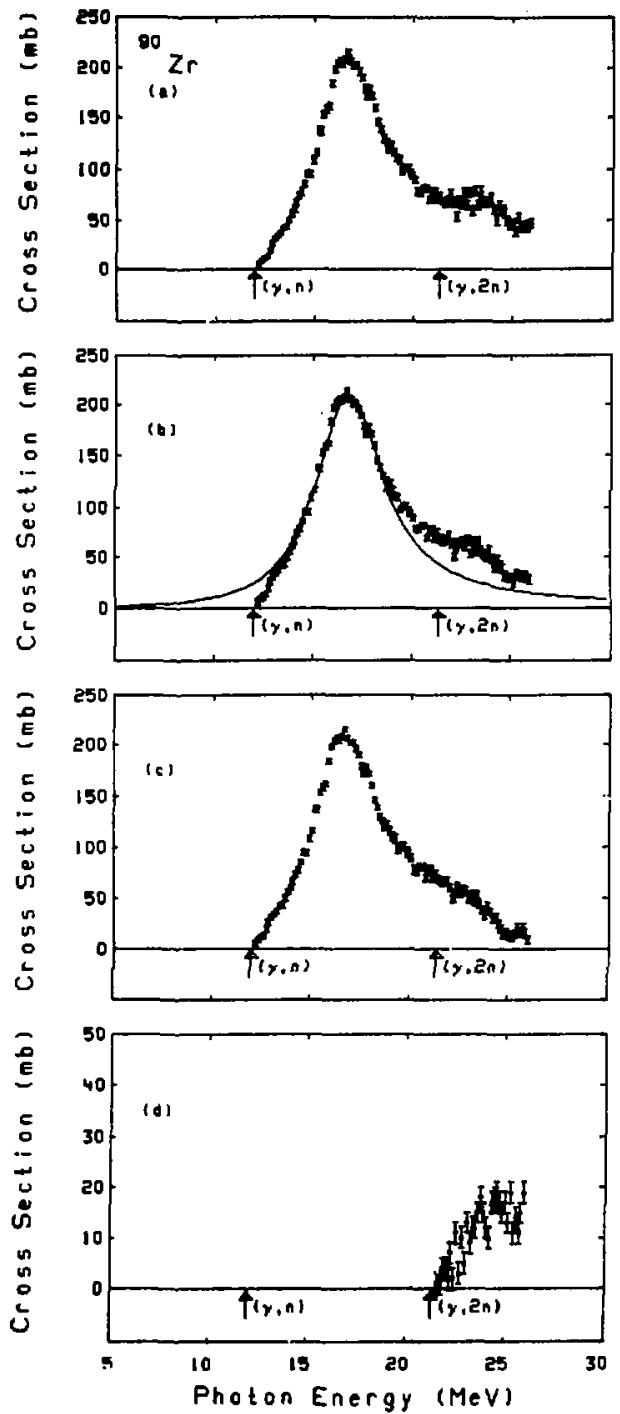

Fig. $73 n$ 


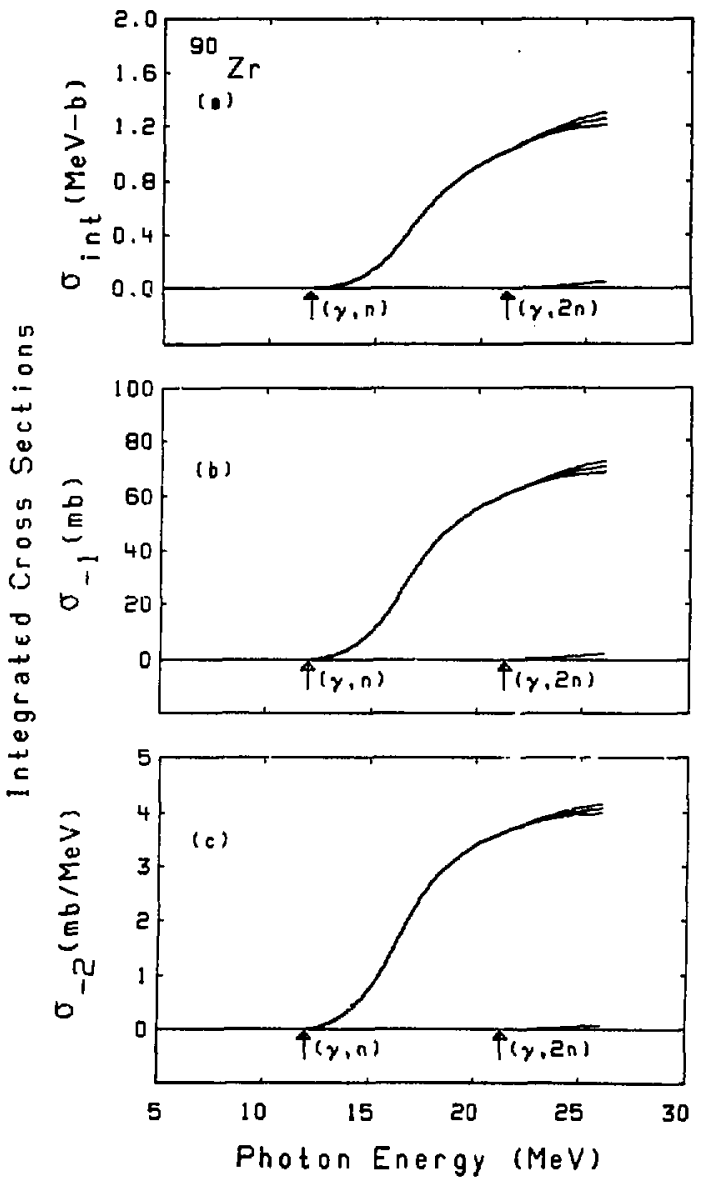

F1g. $73 B$ 

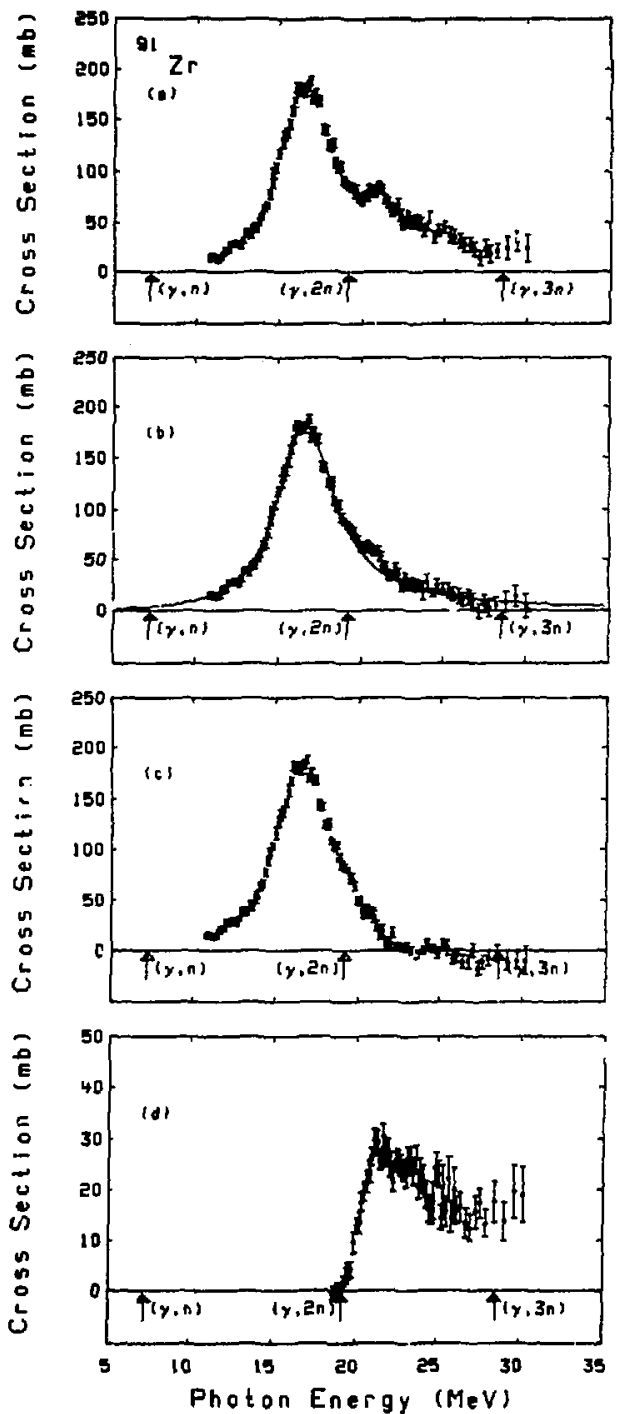

Fig. $74 \mathrm{~A}$ 

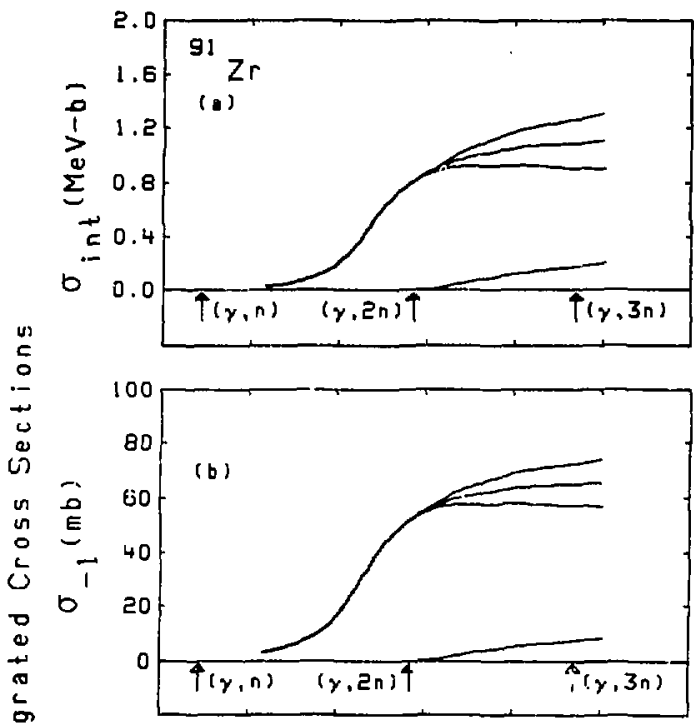

$\stackrel{2}{5}$

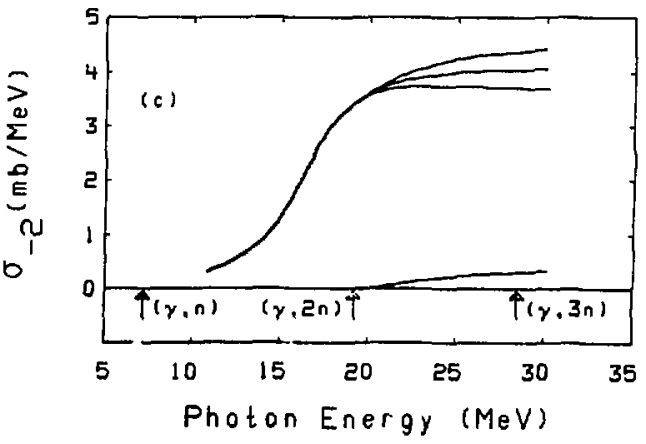

Fig. 748 

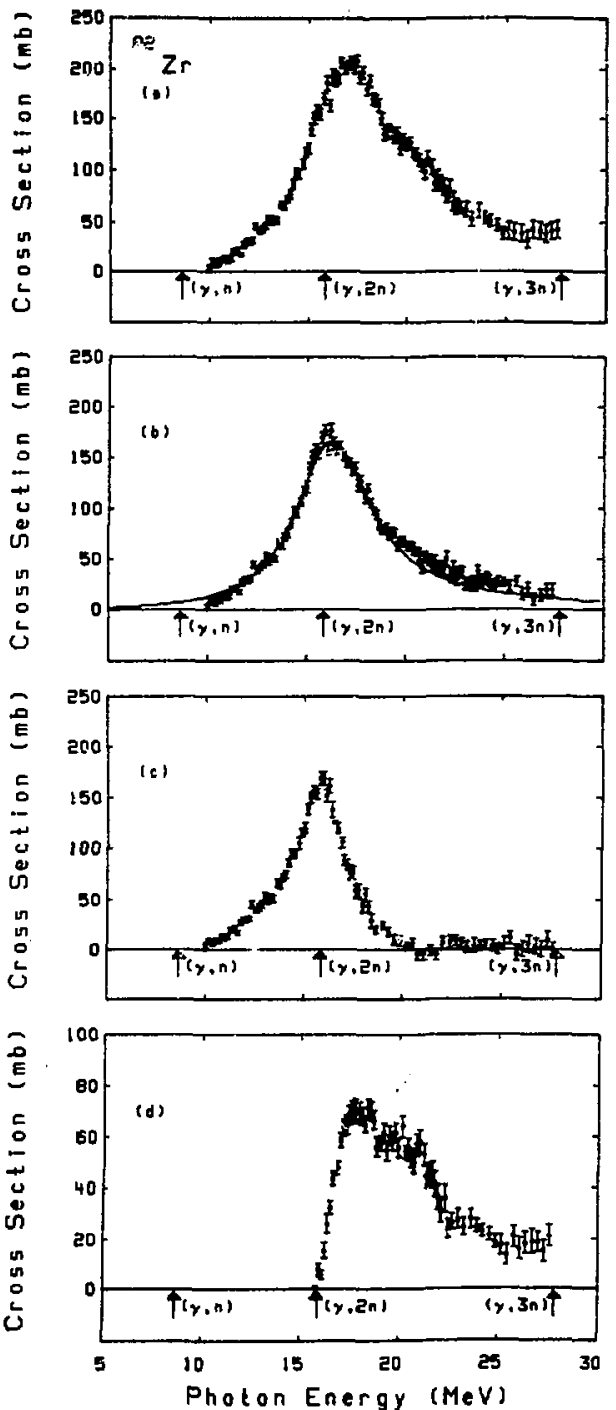

Fig. $75 \mathrm{~A}$ 


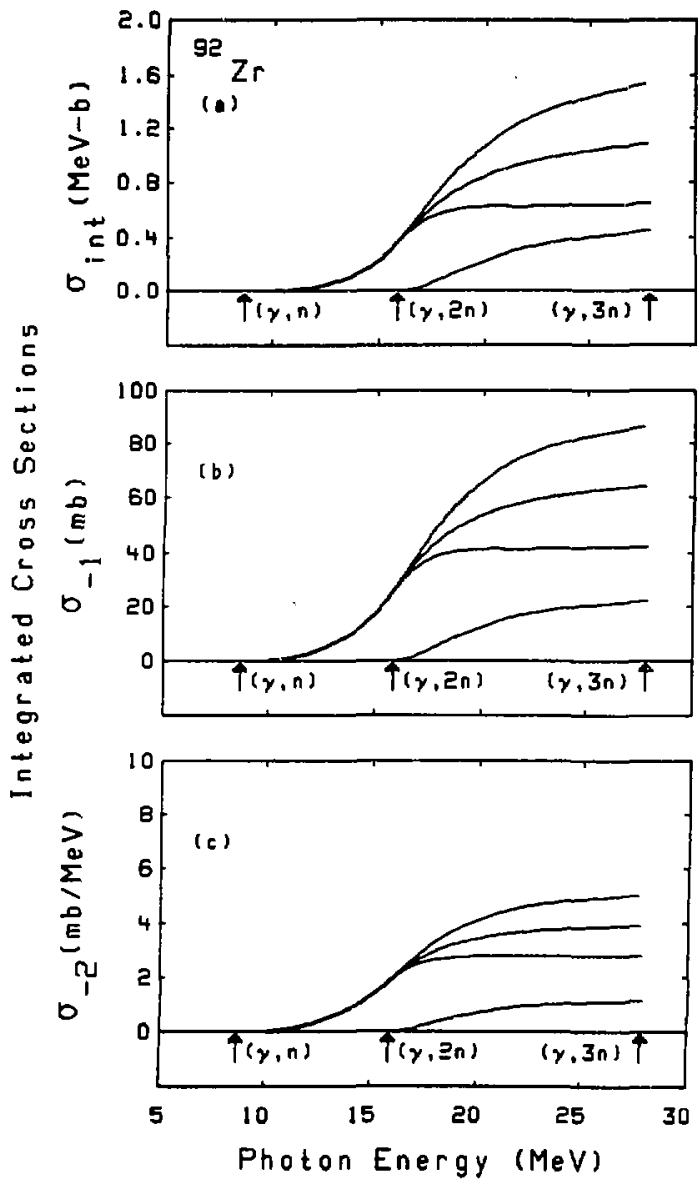

Fig. $75 B$ 

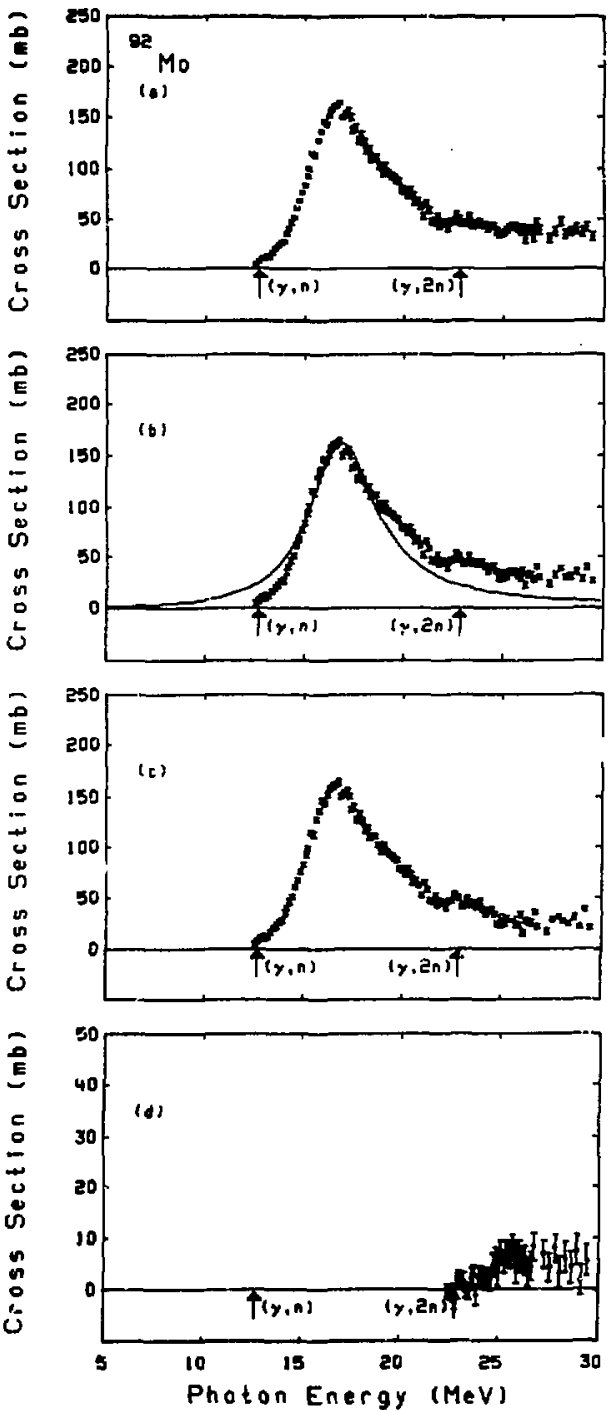

F1g. $76 \mathrm{~A}$ 


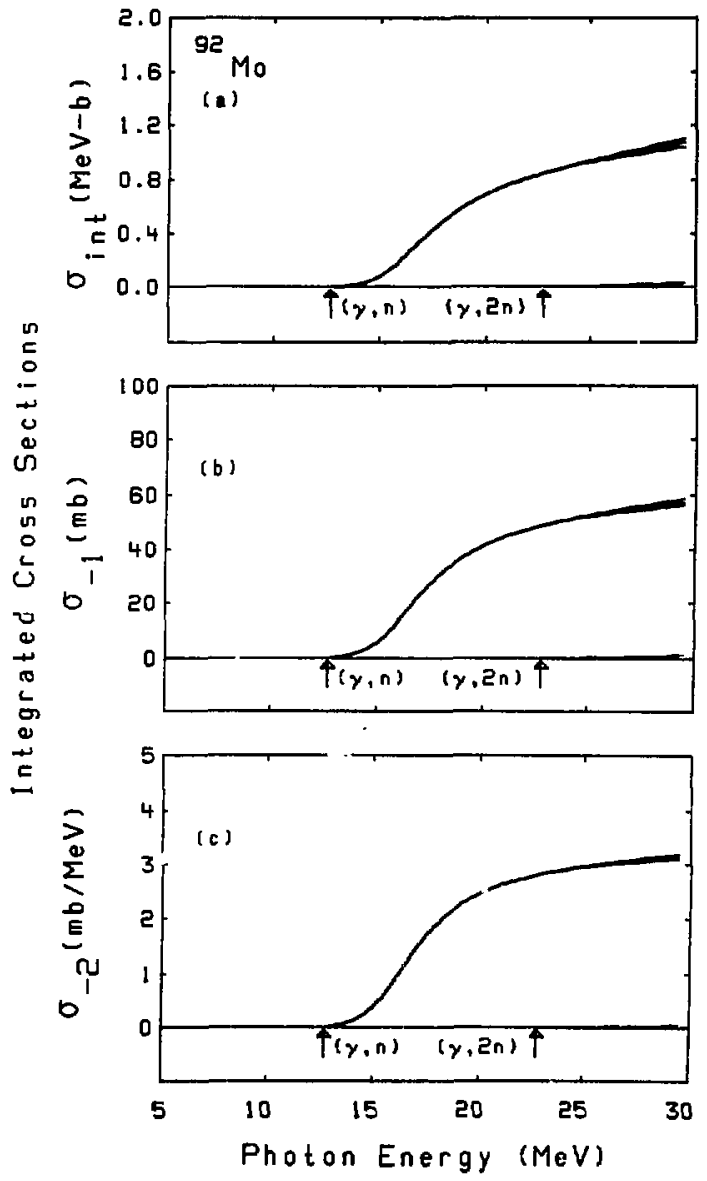

Fig. 768 

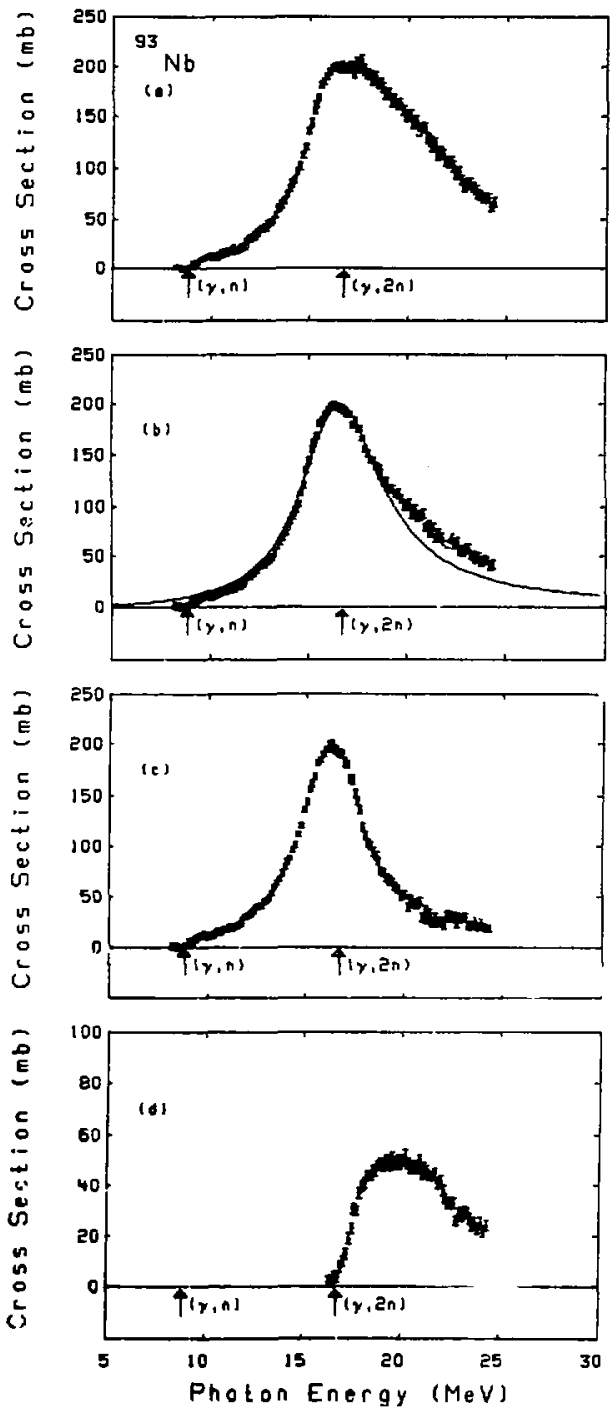

Fig. $77 \mathrm{~A}$ 


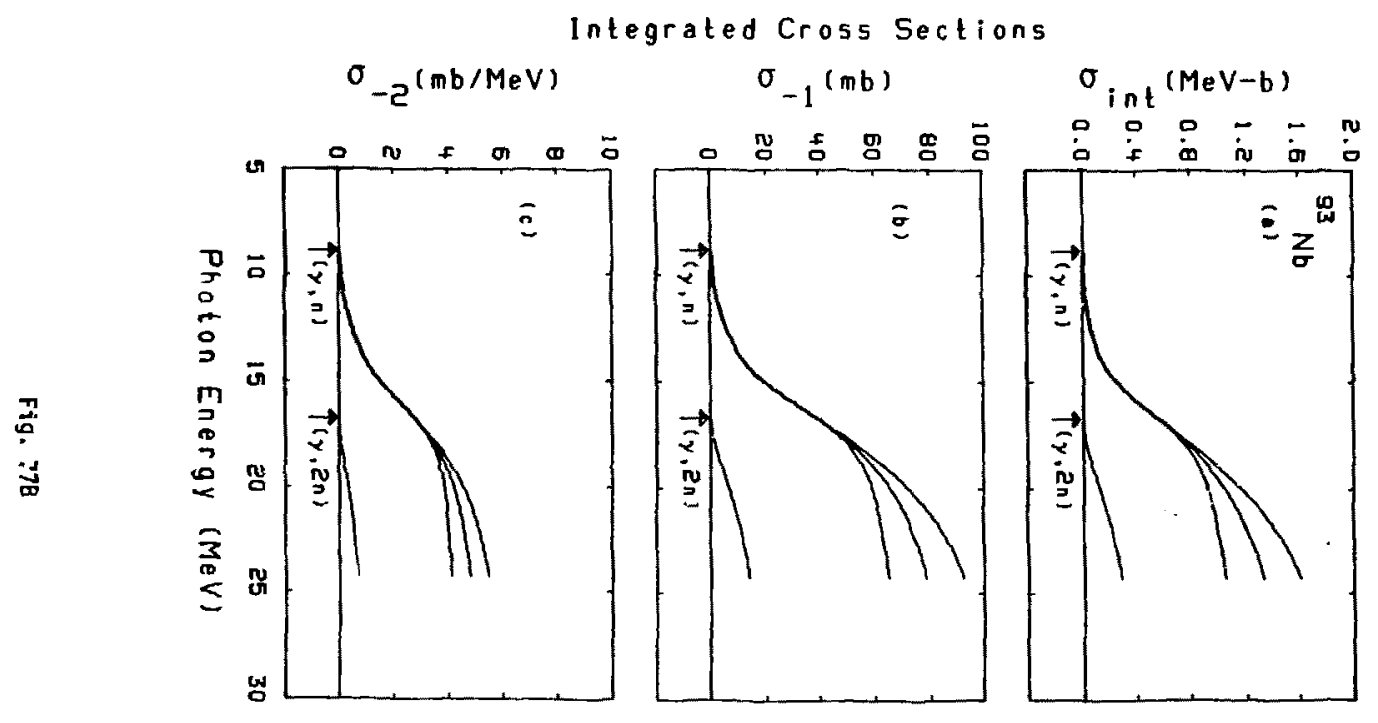



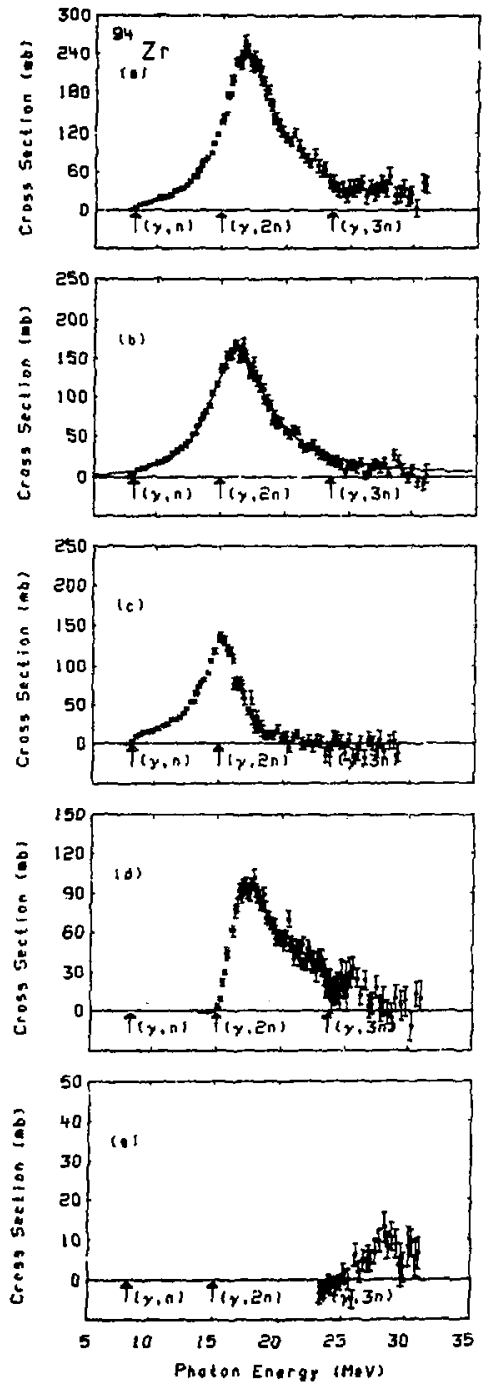

F1g. $78 \mathrm{~A}$ 


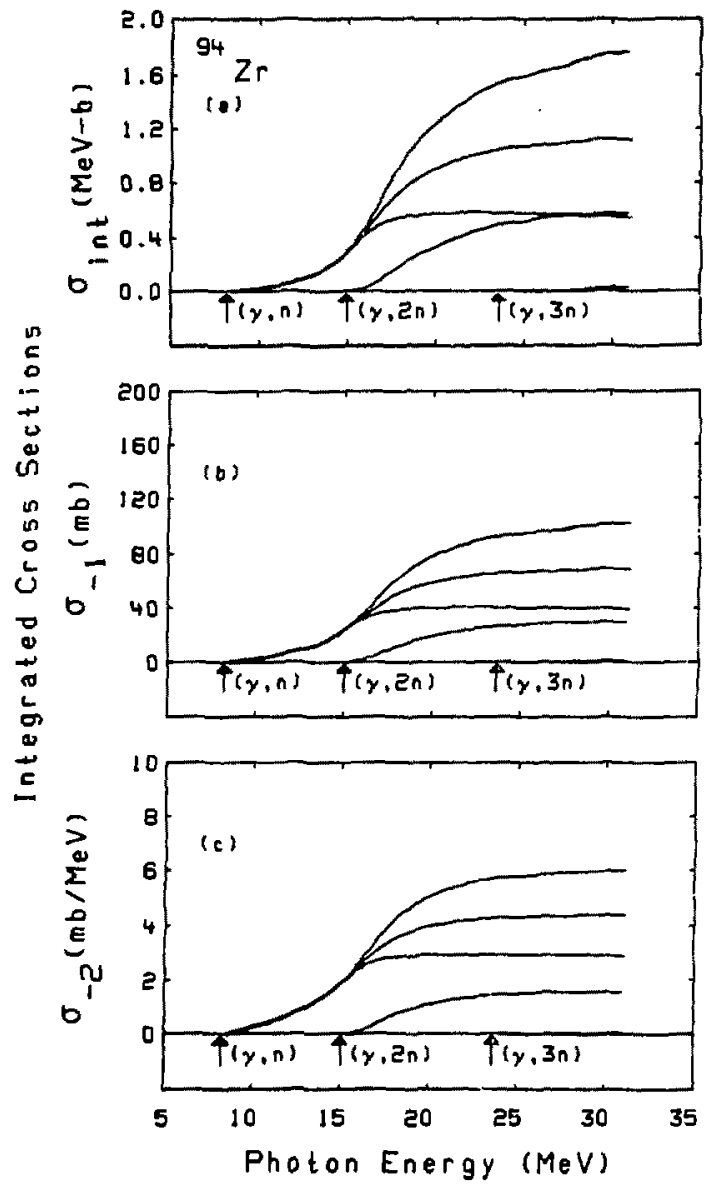

Fig. $78 \mathrm{~B}$ 

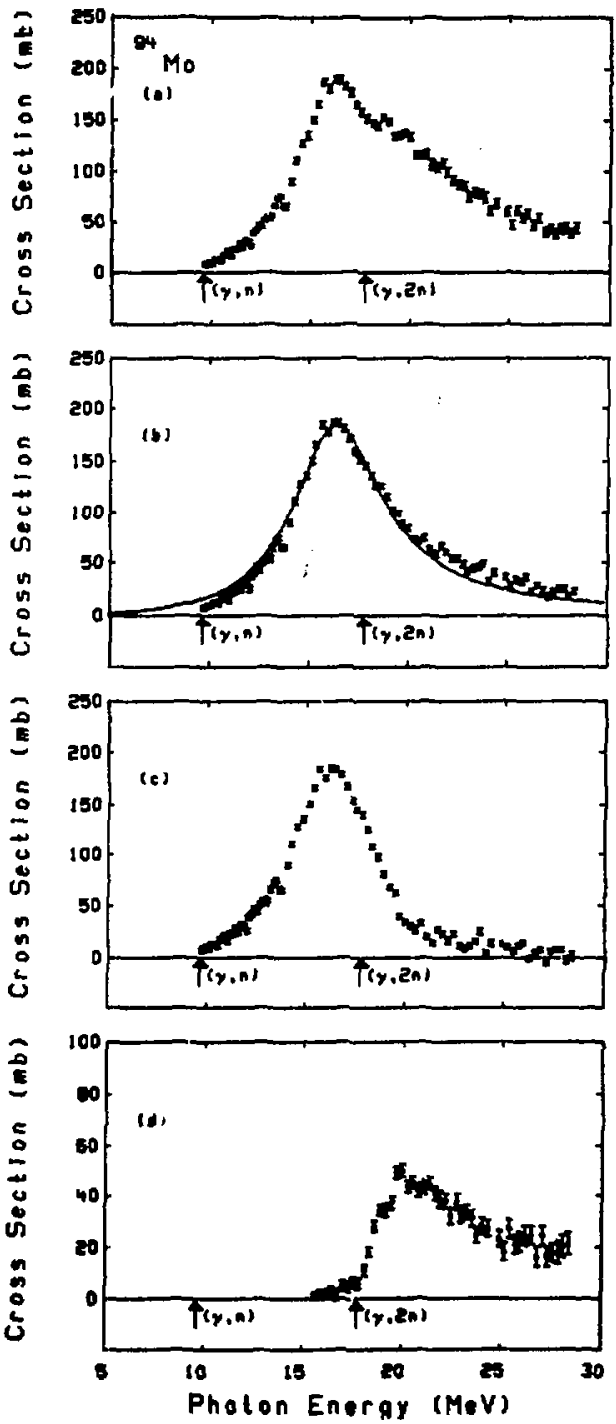


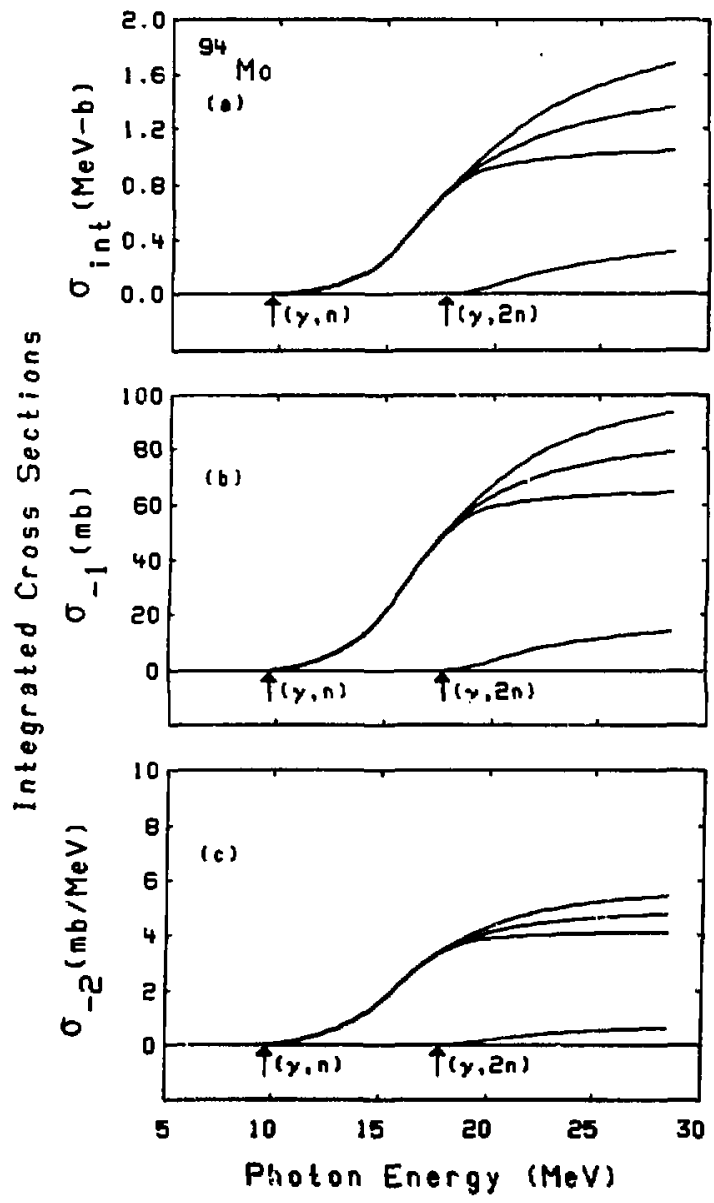

Fig. $19 B$ 

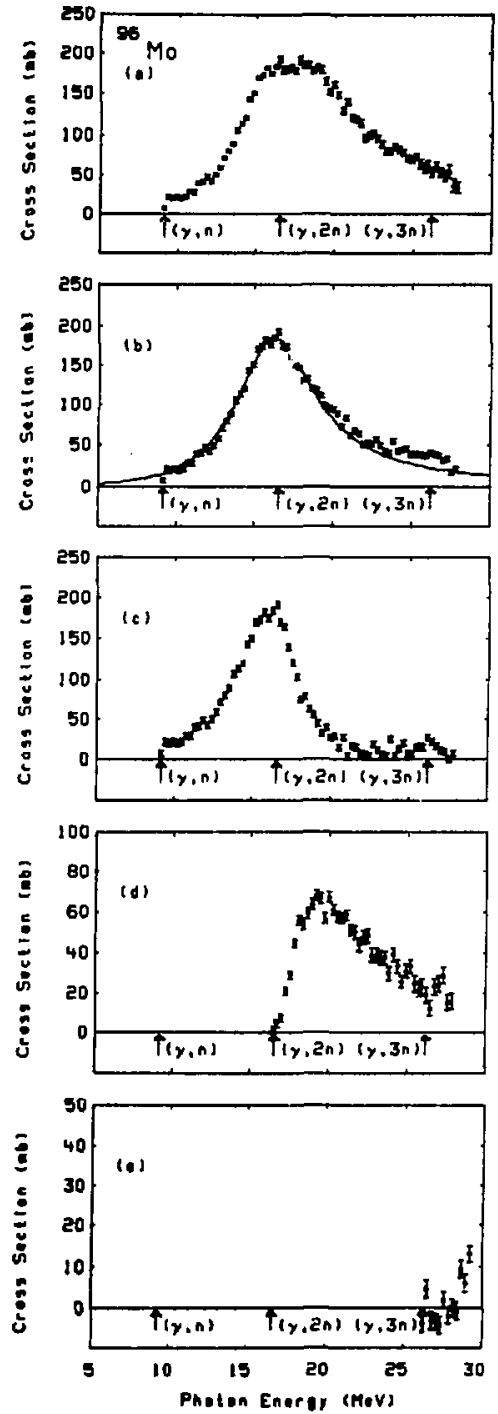

Fig. $80 \mathrm{~A}$ 


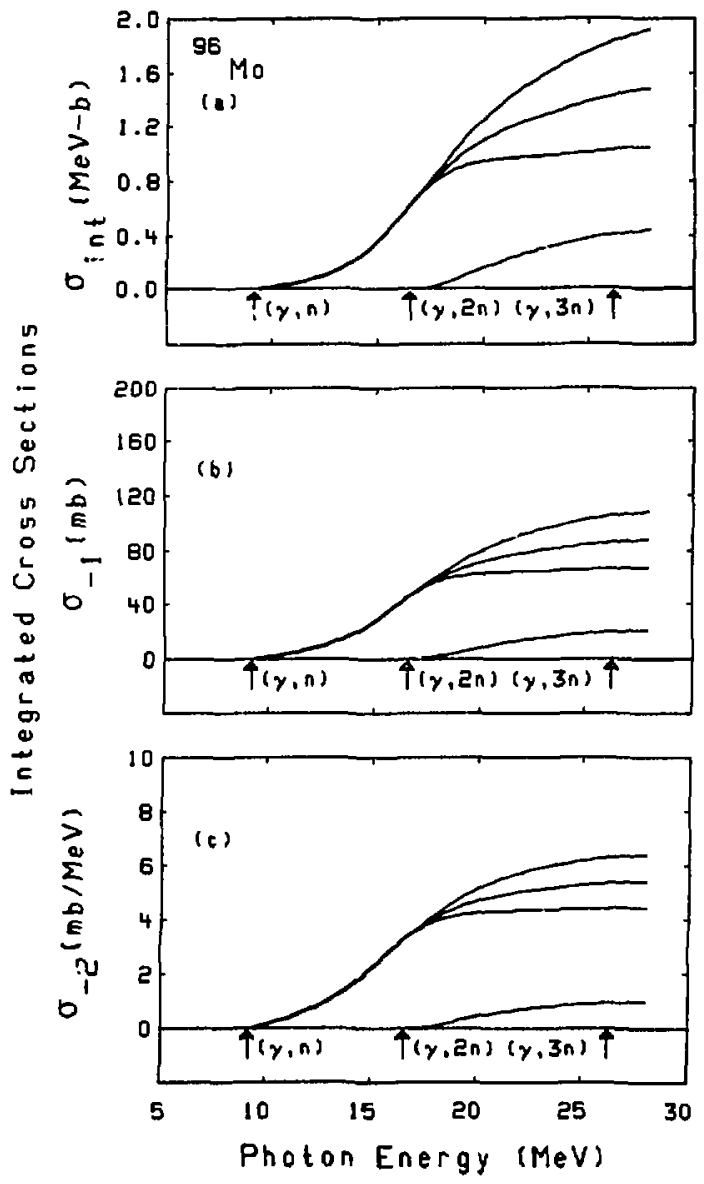

Fig. $80 \mathrm{~B}$ 

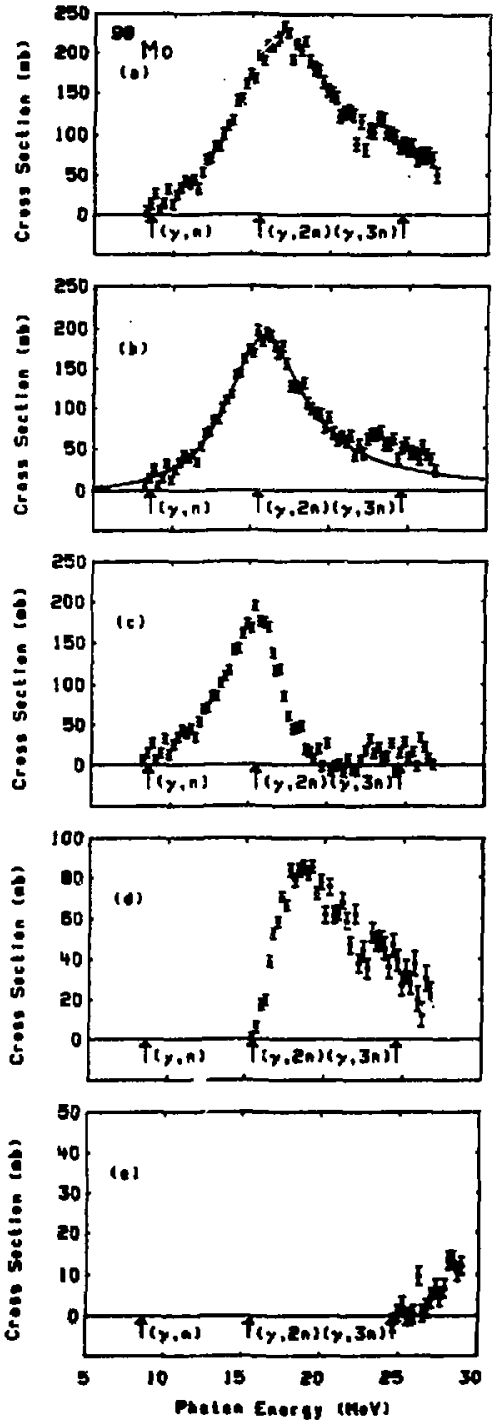

Fig. $81 \mathrm{~A}$ 


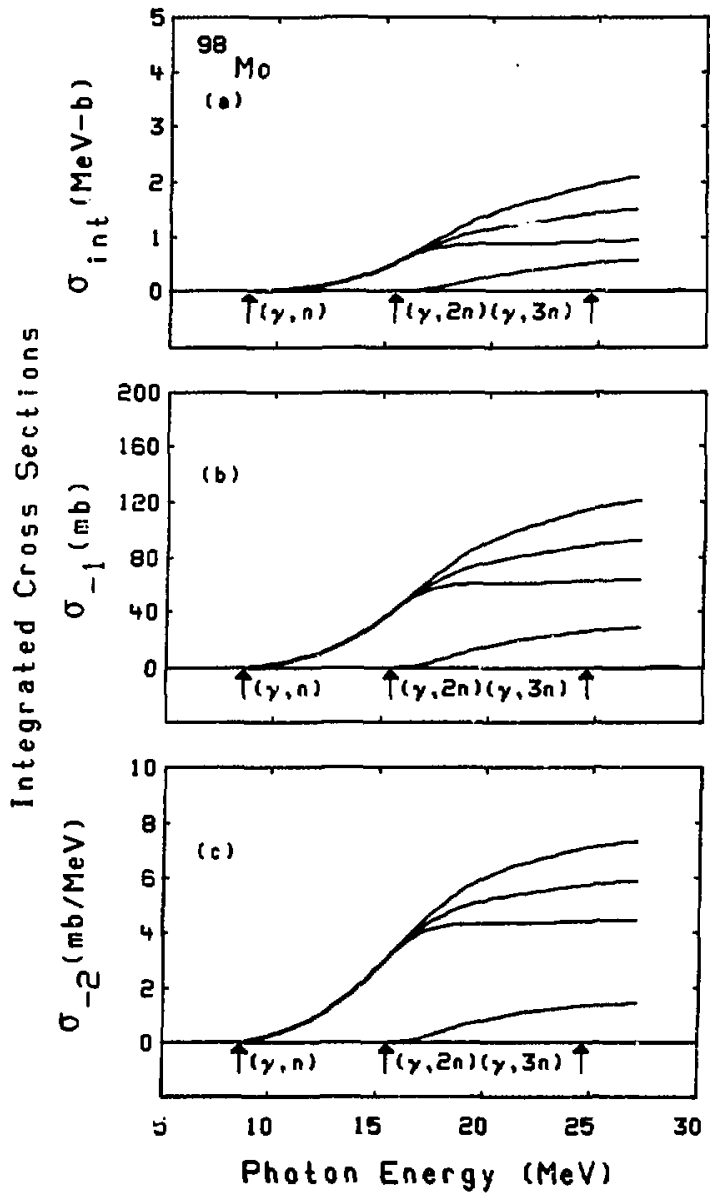

Fig. $81 B$ 

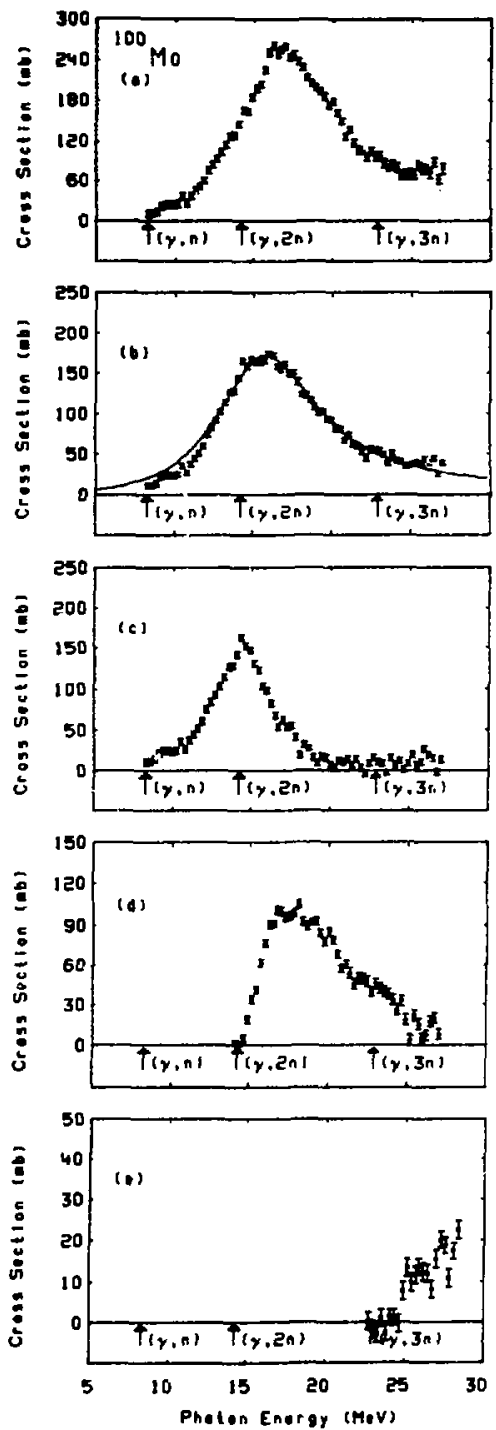

Fig. $82 A$ 


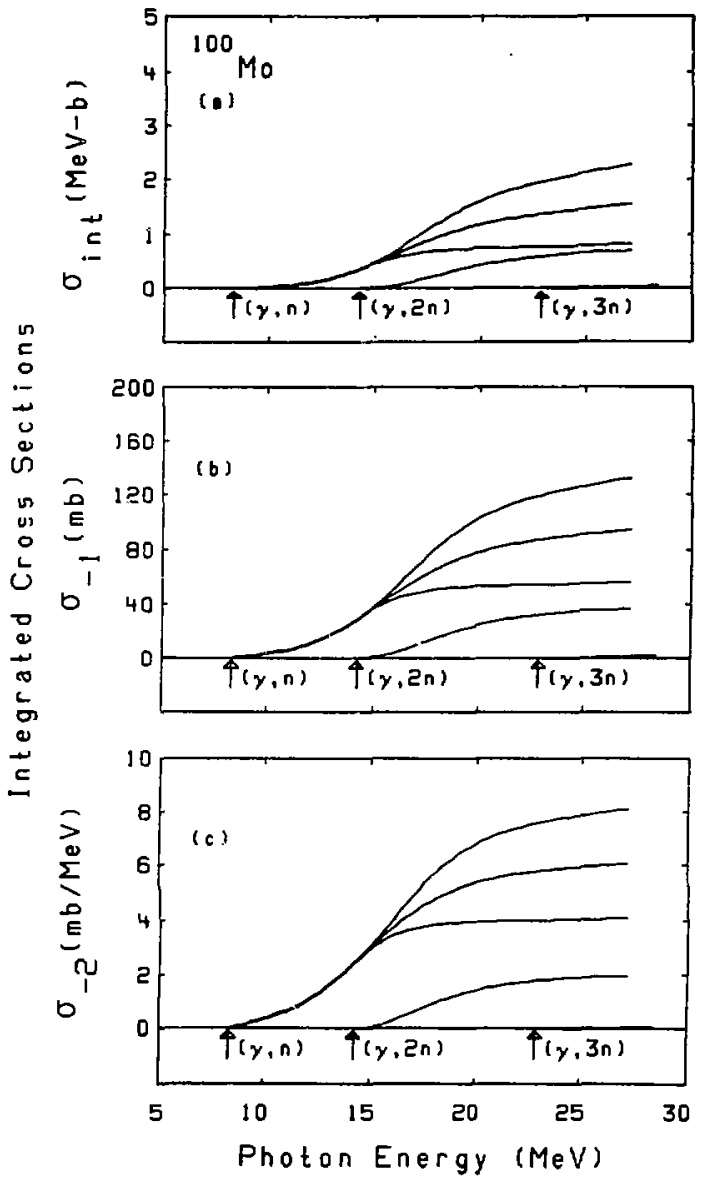

Fig. 82B 

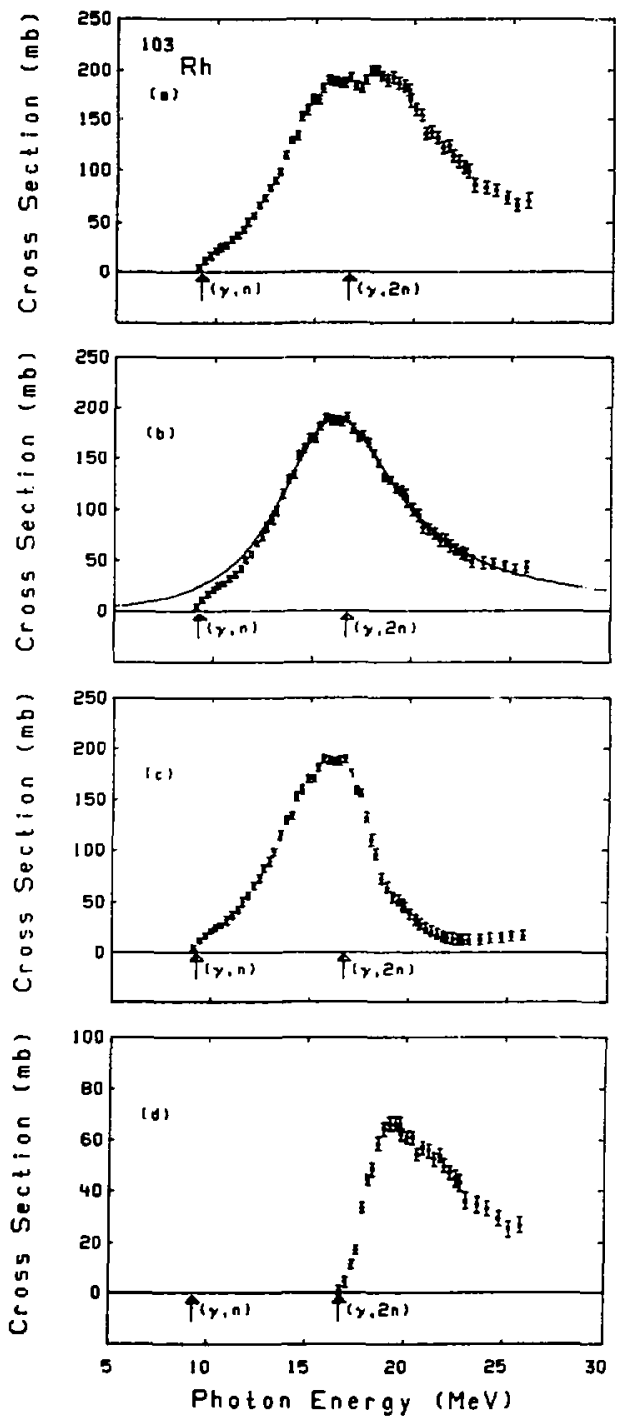

Fig. $83 A$ 


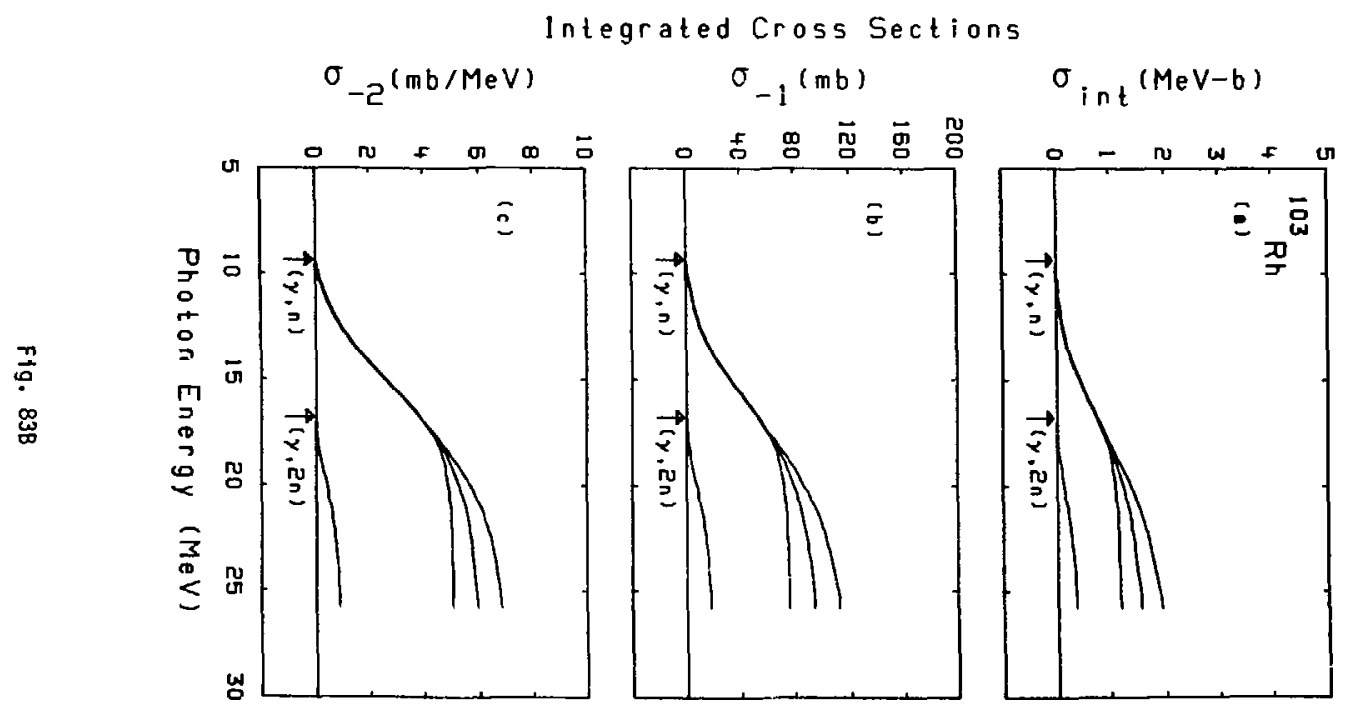



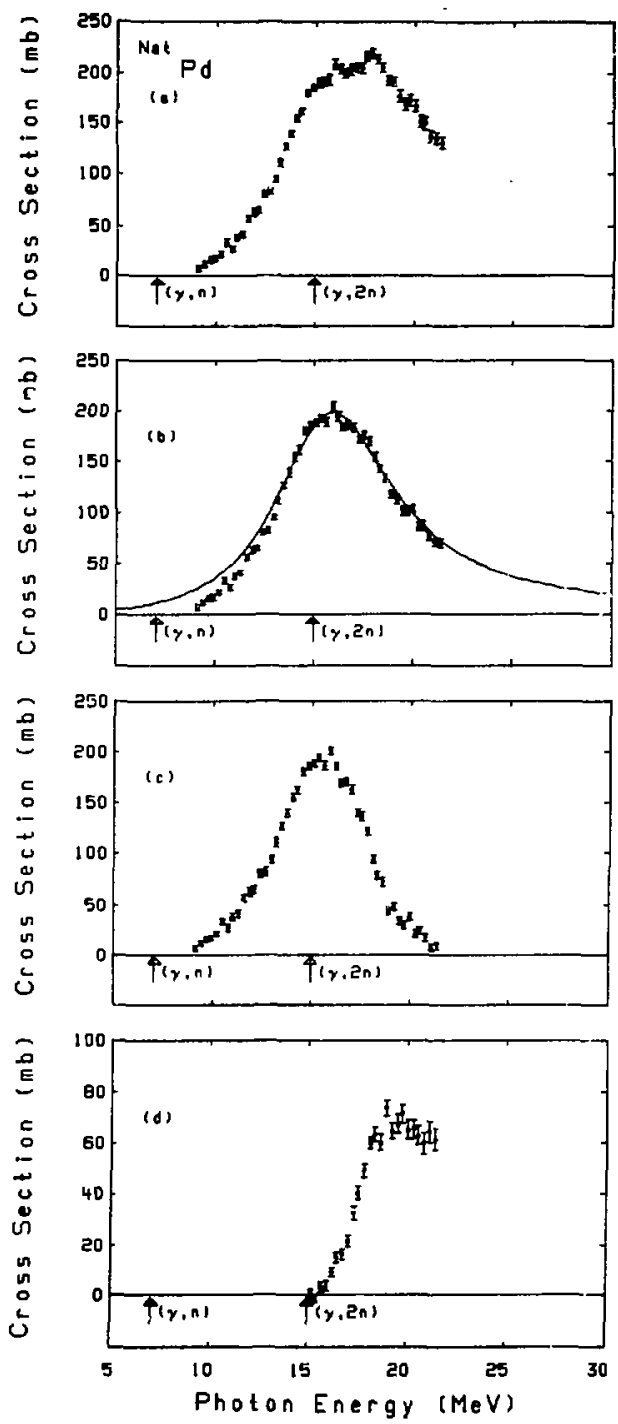

Ftg. $84 \mathrm{~A}$ 


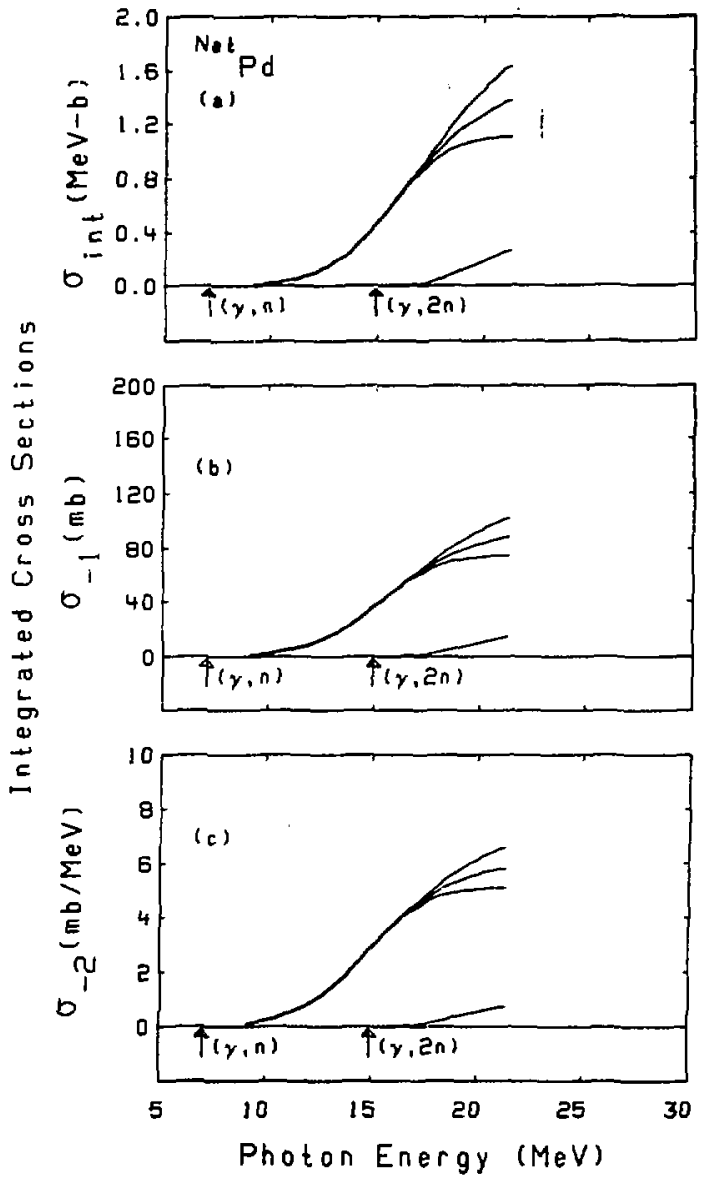

Fig. 848 

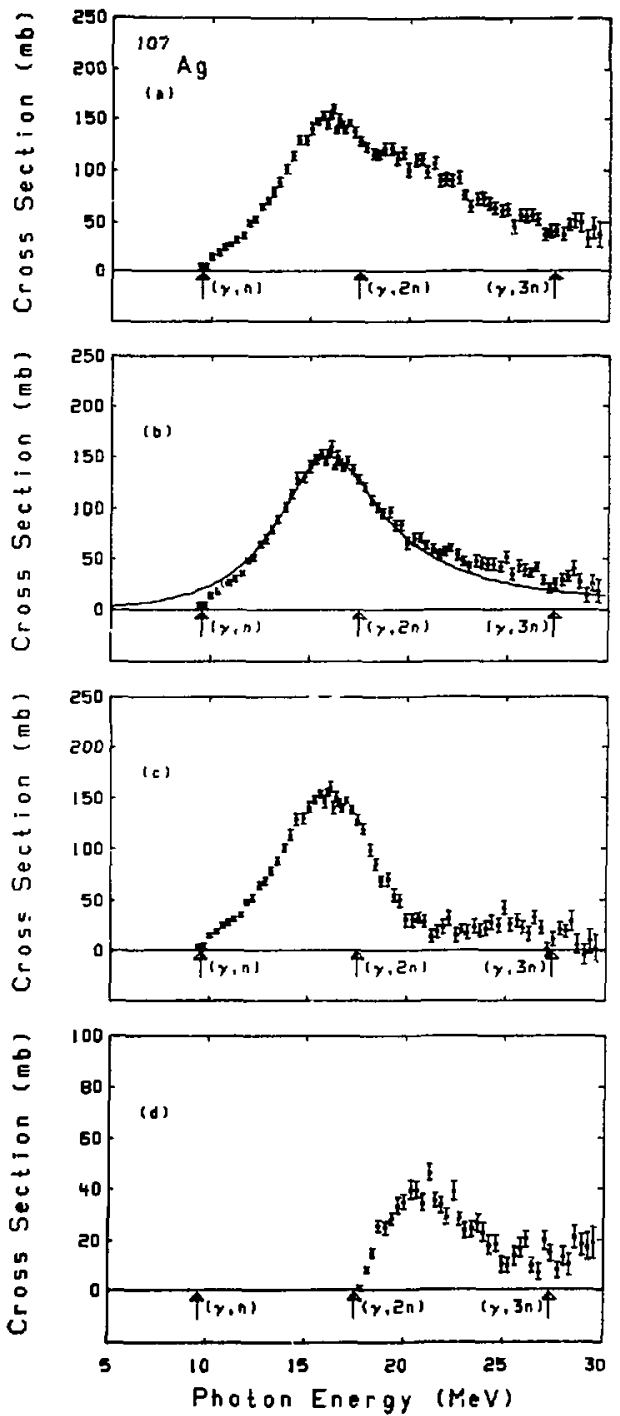

Fig. $85 A$ 


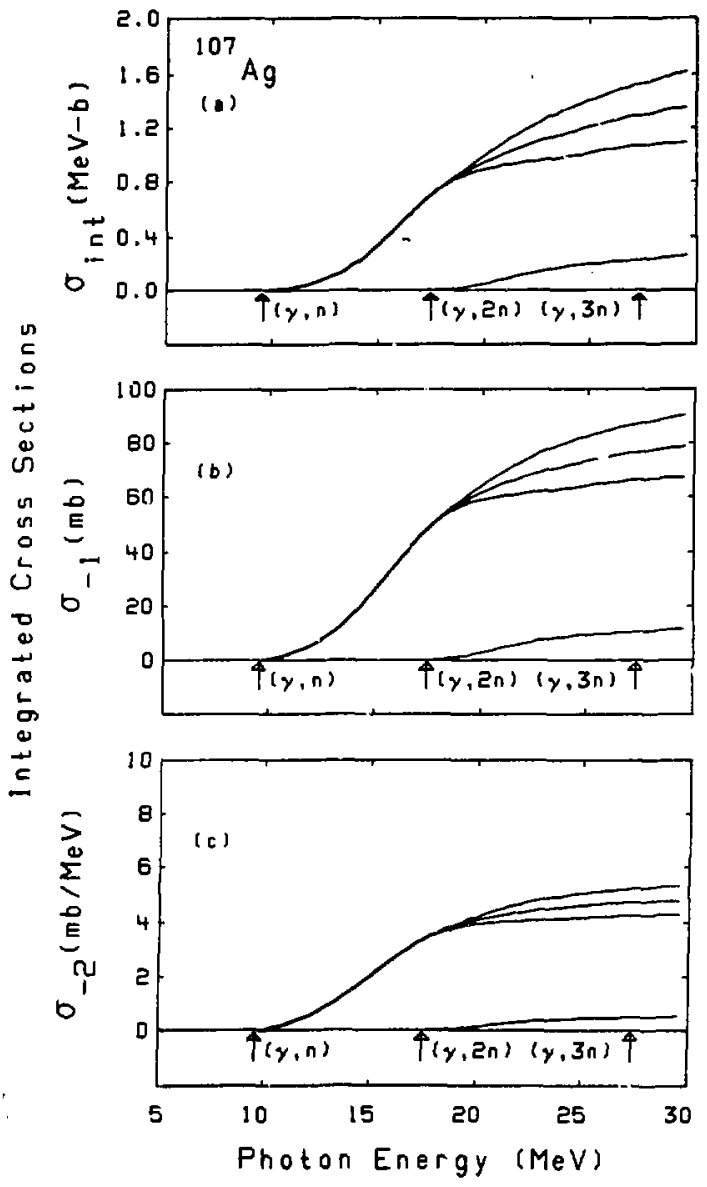

Fig. $85 B$ 

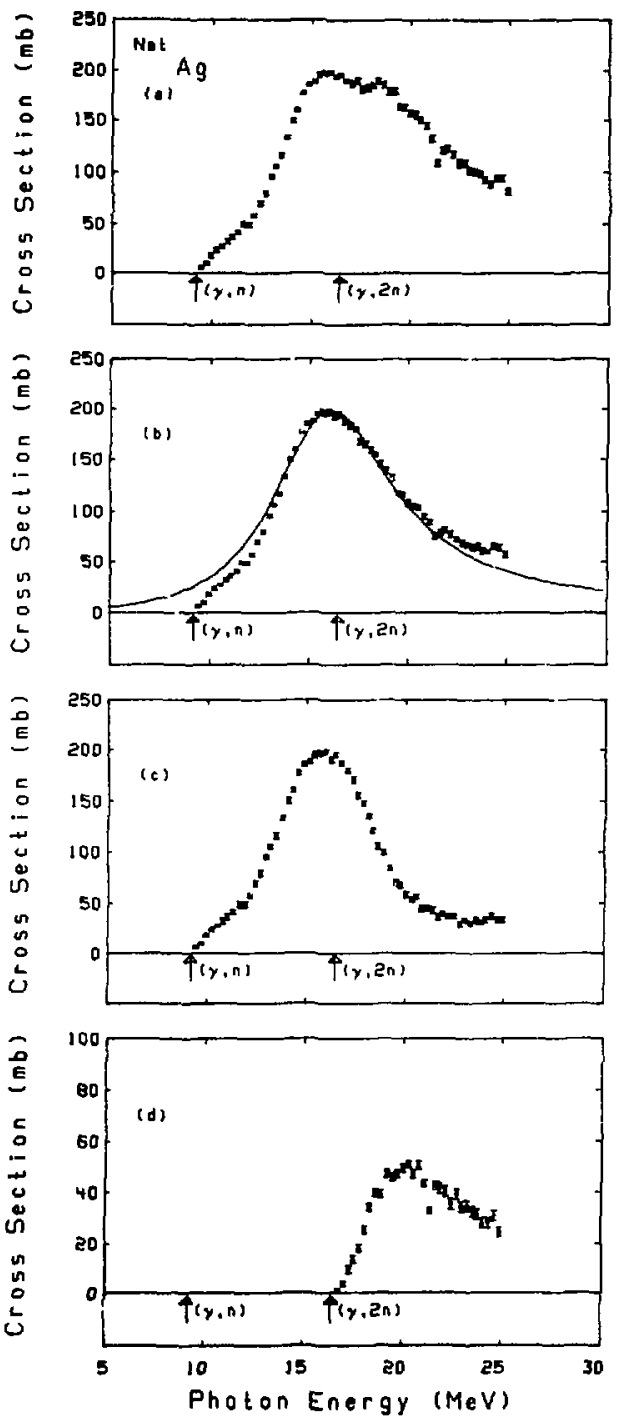

Fig. $86 \mathrm{~A}$ 


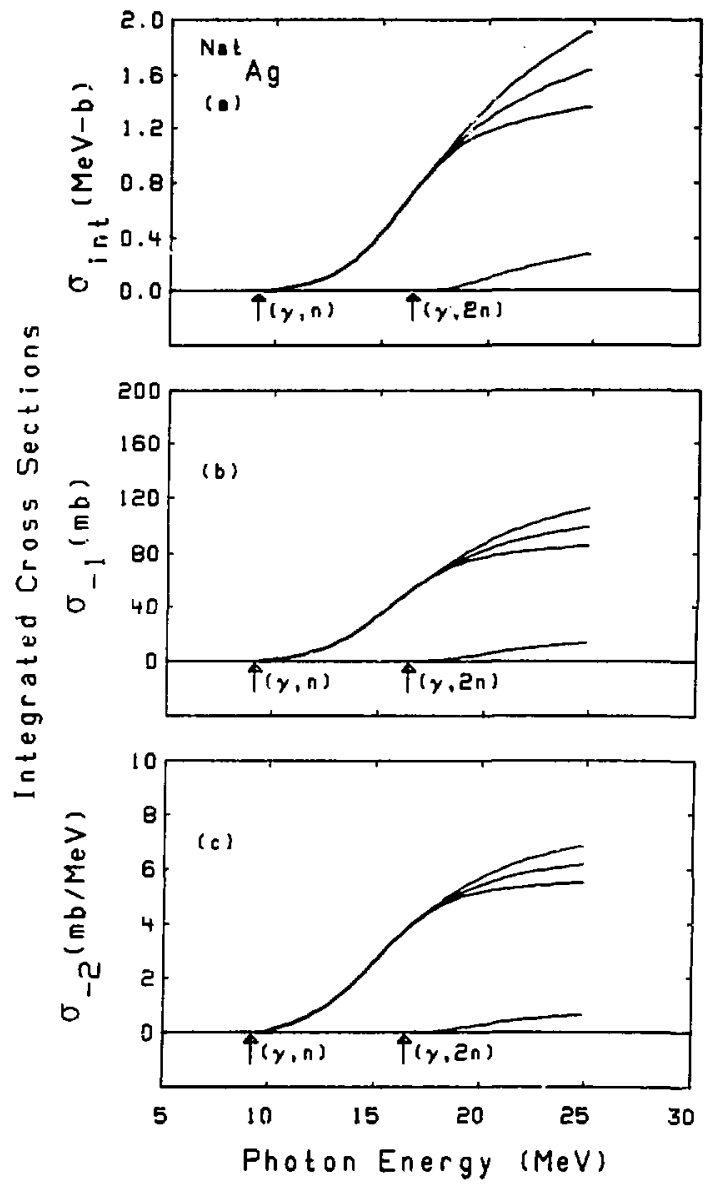

Fig. $86 \mathrm{~B}$ 

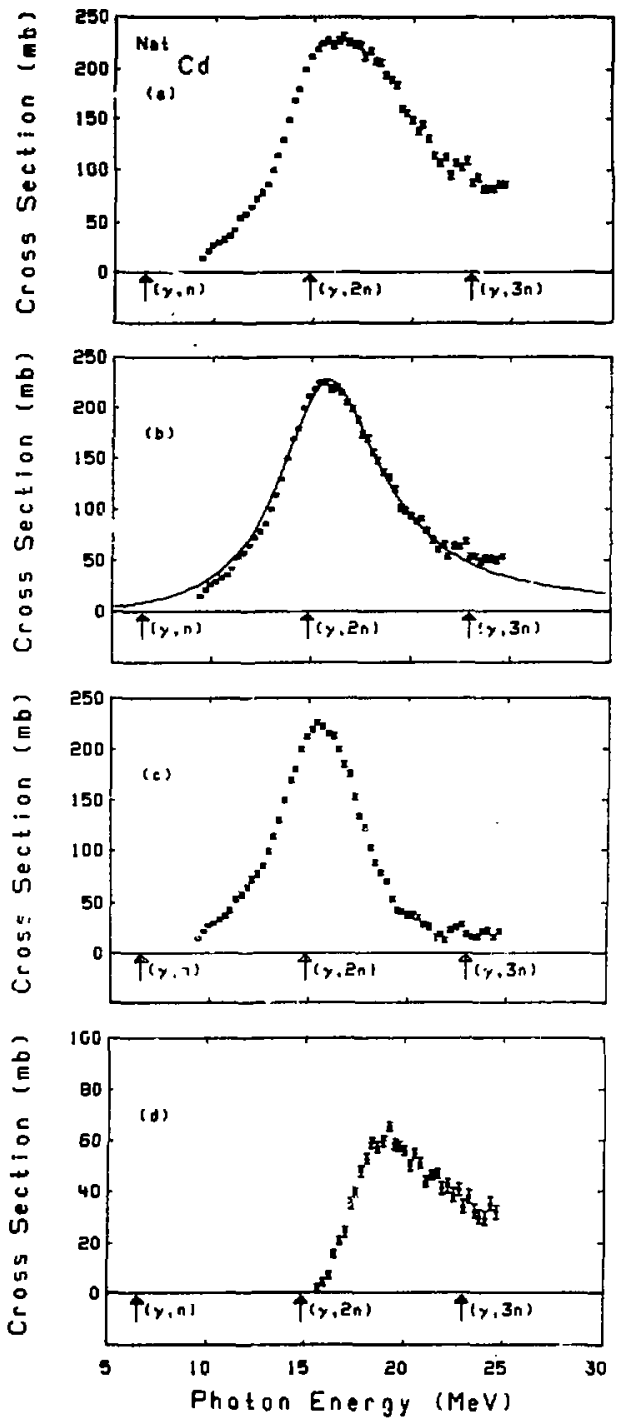

Fig. $87 \mathrm{~A}$ 


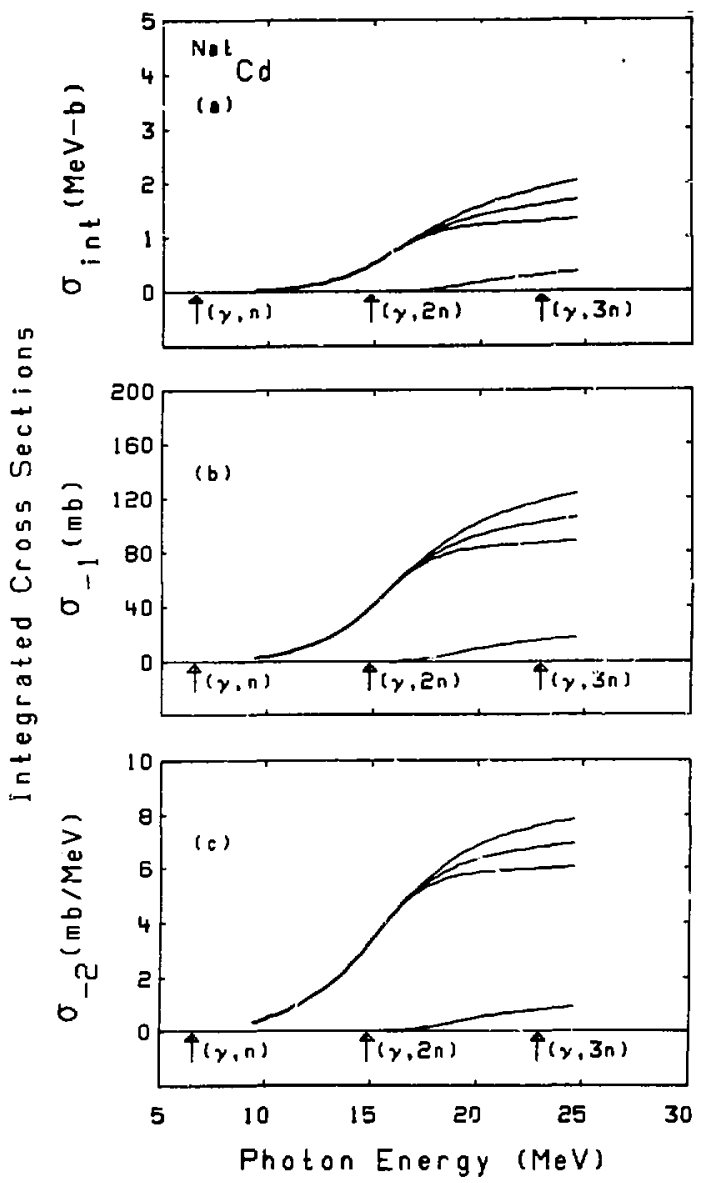

Fig. $87 \mathrm{~B}$ 

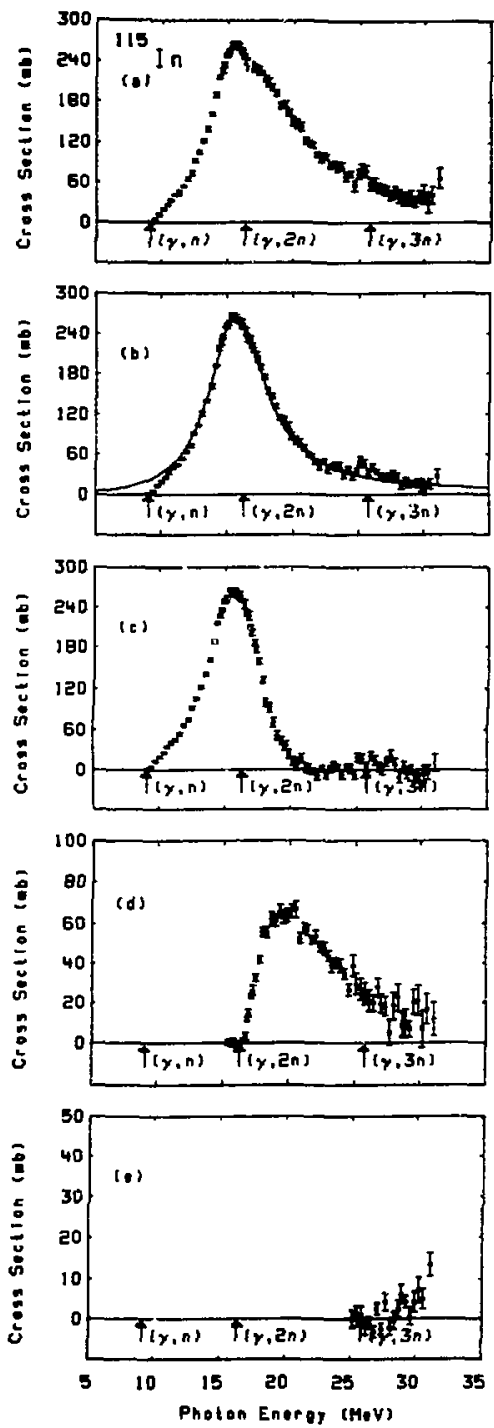

Fig. $88 \mathrm{~A}$ 


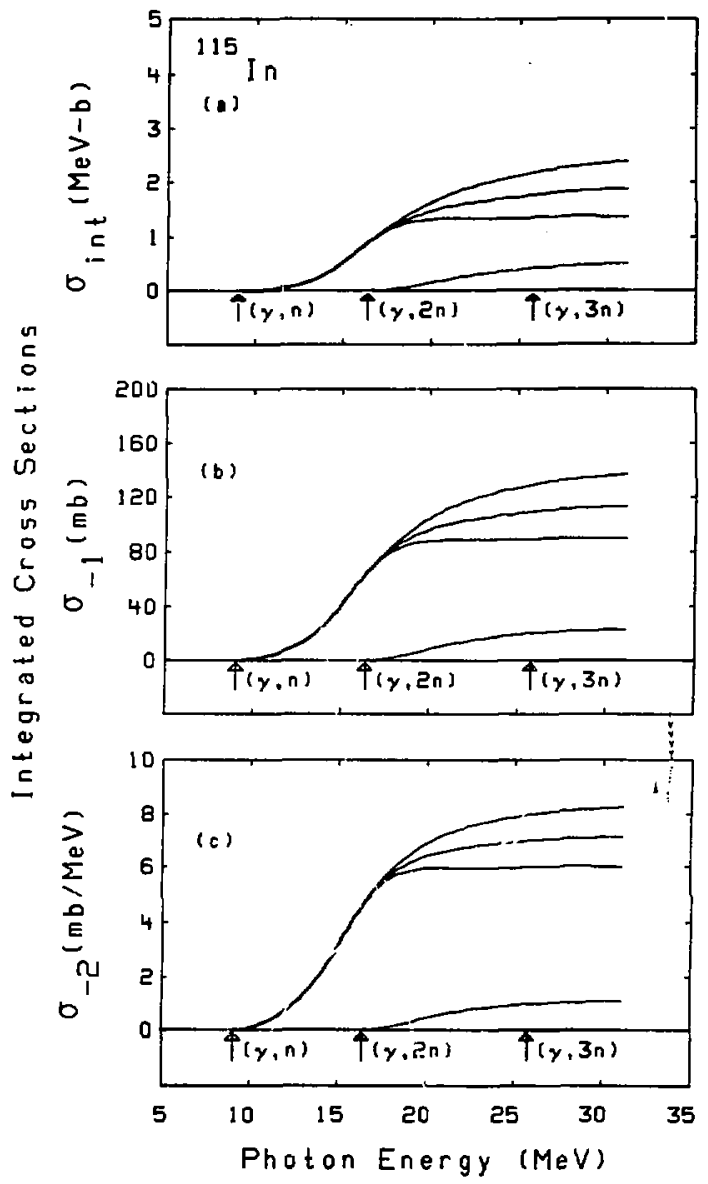

Fig. BBB 

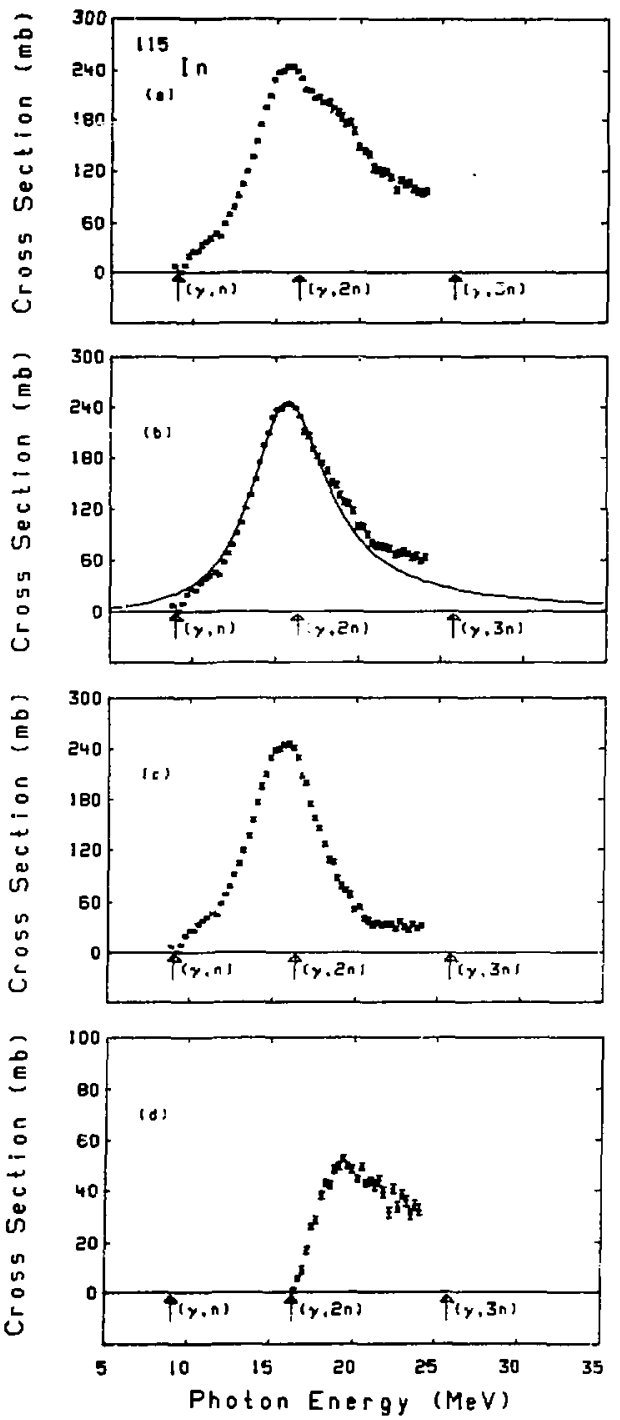

Fig. $89 \mathrm{~A}$ 


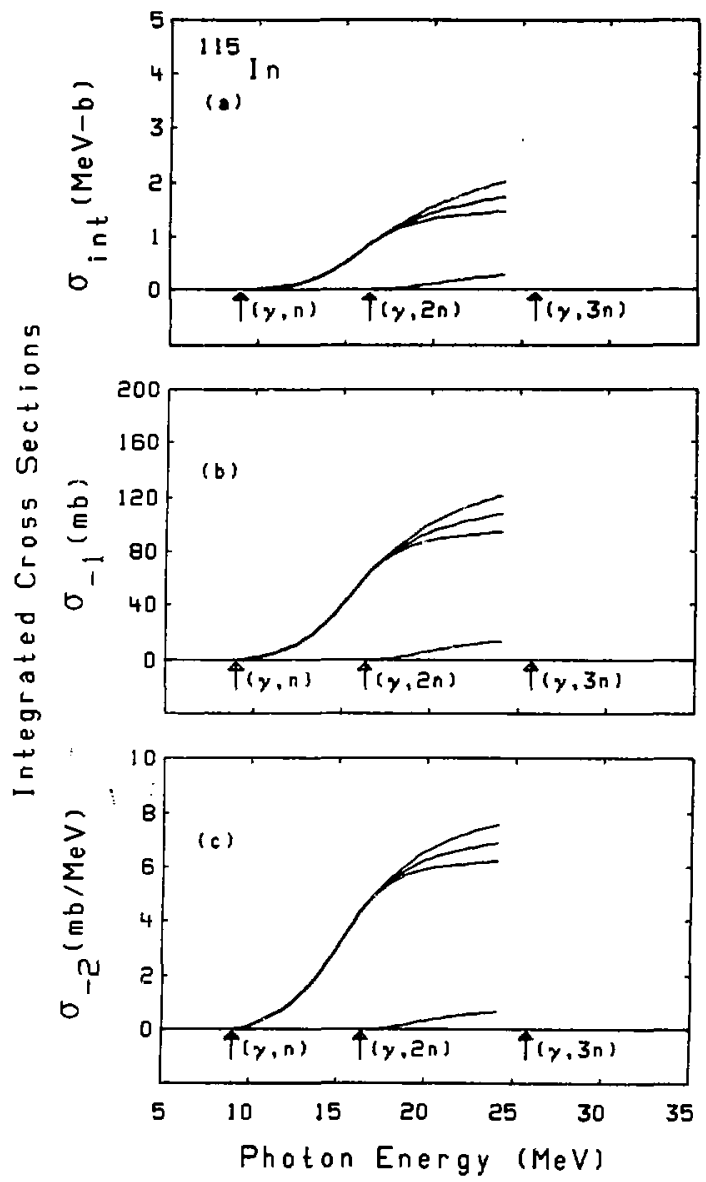

Fig. $89 B$ 

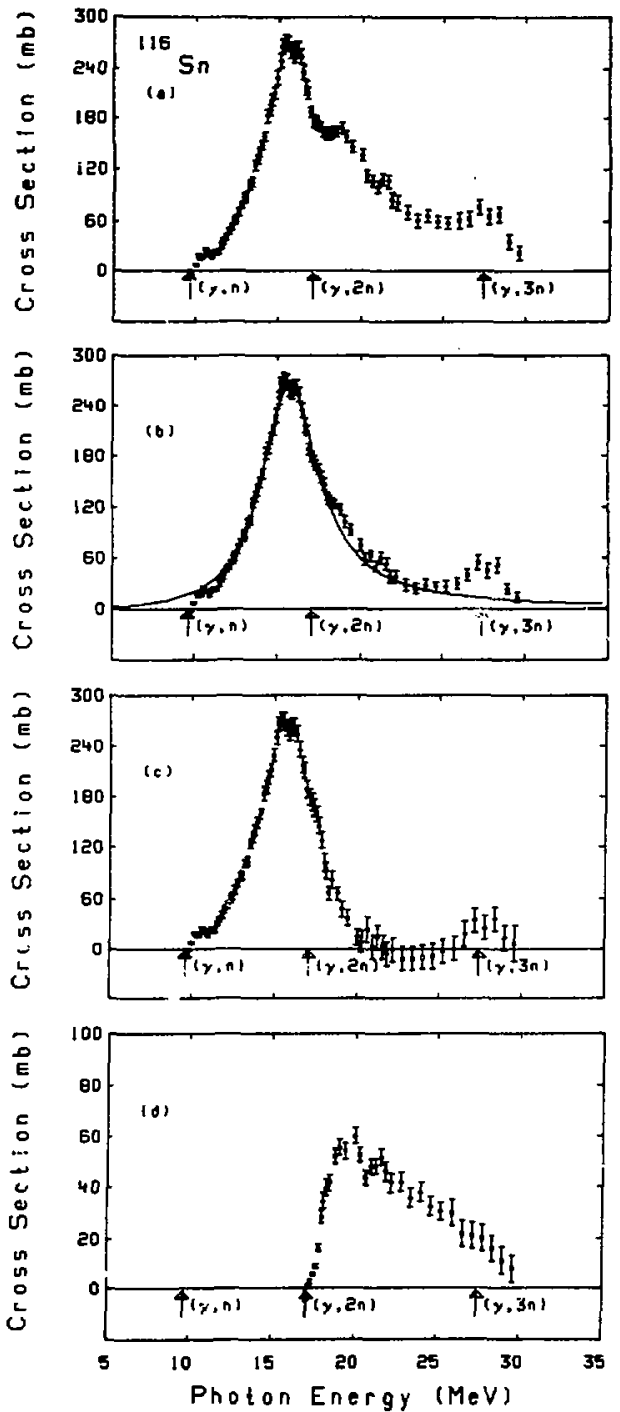

Fig. 90A 


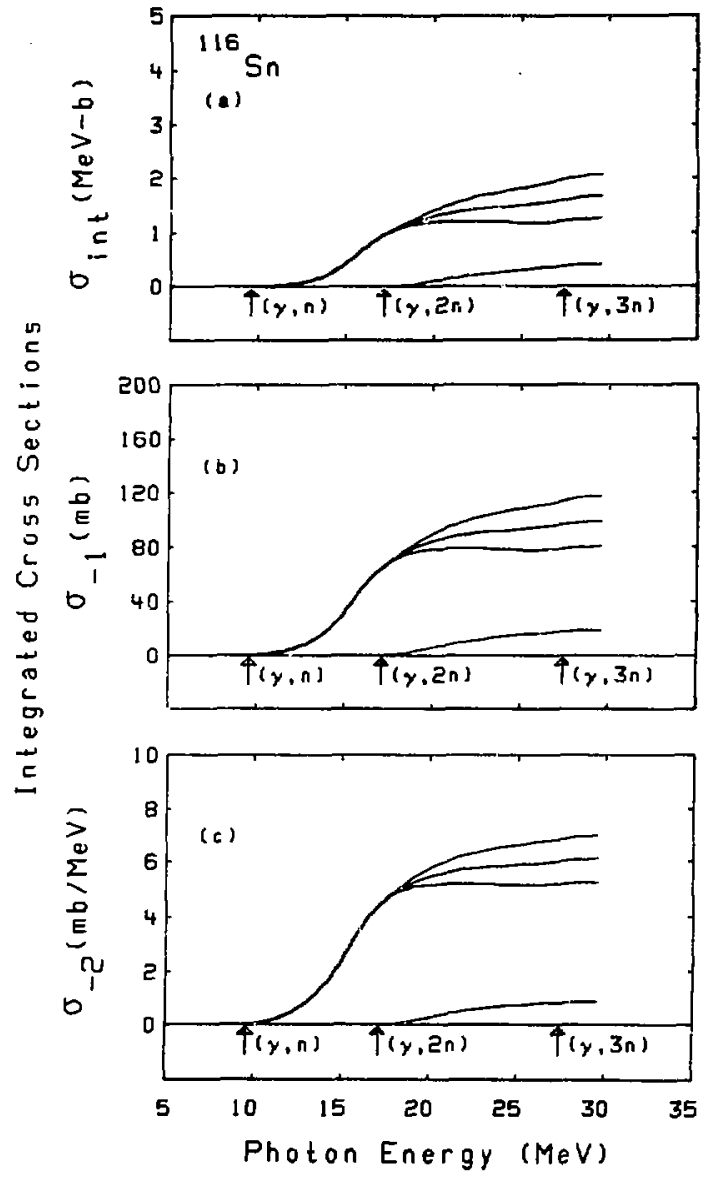

Fig. 90B 

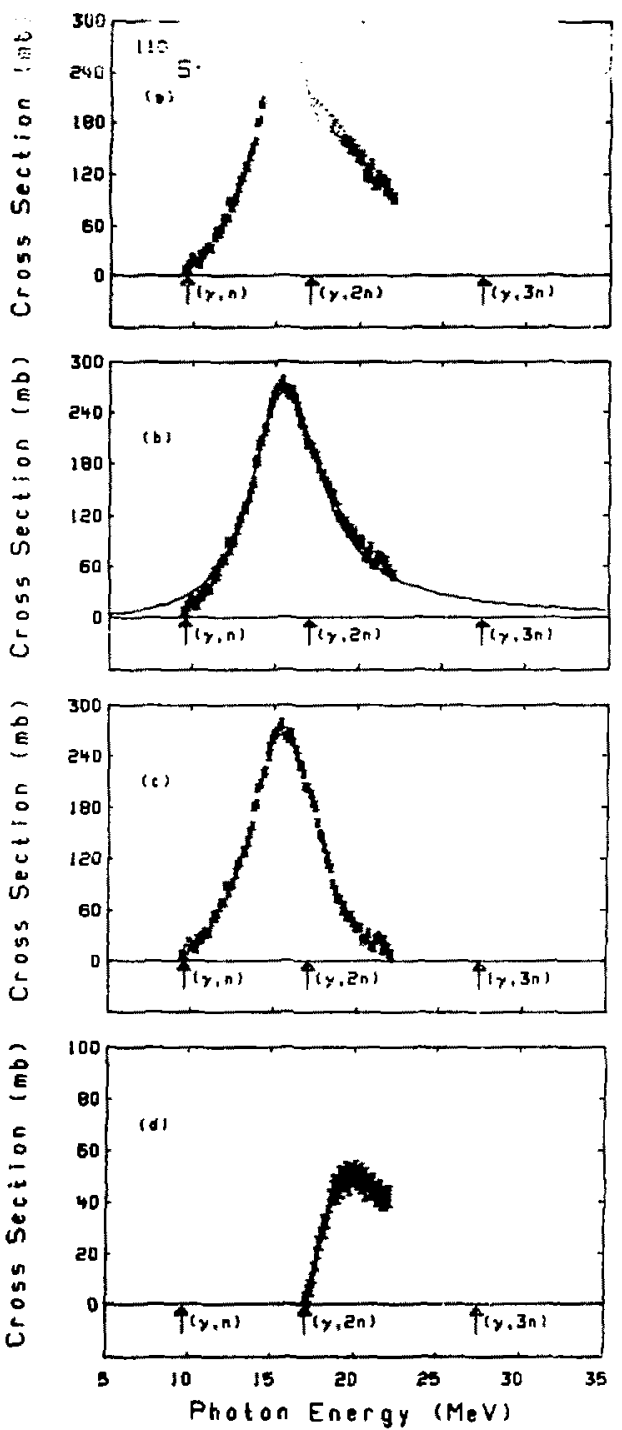

Fig. 91A 


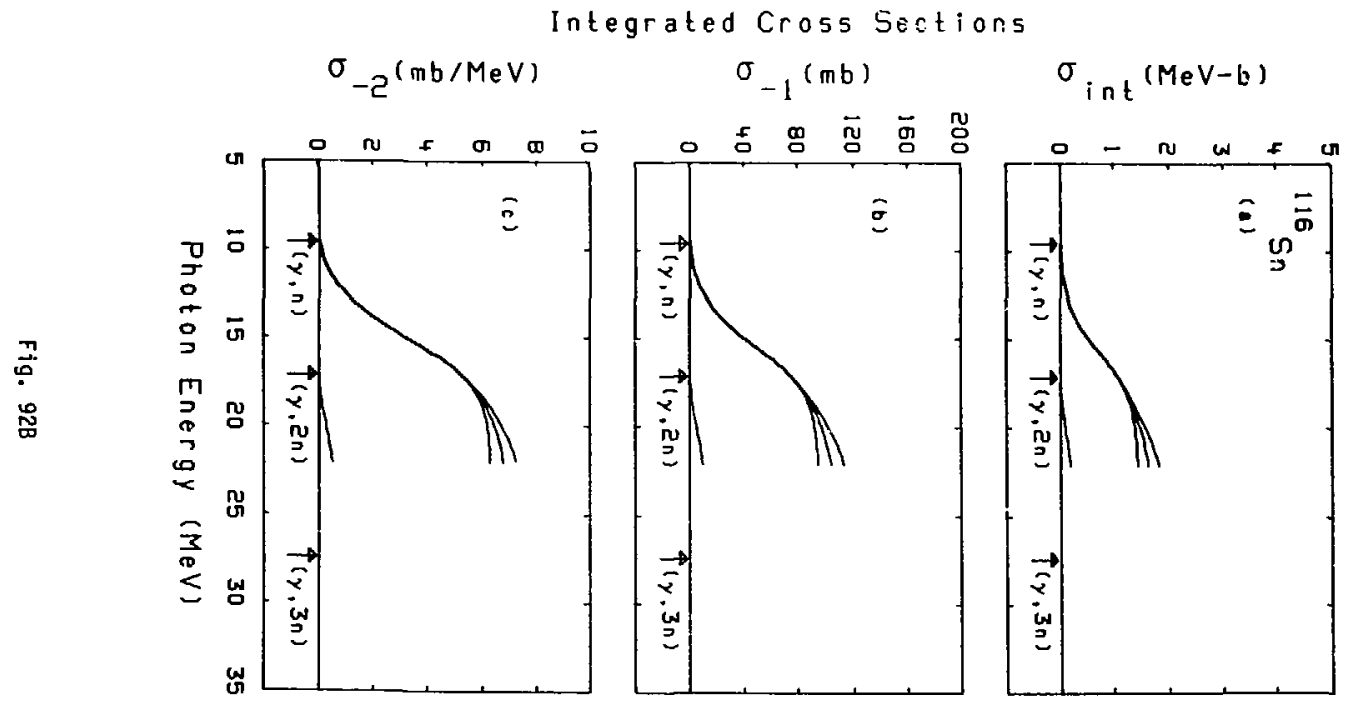



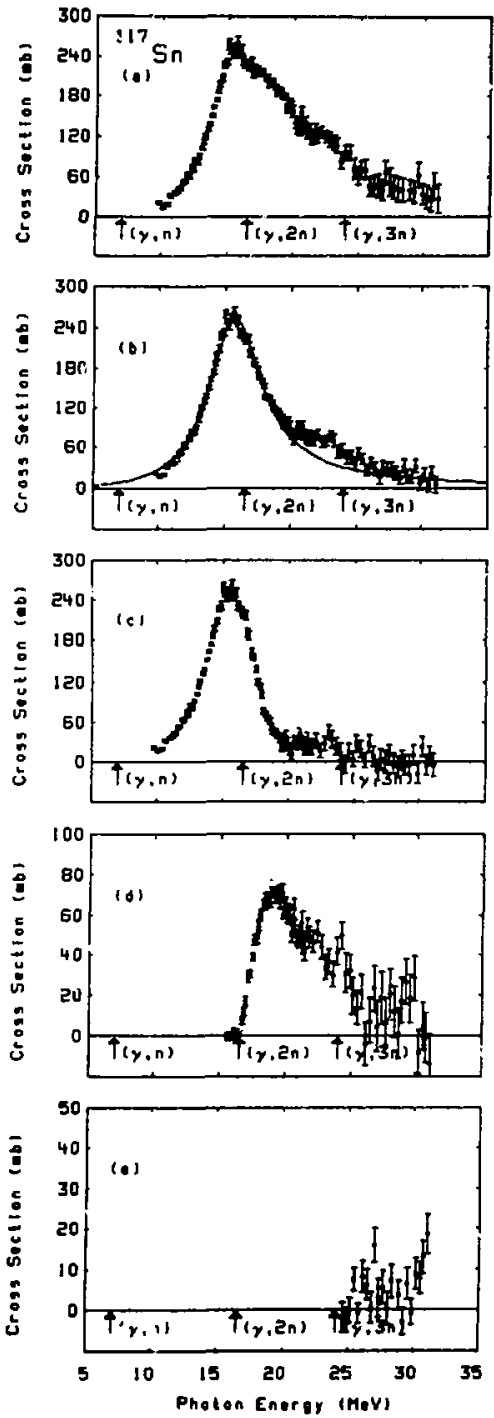

Fig. 92A 


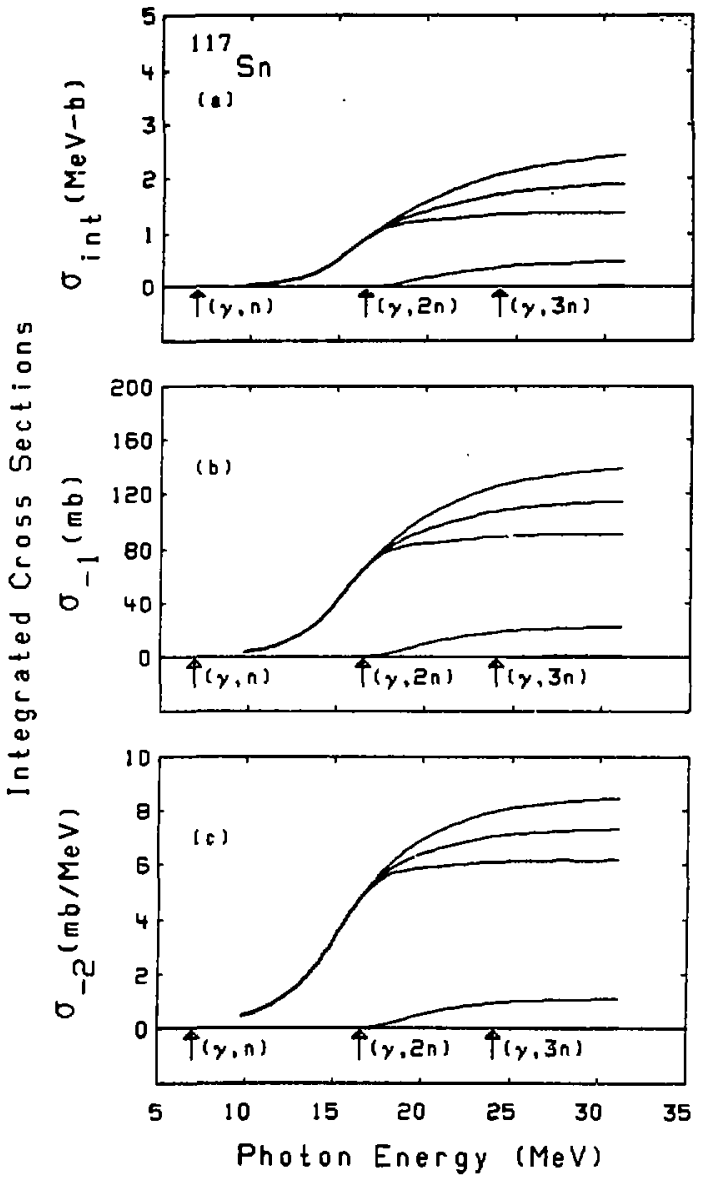

Fig. 92B 

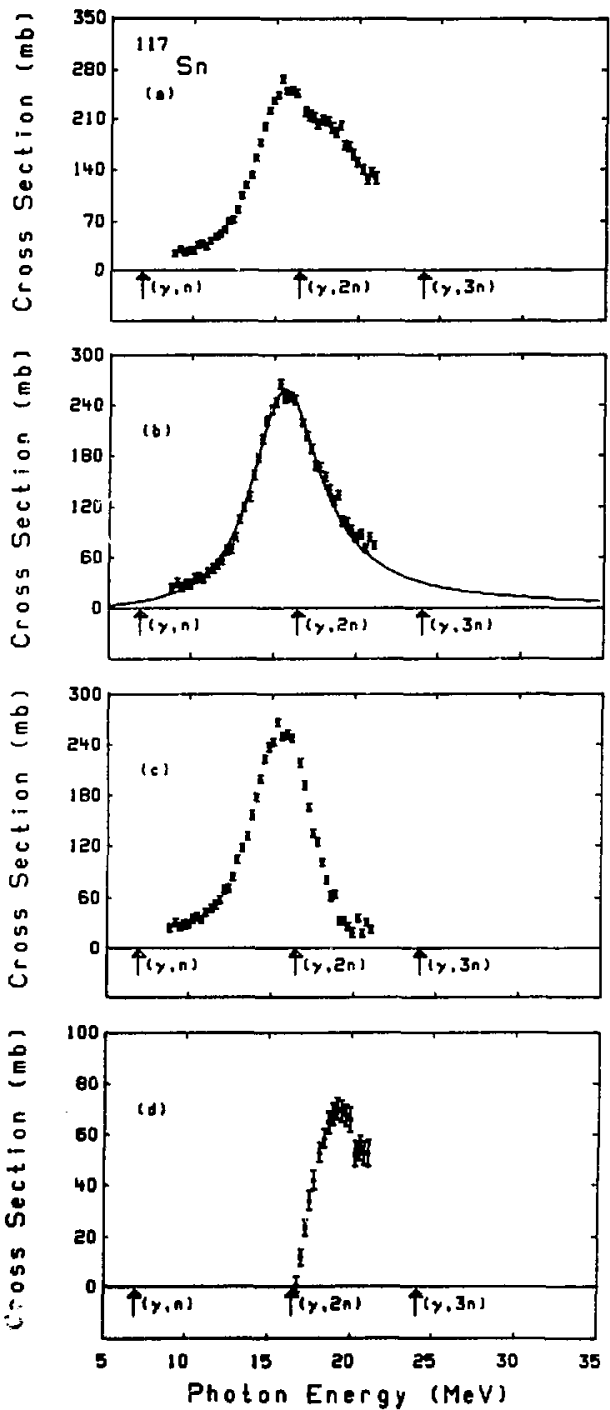

Fig. $93 A$ 


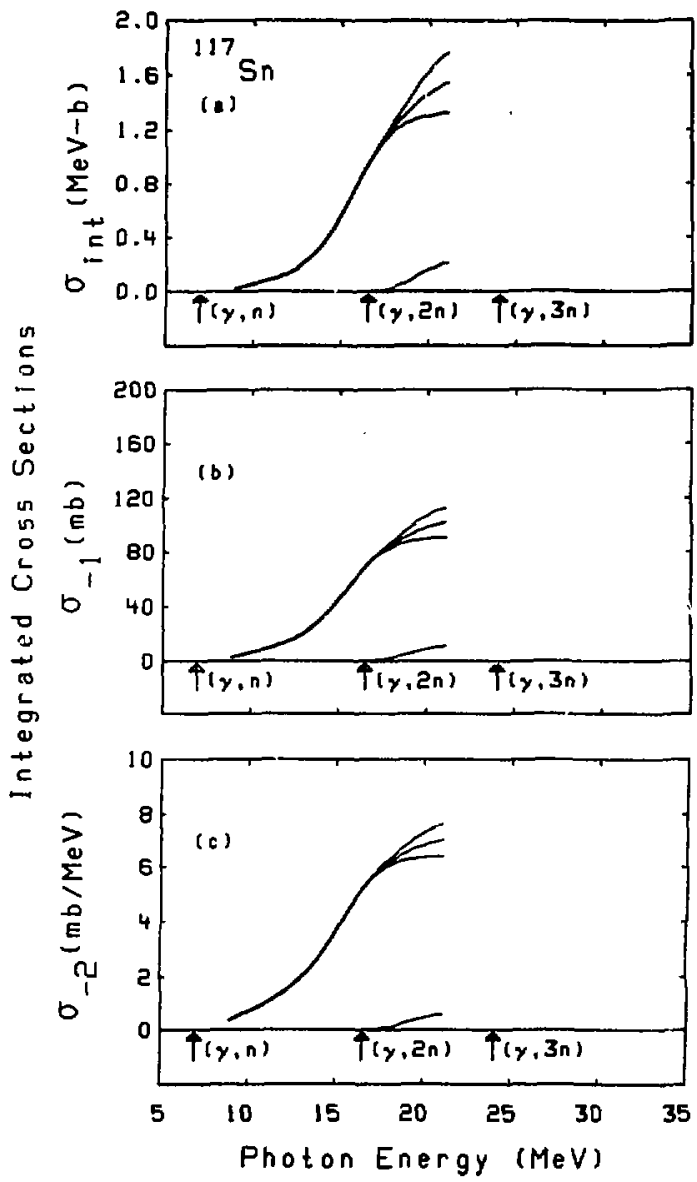

F1g. 93B 

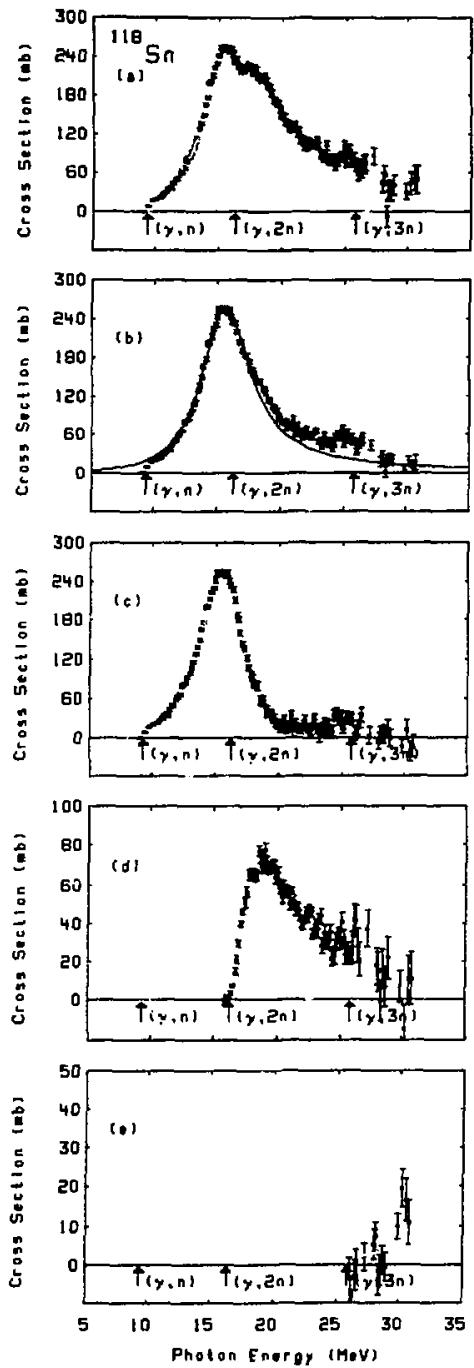

Fig. 94A 


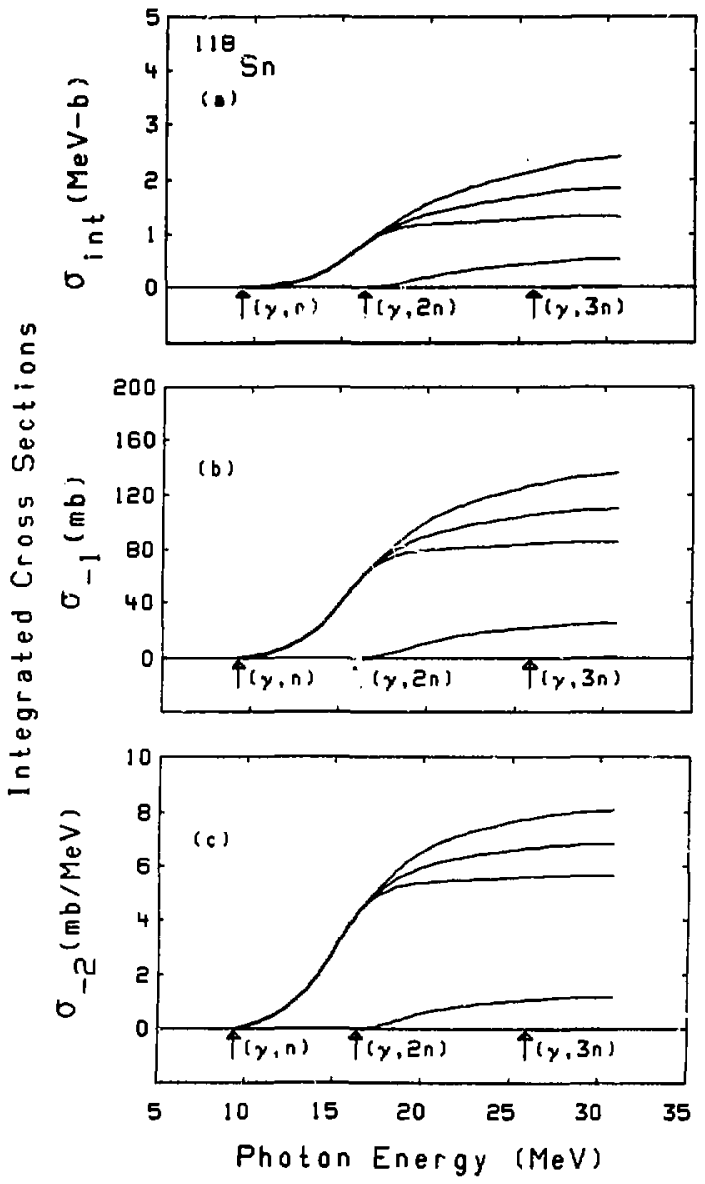

Fig. 948 

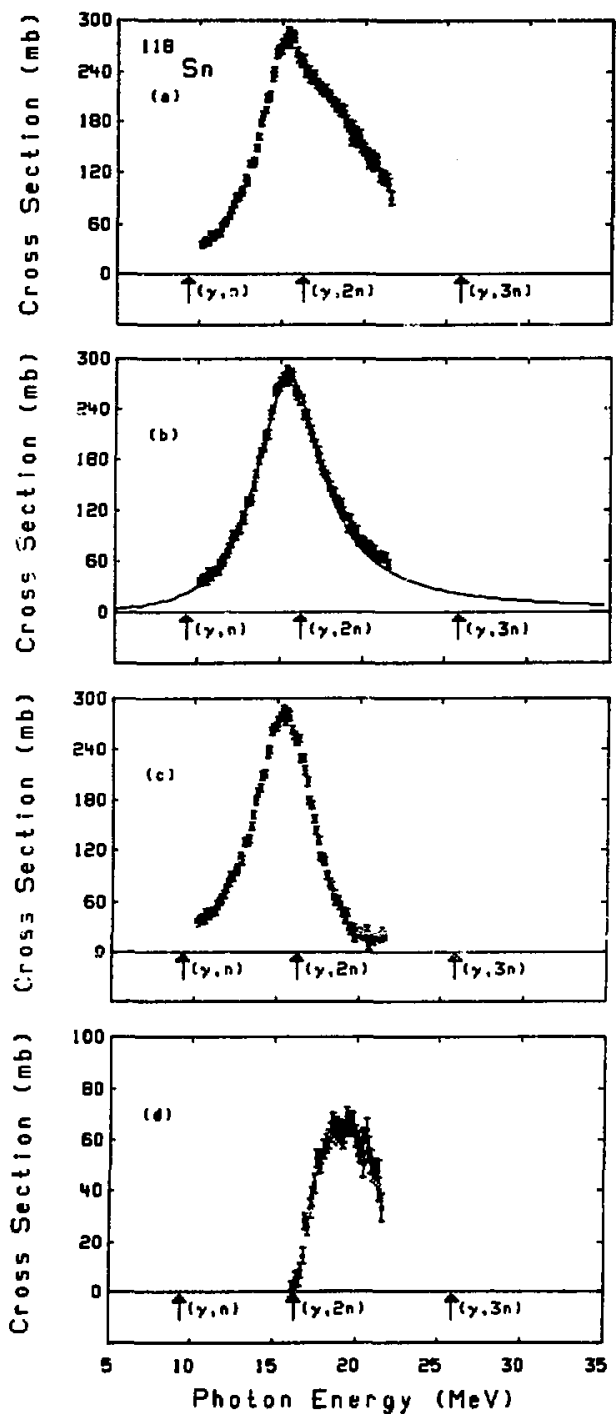

Fig. 95A 


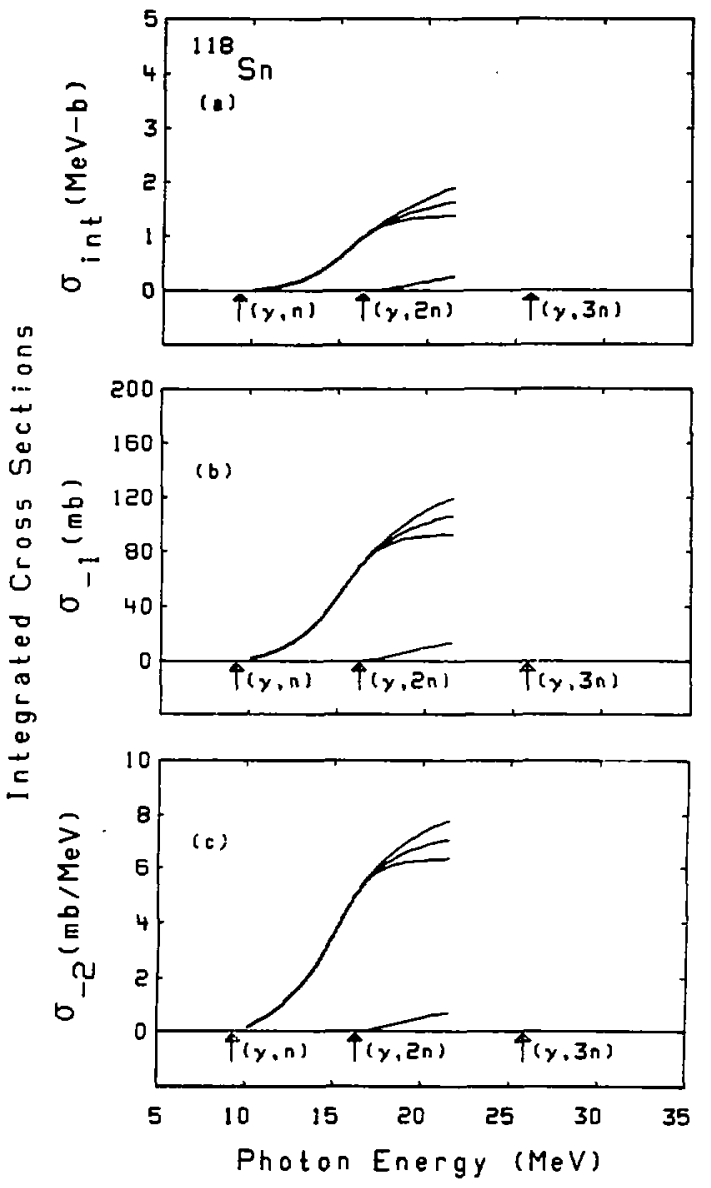

Fig. 958 

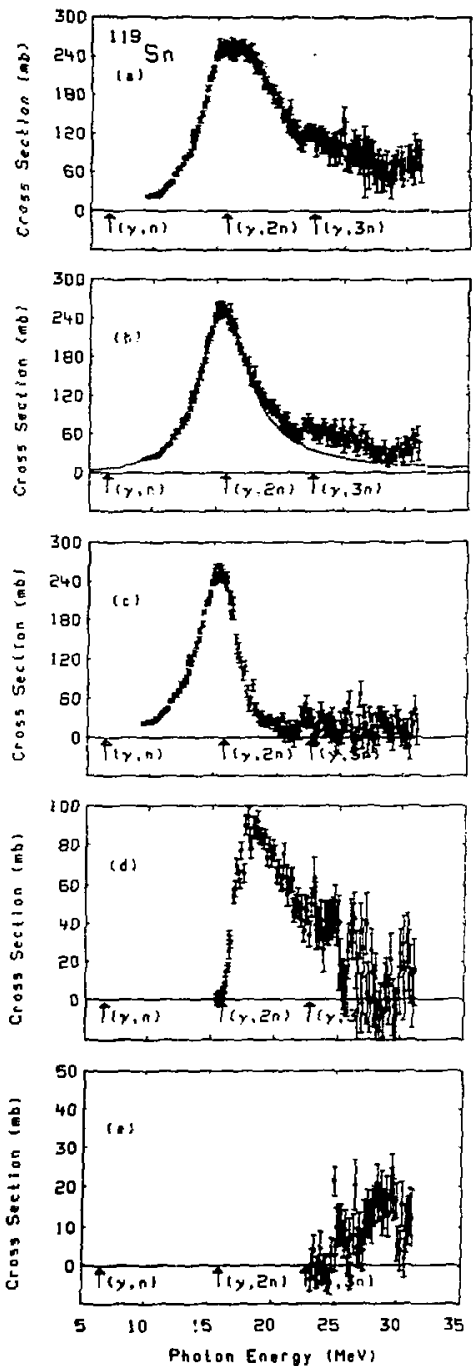

Fig. 96A 


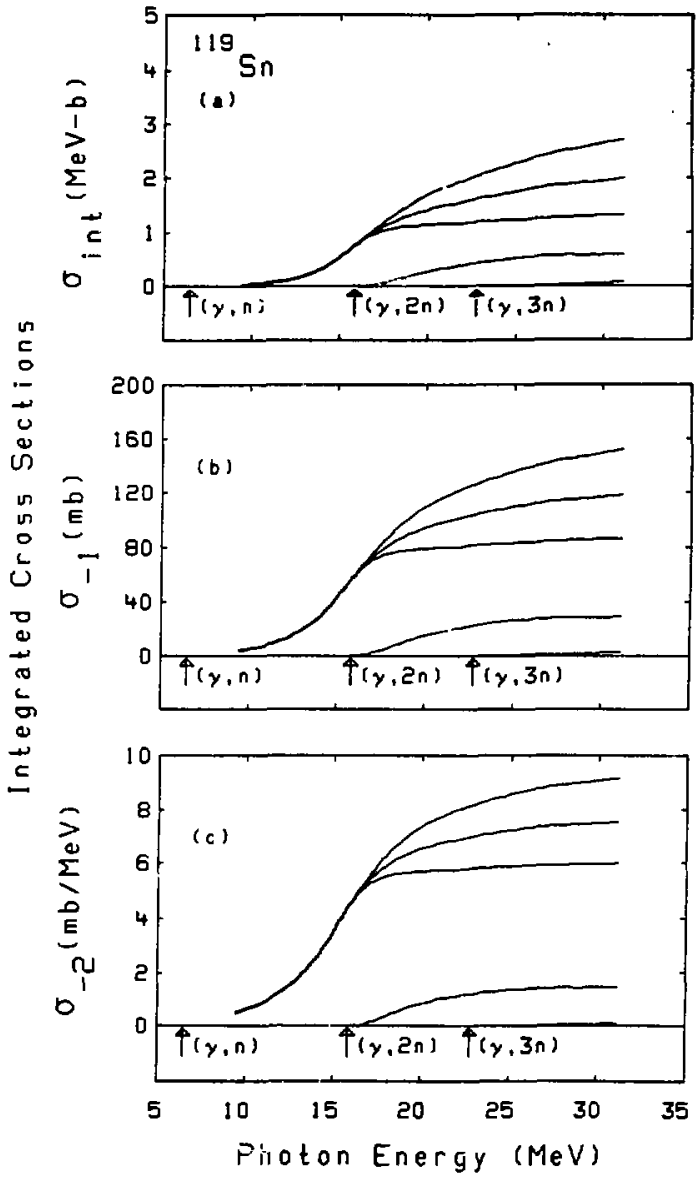

Fig. 96B 

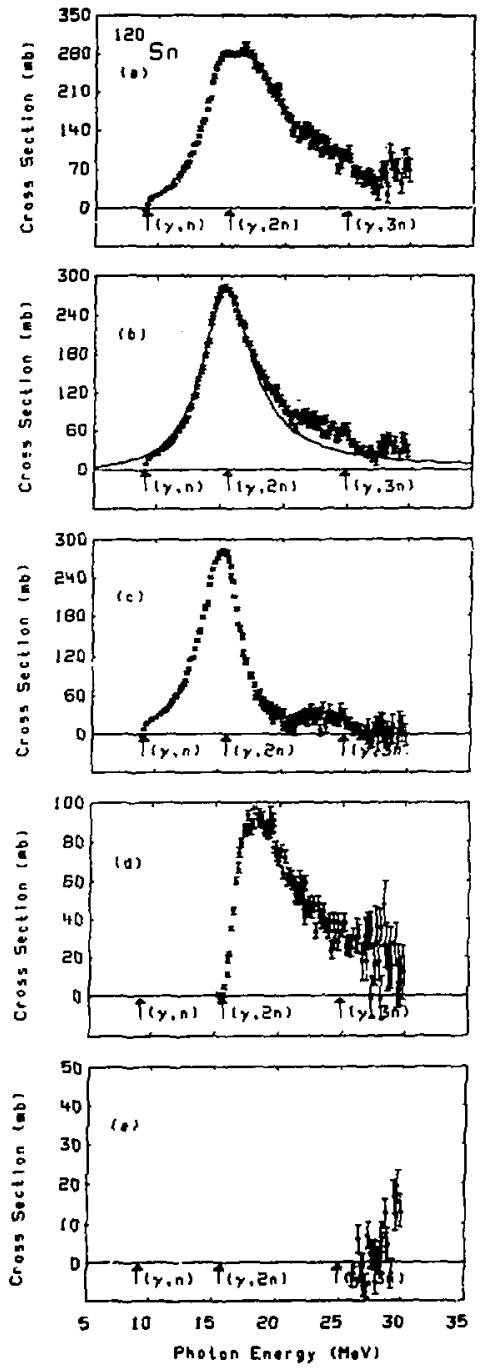

Fig. $97 A$ 


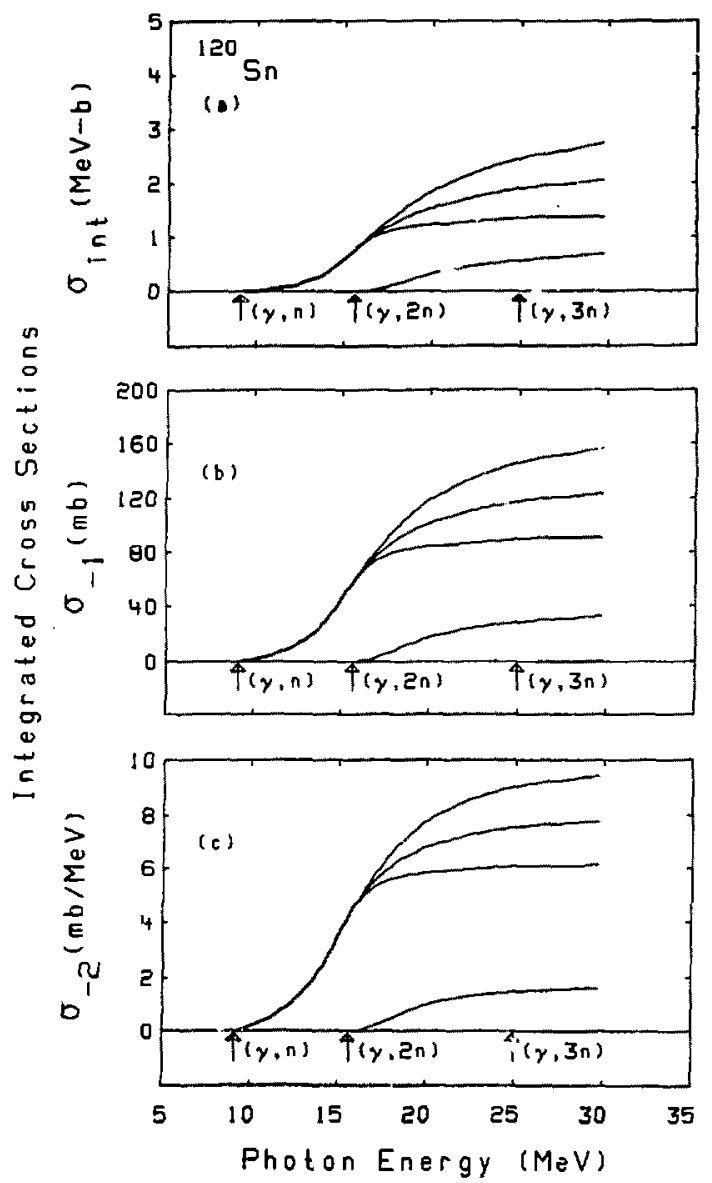

Fig. 97B 

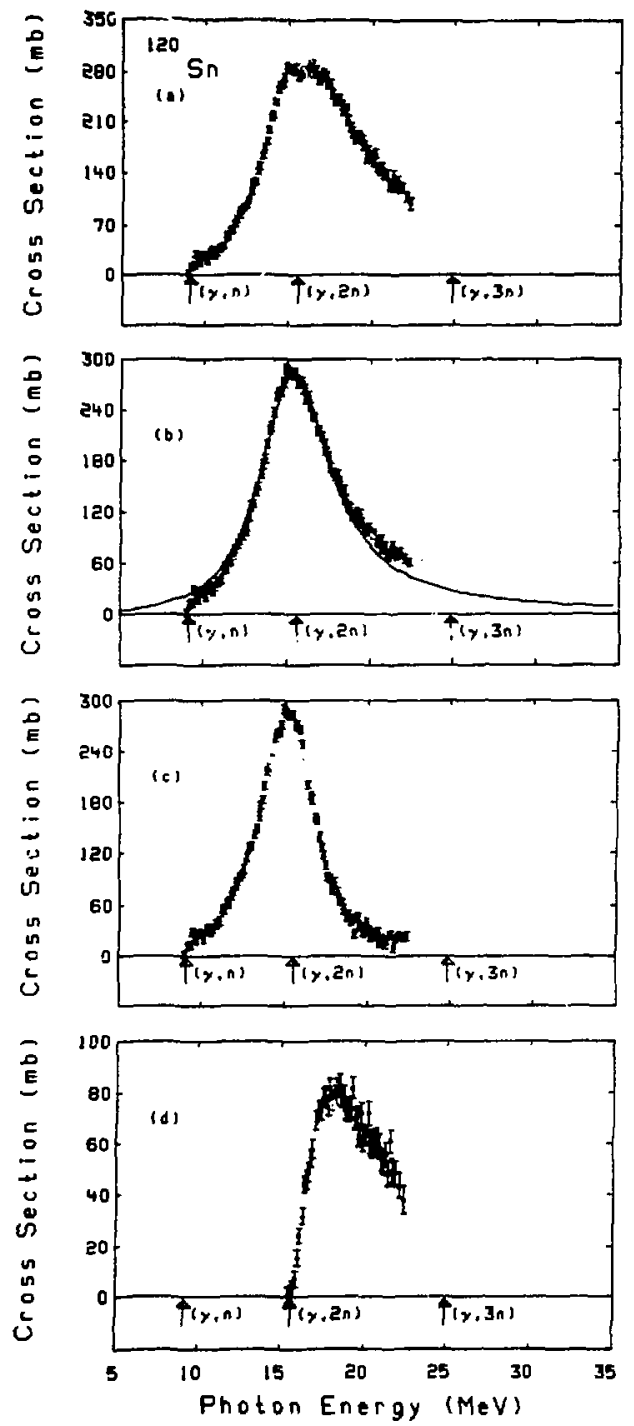

Fig. 98A 


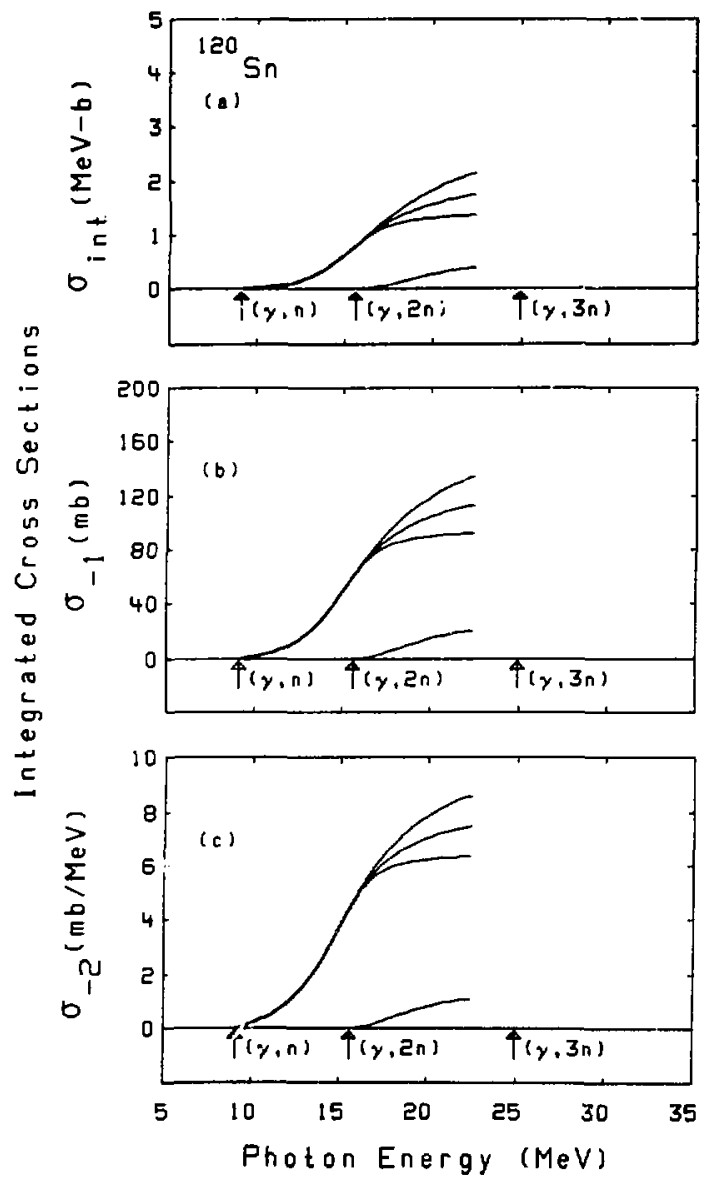

Fig. $98 B$ 

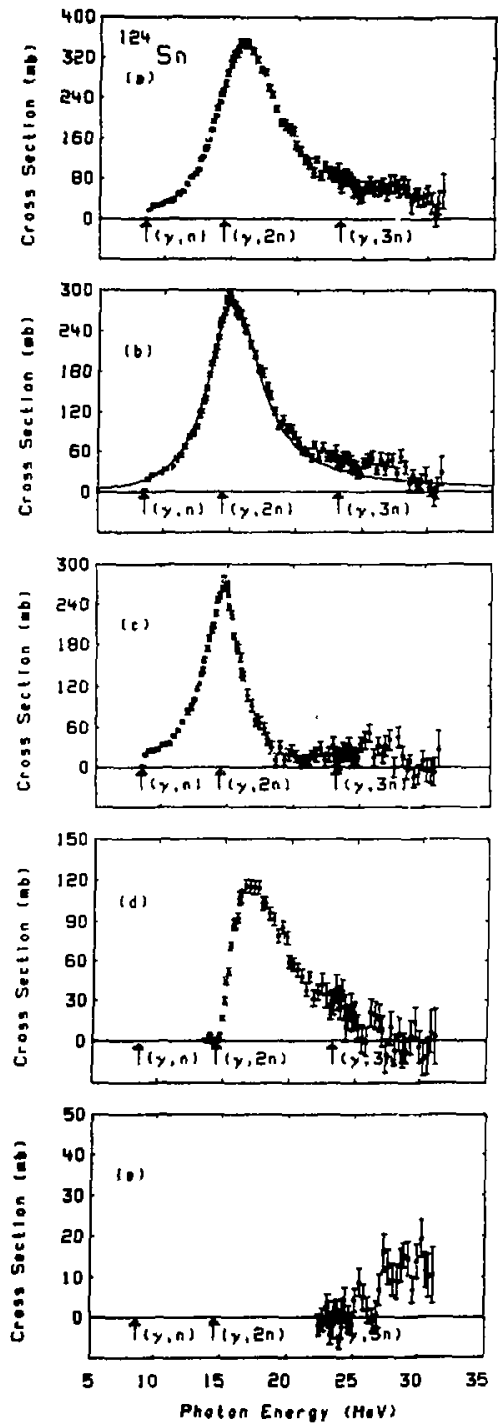

Fig. 99A 


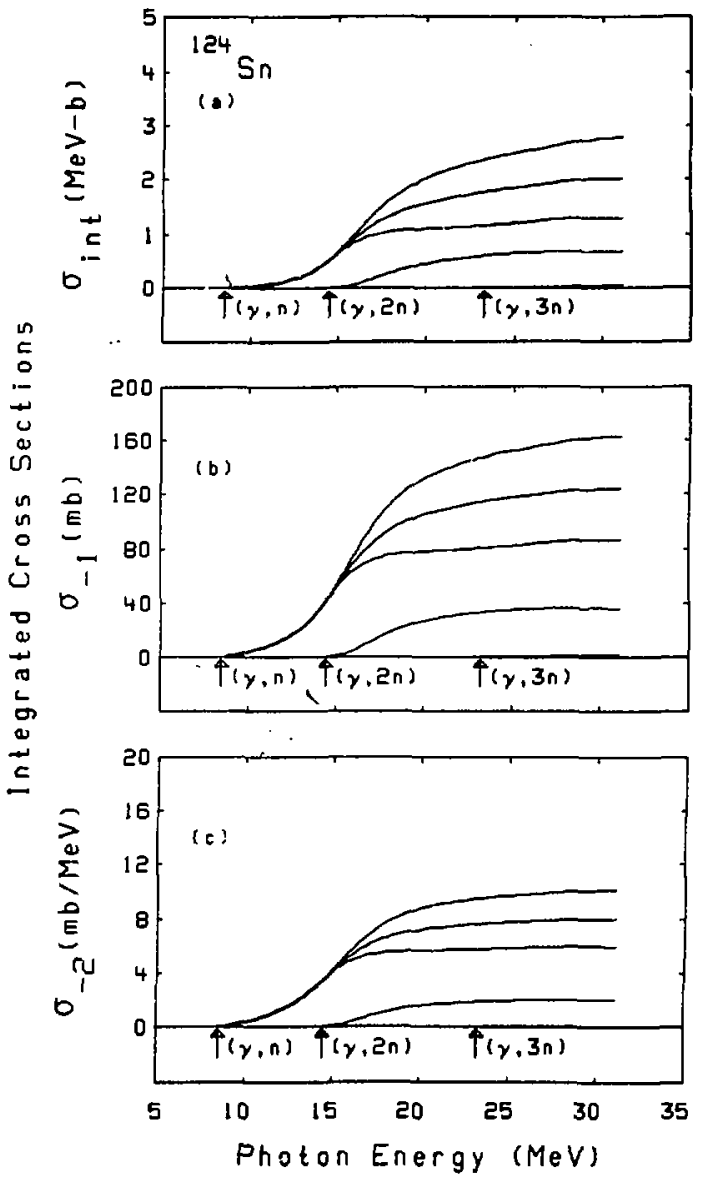

Fig. 99B 

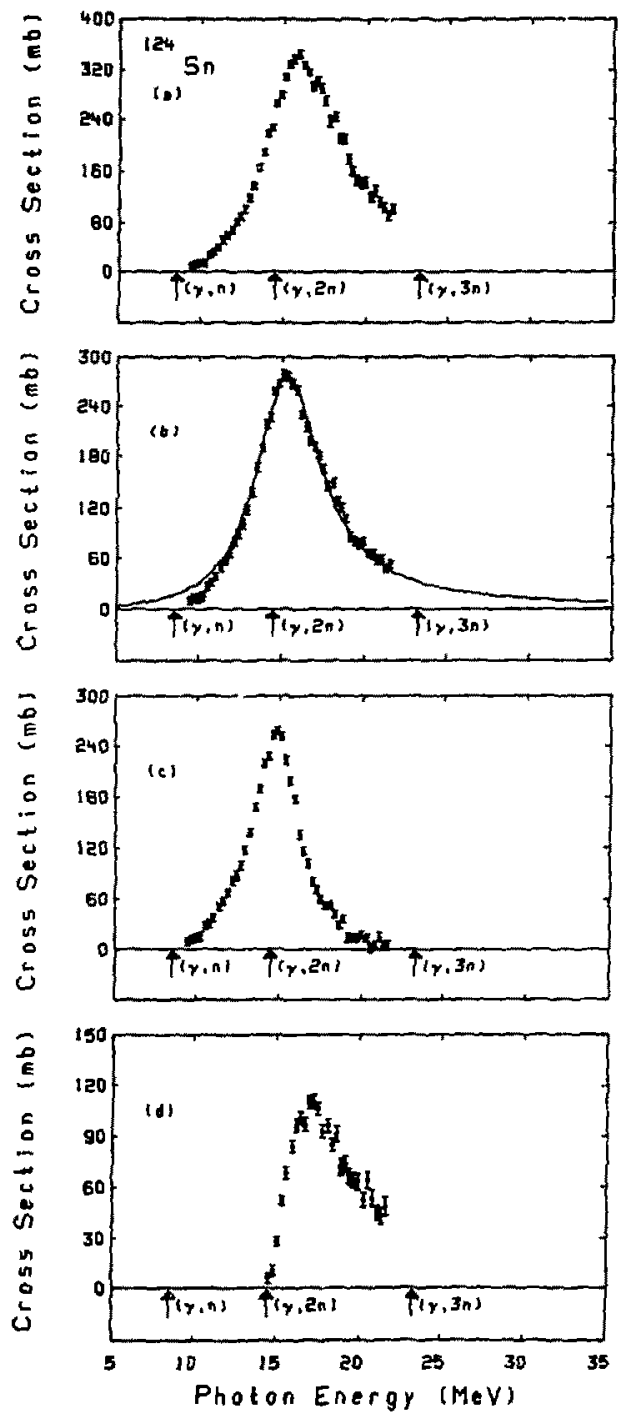

Fig. $100 \mathrm{~A}$ 


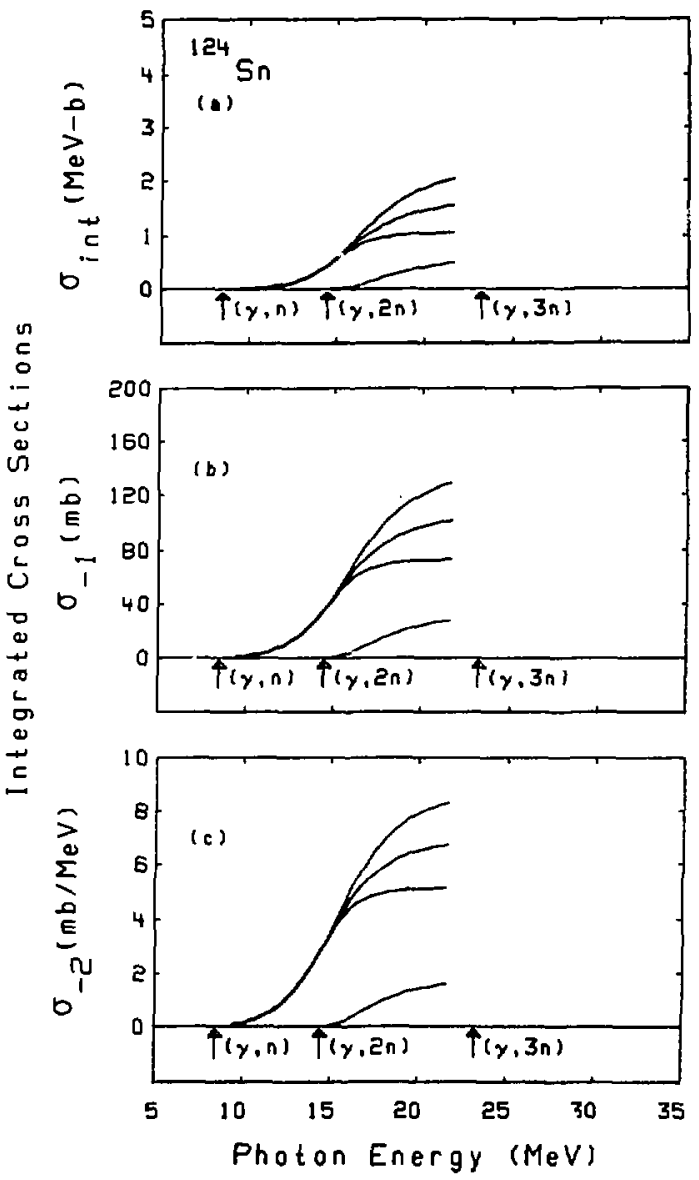

Fig. $100 \mathrm{~B}$ 

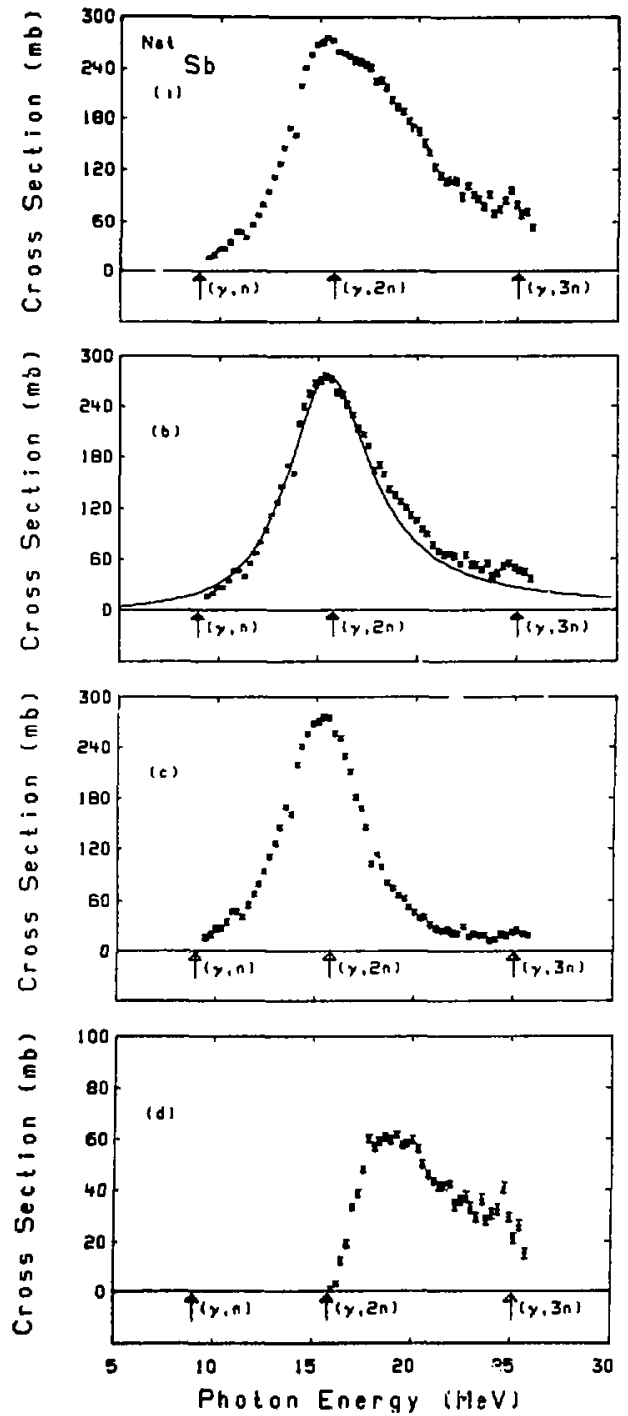

Fig. $101 \mathrm{~A}$ 


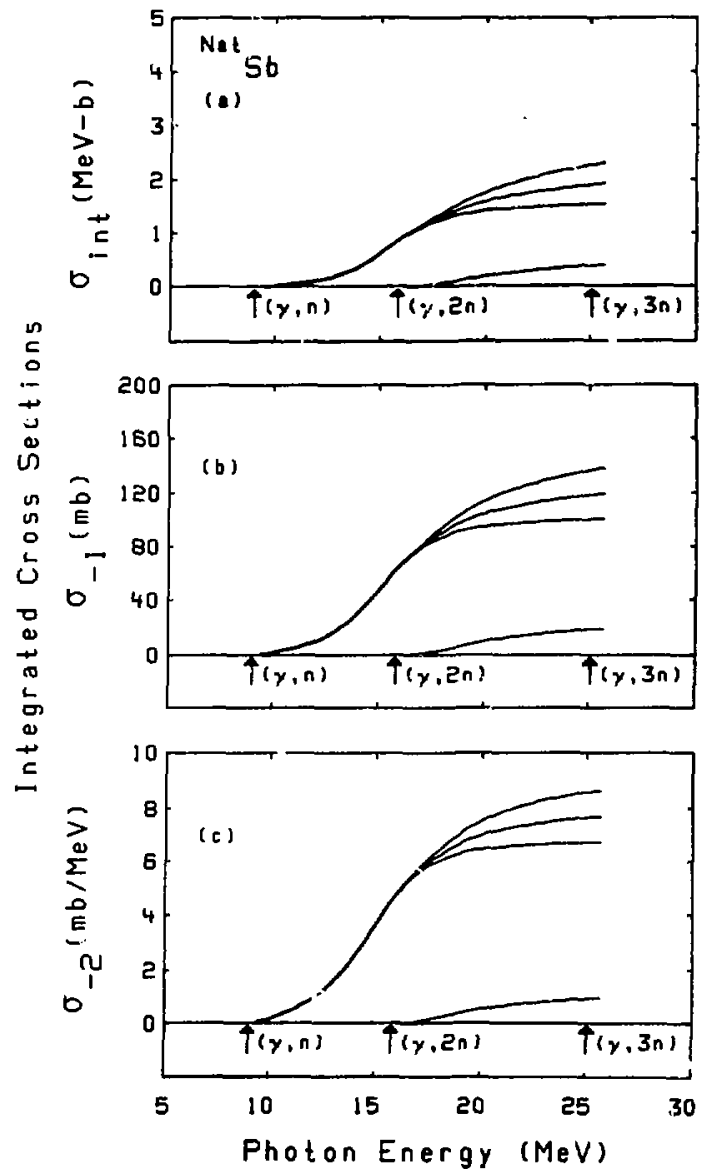

Fig. $101 B$ 

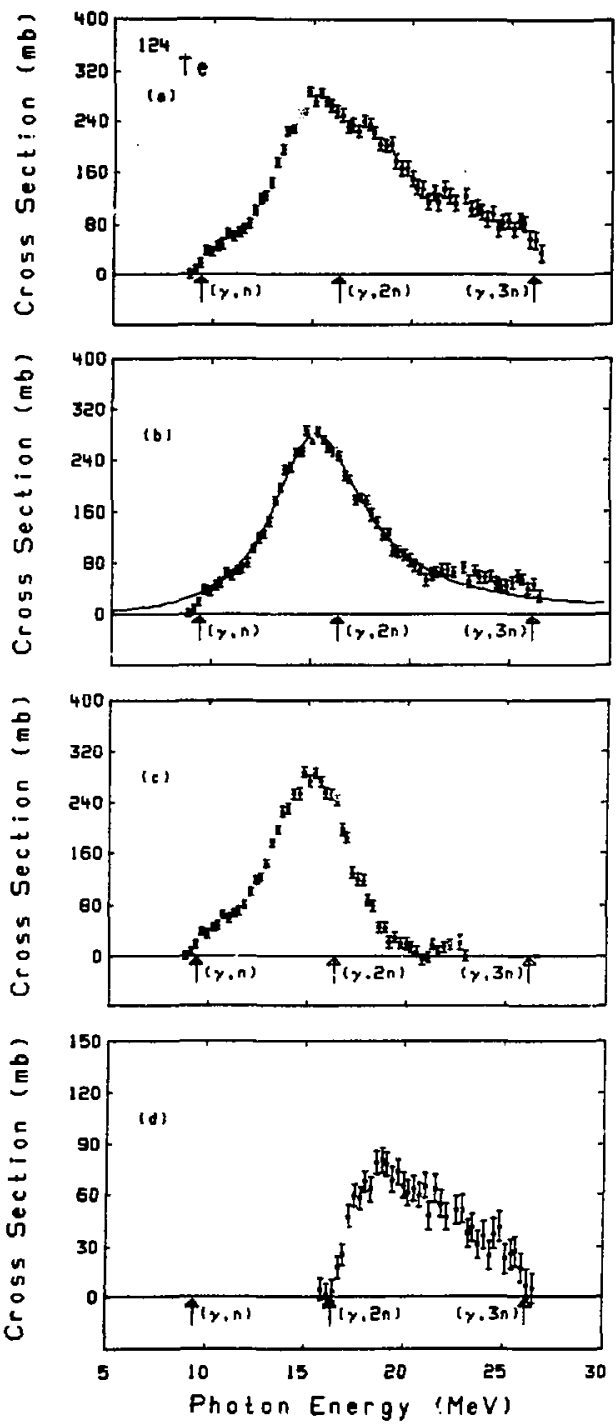

Fig. 102A 


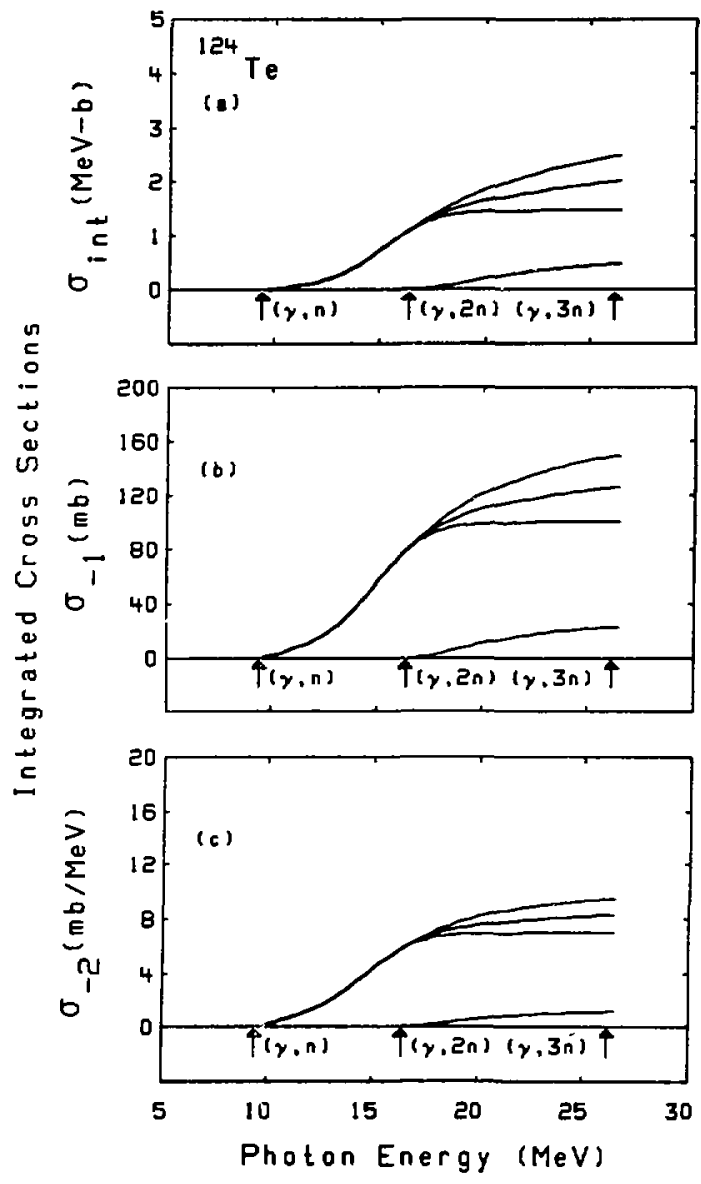

Fig. 102B 

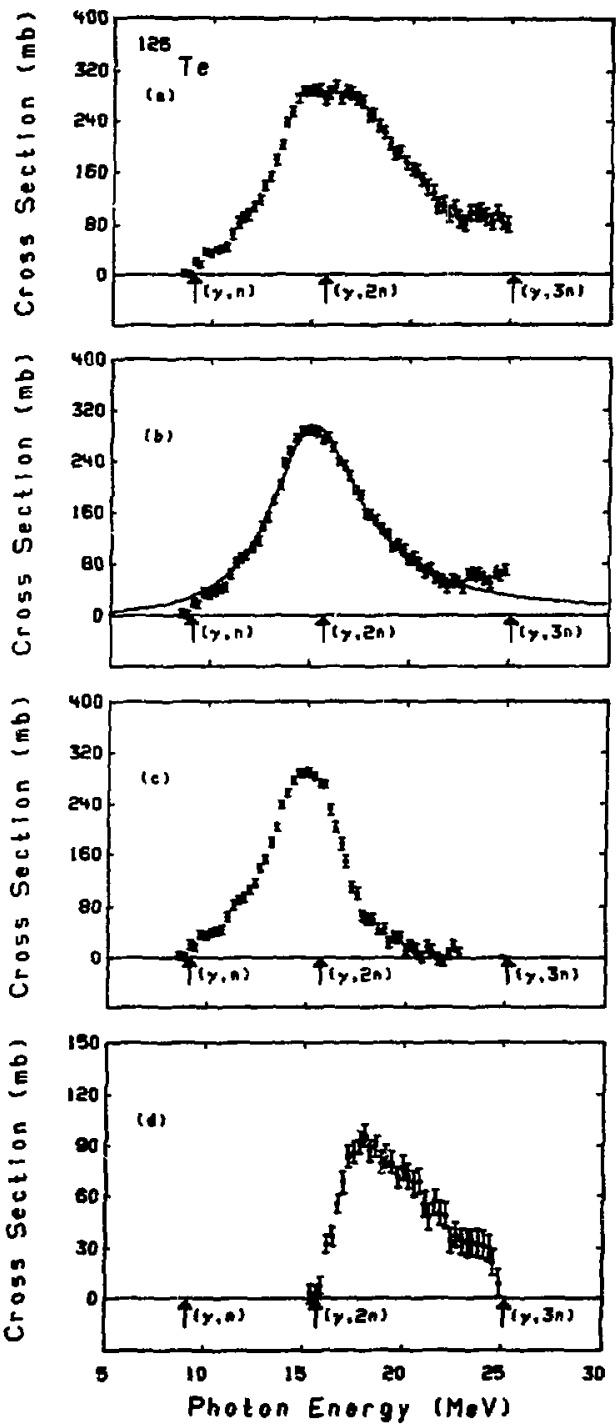

F1g. 103A 


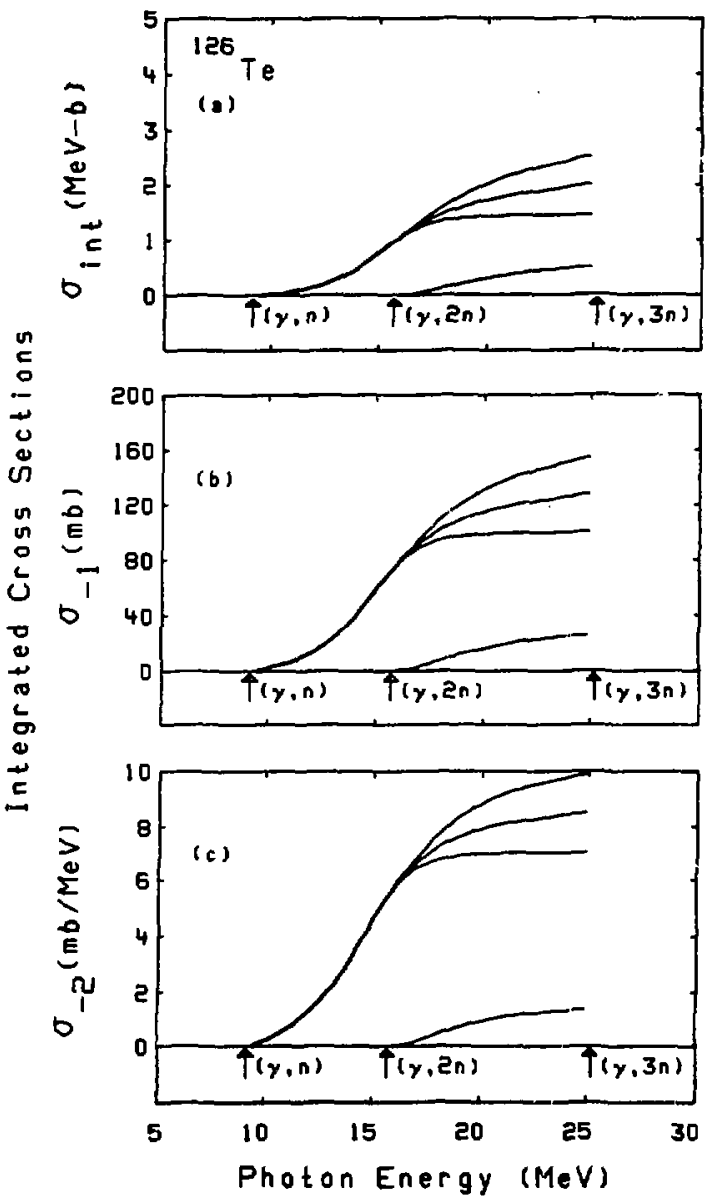

Fig. 103B 


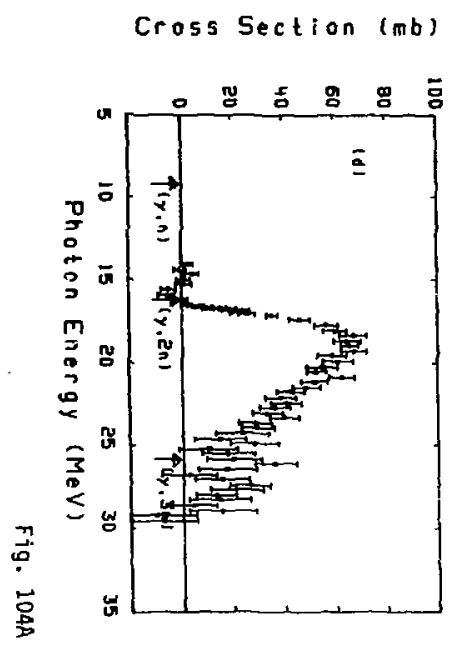

Cross Section (mb)

Cross Section (mb)

Cross Section (mb)
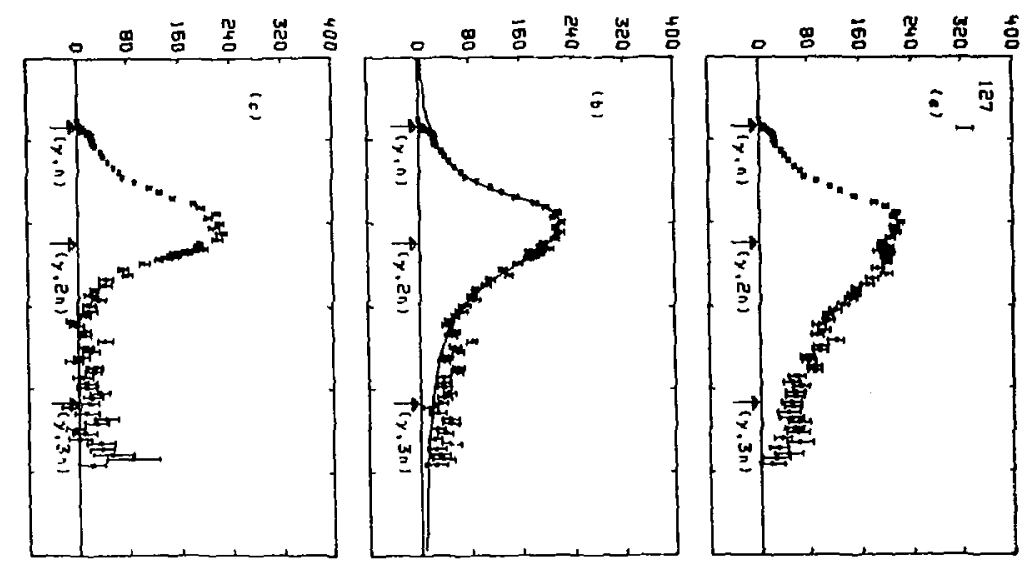


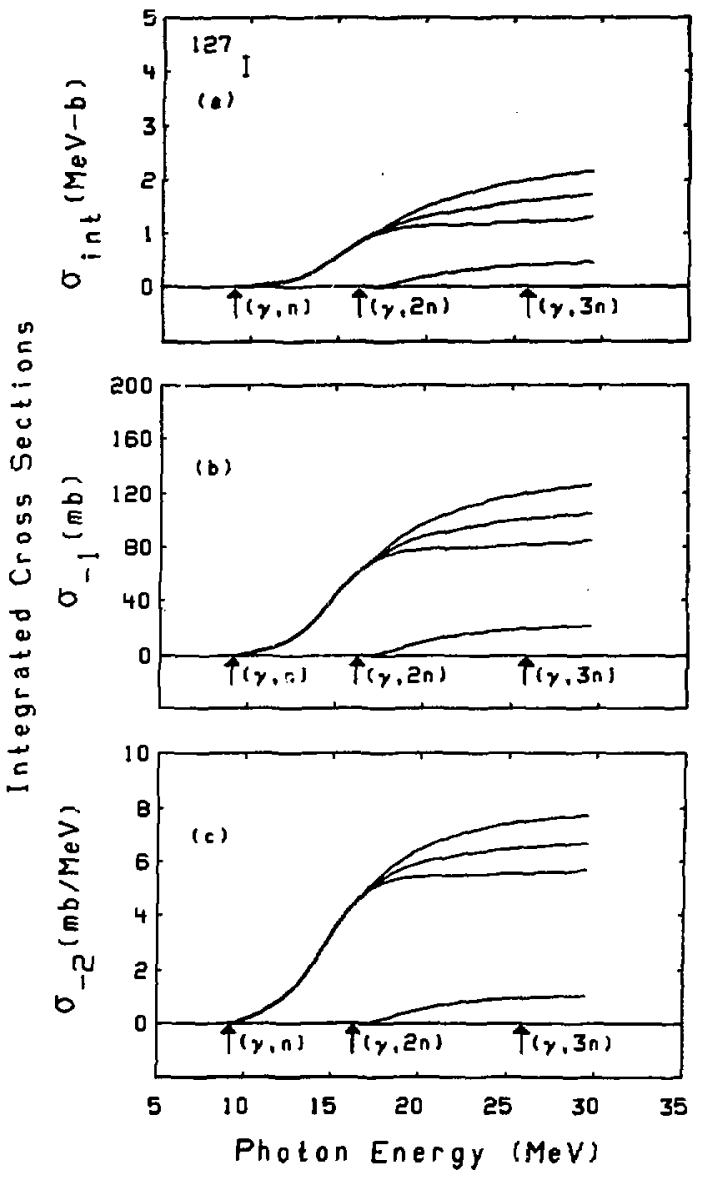

Fig. $104 B$ 

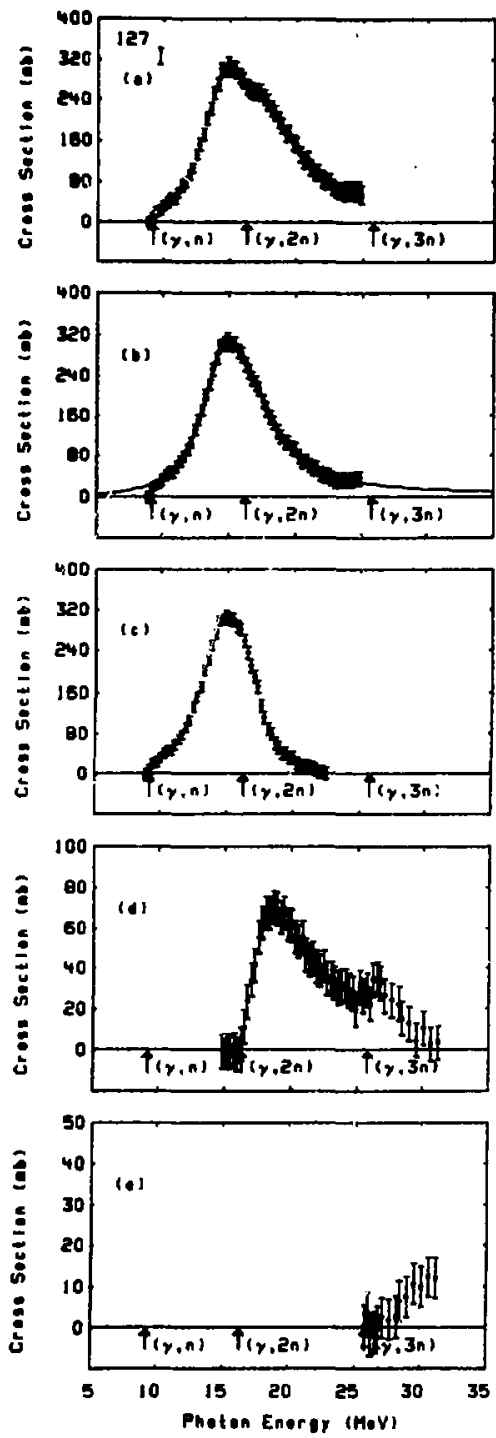

Fig. $105 \mathrm{~A}$ 


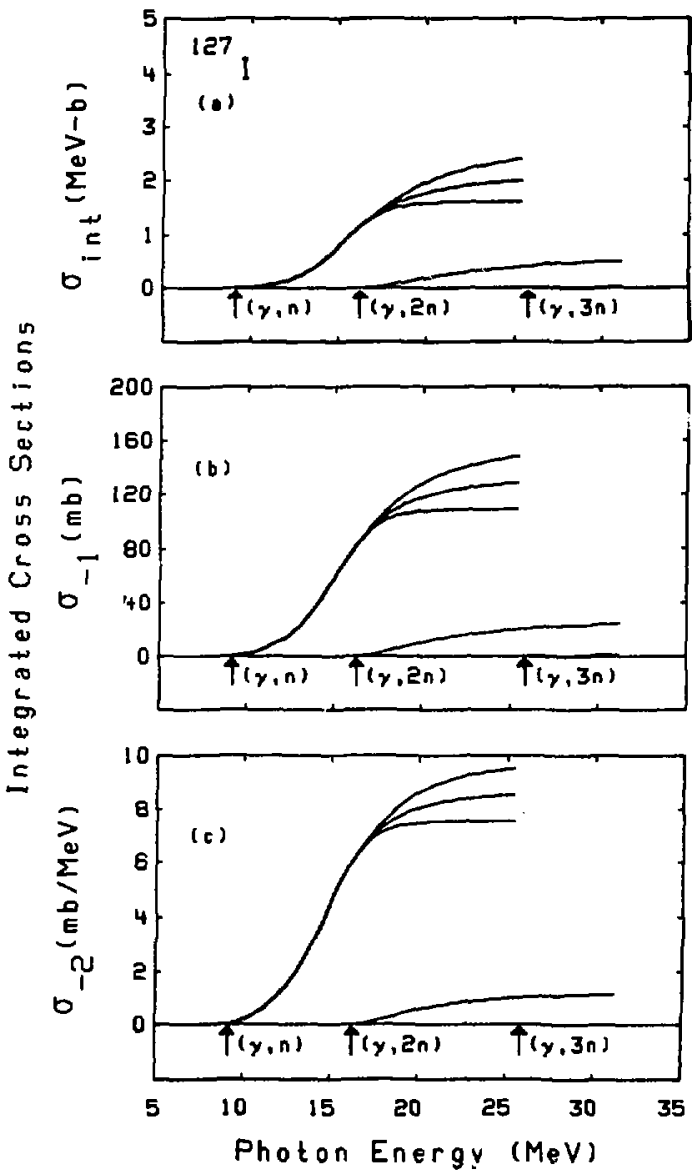

Fig. 105B 

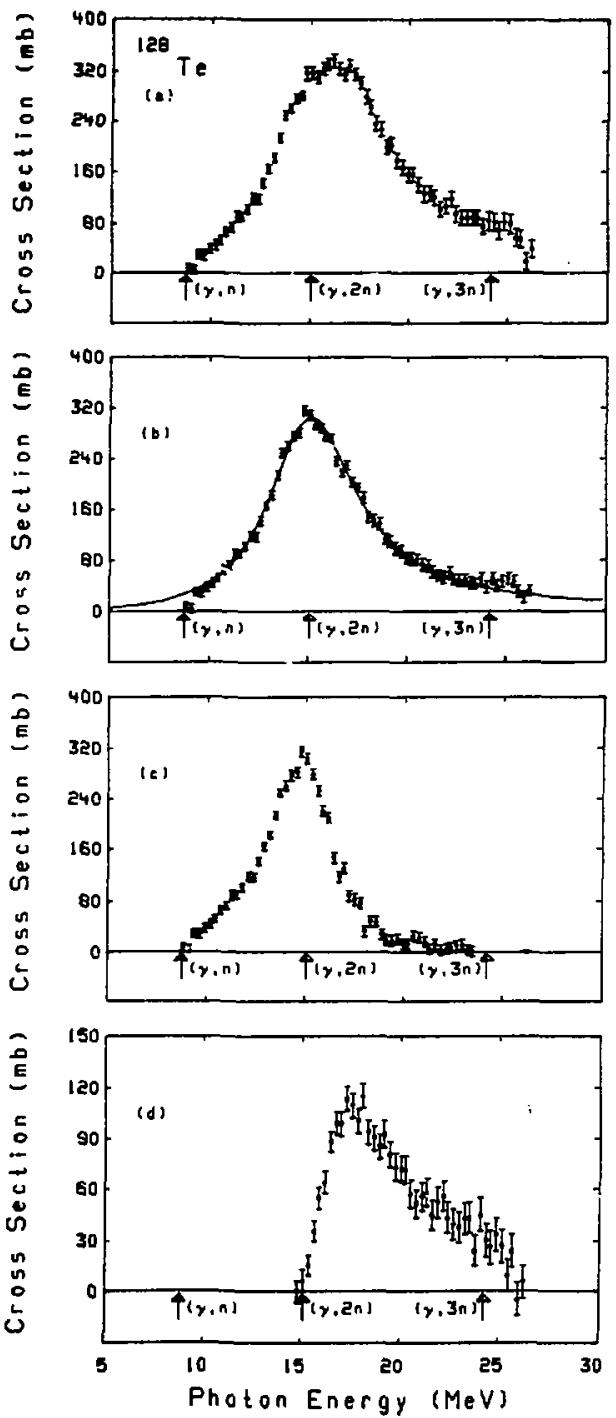

Fig. $106 \mathrm{~A}$ 

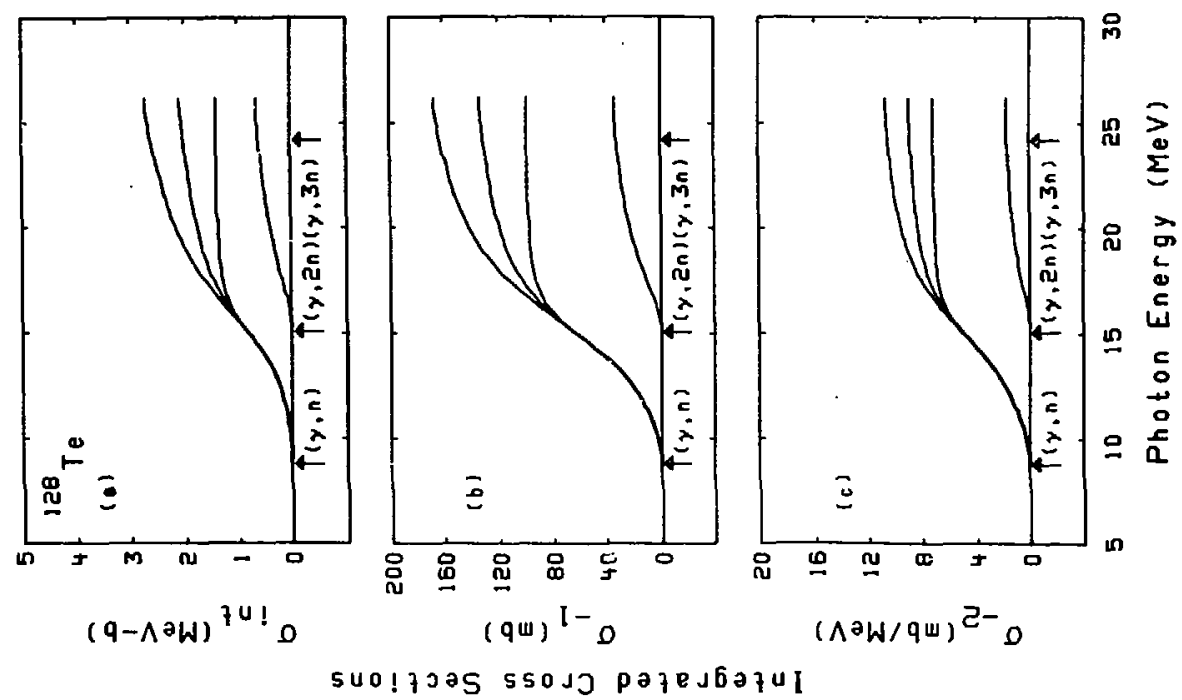

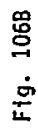

suo! joas ssodj pazejoazuI 

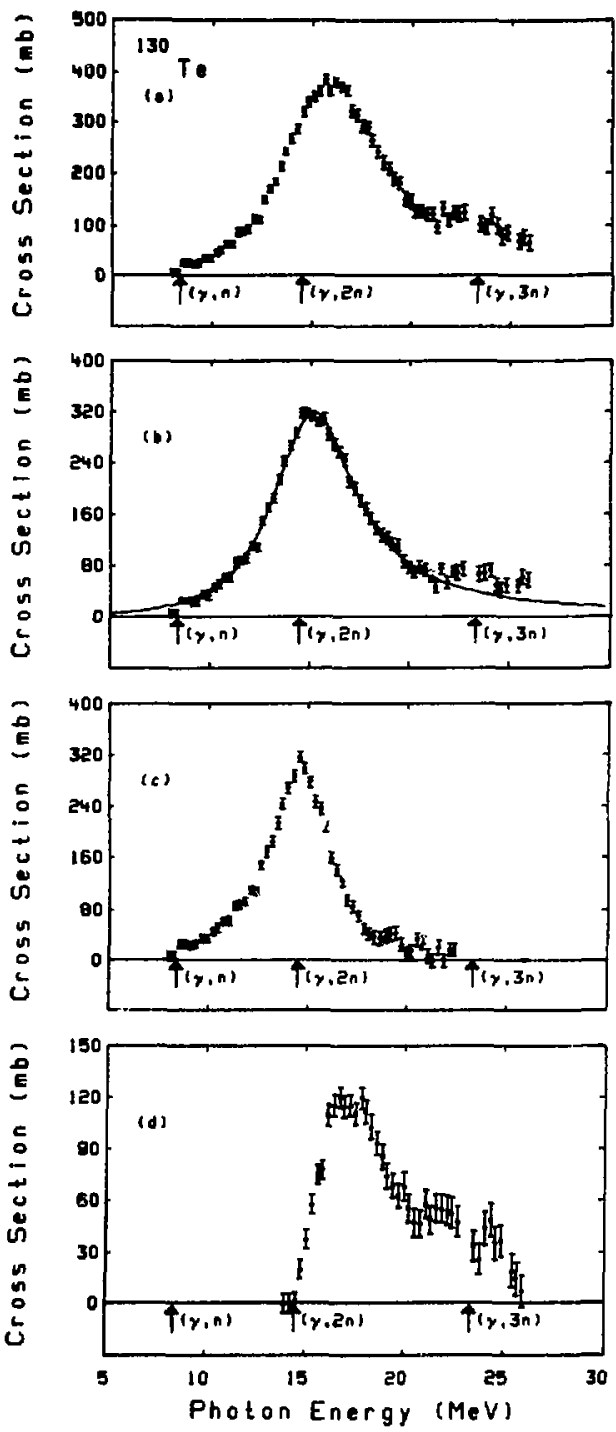

Fig. 107A 


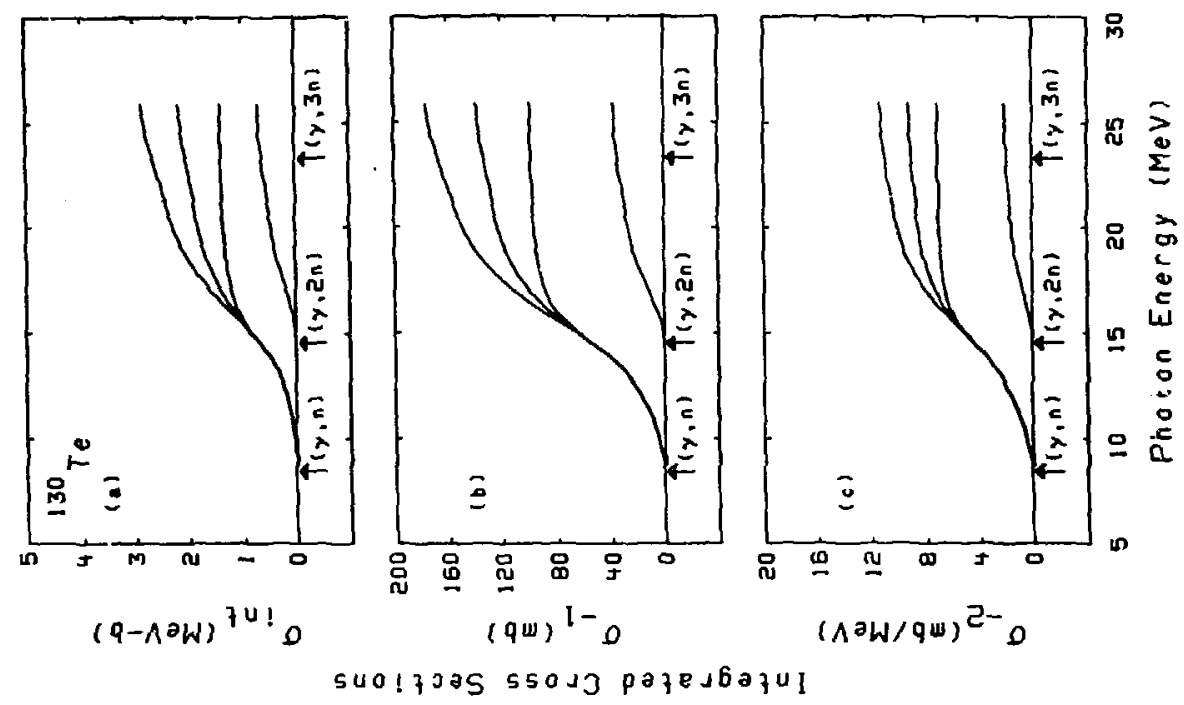

号
它
市 

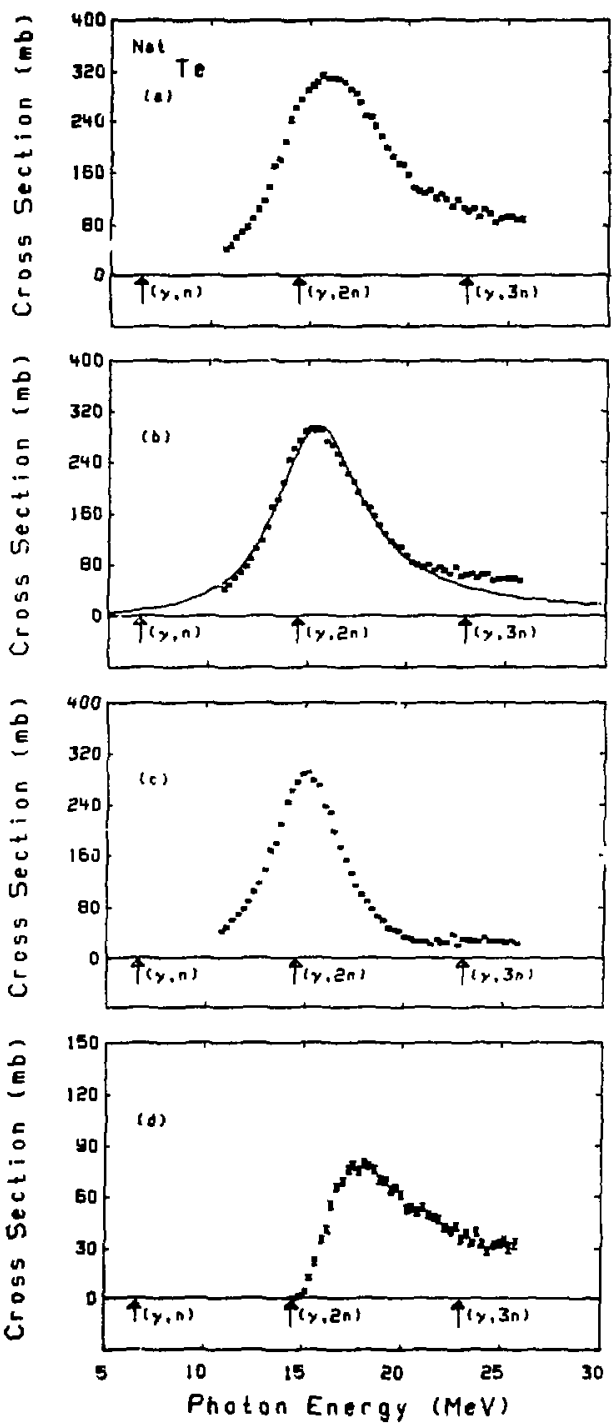

Fig. $108 R$ 


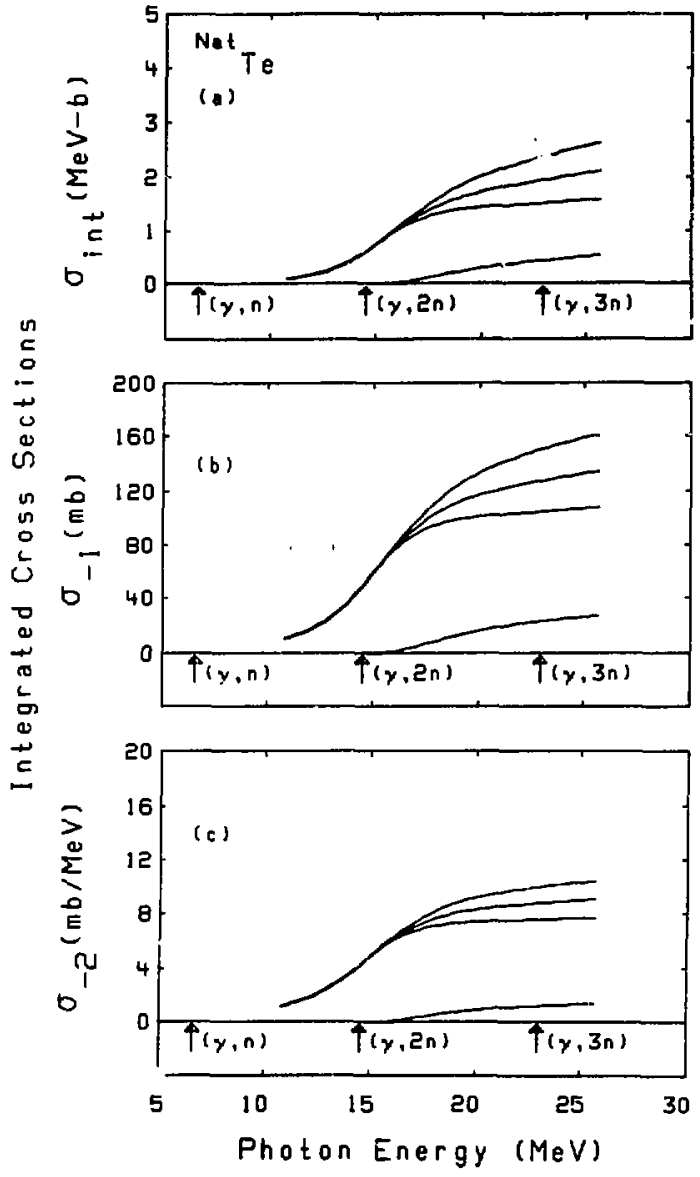

Fig. 108B 

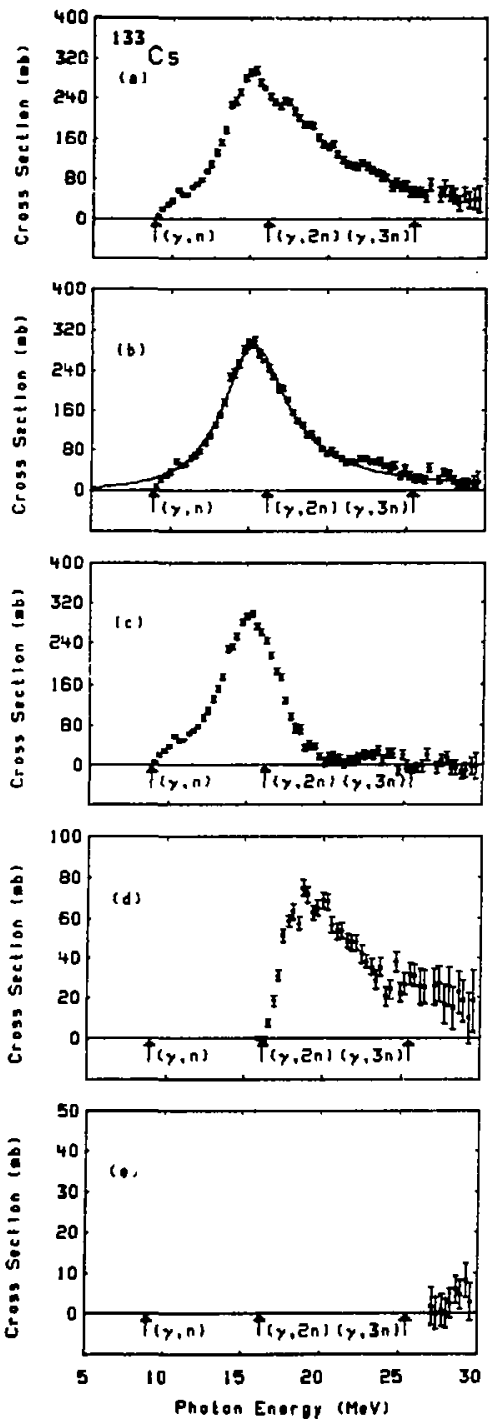

Fig. 109A 


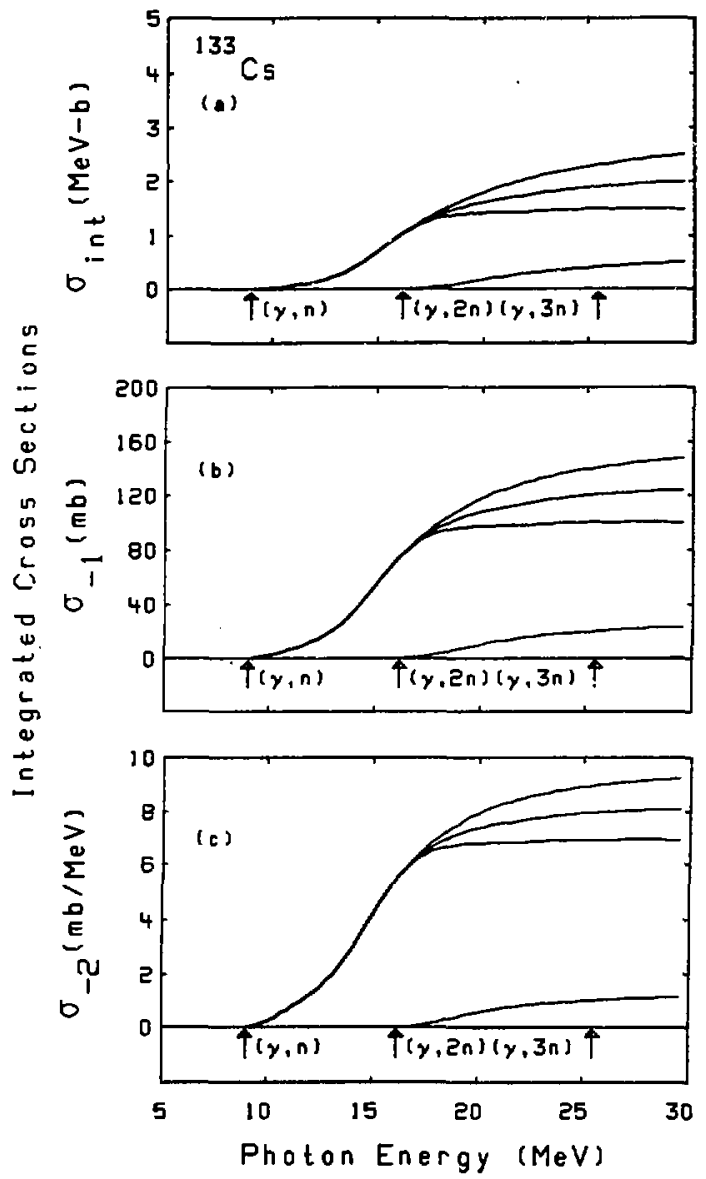

Fig. $209 \mathrm{~B}$ 

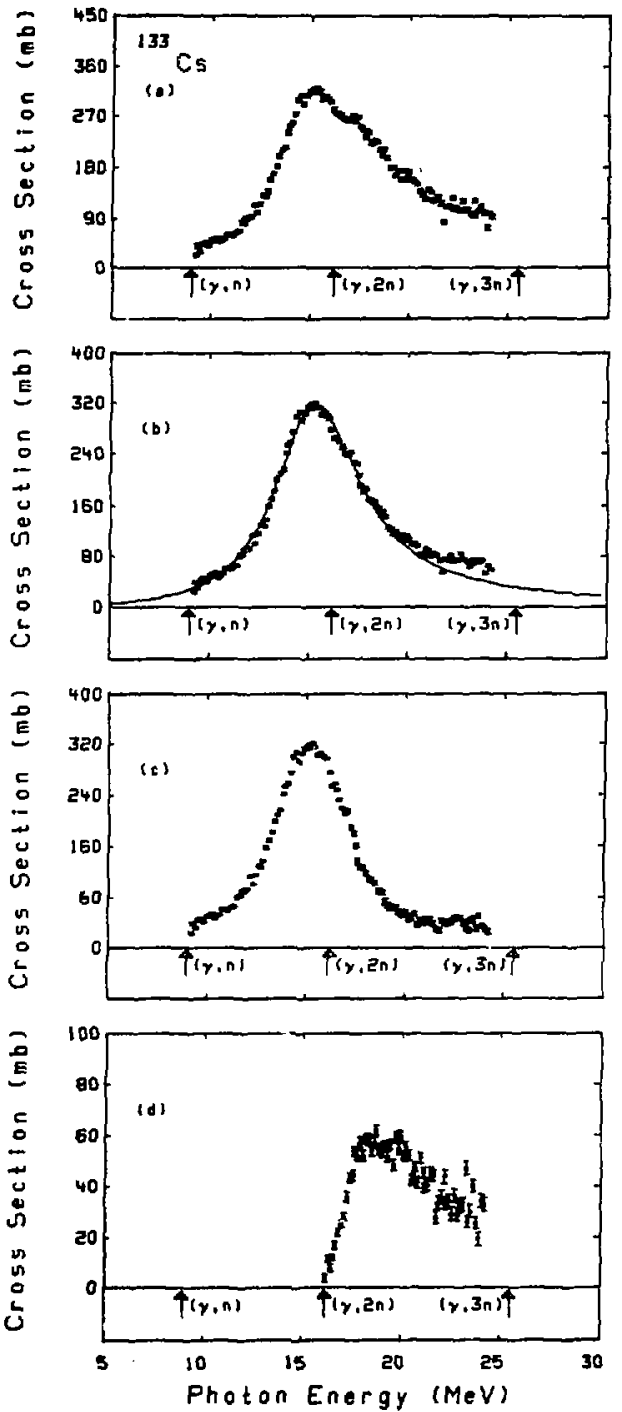

Fig. 110A 


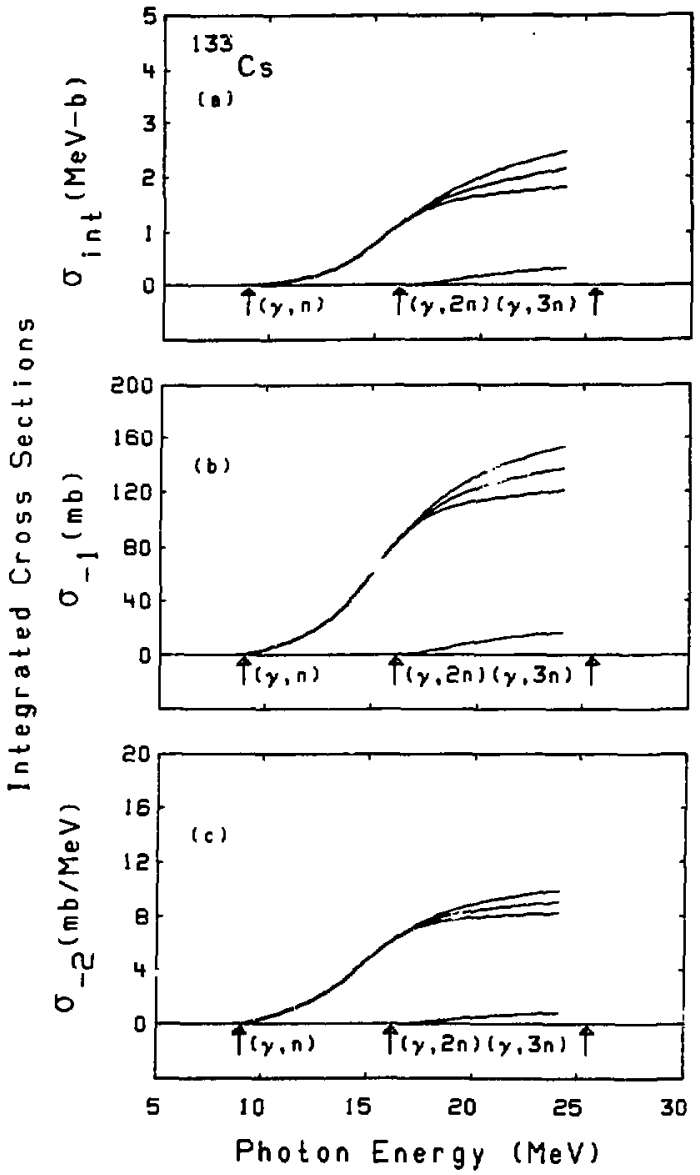

Fig. $110 \mathrm{~B}$ 

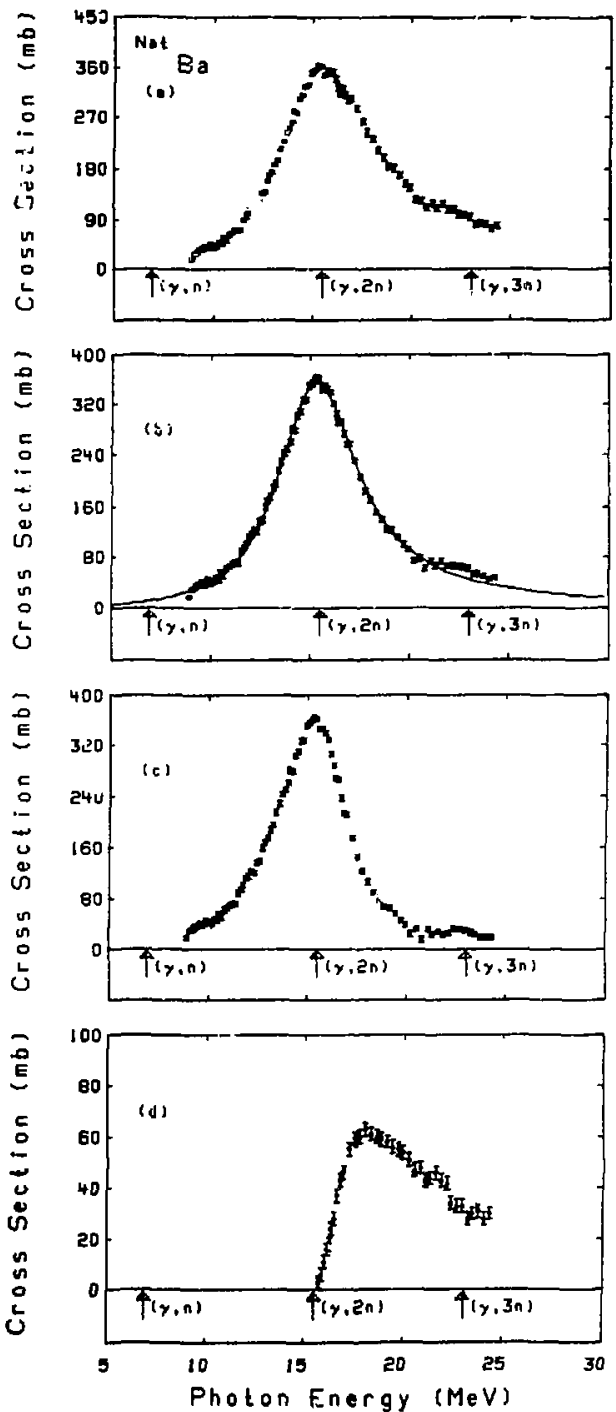

Fig. 111A 


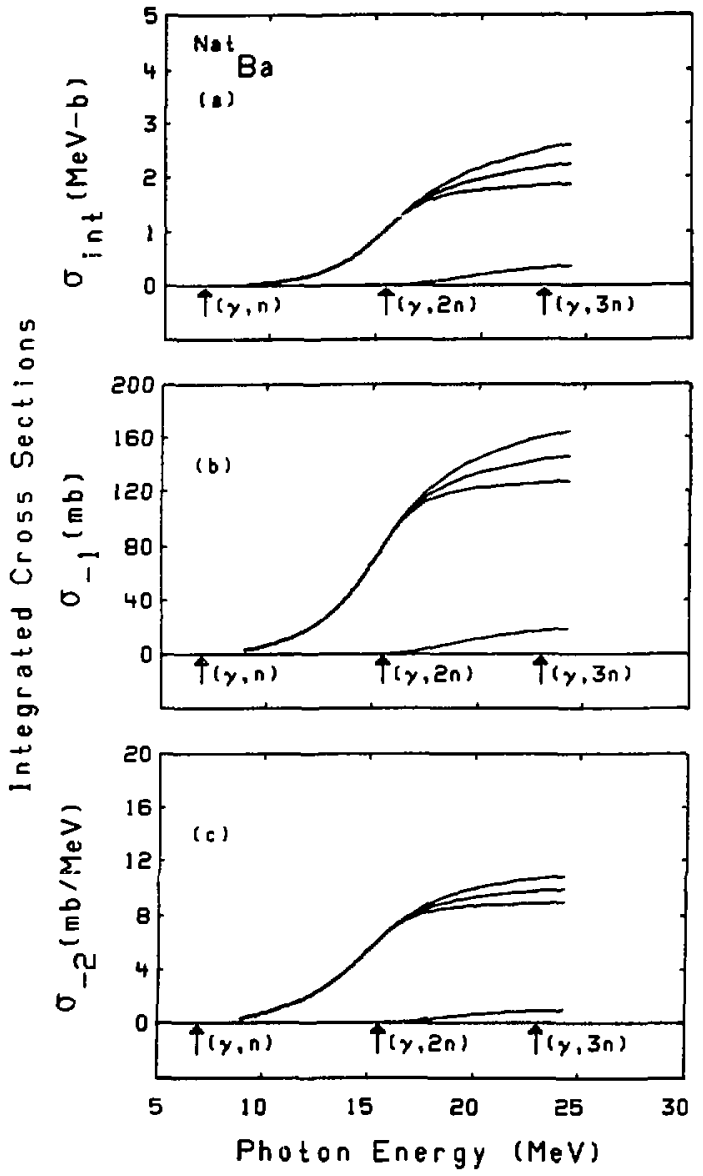

Fig. 111B 

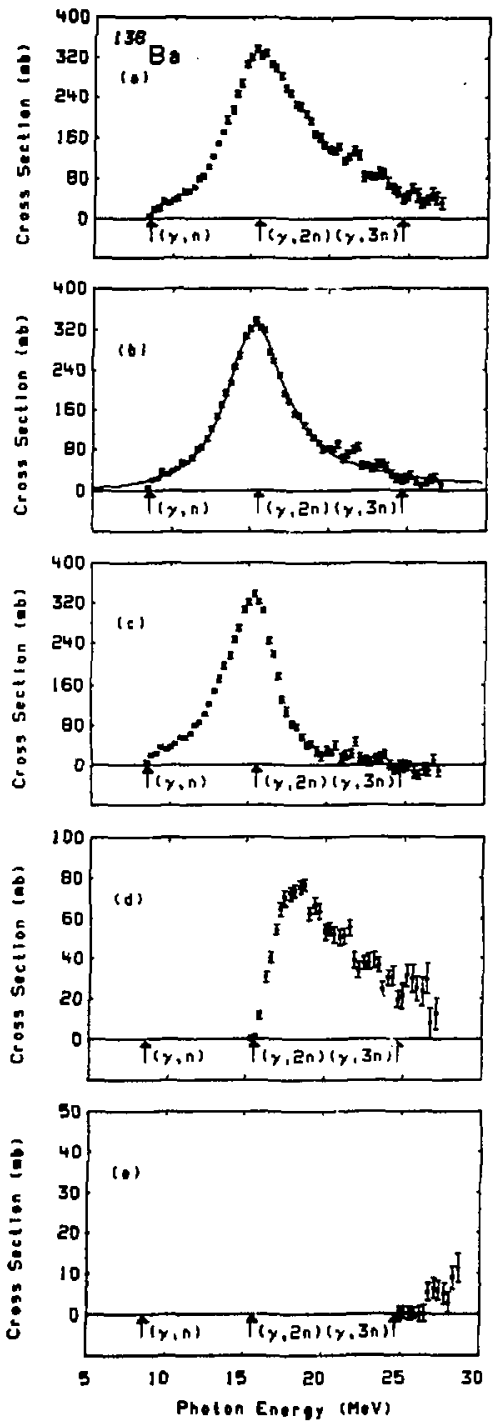

Fig. $112 A$ 


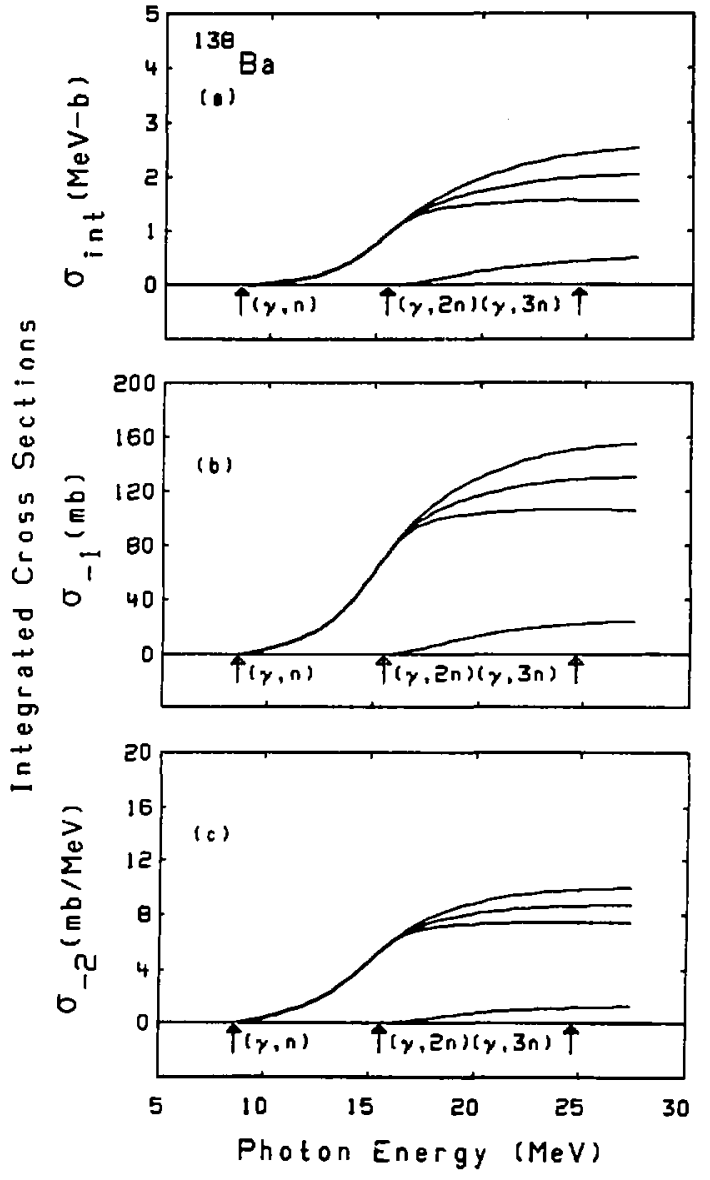

Fig. 112B 

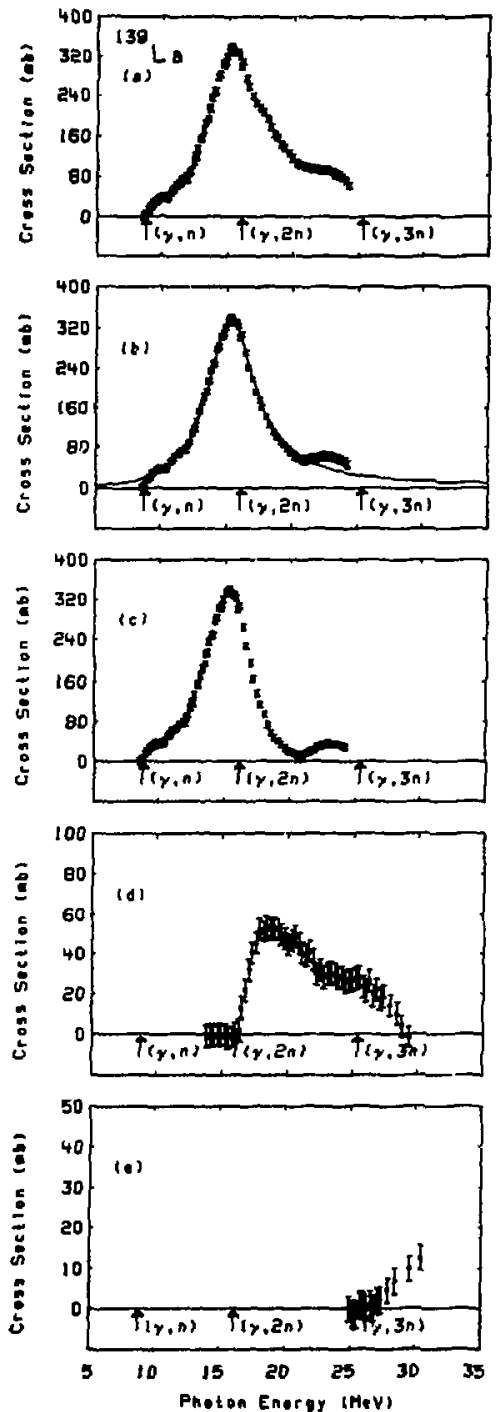

Ffg. $113 \mathrm{~A}$ 


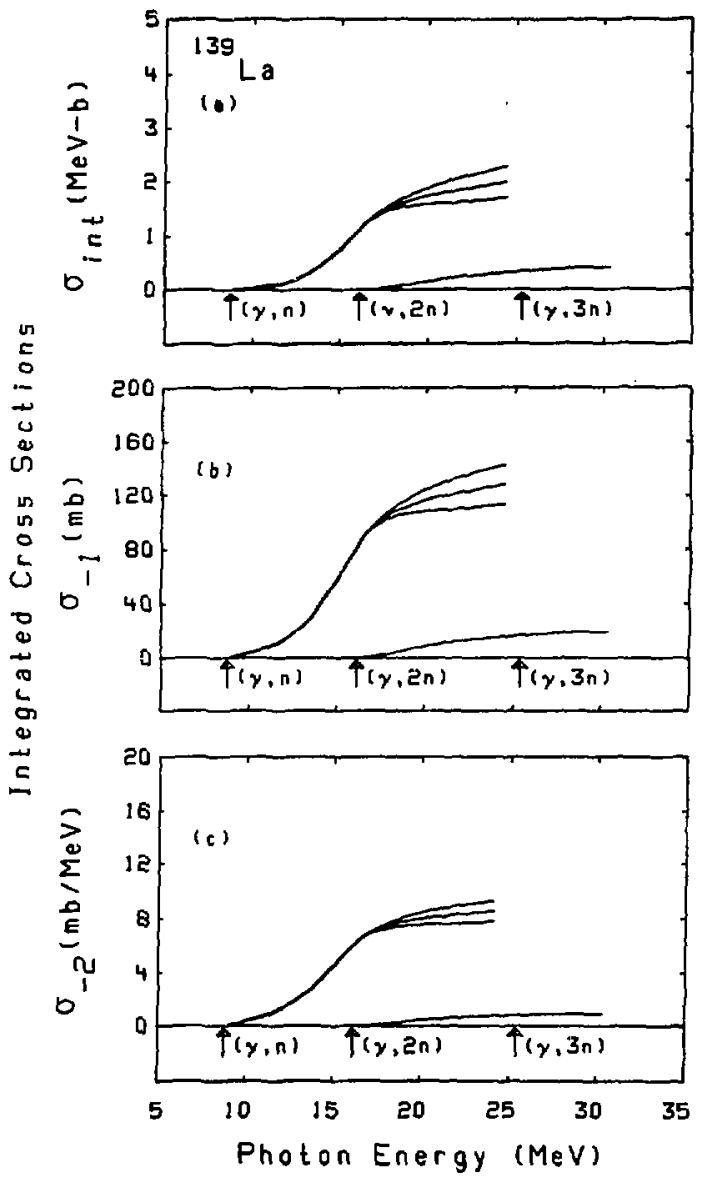

F1g. 113B 

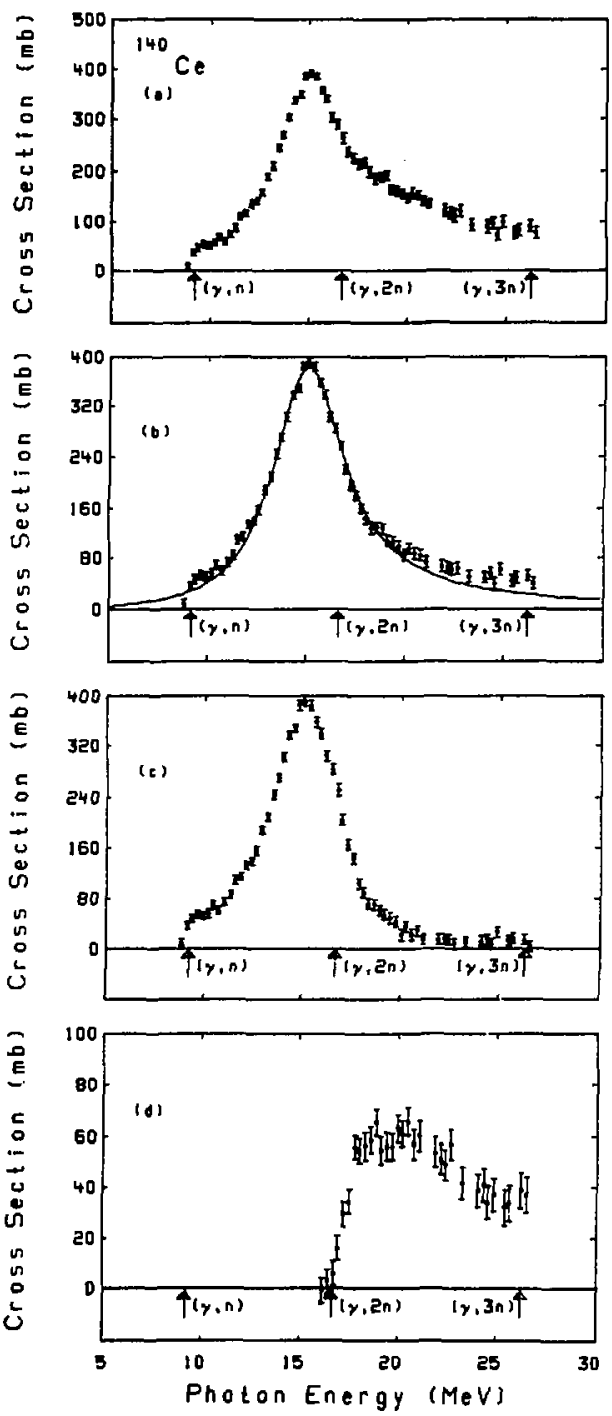

Fig. 114A 


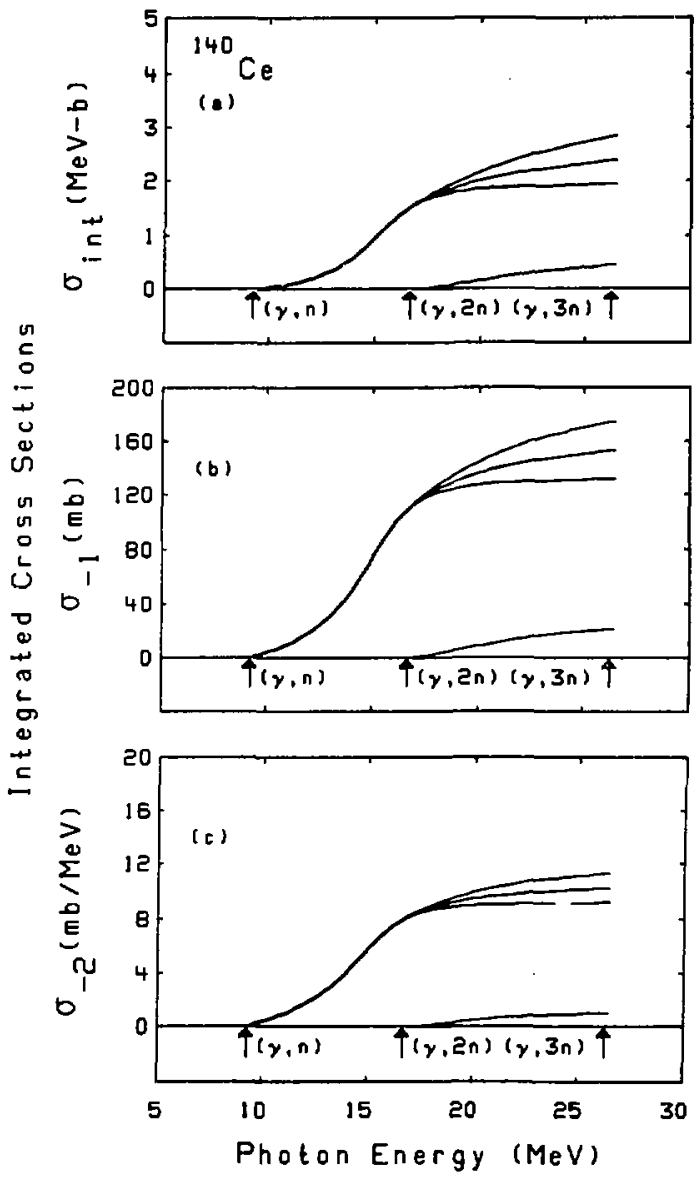

F1g. 1148 

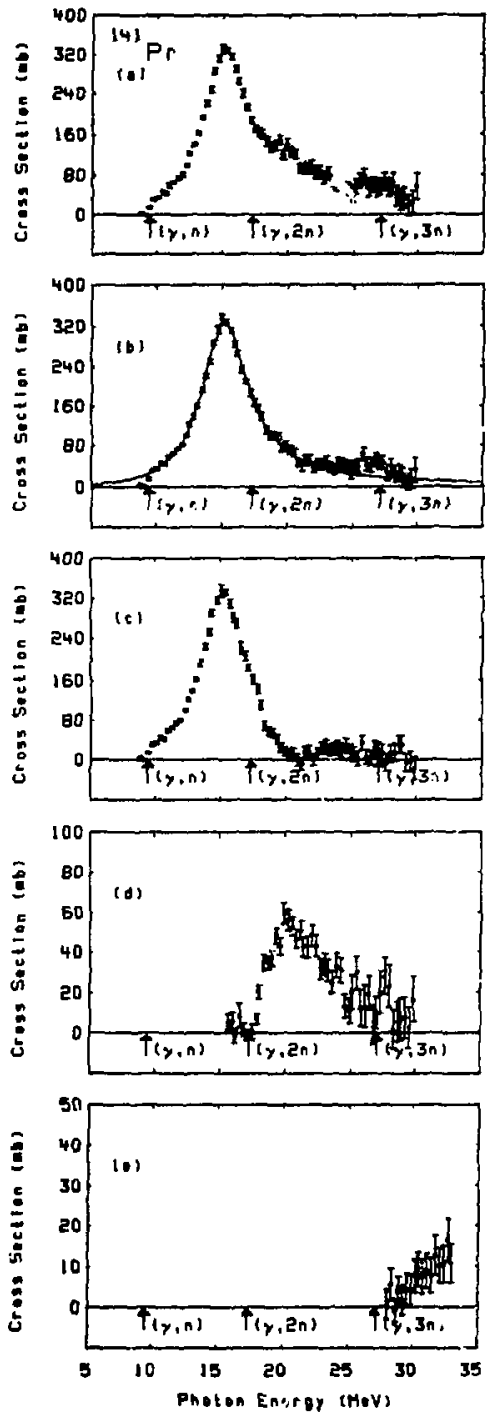

Fig. 115A 


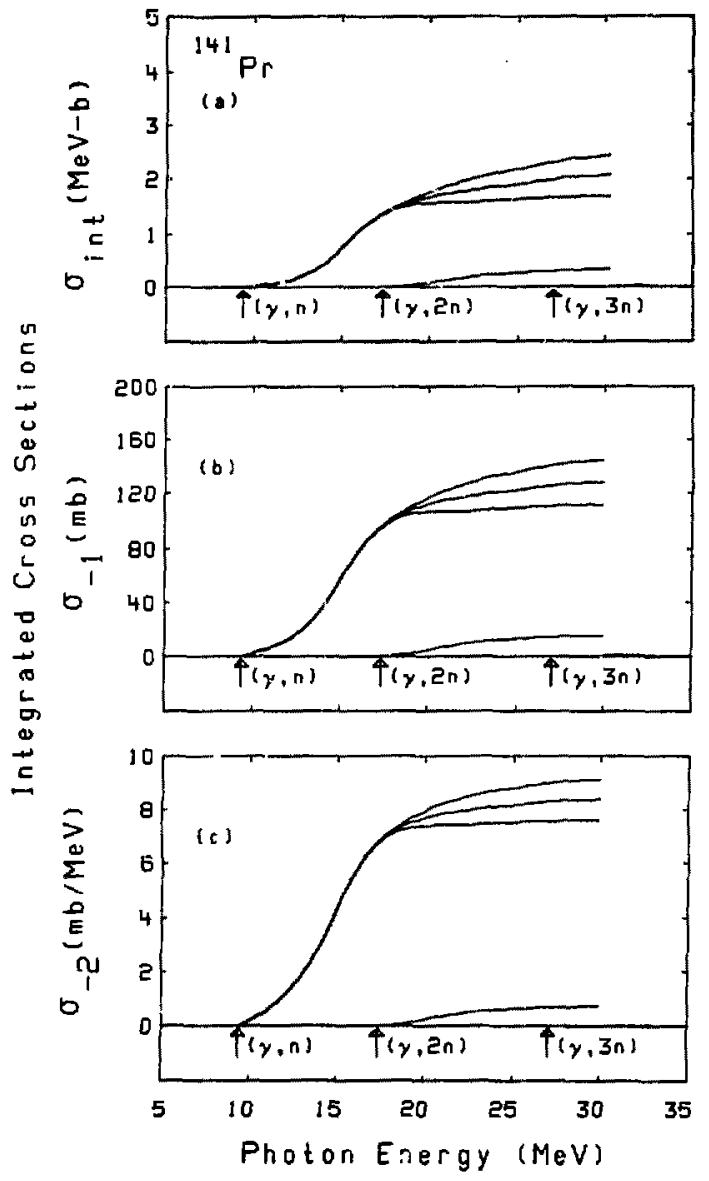

Fig. 115B 


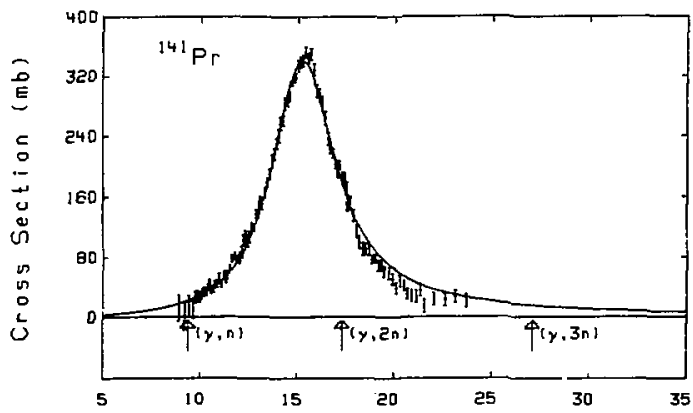

Photon Energy (MeV)

Fig. $116 \mathrm{~A}$ 


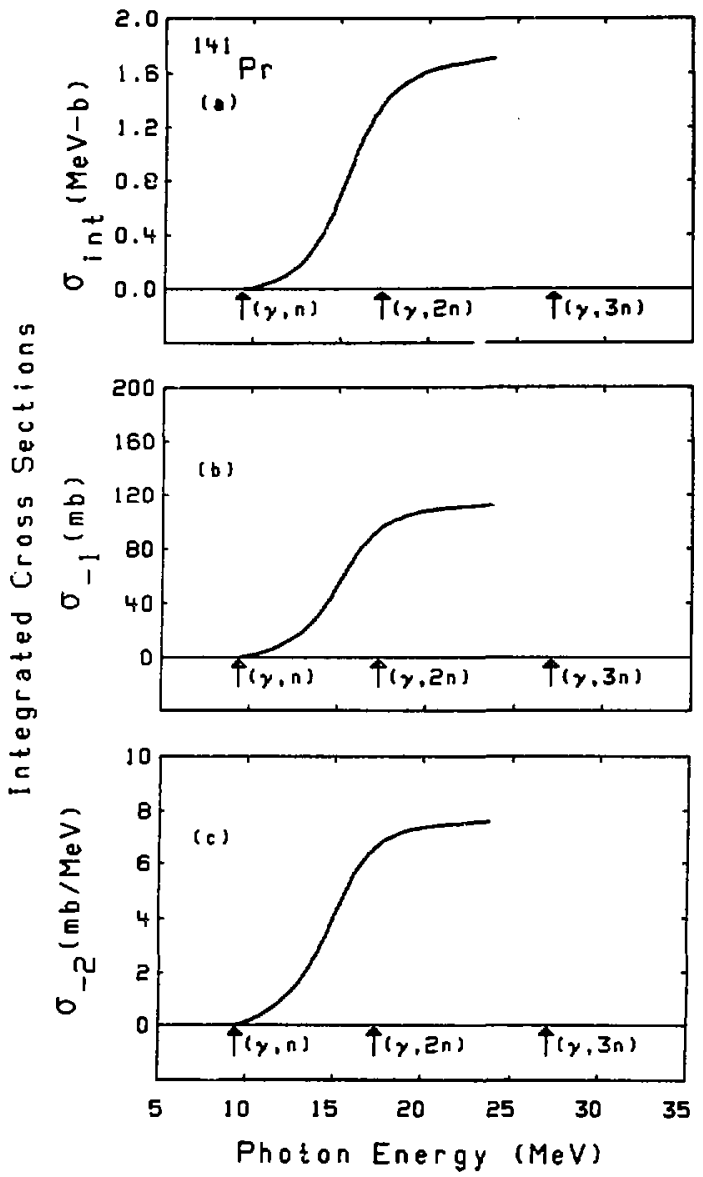

Fig. 116B 


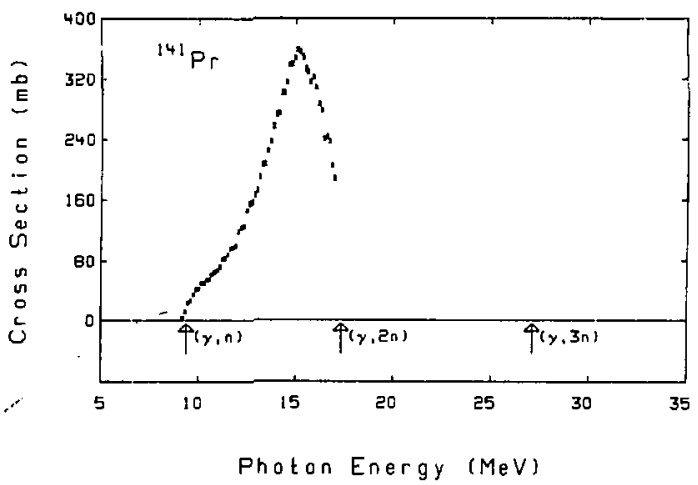

Fig. 117A 


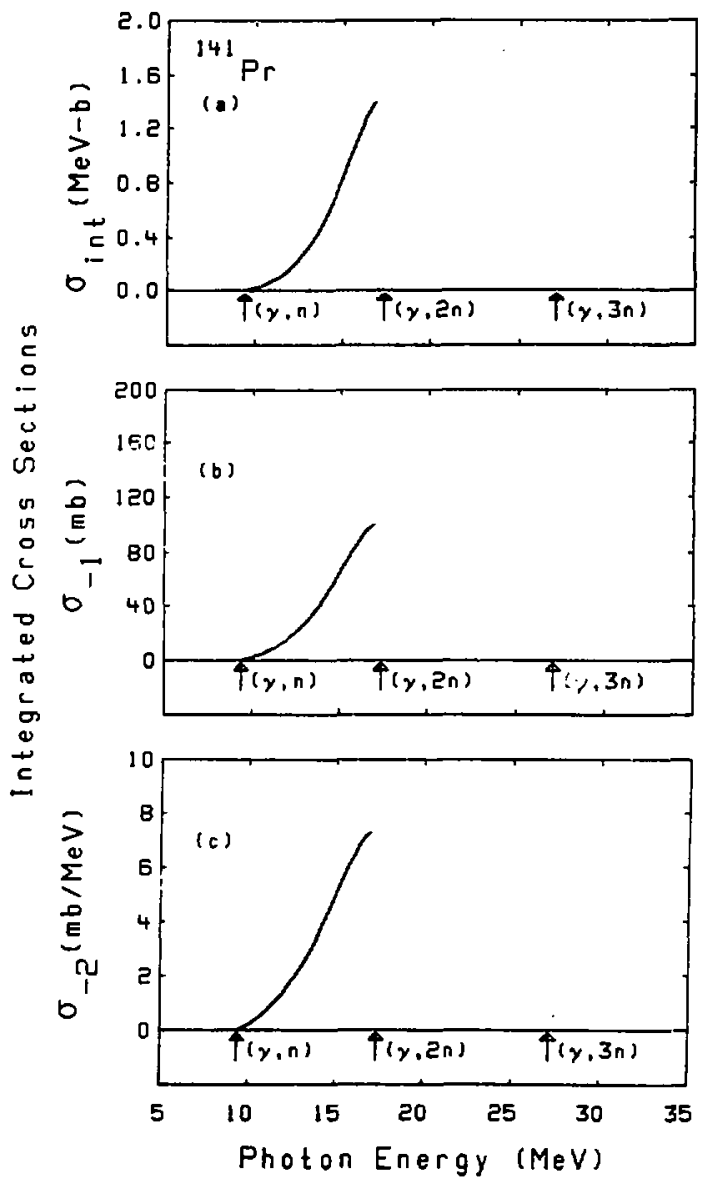

Fig. 1178 


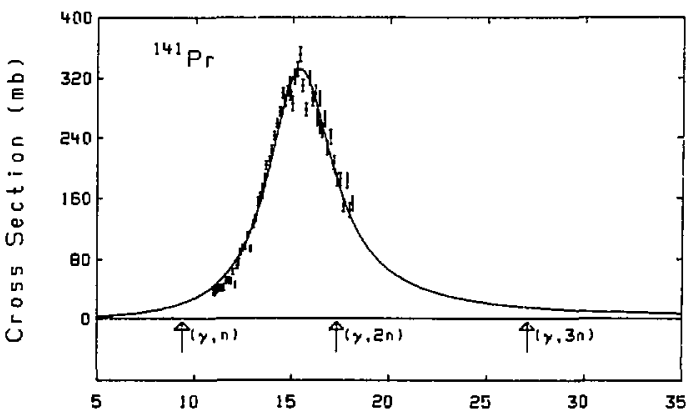

Photon Energy (llev) 


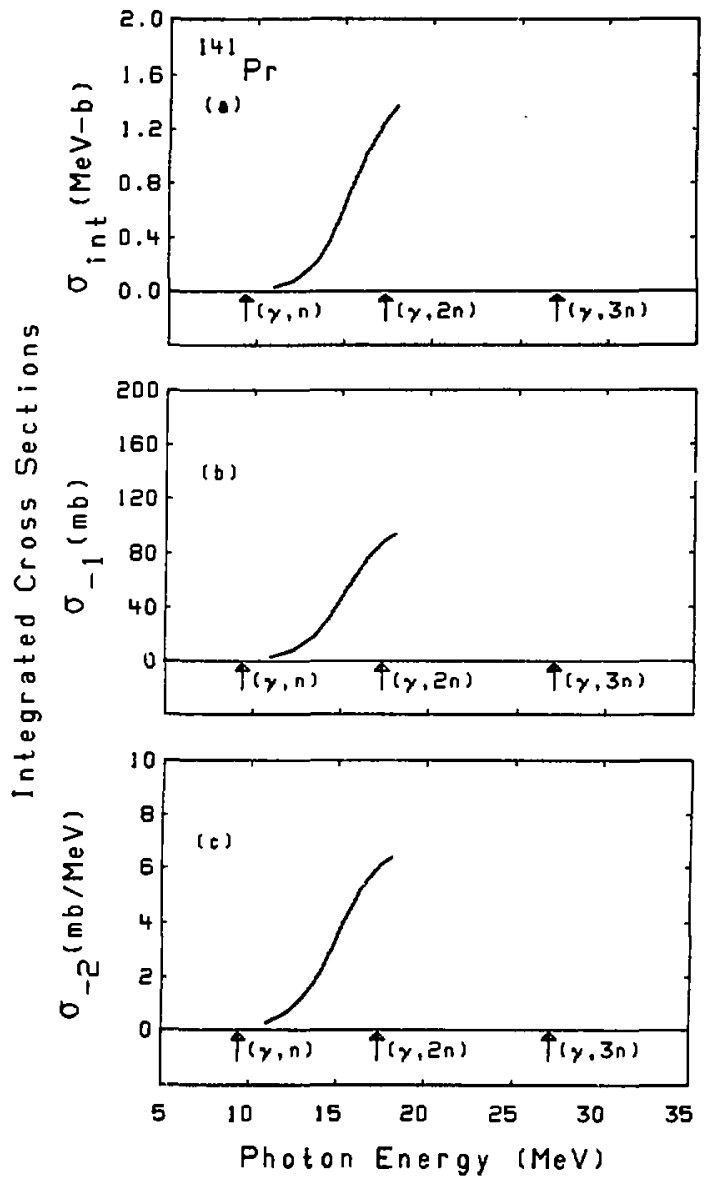

Fig. 118B 


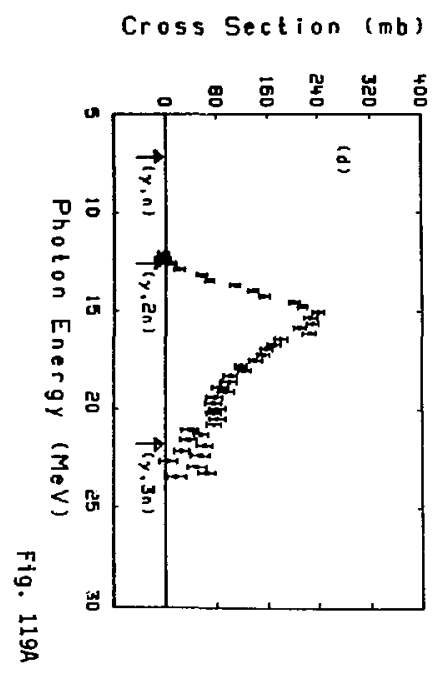

Cross Section (mb) Cross Section (mb) Cross Section (mb)
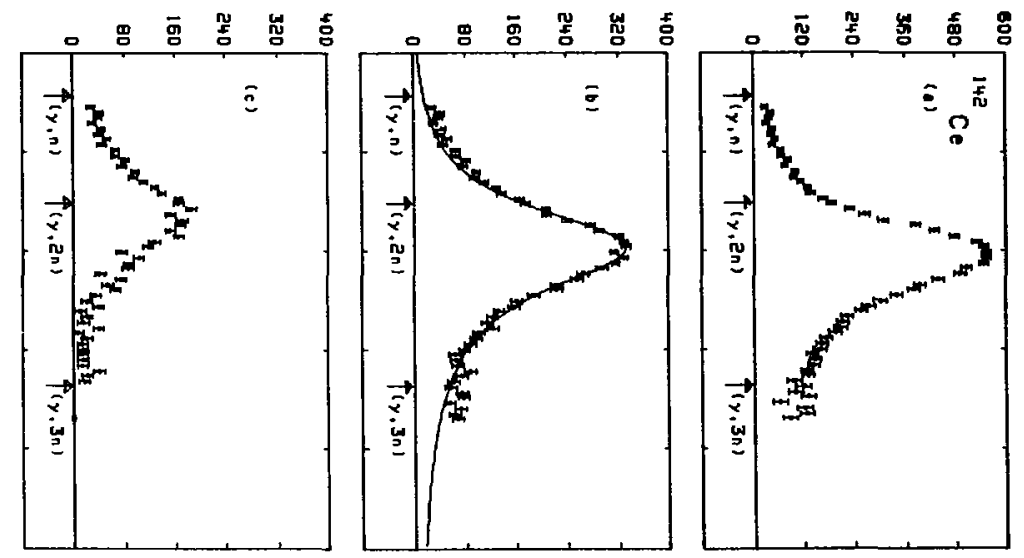


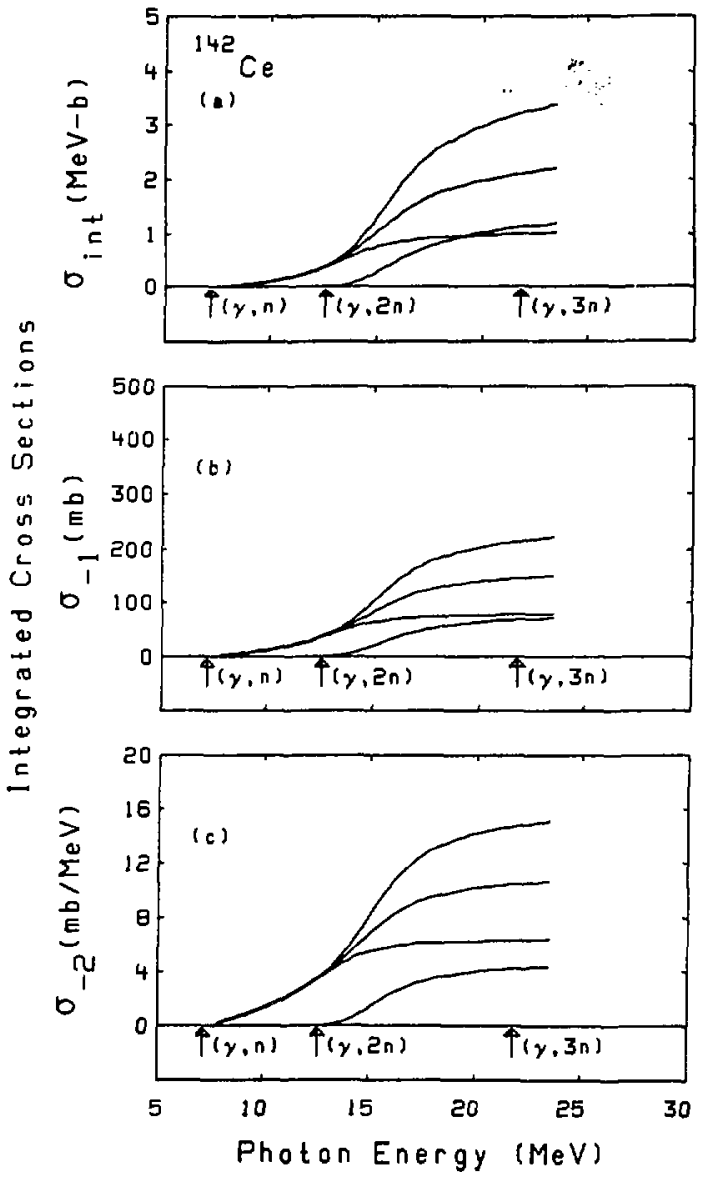

Fig. 119B 

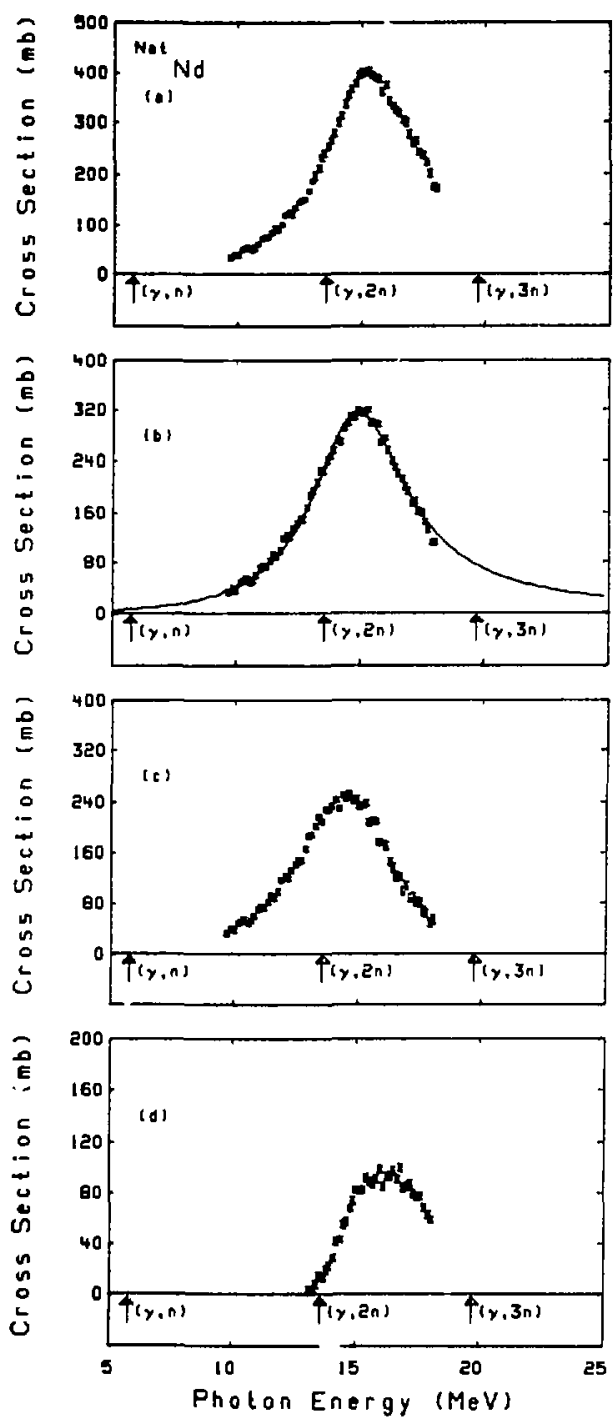

Fig. $120 \mathrm{~A}$ 


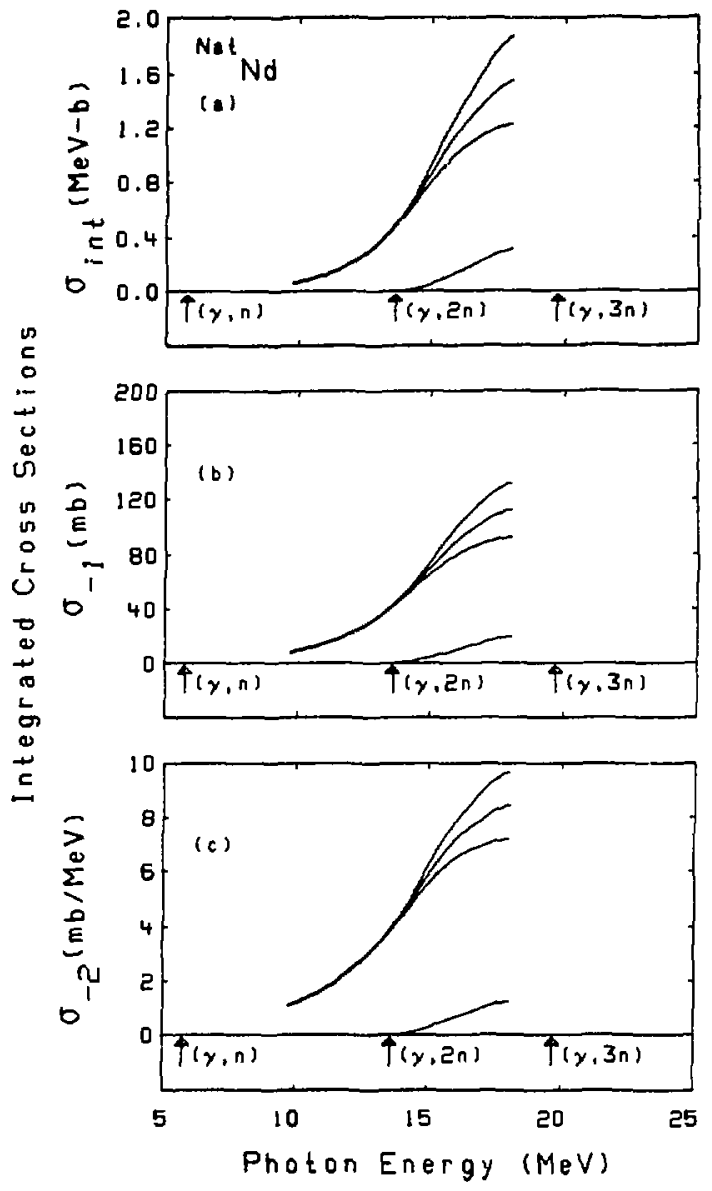

Fig. 120B 

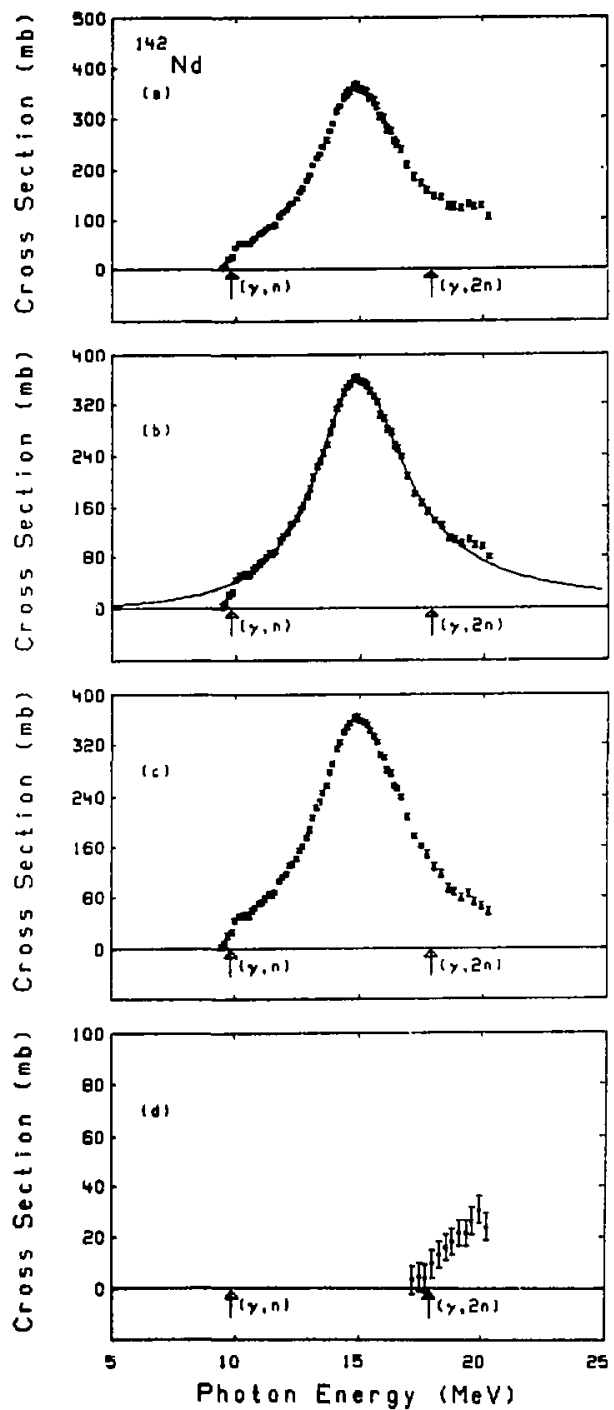

Fig. $121 \mathrm{~A}$ 


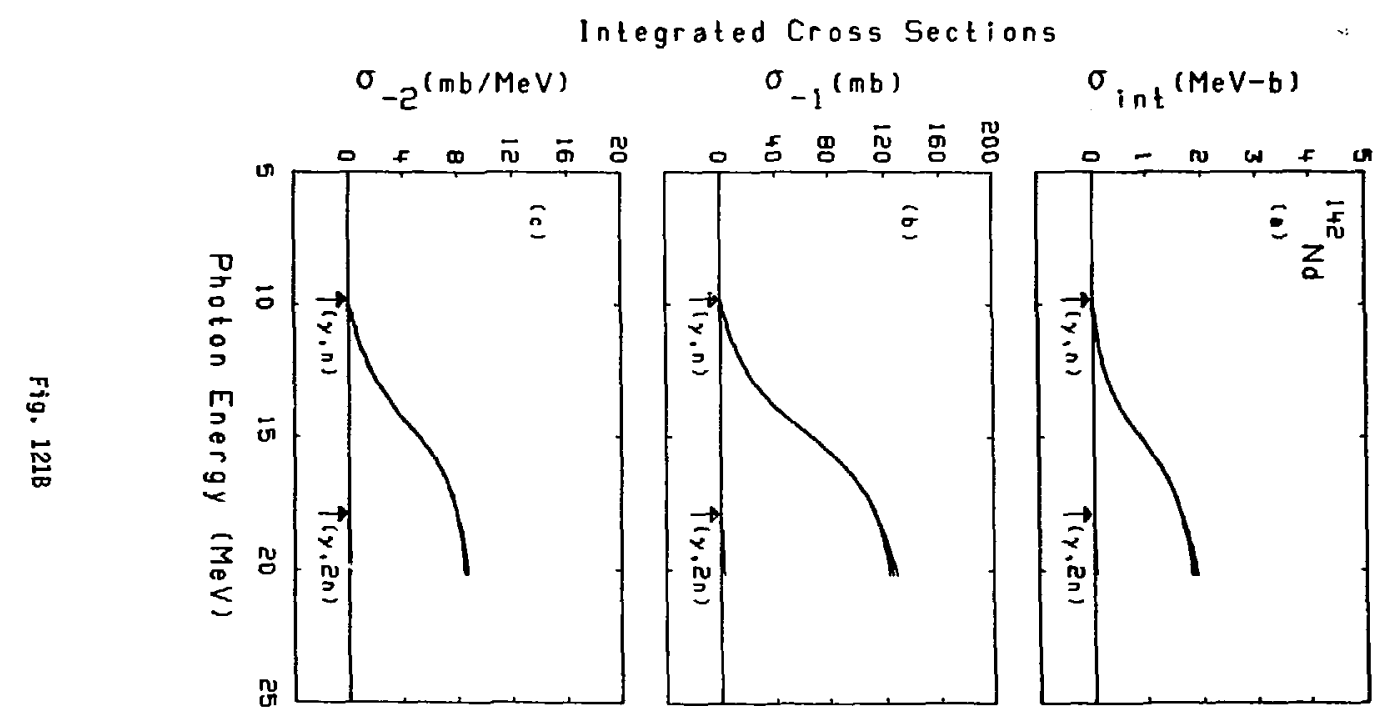



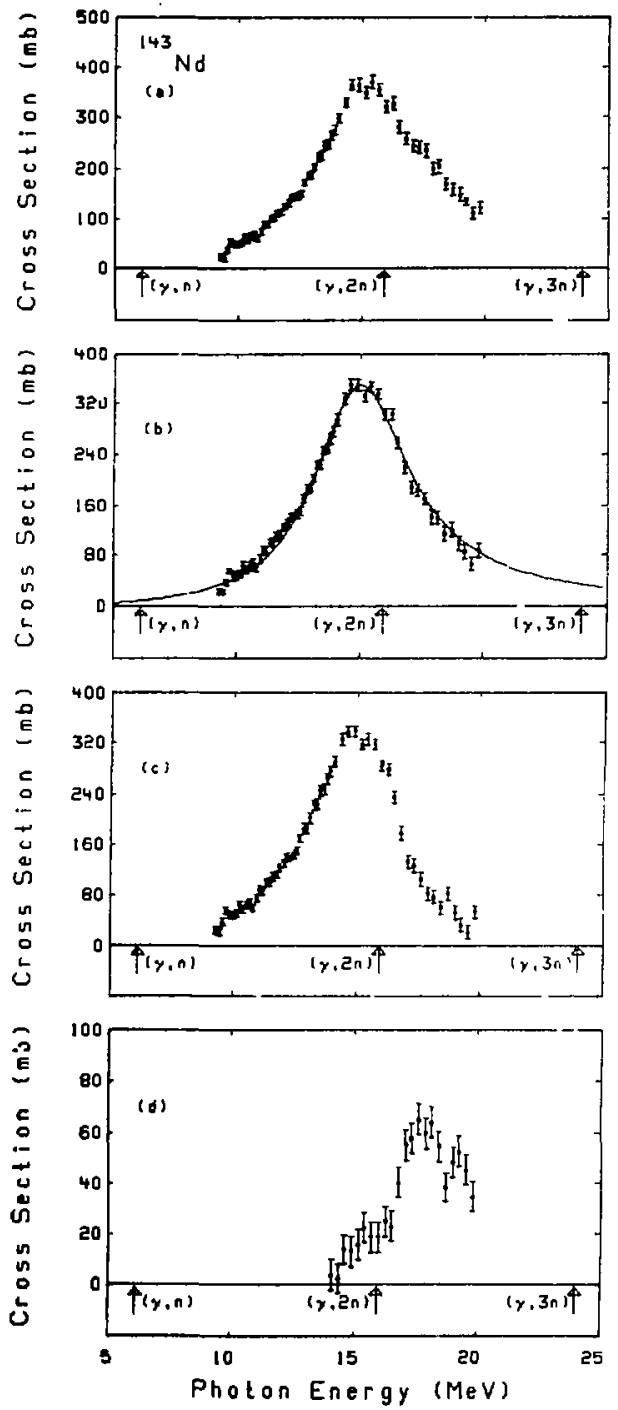

Fig. 122A 


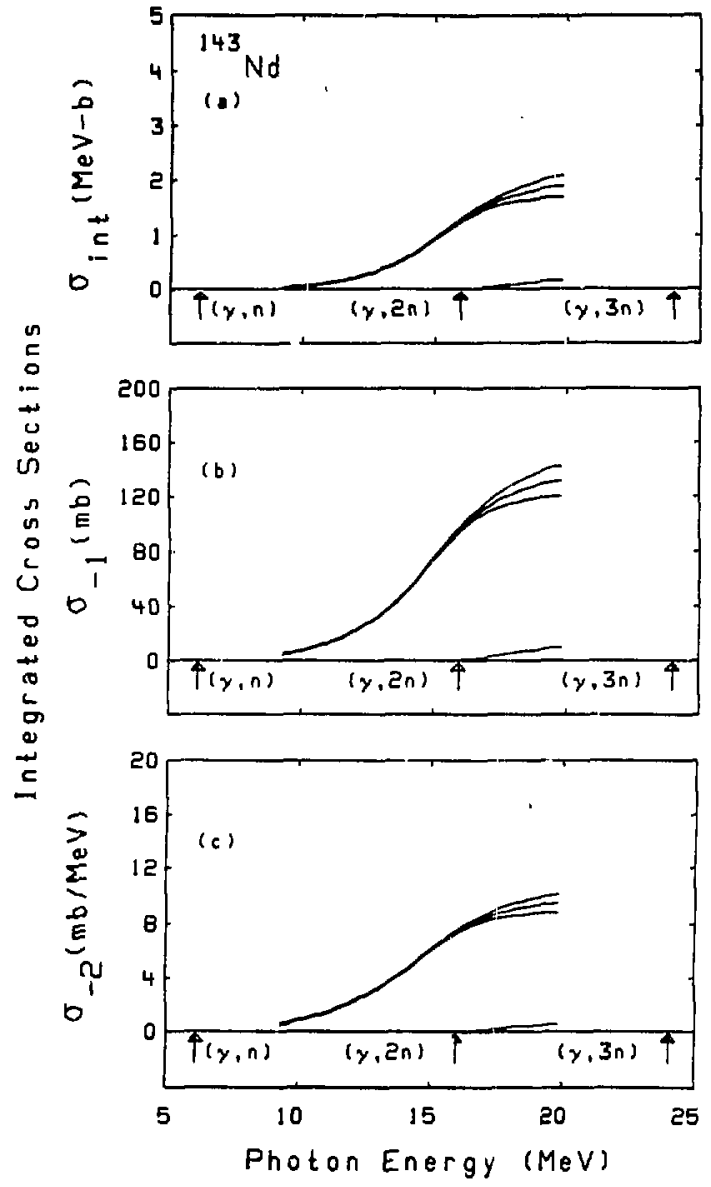

Fig. 1228 

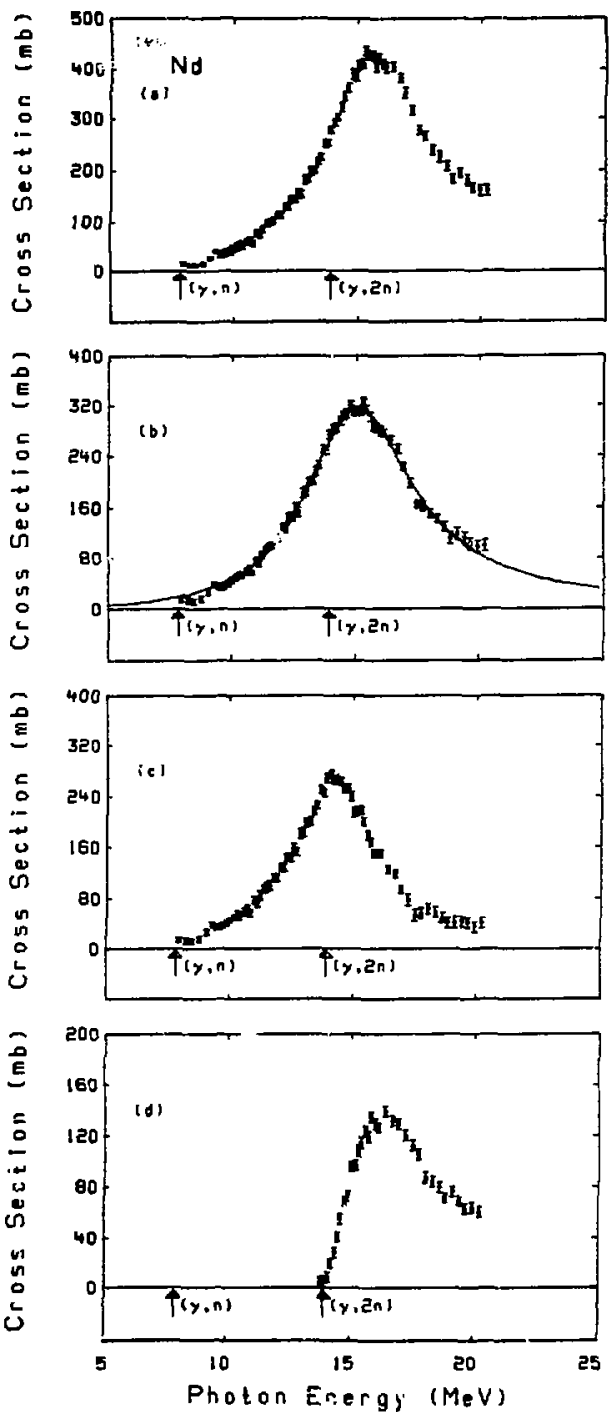

Fig. 123A. 


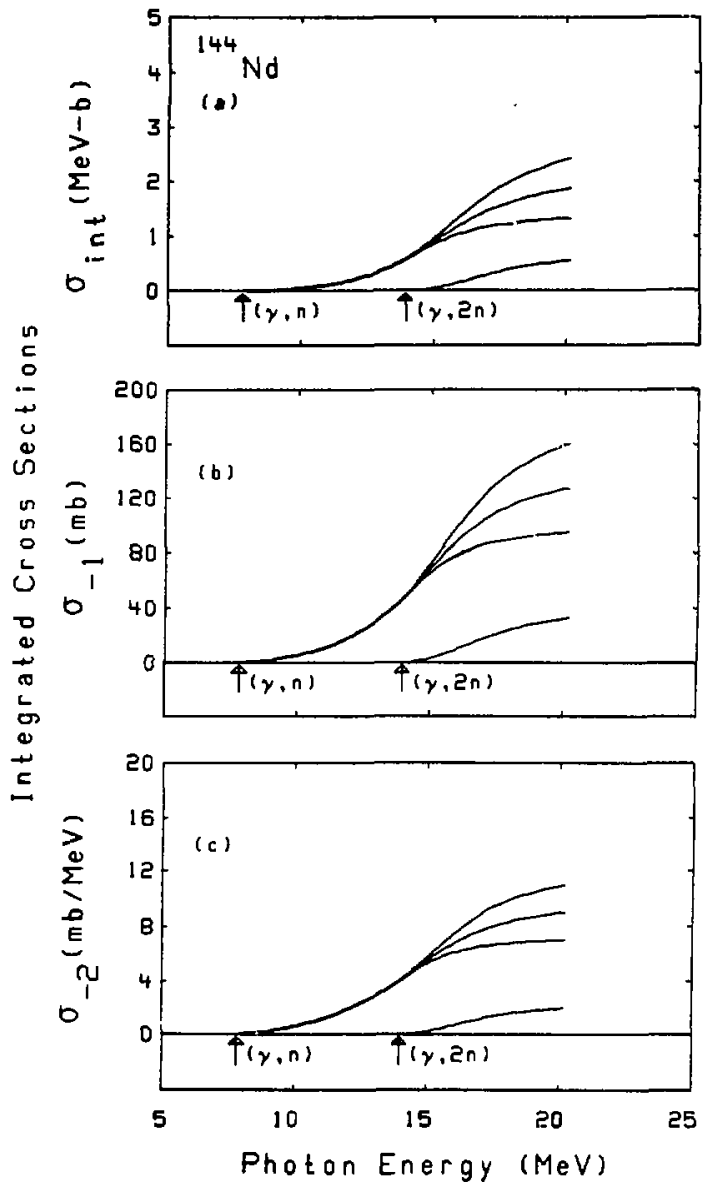

Fig. 123B 

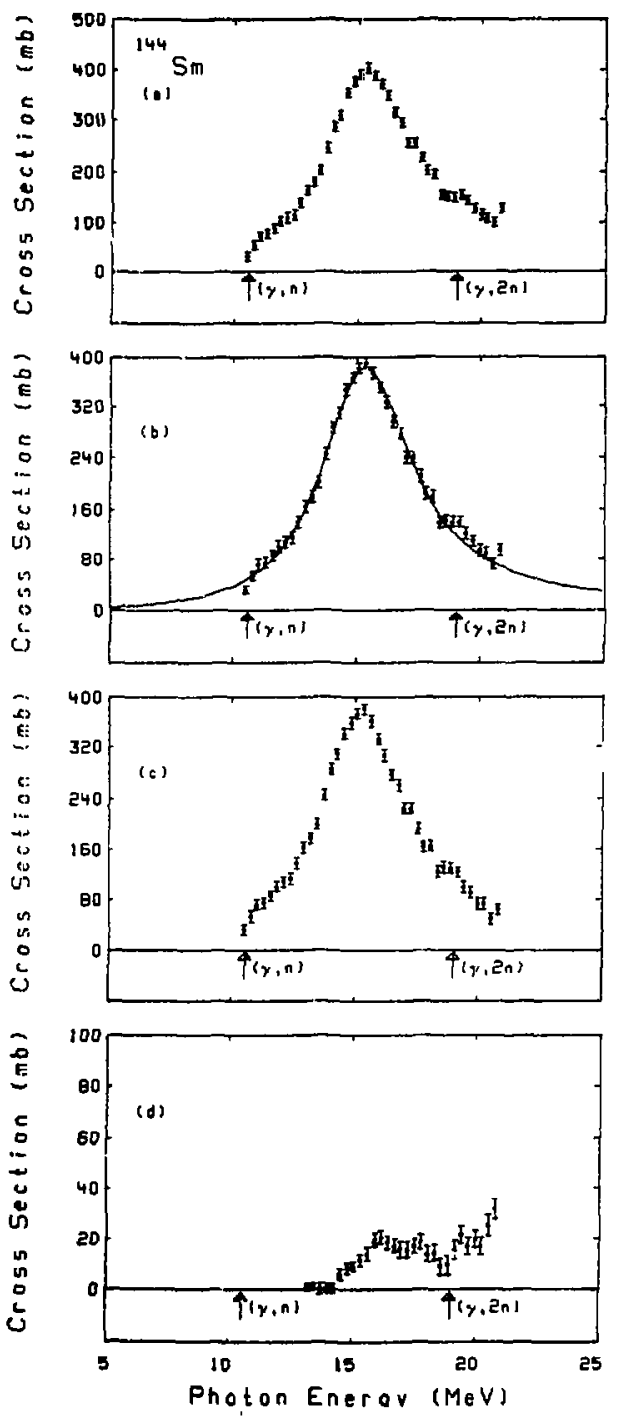

Fig. 124A 


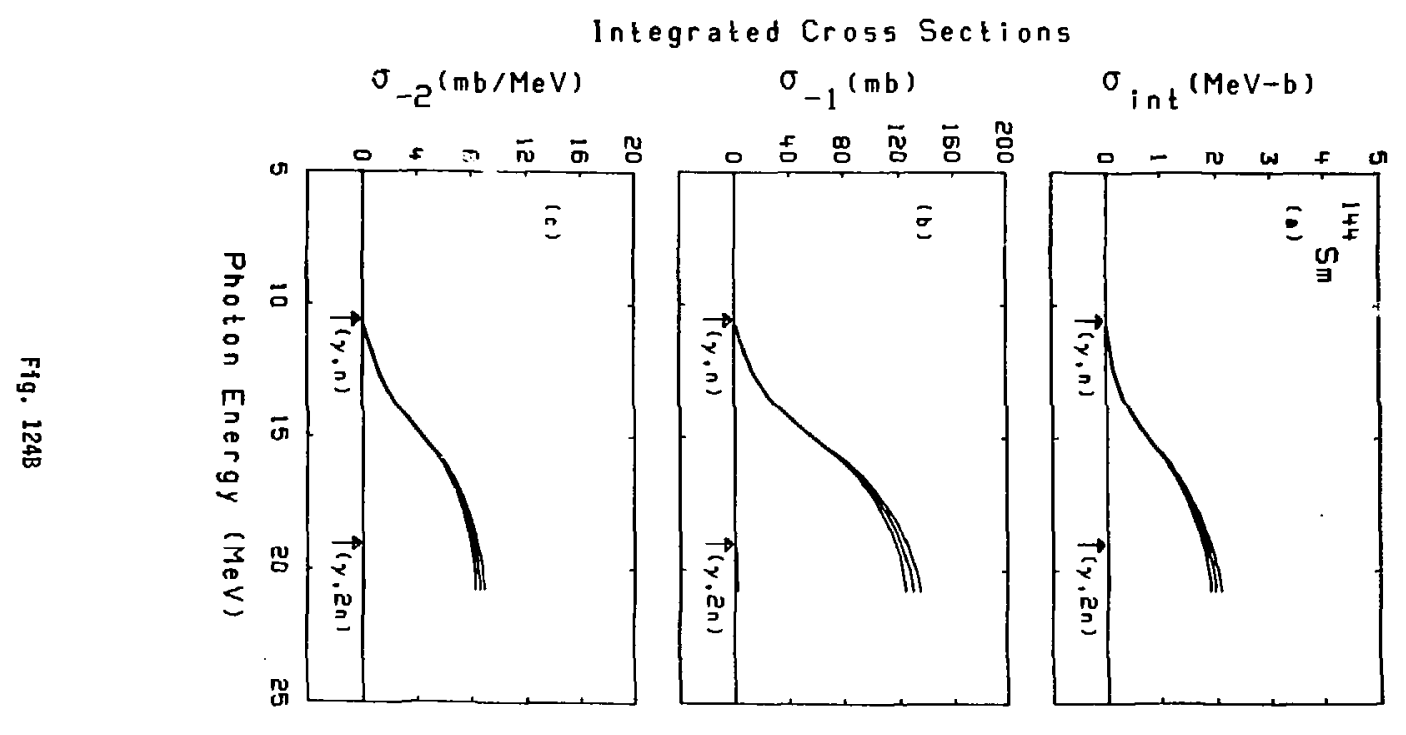



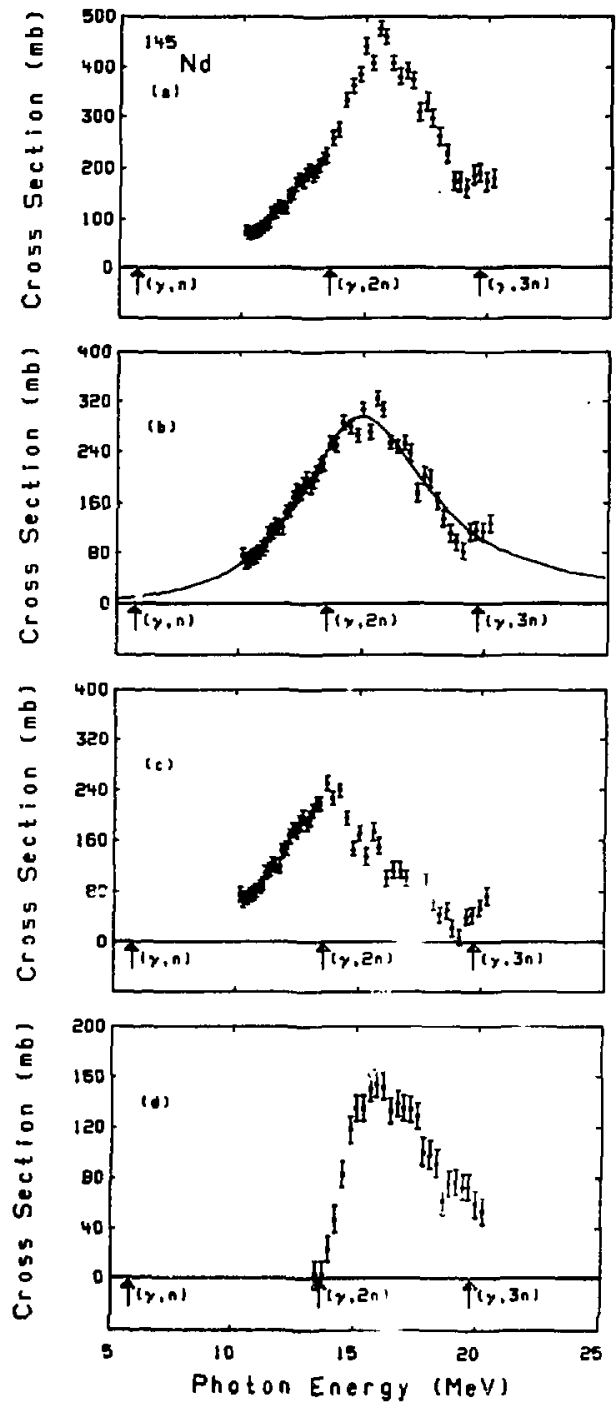

Fig. $125 \mathrm{~A}$ 


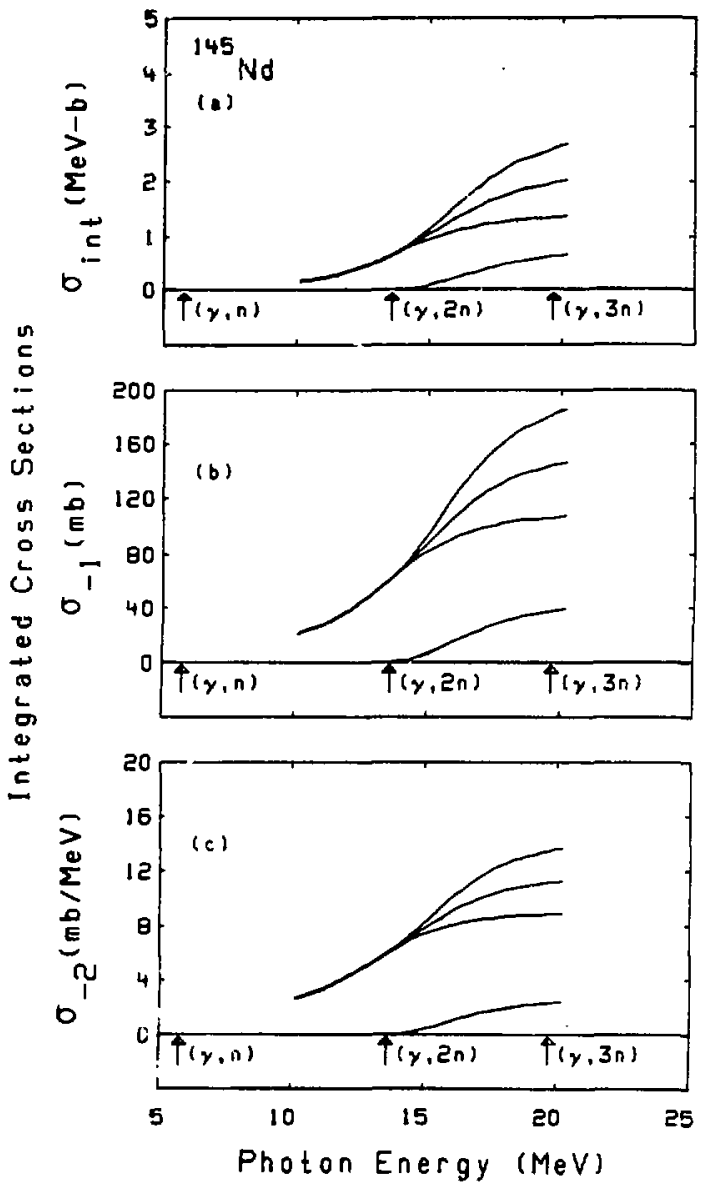

Fig. 1258 

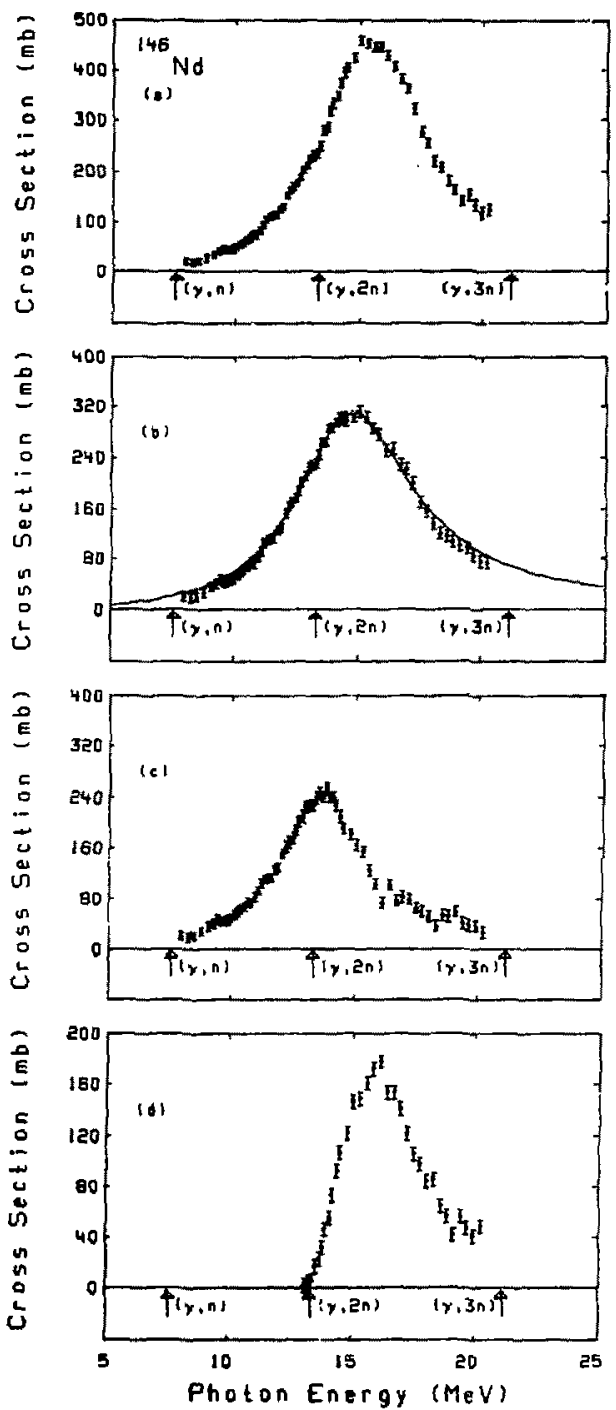

Fig, 126A 


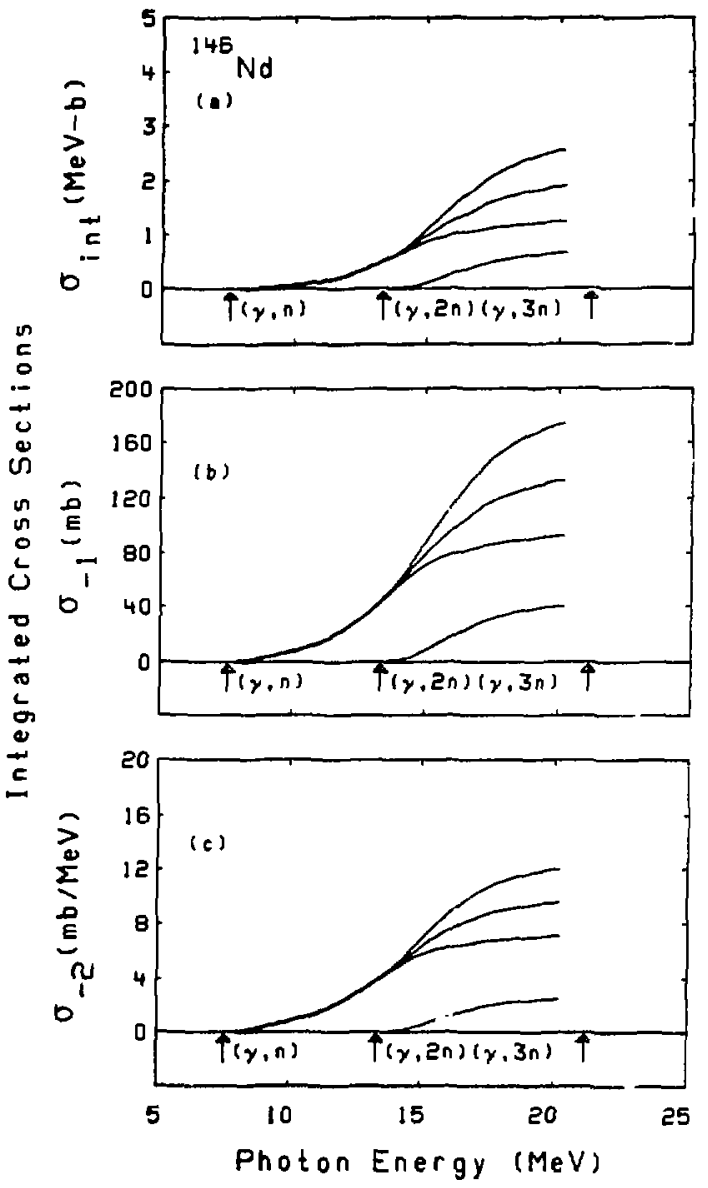

Fig. 1268 

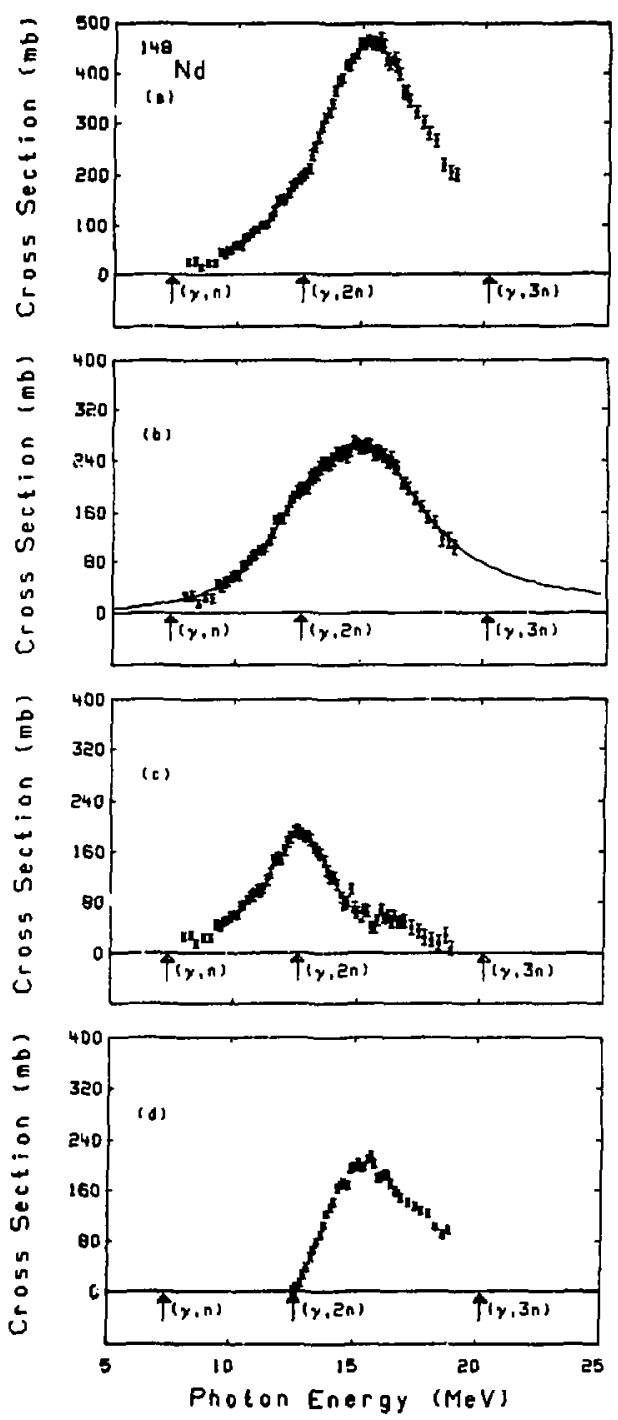

Fig. 127A 


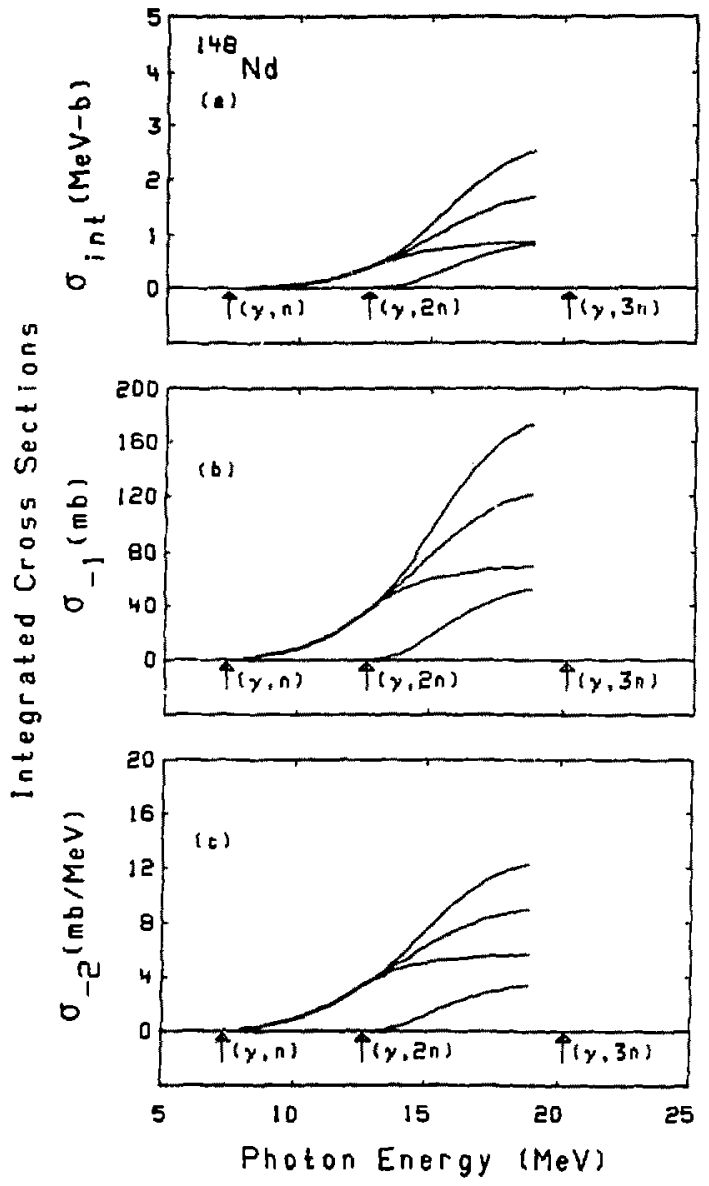

Fig. $127 B$ 

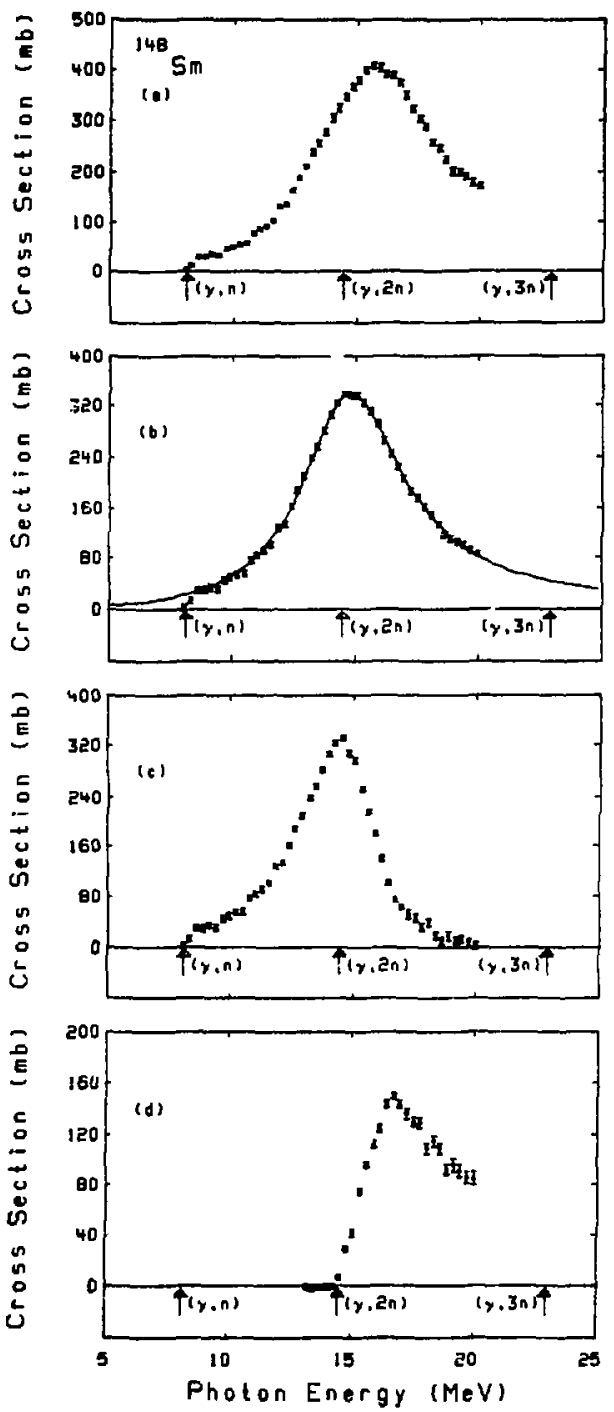

Fig. $128 \mathrm{~A}$ 


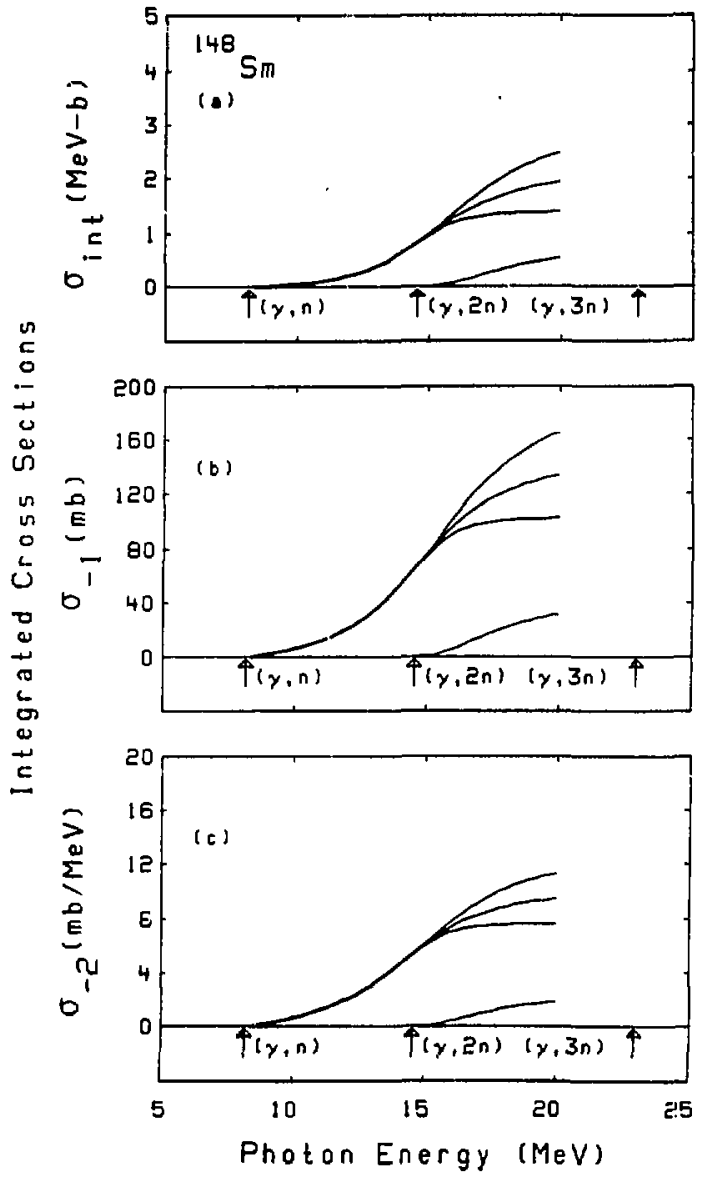

Fig. 1288 

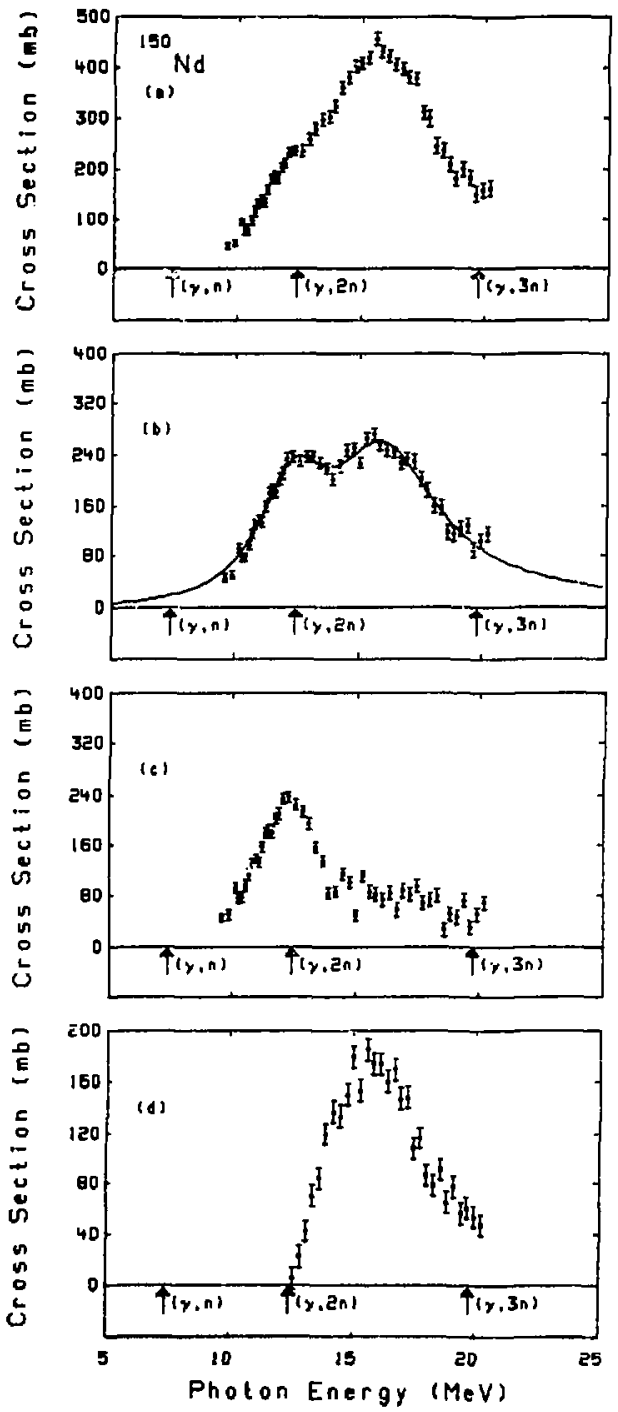

Fig. 129A 

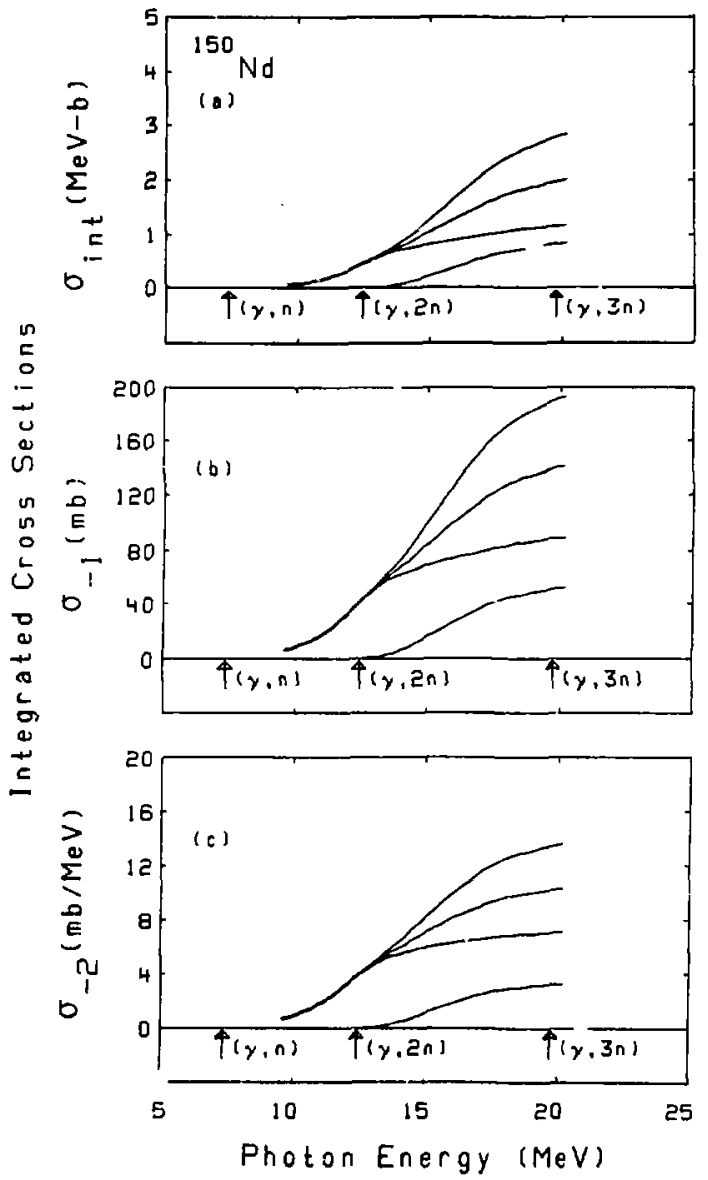

Fig. 1298 

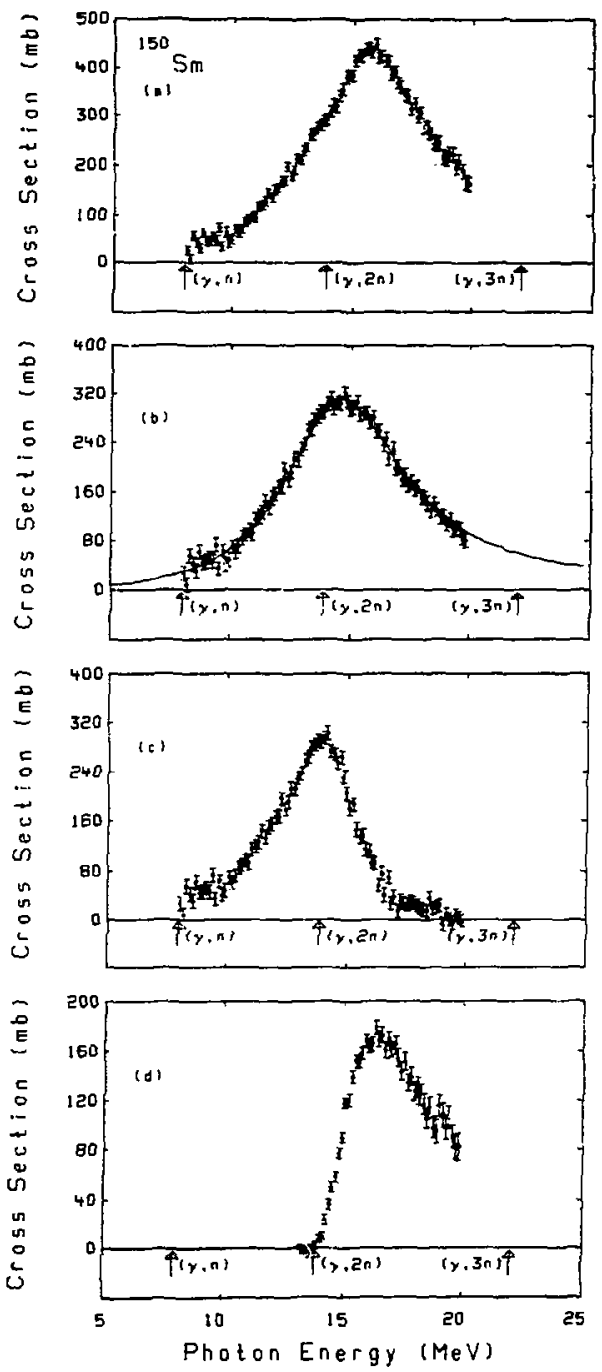

Fig. 130A 


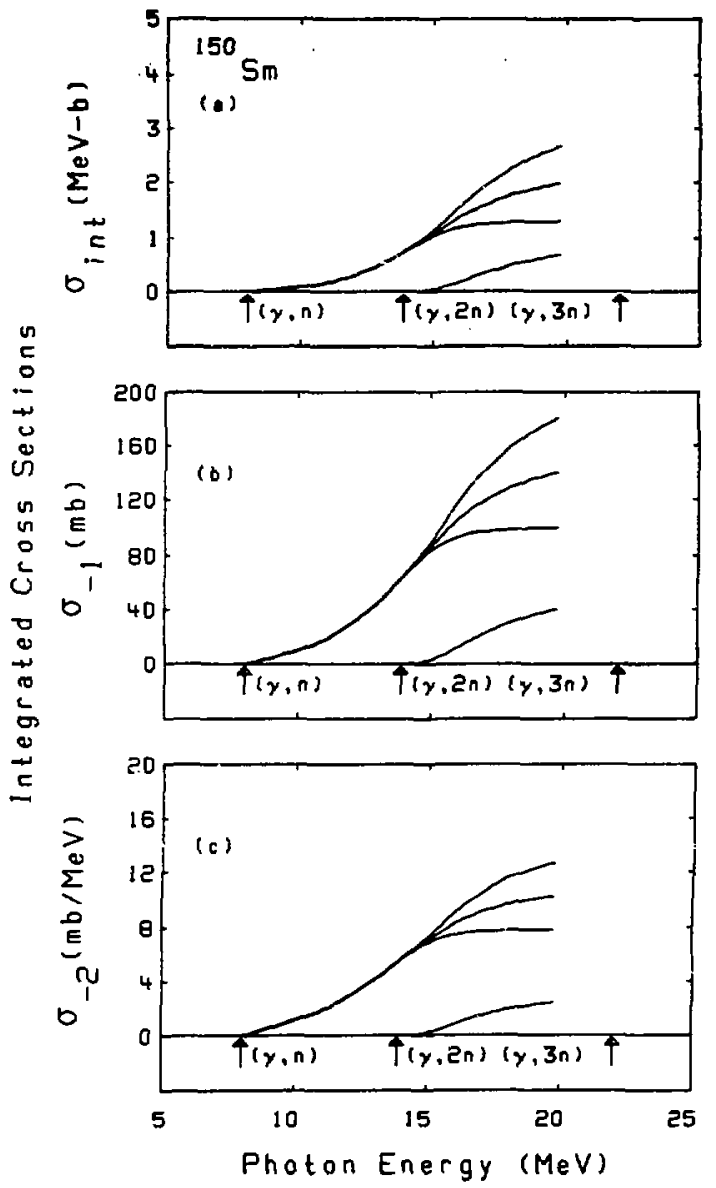

Fig. 1308 

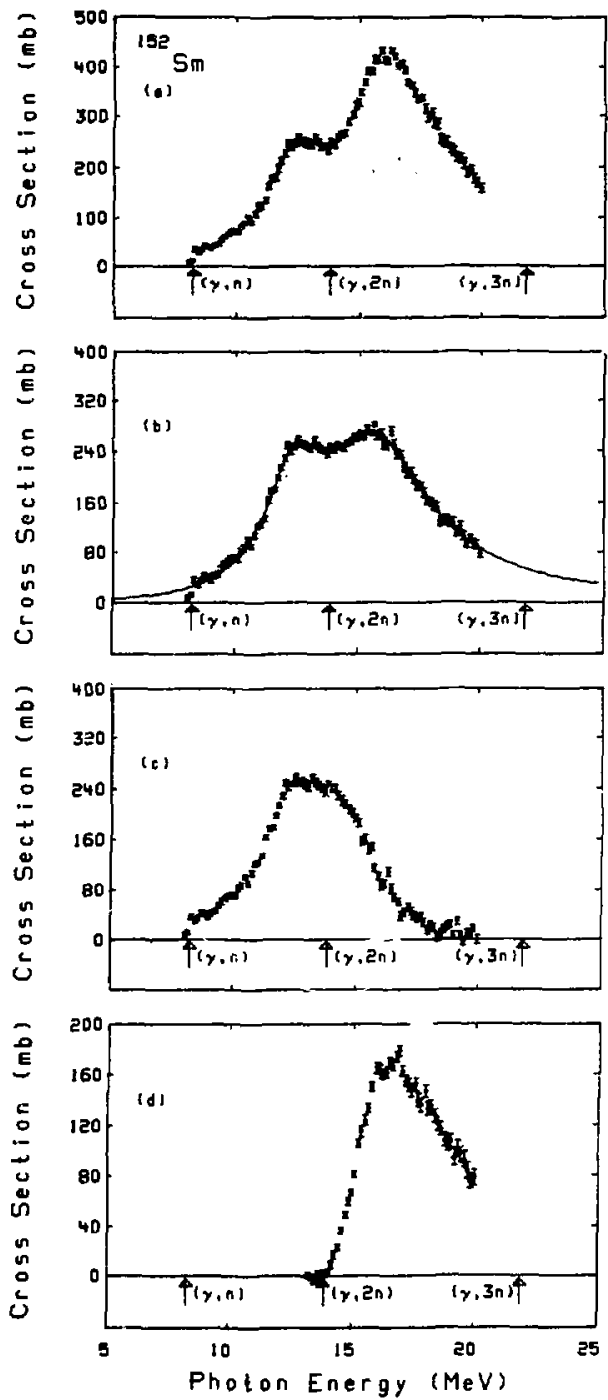

Fig. 131A 


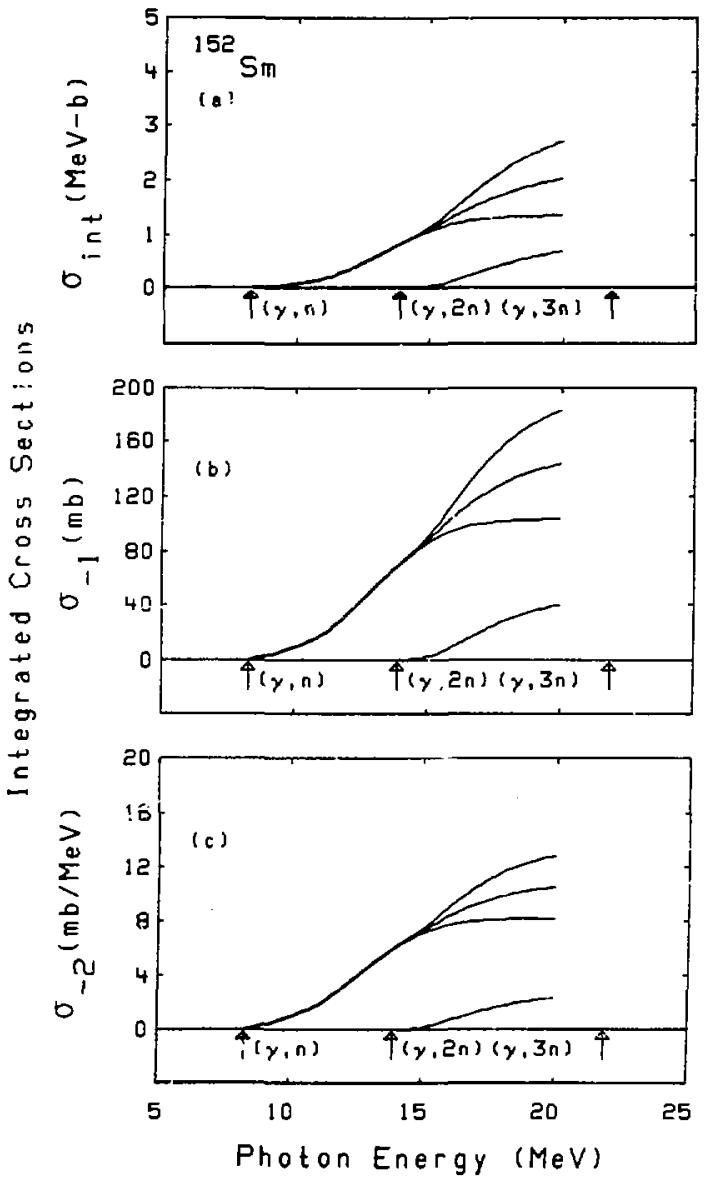

Fig. 131B 

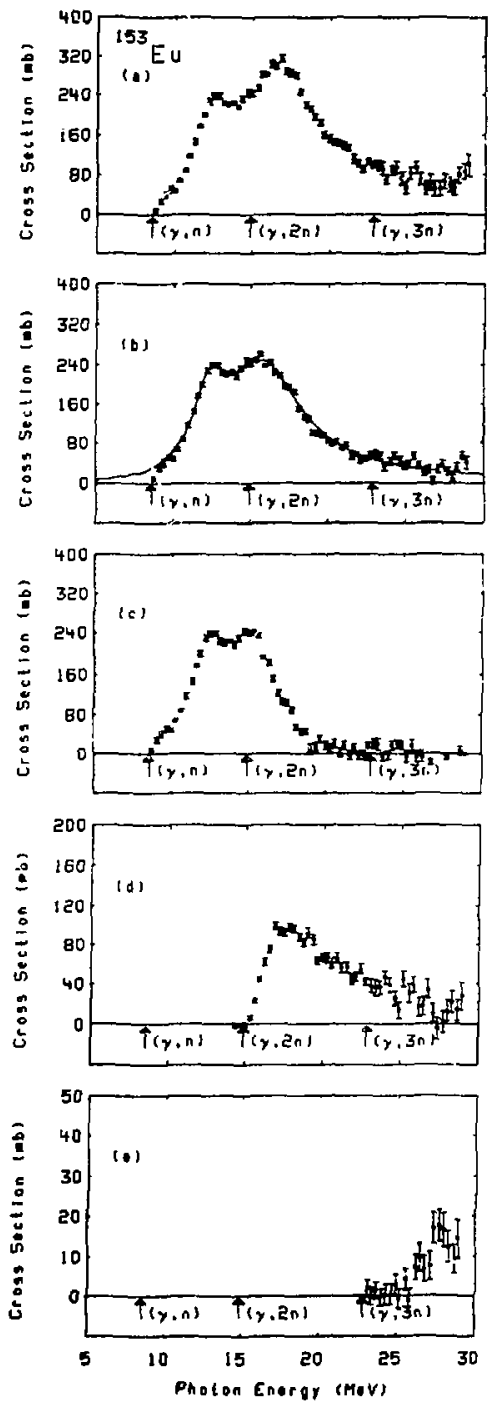

Fig. 132A 


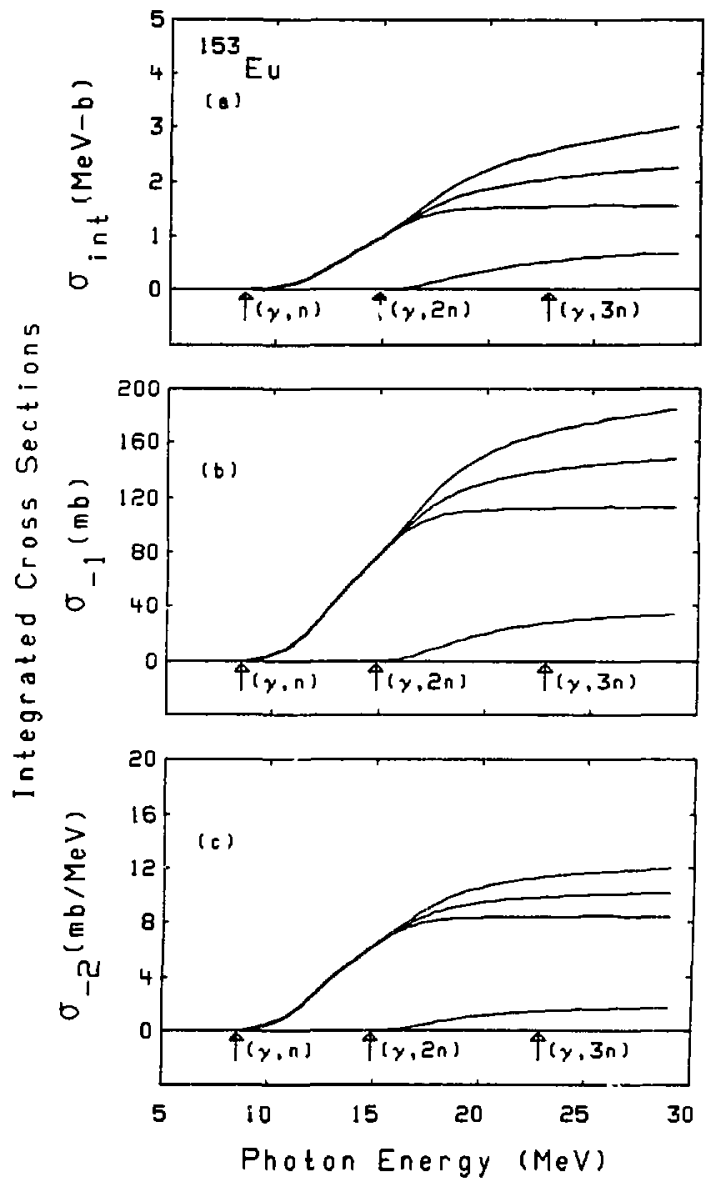

Fig. 132B 

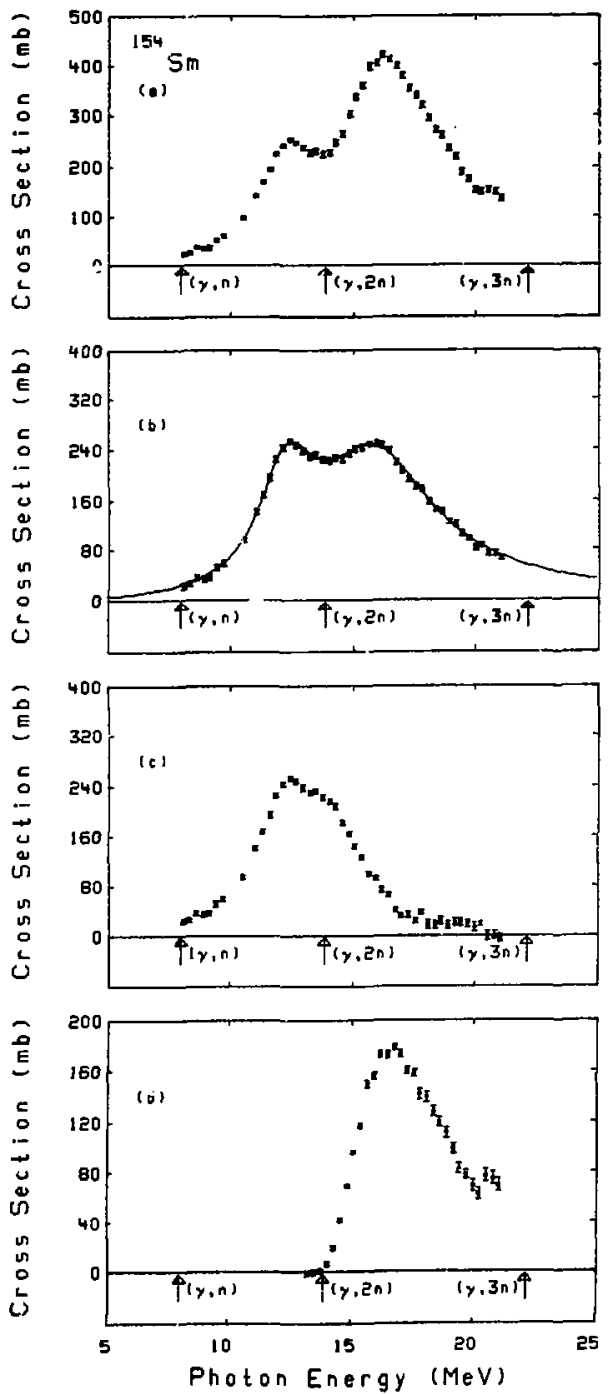

rig. 133A 


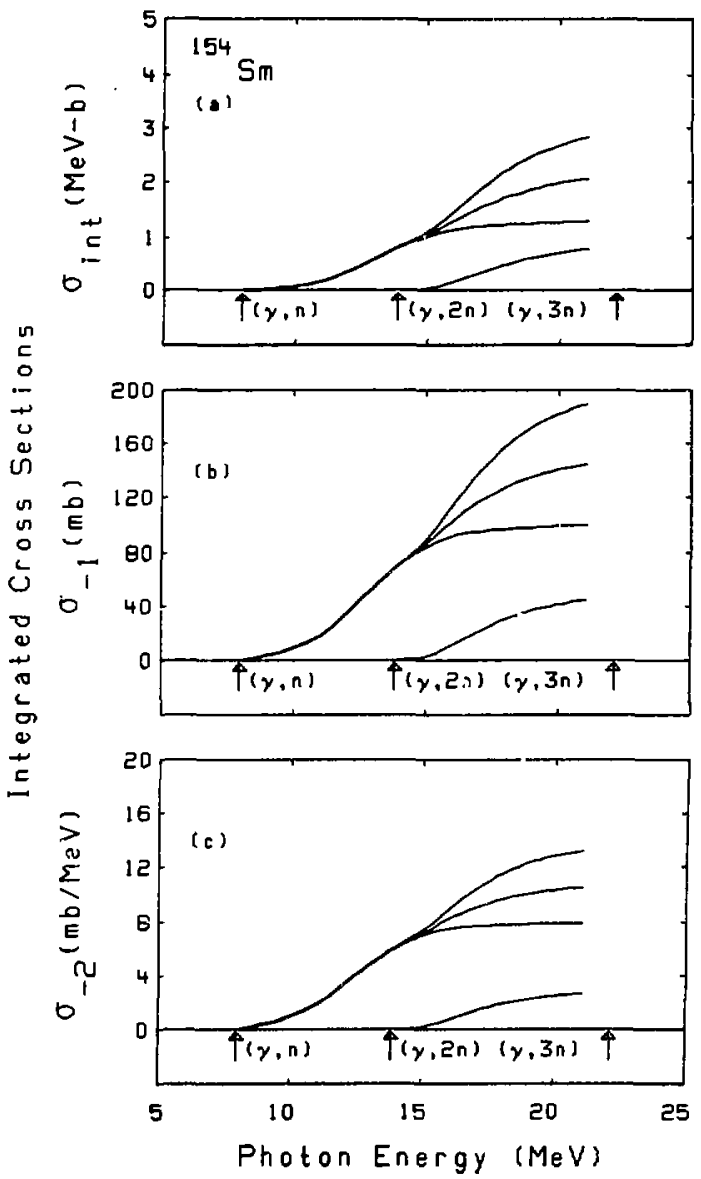

Fig. 133B 

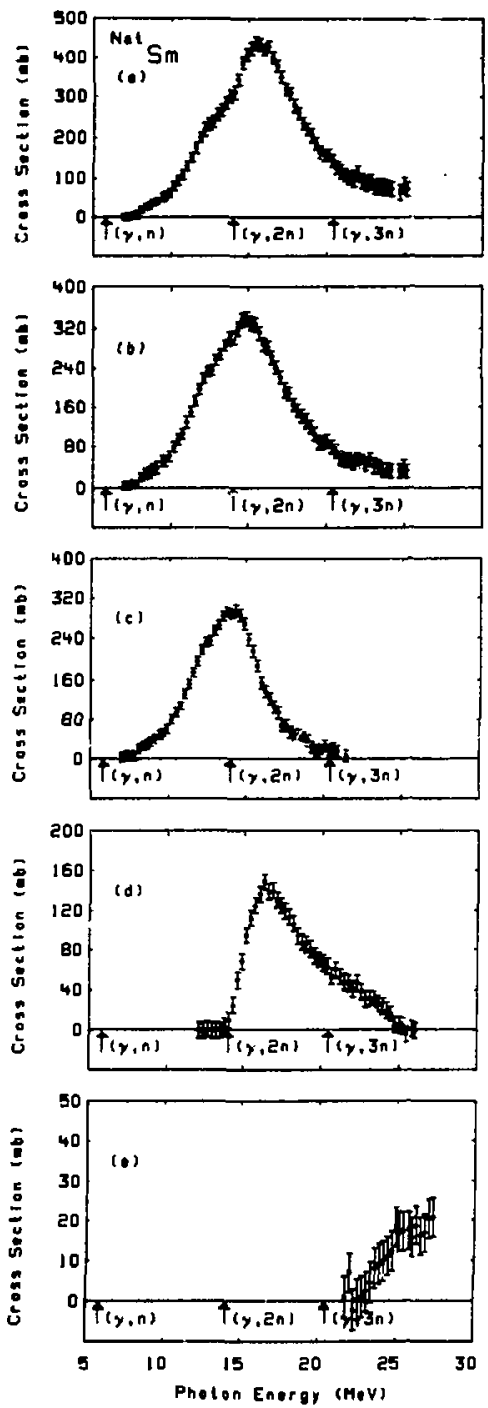

Fig. $134 \mathrm{~A}$ 


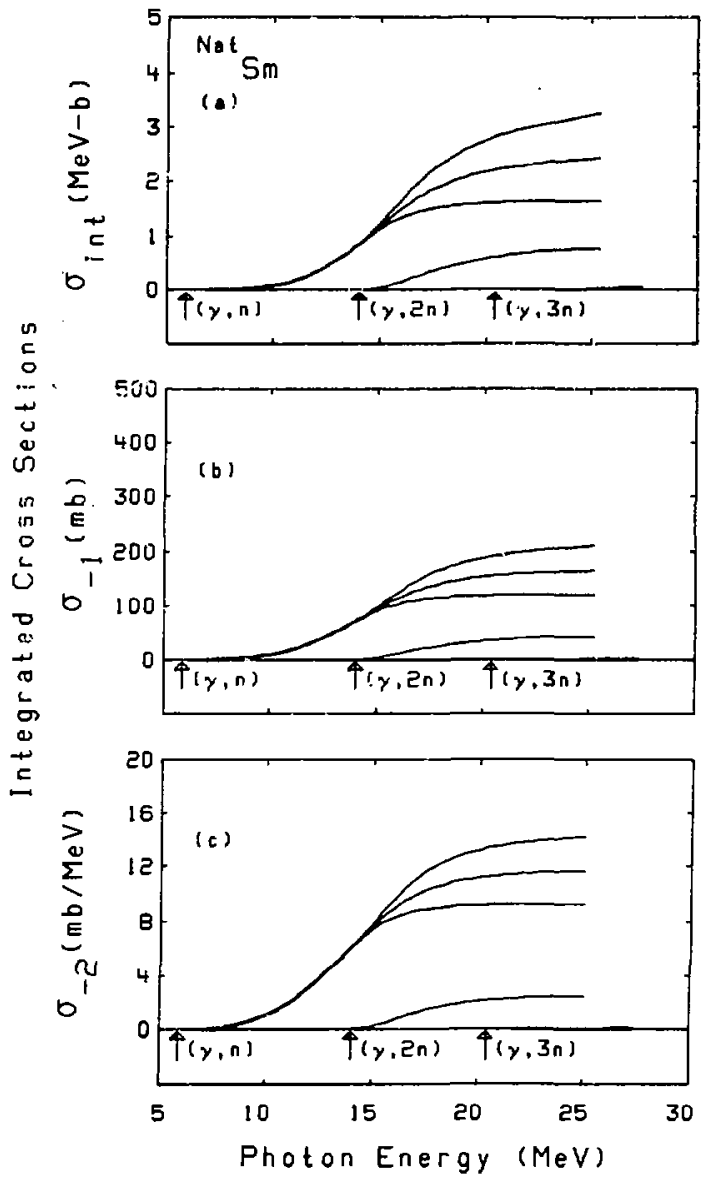

Fig. 1348 

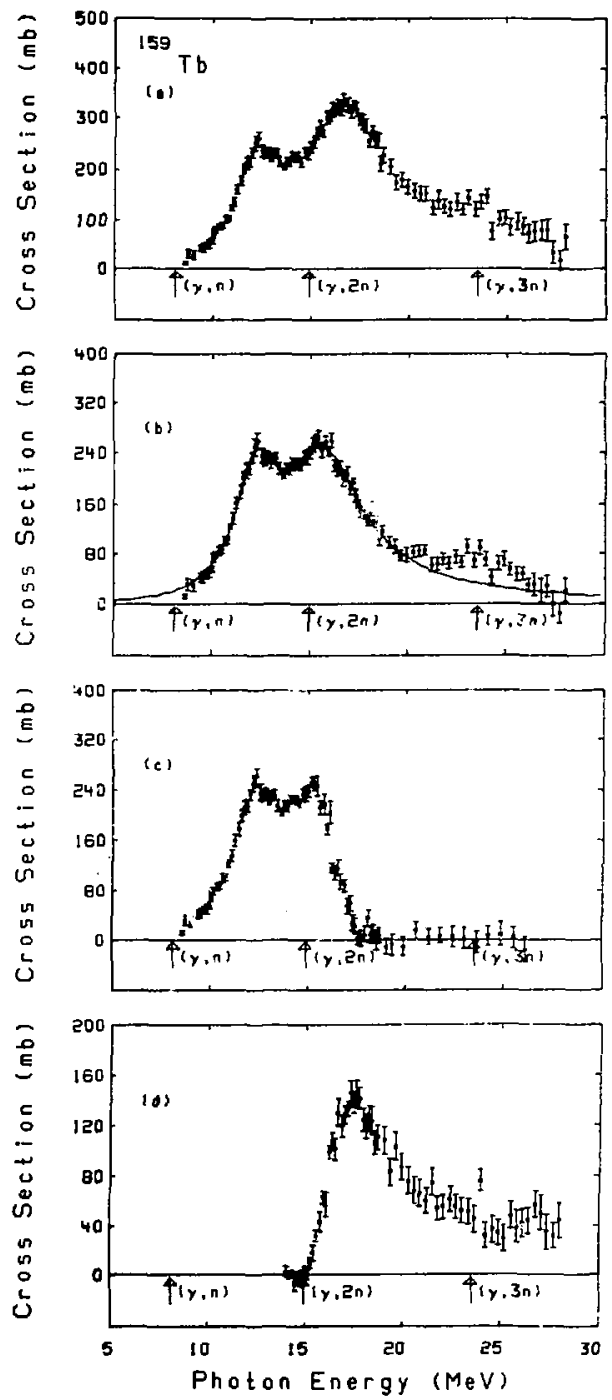

Fig. $135 \mathrm{~A}$ 


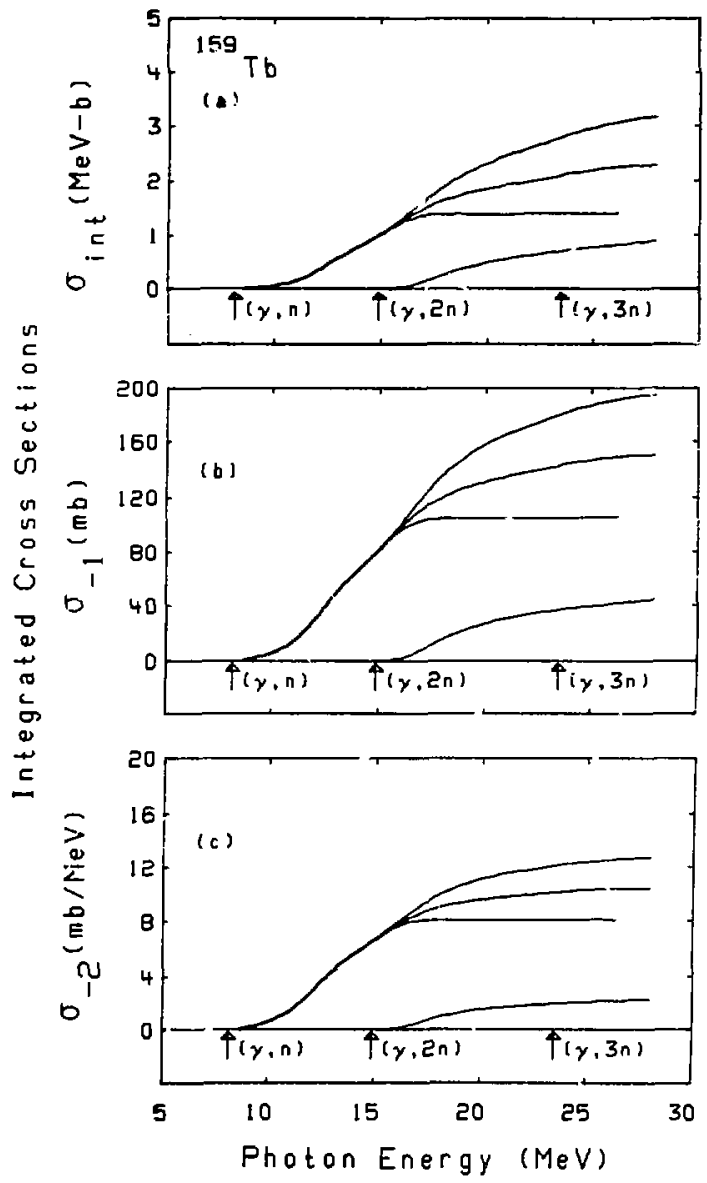

Fig. 1358 

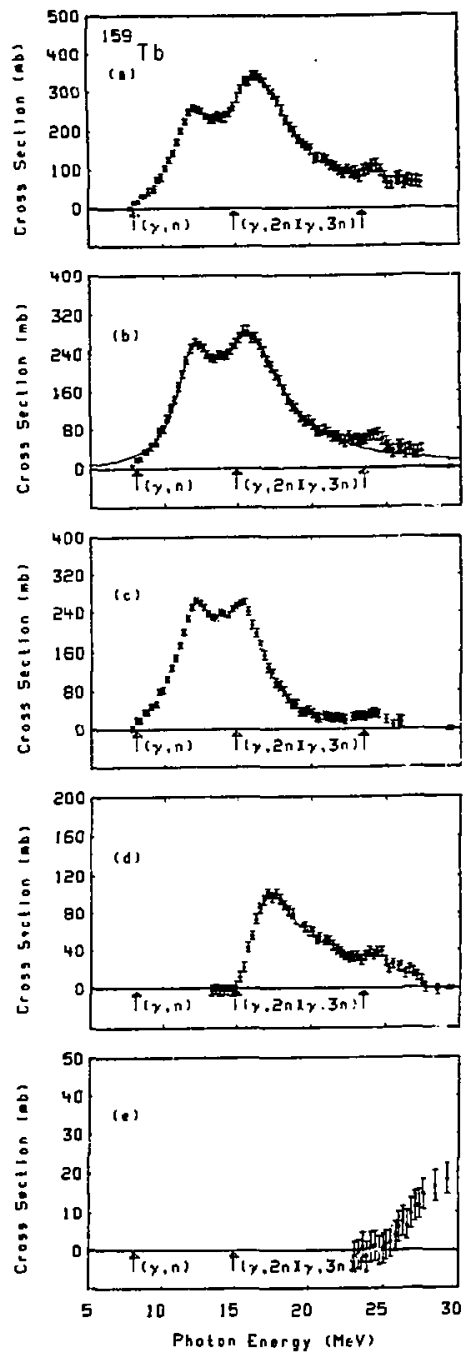

Fig. $136 \mathrm{~A}$ 


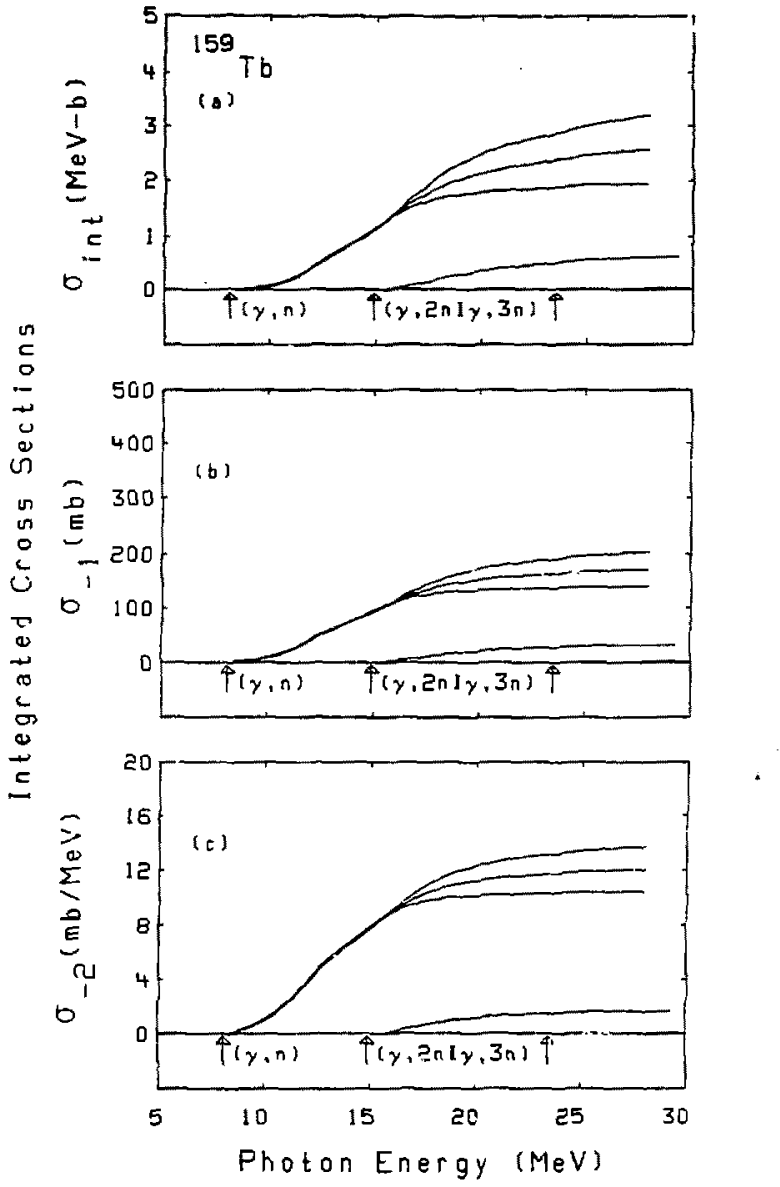

Fig. $136 \mathrm{~B}$ 

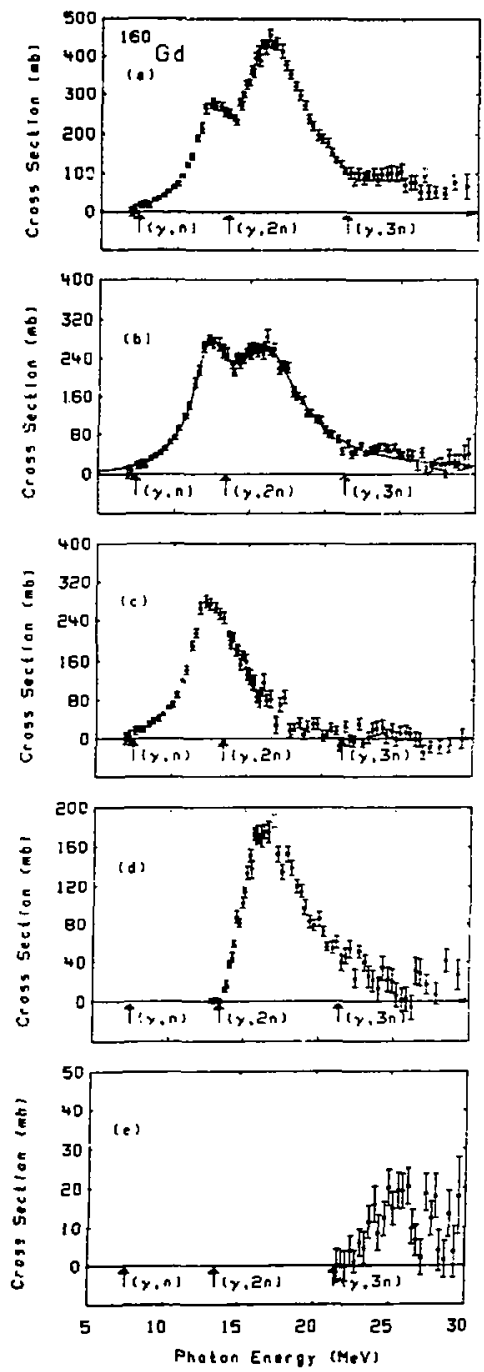

Fig. $137 \mathrm{~A}$ 


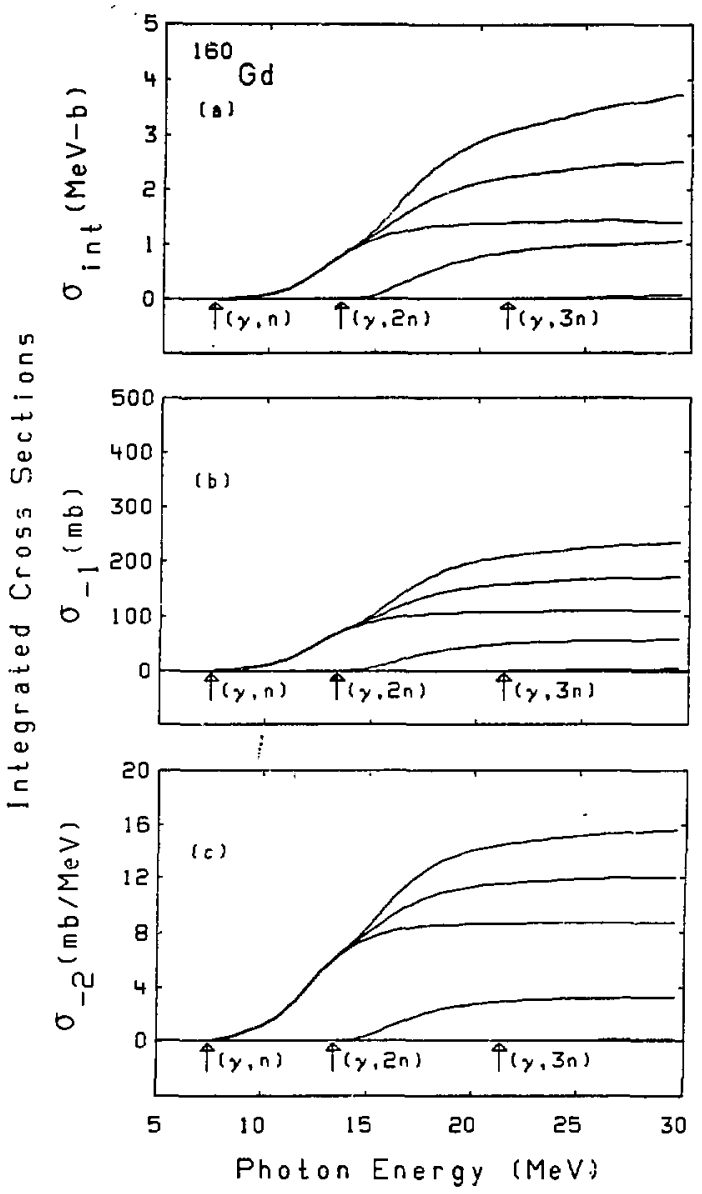

Fig. $137 B$ 

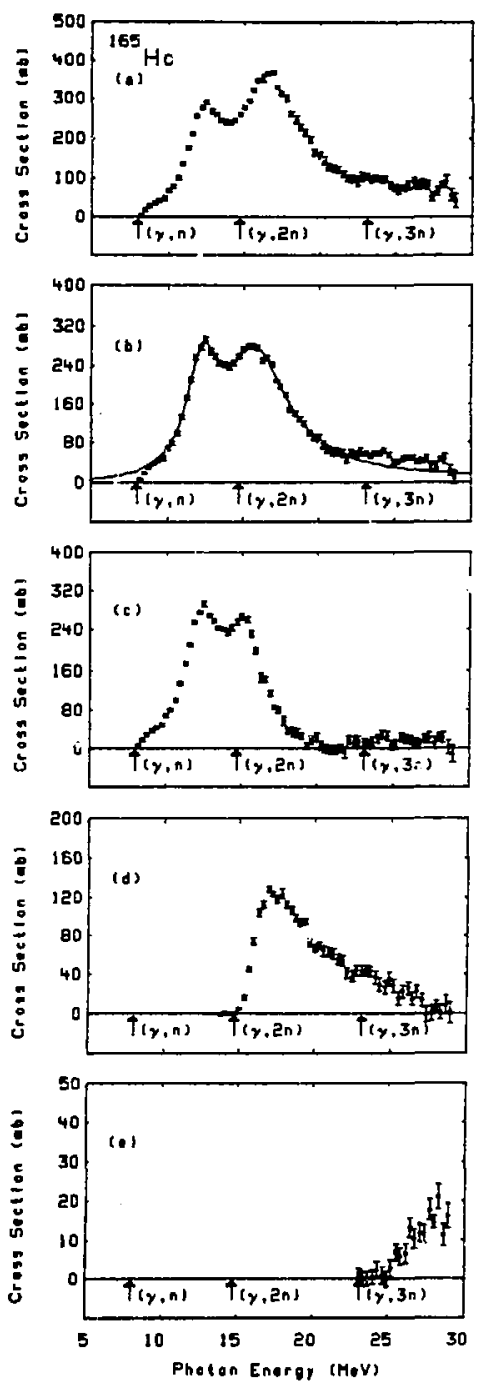

Fig. 138A 


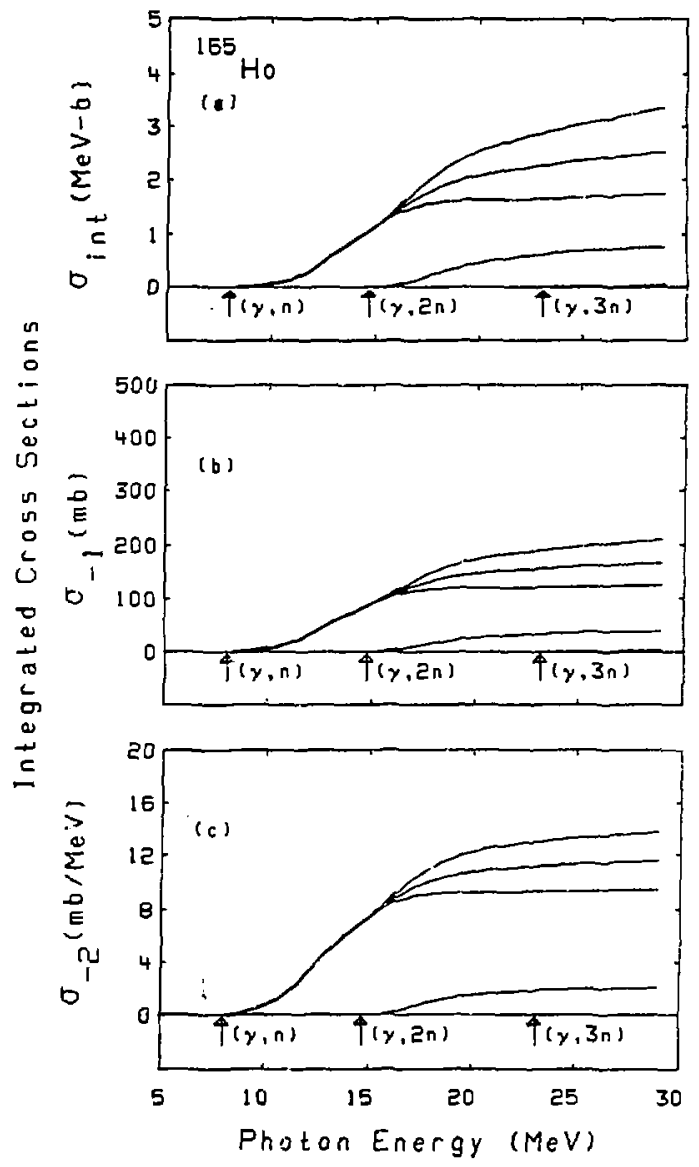

Fig. $138 B$ 

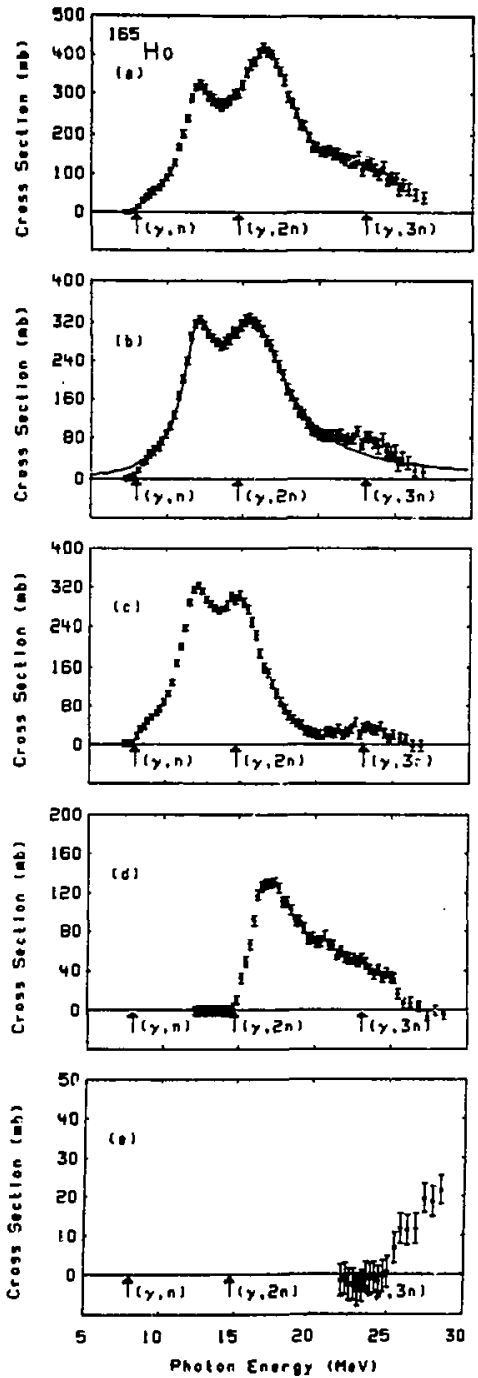

Fig. 139A 

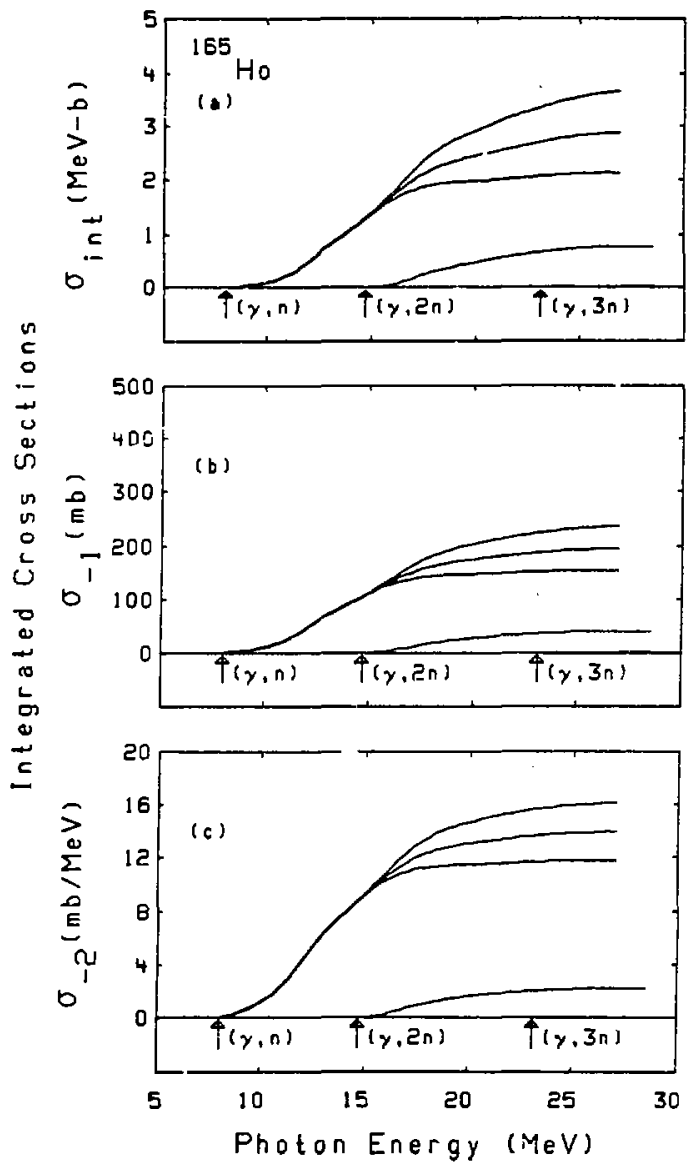

Fig. 139B 

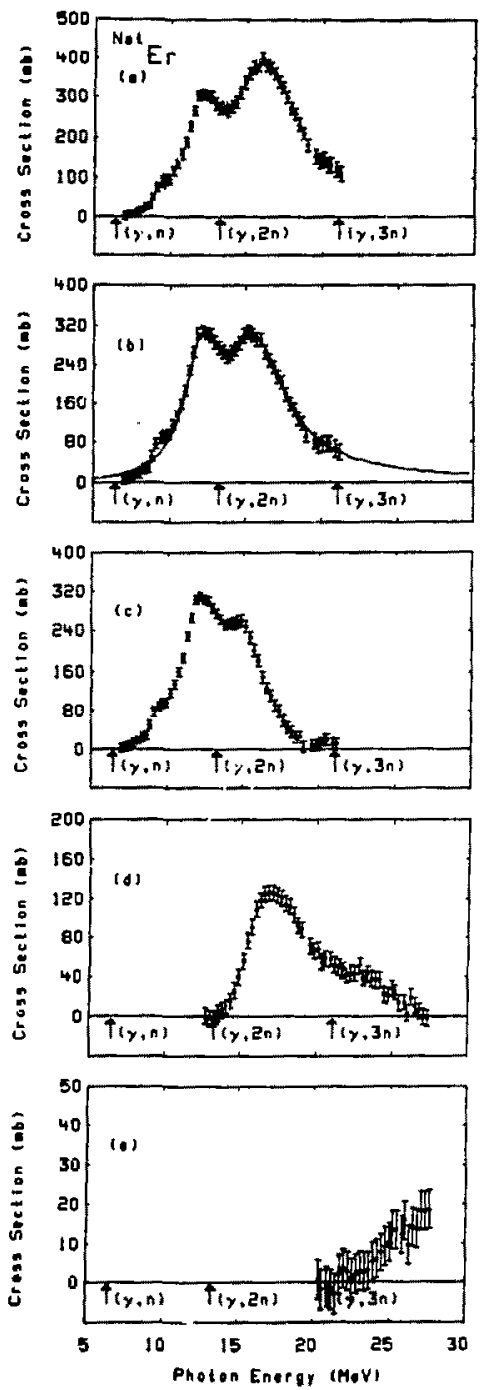

Fig. $140 \mathrm{~A}$ 


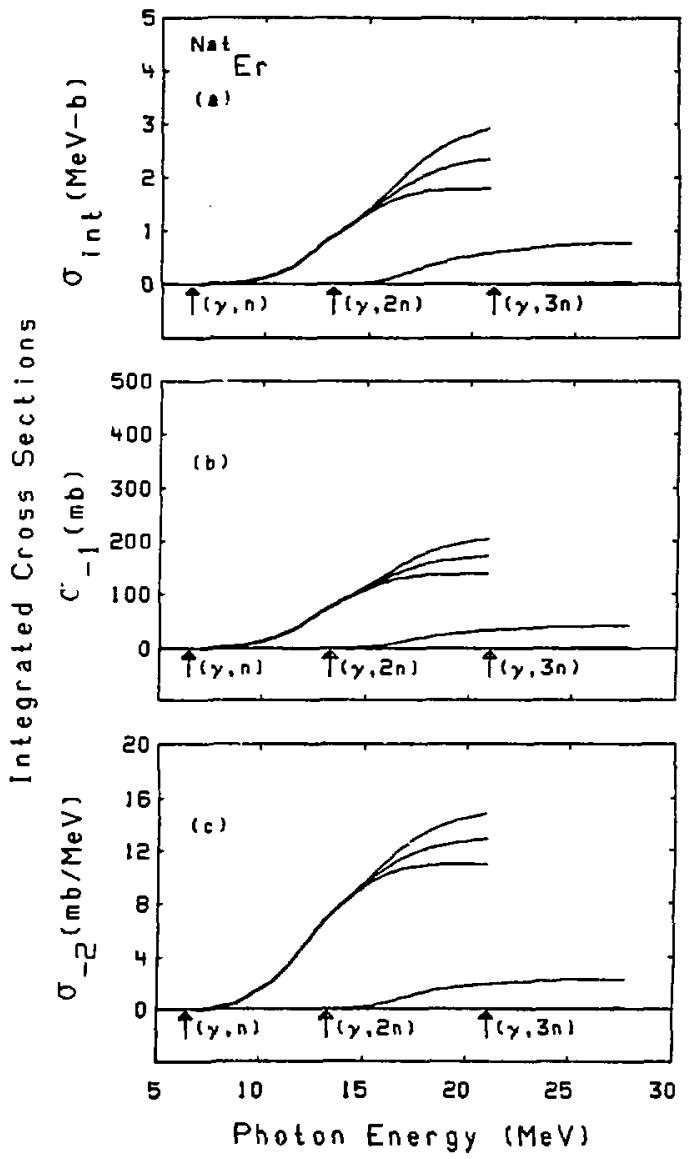

Fig. $140 B$ 

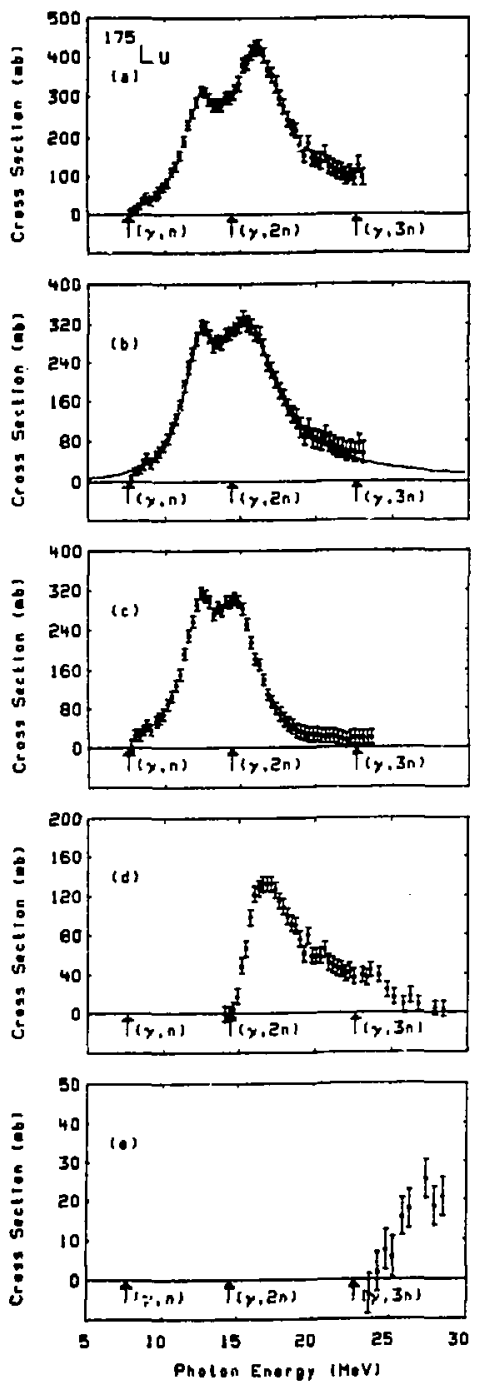

Fig. $141 \mathrm{~A}$ 

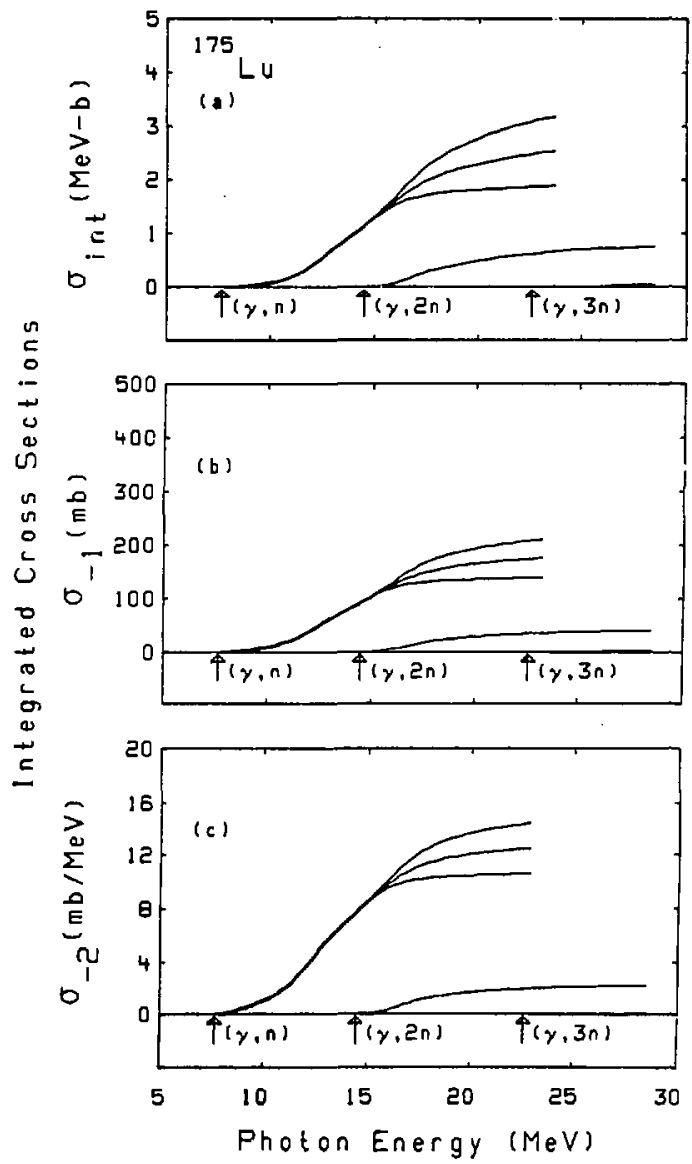

Fig. 141B 

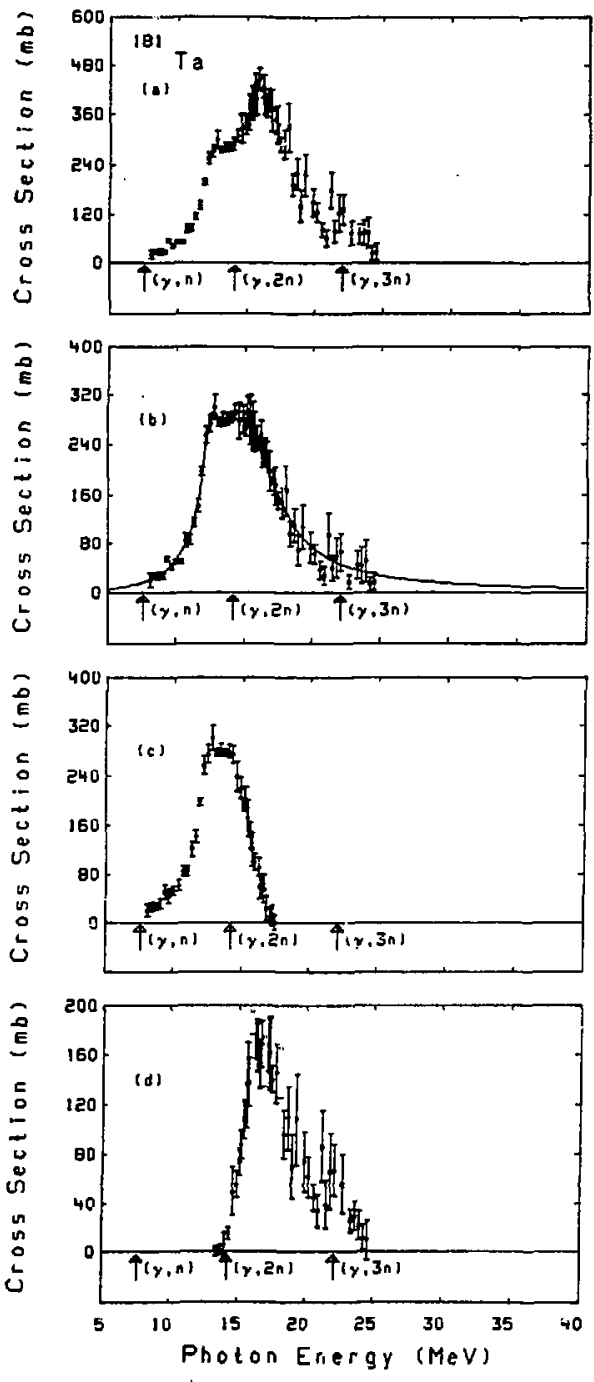

Fig. 142A 


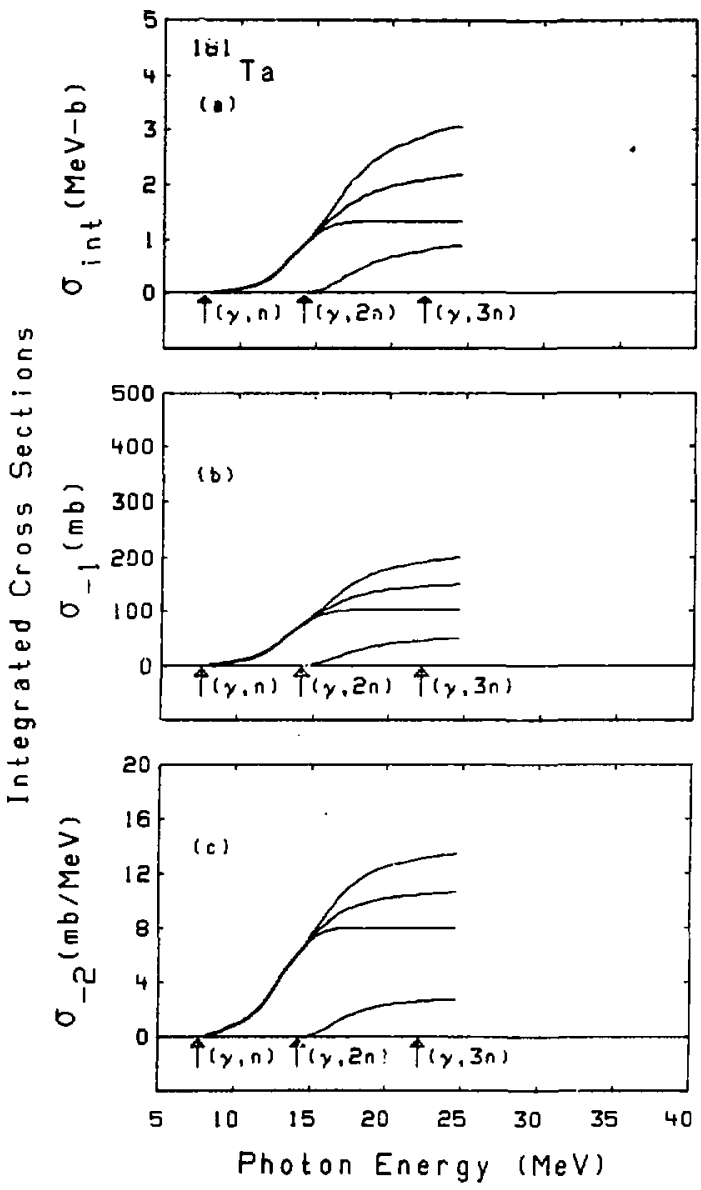

Fig. $142 B$ 

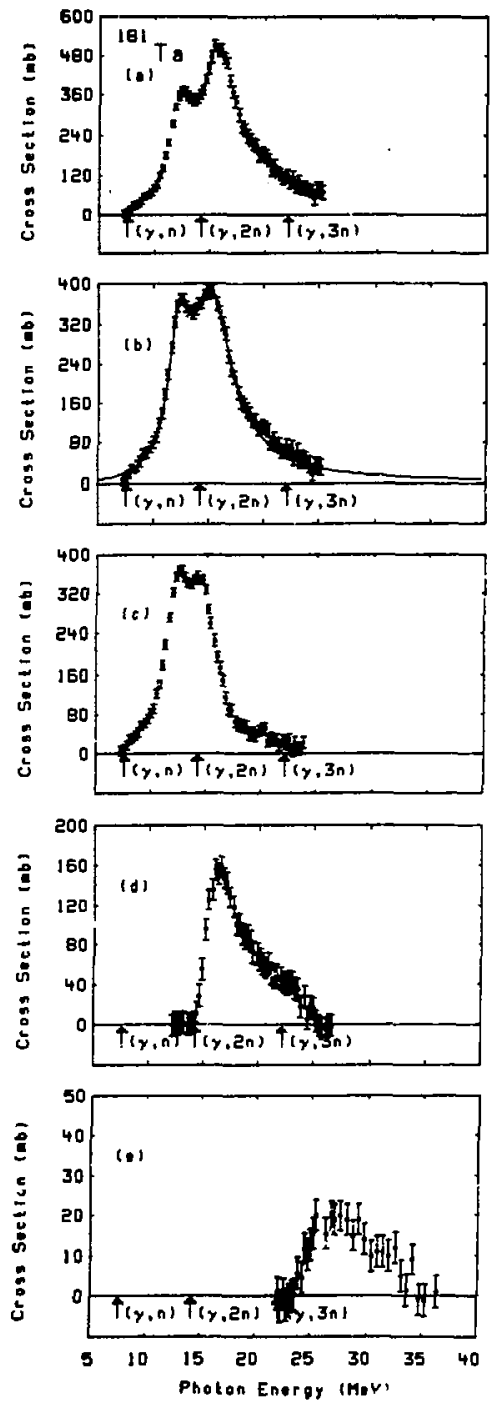

Fig. 143A 

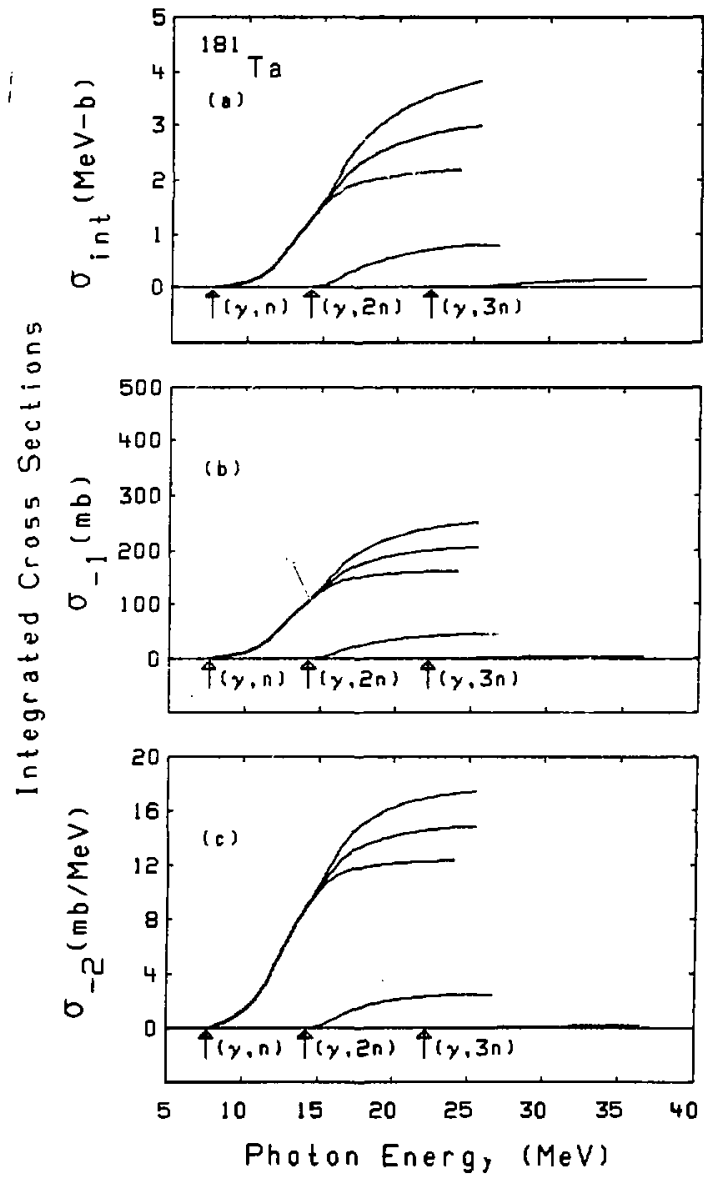

Fig. 1438 

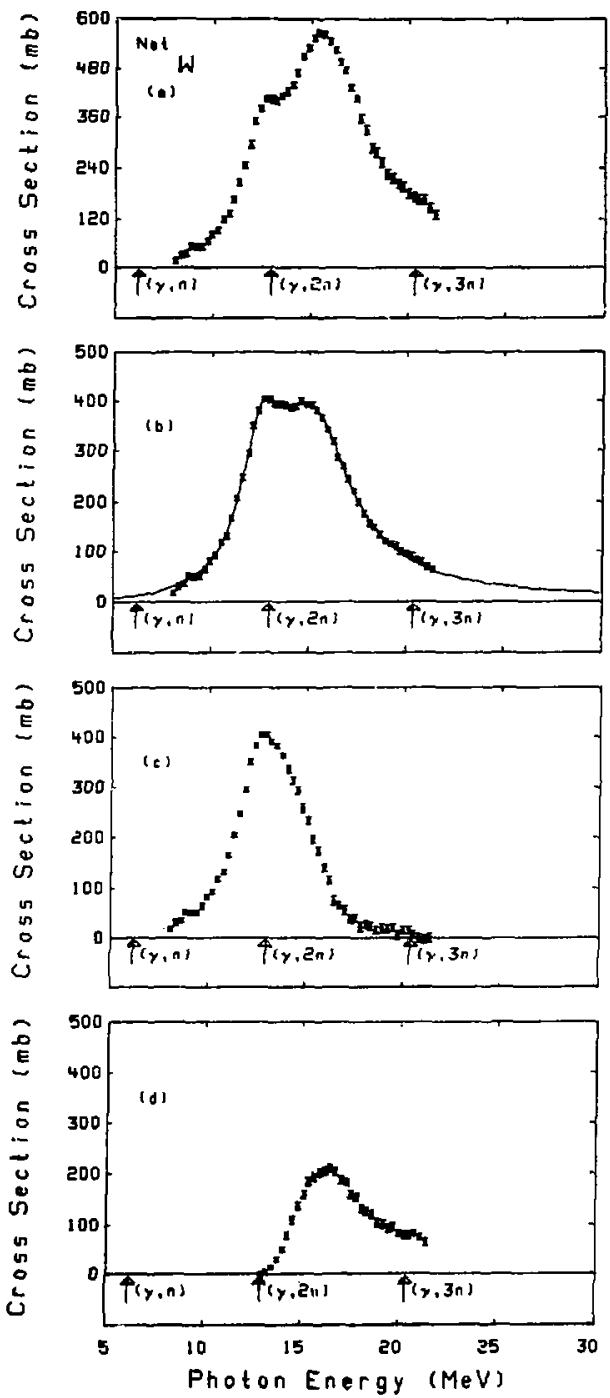

Fig. 144A 

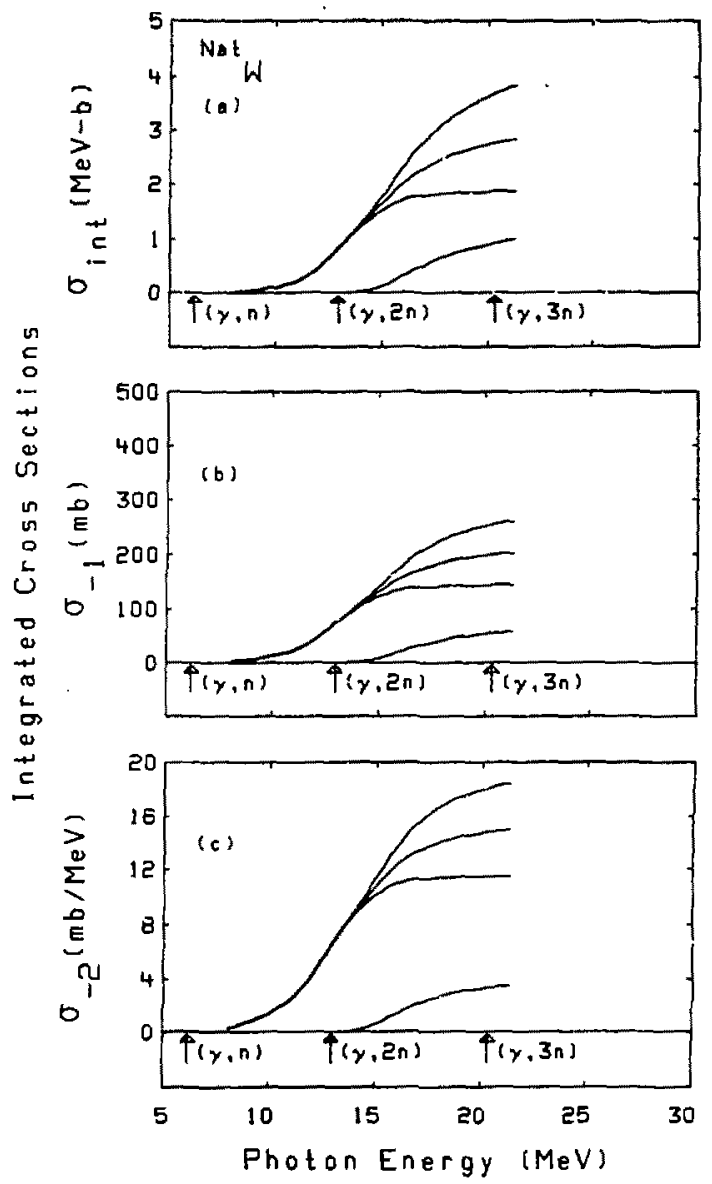

Fig. 144B 

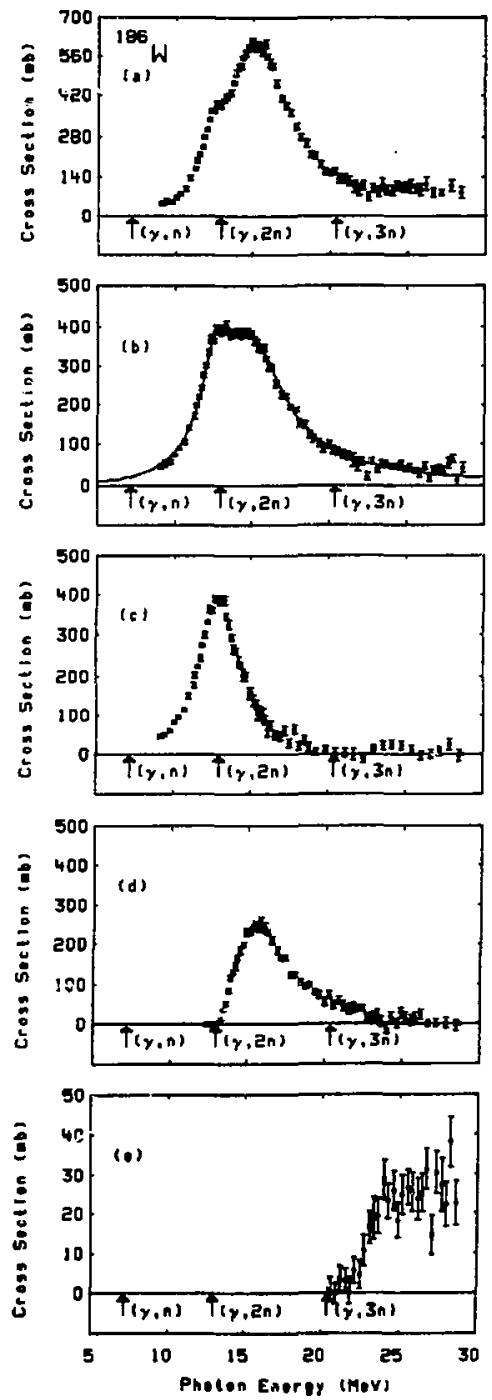

Fig. $145 \mathrm{~A}$ 

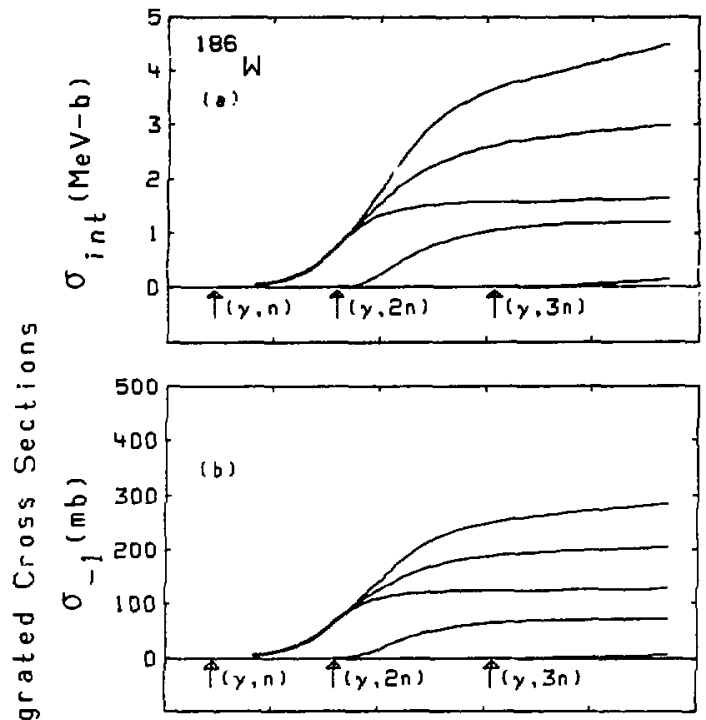

$+$

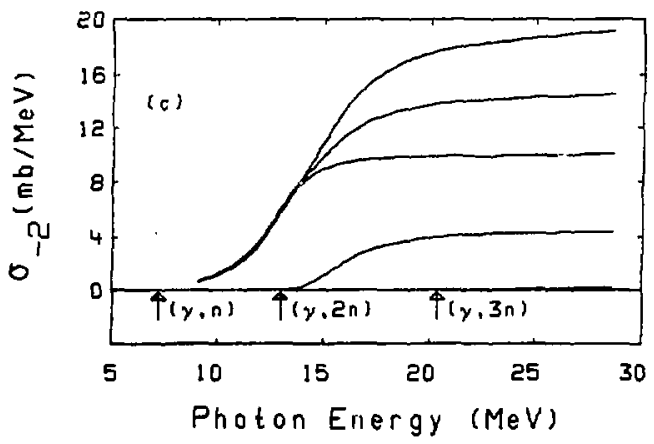

Fig. 145B 

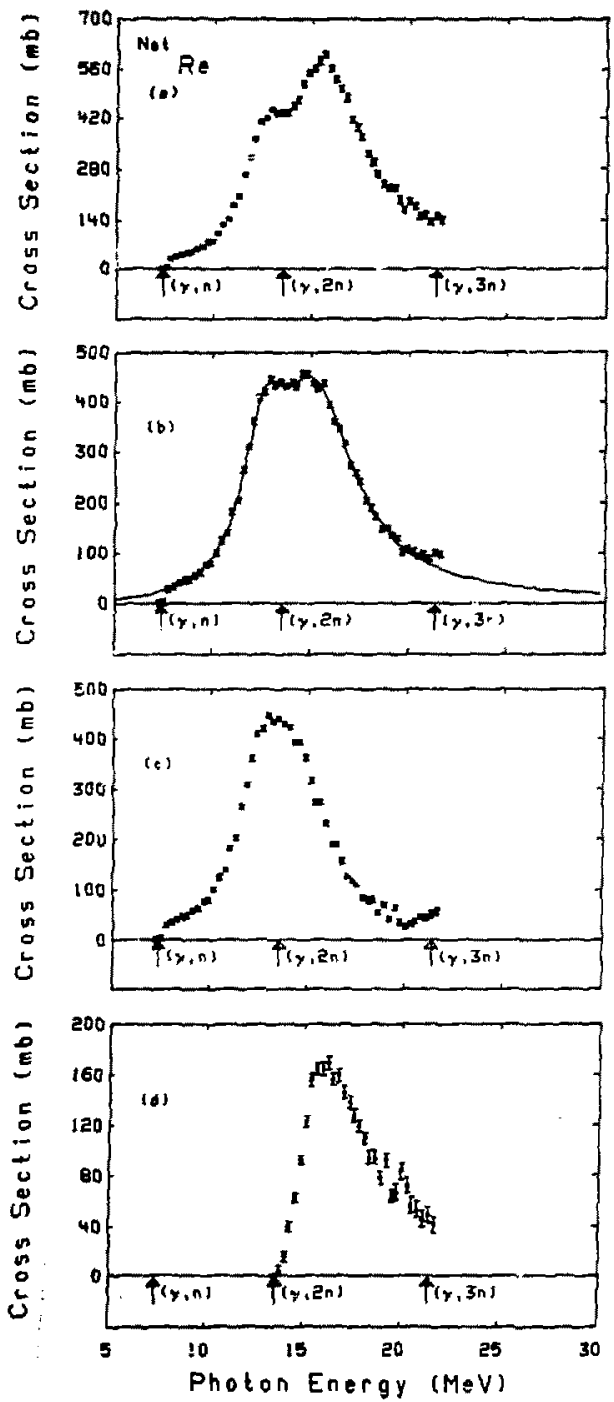

Fig. $246 \mathrm{~A}$ 


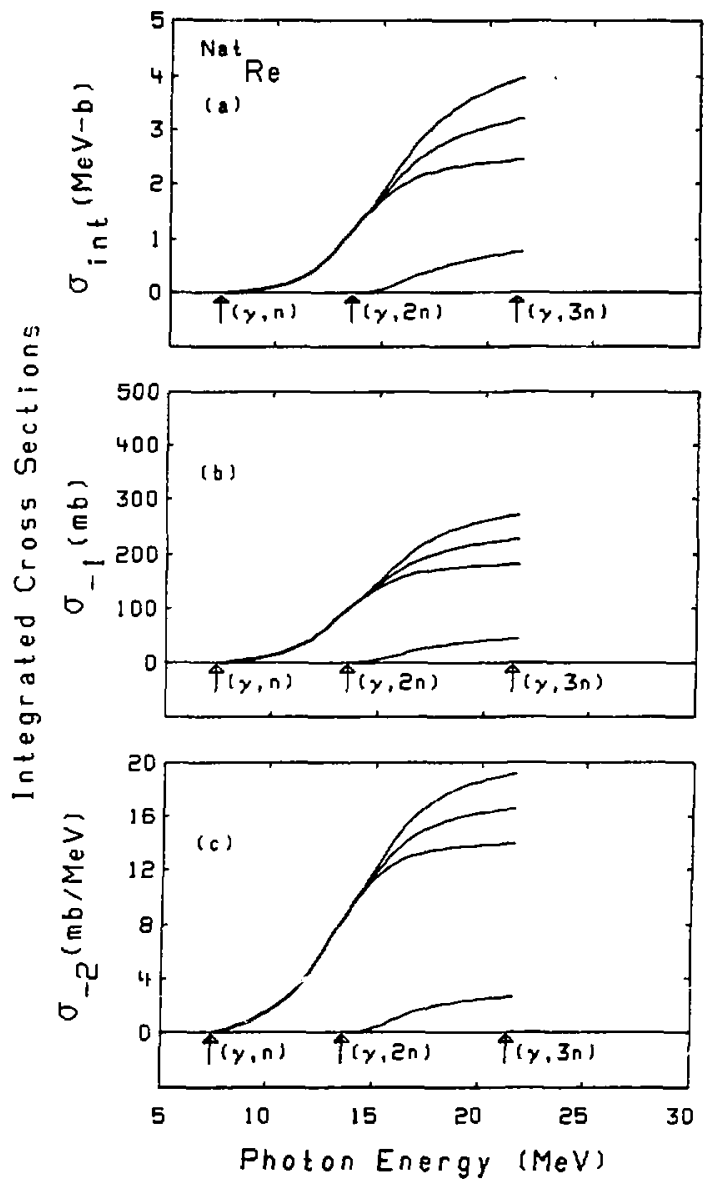

Fig. 146B 


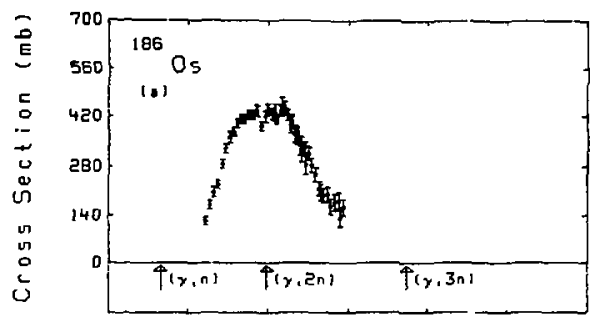

1
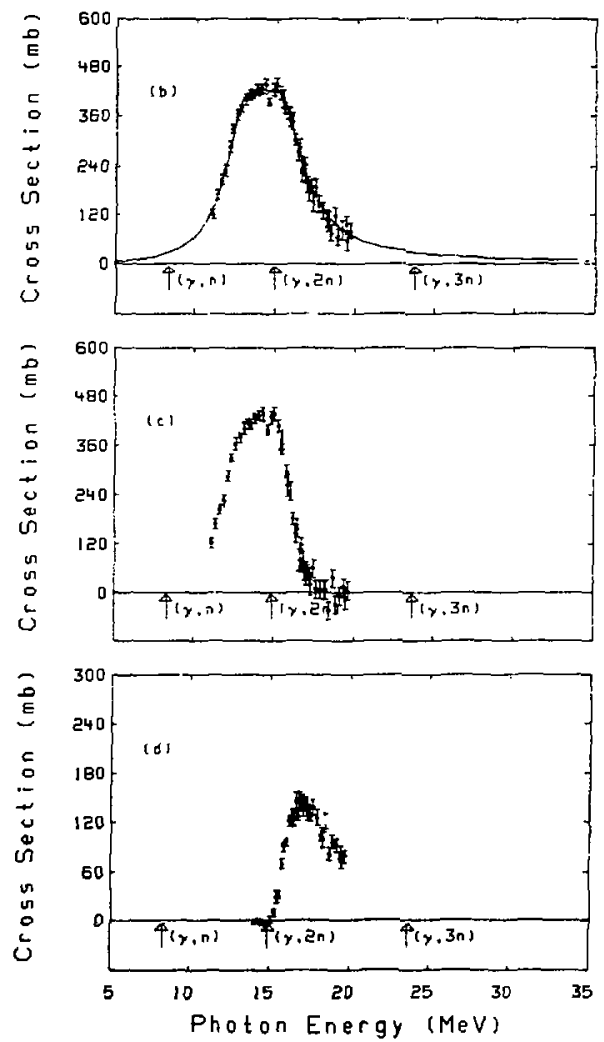

Fig. 147A 

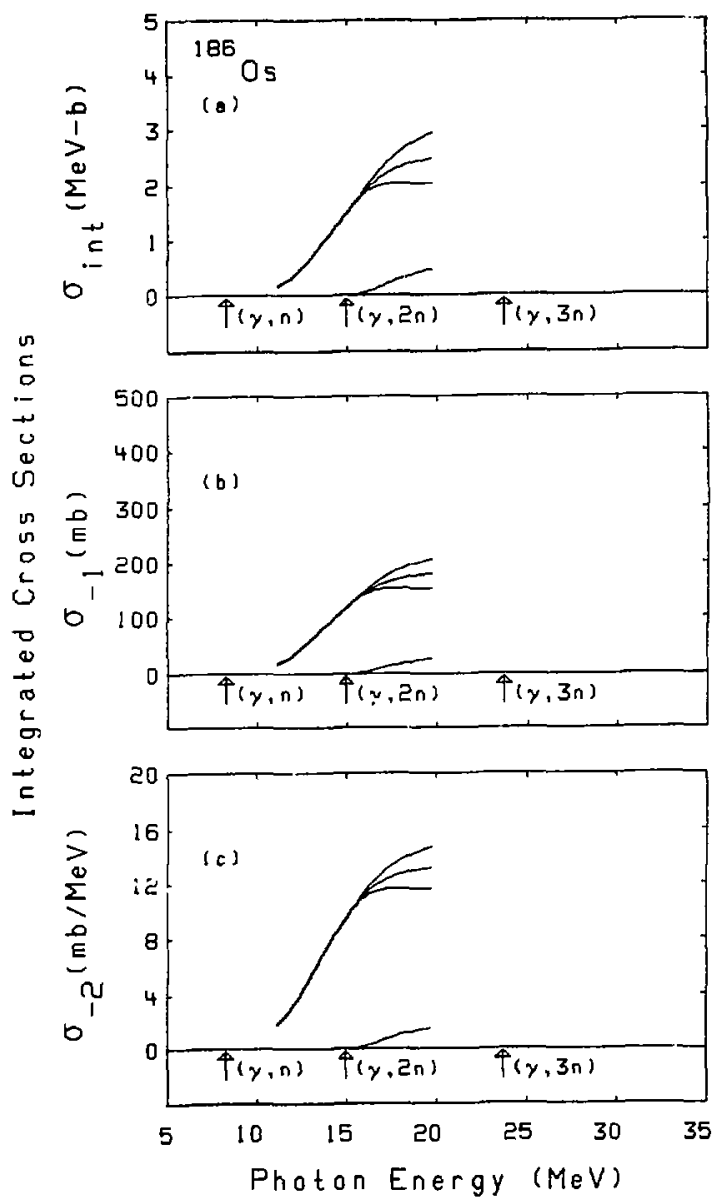

Fig. 147B 

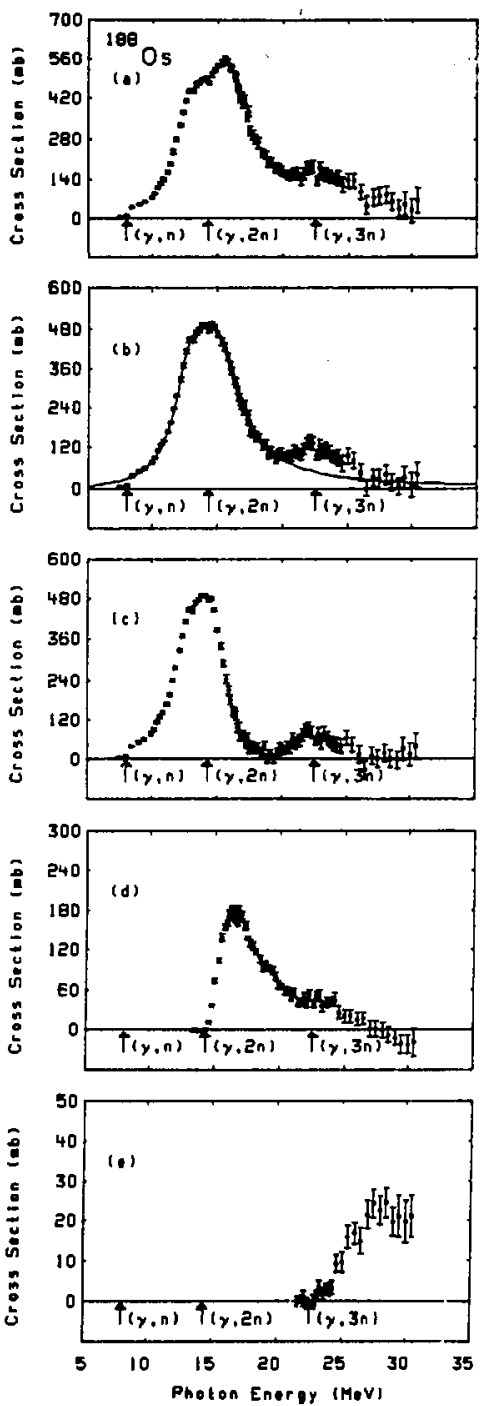

Fig. $148 \mathrm{~A}$ 


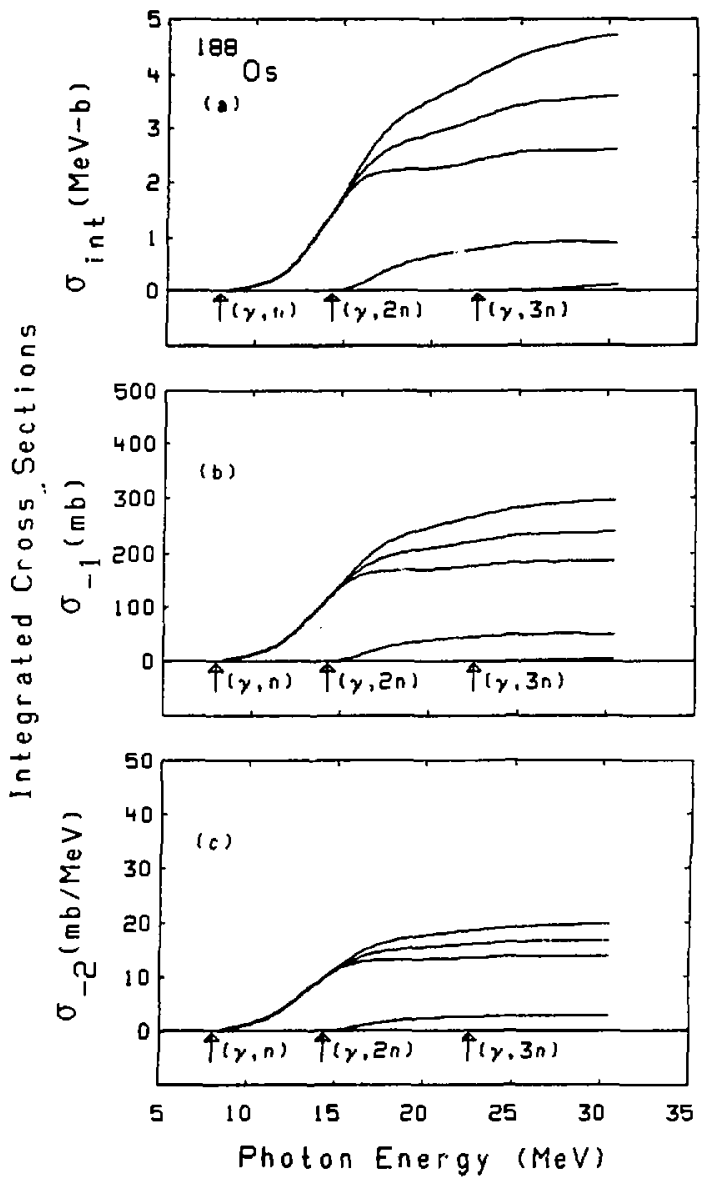

F1g. 1488 

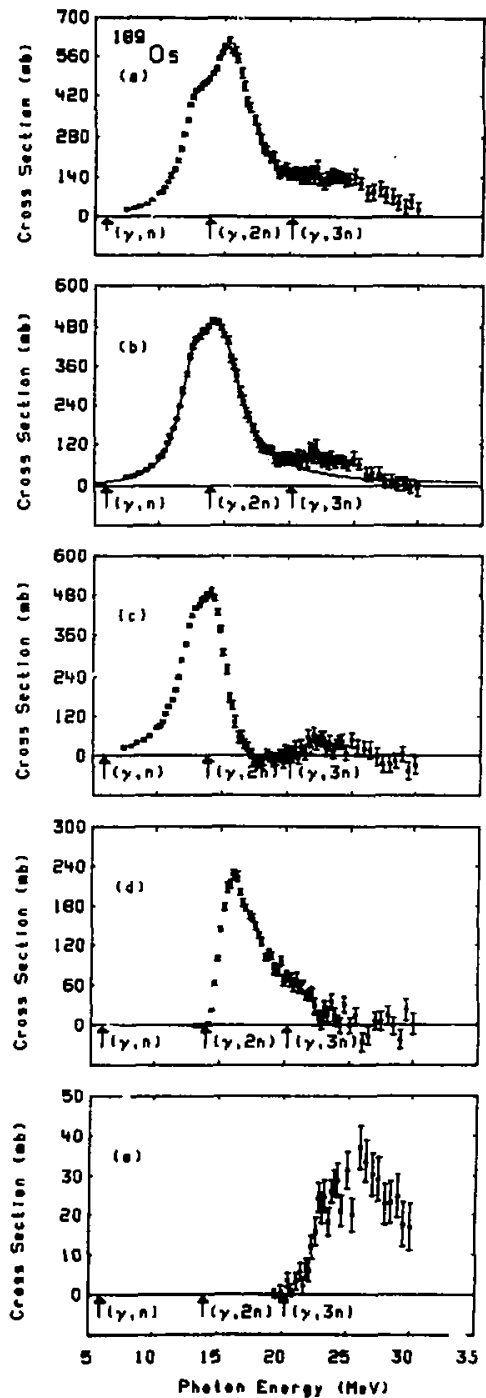

Fig. 149A 


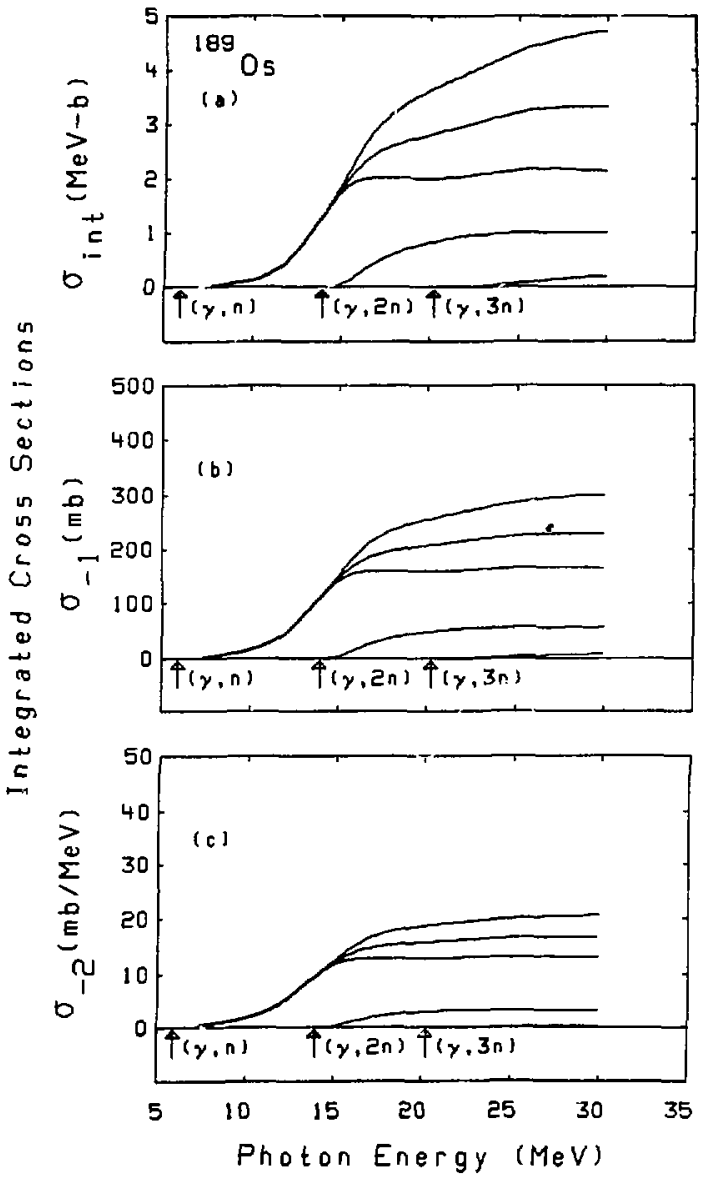

Fig. 1498 

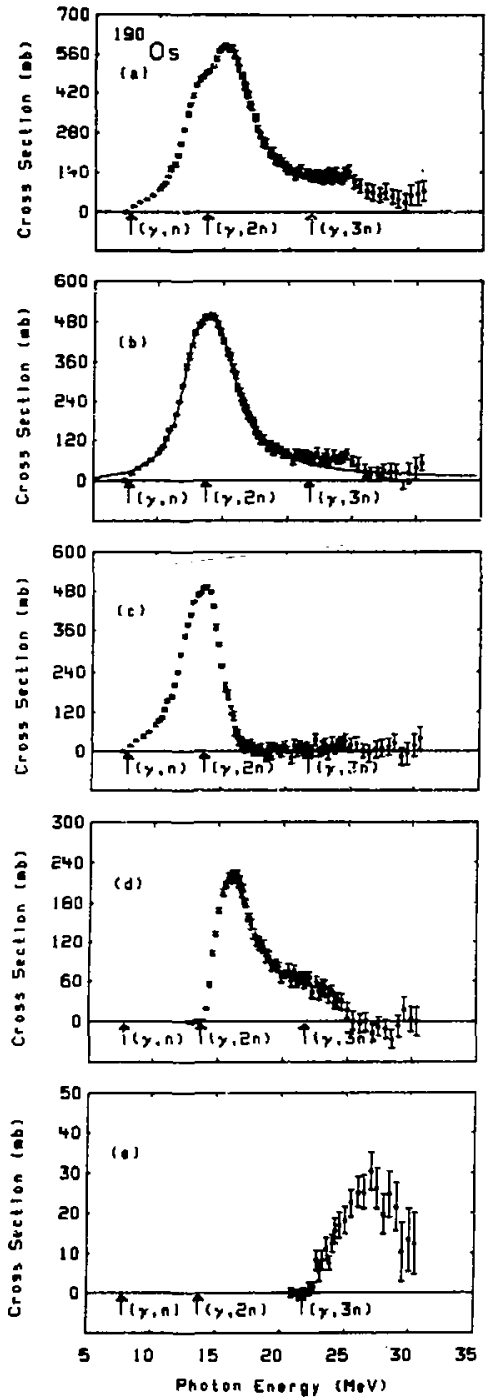

Fig, $150 \mathrm{~A}$ 


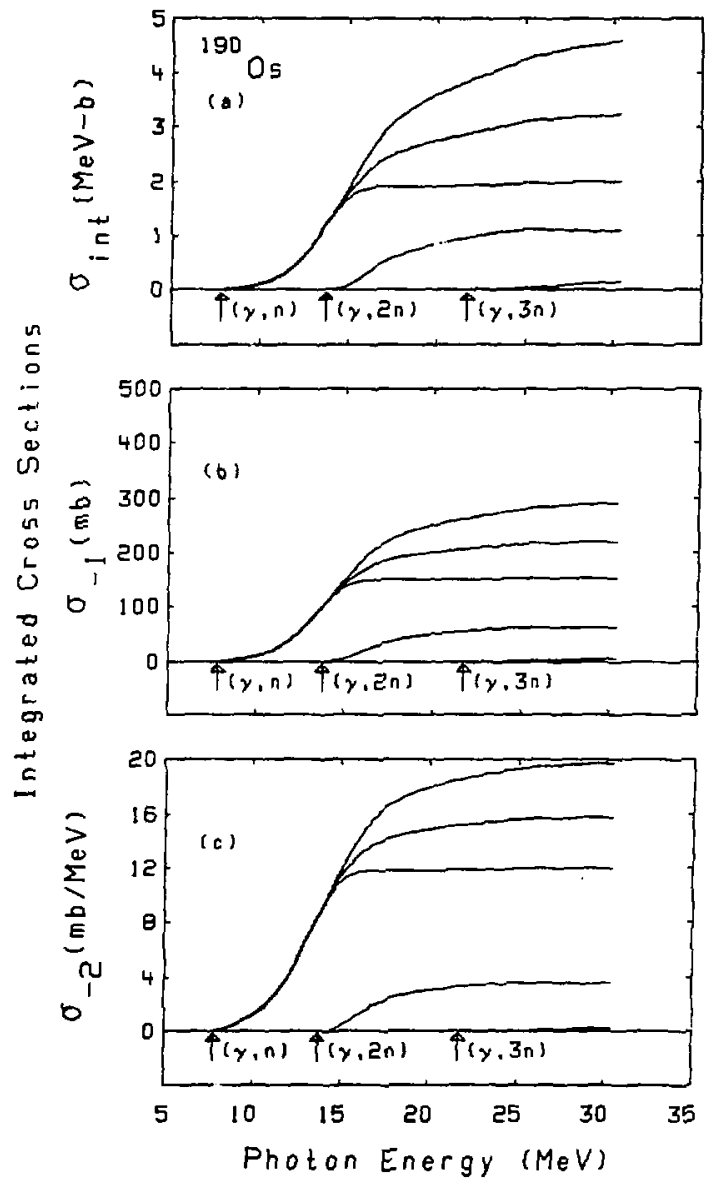

Fig. 150B 

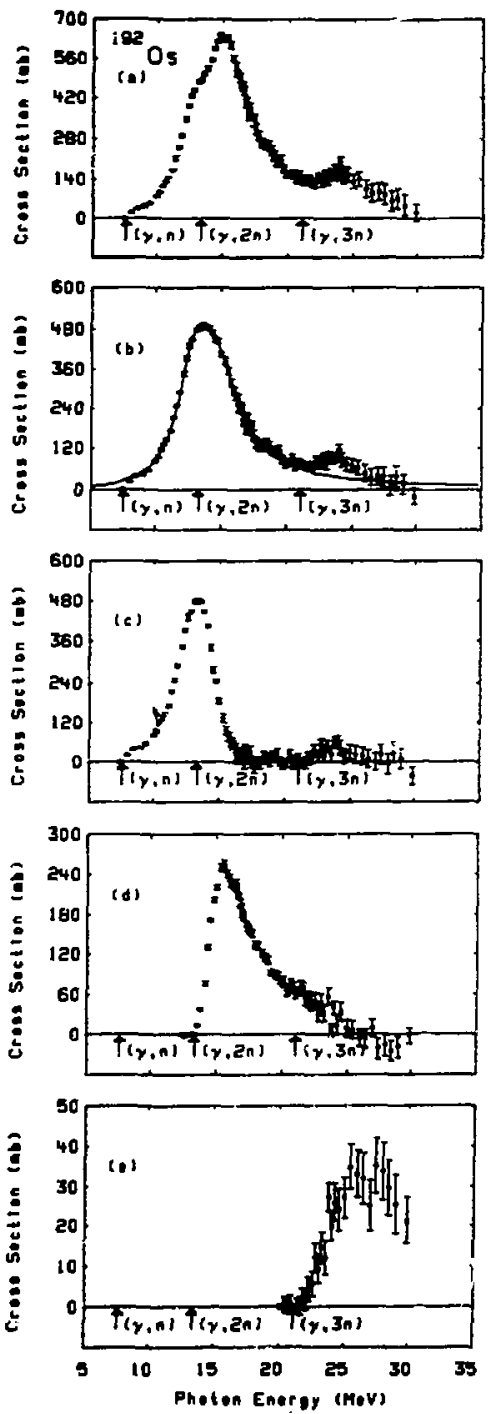

Fin. 151A 

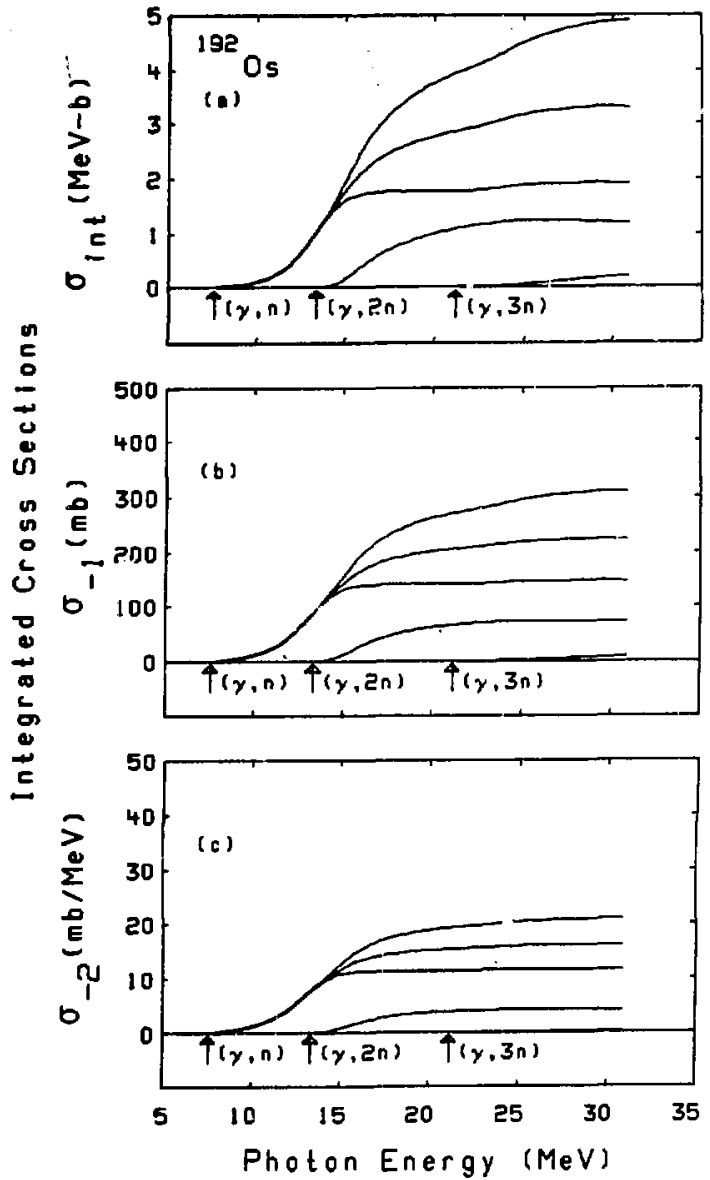

Fig. 151B 

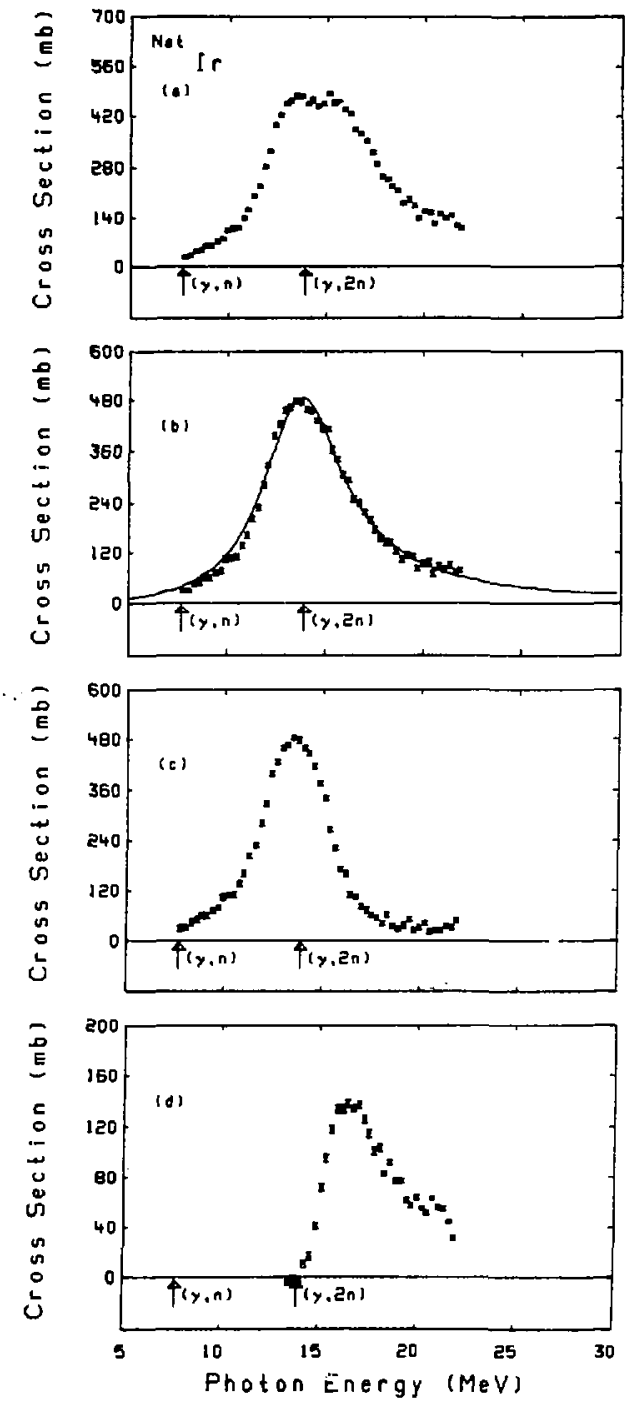

Fig. 152A 


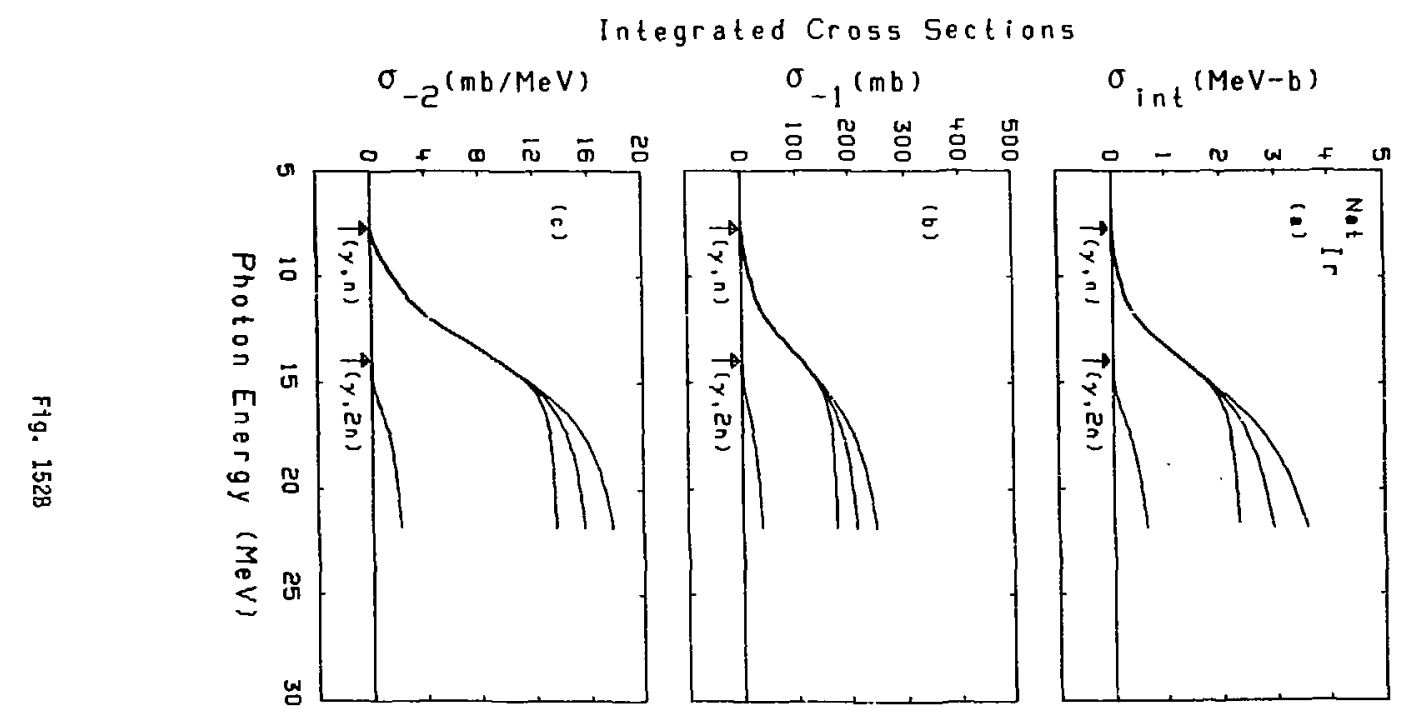



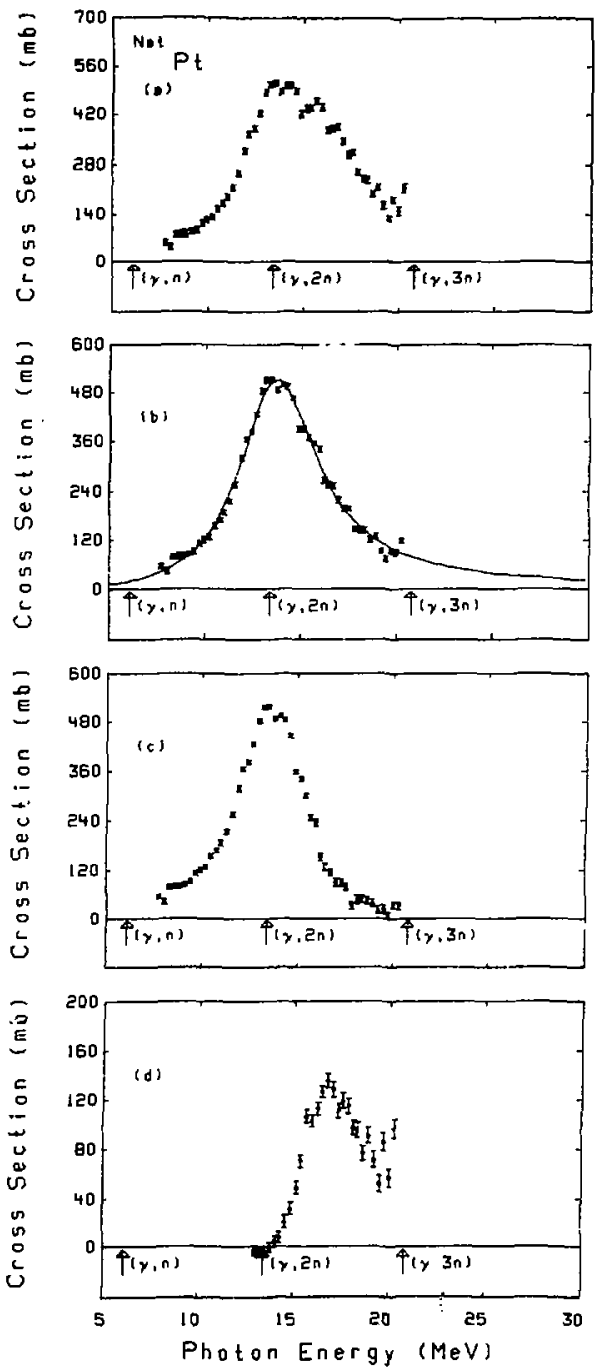

Fig. 153A 


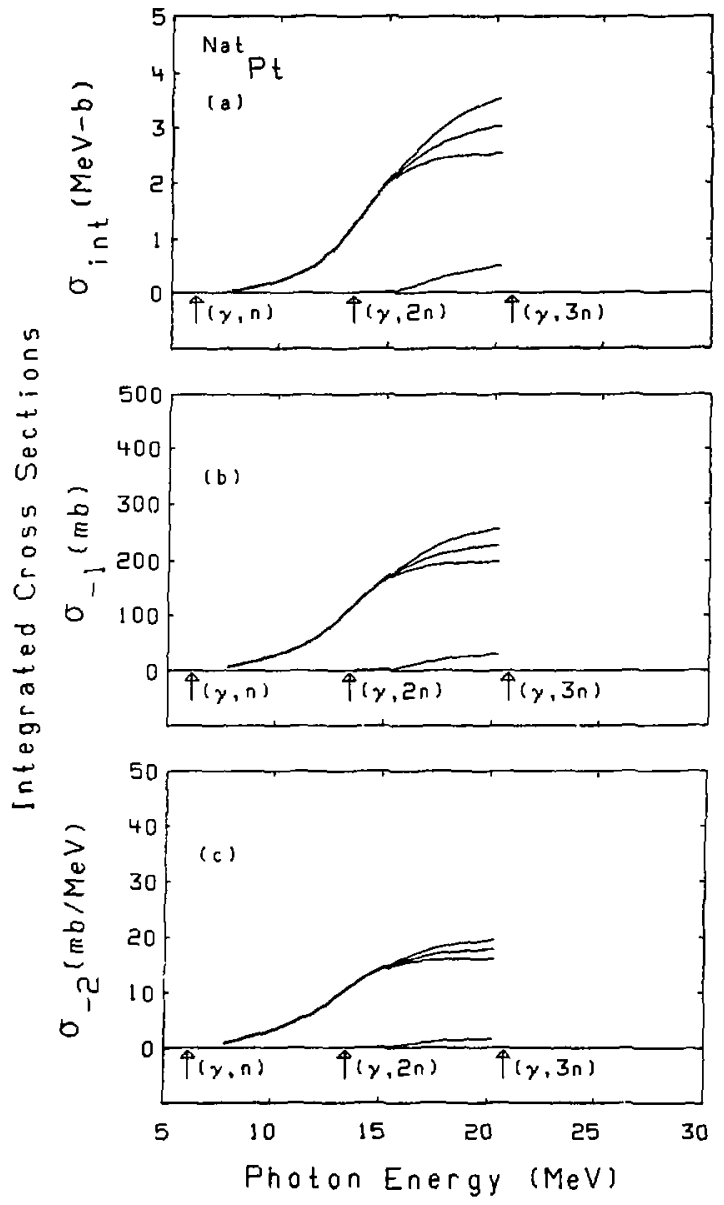

Fig. 153B 

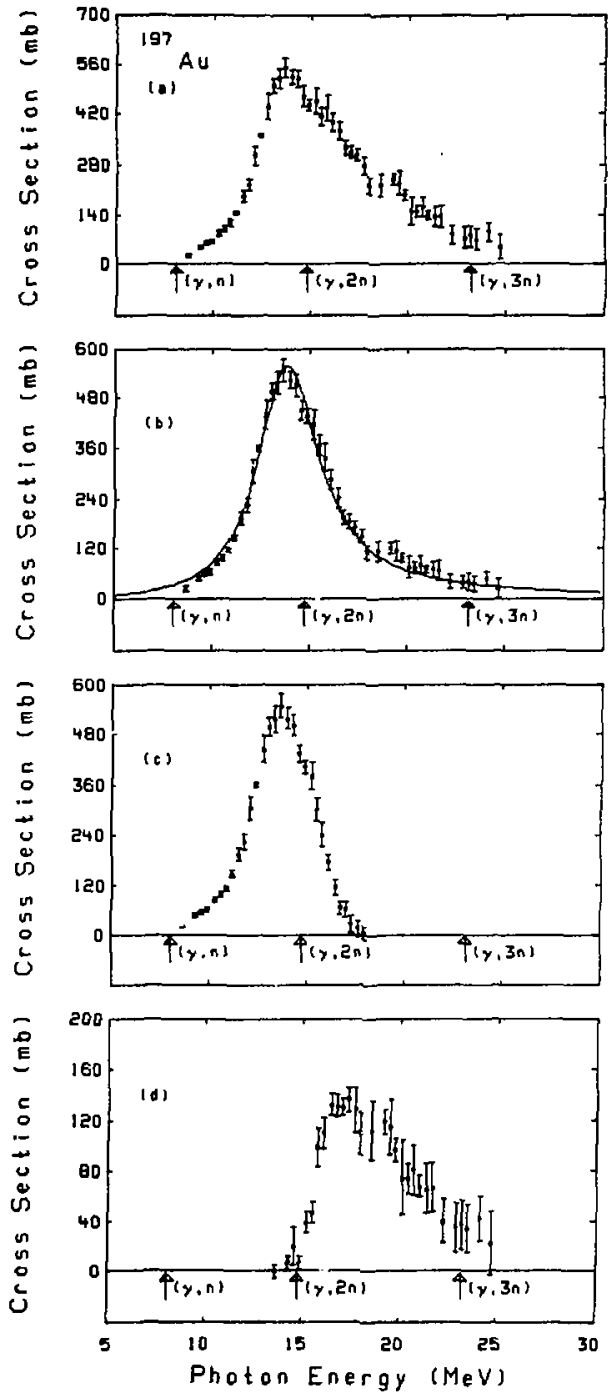

Fig. 154A 


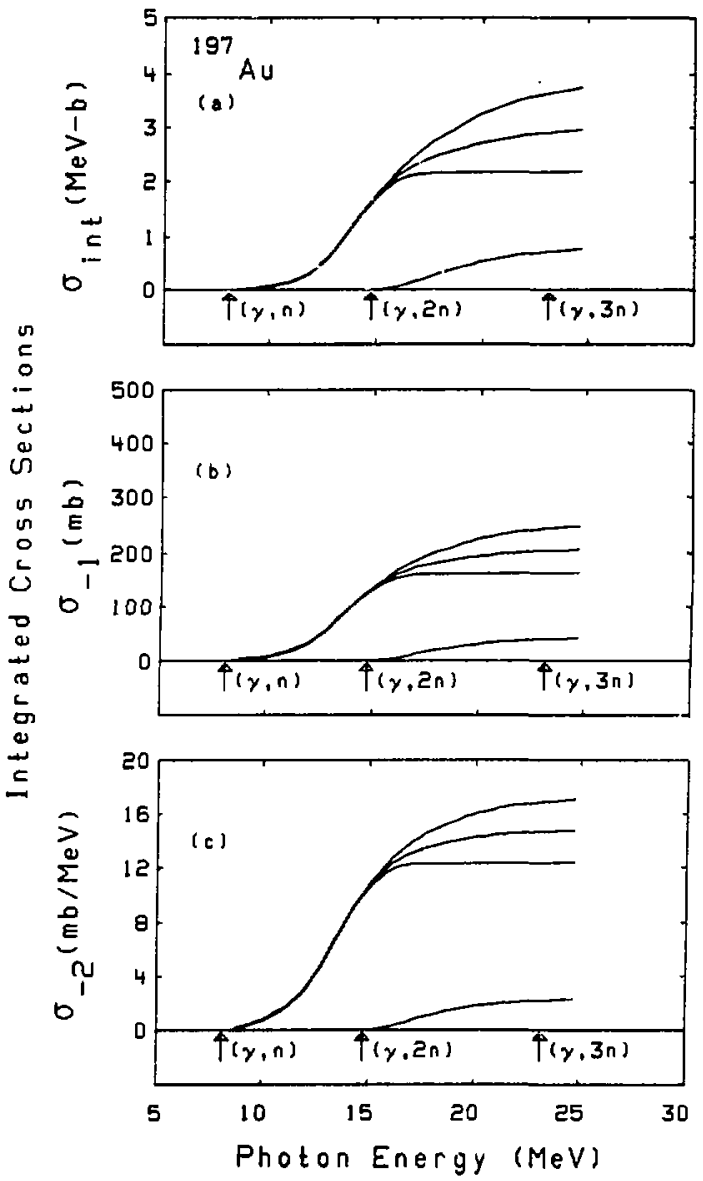

Fig. 154B 

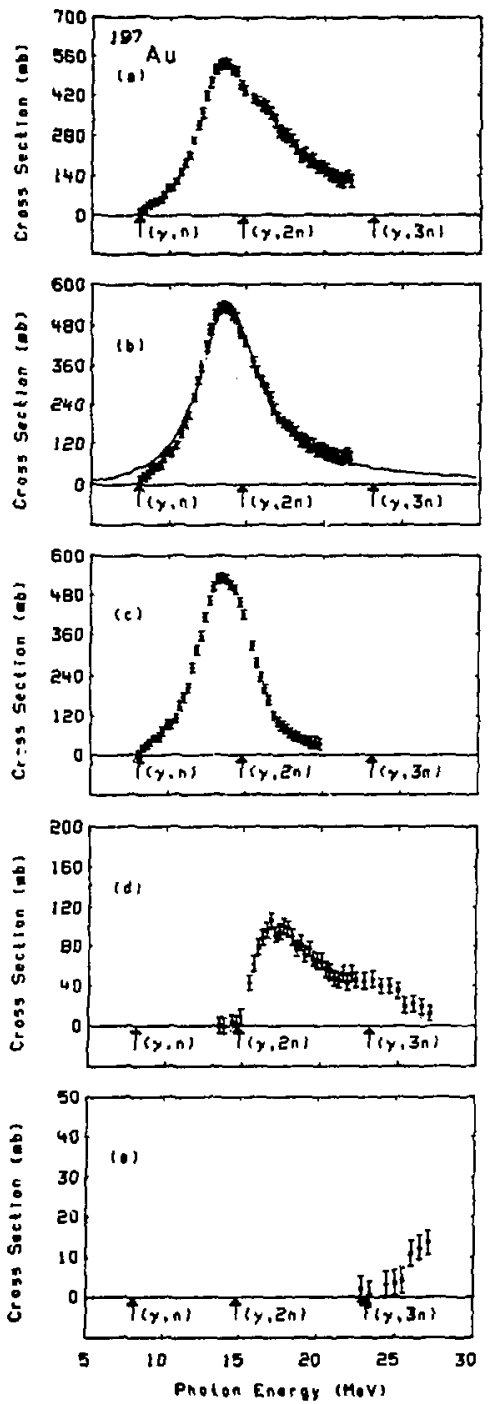

Fig. 155A 


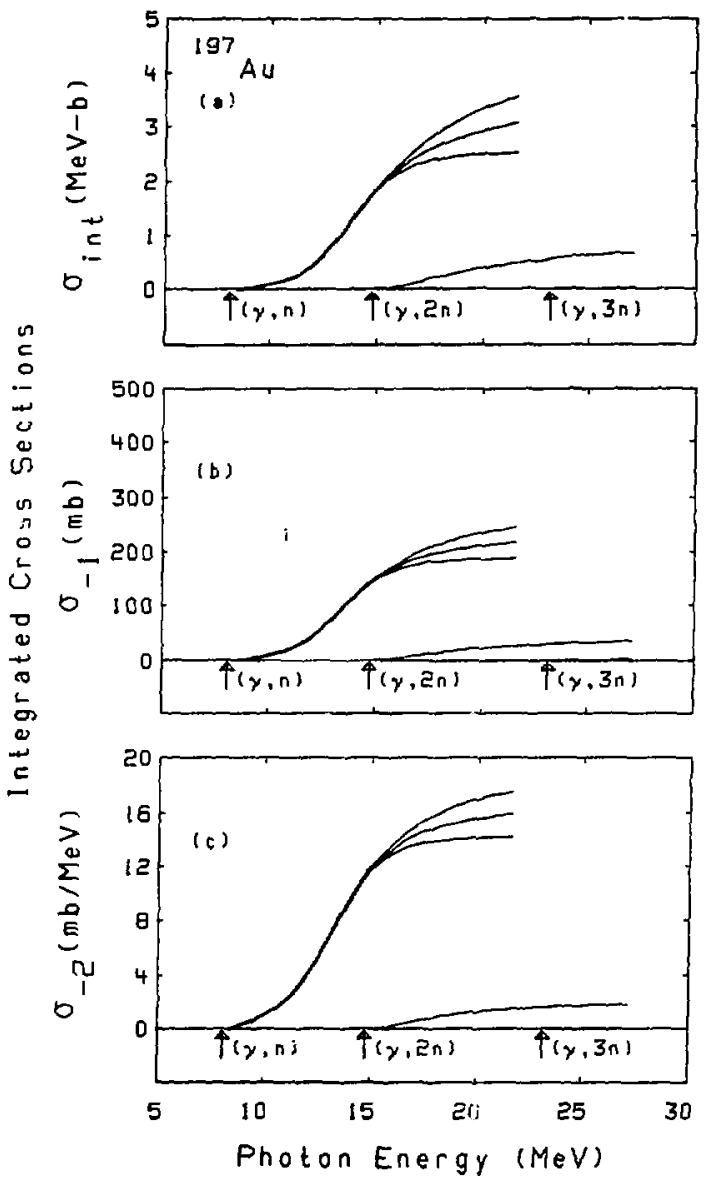

Fig. 155B 

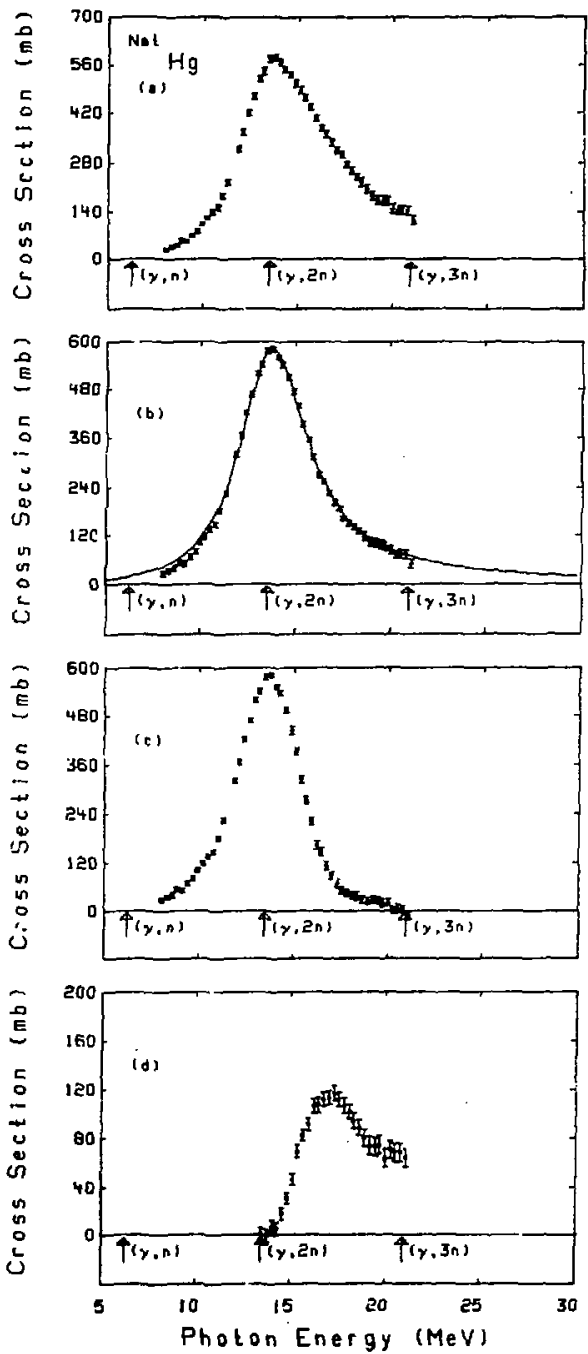

Fig. 156A 


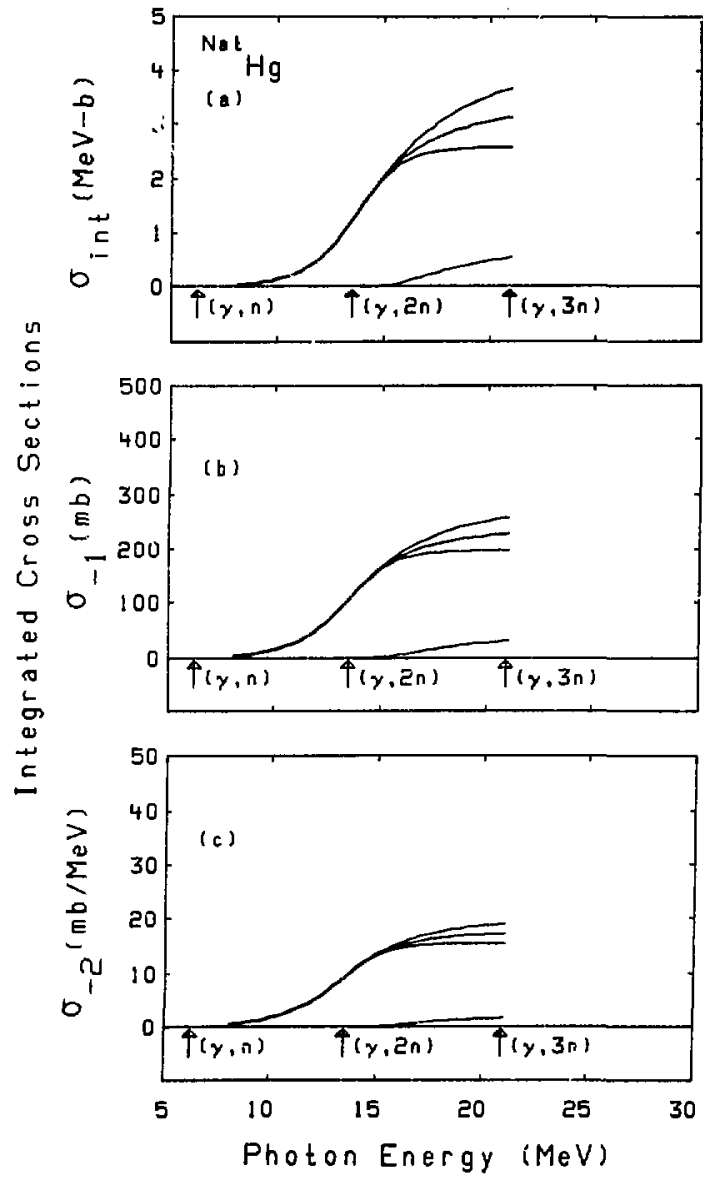

Fig. 156B 

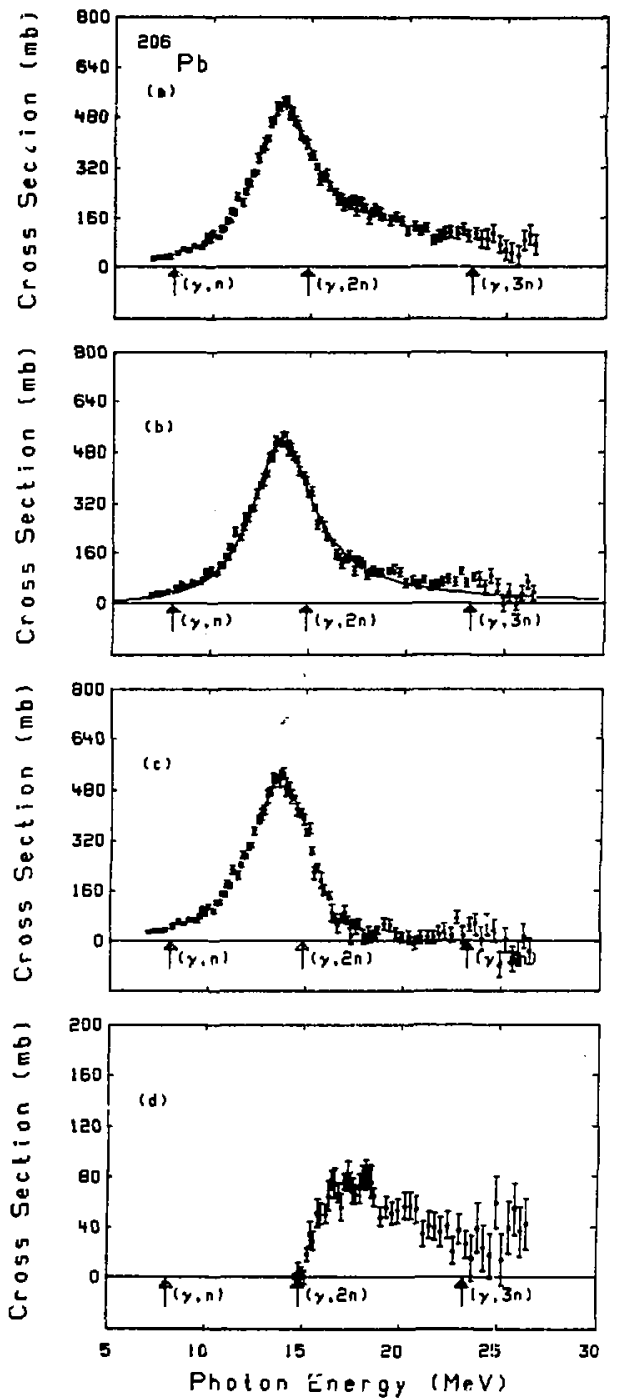

Fig. $157 \mathrm{~A}$ 


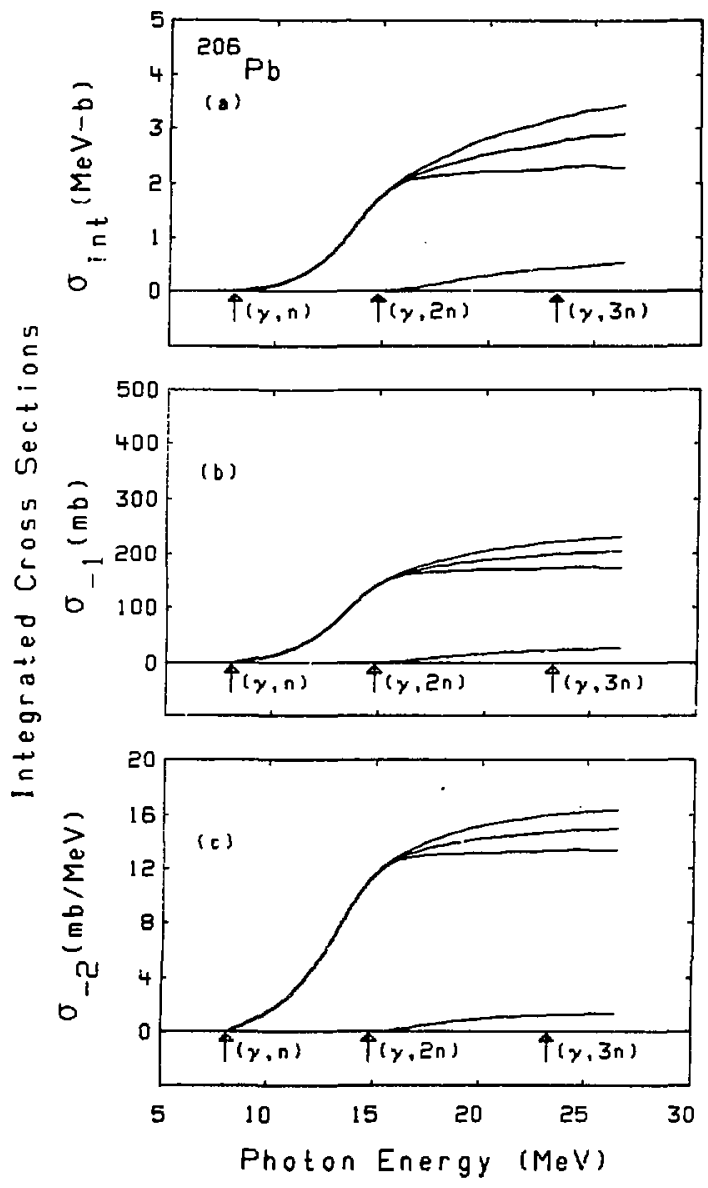

Fig. 157B 

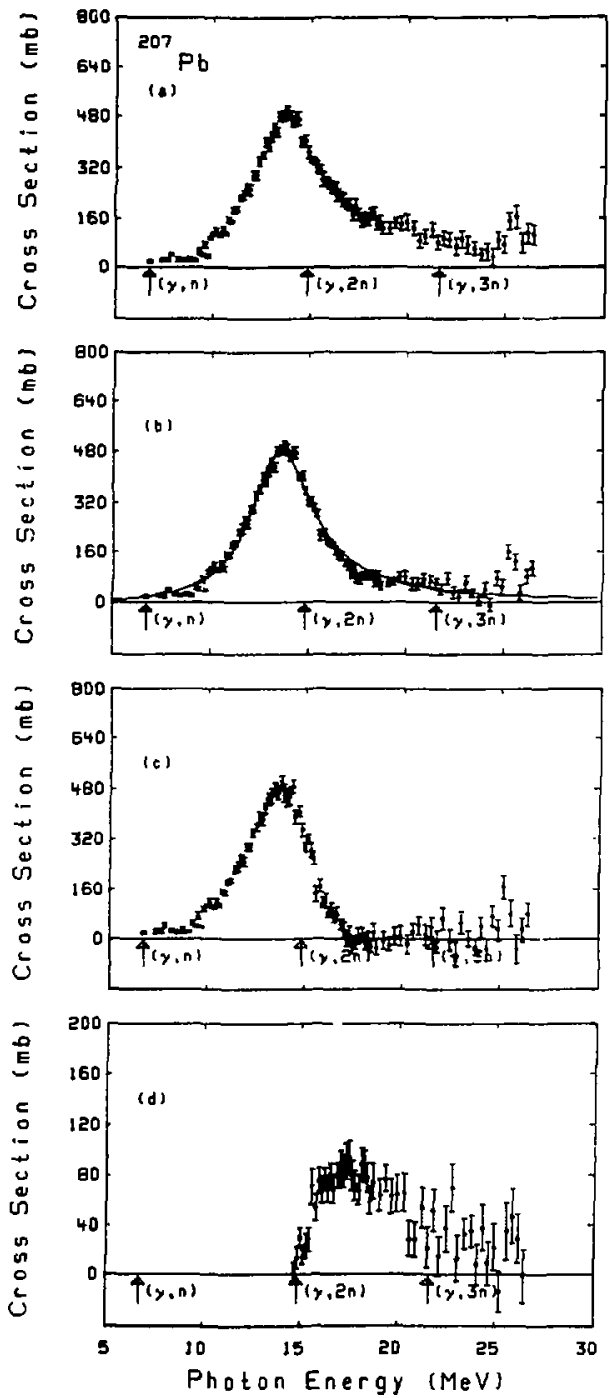

Fig. 158A 

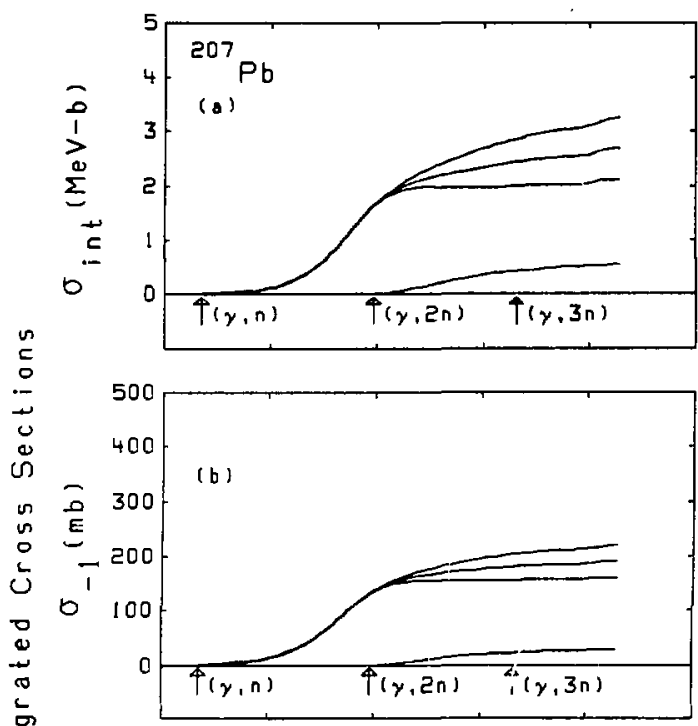

둘

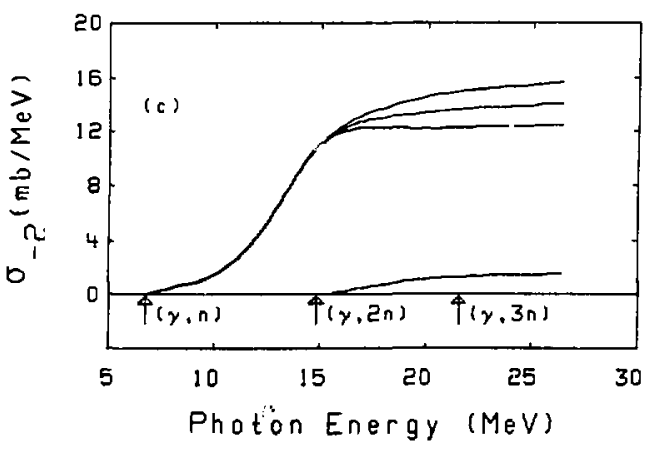

Fig. $158 B$ 

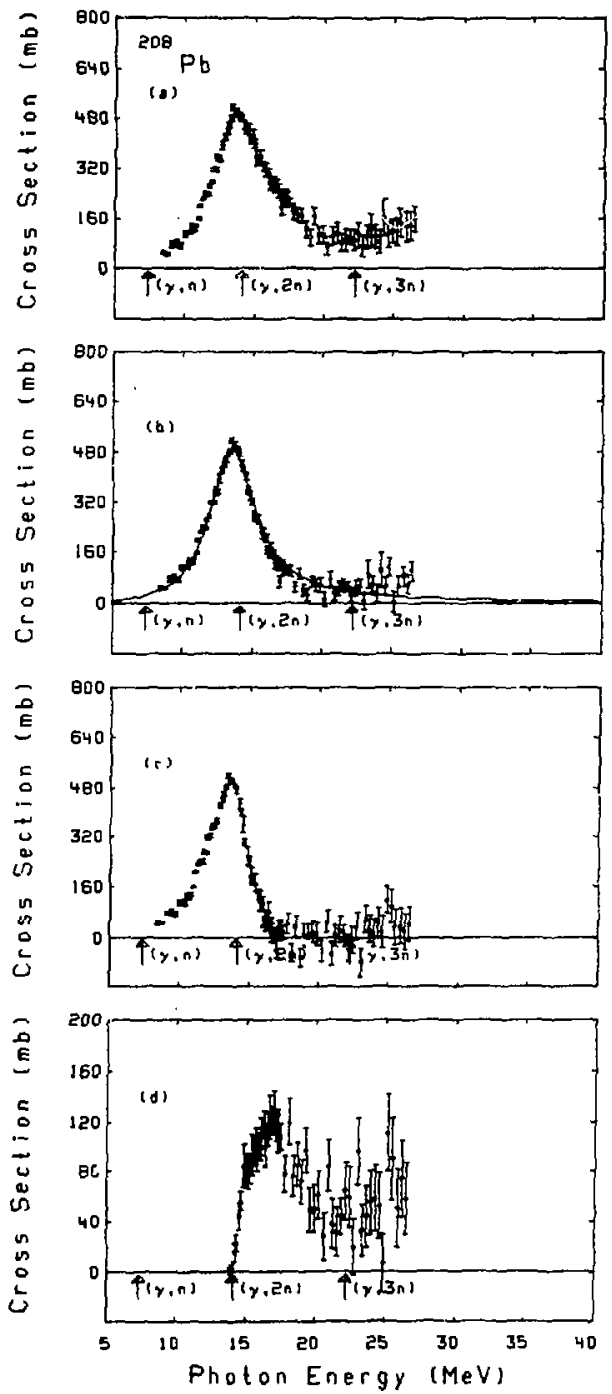

Fig. 159A 


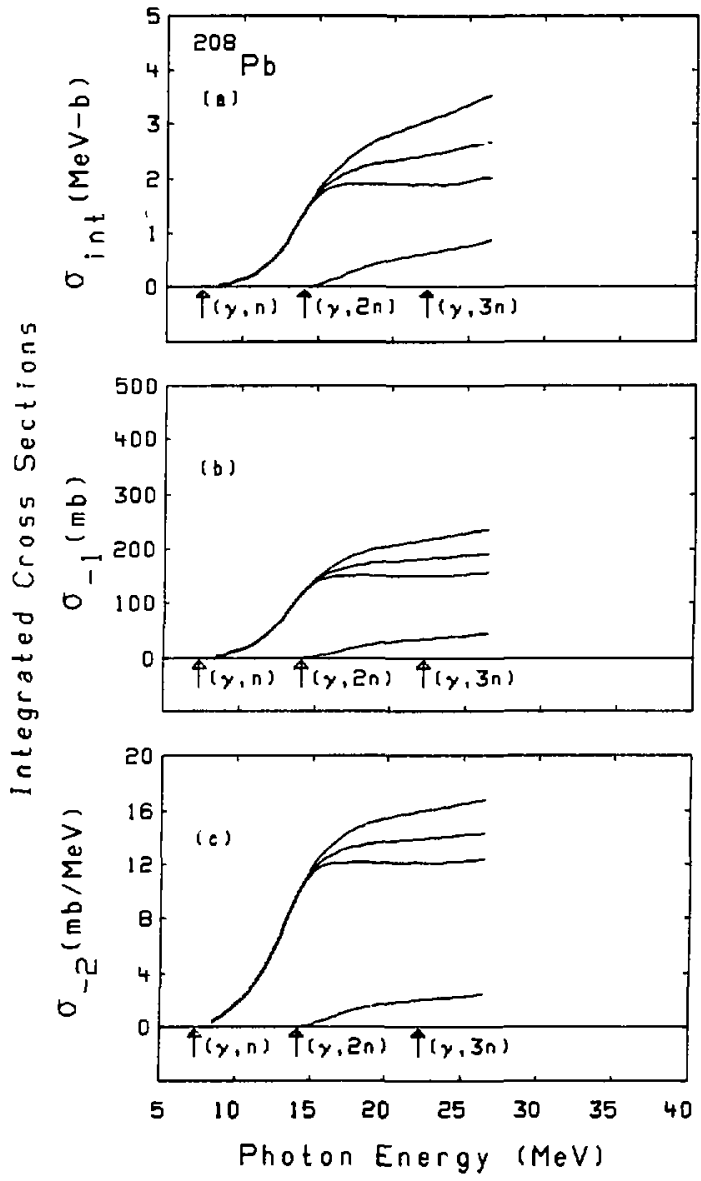

Fig. 159B 

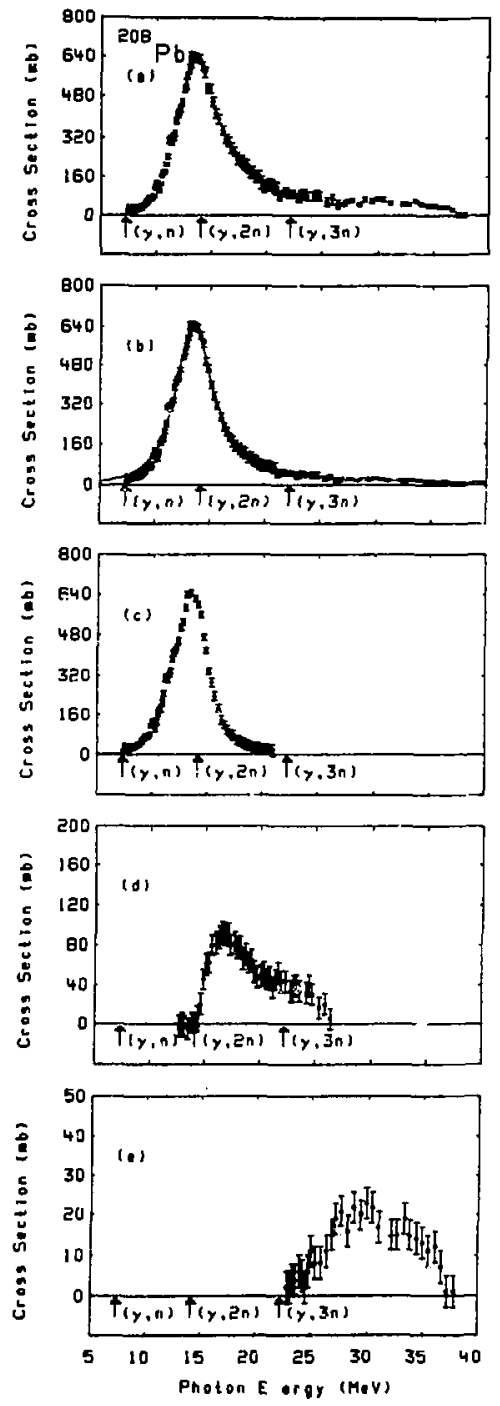

Fig. 160A 

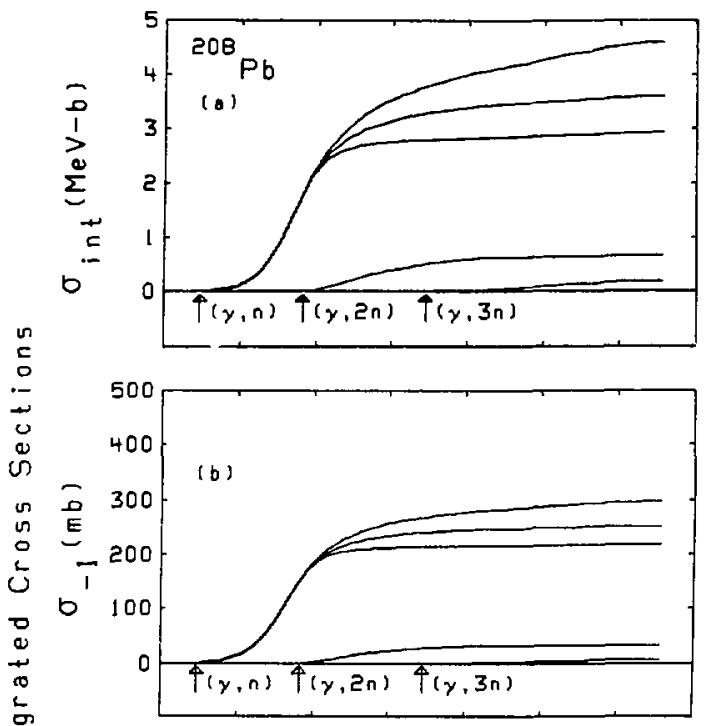

$+$

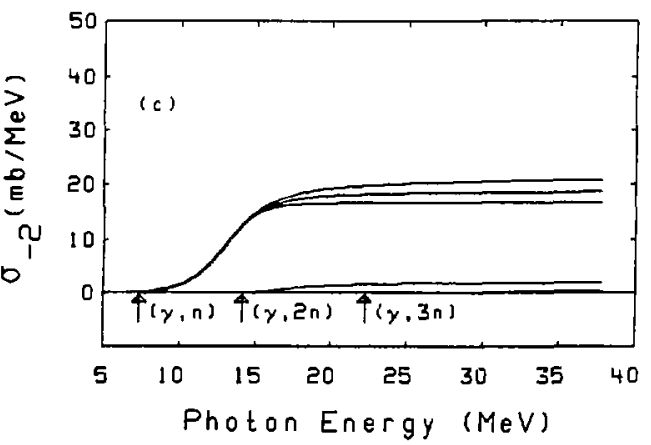

Fig. 160B 


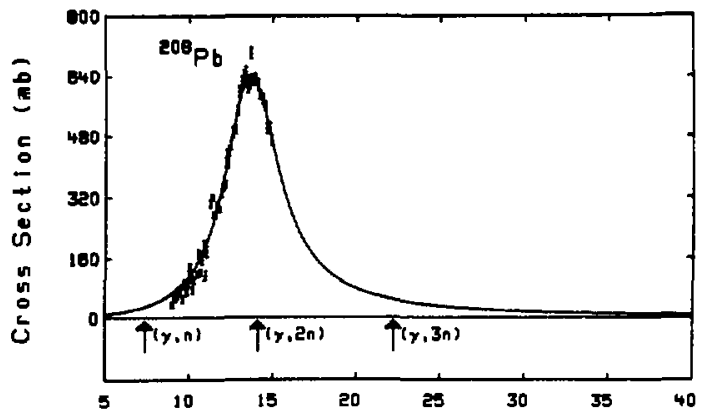

Photon Energy (MeV)

Fig. $161 \mathrm{~A}$ 


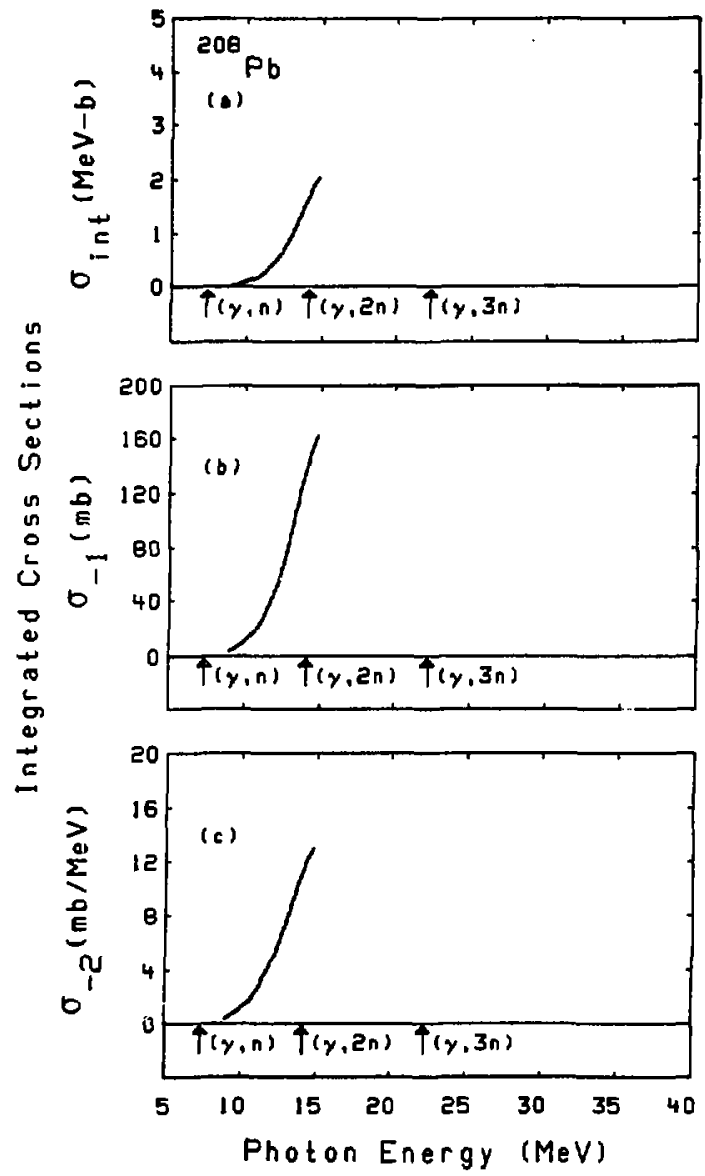

Fig. 161B 

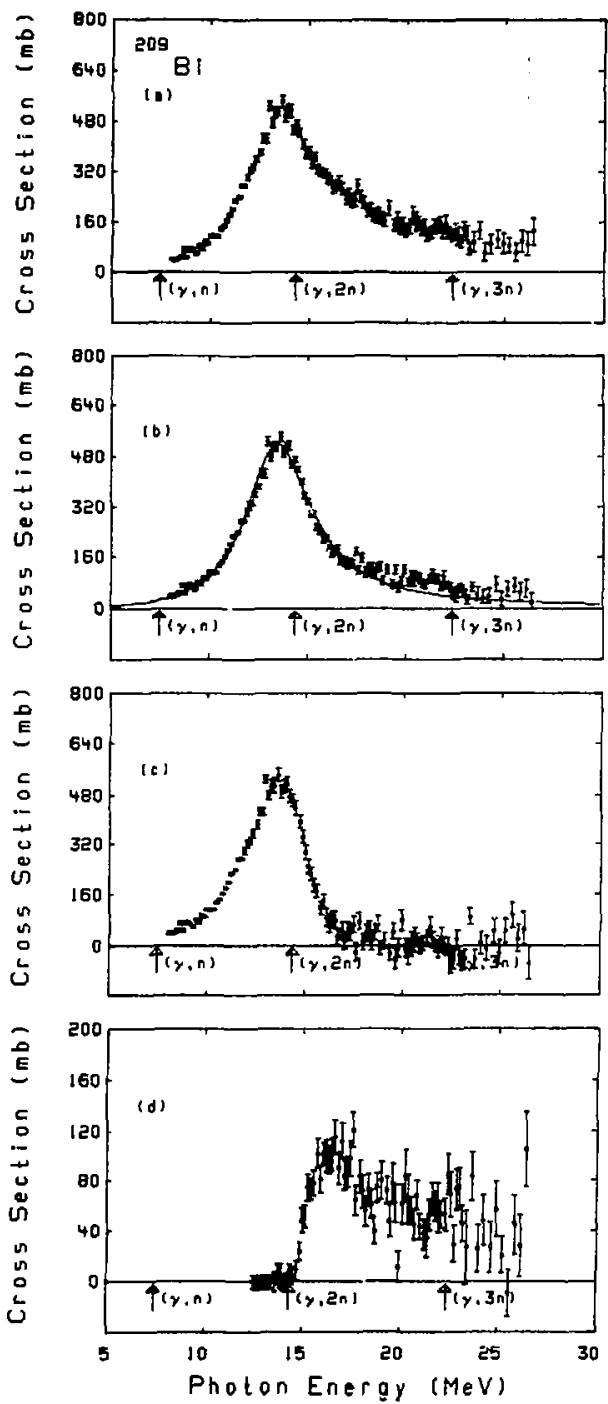

Fig. 162A 

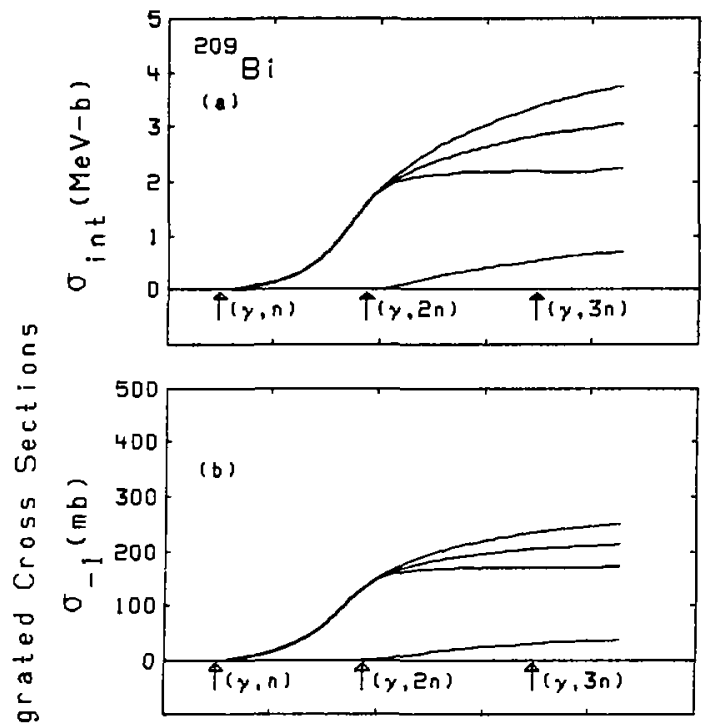

담

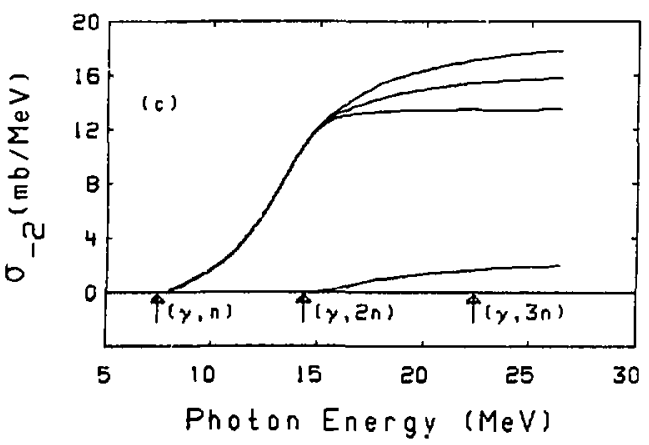

Fig. 162B 


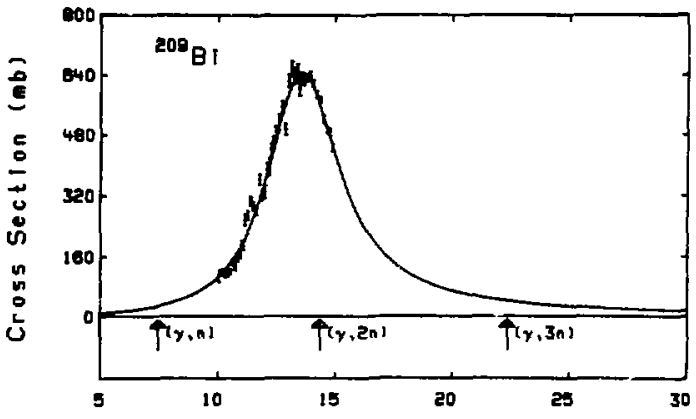

Phokon Energy (MeV)

Fig. $163 A$ 


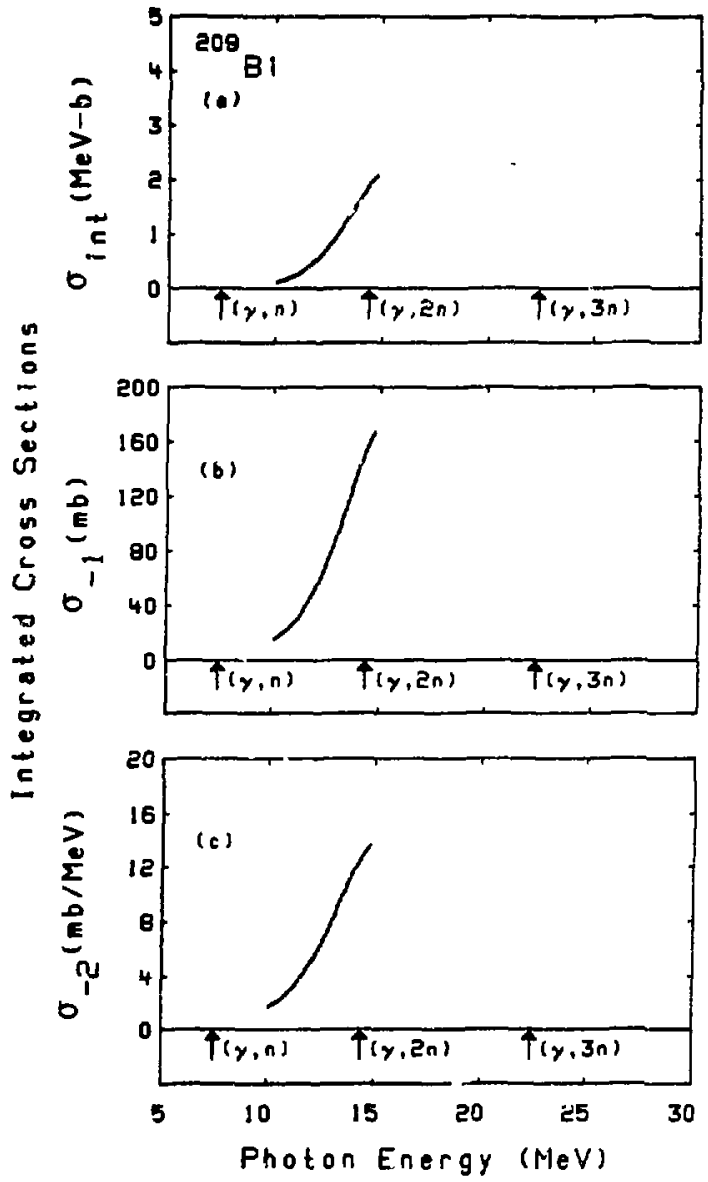

Fig. 1638 

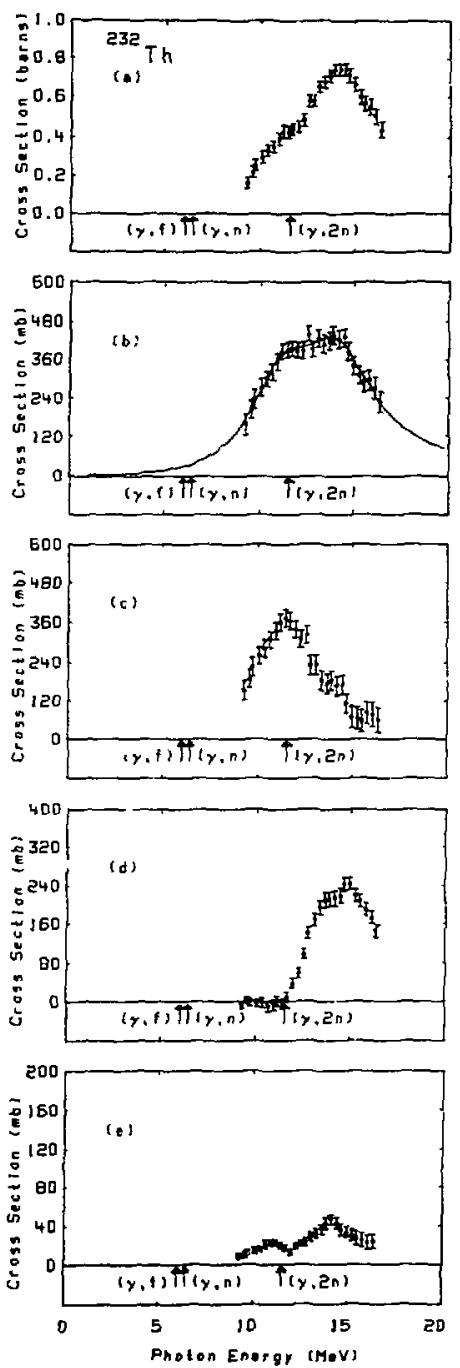

Fig. $164 \mathrm{~A}$ 

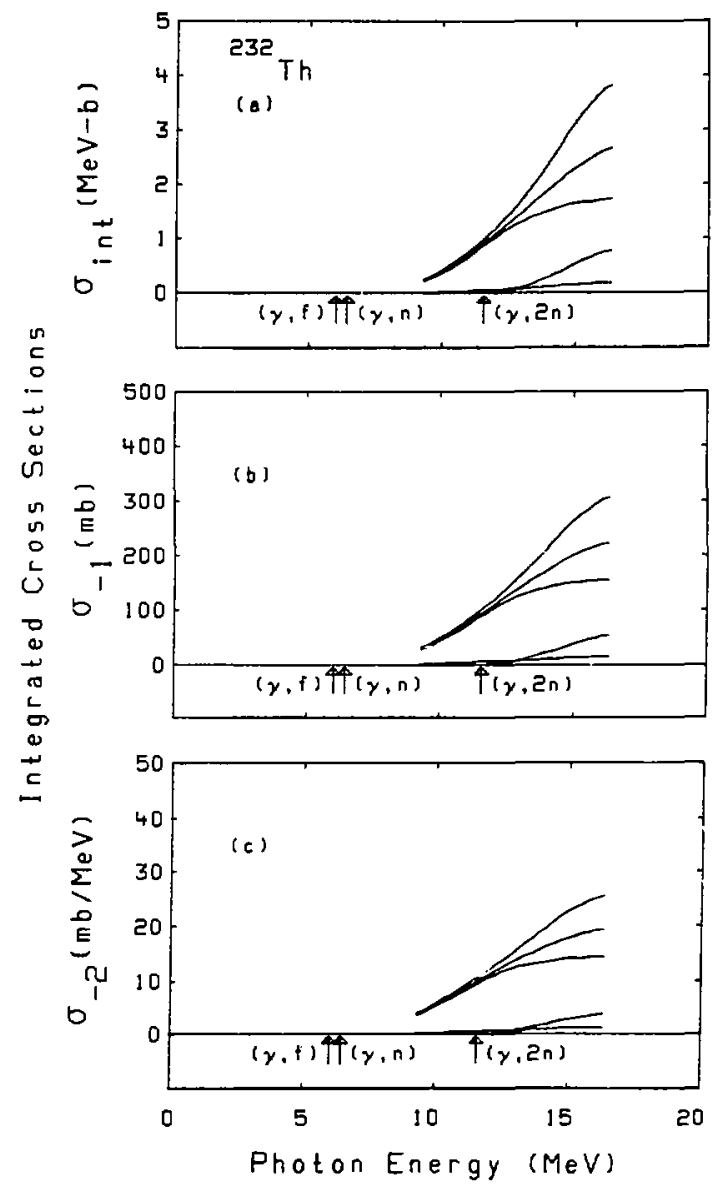

Fig. 164B

$\stackrel{+}{c}$

Photon Energy (MeV) 

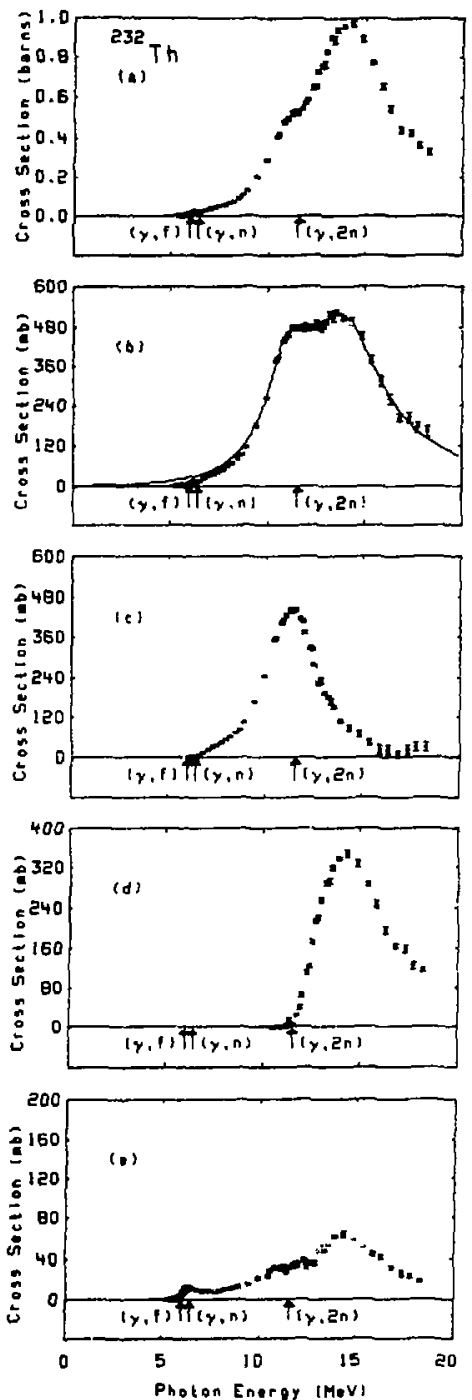

Fig. $165 \mathrm{~A}$ 

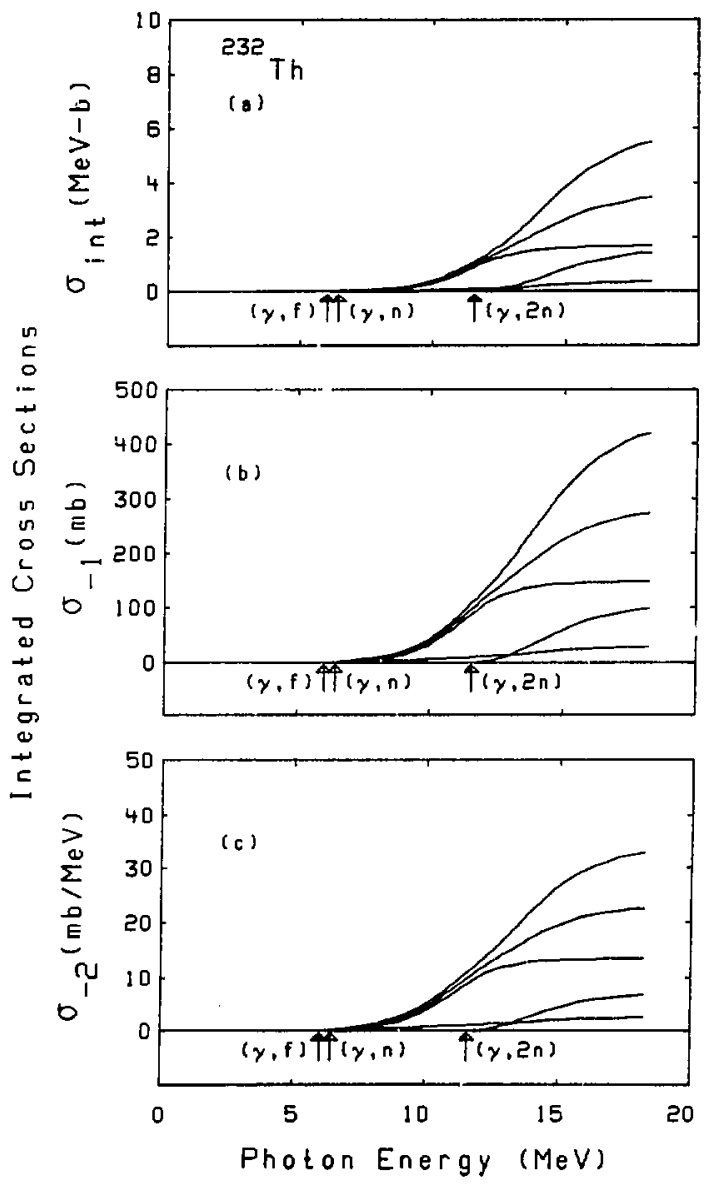

Fig, 165B 

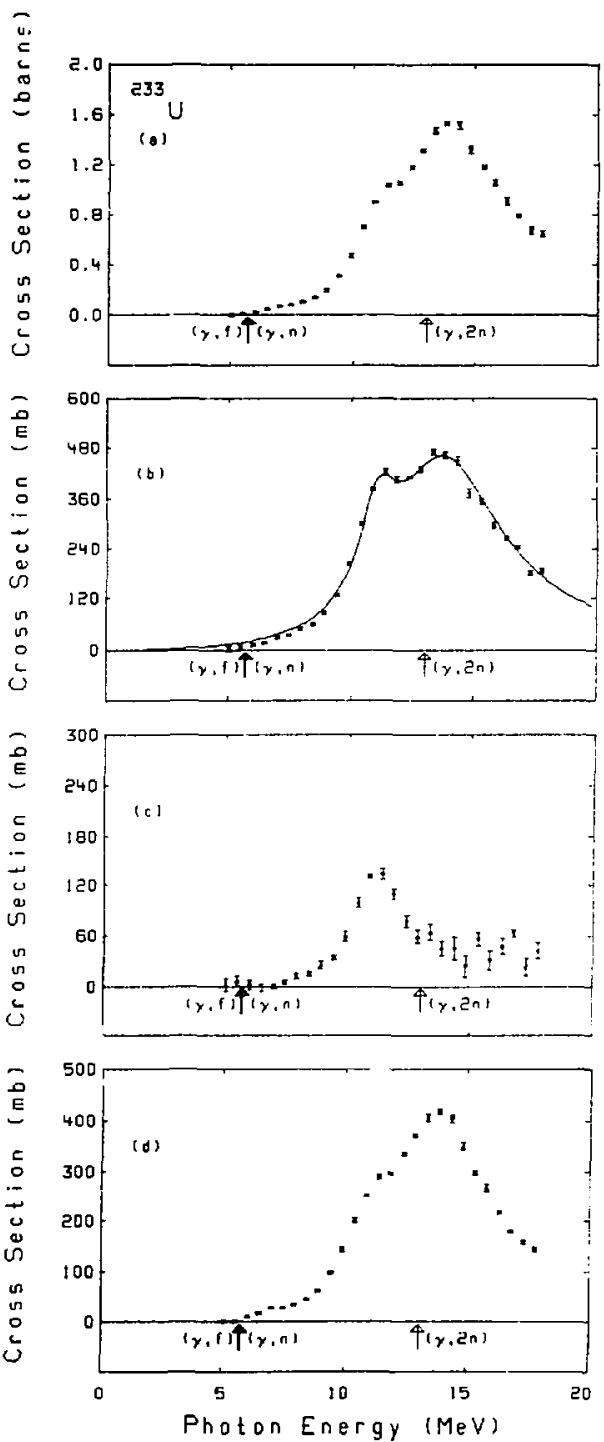

Fig. 166A 


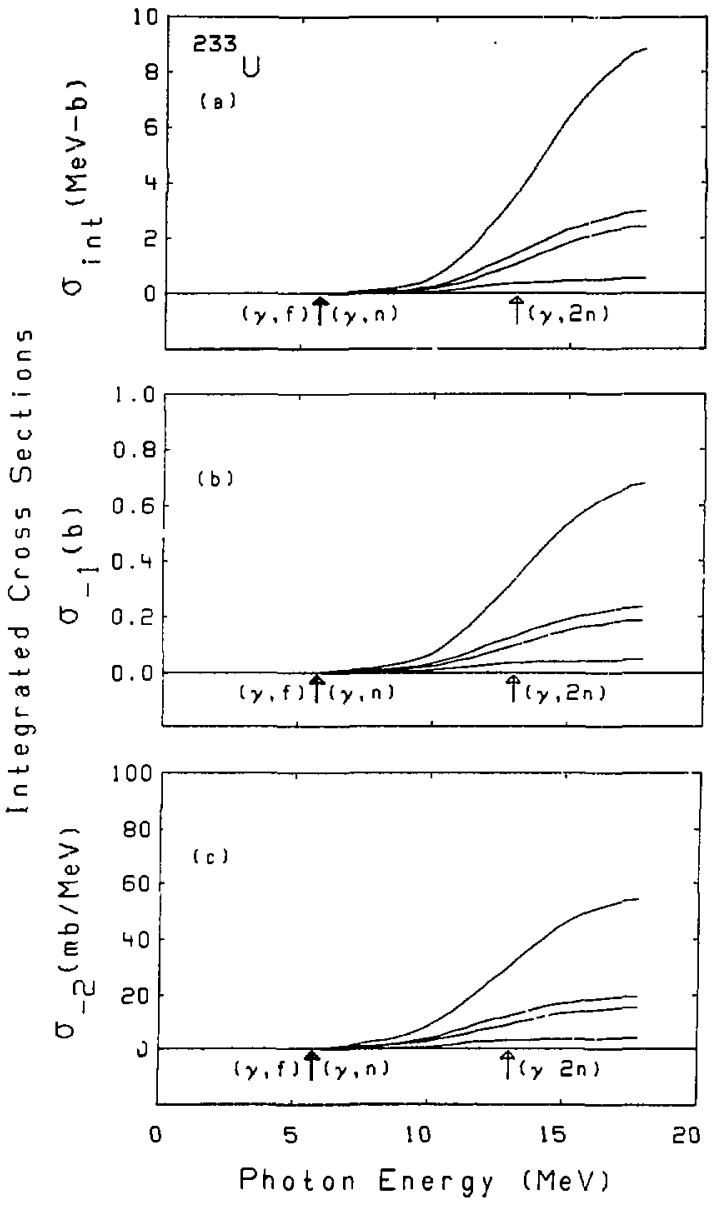

Fig. 166B 

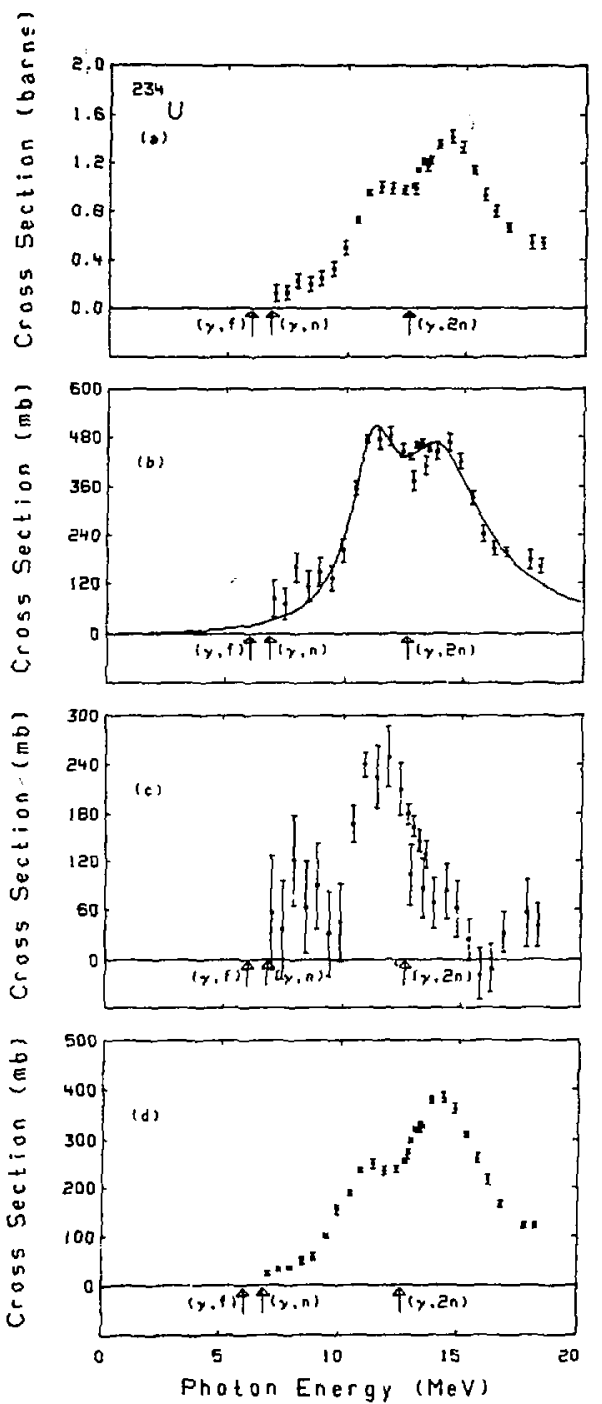

Fig. $167 \mathrm{~A}$ 


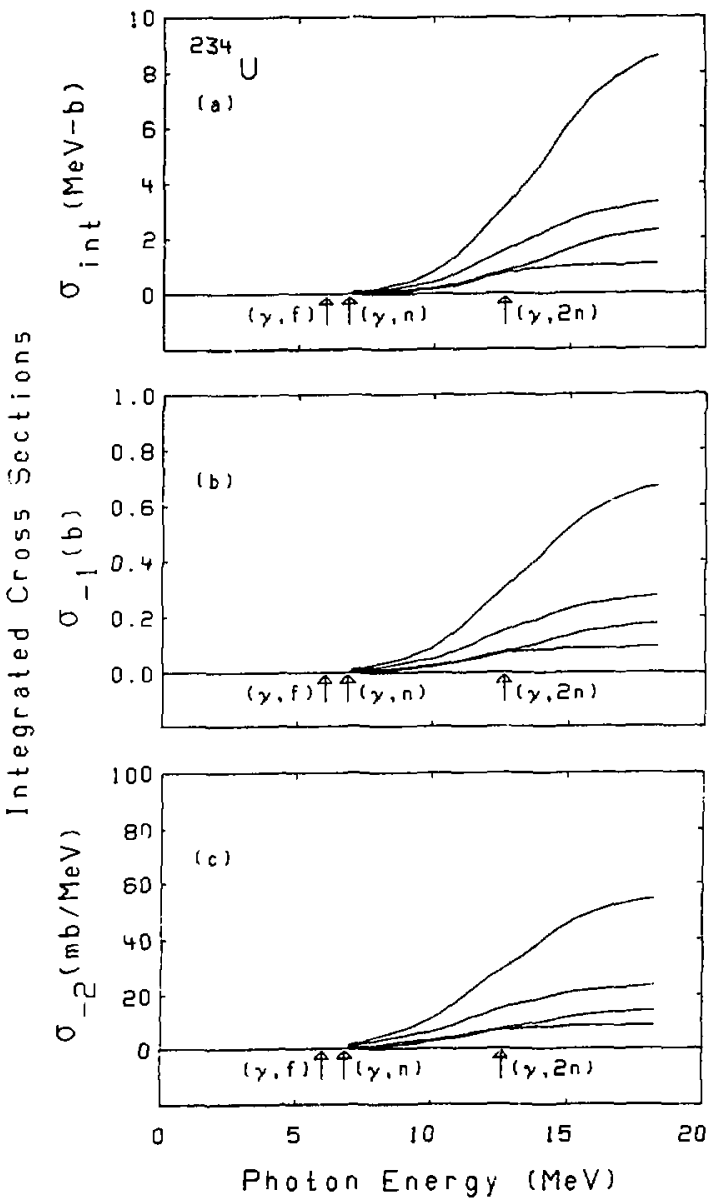

Fig. 1678 

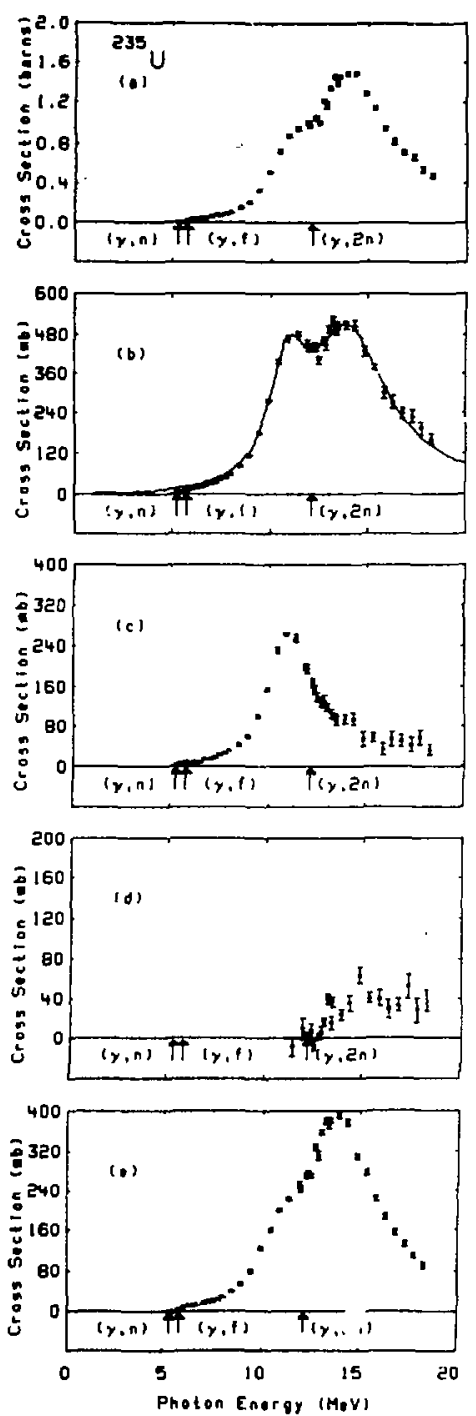

Fig. 168A 

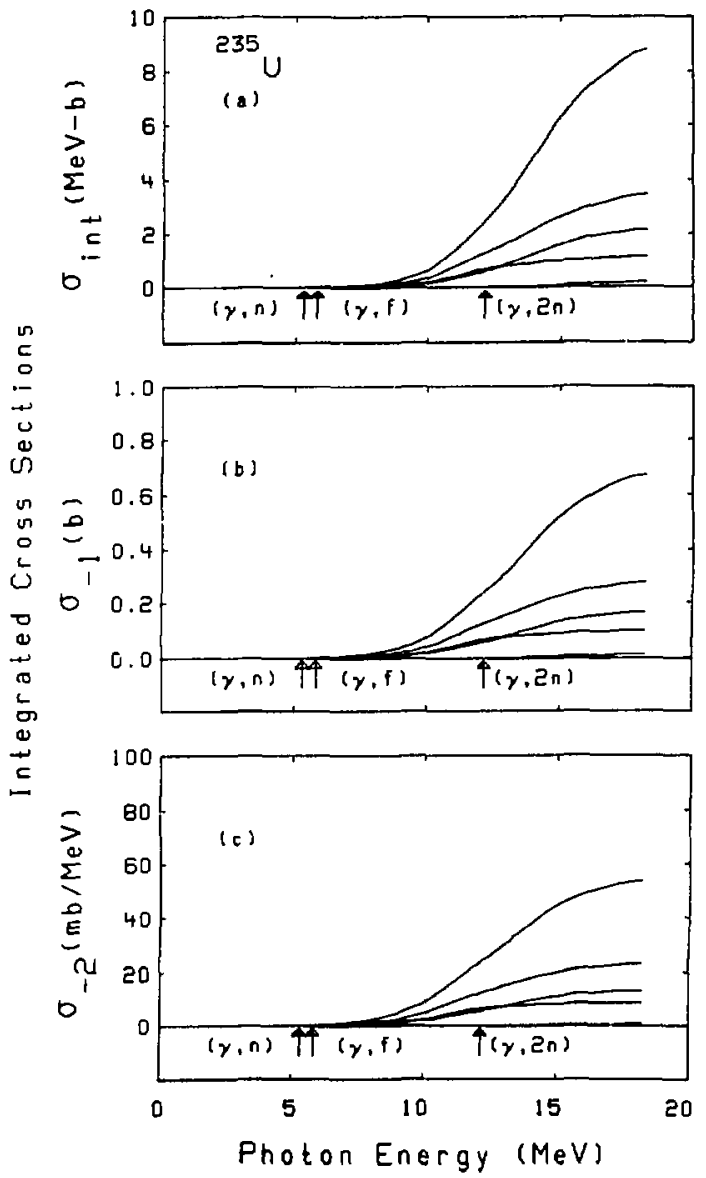

Fig. 168B 

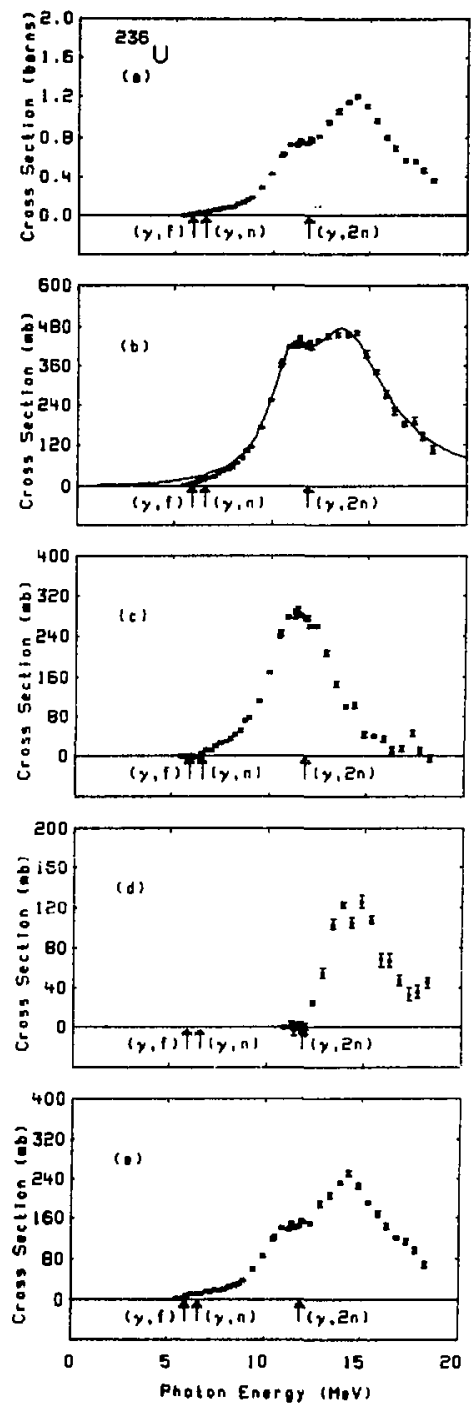

Fig. $169 \mathrm{~A}$ 


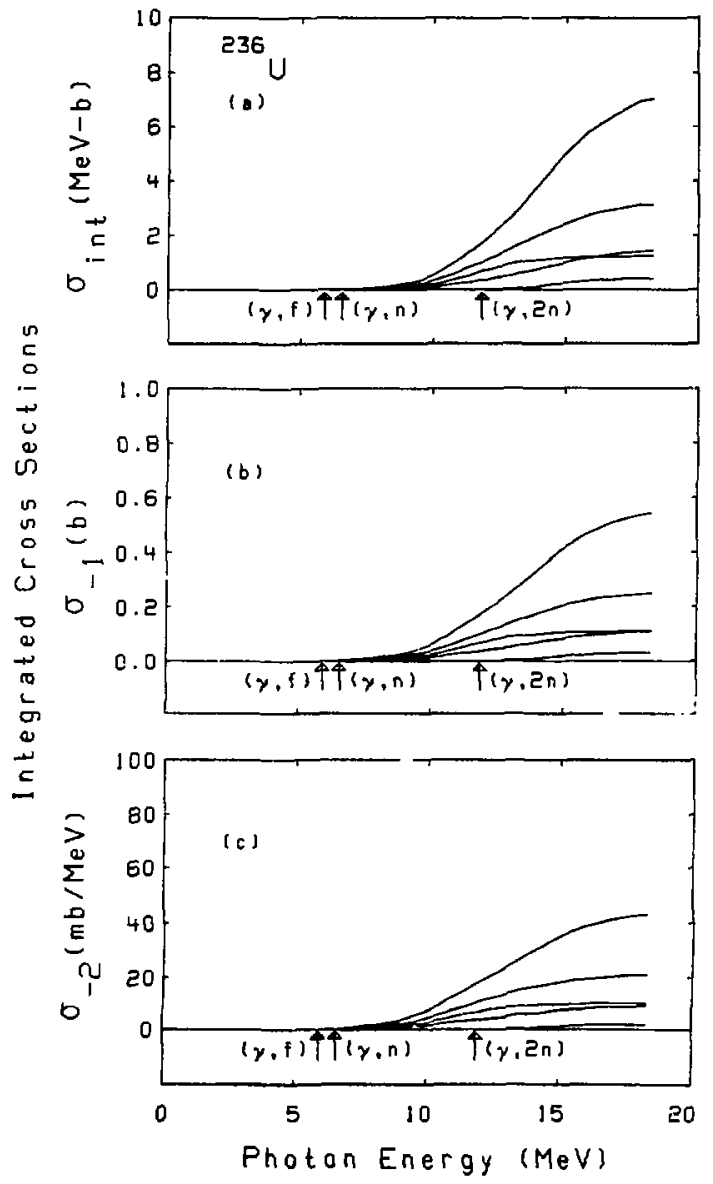

Fig. $169 B$ 


$\frac{M}{m} \frac{M}{m}$




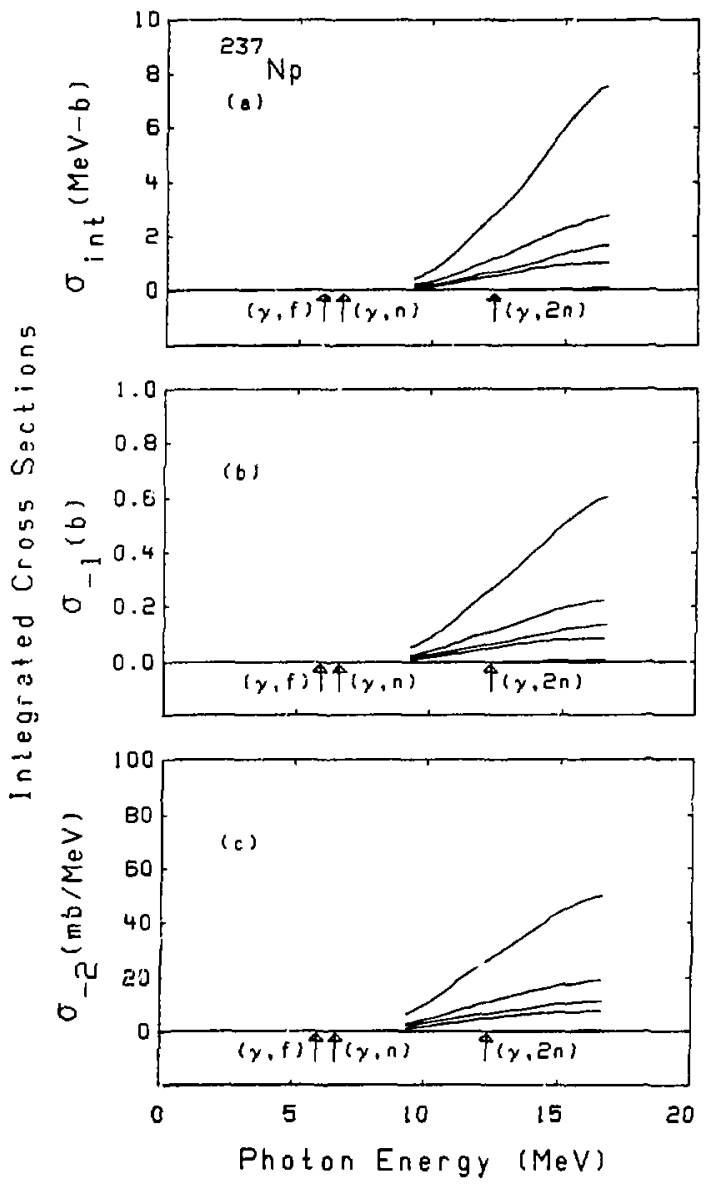

Fig. $170 B$ 

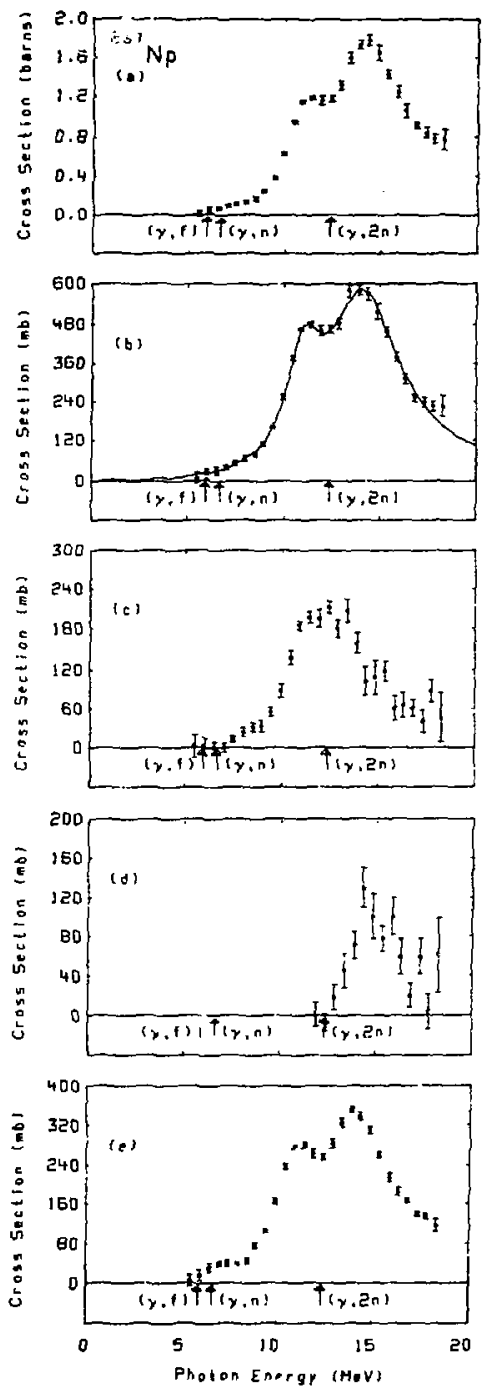

Fig. $171 \mathrm{~A}$ 


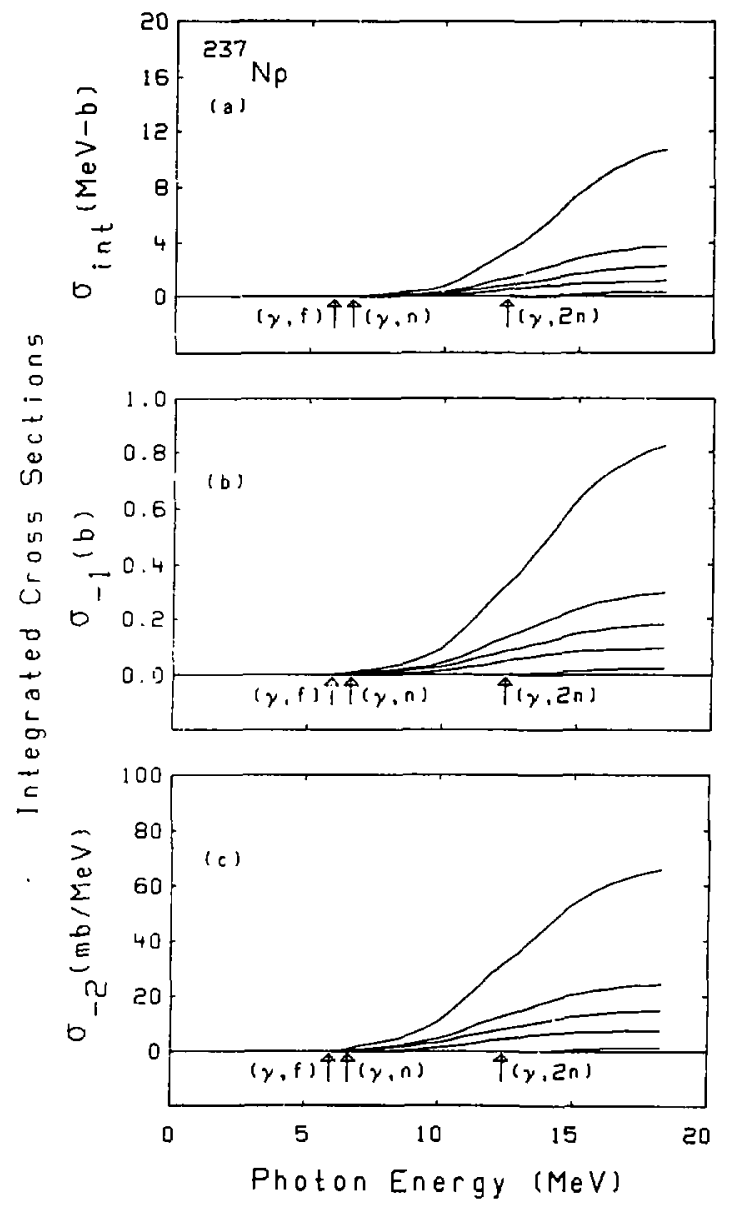

Fig. 1718

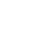
.
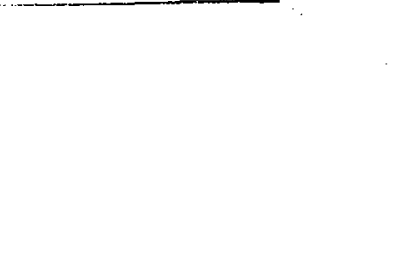

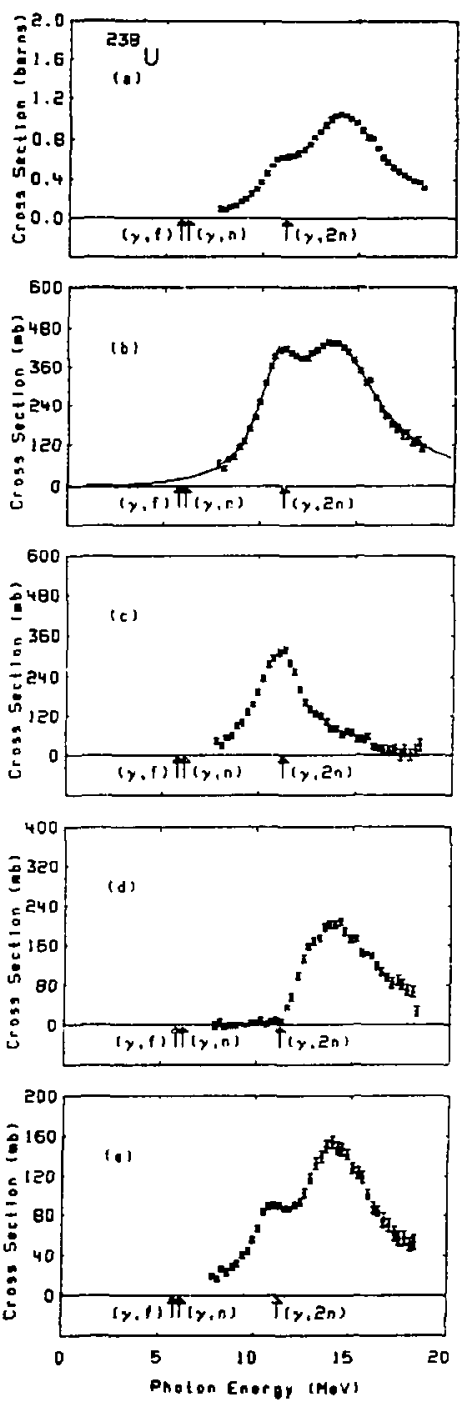

Fig. $172 A$ 


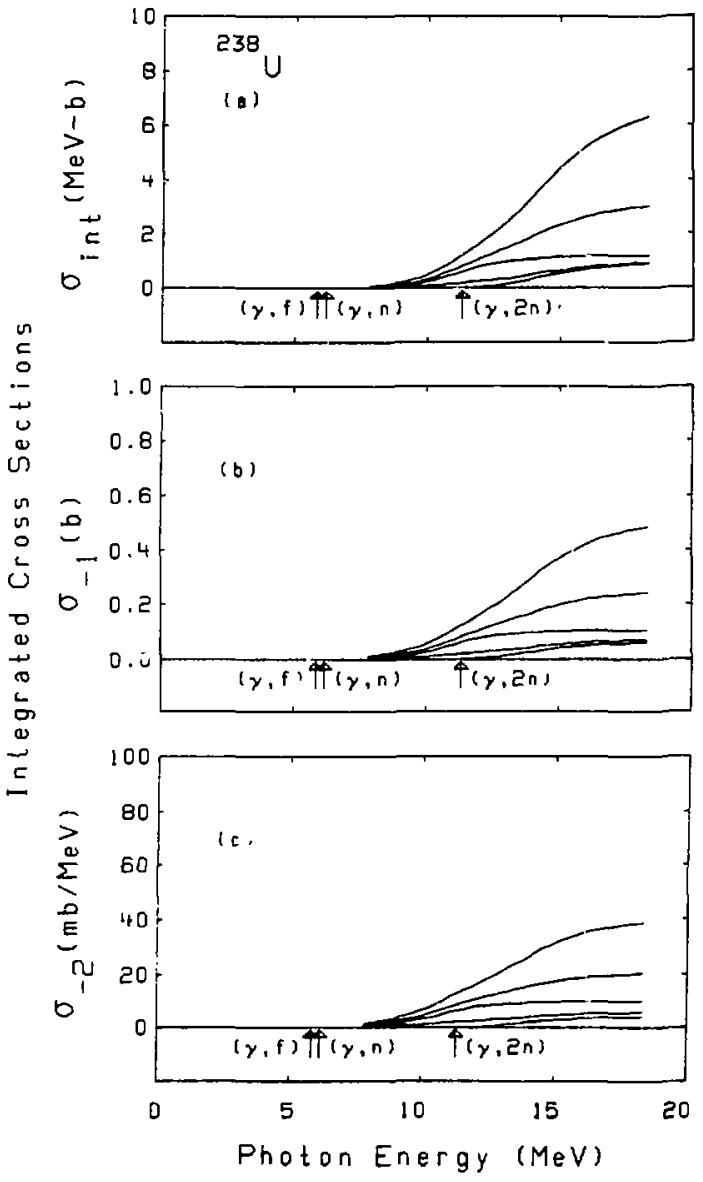

F1g. 172B 

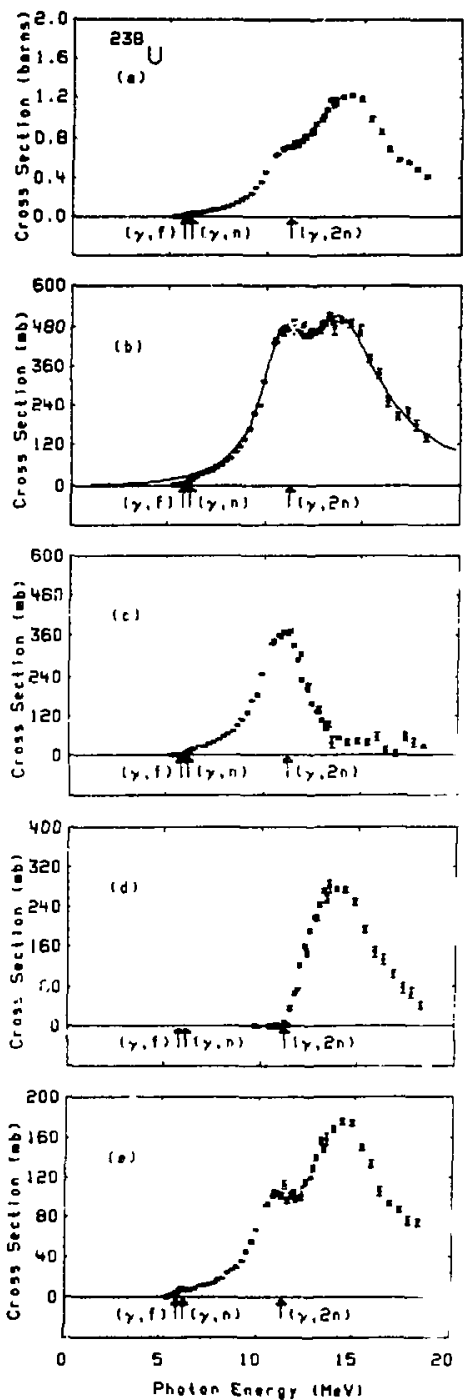

Fig. $173 \mathrm{~A}$ 

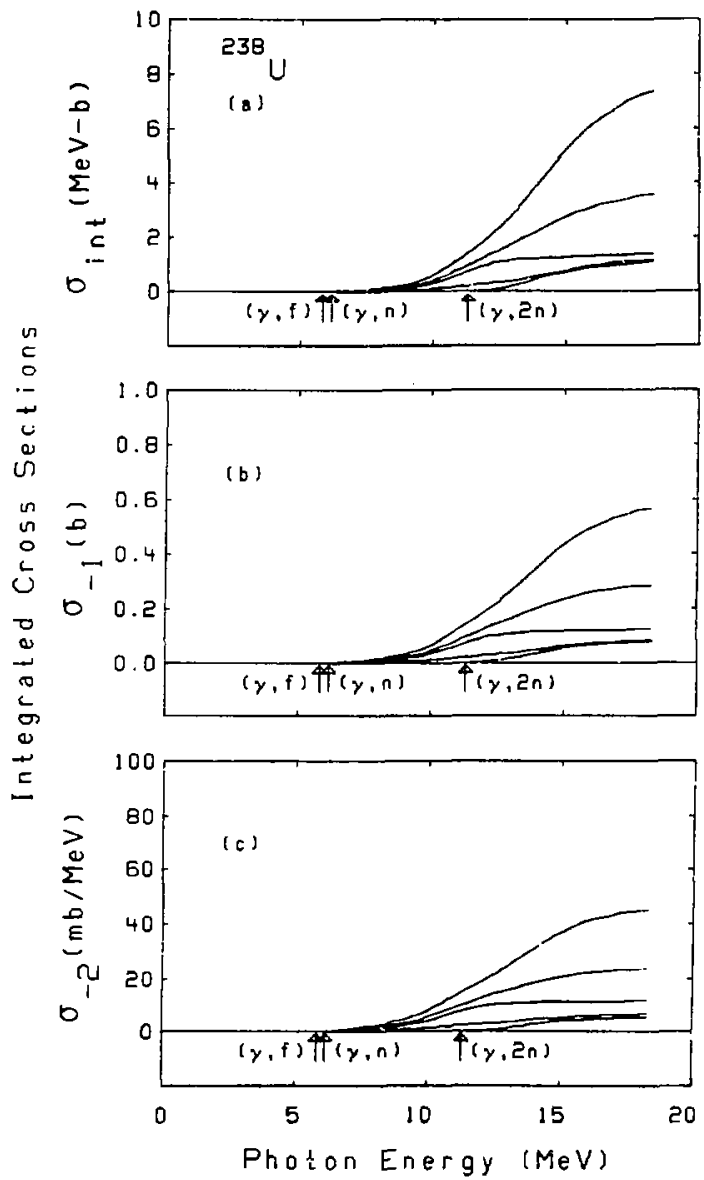

Fig. $273 B$ 


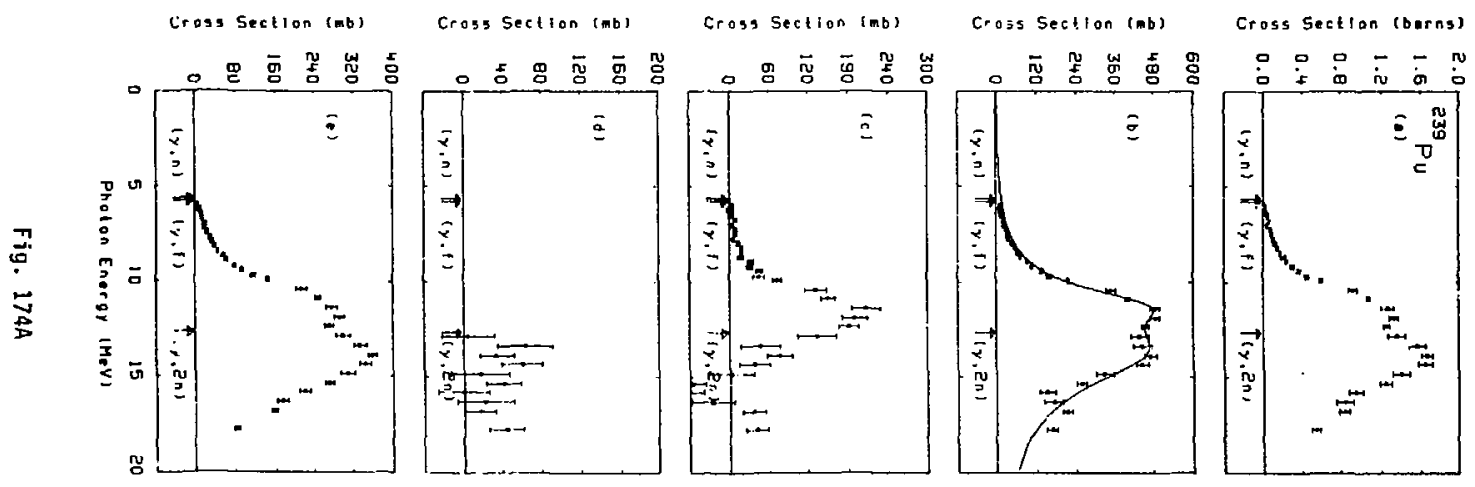




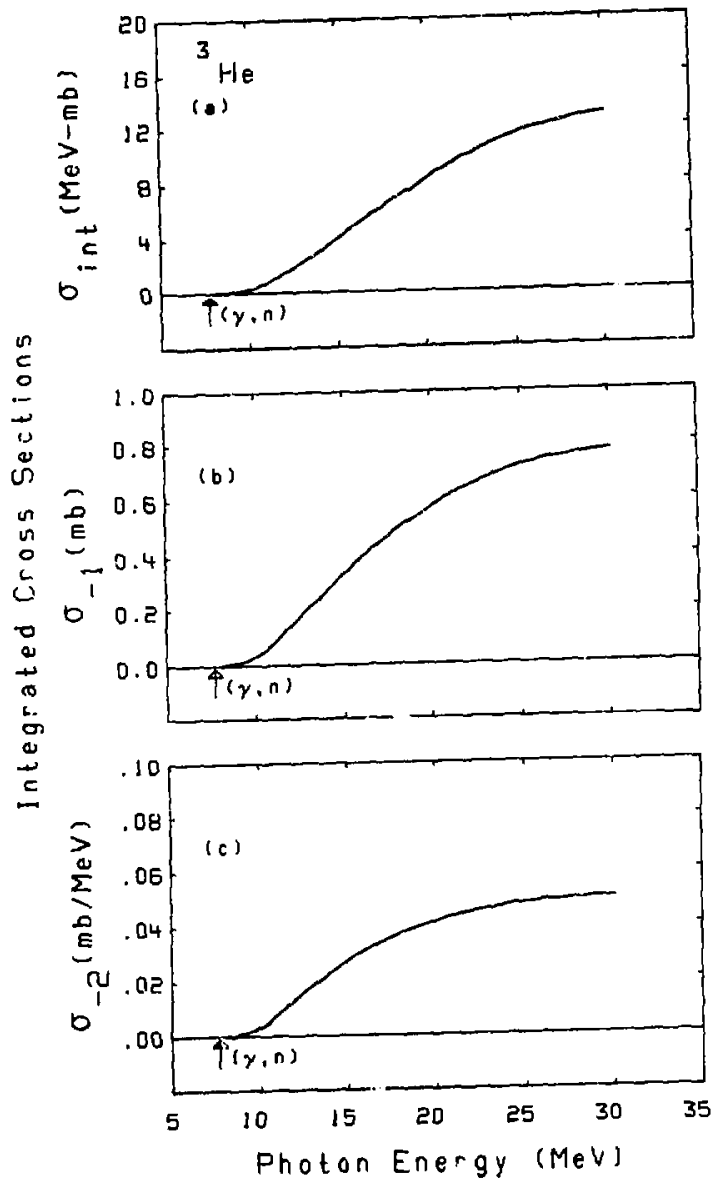

Fig. $2 B$ 


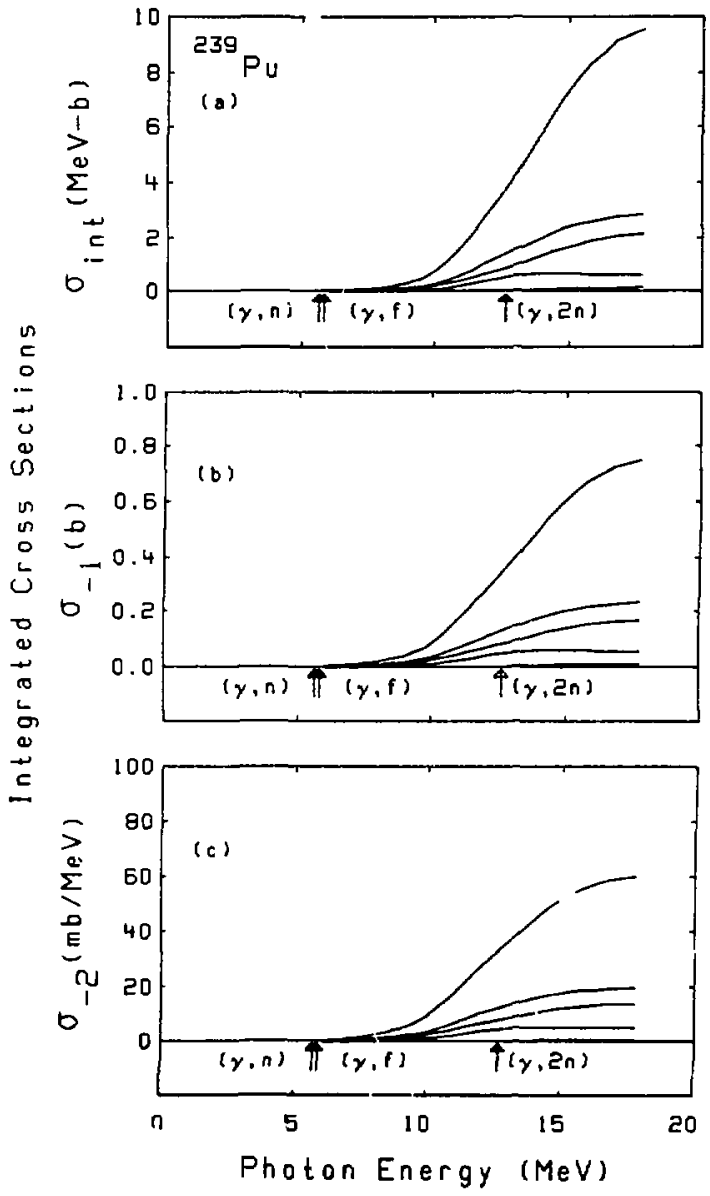

Fig. $274 B$ 